\title{
An Analytical Electron Microscope Study of the Omega Phase Transformation in a Zirconium-Niobium Alloy
}

Nestor John Zaluzec

\section{OAK RIDGE NATIONAL LABORATORY} OPERATED BY UNION CARBIDE CORPORATION · FOR THE DEPARTMENT OF ENERGY 


\section{DISCLAIMER}

This report was prepared as an account of work sponsored by an agency of the United States Government. Neither the United States Government nor any agency Thereof, nor any of their employees, makes any warranty, express or implied, or assumes any legal liability or responsibility for the accuracy, completeness, or usefulness of any information, apparatus, product, or process disclosed, or represents that its use would not infringe privately owned rights. Reference herein to any specific commercial product, process, or service by trade name, trademark, manufacturer, or otherwise does not necessarily constitute or imply its endorsement, recommendation, or favoring by the United States Government or any agency thereof. The views and opinions of authors expressed herein do not necessarily state or reflect those of the United States Government or any agency thereof. 


\section{DISCLAIMER}

Portions of this document may be illegible in electronic image products. Images are produced from the best available original document. 
Printed in the United States of America. Available from National Technical Information Service

U.S. Department of Commerce 5285 Port Royal Road, Springfield, Virginia 22161

Price: Printed Copy $\$ 12.00$; Microfiche $\$ 3.00$

This report was prepared as an account of work sponsored by an agency of the United States Government. Neither the United States Government nor any agency thereof, nor any of their employees, contractors, subcontractors, or their employees, makes any warranty, express or implied, nor assumes any legal liability or responsibility for any third party's use or the results of such use of any information, apparatus, product or process disclosed in this report, nor represents that its use by such third party would not infringe privately owned rights. 


\section{ORNL/TM-6705}

Contract No. W-7405-eng-26

Metals and Ceramics Division

An Analytical Electron Microscope Study of the Omega Phase Transformation in a Zirconium-Niobium Alloy

Nestor John Zaluzec

Submitted in partial fulfillment of the requirements for the degree of Doctor of Philosophy in Metallurgical Engineering in the Graduate College of the University of Illinois at Urbana-Champaign, 1978

Date Published: June 1979

OAK RIDGE NATIONAL LABORATORY

Oak Ridge, Tennessee 37830 operated by UNION CARBIDE CORPORATION for the DEPARTMENT OF ENERGY

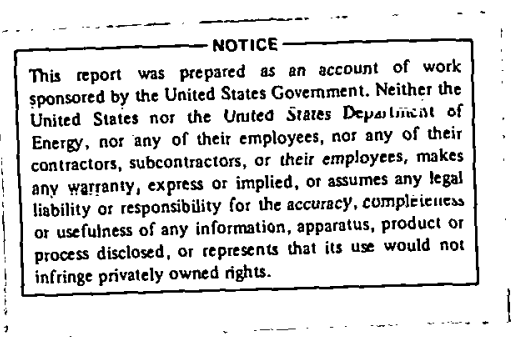

This report was prepared as an aceount of work

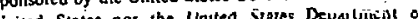
Energy nor any of their employees, nor any of their contractors, subcontractors, or their employees, makes liability of responsibility for the accuracy, compleitele ox usefulness of any information, apparatus, product or process disclosed, owned rights. 
THIS PAGE

\section{WAS INTENTIONALLY}

\section{LEFT BLANK}


AN ANALYTICAL ELECTRON MICROSCOPE STUDY OF THE OMEGA PHASE TRANSFORMATION IN A ZIRCONIUM-NIOBIUM ALLOY*

Nestor John Zaluzec

\begin{abstract}
An in-situ study of the as-quenched omega phase transformation in $2 \mathrm{r}-15 \% \mathrm{Nb}$ was conducted between the temperatures of 77 and $300^{\circ} \mathrm{K}$ using analytical electron microscopy. The domain size of the omega regions observed in this investigation was on the order of $30 \AA$, consistent with previous observations in this system. No aligment of omega domains along <222> directions of the bcc lattice was observed and furthermore insitu thermal cycling experiments failed to produce a long period structure of alternating $\beta$ and $\omega$ phase regions as predicted by one of the most recent theories of this transformation.

During this investigation, several techniques of microstructural analysis were developed, refined, and standardized. Grouped under the general classification of Analytical Electron Microscopy (AEM) they provide the experimentalist with a unique tool for the microcharacterization of solids, allowing semiquantitative to quantitative analysis of the morphology, crystallography, elemental composition, and electronic structure of regions as small as $20 \AA$ in diameter. However, these techniques are not by any means without complications, and it was necessary to study the AEM system used in this work so that instrumental artifacts which invalidate the information produced in the microscope environment might be eliminated. Once these factors had been corrected, it was possible to obtain a wealth of information about the microvolume of material under investigation.

The microanalytical techniques employed during this research include: energy dispersive $x$-ray spectroscopy (EDS) using both conventional and scanning transmission electron microscopy (CTEM, STEM), transmission scanning electron diffraction (TSED), the stationary diffraction pattern technique, and electron energy loss spectroscopy (ELS) using a dedicated scanning transmission electron microscope (DSTEM).
\end{abstract}

${ }^{*}$ Research conducted in the Department of Metallurgy, University of Illinois, Urbana; and as a E. P. Wigner Fellow, Metals and Ceramics Division, Oak Ridge National Laboratory, operated by Union Carbide Corporation under contract W-7405-eng-26 with the U.S. Department of Energy. 


\section{ACKNOWLEDGMENTS}

The author wishes to express a sincere thanks to his advisor, Professor Hamish L. Fraser, for his enthusiasm, constant support, and guidance during this research, and even more so for the friendship which has grown between them. A special note of gratitude is extended to John B. Woodhouse and Ian D. Ward for their invaluable assistance in developing and modifying the instrumentation used during this work, to Professor Harry E. Cook whose lectures and many beneficial discussions on phase transformations influenced this study, and to Peggy Mochel for her help in assembly language prograrming. To his fellow graduate students, particularly Robert D. Trohsdrun Field, the author wishes to acknowledge many hours of valuable assistance and to Frances Scarboro and the Radiation Effects and Microstructural Analysis Group at the Oak Ridge National Laboratory for their contribution to the preparation of this report. Finally, a spccial thanks to the author's family and friends for their continued patience, understanding and help over the years, and to his wife, Karen, and. sons, Christiaan and Nicholaus, whose love makes it all worthwhile.

This research project was supported by the Department of Metallurgy and Mining Engineering and the Materials Kescarch Laboratory at the University of Illinois at Urbana-Champaign under Energy Research and Development contract ERDA(11-1)-1198 and at the Oak Ridge National Laboratory by the Division of Materials Science, U.S. Department of Energy, under contract W-7405-eng-26 with Union Carbide Corporation. 
TABLE OF CONTENTS

Page

INTRODUCTION . . . . . . . . . . . . . . . . 1

1. GENERAL PROPERTIES OF THE OMEGA PHASE IN ZIRCONIUM-NIOBIUM . . 3

1.1 Background ................. . . 3

1.2 Models of the Omega Transformation ........ 10

2. ANALYTICAL ELECTRON MICROSCOPY . . . . . . . . . . . . 31

2.1 Introduction . . . . . . . . . . . . . 31

2.2 X-Ray Microchemical Analysis ........... 32

2.2.1 Theory of Quantitative X-Ray Microanalysis .. . . . 33

2.2.2 The Atomic Number Correction ........... . 35

2.2.3 The Absorption Correction ............. 53

2.2.4 The X-Ray Fluorescence Correction . . . . . . . . 65

2.2.5 Detector Efficiency . . . . . . . . . . . . 70

2.2.6 Optimum Experimental Conditions for X-Ray

2.2.7 Instrumenta1 Factors Influencing X-Ray Analysis . 86

System Peaks . . . . . . . . . . . . . 89

Fluorescence by Uncollimated Radiation . . . . . 99 96

Contamination Effects . . . . . . . . . 118

2.2.8 Data Reduction for Quantitative Analysis . . . . 132

Standardless Analysis . . . . . . . . . 135

Absorption Correction for Standardless Analysis . 140

Quantitative Analysis Using Thin Standards . . . . 141

2.3 Electron Diffraction Techniques ......... 143

2.3.1 Stationary Diffraction Pattern Technique . . . . . 143

2.3.2 Transmission Scanning Electron Diffraction (TSED). $\quad 150$

2.4 Electron Energy Loss Spectroscopy (ELS) . . . . . . . 153

3. EXPERIMENTAL OBSERVATIONS AND RESULTS . . . . . . . . 157

3.1 Instrunentation . . . . . . . . . . . . . . 157

3.2 Sample Preparation . . . . . . . . . . . . . . . . . . . 159

3.3 Microstructural Observations . . . . . . . . . . . 164

3.3:1 As-Quenched Morphology of the Omega Phase . . . 164

3.3.2 Spontaneous Relaxation Effects ........ 167

3.3.3 Phases Resulting from Compositional . . . . . . 173

3.3.4 Fhases Resulting from Incomplete Quenching . . . 176 
Page

3.3.5 Intermediate Structures . . . . . . . . . 176

3.3.6 Precipitation of Coherent Isothermal Omega . . . 189

3.4 In-Situ Thermal Cycling Experiments . . . . . . . 194

4. CONCLUSIONS . . . . . . . . . . . . . 207

LIST OF REFERENCES . . . . . . . . . . . . . 211

APPENDIX A, Computer Program NEDS . . . . . . . . . . 217

APPENDIX B, Subroutine Library NXRYL . . . . . . . . 241

APPENDIX C, Subroutine Library NGRAPH . . . . . . . . 265

APPENDIX D, Computer Program NXRYRT . . . . . . . 285

APPENDIX E, Computer Program NMCS . . . . . . . 297

APPENDIX F, Computer Program MCA . . . . . . . . . 311

APPENDIX G, Circuit Diagram for TSED/TSEM Measurements . . 317

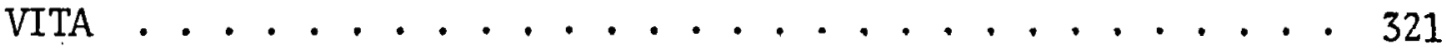




\section{INTRODUCTION}

When alloys of the group IV-B elements $\mathrm{Ti}, \mathrm{Zr}$, and Hf and other transition metals, most notably $\mathrm{V}, \mathrm{Nb}, \mathrm{Cr}$, Mo, and $\mathrm{Fe}$, are quenched from the high-temperature bcc solid solution, the formation of the equilibrium hcp alpha $(\alpha)$ phase is often partially or completely suppressed. Instead, a metastabIe phase, termed omega $(\omega)$ is formed. Two different types of $\omega$ structures have been identified: first, there is reversible athermal transformation which is thought to be a diffusionless displacement controlled reaction; and second, that which develops only upon isothermal aging. Initially, interest in this transformation was generated from the observed embrittlement effects and enhancement of superconducting properties accompanying the precipitation of $\omega$. However, the more recent interest in the athermal transformation stems from the inherent mechanical instabilities of the bcc lattice and its relationship to the bcc-hcp transition. Furthermore, it has been suggested that the $\omega$-like fluctuations serve as precursors to some bcc martensitic transformations. Over the last twenty years several experimental and theoretical studies have been undertaken in an attempt to determine the precise nature of the athermal transformation. The research presented here was initiated in order to test one of those theories.

During the course of this study, several techniques of microstructural analysis were developed, refined, and standardized. Grouped under the general classification of Analytical Electron Microscopy they provide the experimentalist with a unique tool for the microcharacterization of solids, allowing semiquantitative to quantitative analyses of 
the morphology, crystallography, èlemental composition, and electronic structure of regions as small as $20 \AA$ in diameter. As these tools were still in their infancy when this work was initiated, it was necessary to spend considerable time and effort in developing these methods into state-of-the-art analytical techniques, and this work comprises a major portion of the research described herein. The microanalytical techniques employed during this study include: energy dispersive $x$-ray spectroscopy (EDS) using both conventional and scanning transmission electron micrsocopy (CTEM, STEM), transmission scanning electron diffraction (TSED), the stationary diffraction pattern technique, and electron energy loss spectroscopy (ELS) using a dedicated scanning transmission electron microscope (DSTEM). 
CHAPTER 1

1. GENERAL PROPERTIES OF THE OMEGA PHASE IN ZIRCONIUM-NIOBIUM

\subsection{Background}

A partial phase diagram for the $2 \mathrm{r}-\mathrm{Nb}$ system is shown in Fig: 1. At high temperatures there is a continuous series of bcc solid solutions (B) from pure zirconium to pure niobium. On the niobium-rich side of the diagram above the eutectoid temperature there is a miscibility gap extending from 17.5 to approximately $85 \% \mathrm{Nb}$. The terminal zirconium-rich solid solution $(\alpha)$ phase is hexagonal, but for alloys of less than $7 \% \mathrm{Nb}$ the hcp phase is replaced by a martensitic form $\left(\alpha^{\prime}\right)$ of $\alpha$. On quenching the higher niobium concentration alloys from the bcc solid solution, the $\alpha$ and a transformations can be partially or completely suppressed and a bcc phase can be retained. This solid solution then decomposes into a metastable structure called the omega $(\omega)$ phase. The transition temperature $\left(T_{\omega}\right)$ for this transformation ${ }^{1}$ is also shown in Fig. 2.

Early studies using $x$-ray diffraction techniques have shown that $\omega$ is an hexagonal phase with the following orientation relationships $^{2-4}$ to the parent bcc matrix:

$$
[0001]_{\omega} \|[111]_{\beta} \quad \text { and } \quad[11 \overline{2} 0]_{\omega} \|[110]_{B}
$$

The unit cell can be indexed hexagonally with three atoms at the positions $(000)$ and $\pm\left(\frac{1}{3}, \frac{2}{3}, \frac{1}{3}+u\right)$. Here $u$ is the displacement necessary 


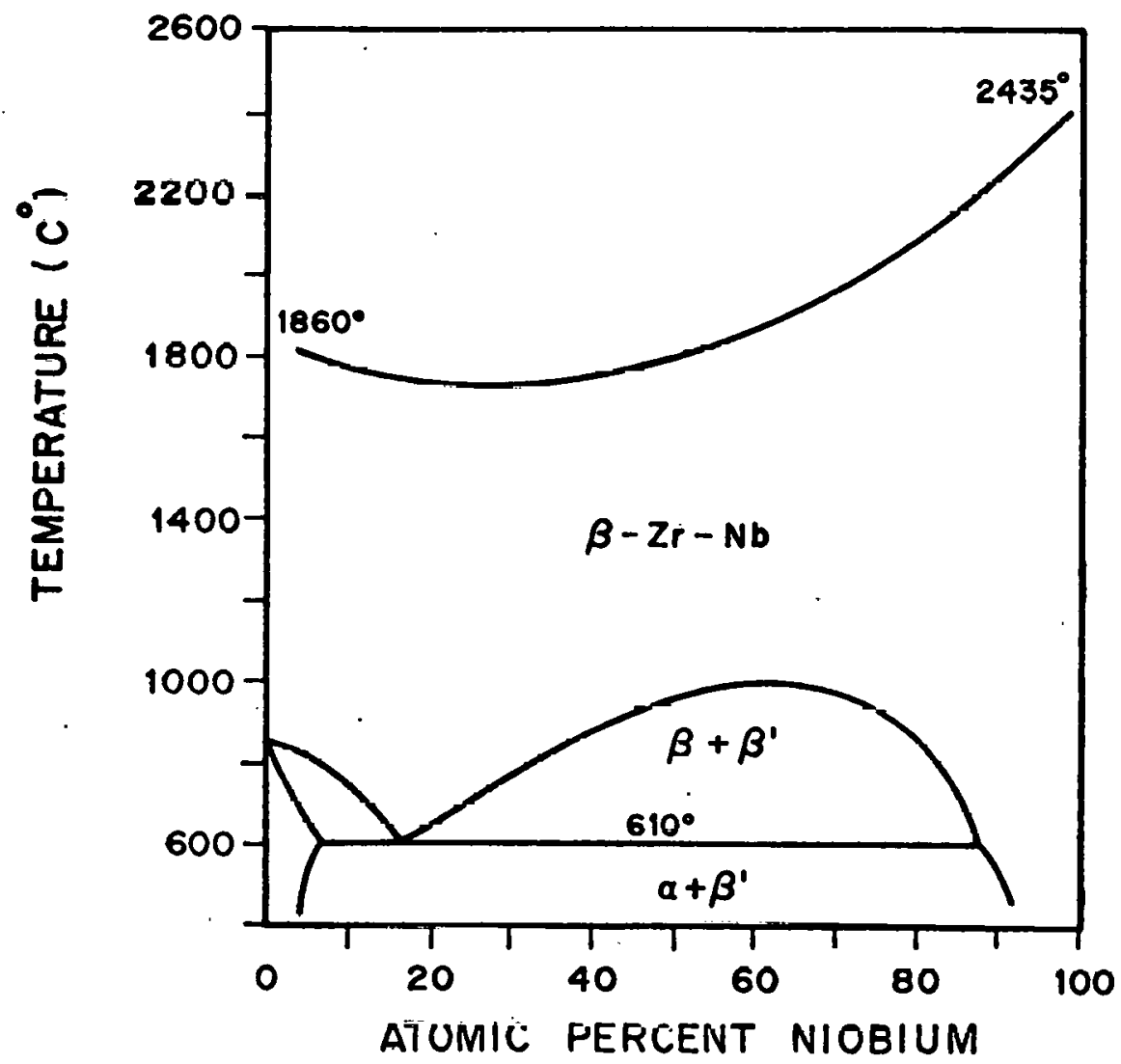

Fig. 1. Phase Diagram for the Zirconium-Niobium System. 


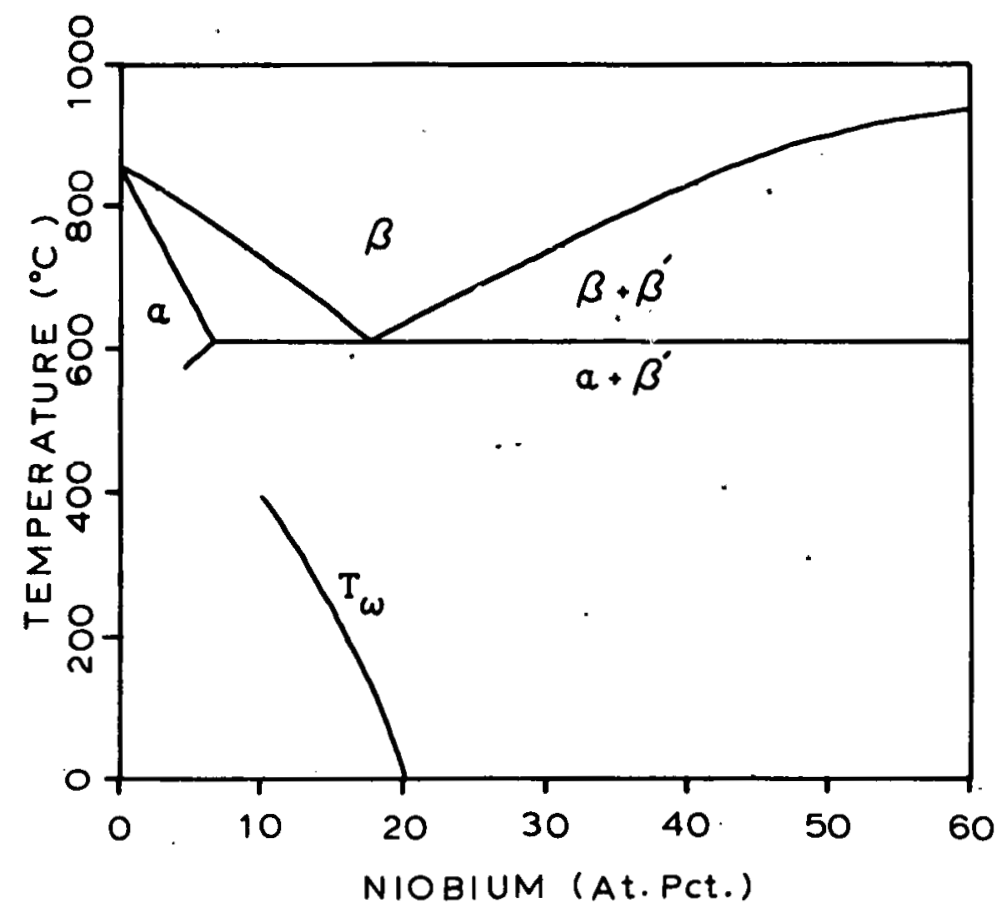

Fig. 2. Partial Phase Diagram for the $\mathrm{Zr}-\mathrm{Nb}$ System Showing the Umega Phase I'ransformation Temperature $\left(\mathrm{T}_{\omega}\right)$. 
along a $<111>$ direction to convert the bcc $(u=0)$ to the hexagonal lattice $\left(u=\frac{1}{6}\right)$. Figure 3 is a sketch of a $\{110\}$ section of a bcc lattice illustrating the relationship between $\omega$ and $\beta$. From this figure, one can see that the rearrangement necessary to convert $\beta$ to $\omega$ involves only the collapse of two adjacent \{111\} planes while the third plane remains fixed. Since there are four equivalent $<111>$ directions in bcc, four different variants of $\omega$ can develop. ${ }^{4}$ Two forms of $\omega$ have been reported: (1) the athermal transformation which occurs by a diffusionless strucEural change of the bcc lattice, and (2) the aged formed by annealing specimens containing either the athermal or as-quenched bec phases at temperatures between $200-400^{\circ} \mathrm{C}$.

Using transmission electron microscopy, studies of the fully aged omega phase in $\mathrm{Zr}-\mathrm{Nb}^{5}$ and $\mathrm{Ti}-\mathrm{Nb}^{6}$ systems have shown that this phase consists of incoherent precipitates lying on $\{111\}$ planes of the retained B phase. A11 four variants were seen to develop equally in both systems; however, the morphology of the precipitate shape varied with alloy system. In $2 \mathrm{r}-\mathrm{Nb}$, the precipitates were in the form of platelets $\sim 1000 \AA$ in diameter and $200 \AA$ thick, while in Ti-Nb ellipsoidal particles ( $\sim 1300 \AA$ long and $500 \AA$ in diameter), with the major axis parallel to $[0001]_{\omega}=[111]_{\beta}$, were observed.

The athemnal transformation in alloys of $\mathrm{Zr}-\mathrm{Nb}, \mathrm{Ti}-\mathrm{Nb}$, and $\mathrm{Ti}-\mathrm{V}$ has been under investigation for a number of years by Sass and his coworkers. ${ }^{7-13}$ From their dark-field. TEM observations they concluded that the morphology of the as-quenched athermal $\omega$ consists of 10-15 $\AA$ diameter 


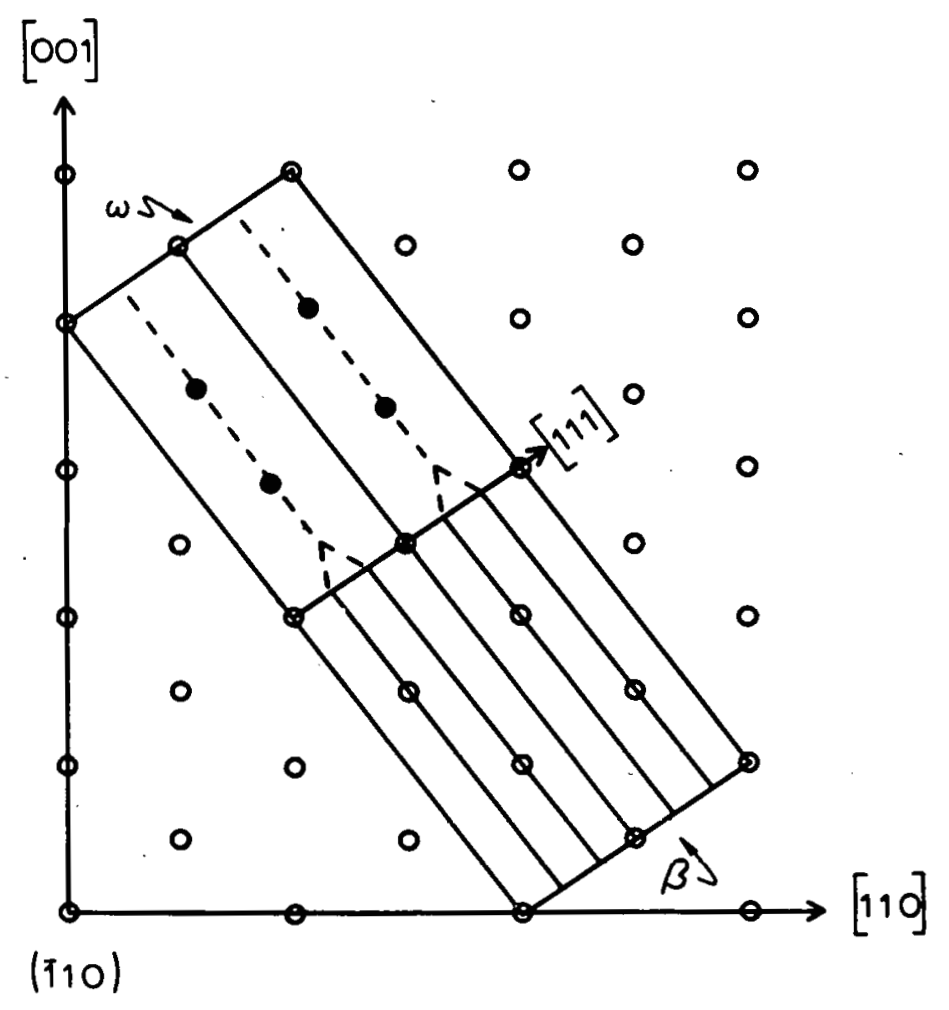

Fig. 3. Schematic Diagram Illustrating the Crystallographic Relationship between the $\beta$ and $\omega$ Phases in an (110) Plane. 
particles spaced 15-25 $\AA$ apart arranged in rows along the $\langle 111\rangle$ directions of the bcc lattice. Furthermore, these rows tended to cluster into different domains, the domain size and number of particles/row decreasing with increasing solute content.

The results of diffraction experiments are summarized in Fig. 4, where a sketch of a typical $\{110\}$ electron diffraction pattern (x-ray and neutron diffraction display similar characteristics) with reflections corresponding to a single [111] $\omega$ variant is shown. Two types of reflections are observed: (1) sharp diffraction spots which are cormon to both the bcc and w structures (solid circles in Fig. 4), and (2) reflections due only to the presence of the whase, hereafter referred to as the $w$ reflections: Above and inmediately below the transition temperature $\left(T_{\omega}\right)$ these $\omega$ reflections appear as diffuse peaks elongated perpendicular to the [111] direction. These peaks are also offset from the ideal hexagonal $\omega$ position $\left(k_{\omega}\right)$, the displacement $(\Delta k)$ being away from the bcc matrix reflections along the [111] direction. Upon cooling the sample below the transformation temperature, these peaks shift toward their ideal positions and corresponding increases in intensity and sharpness have been observed. ${ }^{14}$

Using ncutron diffraction, Moss et a1. ${ }^{15}$ have observed diffuse peaks at the $\omega$ positions at temperatures of $1000^{\circ} \mathrm{C}\left(\sim 975^{\circ}\right.$ above $\left.\mathrm{T}_{\omega}\right)$. Their measurements indicated the existence of a strong elastic component in the diffuse $w$ peaks; this result led them to conclude that a quasi-static w-like particle exists even in the bcc solid solution. They also pointed out that the displacements of the diffuse $\omega$ peaks 


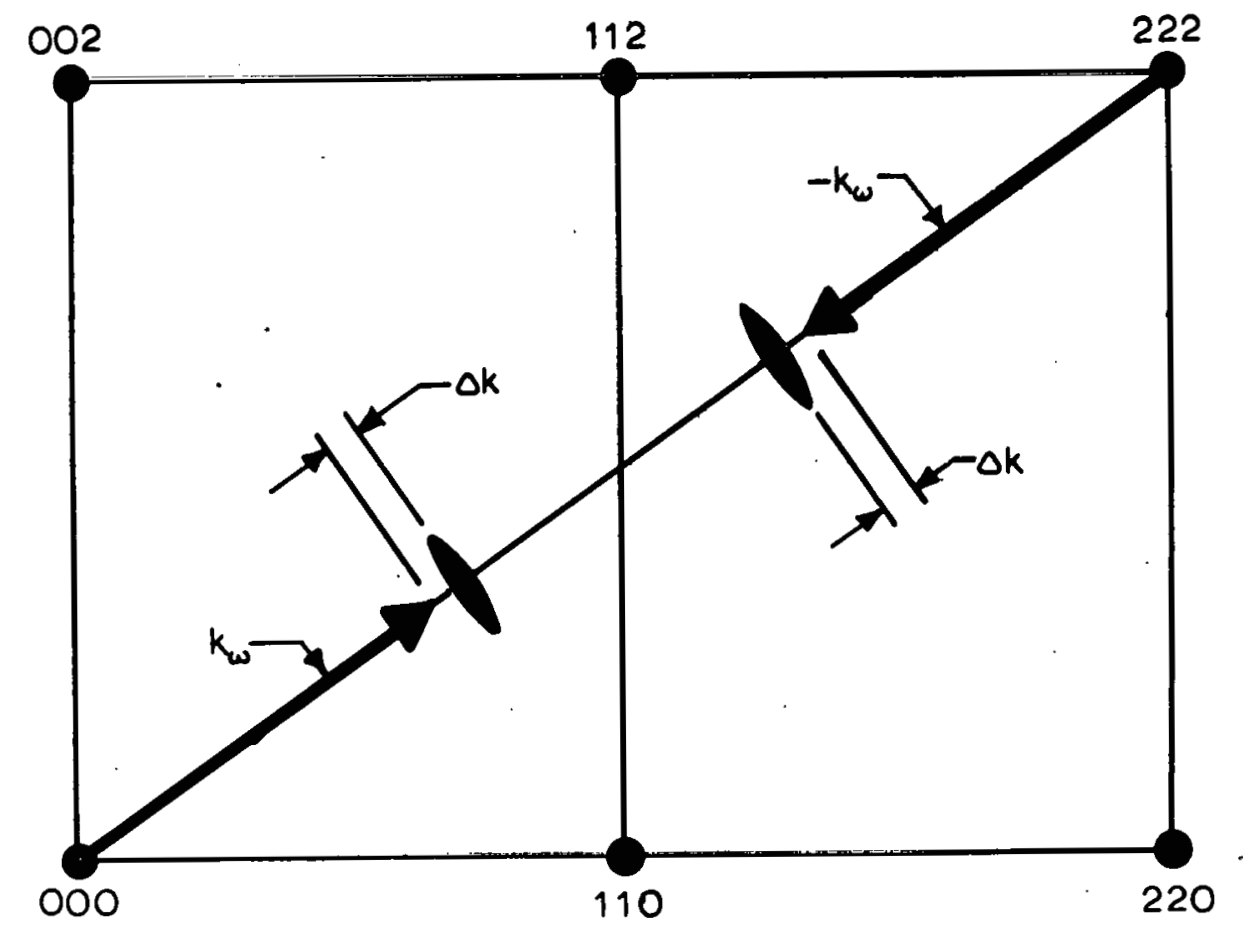

Fig. 4. Schematic Diagram of a $\{110\}$ Electron Diffraction Pattern of a Sample Containing Both the $\beta$. and $\omega$ Phases... Only one $<111\rangle \omega$ variant. is shown for clarity. 
observed are near a point in $k$-space $\left(k_{m}\right)$ which is a minimum in the phonon dispersion curves of the pure bcc transition metals, such as niobium, the suggestion being that lattice vibrations play an important role in determining the nature of the transformation.

Batterman et al. ${ }^{16}$ confirmed the existence of elastic scattering at the $w$ positions using a Mossbauer scattering technique. In addition they also detected an inelastically scattered peak which was revealed by the higher (energy) resolution of this technique compared to neutron scattering $\left(10^{-8}\right.$ versus $\left.10^{-5} \mathrm{eV}\right)$. They were able to show that the inelastic scattering was not merely thermal diffuse scattering (TDS) but was inherently related to the w producing phenomenon. In an extension of this study Lin et al. ${ }^{17}$ determined that in alloys of $\mathrm{Zr}-\mathrm{Nb}$ the elastic scattering is peaked at the positions which corresponded to the offset $\omega$ reflections $\left(k_{m}\right)$ while the inelastic scattering was always centered at the exact hexagonal positions $\left(k_{\omega}\right)$. This implies that not only are quasi-static, particle-like distributions present but also that dynamical (time-dependent) fluctuations exist in the bcc lattice.

\subsection{Mode1s of the Omega Transformation}

Ihe lirst model of the $\omega$ transformation was proposed by Hatt and Roberts $^{4}$ in 1960. Based on their x-ray diffraction measurements, they suggested that the transformation was accomplished by gliding \{112\} planes along $\langle 111\rangle_{\beta}$ directions in a cooperative manner. Figure 5 is a sketch of the $(110)_{\beta}$ plane of the bcc structure showing the $\{112\}$ packing sequence of six planes (labeled $1,2,3,4,5,6,1,2,3, \cdots)$. The 


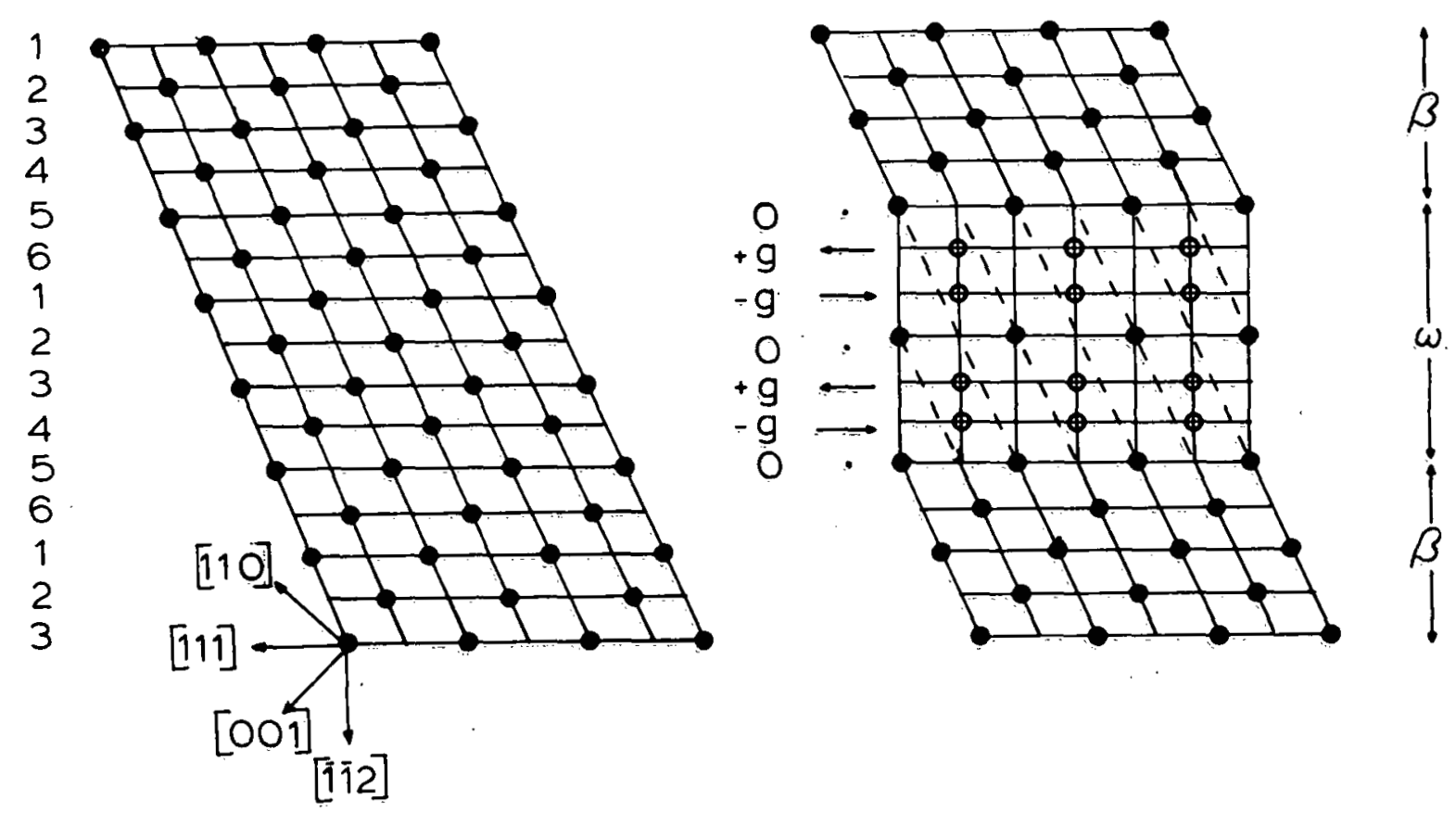

Fig. 5. Glide Sequence Proposed by Hatt and Roberts for the Formation of the $\omega$ Phase. 
w structure can be obtained by gliding specific pairs of $\{112\}$ planes in the [111] and [īīi] directions alternately as shown in Fig. 5, The glide component $g$ is the displacement necessary along $\langle 111\rangle$ to create the $\omega$ phase and for the ideal hexagonal structure is equal to $\frac{1}{12} d_{111}$. $=\frac{1}{6} \sqrt{\frac{3}{2}} a_{\beta}$. In the interpretation of their $x$-ray results they also showed that the w morphology was rod-like with the major dimension being parâllel to a $\langle 111\rangle$ direction.

In 1970 defontaine ${ }^{18}$ formulated a geometrical description of the $\omega$ phase transformation which serves as the basis for all the current theoretical treatments. In this description a $\frac{2}{3}<111>$ longitudinal displacement wave (or equivalently a $\frac{1}{3}<112>$ transverse wave) modulates the bcc lattice by causing a collapse of $\{111\}$ planes as illustrated in Fig. 6. The amplitude of the displacement wave necessary to produce the $\beta \rightarrow \omega$ transformation is a/6 ( $\omega 0.5 \AA$ ). This description has the advantage that it lends itself readily to a lattice dynamics interpretation of the phase transformation.

In the same paper deFontaine ${ }^{18}$ outlined a theory explaining why such an interplanar collapse model was feasible. Expressing the elastic free energy of the bcc solid solution in a harmonic form, he demonstrated that vibrational instabilities (i.e., atomic displacement waves whose amplitude increases with time) would develop for those waves with wave vectors in the vicinity of $k_{\omega}\left(\frac{2}{3}<111>\right)$.

In order to arrive at these results deFontaine had to assume specific sets of atomic force constants which were obtained by using the long wavelength relations of the microscopic theory of inelasticity, 19,20 

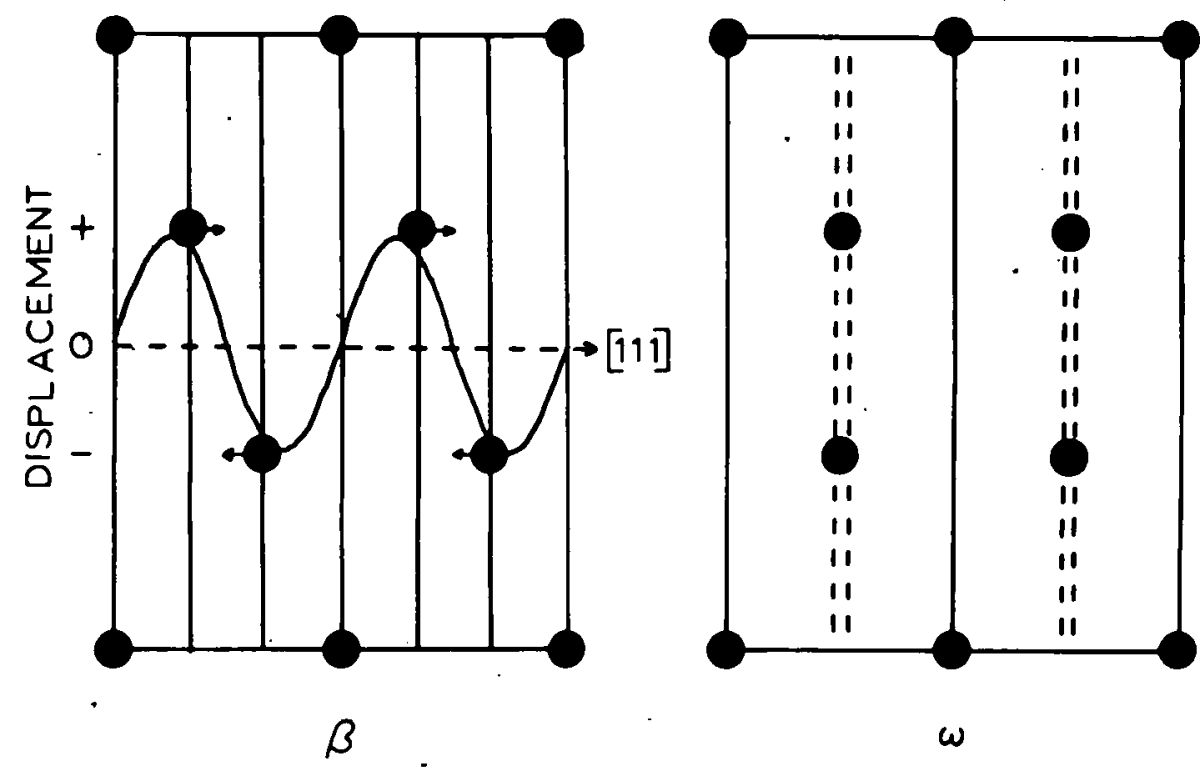

$\omega$

Fig. 6. DeFontaine's Displacement Wave Description for the Formation of the $\omega$ Phase. 
He then showed that such combinations corresponded to a softening or vanishing of the elastic moduli $\mathrm{C}_{44}$ and $\mathrm{C}^{-}\left(=\left[\mathrm{C}_{11}-\mathrm{C}_{12}\right] / 2\right)$. At virtually the same time Fisher and Dever ${ }^{21}$ presented evidence showing that the magnitude of the shear modulus $C^{-}$was closely related to the stability of the bcc transition metals, particularly with regard to the occurrence of martensitic and omega phase transformations. They pointed out (1) that the value of $\mathrm{C}^{-}$is related to the electron population of the d sheil of the transition metals and their alloys, and (2) that the $\beta+\alpha$ and $\beta+\omega$ phase traneformatione were clearly associated with a reduction or vanishing of $\mathrm{C}^{\prime}$. Further work by deFontaine et al.22 pointed out that the transformation is not in fact a lattice instability but rather a metastable state of the $B$ structure. If the bcc structure were truly unstable, which would correspond to a vanishing of the elastic constants, then the entire crystal would transform catastrophically to $w$ once the transition temperature is crossed. In fact, this is never observed experimentally; instead, only localized events are observed. Using the measurements of Fisher and Dever, ${ }^{21}$ deFontaine et a1. ${ }^{22}$ then obtained a consistent set of atomic force constants which yielded contours of low elastic energy in the $k$-space regions near $k_{\omega}$ without forcing the drastic softening of $\mathrm{C}^{\prime}$.

Although deFontaine's theory qualitatively explains the diffraction effects observed in $\omega$ forming alloys, three additional models have been proposed in an attempt to accurately describe the transformation mechanism. They are: (1) Vandermeulen and Deruyttere' $\mathrm{s}^{23}$ proposal where ideal w particles are formed having faulted structures at their 
ends, (2) Borie, Sass and Andreassen's ${ }^{24,25}$ model of ordered subvariants within a single variant, and (3) Cook's ${ }^{26}, 27$ anharmonic Landau model of first-order phase transformations. Each of these will be discussed in succession.

In a study of a Cu-16.5 at. $\%$ Sn alloy, Vandermeulen and Deruyttere ${ }^{23}$ noted that when the high-temperature bcc phase is quenched to room temperature an $\omega$-type transformation occurs, and electron diffraction patterns similar to those reported in titanium and zirconium alloys are observed. In order to explain the diffraction effects (i.e., shifts of the $\omega$ reflections away from $k_{\omega}$ ) they proposed a physical model of the $\omega$ phase which consists of a periodic distribution of ideal $\omega$-like particles separated by small faulted regions. This faulting of the $w$ structure is accomplished by disrupting the $\omega$-forming displacement wave periodically by a displacement function which is incommensurate with the $k_{\omega}$ vibrational wave. This is shown schematically in Fig. 7. The usual $\omega$ wave which modulates the (111) planes of the bcc lattice is shown in Figs. $7(a)$ and (b). If the displacement wave is interrupted by the insertion of a displacement function with wavelength $\frac{1}{3} k_{\omega}\left(\right.$ or $\left.\frac{2}{3} k_{\omega}\right)$, the structure of Fig. $7(c)$ and (d) $[7(e)$ and (f)] is obtained. These two incommensurate displacement functions are essentially faults in the $\omega$ structure and are designated as type $1\left(k-\frac{1}{3} k_{\omega}\right)$ and type $2\left(k=\frac{2}{3} k_{\omega}\right)$ faults, respectively. If one assumes that these faults occur in a periodic manner throughout the crystal, then it is possible to calculate the effects on an electron diffraction pattern. The results of such a 


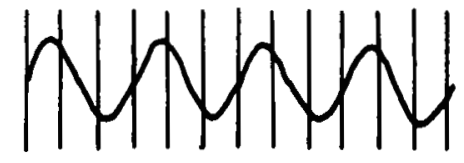

(a)

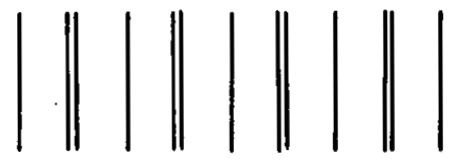

(b)

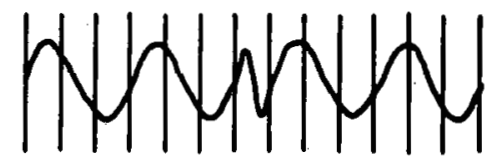

(c)

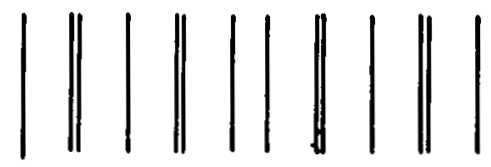

(d)

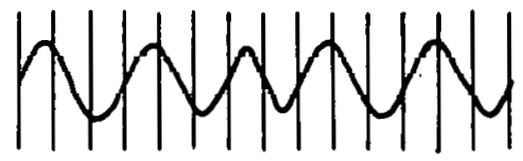

(e)

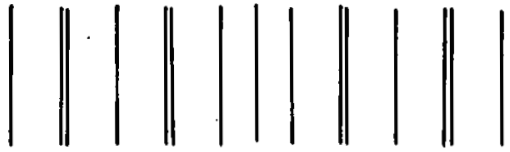

(f)

Fig. 7. Vandermeulen and Deruyttere' $s^{23}$ Model for the Formation of a Faulted Omega Structure. 
calculation of the diffracted intensity for a type 2 fault is illustrated in Fig. 8, where the diffracted intensity along a $\langle 111\rangle$ direction is plotted for regions about the $[0001]_{\omega},[0002]_{\omega}$, and $[0003]_{\omega} \equiv[222]_{\beta}$ reciprocal lattice points. Although the model does correctly predict a shift of the $[0001]_{\omega}$ and $[0002]_{\omega}$ reflections away from the ideal $k_{\omega}$ positions, it also unambiguously predicts the development of satellite peaks off these shifted reflections. In the electron diffraction patterns presented in their work no such satellite intensity is apparent nor to date have such observations been reported in the literature. In addition, Vanderneulen and Deruyttere fail to explain the mechanism of the formation of such faults and their required periodicity.

In a similar attempt to explain the diffraction patterns observed from the athermal $\omega$, Borie et al.24,25 have proposed another purely geometrically based model of $\omega$. Their analysis was based on two assumptions: (1) the entire sample transforms into the $w$ phase (which as pointed out previously has never been achieved experimentally), and (2) within a single $<111>$ variant of $\omega$ a preferential ordering of subvariants occurs. The possibility of the existence of subvariants can be easily demonstrated by the drawing in Fig. 9. Shown here is the $A B C$ packing sequence of $\{111\}$ planes in bcc materials. Clearly there are three equivalent ways in which a given $<111>$ variant of $\omega$ can form - that is, the unshifted bcc plane can be either A, B, or.C and the feasibility of subvariants is apparent. Calculations of diffuse intensity by Borie et al. showed that if a preferred order of subvariants occurs along the [0001] ${ }_{\omega}$ $=[111]_{B}$ direction then shifts of the $\omega$, reflections as experimentally 


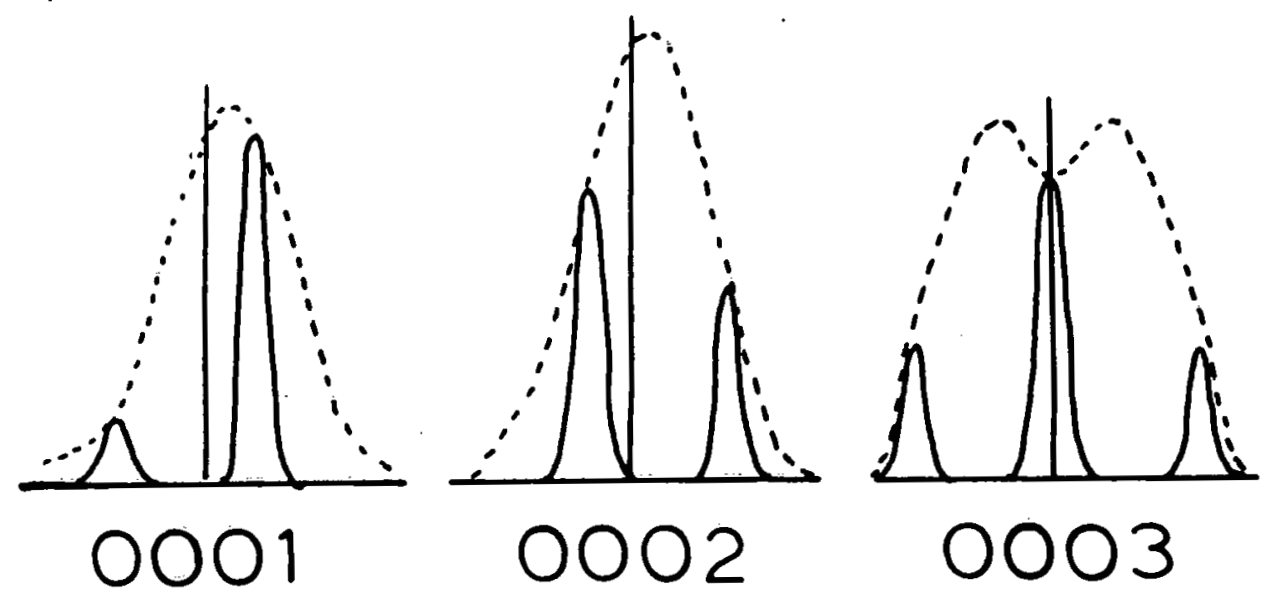

Fig. 8. Calculated Diffraction Intensity Along $<222>_{\beta}$ Direction. in an $\{011\}$ Reciprocal Lattice Plane after Vandermeulen and Deruyttere. ${ }^{23}$ 


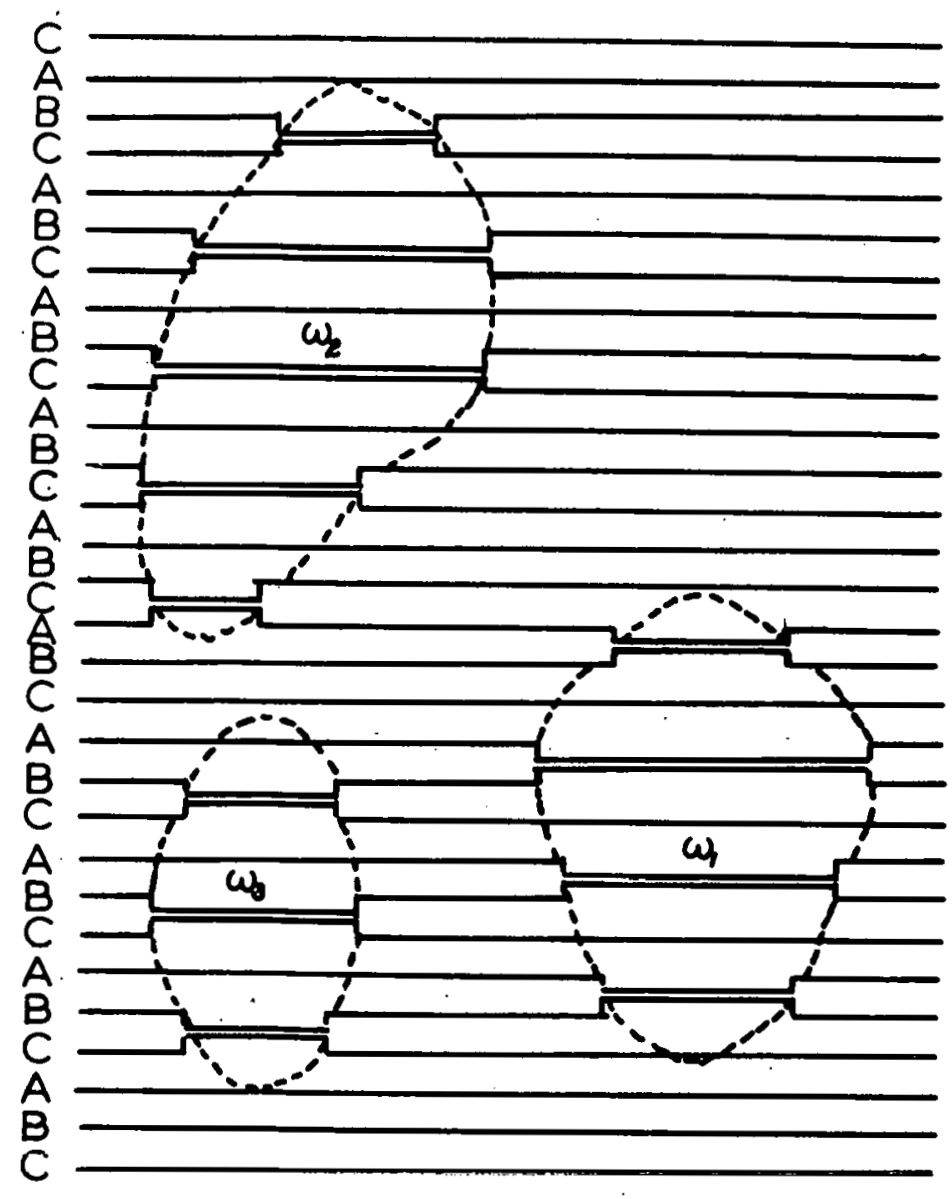

Fig. 9. Formation of Subvariants in the $\omega$ to $\beta$ Transformation after Borie et a1.24 
observed can be obtained. The ordering sequence of subvariants necessary to produce this effect is as follows: $\omega_{1}, \omega_{3}, \omega_{2}, \ldots \ldots$. Here an $\omega_{1}$ subvariant corresponds to the subvariant formed by fixing the $A$ plane and allowing the $B$ and $C$ planes to collapse; likewise the $\omega_{2}$ and $\omega_{3}$ subvariants correspond to the $B$ and $C$ planes remaining fixed, respectively. Recently, using a high-resolution lattice imaging technique, Chang, Krakow and Sass ${ }^{13}$ have observed the existence of subvariants within a single $[111]_{\omega}$ variant; however, they were not able to show the existence of any ordering between subvariants. Although such a purely crystallographic solution to the diffuse intensity problem is valid, it is not unique nor, as with the model of Vandermeulen and Deruyttere, have arguments been presented to justify the existence of such an ordering phenomenon.

In a series of papers published from 1973 through 1975 Cook $^{26-29}$ has presented one of the most unitied explanations of the $\omega$ phase transformation. Beginning with thermodynamic principles he accounts not only for the observed experimental details but also presents a mechanism by which the transformation can develop. For brevity's sake an earlier one-dimensional anharmonic planar lattice mode1 27 will be outlined instead of the three-dimensional formalism presented in his more recent publication. ${ }^{29}$

Of significant importance to the understanding of any phase change in materials is the nature of the transformation - namely, is it of first or second order? Based on symmetry arguments and the Landau criterion for the nature of phase transitions, Cook points out that the 
$\omega$ transformation cannot be of second order. The arguments supporting this assertion are that in the Landau formalism the free energy of the system and hence the structure of the lattice must be symmetric with respect to interchange of the order parameter $(n)$ from positive to negative values (Fig. 10). The order parameter is a means of indicating the degree of completion of the transformation and for the case of omega $\eta$ can be related to the amount of collapse of the $\{111\}$ planes of the bcc phase; when $\eta=1$ the $\omega$ structure is obtained. deFontaines' displacement wave model is readily adapted to this treatment by allowing the amplitude of the displacement wave (hence the degree of collapse) to be governed by the modified expression

$$
u_{<111>}=n \frac{a}{6} \sin \left(k_{\omega} \cdot x\right) .
$$

One can see from Fig. 11 that the structure obtained by modulating the bcc lattice using a positive $n$ (omega) versus negative. $n$ (anti-omega) is clearly different and hence the transformation cannot be of second order.

As in any change of state the most stable structure is that which minimizes the free energy of the system. For the case of $w$, the change in free energy $(\Delta F)$ in going from the $\beta$ to. $\omega$ phases can be written in the form.

$$
\Delta F=\frac{1}{2 !} \phi_{i j} u_{i} u_{j}+\frac{1}{3 !} \phi_{i j k} u_{i} u_{j} u_{k}+\frac{1}{4 !} \phi_{i j k 1} \cdots \ldots
$$

where $u_{i}$ represents the displacement away from the bcc configuration and $\phi_{i j}$ 's are the isothermal coupling parameters (or force constants) 


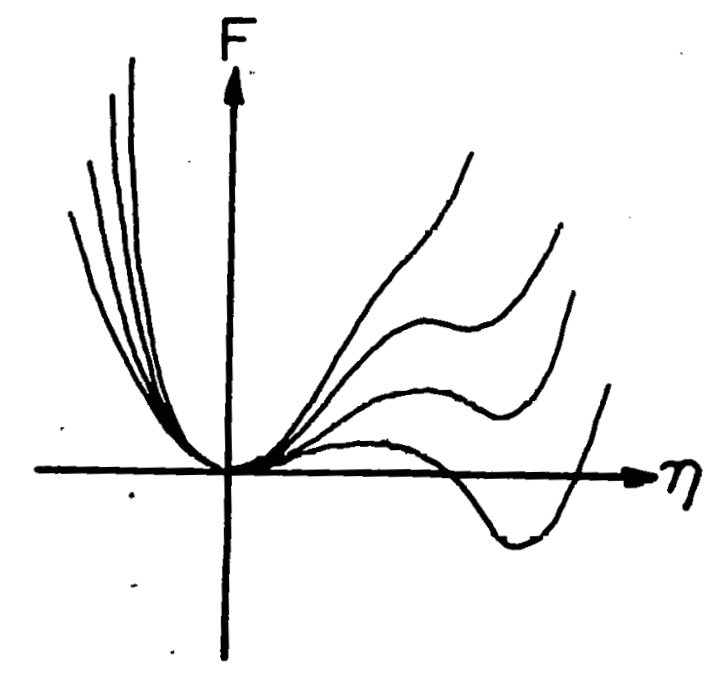

FIRST ORDER

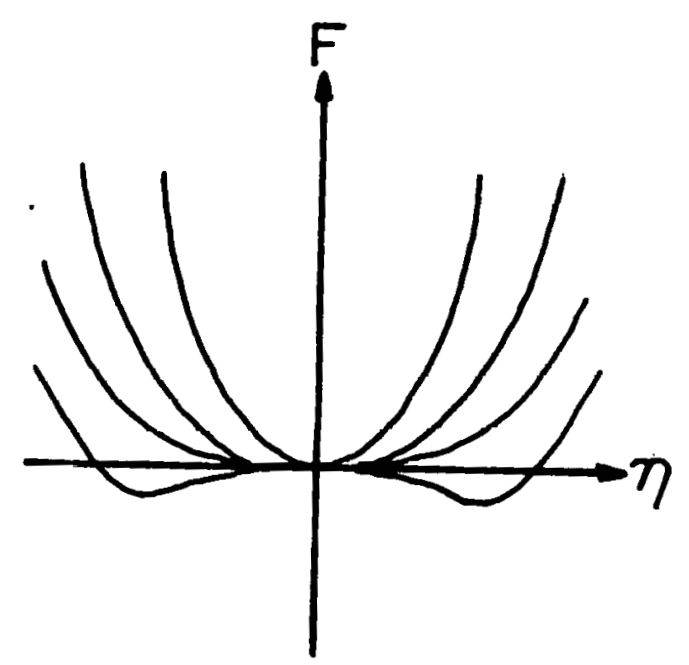

SECOND ORDER

Fig. 10. Free Energy as a Function of Urder Parameter for a First- and Second-Order phase Transformation. 


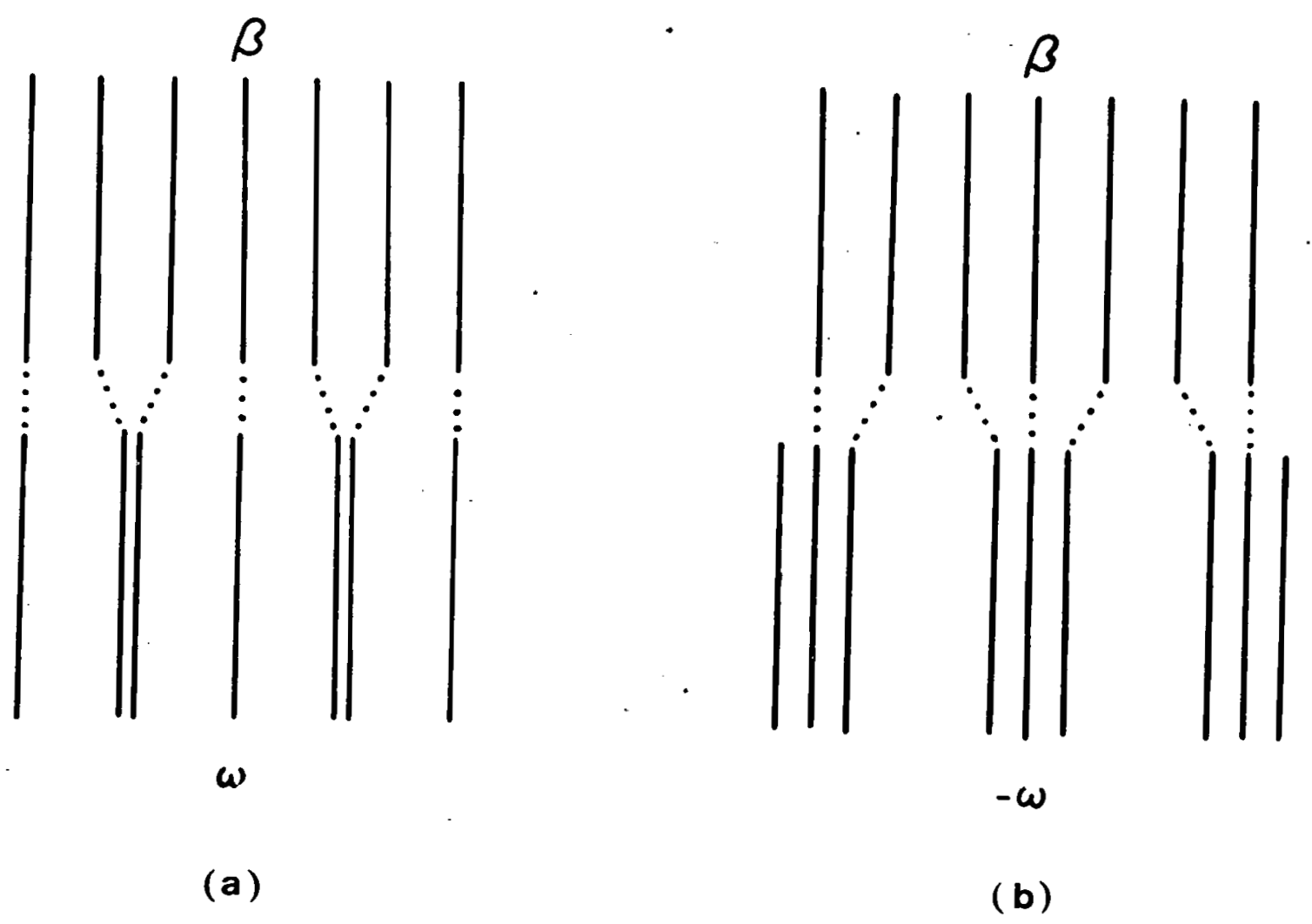

Fig. 11. Formation of $\omega$ (a) and Anti-w (b) Regions Illustrating , the First-Order Nature of the $\omega$ Transformation. 
which describe the response of the system to this displacement. Since deFontaine's model already specifies the displacement field, it remains only to determine the values of the coupling parameters in order to calculate the relative stability of a given configuration.

Values of the $\phi_{i j}$ 's are obtained indirectly usually by fitting theoretical calculations of the response of harmonic or anharmonic crystals to a vibrational wave (i.e., phonon dispersion curves). The results ${ }^{27}$ obtained by fitting the Fourier transform $\Phi(k)$ of the secondorder force constants $\left(\phi_{i j}\right)$ to the square of the longitudinal phonon dispersion curve along a [111] direction in k-space are shown in Fig. 12. As was pointed out by Moss et a1.15 a relative minimum occurs in the $\Phi$ (k) curve for the bcc transition metals, at a wave vector $k_{m}$ which is just greater than the ideal $\omega$ forming wave $k_{\omega}$. Cook was able to show that, as a consequence of this, a displacement wave in the vicinity of $\mathrm{T}_{\omega}$ can actually lower its energy by shifting its wave vector to $\mathrm{k}_{\mathrm{m}}$, rather than remain at $\mathrm{k}_{w}$ (Fig. 13), the ideal $\omega$-forming wave.

This shift of wave vector to $\mathrm{k}_{\mathrm{m}}$ has a pronounced effect on the predicted microstructure of $\omega$; no longer is the crystal subject to a displacement function which is in perfect regist.ry with the lattice but rather to a wave which ultimately creates a modulated structure alternating between the $\beta$ and $\omega$ phases. This can be seen by considering the effects of an $\omega$-like wave,

$$
u_{<111>}=n \frac{a}{6} \sin \left[k_{m} \cdot x\right]=n \frac{a}{6} \cdot \sin \left[\left(k_{\omega}+\Delta k\right) \cdot x\right]
$$

which can be rearranged into 


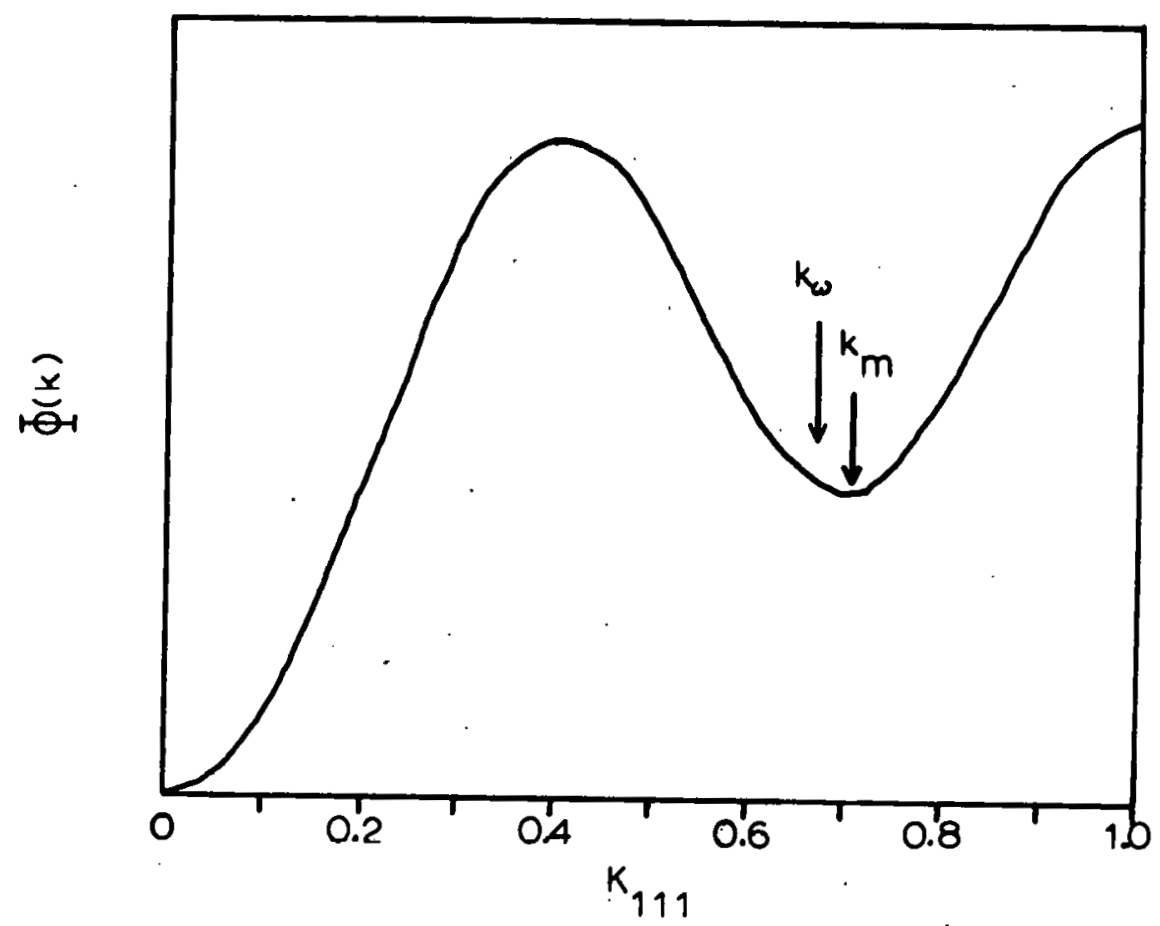

Fig. 12. Fourier Transform $\phi(k)$ of the Second-Order Force Constants after Cook. 27 


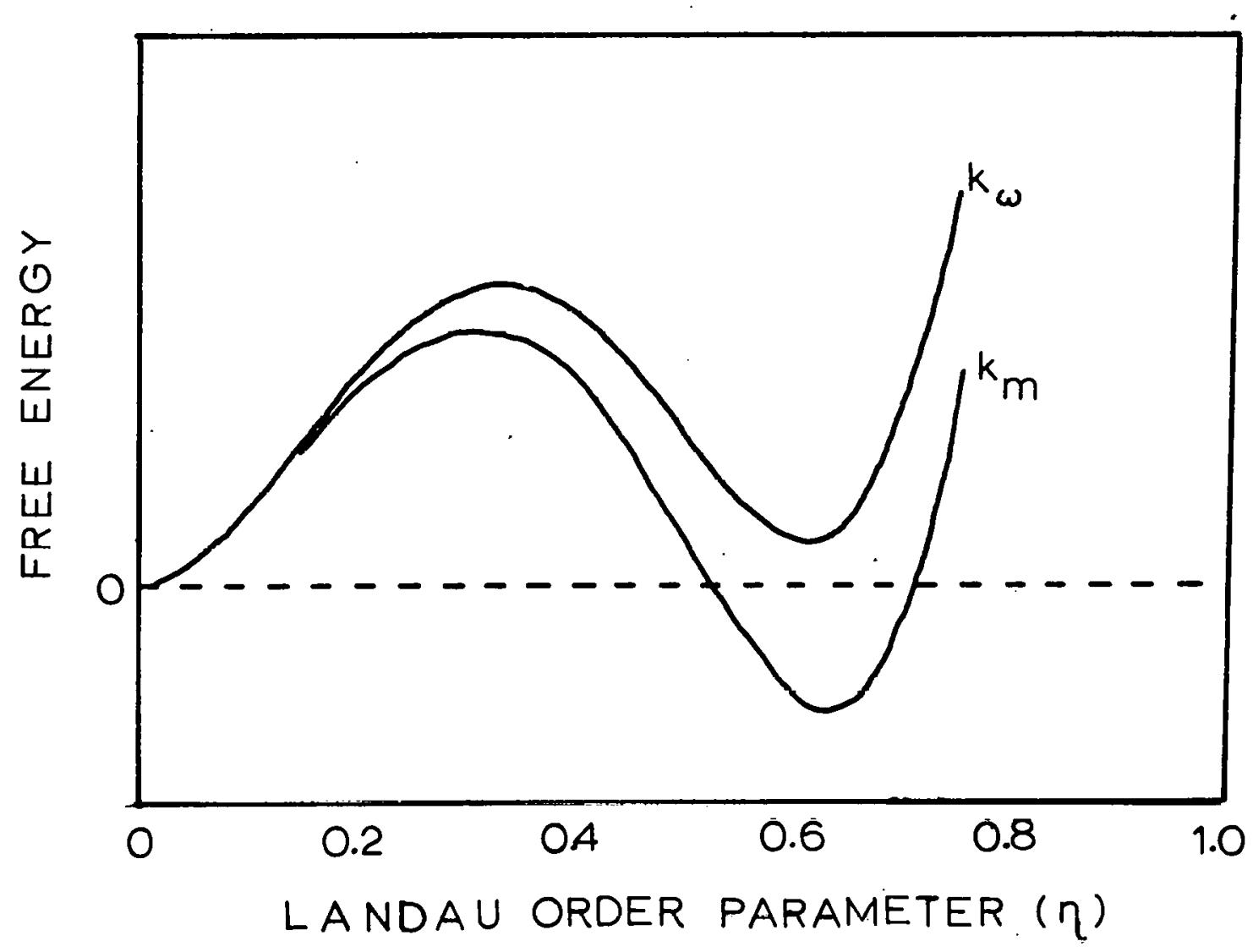

Fig. 13. Ca1milated Free Fnergy Rurves for the Theat "1-Fnrming Wave $\left(\mathrm{k}_{\omega}\right)$ and a Shitted Wave $\left(\mathrm{k}_{\mathrm{m}}\right)$ ) Corresponding to the Minimum in $\phi(\mathrm{k})$ after Cook. 27 


$$
u_{<111>}=n \frac{a}{6}\left\{\cos (\Delta k \cdot x) \sin \left(k_{\omega} \cdot x\right)+\sin (\Delta k \cdot x) \sin \left(k_{\omega} \cdot x\right)\right\} .
$$

The first term of this equation yields a cosine modulated standing wave, which creates locally alternate regions of omega and anti-omega (Fig. 14). The second term peaks between the $\omega$ and anti- $\omega$ regions and represents a transitional structure between the two forms. Using this, cook was able to outline a possible nucleation process for the $\beta$ to $\omega$ reaction; this is shown graphically in Fig. 15. Above the transition temperature $\left(\mathrm{T}_{\omega}\right)$ quasi-static fluctuations in the bcc lattice with wave vector $\mathrm{k}_{\mathrm{m}}$ create alternate regions of $\omega$ and anti-w. Both these structures are unstable relative to the bec phase and are continually forming and decaying (Stage 1). As the temperature reaches $\mathrm{T}_{\omega}$ this unstable structure splits (Stage 2), the $\omega$-forming part becoming stabilized, and the anti- $\omega$ state decaying away rapidly. Interestingly, the formerly anti- $\omega$ region, in reverting back to the bcc state, now becomes eligible for the formation of $\omega$ as the vibrational mode "flips" in sign (Stage 3), allowing it to become $\omega$ forming. Interspaced between these regions of $\omega$ are areas in which the displacement wave has not reached the critical size for nuclean tion and therefore remaining, on average, bcc.

This model predicts a long-range periodic structure immediately below $T_{\omega}$ consisting of alternate $\beta$ and $\omega$ regions, where the period of this structure is governed by the initial offset of $k_{m}$ from $k_{\omega}(\Delta \mathrm{k})$ or about $25 \AA$. As the temperature drops well below $\mathrm{T}_{\omega}$, increases of the thirdorder anharmonic coupling parameter with respect to the harmonic force constants will begin to shift stability toward the ideal $\mathrm{k}_{\omega}$ wave and 


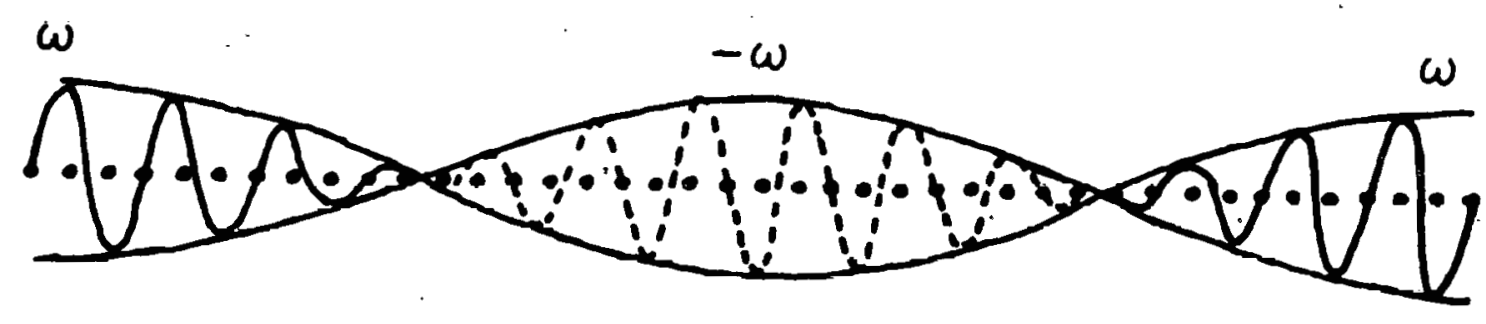

Fig. 14. Cosine-Modulated Standing Wave Creating $\omega$ and Anti- $\omega$ Regions in the $\beta$ Lattice. ${ }^{27}$ 


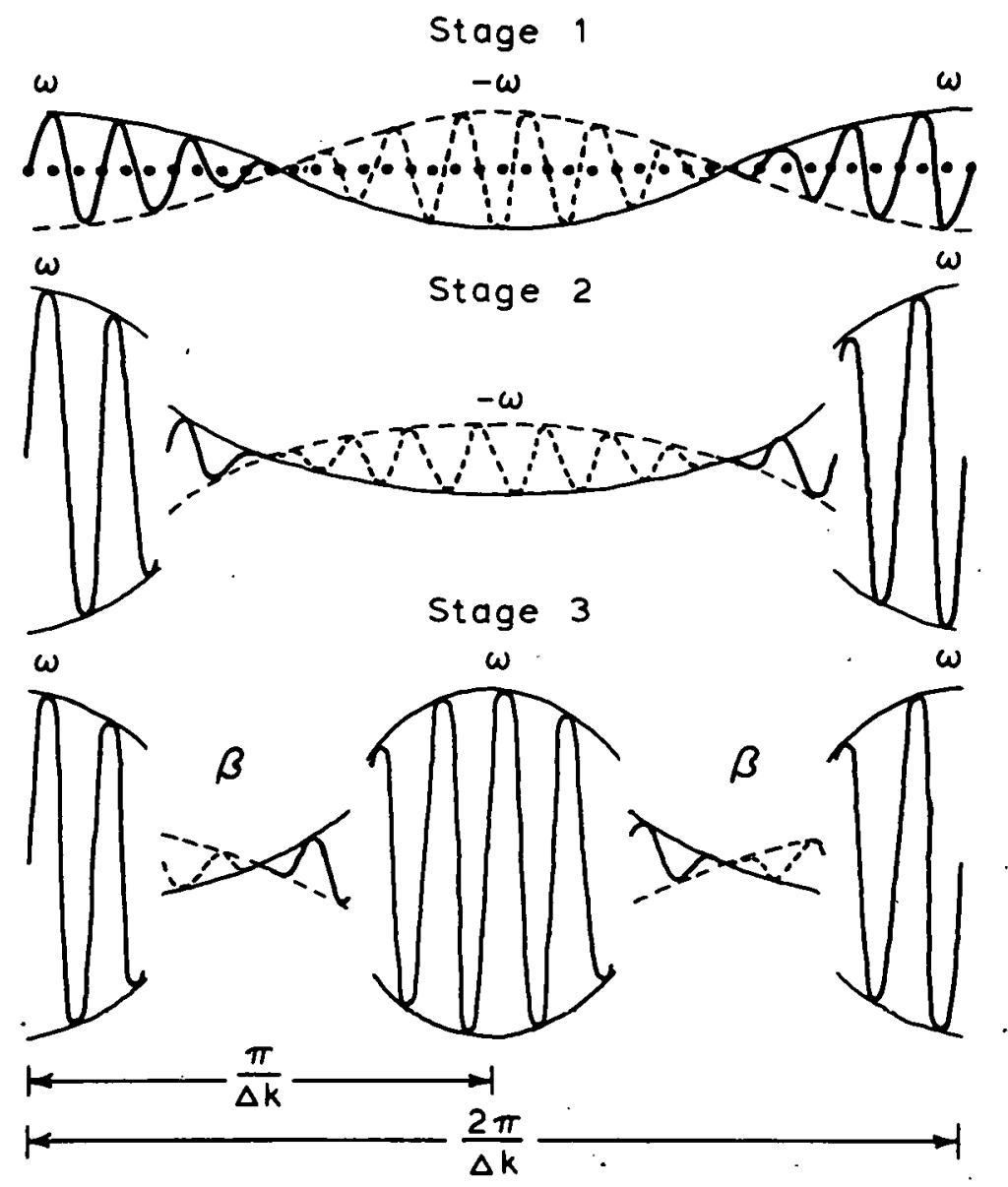

Fig. 15. Nucleation Process for a Long-Period $\beta-\omega$ Structure. after Cook. 27 
the pure w structure will become stable relative to the long period structure.

Using this theory, Cook was able to account for the details of the diffraction experiments discussed previously: namely, (1) the initial offset of scattering $(\Delta \mathrm{k})$ from the ideal wave vector $k_{\omega}$, (2) the pronounced diffuse streaking perpendicular to $\langle 111\rangle$ directions in $\mathrm{k}$ space, and (3) the subsequent shift of the $\omega$ reflections toward $k_{\omega}$ will related increases in inteinsily and sliarpuess un cuoling. In order to test the applicability of Cook's theories on this transformation, a series of in-situ thermal cycling experiments were proposed.' If a longperiod metastable $\omega$ structure develops just below $T_{\omega}$, then it should be possible to detect an ordering/disordering phenomenon in an $\omega$-forming system through in-situ experiments in ar electron microscope.

The nature of phase transformations in most materials is usually complex, generally involving changes in structure, composition, and morphology. It is, therefore, extremely important to be able to completely characterize all the details of a particular transformation. As such, analytical electron microscopy plays an essential role, not only with respect to this study but also in essentially all fields of materials research. Thus the details of analytical techniques investigated during this research are presented in Chap. 2 and comprise a substantial portion of this thesis. 
CHAPTER 2

\section{ANALYTICAL ELECTRON MICROSCOPY}

\subsection{Introduction}

Transmission Electron Microscopy (TEM) has, during the last two decades, proven itself to be an extremely powerful tool for the study of the microstructure of materials. Through the use of electron diffraction experiments it is possible to both observe and characterize a variety of crystalline defects and solid state processes and thus correlate their presence with macroscopic properties. Yet the information obtained about the specimen from such experiments represents only a fraction of that produced by the electron-solid interaction, and the products of inelastic scattering yield additional information concerning the elemental, chemical, and electronic state of the material under investigation. This information is released from the specimen in various forms - for example, the production of secondary, backscattered, and Auger electrons, or in the generation of characteristic and continuum $x$ rays and in fact even within the transmitted electron beam which incurs characteristic energy losses during each of these processes. Analytical Electron Microscopy (AEM) is the term which is now applied to the microcharacterization of materials using as many of these electron-solid interactions as possible.

Although instrumentation has been developed over the years for materials characterization, there has been one common limitation namely; spatial resolution. Prior to a few years ago, analytical spatial 
resolution was limited to the 2 to $5 \mu \mathrm{m}$ range. Recent advances in electron-optical design in the field of scanning transmission electron microscopy (STEM) coupled with the development of field emission electron sources, provide extremely intense and small electron probes $(<10 \AA)$ and have allowed a quantum jump to occur in analytical sensitivity and spatial resolution. State-of-the-art instrumentation now affords the possibility of analysis of regions down to the $20 \AA$ level depending on material, technique and instrumental factors. At the time at which this research was started AEM was in its infancy and the various analytical techniques showed great potential. This chapter describes the work done in developing, refining, and standardizing several of these techniques. In many cases, contributions (i.e., fundamental physical equations) have been collected from the literature, and these sources are appropriately referenced in the text.

\subsection{X-Ray Microchemical Analysis}

The interfacing of a solid-state Si(Li) energy dispersive $x$-ray spectrometer (EDS) to a transmission electron microscope provides a convenient means of measuring the characteristic $x$-ray emission (for elements of atomic number $Z>12$ ) from microvolumes of material. By measuring the relative integrated peak intensities obtained by irradiating various regions of the sample with the electron probe, it becumes a simple matter to obtain qualitative microchemical information. The interpretation 
of these data in terms of quantitative analysis requires, however, careful consideration of both experimental procedure and theoretical corrections, and is the subject of this section.

\subsubsection{Theory of Quantitative X-Ray Microanalysis}

Several methods of reducing measurements of characteristic $x$-ray emission into quantitative chemical analysis of thin samples have been developed over the last decade. ${ }^{30-41}$ Generally, all of these procedures rely on the validity of Philibert's "thin-film" approximation which states that for a sufficiently thin specimen the effects of energy losses in the incident electron beam, electron backscattering, $x$-ray fluorescence (both by characteristic and continuum radiation) and $\mathrm{x}$-ray absorption can be ignored. Using this approximation, one can show that for a homogeneous sample composed of the elements A and B the ratio of any two characteristic $x$-ray peaks $\left(\right.$ e.g., $\left.{ }^{{ }^{k}}{ }_{A}{ }^{\alpha} / I_{B}{ }^{\alpha}\right)$ is independent of sample thickness; it is directly proportional to the relative concentration ratio of those elements; that is:

$$
\frac{I_{A^{\alpha}}^{k}}{I_{B^{\alpha}}^{k}}=\kappa_{A B} \frac{C_{A}}{C_{B}} .
$$

Here $\kappa_{A B}$ is a constant for a given binary system, $I_{A}^{K_{\alpha}}$ the intensity of the $K_{\alpha}$ (or $M_{\alpha}, L_{\alpha}$, etc.) characteristic $x$-ray emission from element $A$ and $C_{A}$ the composition in weight percent of element $A$ in the compound $A B$. In the past it has been the accepted procedure to consider an electron transparent specimen to be "thin" in the Philibert sense; ${ }^{32}$ however, Jacobs and Baborovoska, ${ }^{33}$ Zaluzec and Fraser, $42-44$ and Goldstein et a1. ${ }^{45}$ have shown 
that this is not generally valid. These breakdowns of the simpler analytical models range from failures of the thin film approximation, which can be accounted for theoretically, to experimental details which can only be corrected by instrumental modifications. 43,44

The theory outlined in the following section is concerned with determining the variation with thickness of the relative intensity ratio of two characteristic $x$-ray lines. Since a ratio of intensities is being calculated, rigorous treatment of several parameters such as electron backscattering, transmission, and beam broadening is not required. This is due to the fact that these processes affect the generation of $x$ rays for all elements equally. They will however be considered at the appropriate times.

The generation and subsequent emission of characteristic $x$ rays from a material depend on the product of three terms commnnly referren to in the literature as ZAF corrections. These terms describe the basic processes by which the characteristic $x$-ray intensity is influenced namely the atomic number effect ( $\mathrm{Z}$ ), the absorption correction (A), and finally fluorescence $(F)$ emission. The calculation of the atomic number correction is always necessary as it reflects the inherent efficiency of $x$-ray generation by electrons for various elements. The absorption correction, on the other hand, accounts for decreases in detected intensity dun to the fact that $x$ rays are absorbed in leuving the specimen and thus is directly tied to the sample thickness. Lastly, the fluorescence term deals with the probability that on absorption $x$ rays can also simulate 
characteristic emission. Clearly, if the sample becomes thin enough such that the absorption correction is small, the fluorescence term must in turn be negligible. However, the converse does not always apply, That is, it is possible to have measurable absorption effects with little or no fluorescence correction. The "thin film" approximation reduces to a calculation of the atomic number effect, which for the majority of TEM specimens becomes the principal correction. The inclusion of absorption and fluorescence becomes a specialized but important case of TEM-based microanalysis. Rigorously, the calculation of these effects should be evaluated along each electron's path within the sample. However, within the present set of experimental errors, a reasonable approximation results from an integration of these factors through the sample thickness.

\subsubsection{The Atomic Number Correction}

Consider a homogeneous sample of the elements A and B. The total: number of $K_{\alpha}$ characteristic $x$ rays of element A generated within the infinitesimal $s 1 a b d z$ by electrons which have traveled a path length $z$ within the specimen can be written as:

$$
{ }_{\phi}^{{ }^{k} \alpha}(z) \cdot d z=Q_{A}^{k} \cdot \frac{N_{0}{ }^{-C} C_{A}}{{ }^{W} A} \cdot n_{A B}(z) \cdot \omega_{A}^{k} \cdot F_{A}^{{ }^{\alpha}} \cdot d z
$$

Here $Q_{A}^{K}$ is the ionization cross section of the $K$-shell of element $A$; $\mathrm{N}_{0}, \bar{\rho}, \mathrm{C}_{\mathrm{A}}$, and $\mathrm{w}_{\mathrm{A}}$ are, respectively, Avagadro!'s number, the sample density, the concentration in fractional weight percent, and the atomic weight of $A, \eta_{A B}(z)$ the number of electrons bombarding $d z, \omega_{A}^{\dot{x}}$ the $K$ shell $x$-ray fluorescence yield of $A$ and $f_{A}^{K^{\alpha}}$ and $K_{\alpha}$ fraction of the total 
$\mathrm{K}$-she11 emission. For the remainder of this section the discussion will consider the emission of $K_{\alpha}$ characteristic $x$ rays; the extension of $L_{\alpha}$ or other shells can be obtained by substituting the corresponding terms for that subshell in the appropriate equations.

Various expressions have been suggested for calculating the paraneters in Eq. (2.2). Most of these have resulted from considerations of quantitative $\mathrm{x}$-ray analysis in bulk specimens using electron energies in the 5 to $30 \mathrm{keV}$ range typically found in electron microprobes. Generally these relations were developed to obtain analysis for either the case of infinitely thick (bulk) specimens in which the electron probe stops completely, or infinitesimally thin samples and hence negligible energy 1oss. Although those analyses are indeed applicable to the cases considered, the question of TEM-based $\mathrm{x}$-ray analysis dictates the consideration of the interaction of fast (i.e., relativistic) electrons with not thin, but rather semi-thick targets.

Several analytic expressions have been proposed in recent years as approximations to the ionization cross section for use in the atomic number correction. Generally all of thèse equations can be written in the form

$$
Q_{A}^{K}\left(E_{0}\right)=\frac{\pi c^{4} \cdot z_{A}^{k} \cdot a_{A}^{k}}{E_{0} \cdot E_{A}^{K}} \cdot \ln \left\{b_{A}^{k} \cdot \frac{F_{0}}{E_{A}^{K}}\right\},
$$

which relates the probability of a $K$-shell ionization event $\left(Q_{A}^{K_{*}^{*}}\left(E_{0}\right)\right)$. to the incident electron energy. $\left(E_{0}\right)$, the critical excitation energy of the $K$-shell $E_{A}^{K}$ of $A$, the number of electrons in that shel1 $Z_{A}^{K}$, and two 
constants $a_{A}^{k}$, $b_{A}^{k}$ which are related to the atomic structure of $A$. Since the calculation of the parameters $a_{A}^{K}$ and $b_{A}^{K}$ is non-trivial $1^{46,47}$ for all elements with atomic number greater than 2, considerable discussion over the optimum choices for these constants can be found in the literature. ${ }^{48-54}$ The most recent review of this has been given by Powel1 ${ }^{52}$ for electrons in the 1 to $40 \mathrm{keV}$ range.

It has been the general procedure to obtain values for these constants by fitting Eq. (2.3) to experimental measurements of $Q_{A}^{K}$. Although it is perfectly reasonable to obtain the values of $a_{A}^{k}$ and $b_{A}^{k}$ by this procedure, there is an important limitation to be considered. Thus, the empirical constants obtained using this method are only applicable over a specific energy range and for a specific element. Furthermore, Eq. (2.3) must be reconsidered for application to analytical electron microscopy since relativistic effects become important for electron energies greater than $80 \mathrm{keV}$. Thus for application to AEM, Eq. (2.3) has been reformulated as follows:

$$
Q_{A}^{K}\left(E_{0}\right)=\frac{\pi e^{4} \cdot 2_{A}^{k} \cdot a_{n}^{k}}{E_{0} \cdot E_{A}^{k}}\left\{\ln \left(b_{A}^{k} \frac{E_{0}}{E_{A}^{k}}\right)-\ln \left(1-\beta^{2}\right)-\beta^{2}\right\},
$$

where

$$
\begin{aligned}
a_{A}^{k} & =0.35 \\
b_{A}^{k} & =\frac{0.2}{U_{0} \cdot\{1-\exp (-\gamma)\} \cdot\{1-\exp (-\delta)\}} \\
U_{0} & =E_{0} / E_{A}^{k} \equiv \text { overvoltage ratio } \\
\delta & =\frac{1}{2} \cdot E_{A}^{K}
\end{aligned}
$$




$$
\begin{aligned}
\gamma & =\frac{1.250}{\left\{E_{A}^{k} \cdot U_{0}^{2}\right\}} \\
\pi e^{4} & =6.4924 \times 10^{-20} \\
\beta & =\frac{v}{C}=\text { relativistic correction factor }
\end{aligned}
$$

when all energies are expressed in $\mathrm{keV}$, the units of $Q_{A^{K}}^{K}\left(E_{0}\right)$ are $\mathrm{cm}^{2} /$ atom.

The basic equation (2.4) was taken from the relativistic treatment of Mott and Massey, ${ }^{48}$ while the functions $a_{A}^{k}$ and $b_{A}^{k}$ were determined analytically by fitting Eq. (2.4) to a series of measurements of $Q_{A}^{K}$. Figures 16, 17, and 18 compare the predictions of this equation (solid curve labeled \#1) with experimental measurements of the $\mathrm{K}$-shell cross section of aluminum, 53 nicke1, 54 and silver. 55 Also plotted on this figure for comparison are the calculated values of $Q_{A}^{K}$ using suggested parameters for $a_{A}^{K}$ and $b_{A}^{K}$ using Eq. (2.3).

The broken curve ( $\$ 4)$ in these figures is the Mott and Masscy nonrelativistic Eq. (2.3) with modifications suggested by Worthington and Tomlin ${ }^{49}$ and Burhop 50 using $a_{A}^{k}=0.35$ and $b_{A}^{k}=\frac{4.0}{1.65+2.35 \cdot \exp \left(1-U_{0}\right)}$ which has been applied fairly extensively for several years. From these figures one can see that this expression substantially underestimates the cross-section values at high overvoltages; however, at lower values $(1 \leqslant U \leqslant 4)$ where the expression was formulated the fit is reasonable. The dashed curves (\#2 and \#3) are calculations based on Powe11's fit to a linearized version of Eq. (2.3). Curve \#2 is plotted using the constants $a_{A}^{k}=0.9$ and $b_{A}^{k}=0.79$ which he obtained by fitting Eq. (2.3) to measurements of aluminum. Curve \#3, with coefficients $a_{A}^{k}=1.05$ and 


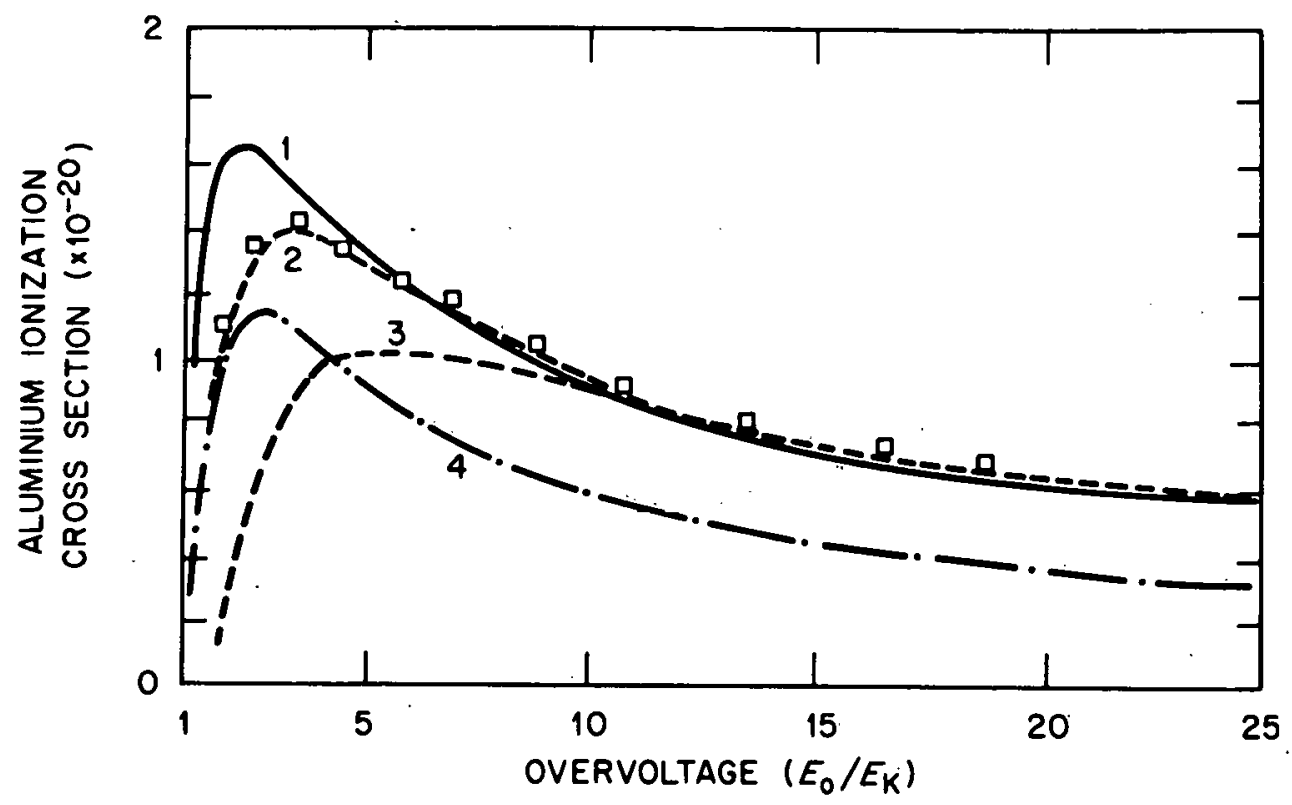

Fig. 16. Comparison of Experimental Ionization Cross Section as a Function of Overvoltage for Aluminum (squares) to Various Calculations.. (1) Using Eq. (2.4); (2), (3) due to Powe11; and (4) due to Mott and Massey. 


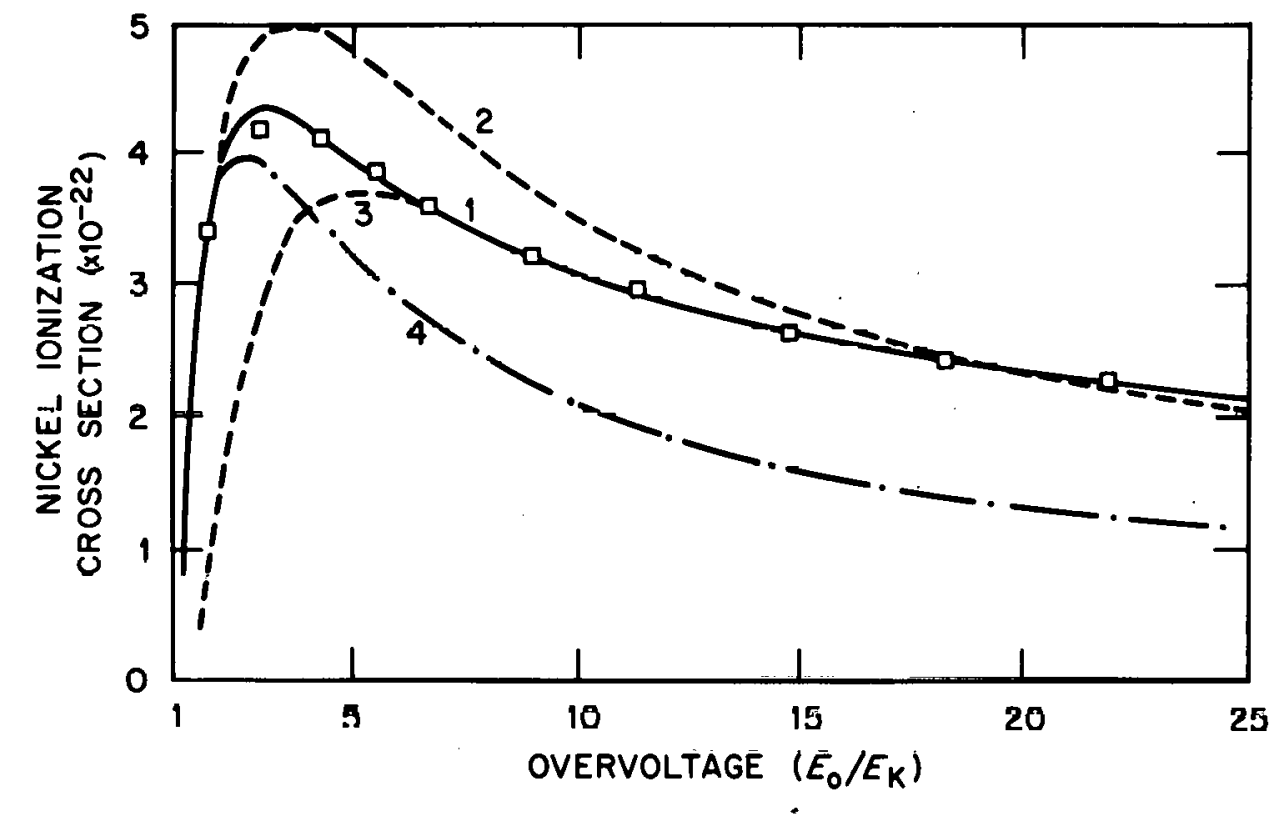

Fig, 17. Comparison of Experimental Innization Crnss Sertinn as a Function of Overvoltage for Nickel (squares) to Various Calculations. (1) Using Eq. (2.4); (2), (3) due to Powell; and (4) due to Mott and Massey. 


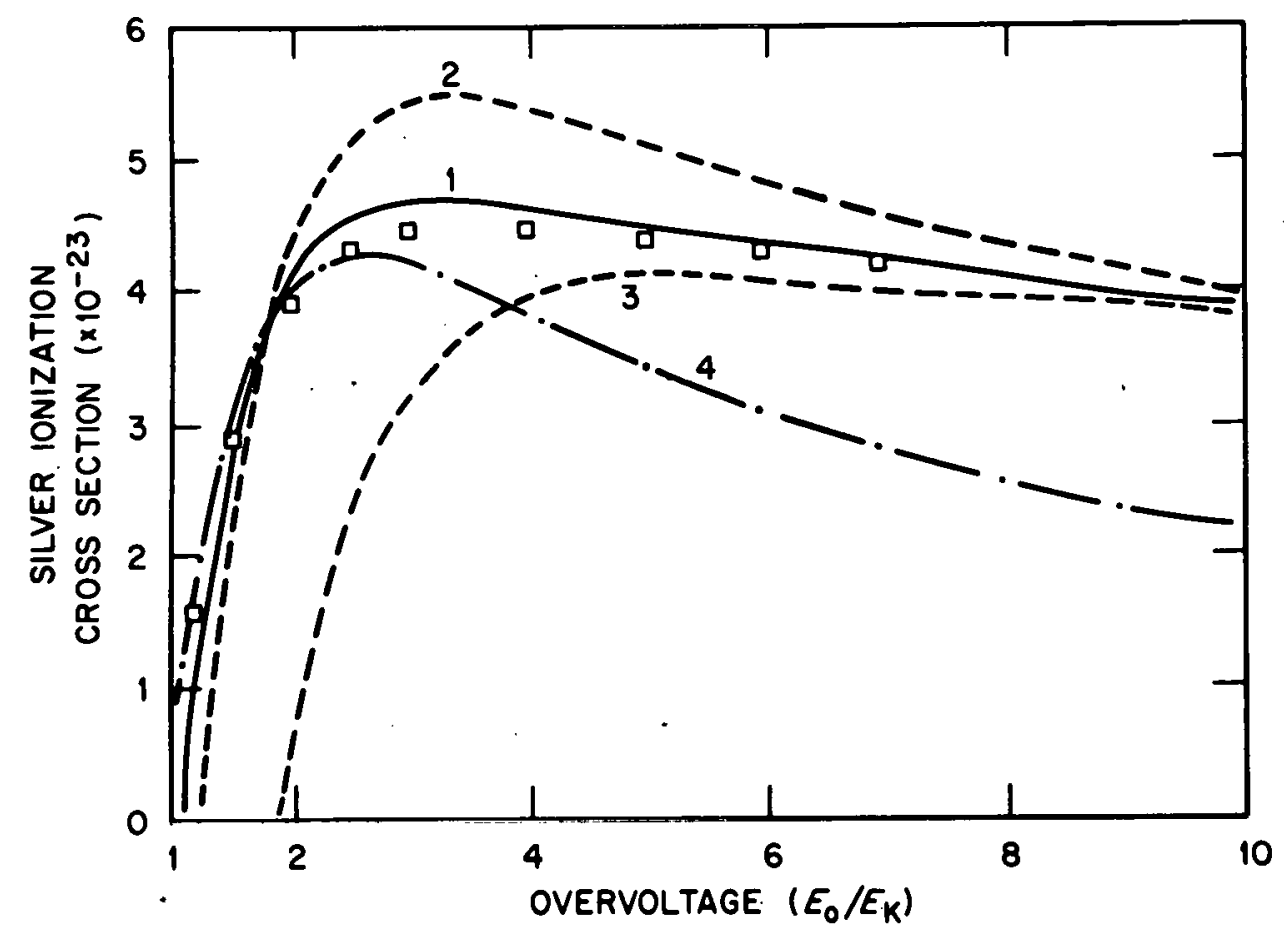

Fig. 18. Comparison of Experimental Ionization Cross Section as a Function of Overvoltage for Silver (squares) to Various Calculations. (1) Using Eq. (2.4); (2), (3) due to Powe11; and (4) due to Mott and Massey. 
$b_{A}^{k}=0.51$ represents his fits to data of nickel. In these figures one can see that although it is possible to find a set of constants $\left(a_{A}^{k}, b_{A}^{k}\right)$ which represents the experimental data reasonably well [\#2, Fig. 1(b)] for one element, it is not generally valid to extrapolate these same constants for elements of a significantly different atomic number (\#2, Fig. 17, $\# 2$, Fig. 18). Thus, to use such an approach requires the parameters $a_{A}^{k}$ and $\mathrm{b}_{\mathrm{A}}^{\mathrm{k}}$ to be determined explicitly for all elements of interest. Unfortunately, there is an insufficicnt amount of experimental data to use the Powe11 coefficients confidently over the wide range of atomic numbers and for typical incident beam energies used in analytical microscopy. Thus, the more complex form of Eq. (2.4) is preferred. It should be noted that in the context used here, the form of $a_{A}^{k}$ and $b_{A}^{k}$ no longer have physical interpretation since they have been used purely as adjustable parameters.

Comparison of these expressions to $L$ shell data for atomic numbers greater than 30 is difficult owing to the lack of measurements in the appropriate energy ranges. Hence; it is assumed that the variation of the $L$ shell cross sections with energy is similar to the $K$ shell. Thus, application of Eq. (2.4) is obtained by changing all superscripts to their respective $L$ she11 equivalents, and the constant $a_{\Lambda}^{L}$ set equal to 0.25 .50 In order to express the ionization cross section as a function of path length it becomes necessary next to consider the rate of electron energy loss with path length $(\mathrm{dE} / \mathrm{dz}) \cdot{ }^{56-59}$ In the context to be used here, it becomes most convenient to use the integral rather than the differential form, which can be written to a good approximation as: 


$$
\left(E_{0}\right)^{m}-\left(E_{A B}\right)^{m}=\overline{k \rho z}
$$

where $E_{0}$ is the incident electron energy, $k$ a constant and $E_{A B}(z)$ the electron energy after traveling a path length $z$ in $A B$. As this equation is only an approximation, the coefficient $m$ varies with the energy; experimental measurements show that it changes from values of 2 at $10 \mathrm{keV}^{58}$ to nearly 1 at $1 \mathrm{MeV} .{ }^{59}$ Rearranging this equation, one obtains the expression for the mean electron energy with path length as

$$
E_{A B}(z)=E_{0}\left(1-\frac{z}{z_{\dot{R}}}\right)^{1 / m},
$$

where $z_{R}$ can now be interpreted as an effective range (evaluated along the electron's path). Archard and Mulvey ${ }^{60}$ have derived the following expression for this range

$$
z_{R}=1.44 \times 10^{-5} \cdot\left(\frac{\overline{\mathrm{w}}}{\overline{\bar{\rho}} \overline{\mathrm{Z}}}\right] \cdot \frac{\mathrm{E}_{0}^{2}}{2 \ln \left[\frac{(101.0) \cdot \mathrm{E}_{0}}{\overline{\mathrm{Z}}}\right]},
$$

where $\bar{w}_{A}, \bar{\rho}$, and $\bar{Z}$ are the average atomic weight, density and number of the compound. Expressing $E_{0}$ in units of $\mathrm{keV}$, the units of $z_{R}$ become centimeters. Calculations of $\mathrm{x}$-ray generation have been performed using various values of the cocfficicnt $m$ in Eq. (2.6) between the values 1 and 2 and as long as the path length $z$ is less than $0.5 z_{R}$ the choice of In has 1ittle effect. This again is due in part to the fact that ratios of intensities as well as high incident beam energies are being considered. A value of 1.5 has been adopted for TEM work.

The $x$-ray fluorescence yield from most elements can be obtained with reasonable accuracy from the expression first proposed by Wentze161 


$$
\omega_{A}^{k}=\frac{z^{4}}{c^{K}+z^{4}}
$$

where $Z$ is the atomic number and $c^{K}$ a constant for the shell in question $\left(c^{K}=1.12 \times 10^{6} ; c^{\mathrm{L}_{\mathrm{III}}} \sim 1 \times 10^{8}\right) .{ }^{62}$ Another and more accurate representation is the empirical expression ${ }^{63}$

$$
\left(\frac{\omega_{A}^{k}}{1-\omega_{A}^{k}}\right)^{\frac{3}{4}}=A+B Z+C Z^{3} \text {, }
$$

in which the coefficients $A, B$, and $C$ are fitted to experimental measurements. In this case the periodic table is broken up into segments and a set of constants is used to determine $\omega_{A}^{k}$ for various ranges of $z$. Since it is usually the case that dedicated minicomputers are used for determining quantitative analyses, it is preferred to use Eq. (2.9). (or tabulated values $^{64,65}$ ) rather than Eq. (2.8). Similarly, as in the case of fluorescence yields, the $K_{\alpha}$ fraction of the total $K$-shell emission $f_{A}^{r_{*}} \alpha$ [defined as $\left.K_{\alpha} /\left(K_{\alpha}+K_{\beta}\right)\right]$ can be obtainèd from polynomial fits to experimental data ${ }^{66}$ or in tabulated form. ${ }^{67-69}$

The last term to consider in the atomic number correction is $\eta_{A B}(z)$, the total electron flux bombarding the infinitesimal element $d z$. This flux is composed of two parts - first, those electrons forwardscattered from the sample volume directly above $\mathrm{dz}$, and second, any electrons which have been backscattered from deeper levels.

The forward-scattered flux at a depth $t$ emitted into a cone of semi-angle, $\pi / 2$, centered about the incident beam direction, can be obtained from Bothe's multiple scattering theory 


$$
f\left(t, \theta=\frac{\pi}{2}\right)=f_{0} \cdot\left\{1-\exp \left(\frac{-\left(\frac{\pi}{2}\right)^{2}}{2 \lambda_{B}^{2}}\right)\right\}
$$

where $f_{0}$ is the incident flux and $\lambda_{B}$ is given by Bothe as

$$
\lambda_{B}^{2}=1.6 \times 10^{5} \cdot\left(\frac{Z}{E_{0}}\right)^{2} \cdot \frac{\bar{p} \cdot t}{w_{A}} ;
$$

here $Z, E_{0}, w_{A}$ are as previously defined, and $\lambda_{B}$ was obtained by an analytical fit to the experimental measurements of Crowther ${ }^{70}$ on fast electron ( $\sim 600 \mathrm{keV}$ ) transmission through various materials. Cosslett and Thomas ${ }^{58}$ in a study of electron solid interactions determined that Eq. (2.10) also fits electron transmission at lower energies (5 to $25 \mathrm{keV}$ ) with $\lambda$ modified to:

$$
\lambda_{\mathrm{CT}}^{2}=1.39 \times 10^{5}\left(\frac{\mathrm{Z}}{\mathrm{E}_{0}}\right)^{\frac{3}{2}} \cdot \frac{\overline{\mathrm{\rho}} \cdot \mathrm{t}}{{ }_{\mathrm{w}_{\mathrm{A}}}} .
$$

In both Eqs. (2.11 and 2.12), $\lambda$ has the units of radians with $E_{0}$ expressed in $\mathrm{keV}$. The difference in $\lambda$ between these two equations is negligible for typical thicknesses $(0.05$ to $2.0 \mu \mathrm{m}$ ) and accelerating voltages (50 to $200 \mathrm{keV}$ ) used in most AEM work; as such, Eq. (2.12), being the more recent, is preferred.

The backscattered flux across $\mathrm{dz}$ will in general be a varying fraction of the number of electrons transmitted through that layer. This effect can be represented by a function of depth, $r_{A B}(t)$, which when multiplied by $f_{A B}(t)$, describes the composite flux due to both sources. For large depths within the sample, where the electrons can be considered as fully scattered, $r_{A B}(t)$ is a constant, since as many electrons will be 
traveling forward as backward. On the other hand, for an infinitely thin layer suspended in vacuo $\mathrm{r}_{\mathrm{AB}}(\mathrm{t})$ must be equal to unity. Philibert ${ }^{37}$ first considered this problem and assumed a simple exponential variation for $r_{A B}(t):$

$$
r_{A B}(t)=R_{\infty}-\left(R_{\infty}-R_{0}\right) \exp \left(-k_{A B} \cdot \rho \cdot t\right)
$$

where $R_{\infty}$ is the bulk (or fully scattered) value and $R_{0}$ the surface contributiun. Assuning a cusine-like angulä distibuliun of electron scattering he obtained a value for $R_{\infty}$ as 4 . Later, based on the measurements of Cosslett and Thomas, which showed the distribution to vary as cosine squared, Reuter determined that. $R_{\infty}$ should in fact be equal to 3 . For $\mathrm{R}_{0}$, Philibert assumed the simple form

$$
\mathrm{R}_{0}=1+\varepsilon
$$

with $\varepsilon$ being chosen to match experimental measurements of the depth distribution of characteristic $x$ rays. Reuter ${ }^{40}$ succeeded in expressing $\mathrm{R}_{0}$ as:

$$
R_{0}=1+2.8\left(1+\frac{0.9}{\mathrm{U}_{0}}\right) \cdot n \text {, }
$$

based on experimental measurements of $x$-ray emission from thin films on bulk substrates. In Eq. (2.15) $U_{0}$ is the overvoltage ratio and $n$ the bulk backscattering coefficient. For the case, of self-supporting thin films, such as TEM specimens, $R_{0}$ should be further modified to account for the fact that $n$ is now a function of sample thickness. 58 
Thus $n$ in Eq. (2.15) should be replaced by the function $n\left(t_{0}\right)$ :

$$
n\left(t_{0}\right)=n\left\{1-\exp \left(-\mu_{A B}^{*} \cdot t_{0}\right)\right\}
$$

where $t_{0}$ is the total sample thickness in the direction of the incident beam. $n$, the bulk backscattering coefficient, can be calculated from the polynomial equation; 40

$$
n=-0.0254+0.0167-1.86 \times 10^{-4} \cdot Z^{2}+8.3 \times 10^{-7} Z^{3} .
$$

The coefficient $\mu_{A B}^{*}$ can be obtained from the results of Cosslett and Thomas. In their measurements they noted that after a sample thickness corresponding to 20 to $30 \%$ of the mean electron range $n$ attains bulk specimen values. Setting $t_{0}$ equal to $25 \%$ of the mean electron range $\left(R_{m}\right)$ and requiring that at this point $n\left(t_{0}\right) \gtrsim 0.95 n$ one then obtains the following:

$$
\mu_{\mathrm{AB}}^{*} \approx \frac{12.0}{\mathrm{R}_{\mathrm{m}}} .
$$

Cosslett and Thomas have also shown that $\mathrm{R}_{\mathrm{m}}$ is always less than the Bethe range $\left(z_{R}\right)$ defined in Eq. (2.7). Thus upon substitution of $z_{R}$ instead of $R_{m}$ into 2.18 one obtains a reasonable approximation for $\mu_{A B}^{*}$ as:

$$
\left.\mu_{A B}^{*} \approx 1.66 \times 10^{-16}\left(\frac{\bar{p} \cdot z}{w_{A}}\right) \cdot \frac{\ln \left\{\frac{101 \cdot E_{0}}{Z}\right\}}{F_{0}^{2}}\right\}
$$

The use of $z_{R}$ instead of $R_{m}$ is not a serious discrepancy since it effectively resets the defining criterion for the point at which $n\left(t_{0}\right)$ is considered a bulk value. 
The last parameter needed to complete the expression for $n_{A B}(z)$ is $k_{A B}$ from Eq. (2.13). It is noted that this coefficient determines the depth at which the total scattering in any direction becomes constant (i.e., the diffusion depth). Philibert and Reuter differ somewhat on this point, basically due to their respective sources of information. Philibert's criterion requires that the most probable scattering angle becomes $\pi / 4$. Reuter, on the other hand, chooses $38^{\circ}$ to be consistent with the data of Cosslett and Thomas. Furthermore, Philibert solves for the diffusion depth using Bothe's equation for the most probable scattering angle $\theta_{\rho}$

$$
\theta_{\rho}^{B}=\frac{400}{E_{0}}\left(\frac{Z^{2} \cdot \rho t}{A}\right)^{\frac{1}{2}}
$$

while Reuter uses the analytic fit proposed by Cosslett and Thomas

$$
{ }_{p}^{C T}=109.5\left(\frac{z^{\frac{3}{2}} \cdot p t}{A \cdot E_{Q}}\right)^{\frac{1}{2}} .
$$

Unfortunately, there has been no experimental work to date done on this question in the 50 to $200 \mathrm{keV}$ range, which straddles these two extremes. Thus for low voltage work ( $\lesssim 100 \mathrm{keV}$ ), Eq. $(2.21)$ is used, while for high voltage measurement $(\gtrsim 200 \mathrm{keV}$ ) Eq. (2.20) becomes more appealing. In both cases, however, the Philibert choice of most probable scattering angle $\pi / 1$ is chosen, since it represents the condition of cqual scattcring in both the horizontal and forward directions. Upon rearranging, one obtains the following equations for the onset of the diffusion depth for high and low energies, respectively, as: 


$$
\begin{aligned}
& \underset{\text { diff }}{\text { high } E}=\frac{w_{A}}{\bar{\rho} \cdot \bar{z}^{2}} \cdot\left(\frac{\pi E_{0}}{1600}\right)^{2} \\
& t_{\text {diff }}^{\text {low } E}=\frac{{ }{ }_{A} \cdot E_{0}}{{\bar{\rho} \cdot \bar{Z}^{3 / 2}}_{2}} \cdot\left(\frac{\pi}{438}\right)^{2} .
\end{aligned}
$$

As with the backscatter coefficient, $k_{A B}$ is then determined by setting $r_{A B}(t)$ equal to $95 \%$ of the bulk value. Substituting into Eq. (2.13) and rearranging terms, one can show:

$$
k_{A B}=\frac{1}{\bar{\rho} t_{\operatorname{diff}}} \cdot \ln \left(\frac{0.05}{1-\frac{R_{0}}{R_{\infty}}}\right)
$$

where $t_{\text {diff }}$ is determined from either 2.22 or 2.23 , whichever becomes most appropriate.

Combining Eqs. (2.2) through (2.24) one can begin the calculation of $x$-ray generation for a thin sample. Consider first the case of infinitely thin slab, dz, from Eq. (2.2); the generated intensity ratio of element $A$ to $B$ is:

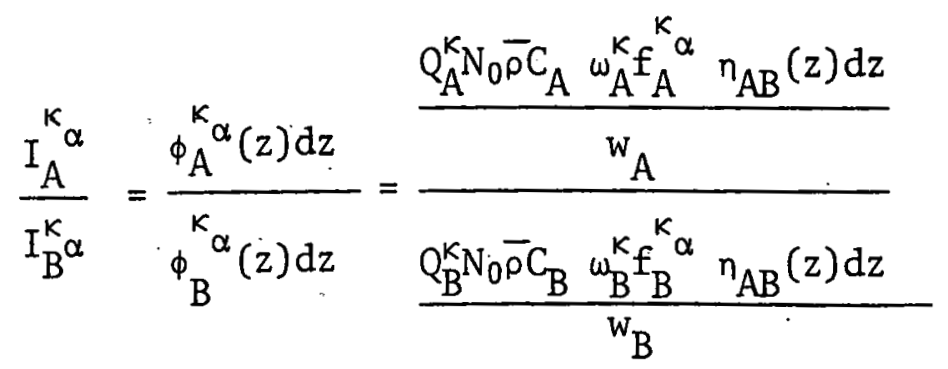

which simplifies to 


$$
\frac{I_{A}^{{ }^{\alpha} \alpha}}{I_{B}^{k}{ }^{\alpha}}=\frac{Q_{A}^{k}}{Q_{B}^{k}} \cdot \frac{w_{B}}{w_{A}} \cdot \frac{\omega_{A}^{{ }^{k}} f_{A}^{{ }^{k}}{ }^{\alpha}}{\omega_{B}^{k^{k} f_{B}{ }^{k}}} \cdot \frac{C_{A}}{C_{B}},
$$

resulting in the simple relation that for an infinitely thin film the relative intensity ratio is directly proportional to the concentration ratio. The constant of proportionality here is a function of the ionization cross sections, the atomic weights, and the $x$-ray yields of the elements under excitation.

For a sample of finite thickness $t_{0}$ the intensity ratio can be written as a ratio of integrals of Eq. (2.2)

$$
\frac{I_{A}^{{ }^{\alpha}}\left(t_{0}\right)}{I_{B}^{K^{\alpha}}\left(t_{0}\right)}=\frac{\int_{0}^{t_{0}}{ }_{\phi}^{k^{\alpha}}(z) d z}{\int_{0}^{t_{0}{ }_{\phi}{ }^{k} \alpha}(z) d z},
$$

both of which must be evaluated along each electron's path within the sample. It is important at this point to recall that ${ }_{\phi_{A}}^{K_{u}}(z)$ has been formulated as several constants multiplied by two functions $Q_{A}^{k}(z)$ and $\eta_{A B}(z)$. $Q_{A}^{K}(z)$ is a function of path length; however $\eta_{A B}(z)$ has been expressed as a function of depth $(t)$. The two quantities are in general not equal (path length $\geqslant$ thickness); however, for the special case of thin films and high incident beam energies the difference will be small. Thus to a good first approximation (for TEM specimens) one can equate the two variables and proceed with the integration.

The results of such an integration are shown in Fig. 19, which was evaluated by numerically integrating Eq. (2.27) for a 50/50 atomic 


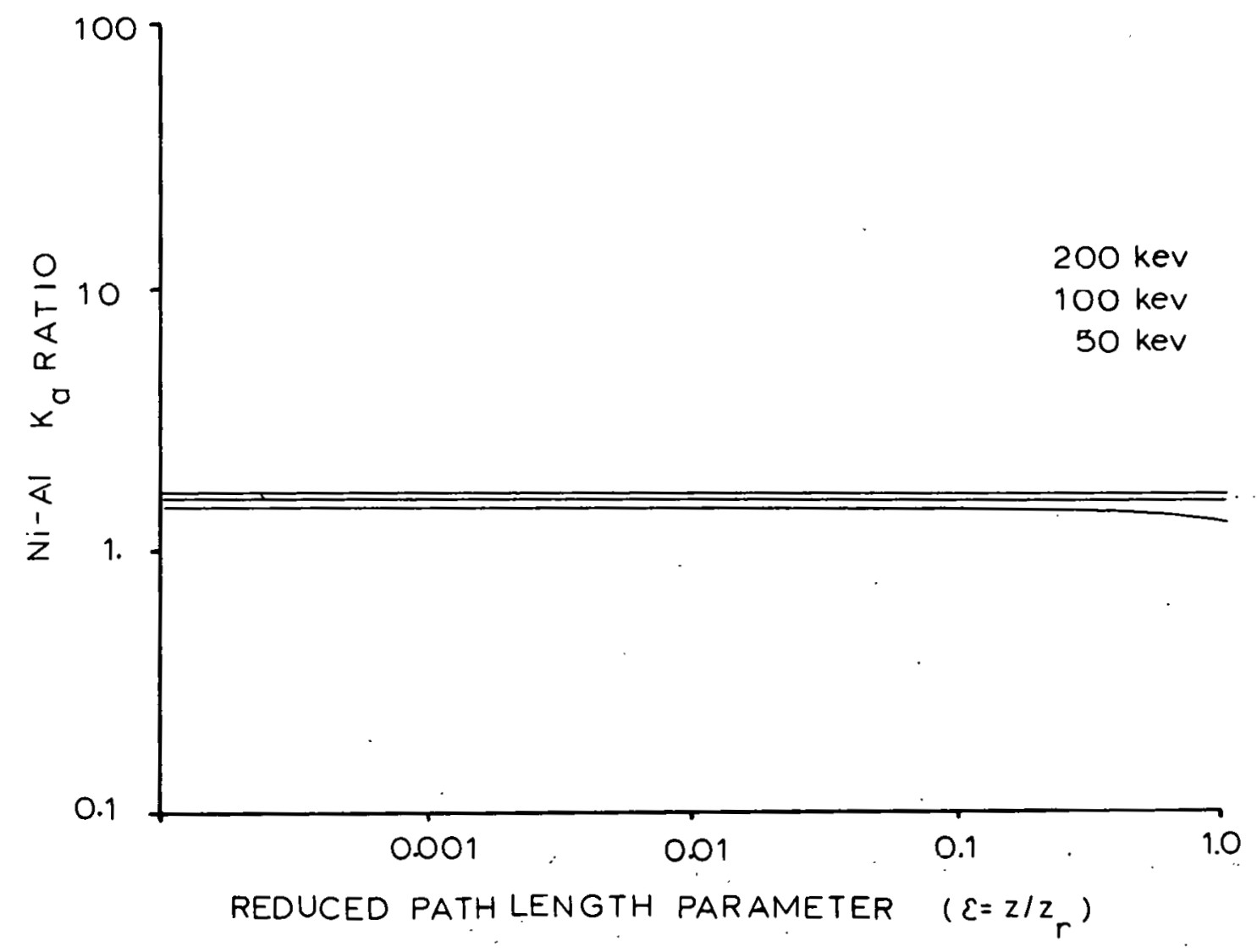

Fig. 19. Results of Calculation of Ni/A1 Ratio by Numerical Integration of Eq. (2.27) for Incident Electron Energies of 200, 100, and $50 \mathrm{keV}$ (top, midale, and bottom curves, rcspectivcly). 
percent alloy of NiAl. The ordinate $\zeta$ is a reduced path-length parameter $\left(\zeta=z / z_{R}\right)$ and is defined such that when its value becomes unity the mean electron energy has dropped below the $x$-ray critical excitation edge. At this point the electrons have reached their maximum usable range in the material. Obviously these calculations have been extrapolated significantly beyond the point where the path length/thickness approximation breaks down. Furthermore, once the electron diffusion depth is reached, the forward scattcred flux $f(t, \pi / 2)$ in Eq. (2.10) should in reality be replaced by Lenard's law of electron transmission

$$
f(t, \pi / 2)=f_{0} \exp (-\sigma t)
$$

rather than Bothe's multiple scattering theory for the sample (here $\sigma$ is the electron attenuation coefficient). Although both of these effects should be included in order to accurately describe the generation process, their emission at this point will not alter the calculated ratio. The simple reason for this is that the inclusion of these terms merely changes the net electron flux crossing a given layer and therefore affects the generation process equally for both elements. Thus there will be no net change in the calculated ratio.

The theory up to this point has yiclded a useful result - namcly that the generated $x$-ray ratio is nearly independent of path length (hence also thickness) for a wide range of incident energies (50 to $200 \mathrm{keV})$. The slow decrease in the calculated ratio predicted in the $50 \mathrm{keV}$ calculations of Fig. 19 reflects the fact that as the electrons approach their respective maximu ranges $(\zeta \equiv 1)$ changes in the ioniza*. tion cross section begin to favor the element with the lowest critical 
excitation energy $\left(E_{A}^{k}\right)$. Similar results are also predicted in the 100 and $200 \mathrm{keV}$ calculations; however, scale factors prohibit their observation. Thus it would appear that for samples whose thickness is such that the total path length traveled is less than $0.25 \mathrm{z}_{\mathrm{R}}$, Eq. (2.26) is ideally suited for quantitative analysis. Unfortunately, the effects of $x$-ray absorption must frequently be included in order to correctly interpret results from many TEM-based analyses.

\subsubsection{The Absorption Correction}

The inclusion of $x$-ray absorption effects to the calculation of $x$-ray emission is fundamentally a simple concept. The generated intensity, at each point within the specimen, must travel through a finite amount of material in order to be detected and, as such, suffers a reduction in intensity due to absorption. This can be expressed as

$$
{ }_{\phi_{A}}^{{ }^{\alpha}}(\mathrm{d}, \mathrm{t})={ }_{\phi_{A}}^{\kappa_{\alpha}}(\mathrm{t}) \cdot \exp \left\{-\left(\frac{\mu}{\rho}\right)_{A B}^{\kappa_{\alpha}, A} \cdot \bar{\rho} \cdot d\right\}
$$

where $\phi_{A}^{K_{\alpha}}(d, t)$ is the number of $K_{\alpha}$ photons of $A$ generated at depth $t$ leaving the specimen after having traveled a distance $d$ within a medium of mass absorption coefficient $\left(\frac{\mu}{\rho}\right)_{A B}^{K_{\alpha}, A}$, and $\phi_{A}{ }^{\alpha}(t)$ is the initial generated intensity at that depth. The task at hand is clearly to determine the relationship between $t$, the depth of production, and $d$, the exiting path length, which is solely dictated by the system/sample geometry.

The geometry found in most electron microprobes is relatively simple. The electron probe strikes a plane surface usually at normal 
incidence as is shown in Fig. 20. Only those $x$ rays which leave the specimen in the direction of the x-ray detector, which is oriented at an elevation (or takeoff) angle $\theta_{E}$ relative to the sample surface, are recorded by the measuring system. Thus it is an easy exercise to show that the exiting path length $d$ is given by the following equation:

$$
\mathrm{d}=t \operatorname{cosec}\left(\theta_{E}\right) \text {, }
$$

where $t$ is the depth of production. Unfortunately, due to the constraints on detector size and position imposed by a transmission electron microscope objective lens pole piece, the geometry found in TEM is not as simple as this. In most TEM instruments the detector axis lies in the same plane as the specimen $\left(\right.$ hence, $\left.\theta_{E} \simeq 0^{\circ}\right)$ and it therefore becomes necessary to tilt the sample through some angle in order that characteristic $x$ rays generated by the electron probe can leave the sample and reach the detector system. This angle can be as much as 20 to $30^{\circ}$ owing to the added complications that the specimen stage and supporting mechanisms also tend to block the line of sight of the detector to the specimen. In some of the newer designs, AEM systems (both CTEM/STEM and DSTEM) have been modified such that the elevation angle, $\theta_{E}$, is nonzero $\left(\sim 15^{\circ}\right)$; however, one must still tilt the sample in order to optimize experimental conditions. Therefore, the $\mathrm{x}$-ray geometry found on most analytical electron microscope is shown in Fig. 21, which represents a plane section through the sample containing the incident beam direction and the detector axis. The elevation angle, ${ }_{E}$, is defined relative to an imaginary plane perpendicular to the incident beam direction and is nominally located at 


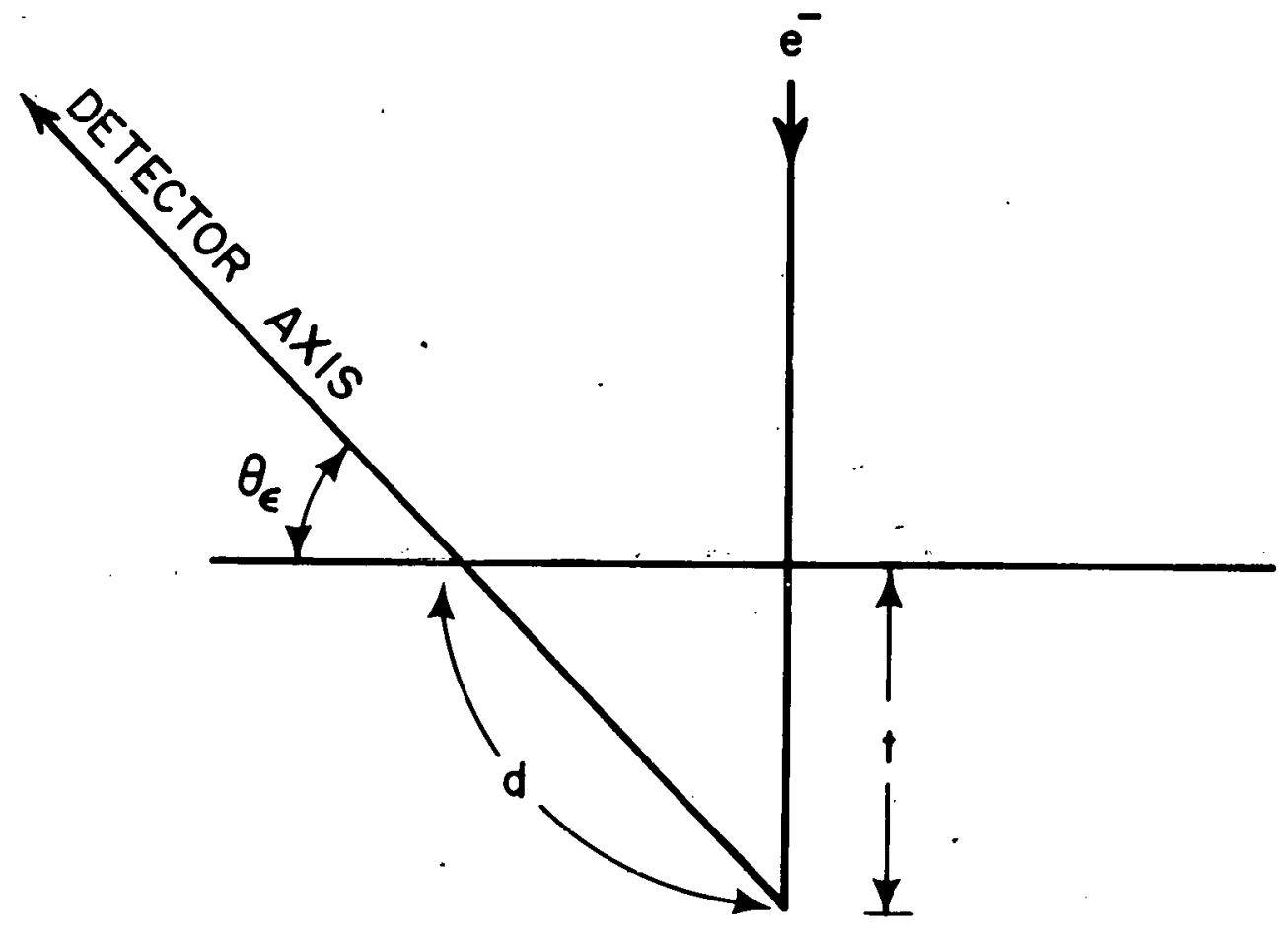

Fig. 20. Geometry of the Absorption Correction Commonly Found in Electron Microprobes. 


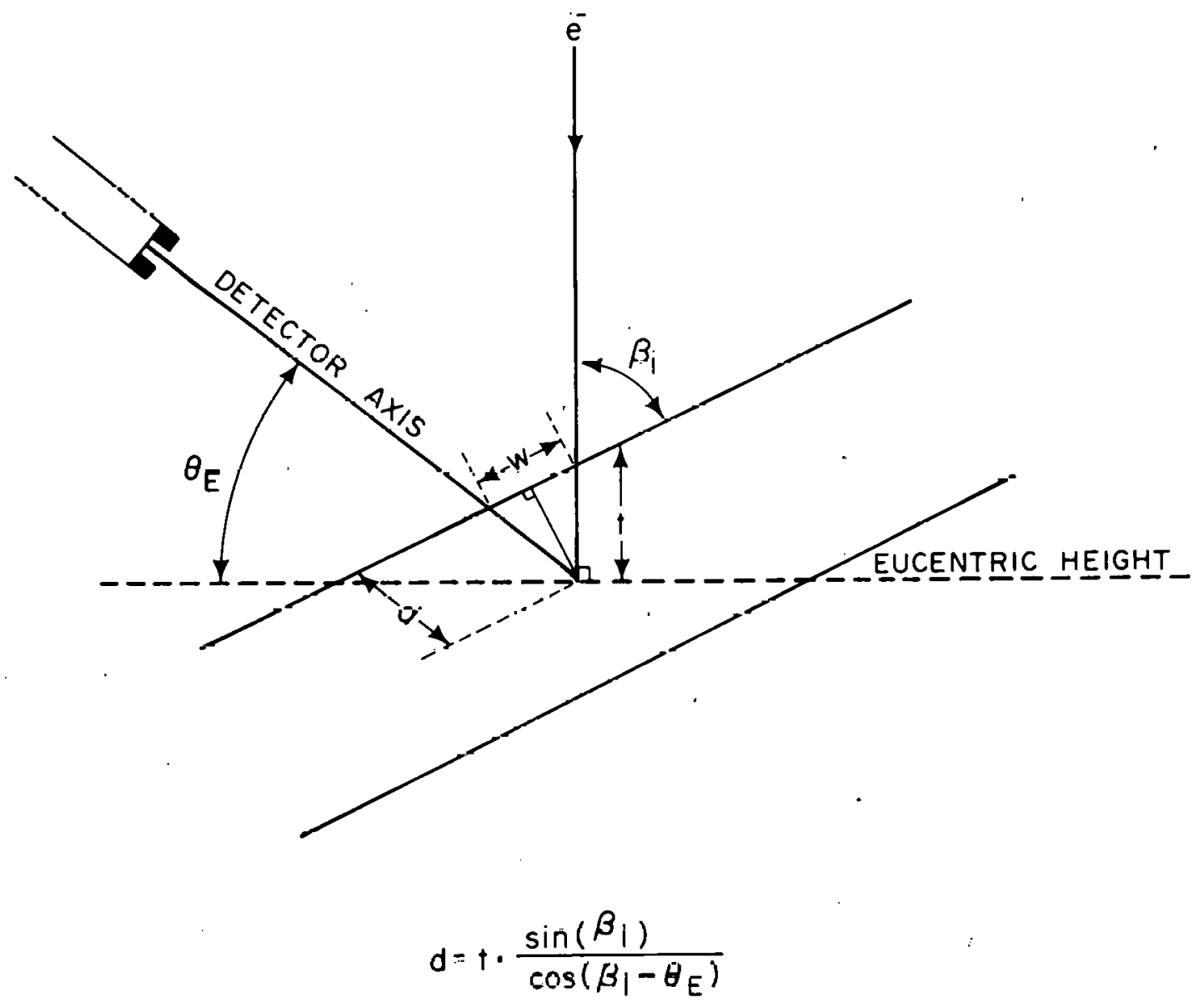

Fig. 21. Detector/Specimen Geometry for an AEM Instrument. 
the height of the midplane of the untilted specimen position. The inaccuracies involved with this definition of $\theta_{E}$ are obvious since for a tilted specimen $\theta_{E}$ actually is a function of $t$; however, for typical detector-specimen distances $(\sim 2 \mathrm{~cm})$ and sample thicknesses $(0.1$ to $1.0 \mu \mathrm{m})$ this error is insignificant. The electron incidence angle $\beta_{i}$ is also defined in Fig. 21, as the angle between the electron probe and the sample surface. From this figure one can show that the relationship between depth of production and exiting path length is given by:

$$
d=t \cdot \frac{\sin \left(\beta_{i}\right)}{\cos \left(\beta_{i}-\theta_{E}\right)} \text {. }
$$

It can be seen that for the special case of normal incidence $\left(\beta_{i}=\pi / 2\right)$ Eq. (2.31) reduces to (2.30). Implicit in this equation is the assumption that the sample can be considered to be a plane slab of uniform thickness. Obviously on a macroscopic scale most TEM specimens do not satisfy this criterion; however, again considering the scale on which most analytical work is done $(<1000 \AA)$ the approximation is reasonable. Care must, however, be taken in using Eq. (2.31) on samples which are severely bent or having significant thickness changes over distances whose dimensions are on the order of $\omega_{0}$ :

$$
\omega_{0}=t_{0}\left[\sin \beta_{i} \tan \left(\beta_{i}-\theta_{E}\right)+\cos \beta_{i}\right]
$$

where $\omega_{0}$ is the distance between the point at which the incident probe strikes the sample and the exit point for $x$ rays emitted from the maximum 
depth $\left(t_{0}\right)$ of production measured parallel to the incident beam direction (sec Fig. 21).

The angle of electron incidence, $\beta_{i}$, is similarly a function of the detector/specimen geometry. Although it is possible to determine $\beta_{i}$ using solid geometry it is more convenient to plot the various parameters on a stereographic projection from which the value of $\beta_{i}$ can be easily read. In urder to proceed it is necessary to first define a cartesian coordinate system within the microscope. The logical choice for such a system is the set of vectors defined by the two specimen translate direc: tions and the electron-optical axis, labeled $x, y$, and $z$, respectively, in Fig. 22. Relative to this coordinate system, one can uniquely specify the position of the $x$-ray detector using two angles. The azimuthal angle $\left(\theta_{A}\right)$ describes the relative position of the detector plane (i.e., the plane containing the detector axis and the electron-optical axis), and is defined as the rotation in the $x-y$ plane about the $z$ axis of this plane relative to either the $x$ or $y$ direction. The elevation angle, ${ }_{\mathrm{E}}$, was introduced previously and in this coordinate system is defined as the angle between the detector axis and the $x-y$ plane (measured in the detertor plane). Figure 22 illustrates these relationships using a standard cartesian coordinate system, and Fig. 23(a) shows the same relationships on a stereographic projection.

In the initial nontilted position the sample normal is oriented parallel to the electron optical axis. Knowing the amount of tilt and the relationship of the respective tilt axes to the reference coordinate 


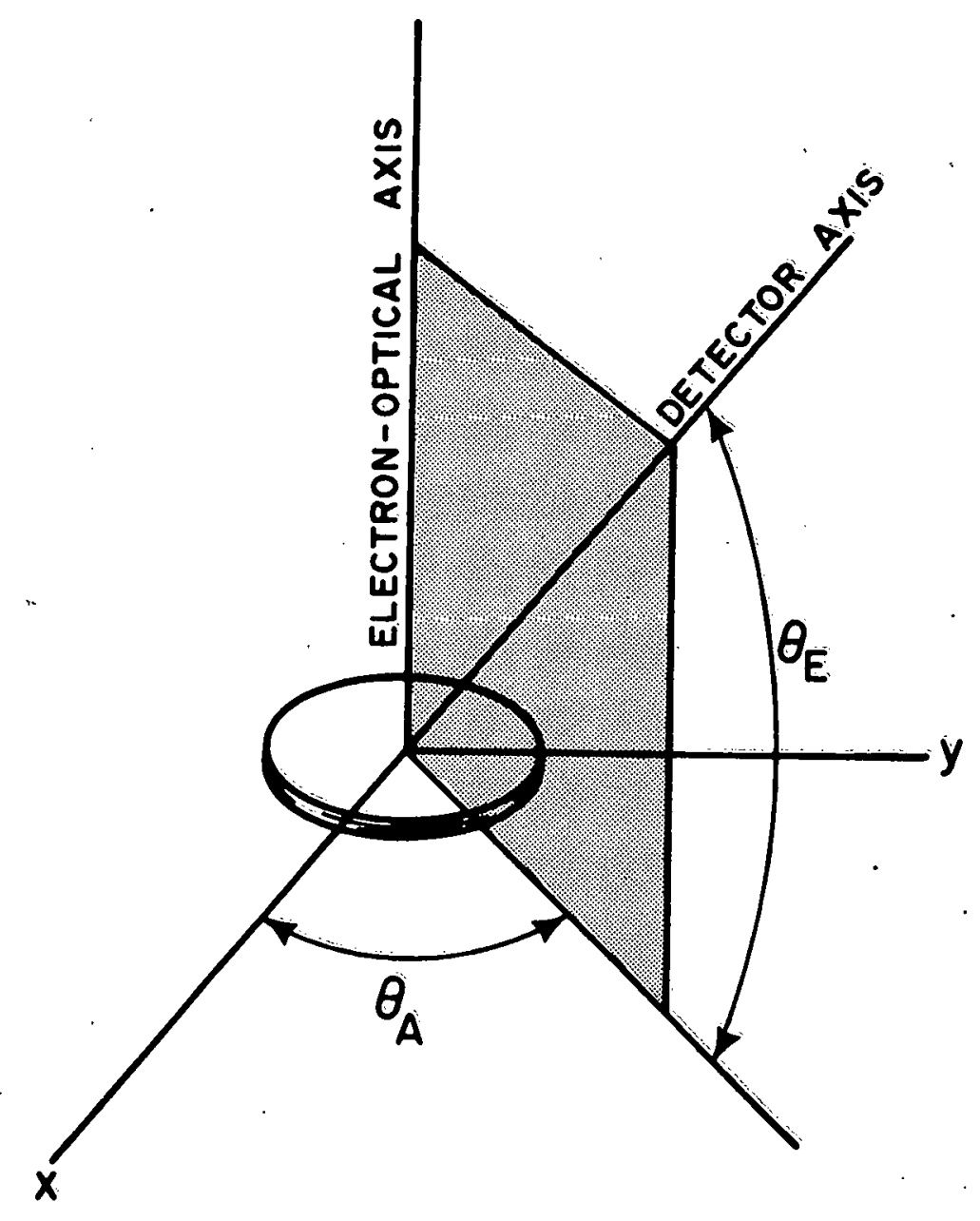

Fig. 22. Coordinate System Used in the Absorption Correction for AEM X-Ray Work. 

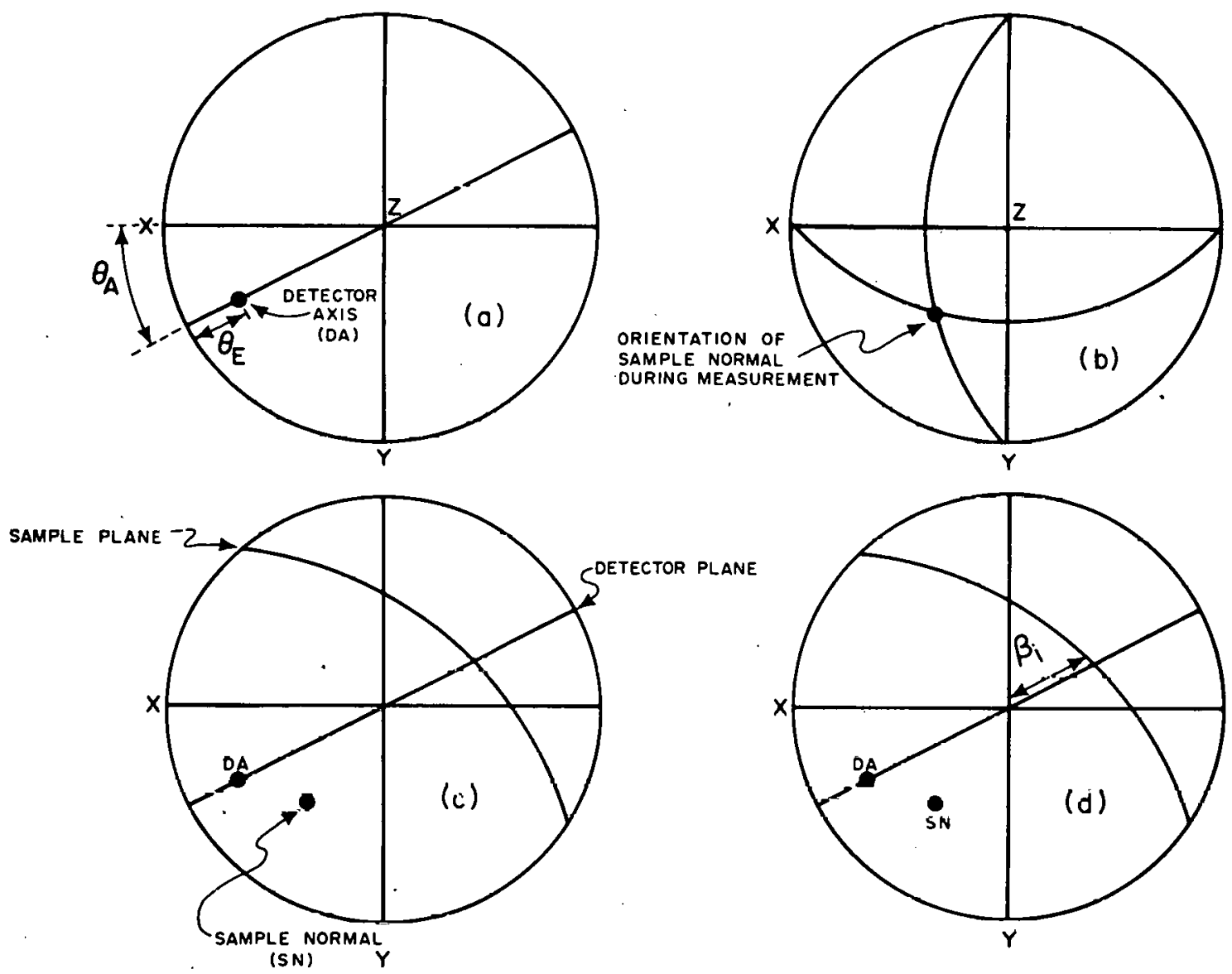

Fig. 23. Stereographic Projection Indicating the Absorption Correction Genmetry. 
system it is possible to plot the position of the sample normal on the stereographic projection [Fig. 23(b)]. Care must be taken in plotting the normal, particularly in determining the position of the tilt axes. This is due to the fact that in most double-tilt stages, the secondary tilt axis is usually not independent of the primary axis. This is an important point to remember when determining $\beta_{i}$, and is readily accounted for using the standard rules of stereographic projection. ${ }^{71}$ Having plotted the sample normal, one proceeds to draw in the detector plane and sample plane [Fig. 23(c)]. The angle of incidence, $\beta_{i}$, is then determined as the angle between the sample plane and $z$ axis measured in the detector plane [Fig. 23(d)].

The total characteristic intensity emitted in the direction of the detector system, including absorption effects, is given by:

$$
I_{A}^{{ }^{k} \alpha}=\int_{0}^{t_{0}{ }^{k}{ }^{k} \alpha}(z) \cdot \exp \left\{-\left(\frac{\mu}{\rho}\right)_{A B}^{\kappa_{\alpha}, A} \cdot \bar{\rho} \cdot d\right\} d z .
$$

and the intensity ratio is, upon substitution,

$$
\frac{I_{A}^{k_{\alpha}}}{I_{B}^{k_{\alpha}}}=\left(\frac{C_{A}}{C_{B}}\right) \cdot\left(k_{A B}\right) \cdot\left\{\frac{\int_{0}^{t_{0}} Q_{A}^{k}(Z) \cdot n_{A B}(Z) \cdot \exp \left\{-\left(\frac{\mu}{\rho}\right)_{A B}^{K_{\alpha}, A} \cdot \bar{\rho} \cdot t \cdot \frac{\sin \left(\beta_{i}\right)}{\cos \left(\beta_{i}-\theta_{E}\right)}\right\} \cdot d z}{\int_{0}^{t_{0}} Q_{B}^{k}(Z) \cdot n_{A B}(Z) \cdot \exp \left\{-\left(\frac{\mu}{\rho}\right)_{A B}^{K_{\alpha}, B} \cdot \bar{\rho} \cdot t \cdot \frac{\sin \left(\beta_{i}\right)}{\cos \left(\beta_{i}-\theta_{E}\right)}\right\} \cdot d Z}\right\}
$$

where

$$
k_{A B}=\frac{w_{B} \cdot \omega_{A}^{k} \cdot f_{A}^{k}}{w_{A} \cdot \omega_{B}^{k} \cdot f_{B}^{k}}
$$


The mass absorption coefficient $\left(\frac{\mu}{\rho}\right)_{A B}^{\kappa_{\alpha}}{ }_{A}$ for $K_{\alpha} x$-ray photons of element $A$ of the alloy $A B$ is defined as:

$$
\left(\frac{\mu}{\rho}\right)_{A B}^{\kappa} \alpha, A=\sum_{i=1}^{N}\left(\frac{\mu}{\rho}\right)_{i}^{K} \alpha, A C_{i}
$$

which is clearly a function of the composition of the compound under investigation. $C_{i}$ is the composition in weight percent of the $i^{\text {th }}$ element in the sample and $\left(\frac{\mu}{\rho}\right)_{i}^{\kappa} \alpha, A_{i}$ is the absorption coefficient for the $K_{\alpha} x$ ray of $A$ in the pure element $i$. Obviously the composition of the alloy is initially an unknown quantity; however, it is possible to determine $\left(\frac{\mu}{\rho}\right)^{\kappa} \alpha$ iteratively. As a first approximation the composition of the alloy is determined solely using the atomic number correction. Using these values, a first-order absorption correction is calculated. The new values of composition are then backsubstituted into the absorption term and the procedure is repeated until a converence is obtained. Figure 24 illustrates the change in the intensity ratio expected from the NiAl compound previously described with the inclusion of absorption [Eq. (2.34)]. This figure can be directly compared with 19, which represents a calculation of only the atomic number effect. In order to perform these computations the same approximations used in obtaining Fig. 19 were applied here. Of these, the most important is the path length/thickness approximation and, as in the case of the atomic number calculation, the computations have been extended well beyond their valid ranges. Because of this the results presented for thick filnts $(\xi \geqslant 0.1)$ 


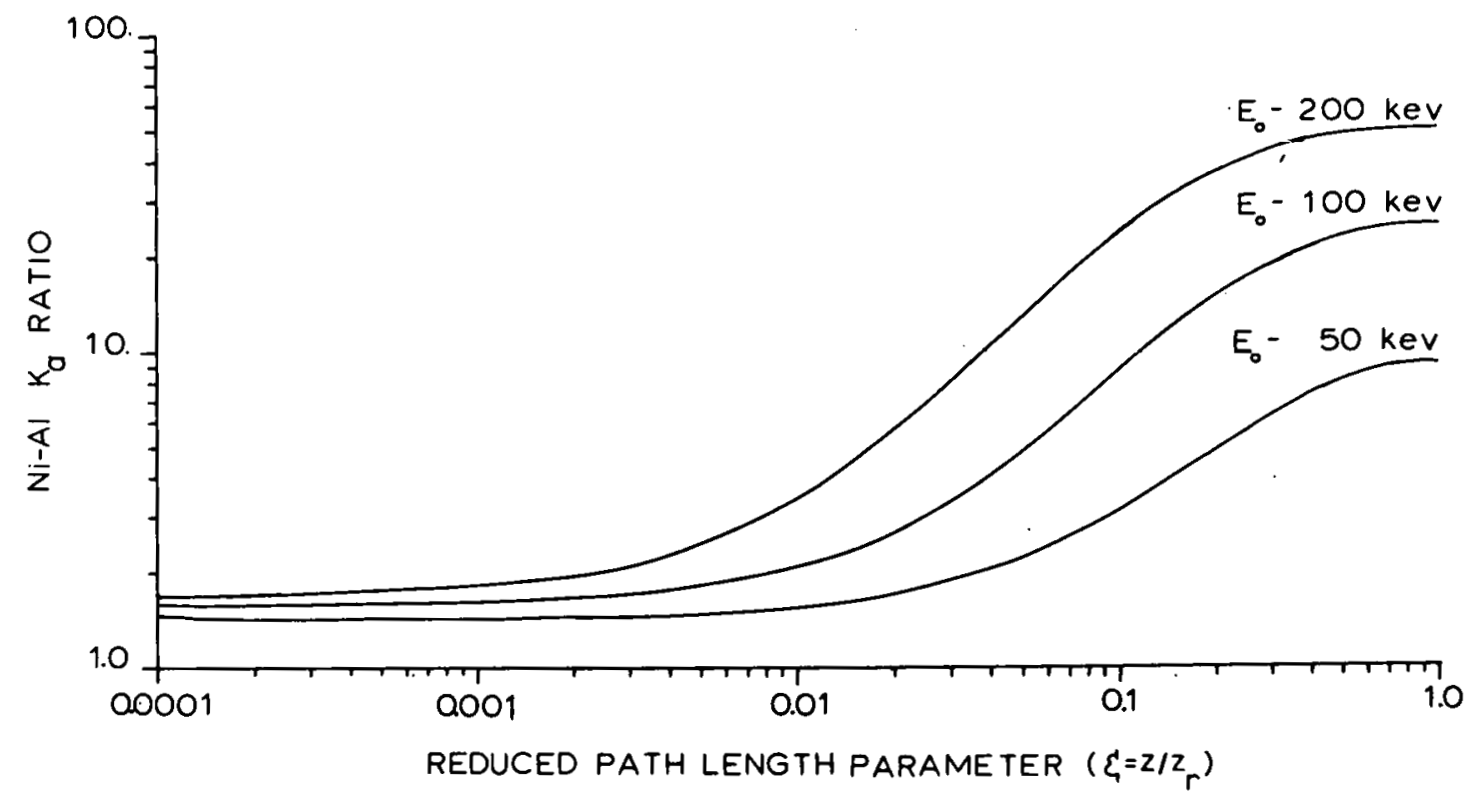

Fig. 24. Calculated Variation in Ni/A1 $\mathrm{K}_{\alpha}$ Ratio Including Absorption Effects for Various Incident Beam Energies. 
should be considered only as a qualitative illustration of absorption. In this range the intensity ratio initially increases due to the preferential absorption of the lower energy aluminum $K_{\alpha} \times$ rays $(1.48 \mathrm{keV})$ while the higher energy nickel $\mathrm{K}_{\alpha}$ line $(7.45 \mathrm{keV})$ is easily transmitted. U1timately, however, the ratio levels out as the sample thickness becomes sufficient to absorb a substantial portion of the nickel intensity. The variation below $\zeta=0.1$, the region of interest in most transmission work, however, merits further consideration particularly with respect to path length/thickness assumptions.

This approximation was initially invoked because the ionization cross section was formulated as a function of path length through the use of the rate of energy loss equations. On the other hand, the net electron flux across a given layer $\mathrm{dz}$ was expressed as a function of the depth, $t$, below the electron entrance surface. Obviously these two variables are in general not equivalent; however, because of the relatively slow variation in the values of the cross section for the energy ranges typically encountered in AEM, the discrepancy between these parameters can be ignored. This can be seen by considering the change in cross section $\left(Q_{k}\right)$ predicted by Eq. (2.4) for moderate $(\sim 10 \%)$ changes in energy in the vicinity of $100 \mathrm{keV}$. Reference to curve 1 of Figs. 16, 17 , and 18 shows that only minor changes are observed in the value of $\mathrm{Q}_{\mathrm{k}}$ in the region of $100 \mathrm{keV}(\mathrm{U}=64,12$, and 4 for aluminum, nickel, and silver, respectively). Thus, the inaccuracies involved in the calculations for $\zeta \leq 0.1$ obtained by equating path length and thickness are 
sma11. Furthermore, these inaccuracies decrease as the incident energy increases.

\subsubsection{The X-Ray Fluorescence Correction}

Up to this point we have been concerned with calculating the contributions to characteristic $x$-ray emission resulting solely from electron excitation of the sample. It is, however, equally possible to induce characteristic emission through an $x$-ray fluorescence process. Thus whenever an $x$-ray quantum of sufficient energy is absorbed by the sample there is a finite probability that it will ionize an inner core level and a certain fraction of these events will result in the emission of characteristic $x$ rays from that she11. There are a variety of sources of $x$-ray radiation which bombard the sample in a conventional TEM, all of which are nonlocalized and are thus detrimental to microanalysis. Most of these sources can be eliminated or reduced to negligible intensities by suitable modifications to the microscope column. However, those $x$ rays generated within the sample itself. cannot be eliminated; hence, the calculations outlined in the following section are presented in order to estimate their influence on characteristic emission.

Figure 25 illustrates. a schematic $x$-ray spectrum from a multielement sample composed of the elements A, B, and C. For simplicity, only the $\mathrm{K}$ lines of each element along with their respective absorption edges are indicated. In order to induce $x$-ray fluorescence of element $A$ the fluorescing radiation must have an energy greater than the critical excitation edge $\left(E_{A}^{k}\right)$; thus a11 $x$-ray photons in Fig. 25 , both 


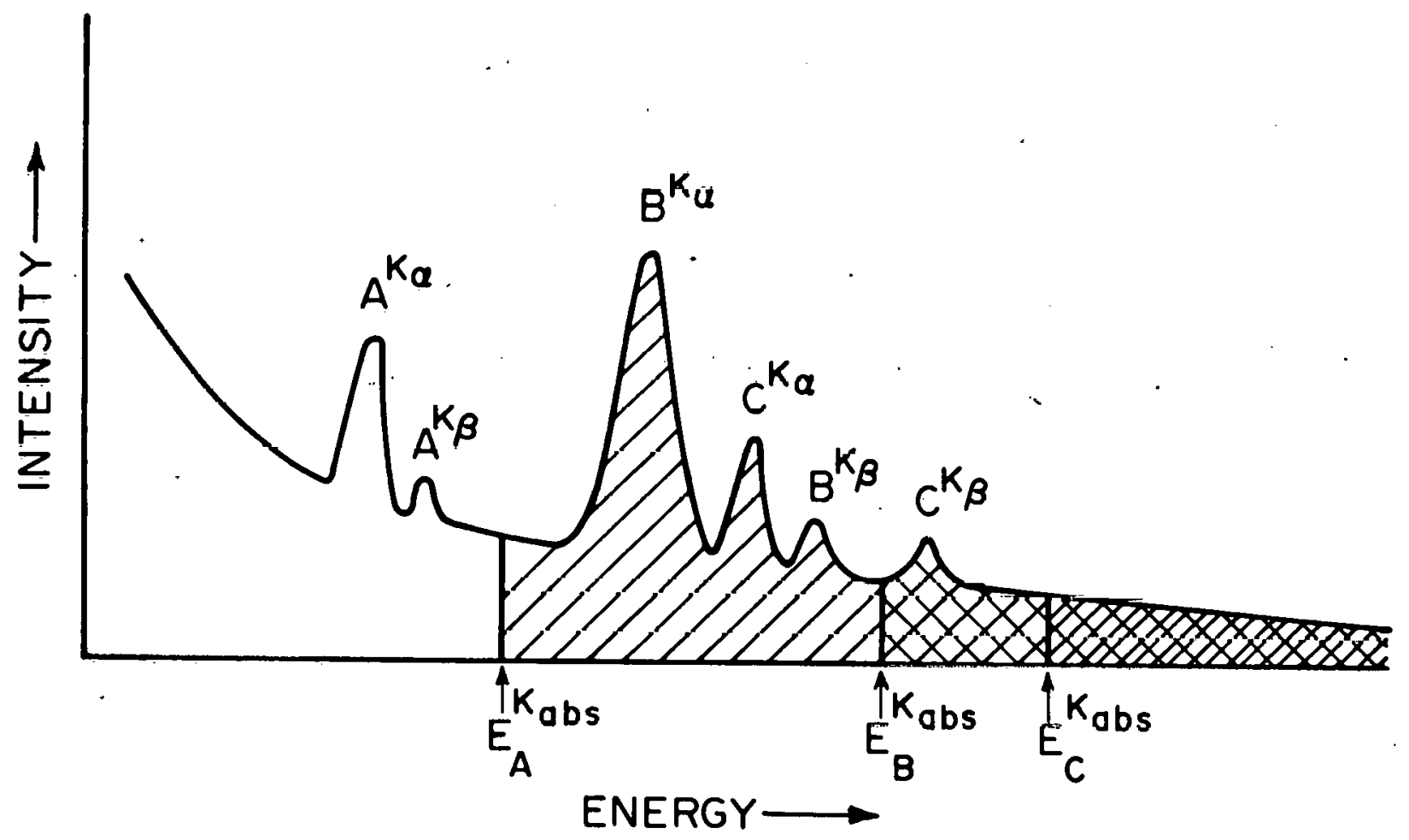

Fig. 25. Schematic X-Ray Spectrun Indicating Relative Absorption Edge Positiuns for Use in the X Ray Fluoroscence Correction. 
characteristic and continuum, whose energy is greater than $E_{A}^{K}$ are eligible to excite A. Element $B$ represents a slightly different case of fluorescence. Here the $K_{\alpha}$ line of element $C$ lies just below the $K$ edge of $B$; hence only the $K_{B}$ line of $C$ together with the remaining continuum spectrum can excite B. Finally, element C cannot be fluoresced by any characteris: tic lines of A or B and thus can only be enhanced via continurm fluorescence. For an electron microscope, correctly aligned and modified for $x$-ray microanalysis, the continum intensity generated by electron excitation of TEM specimens is small and fluorescence due to this source can generally be neglected. The following calculations are presented, therefore, to estimate the influence of only characteristic fluorescence. The total $K_{\alpha} x$-ray intensity of A emitted by samples can be written as:

$$
\mathrm{I}_{\mathrm{A}}^{\mathrm{Tota} 1}=\mathrm{I}_{\mathrm{A}}^{\mathrm{el}}+\mathrm{I}_{\mathrm{A}}^{\mathrm{fl}} \text {, }
$$

where $\mathrm{I}_{\mathrm{A}}^{\mathrm{el}}$ is the contribution due to electron excitation and $\mathrm{I}_{\mathrm{A}}^{\mathrm{fl}}$ the fluorescence contribution. Since the relative intensity due only to electron excitation is the parameter of interest for quantitative analysis the fluorescence correction can be written as:

$$
I_{\mathrm{A}}^{\mathrm{e} 1}=\frac{\mathrm{I}_{\mathrm{A}}^{\text {Total }}}{\left(1 .+\mathrm{I}_{\mathrm{A}}^{\mathrm{fl} / \mathrm{I}_{\mathrm{A}}^{\mathrm{e}}}\right)},
$$

and thus it is necessary to calculate the ratio $I_{A}^{f 1} / I_{A}^{e}$. In order to reduce the complexity of the formulae which follow we shall consider the case of fluorescence of element A only by a single characteristic line 
$\left(K_{\alpha}\right)$ of element $B$. The extension to the $K_{B}$ line or other lines as well as the addition of multiple elements is straightforward.

Let us assunie that $\mathrm{I}_{\mathrm{B}}^{\mathrm{ab}}$ is the total amount of $\mathrm{B} \mathrm{K} \mathrm{K}_{\alpha}$ radiation absorbed within the multi-element sample. Of this absorbed intensity the fraction

$$
C_{A} \cdot\left\{\frac{(\mu / \rho)_{A}^{\alpha, B}}{(\mu / \rho)_{B}^{\alpha, B}}\right\}
$$

will be absorbed by atoms of element $A$, where $C_{A}$ is the concentration of $A$ in fractional weight percent and $(\mu / \rho)_{C}{ }^{\alpha, D}$ is the mass absorption coefficient for $\mathrm{K}_{\alpha} \mathrm{x}$ rays of element $\mathrm{D}$ in the pure element C. Furthermore the fraction

$$
\left(\mathrm{r}_{\mathrm{K}}^{\Lambda}-1\right) / \mathrm{r}_{\mathrm{K}}^{\mathrm{A}}
$$

of this intensity will be absorbed by the $K$ shell of $A$, and hence the total number of ionization events of the $K$ shel1 of $A$ due to $x$-ray fluorescence $\left(Q_{A}^{K}, A b\right)$ is given by:

$$
Q_{A}^{K, A b}=C_{A} \cdot\left\{\frac{(\mu / \rho)_{A}^{K_{\alpha}, B}}{(\mu / \rho)_{B}^{{ }^{k}, B}}\right\} \cdot\left\{\frac{r_{K}^{A}-1}{r_{K}^{A}}\right\} \cdot I_{B}^{a b}
$$

with the quantity $r_{K}^{A}$ defined as the $K$ absorption edge jump ratio of element A.

The total yicld of $\mathrm{K}_{\alpha} \times$ rays from clement $\Lambda$ due to this ionization is given by the product of $Q_{A}^{K, A b}$ and the relative yield of $K_{\alpha} \times$ rays from the $K$ she11 $\left(\omega_{A}^{K} \cdot f_{A}^{K}\right)$ 


$$
I_{A}^{f 1}=C_{A} \cdot\left\{\frac{\left(\frac{\mu}{p}\right)_{A}^{K}}{\left(\frac{\mu}{\rho}\right)^{K}{ }_{B}{ }_{\alpha}, B}\right\} \cdot\left\{\frac{r_{K}^{A}-1}{r_{K}^{A}}\right\} \cdot \omega_{A}^{K} \cdot f_{A}^{K_{\alpha}} \cdot I_{B}^{a b}
$$

An exact calculation of $\mathrm{I}^{\mathrm{fl}}$ actually requires that $\mathrm{I}_{\mathrm{B}}^{\mathrm{ab}}$ be calculated at each point in the sample. The fluorescence intensity at each point having been determined, an absorption correction to the intensity emitted in the direction of the detector system is then calculated using the procedure outlined previously. Realistically the fluorescence correction is small; hence it becomes reasonable to make certain simplifying approxinations. Firstly the total absorbed intensity of the characteristic line of $B$ can be approximated by:

$$
I_{B}^{a b}=\frac{1}{2} \cdot I_{B}{ }^{\kappa_{\alpha}} \cdot \exp \left\{\left(-\frac{\mu}{\rho}\right)^{{ }^{\kappa}}{ }^{\alpha, B} \cdot \bar{\rho} \cdot t_{0}\right\}
$$

where $I_{B}{ }^{k}{ }^{\alpha}$ is the total characteristic emission generated in the sample of thickness to purely by electron excitation, and $(\mu / \rho)_{A B}^{K} \alpha, B$ the mass absorption coefficient of the compound for $B \mathrm{~K}_{\alpha}$ radiation as previously defined in Eq. (2.35). Secondly; let. us. further assume that all the fluorescence intensity is emitted in the direction of the detector system from the midpoint of the sample thickness which results in the following expression for $\mathrm{I}_{\mathrm{A}}^{\mathrm{f1}}$

$$
\begin{aligned}
& I_{A}^{f 1}=\frac{1}{2} \cdot C_{\Lambda} \cdot\left\{\frac{\left(\frac{\mu}{\rho}\right)_{A}^{K}{ }_{\alpha, B}}{\left(\frac{\mu}{\rho}\right)_{B}^{K_{\alpha, B}}}\right\} \cdot\left\{\frac{r_{K}^{A}-1}{r_{K}^{A}}\right\} \cdot \omega_{A}^{K} \cdot f_{A}^{K^{\alpha}} \cdot \exp \left\{-\frac{3}{2} \cdot\left(\frac{\mu}{\rho}\right)_{A B}^{K_{\alpha, B}} .\right. \\
& \left.\cdot \bar{\rho} \cdot t_{0} \cdot\left(1+\frac{\sin \beta_{i}}{\cos \left(\beta_{i}-\theta_{E}\right)}\right)\right\} \cdot I_{B}^{K^{\alpha}}
\end{aligned}
$$


Fina11y, dividing by $\mathrm{I}_{\mathrm{A}}{ }^{\alpha}$ we obtain the following approximate expression for $\mathrm{I}_{\mathrm{A}}^{\mathrm{fl}} / \mathrm{I}_{\mathrm{A}}^{\mathrm{el}}$ for a $\mathrm{TEM}$ specimen as:

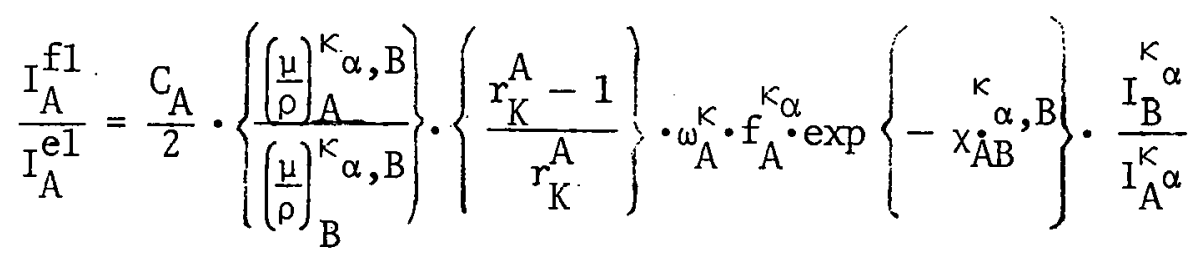

with

$$
\chi_{A B}^{\kappa_{\alpha, B}}=\frac{3}{2} \cdot\left(\frac{\mu}{\rho}\right)_{A B}^{\kappa}{ }_{\alpha, B} \cdot \bar{\rho} \cdot\left(1+\frac{\sin \beta_{i}}{\cos \left(\dot{\beta}_{i}-\theta_{\bar{E}}\right)}\right) \cdot t_{0} .
$$

Al1 quantities have been previously defined. Clearly from Eq. $(2,41)$ if absorption effects within the sample are sma1l the fluorescence intensity will be minimal. It is seldom the case that this correction amounts to more than a few percent change and is usually negligible for atomic numbers less than 20. For a given element $A$, the magnitude of $\mathrm{I}_{\mathrm{A}}^{\mathrm{fl}} / \mathrm{I}_{\mathrm{A}}^{\mathrm{el}}$ decreases with increasing separation of the atomic number $B$. The mosst important range for this correction is the transicton mletals lilauiun through zirconium, particularly combinations such as $\mathrm{Ni}-\mathrm{Fe}$.

\subsubsection{Detector Efficiency}

After being emitted from the sample, the $x$-ray inlensity must be measured by some type of detector system. Usually. in AEM systems solid state $\mathrm{Si}(\mathrm{Li}) \mathrm{x}$-ray detectors are used because of their compact size. These detectors have an inherent efficiency in their response to $x$-ray events, the most important of which is due to absorption effects in the window material limiting the detector sensitivity to low-energy $\mathrm{x}$ rays. 
This absorption must be accounted for in order to correctly convert intensity measurements to quantitative results. The reduction in intensity can be calculated from the expression:

$$
I^{\text {Measured }}=I(E) \cdot \underbrace{N}_{i=1} \exp \left(-\left(\frac{\mu}{\rho}\right)_{i}^{E} \cdot \rho_{i} \cdot \frac{t_{i}}{\cos \alpha}\right)
$$

where $\pi$ is the usual notation for the product of $i$ terms, $(\mu / \rho)^{E}$ is the mass absorption coefficient of element $i$ for $x$ rays of energy $E, \rho$ the density of $i$, and $t_{i}$ the thickness of the respective absorbing medium along the detector axis. The factor $\cos \alpha$ accounts for increases in path length which will be experienced if the $\mathrm{x}$ rays do not enter the detector parallel to its axis. The angle $\alpha$ is defined in Fig. 26. Figure 27 plots the calculated efficiency for a typical solid state Si(Li) detector as a function of incident $x$-ray energy using the following typical detector parameters:

$$
\begin{aligned}
\text { Window thickness } & \mathrm{Be} & =0.008 \mathrm{~mm} \\
\text { Window thickness } & \mathrm{Au} & =0.02 \mu \mathrm{m} \\
\text { Si dead layer } & & =0.1 \mu \mathrm{m} \\
\alpha & & =0^{\circ}
\end{aligned}
$$

The steps in the curve correspond to absorption edges of $\mathrm{Au}$ and $\mathrm{Si}$. Above $20 \mathrm{keV}$ the efficiency of the detector begins to fall again due to transmission of the high-energy $x$ rays through the silicon crystal (nominally $4 \mathrm{~mm}$ thick); however, it is usually the case that for these elements one changes to the $L$ lines rather than the higher energy K series. 


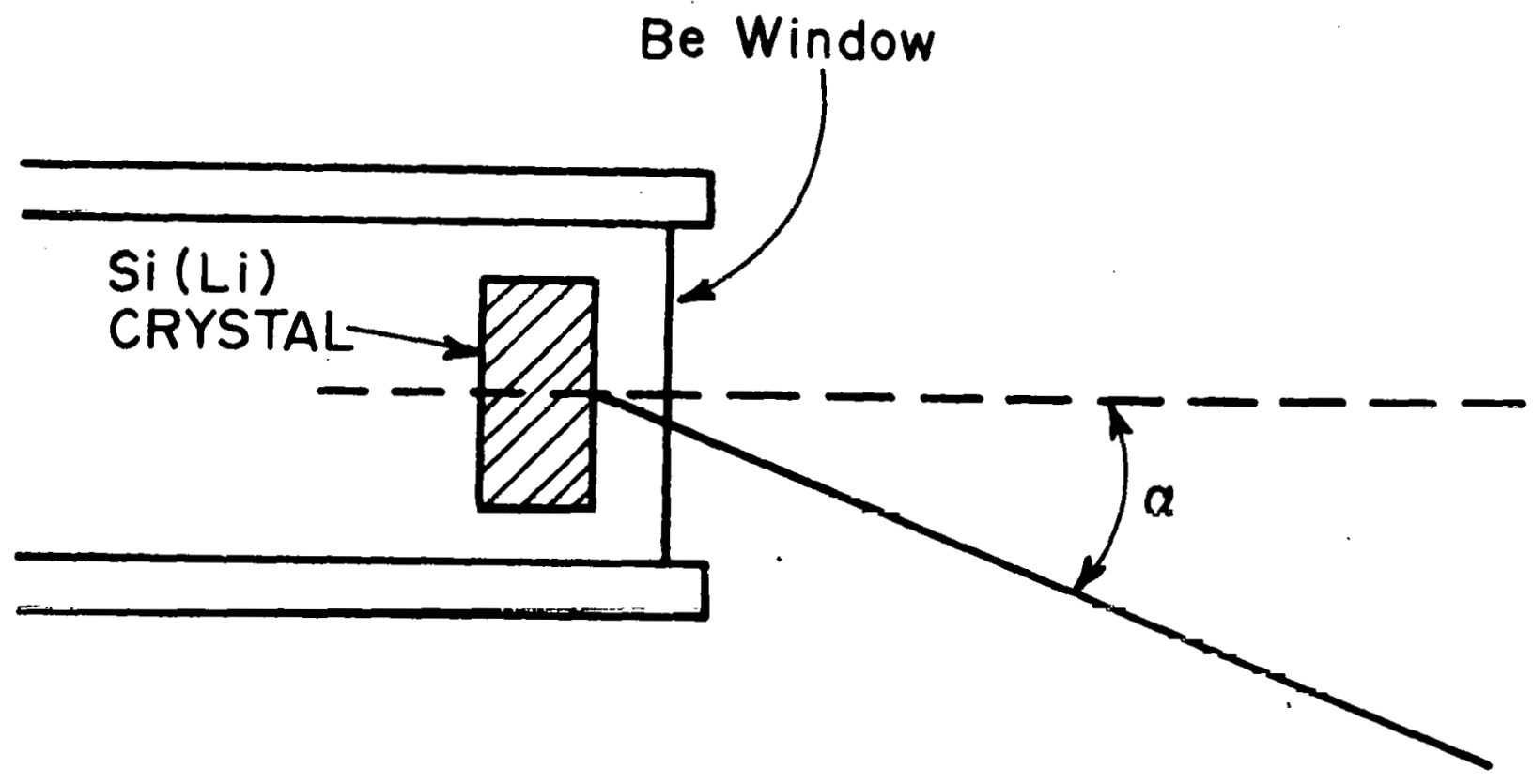

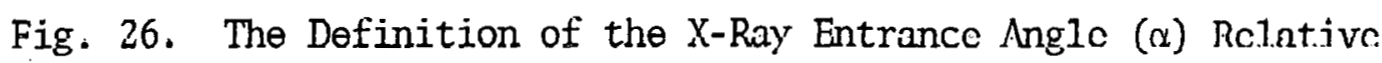
to Detector Axis. 


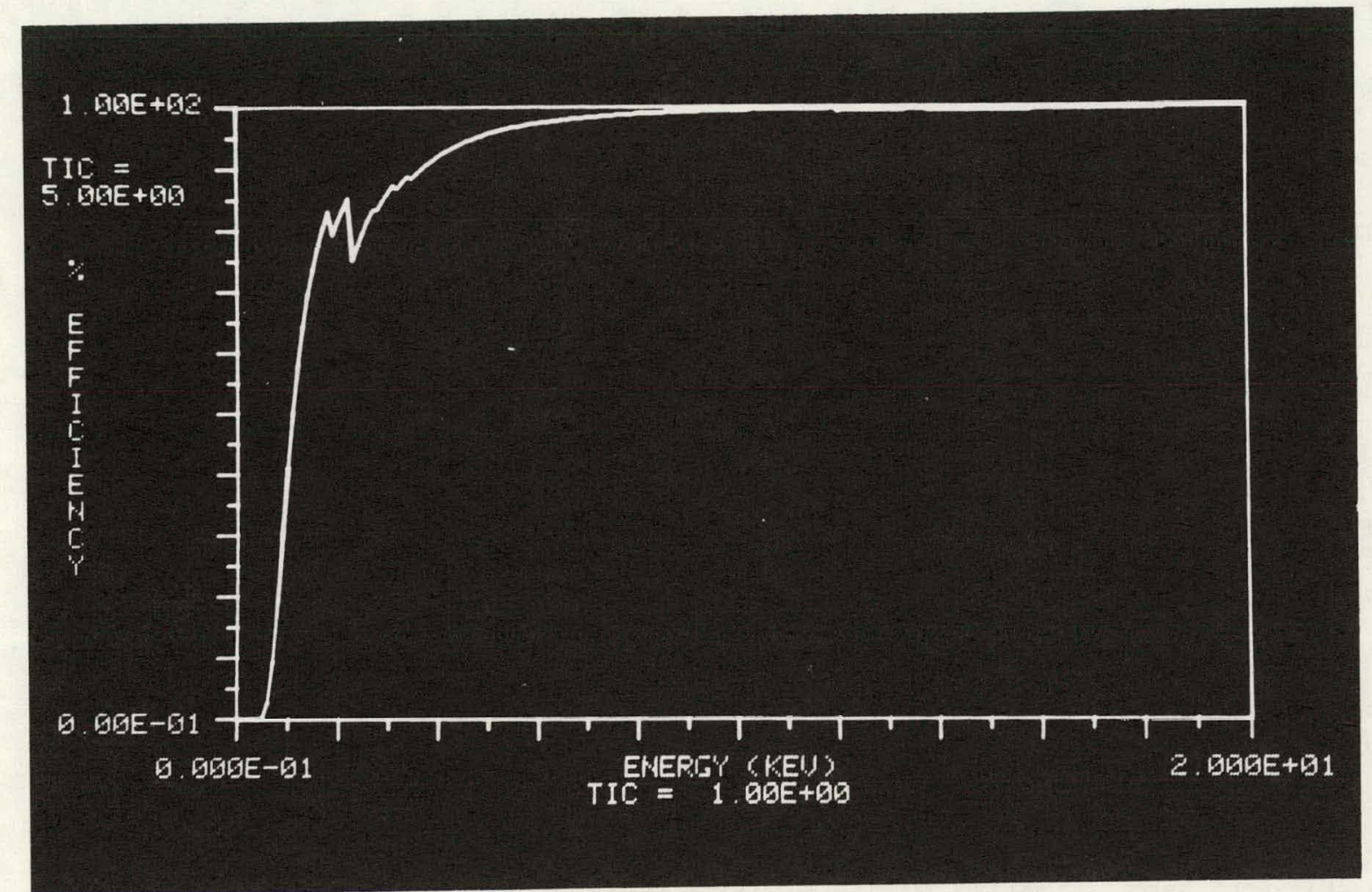

Fig. 27. Calculated Detector Efficiency for a Si(Li) Detector Due to Absorption Effects in the Be Window Au and Si Dead Layers, 


\subsubsection{Optimum Experimental Conditions for X-Ray Analysis}

The ultimate sensitivity of $x$-ray microchemical analysis in an electron microscope is a function of the specific operating conditions which exist during the experimental measurements. In general one wants to maximize the characteristic information measured from the specimen and minimize all other emission. The parameter which is used to express the sensitivity of $x$-ray analysis is then the ratio of characteristic (peak) to continum (background) intensities. Several factors influence this ratio and the calculations outlined in this section are specifically formulated to assess the effects of incident beam energy, specimen/ detector geometry and sample composition on the peak-to-background (P/B) ratio. These computations will assume that the sample is thin enough so that complications resulting from electron scattering, $x$-ray absorption and x-ray fluorescence can be neglected. Furthermore, instrumental factors associated with systems peaks, bremsstrahlung fluorescence, electron tails, and sample contamination are not considered since such artifacts must be eliminated in order to obtain meaningful $x$-ray results in all cases. A discussion of these artifacts and their minimization can be found in Sect. 2.2.7.

Let us consider an infinitely thin sample of thickness, dt. The number of characteristic $x$-ray photons emitted into the solid angle $d \Omega$ can from the previously described formalism be written as:

$$
I_{p}^{A}=\frac{Q_{A}^{k} \cdot N_{0} \cdot \bar{\rho} \cdot C_{A} \cdot{ }^{k}{ }_{A}^{k} \cdot f_{A}{ }^{\alpha} \cdot n_{A B}(t) \cdot d t \cdot d \Omega}{{ }_{A}{ }^{\cdot 4 \pi}},
$$


where all parameters in Eq. (2.47) have been previously defined, the emission being isotropic over $4 \pi$ steradians. The continuum intensity is, on the other hand, highly anisotropic and is strongly influenced by relativistic effects. The number of continuum photons within the energy window $E_{B}$ to $E_{B}+d_{B}$ emitted at an angle $\Omega$ with respect to the forward scattering direction is given by:

$$
I_{B}=I_{\Omega} \cdot \frac{N_{0} \cdot \bar{\rho} \cdot n_{A B}(t) \cdot d E_{B} \cdot d t \cdot d \Omega}{W_{A} \cdot E_{B}},
$$

with

$$
\begin{aligned}
& \left.I_{\Omega}=I_{X}\left\{\frac{\sin ^{2} \Omega}{(1-\beta \cos \Omega}\right)^{4}\right\}+I_{y}\left\{1+\frac{\cos ^{2} \Omega}{(1-\beta \cos \Omega)^{4}}\right\} \\
& \beta=v / c=\text { relativistic correction factor. }
\end{aligned}
$$

$I_{x}$ and $I_{y}$ in Eq. (2.47) are the continuum radiation components resulting from the slowing down of the electrons in the sample. The calculation of these components has been determined from the theory of Sommerfeld ${ }^{72}$ by Weinstock; ${ }^{73}$ however, the routine evaluation of his expressions is nontrivial. Kirkpatrick and Weidmann ${ }^{74}$ have succeeded in obtaining the following analytic expressions for $I_{x}$ and $I_{y}$ which are accurate to $2 \%$ relative to the more complex computations.

$$
\begin{aligned}
I_{x} & =\left\{\frac{0.252+a_{2}\left(d_{1}-0.135\right)-b_{2}\left(d_{1}-0.135\right)^{2}}{c_{1}}\right\} \cdot 1.51 \times 10^{-28} \\
a_{2} & =1.47 b_{1}-0.507 a_{1}-0.833 \\
b_{2} & =1.70 b_{1}-1.09 a_{1}-0.627 \\
a_{1} & =\exp \left(-0.223 c_{1}\right)-\exp \left(-57 c_{1}\right)
\end{aligned}
$$




$$
\begin{aligned}
b_{1} & =\exp \left(-0.0828 c_{1}\right)-\exp \left(-84.9 c_{1}\right) \\
c_{1} & =\frac{E_{0}}{300 z^{2}} \\
d_{1} & =\frac{E_{B}}{E_{0}} \quad I_{y}=\left\{-j_{1}+\frac{k_{1}}{\left(d_{1}+h_{1}\right)}\right)\left(\frac{1.51 \times 10-28}{c_{1}}\right) \\
k_{1} & =\frac{-0.214 e_{1}+1.21 f_{1}-g_{1}}{1.43 \quad e_{1}-2.43 f_{1}+g_{1}} \\
j_{1} & =\left(1+2 h_{1}\right) f_{1}-2\left(1+h_{1}\right) g_{1} \\
k_{1} & =\left(1+h_{1}\right)\left(g_{1}+j_{1}\right) \\
e_{1} & =0.220\left[1-0.39 \text { exp }\left(-26.9 c_{1}\right)\right] \\
f_{1}= & 0.067+\frac{0.023}{\left(c_{1}+0.75\right)} \\
g_{1}= & -0.00259+\frac{0.00776}{\left(c_{1}+0.116\right)} \\
L= & \text { atomic number uf specimen. }
\end{aligned}
$$

It should be noted that the preceding equations $(2.48,2.49,2.50$, and 2.51) have been rewritten using the MKS system of units rather than the Gaussian units found in the original work. The units of $I_{\Omega}$ become $\mathrm{m}^{2} /$ steradian/atom/electron when all energies are entered in electron volts.

A polar plot of the angular distribution of continuum $x$-ray radiation generated by 10 and $100 \mathrm{keV}$ electrons incident on a pure nicke1 specimen $(Z=28)$ is shown in Figs. 28 and 29, respectively. The intensity in these figures has been normalized to $I(\Omega) / I_{\max }$; thus in all 


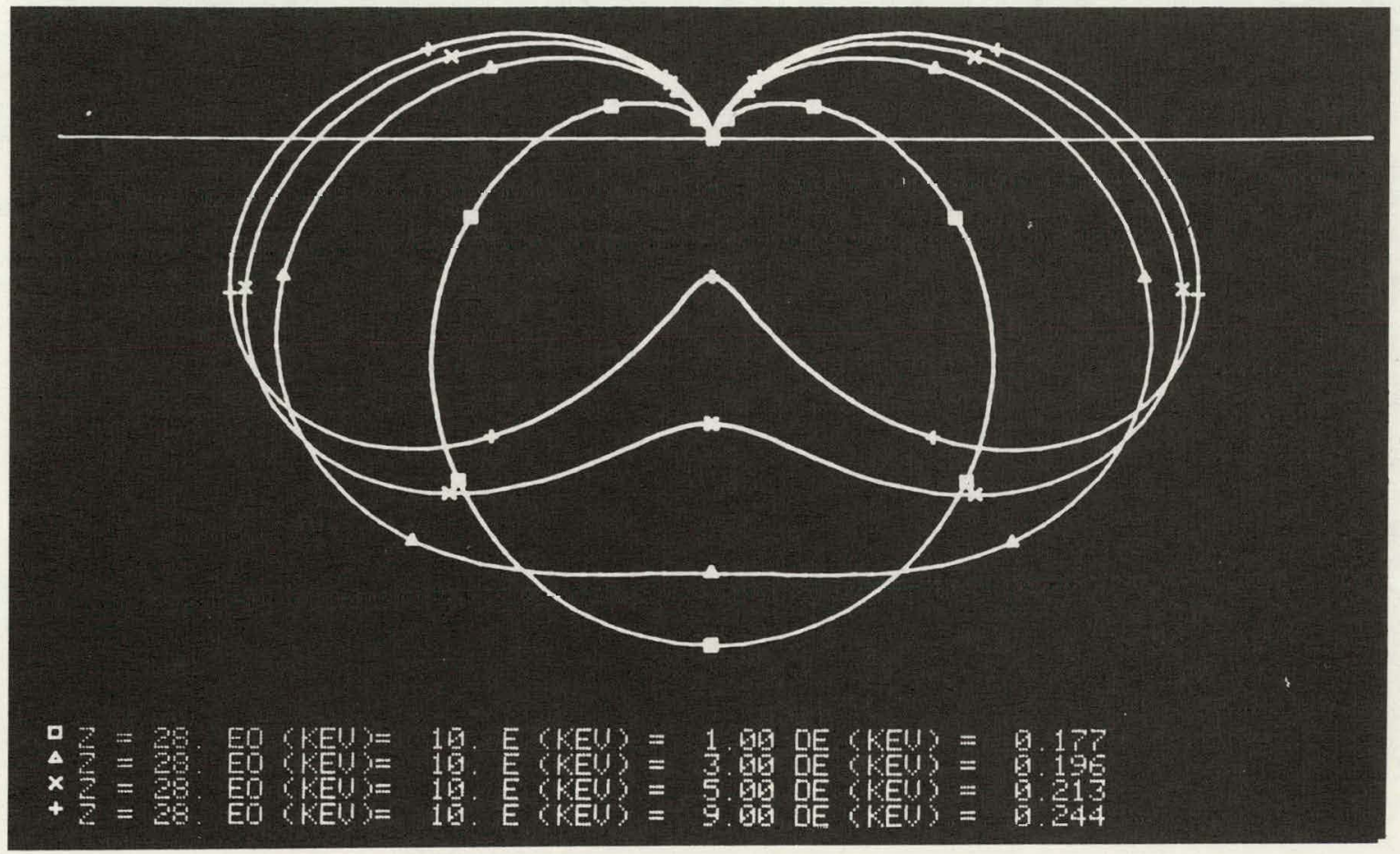

Fig. 28. Polar Plot of the Continum Intensity Generated by a $10 \mathrm{keV}$ Incident Energy Electron Beam on a Thin Foil at Various Values of Continuum Energy (E). 


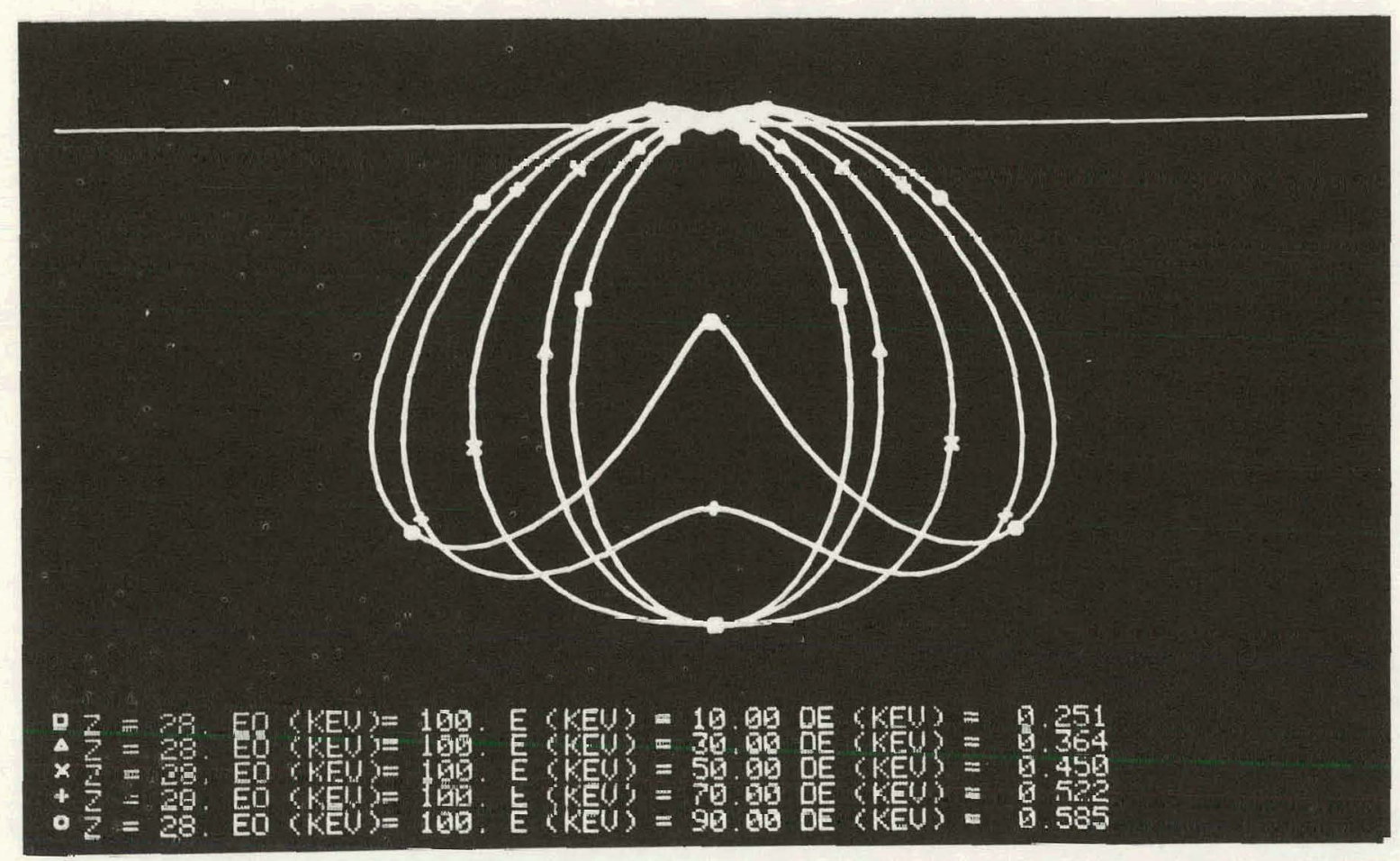

Fig. 29. Polar Plot of the Continumn Intensity by Electron Beam of Incident Energy of $100 \mathrm{keV}$ on a Thin Foil for Various Values of Continuum Energy (E). 
curves the maximum intensity plotted is unity. This is done in order that relative changes in the distribution can be easily observed. The intensity distribution is rotationally symmetric about the electron beam axis and is proportional to the radial distance from the point of incidence. These figures show that the continum intensity on the electron entrance surface of the specimen $\left(\Omega \geqslant 90^{\circ}\right)$ is in general less than the exit surface, and also that relativistic effects (100 keV results) clearly shift the emission in the forward scattered direction. This is illustrated in more detail in Fig. 30. This figure shows the' normalized continuum distribution of $\mathrm{x}$ rays in the vicinity of the nickel $\mathrm{K}_{\alpha}$ peak $\left(\sim 7.5 \mathrm{keV}\right.$ ) as a function of incident electron energy $\left(\mathrm{E}_{0}\right)$ over an energy window $d E$ roughly corresponding to the full-width half maximum (FWHM) of the nicke $1 \mathrm{~K}_{\alpha}$ line. The relative intensity in any direction on the electron entrance surface clearıy decreases with increasing $E_{0}$. Figure 31 reproduces the same calculations on an absolute scale; hence a direct comparison of the various intensities can be made. Note that in plotting this figure the 200 and $100 \mathrm{keV}$ calculations have been truncated in the forward scattering direction for clarity. From these calculations it is apparent that the placement of the detector relative to the electron entrance surface is an important consideration.

The optimum position of the detector can now be evaluated by calculating the characteristic peak-to-background (P/B) ratio as a function of $s$ using Eqs. (2.47) through (2.51). This is plotted in Fig. 32 for the same pure nickel sample as a function of observation angle $(\Omega)$ at various values of incident beam energy. We recall that the forward 


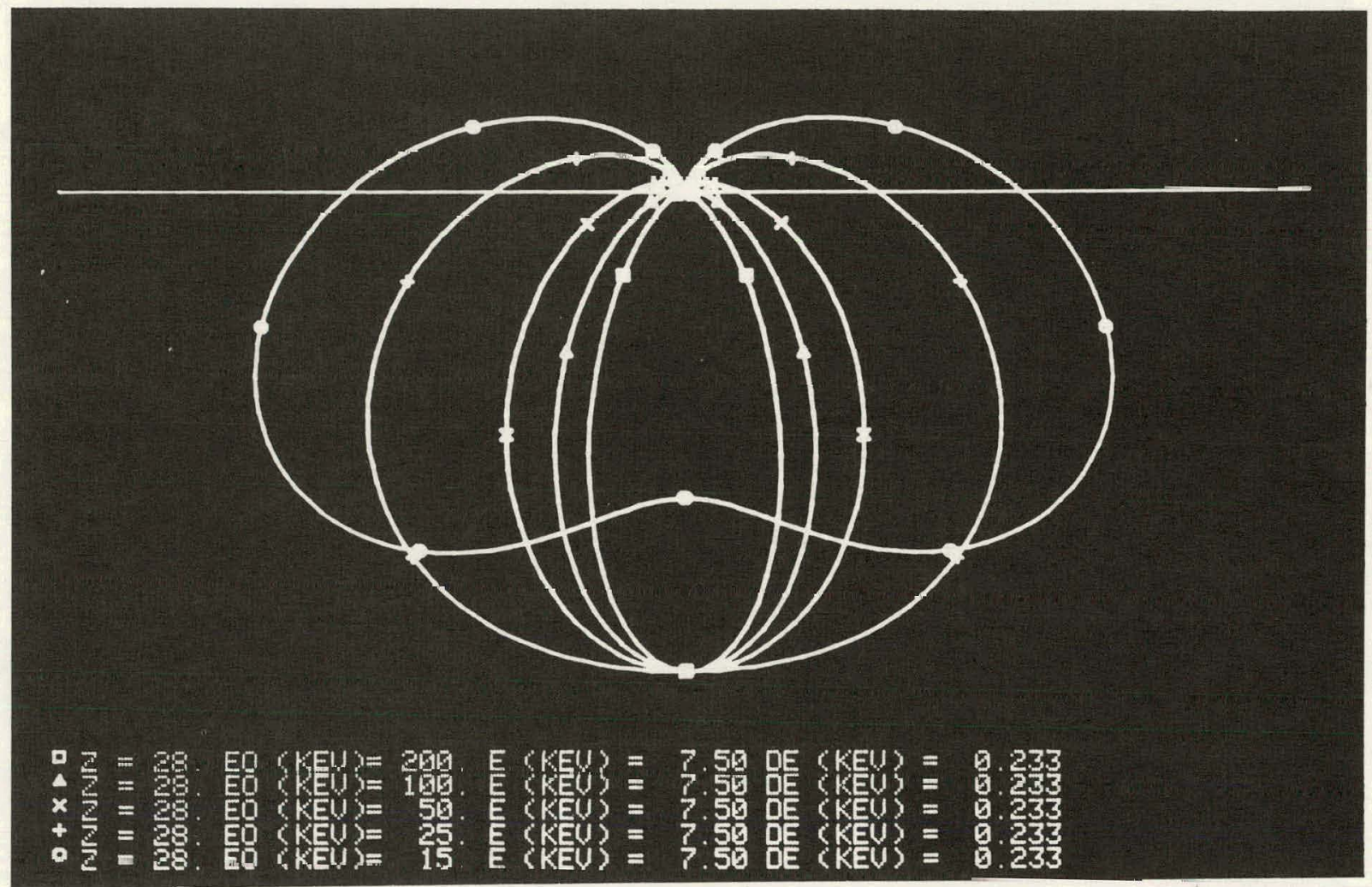

Fig. 30. The Nngular Distribution of Continuum Intensity Generated (at $7.5 \mathrm{keV}$ ) by Various Energy Electron Beams. (All curves normalized to unity maximum intensity.) 


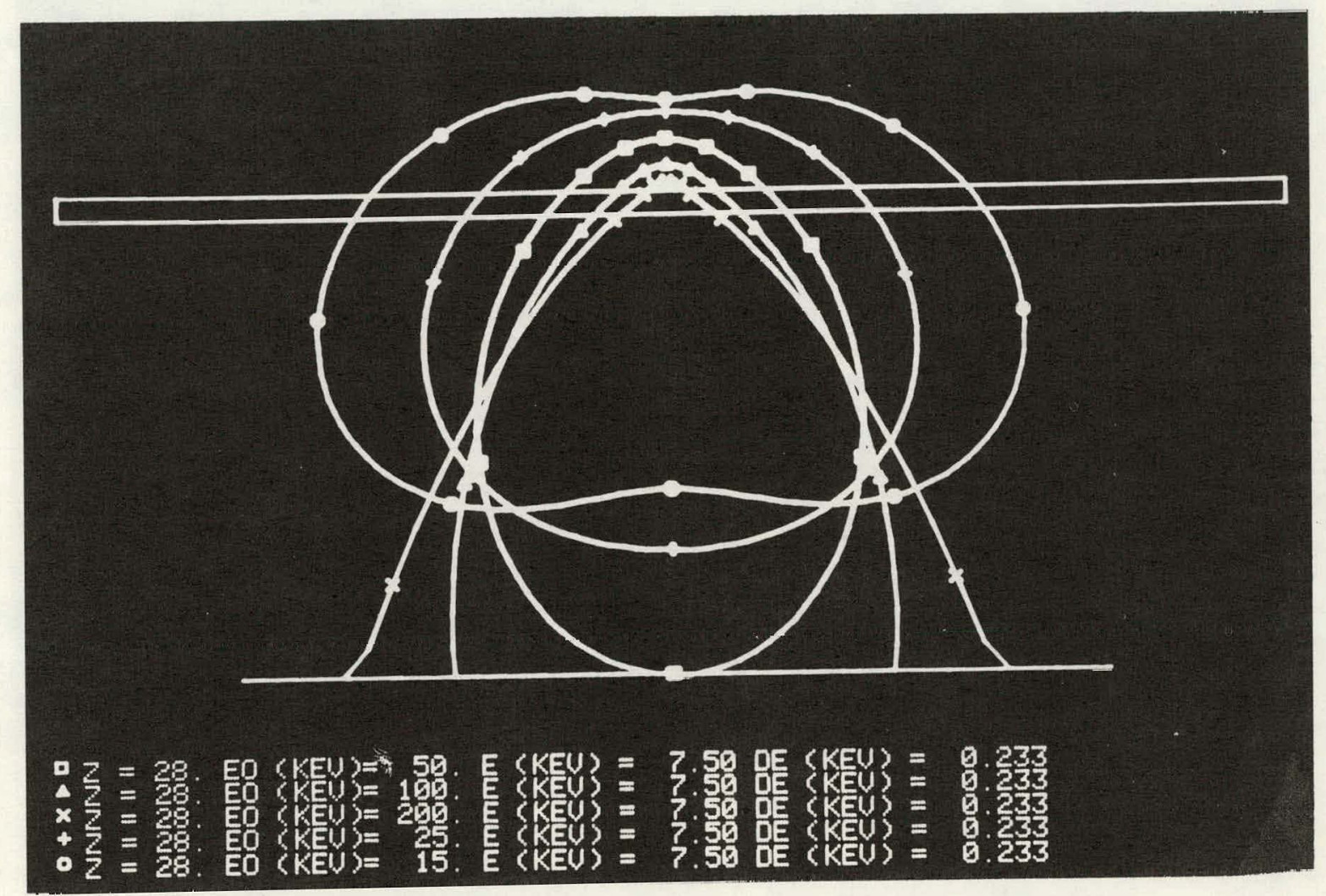

Fig. 31. The Angular Distribution of Continuum Intensity Generated (at $7.5 \mathrm{keV}$ ) by Various Energy Electron Beams. 


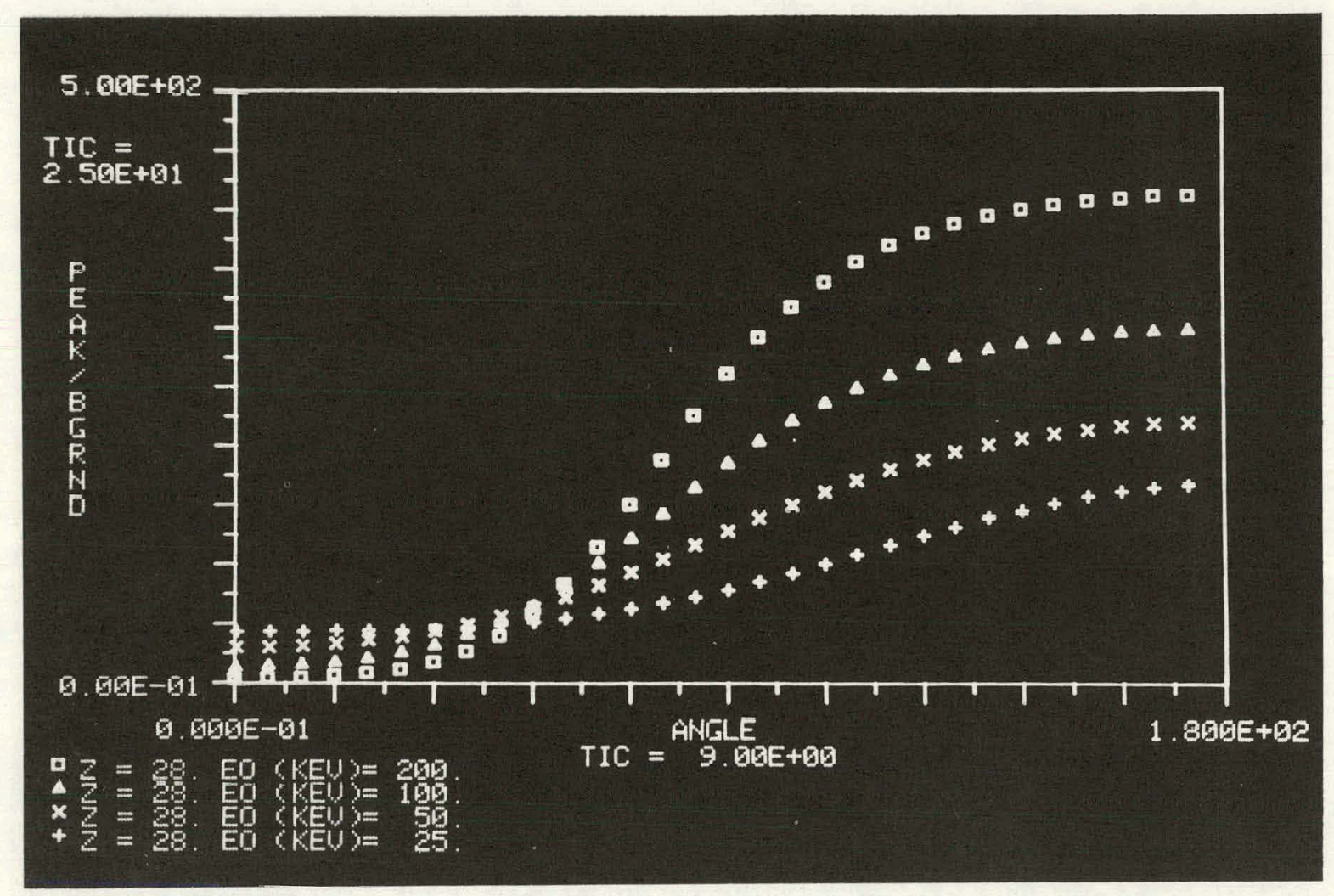

Fig. 32. Calculated P/B Ratio as a Function of Detector Observation Angle $\Omega$ at Various Values of Incident Electron Beam Energy. 
scattering direction corresponds to an observation angle of $0^{\circ}$ and in this position the detector axis is perpendicular to the electron exit surface. Similarly, $\Omega=180^{\circ}$ corresponds to the detector axis being colinear with the incident beam direction. Referring to Fig. 32 one can see that in all cases the $\mathrm{P} / \mathrm{B}$ ratio is maximized when the $\mathrm{x}$-ray detector is positioned on the electron entrance surface $(\Omega \geqslant \pi / 2)$. It is, however, interesting to note that the variation in $\mathrm{P} / \mathrm{B}$ begins to level out at angles above $125^{\circ}$ (at $100 \mathrm{keV}$ ). Thus, several of the new AEM instruments commercially available, such as the Vacuum Generators HB5, Hitachi H-500, and Philips EM-400 microscopes whose detector observation angles are $\geqslant 105^{\circ}$, are approaching the optimum geometry for $x$-ray analysis.

Using the equations presented in this section, it is also possible to calculate the variation in $P / B$ as a function of several other parameters. Figure 33 plots the change in the P/B ratio for the $K_{\alpha}$ line of pure elements (atomic number $Z$ ) at various values of incident energy. In computing these curves a detector observation angle of $\pi / 2$, the geometry found in most CTEM/STEM instruments, was used. These results show a monotonic increase in $\mathrm{P} / \mathrm{B}$ with increasing $\mathrm{E}_{0}$ for all elements. The sharp drop at $Z=45$ corresponds to a change in the calculation from $K_{\alpha}$ to the $\mathrm{L}_{\alpha}$ 1ine. Extended calculations for incident energies greater than $1 \mathrm{MeV}$ indicate a continuous increase in $\mathrm{P} / \mathrm{B}$ to nearly 2.5 times the $100 \mathrm{keV}$ values (see Fig. 34). However, the extrapolation of these equations to such energies is questionable; thus only the trends should be considered. 


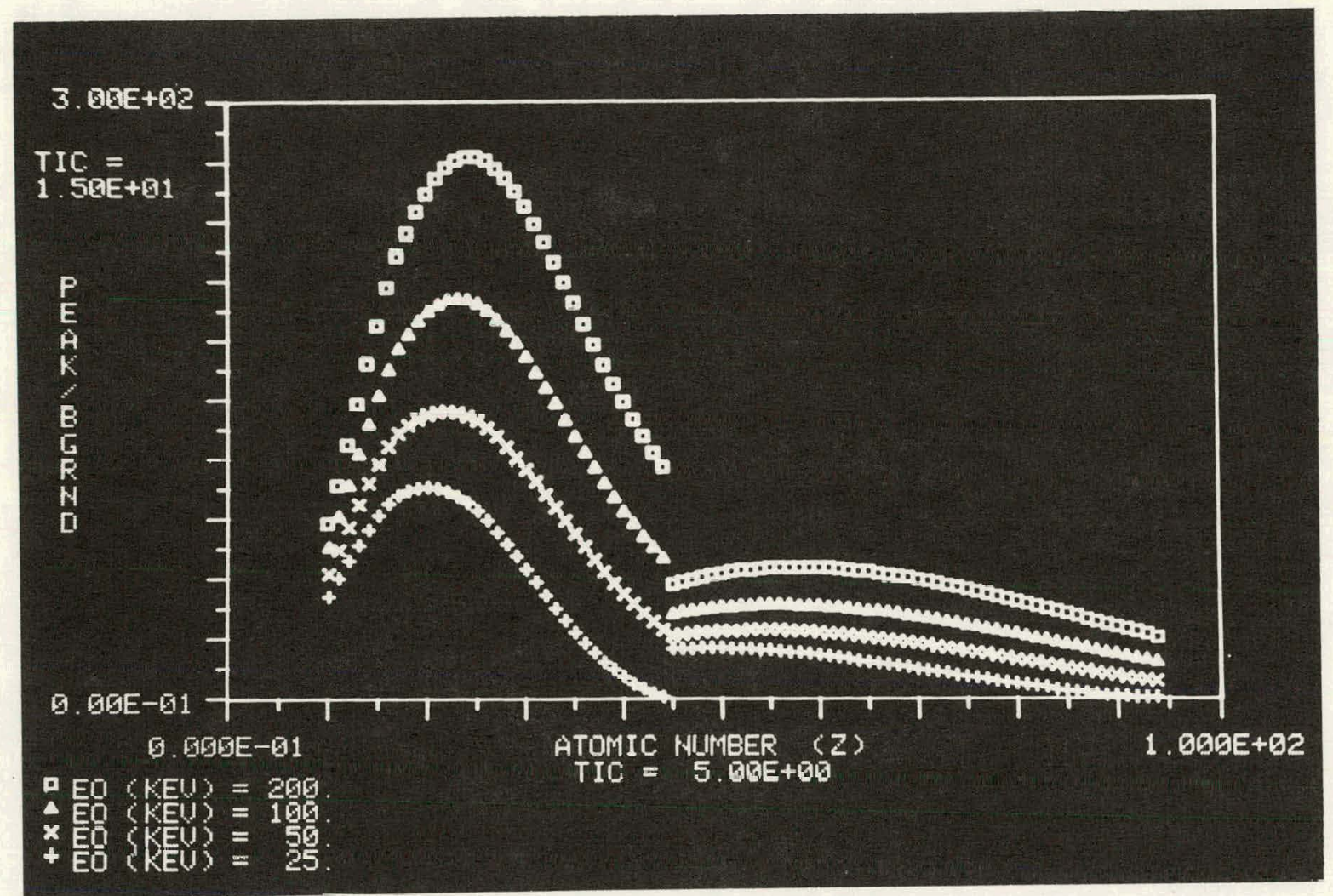

Fig. 33. Calculated P/B Ratio for Pure Element Samples as a Function of Incident Beam Energy. 


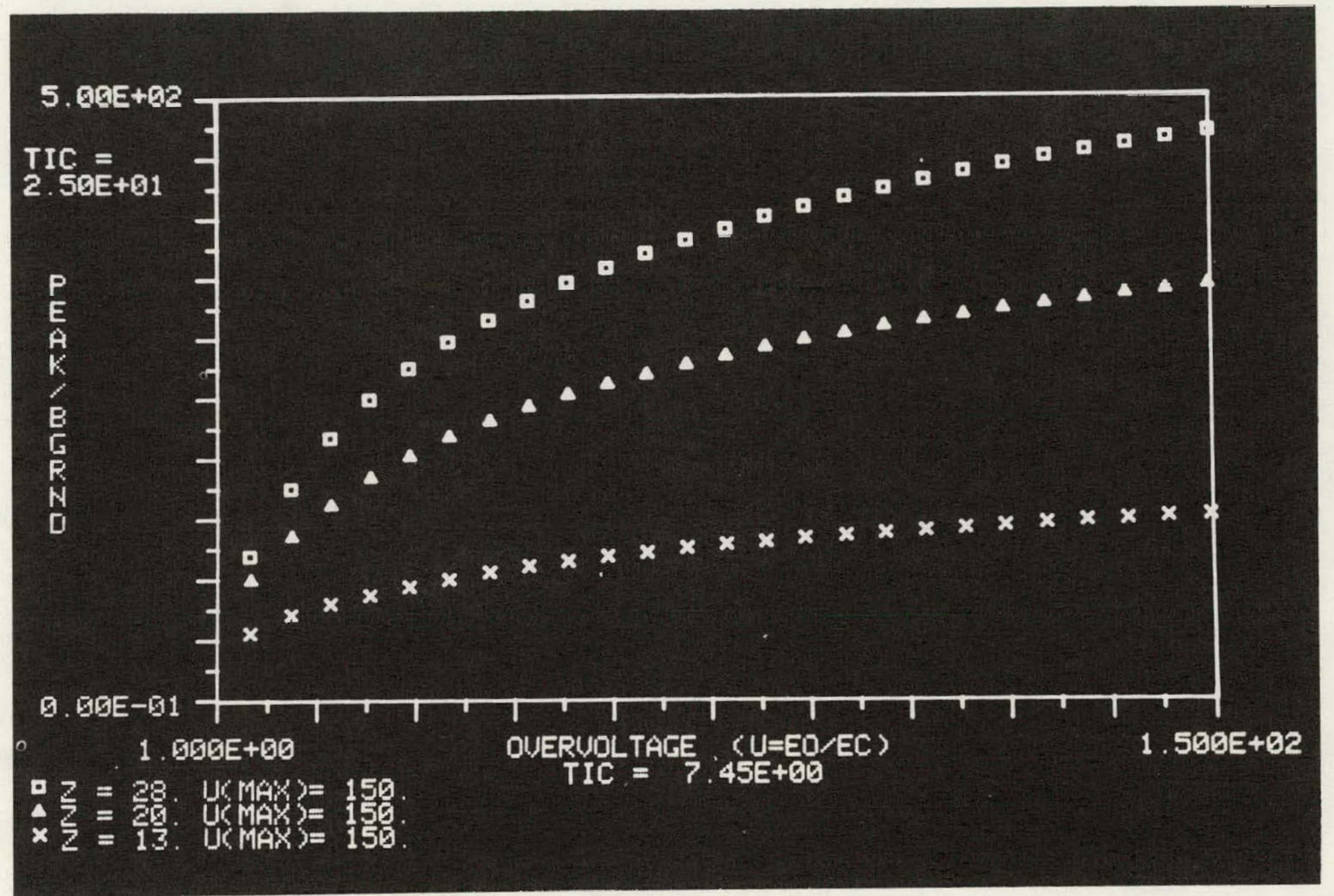

Fig. 34. Calculated P/B Ratio as a Function of Overvoltage for Various Elements. 
Finally by substituting different values of $\mathrm{Z}, \mathrm{W}$, and $\bar{\rho}$ into the characteristic and continum equations, respectively, it is possible to evaluate the $\mathrm{P} / \mathrm{B}$ ratio for one element in a matrix of different (or average) atomic number $\left(\mathrm{z}_{\mathrm{av}}\right)$. Such a calculation is presented in Fig. 35 which shows, as would be expected, a monotonic decrease in the $\mathrm{P} / \mathrm{B}$ ratio with increasing matrix atomic number $\left(\mathrm{Z}_{\text {av }}\right)$ at constant electron energy. Similarly, Fig. 36 shows a monotonic decrease in the $\mathrm{P} / \mathrm{B}$ ratio with decreasing $\mathrm{E}_{0}$ for a constant matrix composition. In calculating both these figures, $\mathrm{C}_{\mathrm{A}}$ in $\mathrm{Eq}$. (2.47) was set to unity; this, in any comparison with experimental data the value of $\mathrm{P} / \mathrm{B}$ predicted should be multiplied by the composition in weight percent.

In summary, these calculations have demonstrated that the maximum theoretical sensitivity is obtained by placing the $\mathrm{x}$-ray detector with an observation angle $\Omega \geqslant \pi / 2$, using the highest value of incident beam energy, and minimizing (when possible) the average atomic number of the matrix.

\subsubsection{Instrumental Factors Influencing X-Ray Analysis}

Although the coupling of a solid state $\mathrm{Si}(\mathrm{Li})$ x-ray detector to a transmission electron microscope attords the possibility of per forming microchemical analysis of thin specimens on a scale of several hundred angstroms or less, there are several experimental aspects of this technique which limit its applicability. Furtunately, these factors are purc ly instrumental in nature and can be reduced or eliminated by judiciously modifying the analytical system. Three topics will be considered in this 


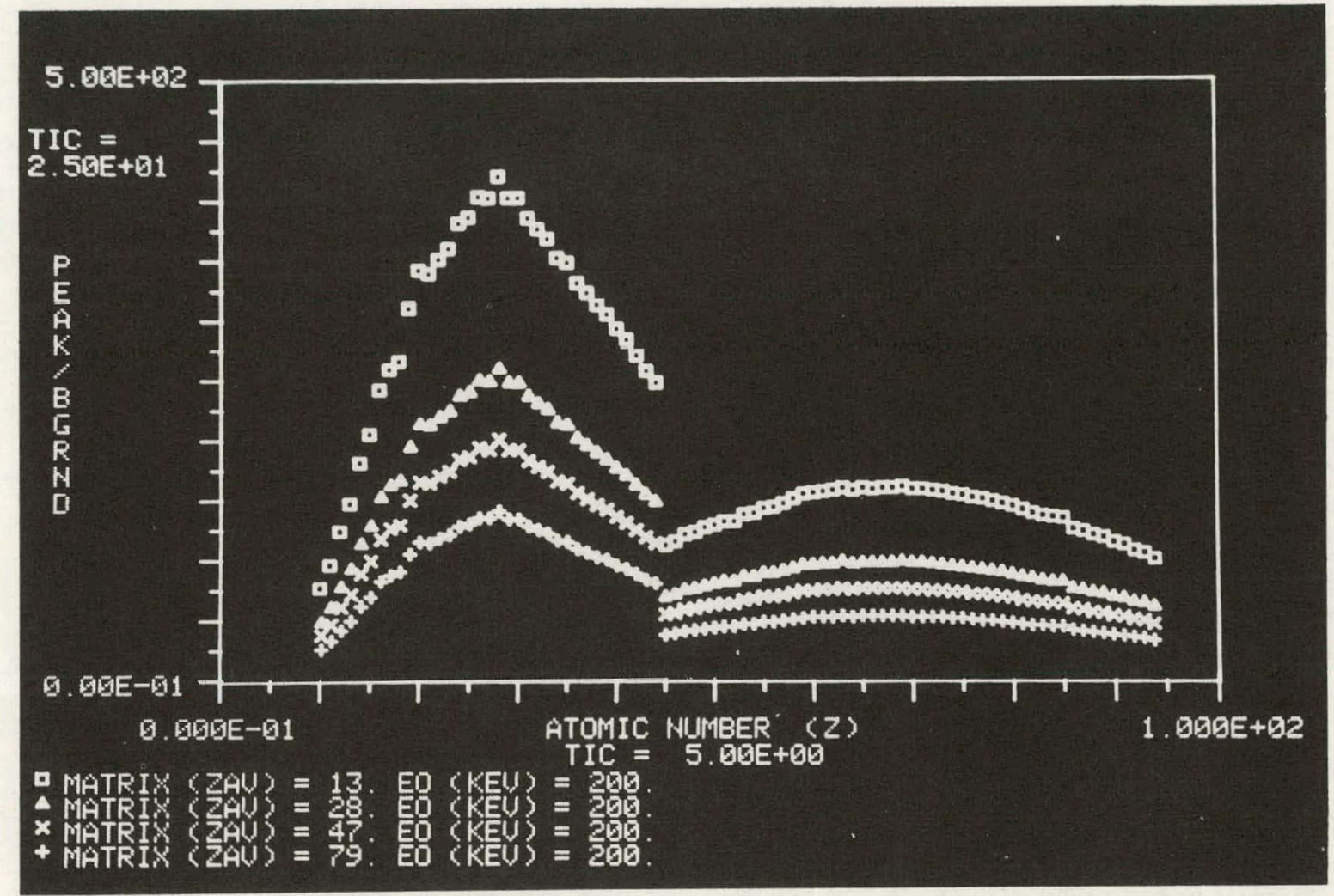

Fig. 35. Calculated P/B Ratio for an Element of Atomic Number $(Z)$ in a matrix of Average Atomic Number $\left(Z_{a v}\right)$ at Constant Incident Electron Energy, 


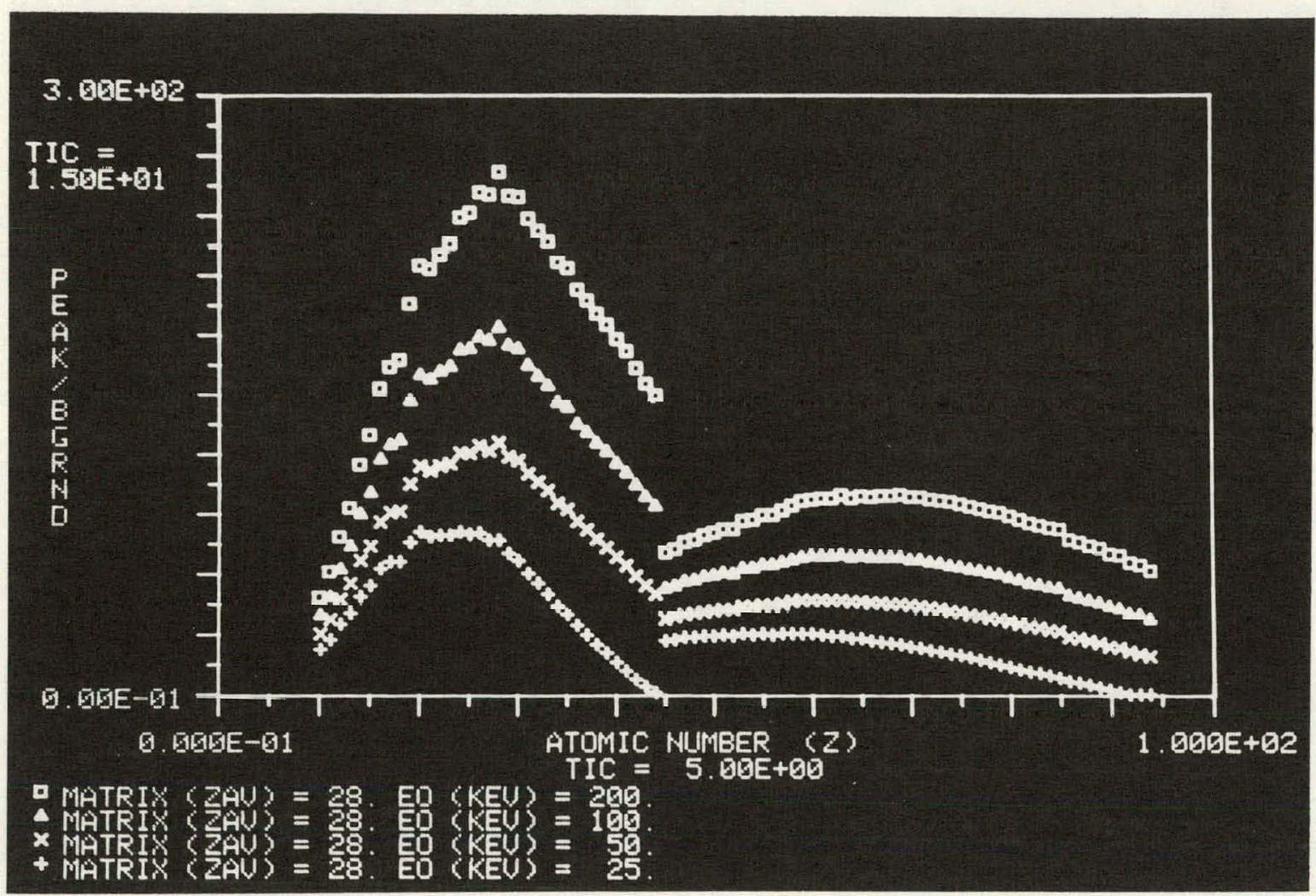

Fig. 36. Calculated P/B Ratio for an Element $Z$ in a Matrix of Average Atomic Number $z_{a v}$ at Various Incident Beam Energies. 
section - namely: systems peaks; fluorescence by uncollimated radiation; and specimen contamination effects. These problems are found in all electron microscopes; however, their relative magnitude changes from one instrument to another. In all cases, it is important that these artifacts be identified and eliminated since they affect the qualitative as well as quantitative analysis detrimentally.

System Peaks: When a solid state $\mathrm{Si}(\mathrm{Li}) \mathrm{x}$-ray detector is used to measure the $\mathrm{x}$-ray emission produced in an electron microscope, a spectrum consisting of several characteristic peaks superimposed on a continuum or background intensity distribution is observed. Ideally, this spectrum is a result of electron-induced excitation of the sample; unfortunately, $\mathrm{x}$ rays are also produced in the material immediately surrounding the sample (i.e., the sample stage, aperture drives, cold finger, etc.) by mechanisms other than direct electron excitation, and hence are recorded simultaneously with the sample spectrum. Although it is usually possible to identify these "systems peaks" it is important to eliminate them rather than simply account for their presence.

The reduction of system peak intensity is accomplished by replacing the material surrounding the specimen by an element which does not produce detectable $x$ rays. Several stages have been designed for this purpose; they were constructed from graphite ${ }^{75}$ or plastic (PMMA). ${ }^{76}$ However, they are severely limited in their in-situ tilting capabilities making them unsuitable for analytical microscopy. Thus, a modified design of gimbal cup was developed for use in the double-tilt stage supplied with the JEOL JSEM $200 \mathrm{keV}$ CTEM/STEM. 77 Machined totally out 
of graphite this cup provides full two-axis-tilting capabilities along with the reduction of systems peaks. Since this time, several researchers have developed total specimen stages machined from bery1$\operatorname{lium}^{78,79}$ and graphite ${ }^{80}$ which provide equivalent capabilities as we11 as high mechanical stability. A brief description of the modified gimbal cup for the JEOL double-tilt stage developed in this research follows.

Shown in Fig. 37 is a schematic diagram of the x-ray geometry in the JEOL JSEM 200 with the incident electron beam being normal to the plane of the page. Optimization of the $x$-ray count rate is accomplished through the use of a double-tilt stage, which allows tilting of the specimen about two mutually perpendicular axes. The primary axis (A-Ain Fig. 37) can be tilted by $\pm 55^{\circ}$ and the secondary axis ( $\mathrm{B}-\mathrm{B}^{*}$ in Fig. 37) by $\pm 45^{\circ}$. An ORTEC Minitec Si(Li) x-ray detector is mounted on the microscope column normal to the incident electron beam (i.e., a detector observation angle $\Omega=\pi / 2$ defined in Secl. 2.2.6) and at an azimuthal angle of $45^{\circ}$ to the primary tilt axis. The front of the detector is protected by a 7.5 - $\mu$ m-thick beryllium window and the specimen-to-window distance is approximately $2 \mathrm{~cm}$. Through the use of a graphite collimator tho $x$-ray acceptanre ang1e ( $\theta$ in Fig. 37 ) is $\sim 15^{\circ}$; i.e., the detector accepts radiation from roughly a $0.5-\mathrm{cm}$-dia disk at the specimen surface. There were two purposes in redesigning the specinlen holder. The first was to remove the interference in the recorded $x$-ray spectrum due to the "system peaks" generated in the specimen cup. The original cup is machined from a $\mathrm{Cu}-\mathrm{Be}$ alloy and removal of the "system peaks" from it 


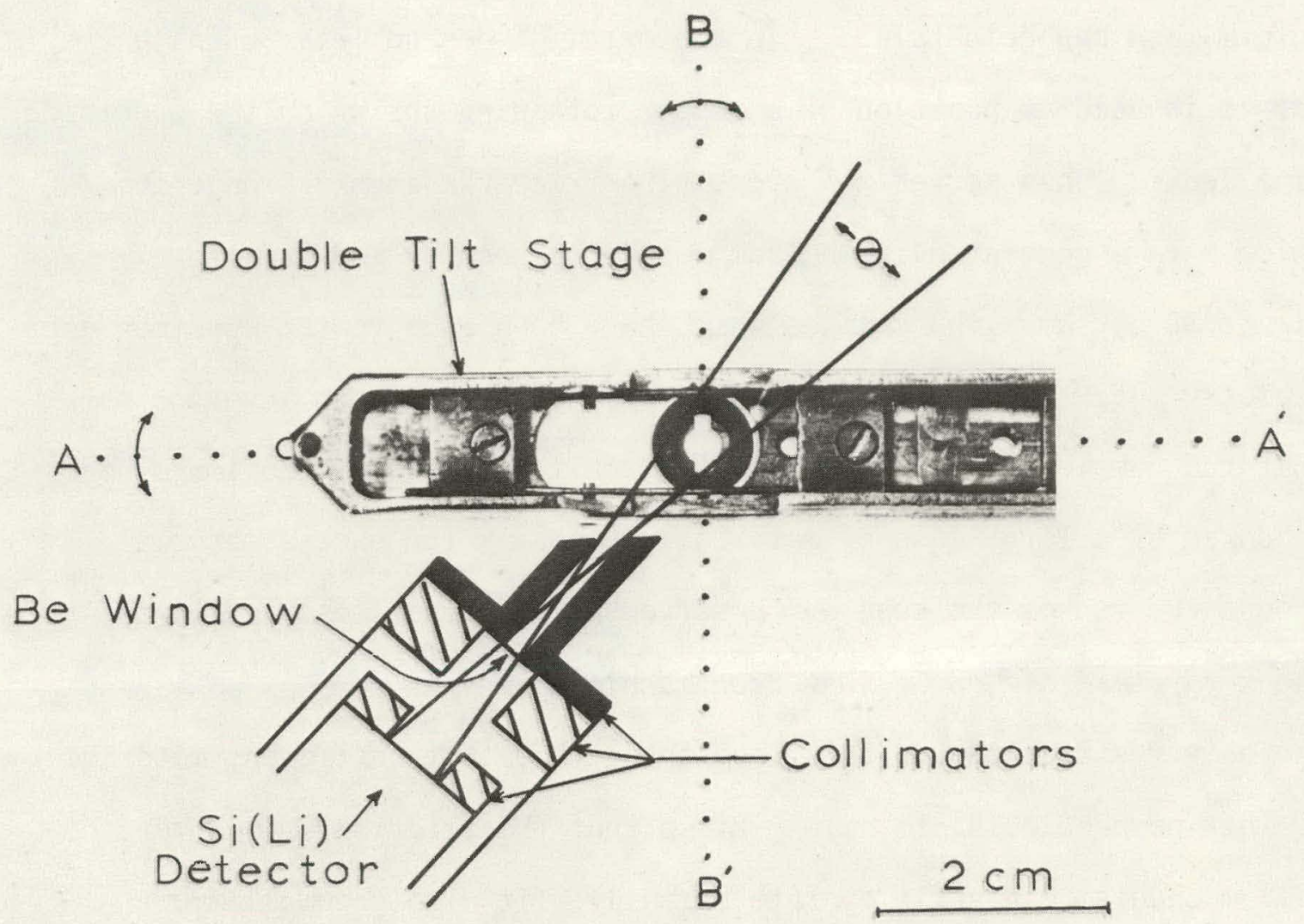

Fig. 37. Schematic Diagram of the X-Ray Geometry in the JEOL JSEM 200. 
is accomplished by replacing this alloy by an alternate material. Bery1lium would be the best choice; however, due to the hazards involved in its machining, graphite was chosen as an alternative. The second purpose was to increase the range of take-off angles in which usable $x$-ray information can be obtained. In the original design [Fig. 38(a)], the sample is held in position by a copper retaining spring on the underside of a ledge. This scrves to severely restrict. the angular range from which $x$ rays generated in the sample can be seen hy the detector. Figure 38(b) shows the changes which have been made to the specimen cup geometry. First the interior design of the cup has been inverted so that the sample now rests on top of a small ledge and is no longer overshadowed by a large mass of material. Secondly the excess material around the top of the cup, which served as a further restriction to the $\mathrm{x}$-ray take-off angle, has been machined away as much as possiblc. The only restrictions on this last change are that sufficient material be left perpendicular to axis $\mathrm{B}^{-\mathrm{B}^{*}}$ so that the friction drives for the tilt mechanism can still operate normally. It also becomes important to replace the copper retaining spring used to hold the sample rigidly in position. Since no suitable spring substitute is available, the sample is now held in place by the judicious application of a colloidian of graphite along the specimen's edges, or on a thin graphite washer used to hold the sample in position.

A. homogeneous alloy of $\beta$-NiAl was used as a test specimen to evaluate the characteristics of the two types of specimen cups (Cu-Be versus graphite). When determining the characteristics of both cups, 

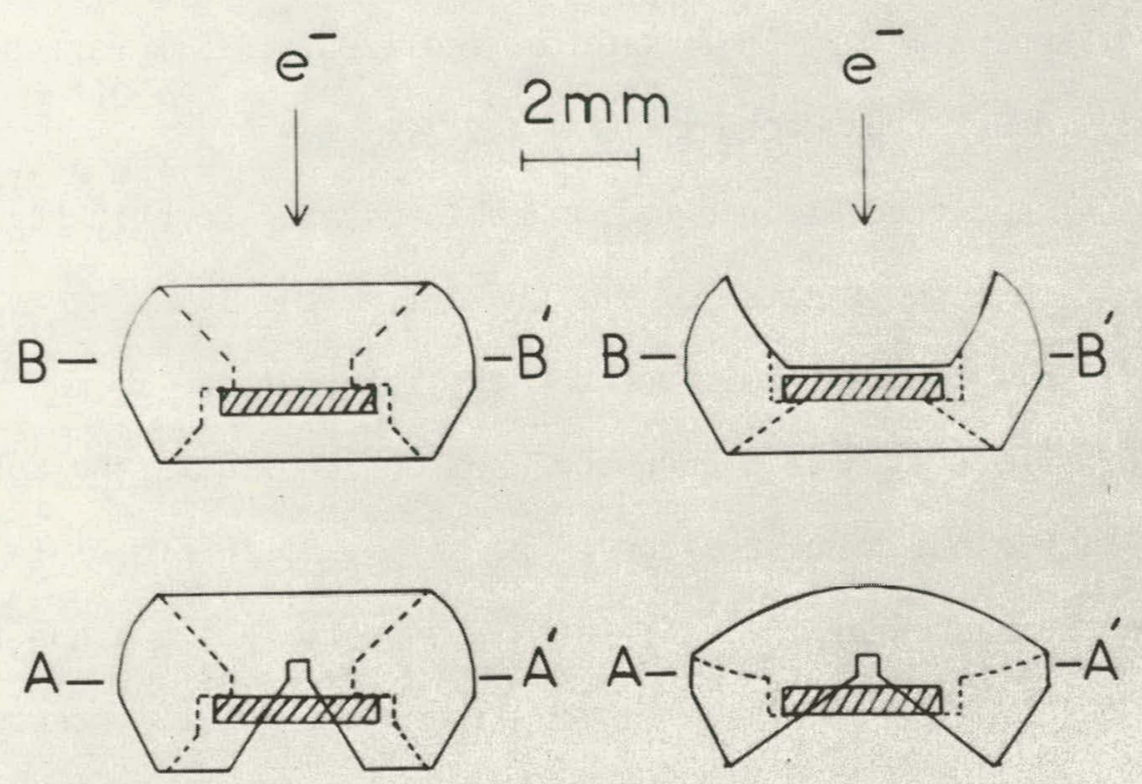

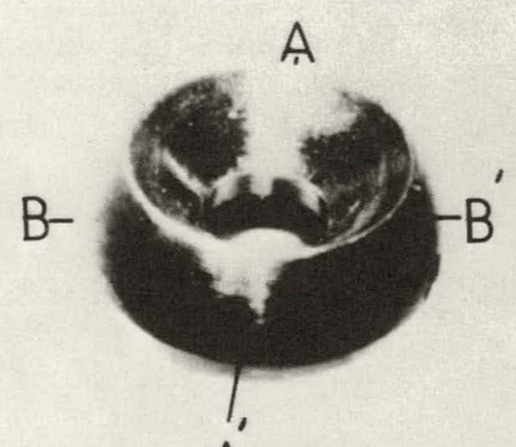

A

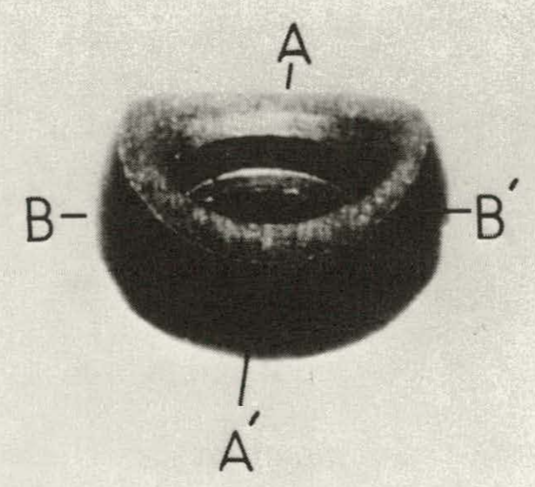

(b)

(a)

Fig. 38. Modified Design of Specimen Cup to Reduce System Peaks. 
care was taken so that the sample was imaged under identical operating conditions and orientations. The microscope was operated with an accc1erating voltage of $200 \mathrm{keV}$ and in the STEM mode, so that electron probe diameters of approximately $50 \AA$ could be realized. A11 x-ray spectra were recorded under these conditions.

Typical spectra obtained using both cups are shown in Fig. 39. It is evident that the removal of the $\mathrm{Cu}$ - $\mathrm{Be}$ from the immediate vicinity of the sample has substantially reduced the interference from "system peaks." The effect is most pronounced when one considers the $\mathrm{CuK}_{\alpha}$ and $\mathrm{NiK}_{\beta}$ lines in the emission spectrum. The $\mathrm{NiK}_{\beta}$ line in Fig. 38(a) has been completely masked by the $\mathrm{CuK}_{\alpha}$ peak. However, when a graphite cup is used, the $\mathrm{NiK}_{\beta}$ line becomes clearly resolved and the interference due to the $\mathrm{CuK}_{\alpha}$ line is substantially reduced [Fig. 39(b)]. Using a computational technique for profile fitting, ${ }^{b u}$ it is possible to analyze the extent of overlap of these two peaks. The measured $\mathrm{CuK}_{\alpha} / \mathrm{NiK}_{B}$ ratio is $2.27 \pm 0.28$ and $0.19 \pm 0.03$ for the $\mathrm{Cu}-\mathrm{Be}$ and graphite gimbals, respectively. Thus, a net reduction by a factor of 12 of the $\mathrm{CuK}_{\alpha}$ intensity is achieved. Such reductions are typical; however, "system peaks" from the $\mathrm{Cu}-\mathrm{Be}$ cup are dependent on the orientation of that cup with respect to the incident electron beam and detector system and changes in this ratio can be expected.

It should be noted that even after removal of one of the most important sources of "system peaks" it is still possible to detect interference. This indicates that the scattered radiation (elastic and inelastically scattered electrons and bremsstrahlung) in the microscope 


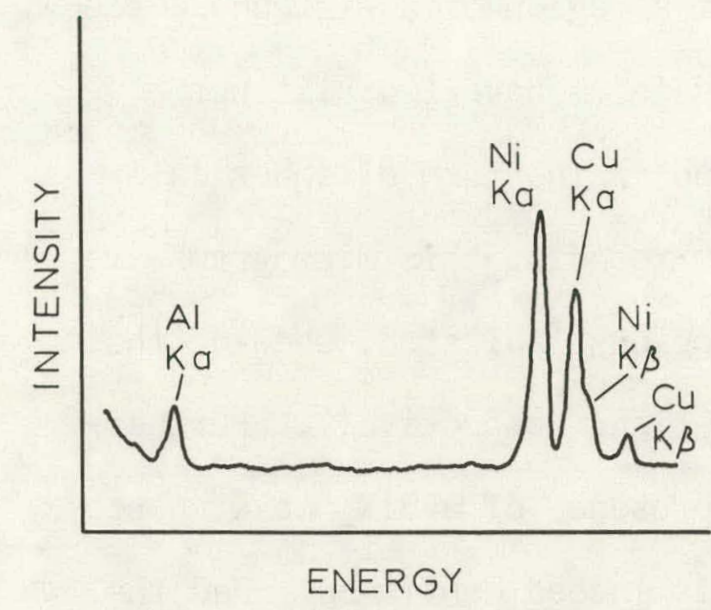

(a)

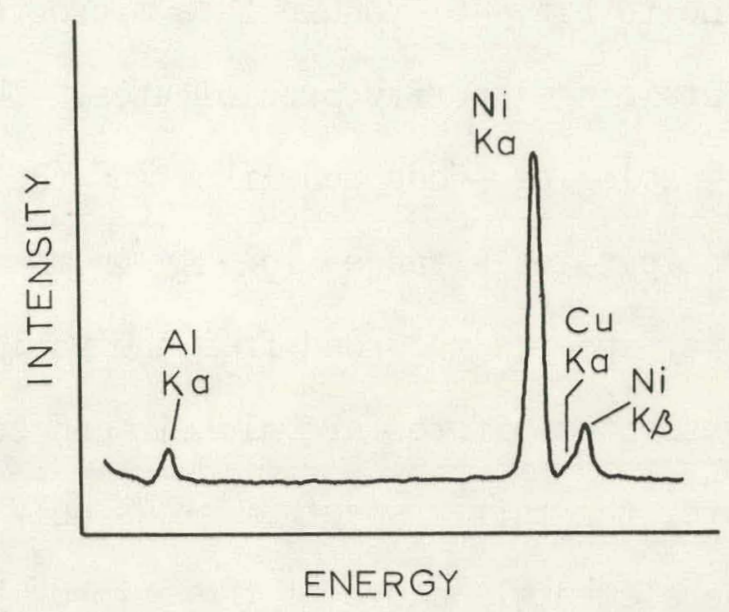

(b)

Fig. 39. Comparison of Systems Peak Intensity (a) Before, and (b) after Modified Specimen Cup Design was Introduced into JSEM 200. 
is a significant source of $x$-ray excitation as seen by the detector, and, in addition, it shows that large areas in the specimen region are still being excited.

The importance of eliminating system peaks can be seen by reference to Fig. 40. Shown is a micrograph of a region of a silicon sample containing impurity precipitates. These defects have two distinct murphologies - onc rod-like, and the second in the form of spherical precipitates arranged roughly in rows. Surrounding this micrograph are $\mathrm{x}$-ray spectra recorded from the various regions indicated, showing that these precipitates are nicke1- and copper-rich, respectively. Furthermore, the matrix spectrum shows only the presence of a SiK $\alpha$ peak; hence the elemental nature of these impurities is indeed confirmed. Had the copper system peaks not been eliminated in this experiment, it would not have been possible to uniquely identify the copper-rich impurity because of the minute amount present. In addition, due to the similarly weak intensity of the nicke1 line analysis in the presence of a large cupper systems peak would have been difficult if not impossible. Thus, for the case of low concentration or small volume fraction precipitates, it is essential to remove all systems peaks.

Fluorescence by Inrnilimated Radiation: One of the serious instrumental problems associated with TEM-based microanalysis arises due to nonlocalized excitation of characteristic $\mathrm{x}$ rays frum the sample. Referring to Fig. 39, it can be noticed that even though a $50 \AA$ electron prohe was used to generate $x$ rays in the sample a significant volume of material must have been irradiated in order to produce the magnitude of 


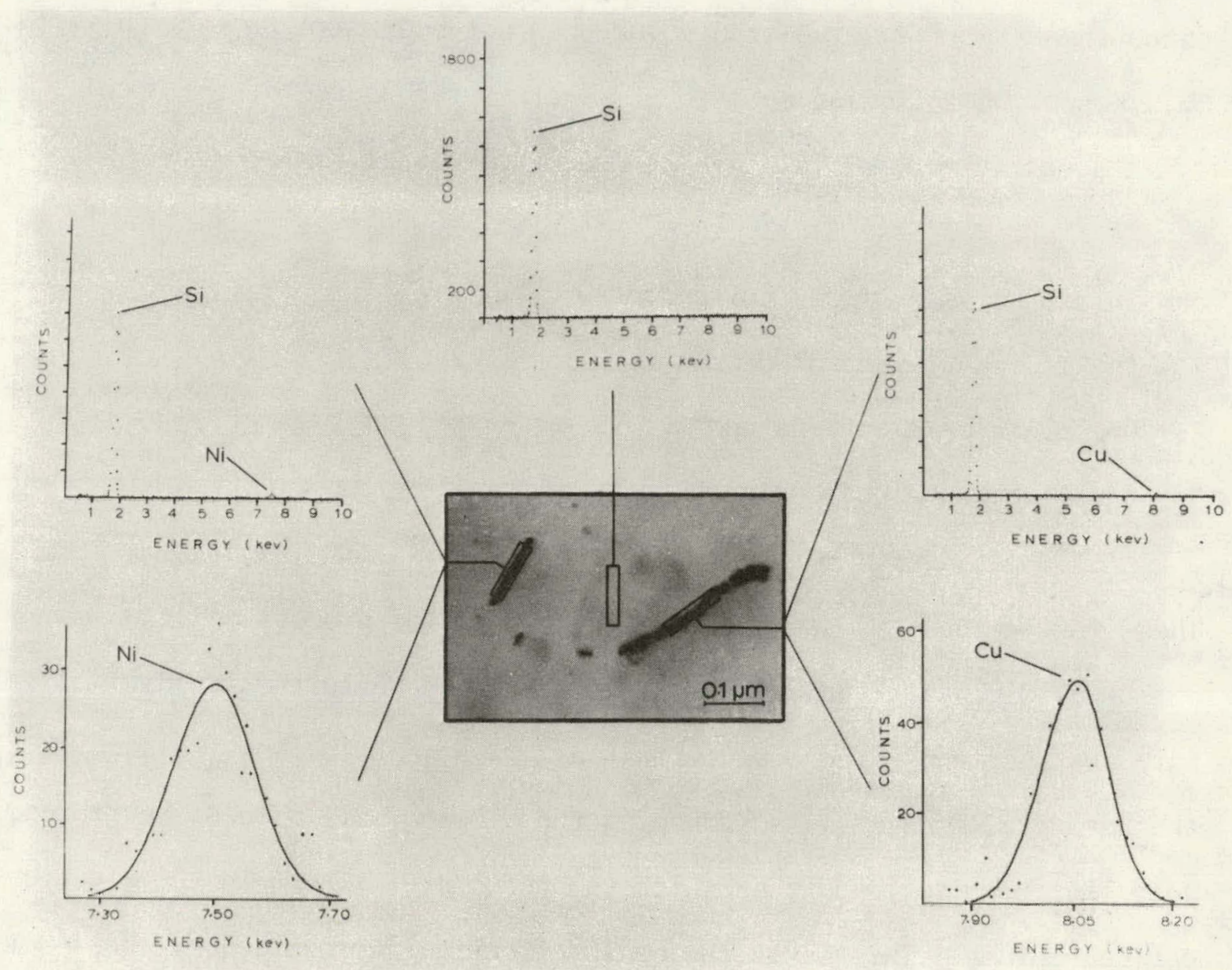

Fig. 40. X-Ray Microanalysis of Impurity Precipitates in Si, Indicating the Necessity for Removal of Systems Peaks. 
systems peaks which were detected. The removal of medium to high atomic number material from the inmediate vicinity of the sample clearly reduces the intensity of systems peaks; however, it is only a cosmetic solution since the effective irradiated volume has not been significantly altered. This poses the following questions. What is the source of the uncollimated radiation, and how does it affect microanalysis?

There are essentially three potential sources of radiation which can induce emission from the specimen. They are:

1. the incident electron probe,

2. uncollimated electrons and $x$ rays produced by the sample,

3. uncollimated electrons and $x$ rays produced by the electron microscope hardware.

The first two sources are basic to the generation process and thus cannot be eliminated. The last term, however, can be further subdivided into two sections - those uncollimated $x$ rays and clcctrons related to the hardware immediately surrounding the sample (pole pieces, apertures, cold fingers, etc.) and that which is produced elsewhere in the electronoptical column. This first factor is effectively minimized by constructing suitable low atomic number substitutes for various parts of the microscope, ${ }^{75-81}$ and amounts to the systems peak correction discussed in Systems Peaks. The second term is extremely important and has been shown to be one of the most significant sources of nonlocalized radiation. ${ }^{43}$ When present, it can become the limiting factor in both spatial resolution and quantitative analysis. This effect can become so great in some instruments that errors in excess of several hundred 
percent can easily develop. The remainder of this discussion will center upon the identification of this radiation and its effects on microanalysis. The examples given were obtained using a JEOL JSEM 200 CTEM/ STEM instrument, but can also be observed in other electron microscopes with varying magnitude.

When an electron beam is positioned on a specimen, it is generally assumed that all characteristic $x$ rays emitted from the sample are generated in the volume defined by that probe. If this is the case; then, when the probe is translated off the sample all $x$-ray emission should cease (assuming, of course, that the electron column is properly aligned). More often than not an $x$-ray spectrum from the sample will still be detected and the magnitude of this emission can be substantial. This is illustrated in Figs. 41, 42, and 43, which are typical spectra obtained when the probe is incident on the sample (a) and then allowed to pass through the perforation formed during the sample preparation (b) for incident electron energies of 200,100 , and $50 \mathrm{keV}$, respectively.

These spectra were accumulated using the experimental geometry described in Systems Peaks. The sample used was a three millimeter single-crystal disc of $\mathrm{B}$-NiAl of the 50/50 at. \% composition. The specimen was electropolished to TEM quality using standard techniques ${ }^{82}$ and had a small perforation approximately $40 . \mu \mathrm{m}$ in diameter near the center of the foil. All spectra were recorded for $200 \mathrm{~s}$ with the microscope operating in the STEM mode and using electron probe diameters of approximately $50 \AA$. Measurements were made by positioning the probe 

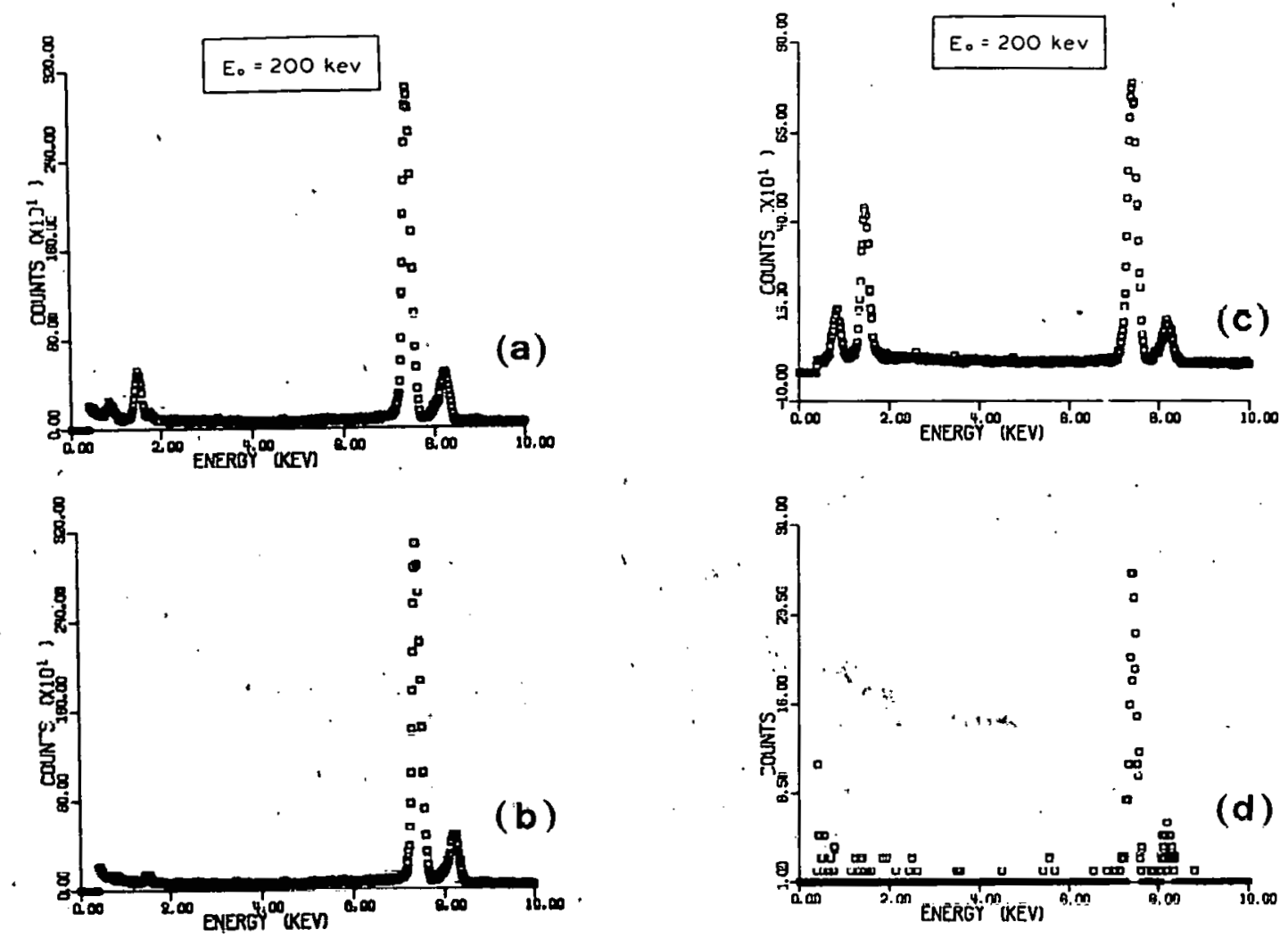

Fig. 41. Sample (a) and In-fiole Spectra (b) before Installation of Bremsstrahlung Aperture. Sample (c) and in-hole spectra (d) after installation of bremsstrahlung aperture. $E_{0}=200 \mathrm{keV}$. 

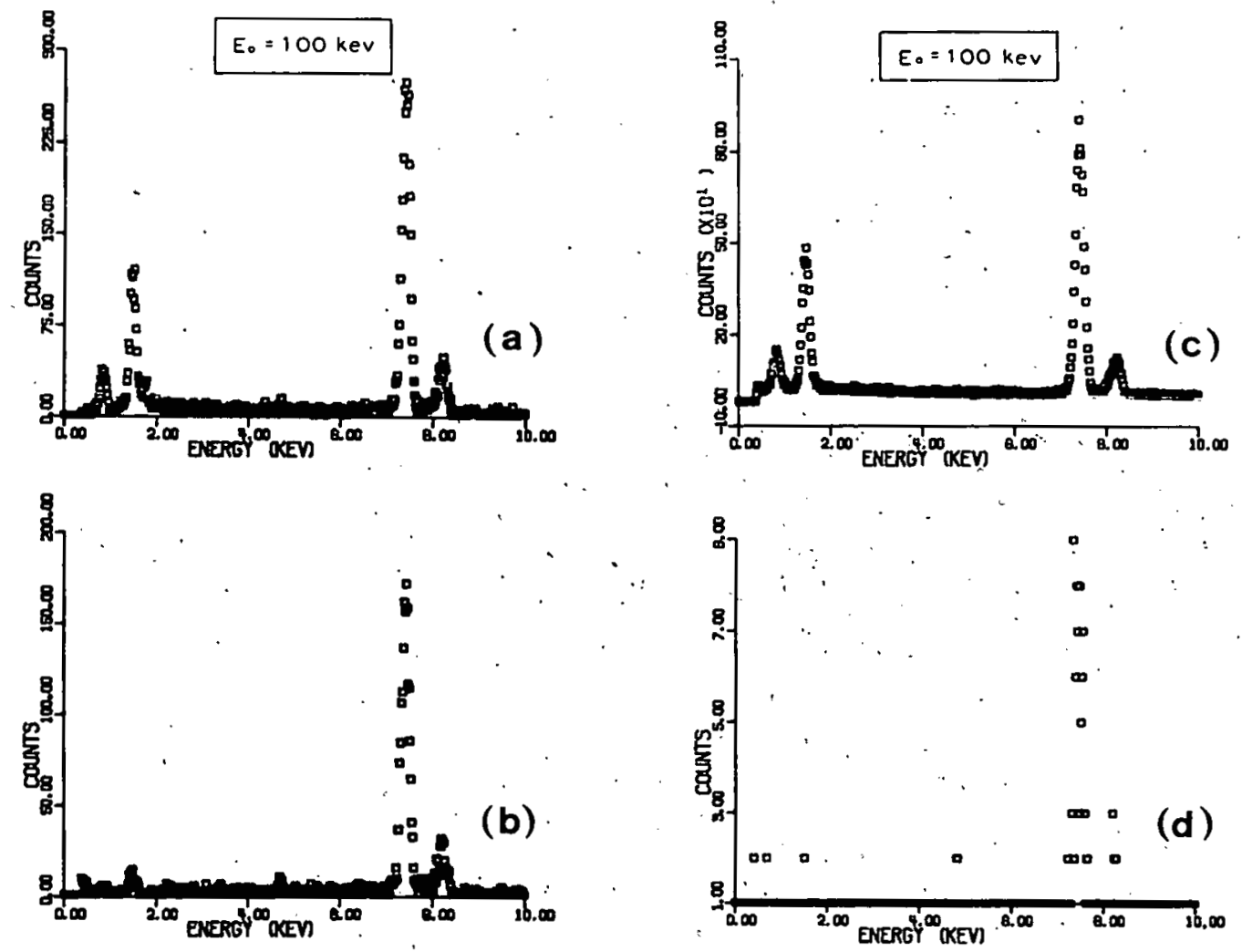

Fig. 42. Sample (a) and In-Hole Spectra (b) before Installation of Bremsstrahlung Aperture. Sample (c) and in-hole spectra (d) after installation of bremsstrahling aperture. $E_{0}=100 \mathrm{keV}$. 

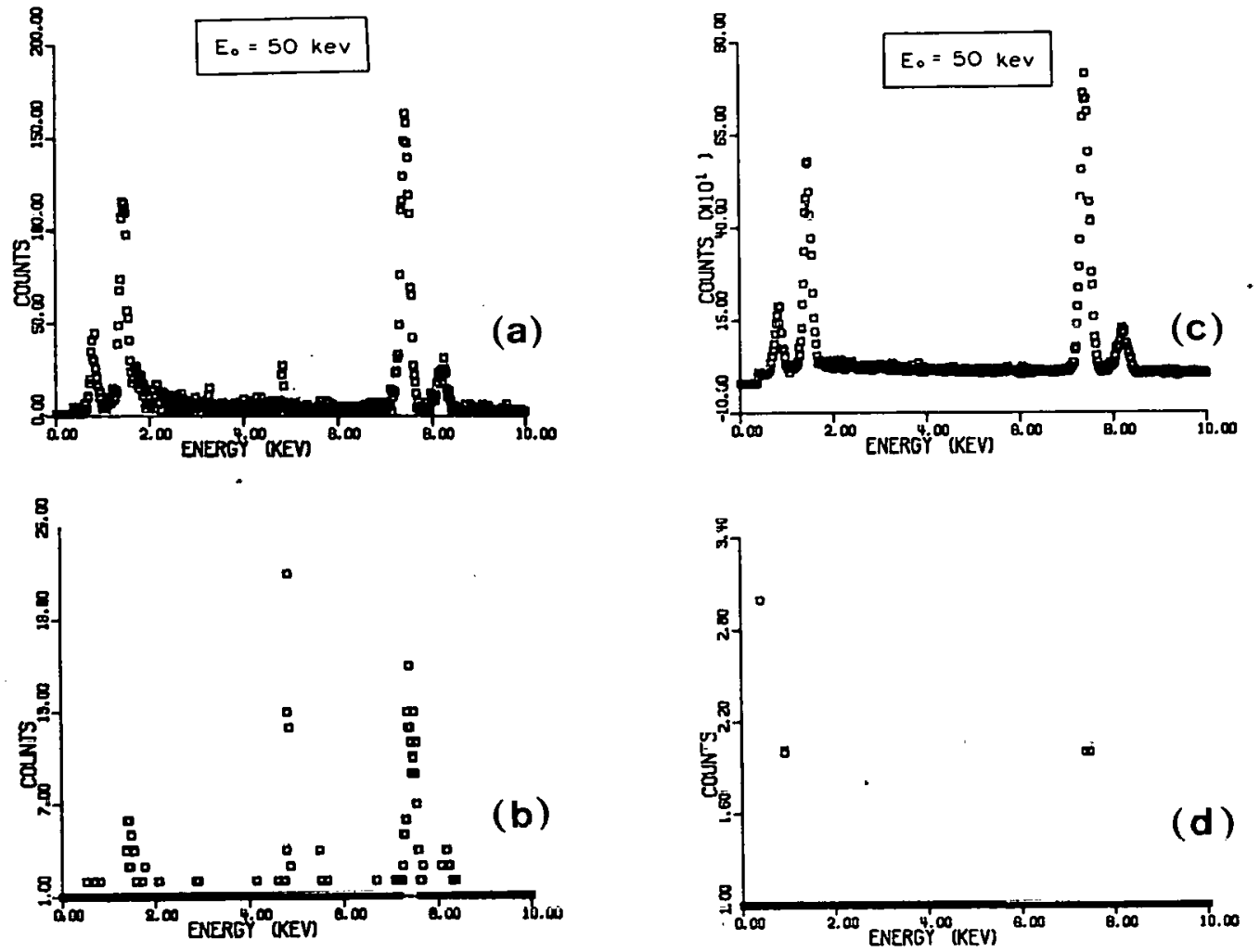

Fig. 43. Sample (a) and In-Hole Spectra (b) before Installation of Bremsstrahlung Aperture. Sample (c) and in-hole spectra (d) after insta1lation of bremsstrahlung aperture, $E_{0}=50 \mathrm{keV}$. 
on an electron transparent region near the edge of the foil (position A, Fig. 44) while "in-hole" counts were obtained by transiating the probe into the perforation at least $2 \mu \mathrm{m}$ from the foil edge (indicated as B, Fig. 44).

Examining these spectra one can see that under certain operating conditions the "in-hole" spectrum can exceed $90 \%$ of the total emission measured from the sample. Furthermore, one can detect an appreciable atomic number effect in the excitation of characteristic $x$ rays by this uncollimated radiation. The excitation of the nickel $\mathrm{K}$ lines is several orders of magnitude greater than aluminum. The effects of this radiation on microanalysis are even more pronounced and are shown in Fig. 45. Plotted is the experimental variation in the $\mathrm{Ni} / \mathrm{Al} \mathrm{K}_{\alpha}$ intensity ratio as a function of sample thickness for various electron energies. The broken curves under each set of experimental measurements represent the theoretical calculations based on the formalism previously described. One would expect the intensity ratio to be constant initially until a sample thickness is reached when absorption effects become appreciable. At this point, absorption of the lower energy alıminum $K_{\alpha}$ $x$ rays should cause the ratio to increase monotonically. Experimentally this is not observed. Initially one measures a decrease in the Ni/A1 ratio with increasing thickness followed by an inflection point and then a gradual increase in the ratio. This monotonically increasing region interestingly roughly parallels the calculated variation corresponding to the region of high aluminum absorption. Furthermore, a systematic shift in the inflection point toward thinner regions occurs with decreasing 


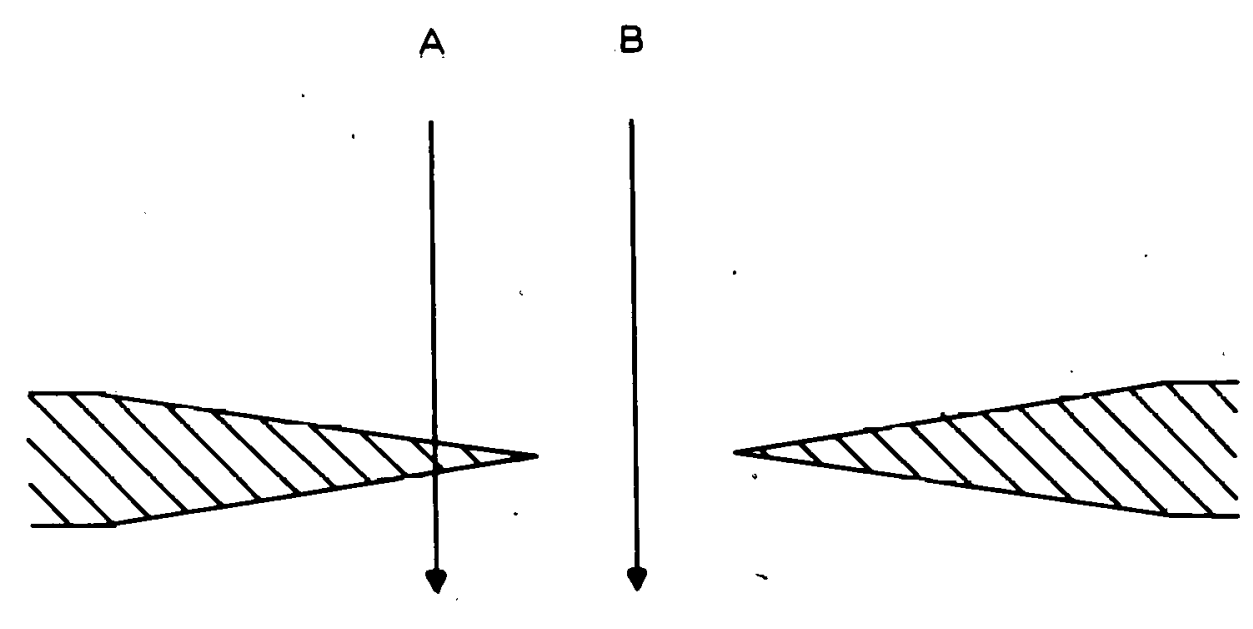

Fig. 44. Srhematic Illustration of Electron Probe Position during Sample Measurement (A) and In-Hole Measurement (B). 

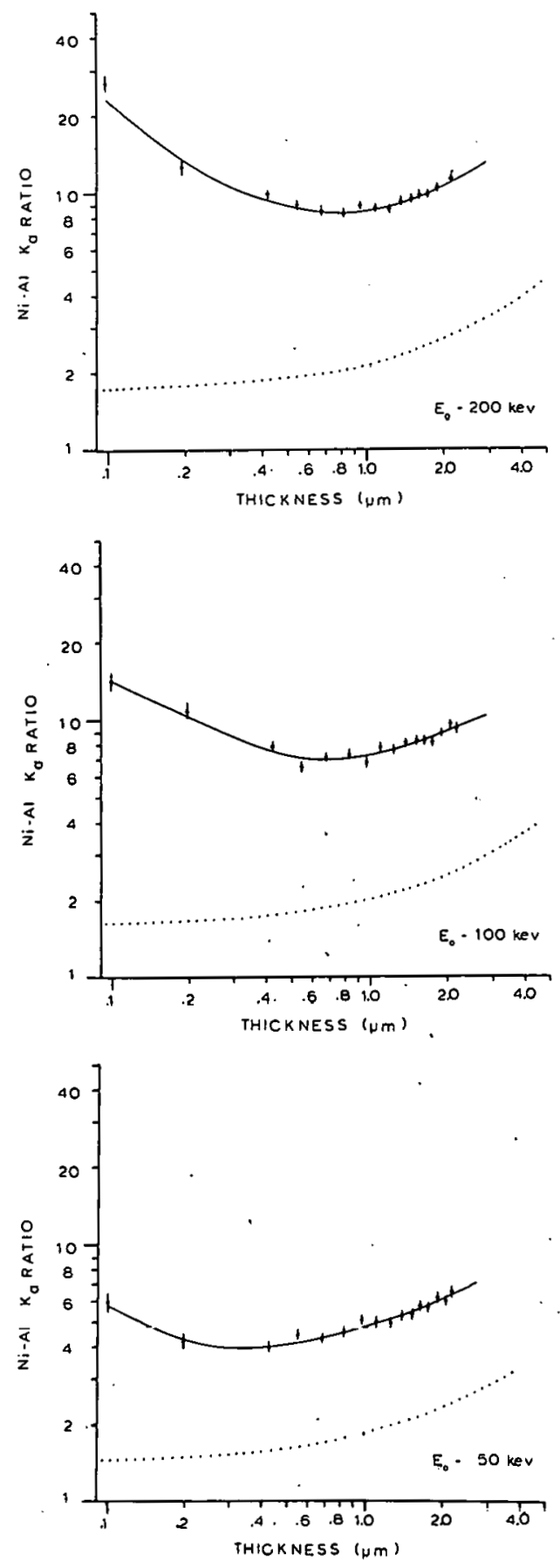

Fig. 45. Plot of Measured Ni/Al Ratio as a Function of Sample Thickness at Various Incident Beam Energies. Broken curve indicates calculated variation. 
incident energy. It should be noted that at $200 \mathrm{keV}$ the sample was just barely transparent to electrons at the last data point plotted $(\sim 2 \mu \mathrm{m})$. Thus, to measure the intensity ratio at these last few points using lower accelerating voltages it was necessary to operate the STEM unit in the SEM mode, the electron probe being positioned by observing the contamination marks on the sample surface produced during the $200 \mathrm{keV}$ measurements. It is apparent from these data that serious discrepancies exist between experimental and calculated intensities, and it would seem that quantitative analysis under such conditions is impossible.

The breakdown of the theory of quantitative analysis in this case can be traced directly back to the first assumption made in the calculations. That is, it was assumed that all $\mathrm{x}$-ray emission from the sample is a direct result of electron excitation of a microvolume of material. Should there be any additional sources of $x$-ray excitation they must either be eliminated from the instrument or included in the calculations. The question then still remains as to the nature and the source of this anomalous excitation. Considering closely the characteristics of the "inhole" spectra as well as their variation with accelerating voltage it was postulated that high energy bremsstrahlung radiation generated in the condenser-aperture system of the electron microscope could account for these experimental results. The following experiment was then devised to test the feasibility of this hypothesis. ${ }^{83}$

The microscope was aligned in the usual manner and an in-hole spectrum from B-NiAl was confirmed. The variable condenser-aperture drive was then removed and the aperture assembly, which was originally 
constructed from a $\mathrm{Cu}$-Be alloy, was replaced by a 3-mm-thick lead substitute having a single 200- $\mu$ m aperture. This lead aperture was replaced in the column and the in-hole effect reproduced. The next step was to cover the $200 \mu \mathrm{m}$ aperture by a thin lead foil approximately $15 \mu \mathrm{m}$ thick and replace the entire assembly. This foil is of sufficient thickness that all electrons bombarding the condenser aperture are stopped; however high energy bremsstrahlung radiation generated by the electrons slowing down in this foil can still be transmitted. When this modified aperture was inserted and the instrument returned to normal operating conditions it was still possible to measure an $x$-ray emission spectrum of NiAl even though all electrons were prevented from striking the sample. From this result one can suggest an explanation of the anomalous results obtained previously as well as propose an effective solution.

Bremsstrahlung radiation, created principally in the thin regions of the condenser apertures, is continuously bombarding the sample area. This radiation is highly peaked in the forward scattering direction and is limited spatially only by the bore of the microscope column. It is then entirely likely that characteristic emission from the sample and surrounding material is induced by an $x$-ray fluorescence mechanism. This emission will be recorded simultaneously with that produced by electron irradiation of the specimen and is independent of electron probe position on the sample. It is also probable that this fluorescence emission will exceed that produced by direct electron excitation since the fluorescence is of essentially a bulk specimen while the electron-induced fraction results only from the microvolume of material defined by a $1.00 \AA$ electron 
probe. It would also be expected that the intensity ratio of Ni/Al will be relatively high, when compared to electron excitation, since the efficiency of $x$-ray fluorescence of low atomic number material $(z<20)$ is poor.

One can then postulate the following sequence of events. In the thinnest region of the foil the measured ratio is dominated by the bulk $x$-ray fluorescence effects. As the electron probe begins to sample thicker and thicker regions the relative contribution of the electron excitation will increase and eventually exceed the bremsstrahlung contribution. Initially, one would expect a decrease in the measured ratio of $\mathrm{Ni} / \mathrm{Al}$ to a point at which electron excitation begins to dominate and then it would be expected that the variation in the ratio would begin to follow the theoretically predicted curves. Furthermore, if the relative cunlribution of the bremsstrahlung radiation is decreased - for example, by lowering the accelerating voltage - then the crossover point of dominance of bremsstrahlung versus electron excitation should shift to thinner regions. All of these effects are clearly demonstrated in Figs. 41, 12, 43 , and 45, and when combined with the results of the lead aperture experiment suggest stongly that the source of anomnlous cmission is $\mathrm{x}$ ray fluerescence.

Tn attempt a theoretical calculation of a correction to this phenomenon is difficult due to a lack of sufficient information concerning the precise source and magnitude of this radiation. Thus, one must attempt to modify the electron-optical colum in such a way as to minimize 
or eliminate this radiation from reaching the specimen. The ideal solution to such a problem is to deflect the incident electron probe around a thick $x$-ray stop, much as is used in high voltage electron microscopes (HVEM) to prevent ion bombardment of the sample. Realistically this requires significant modifications of the electron-optical column, and furthermore would probably result in significant aberrations, and so a simpler solution is necessary. An alternate solution would be to reduce the total $x$-ray flux bombarding the sample by special collimators $;^{83}$ it is by far the easier method, as illustrated in Fig. 46.

This has been accomplished successfully on the JSEM 200 using two different designs of collimators. The first and simplest design is to insert a thick $(>2 \mathrm{~cm})$ aperture in the column below the condenser aperture, which reduces the bore of the column from $1 \mathrm{~cm}$ to $0.5 \mathrm{~mm}$. This does not interfere with the electron optics, yet substantially reduces fluorescence effects. Two eccentric cylinders are used in this case as shown in Fig. 47. The center and innermost cylinder is aligned with the electron-optical axis by rotating the two cylinders with respect to each other and the column. Such an arrangement was necessary because in this instrument the mechanical axis and the electron-optical axis were not precisely aligned. Later, a more elaborate aperture system was installed and is shown in Fig. 48. Externally adjustable, this bremsstrahlung aperture is mounted on the column in the vacant region normally occupied by a top-entry stage insertion mechanism (Fig. 49). The housing is composed of lead, while the actual aperture $(0.5 \mathrm{~mm} \times 2 \mathrm{~cm})$ was machined from brass to facilitate removal and cleaning when necessary. Also mounted on the aperture mechanism is a Faraday cage to monitor the incident probe current. 

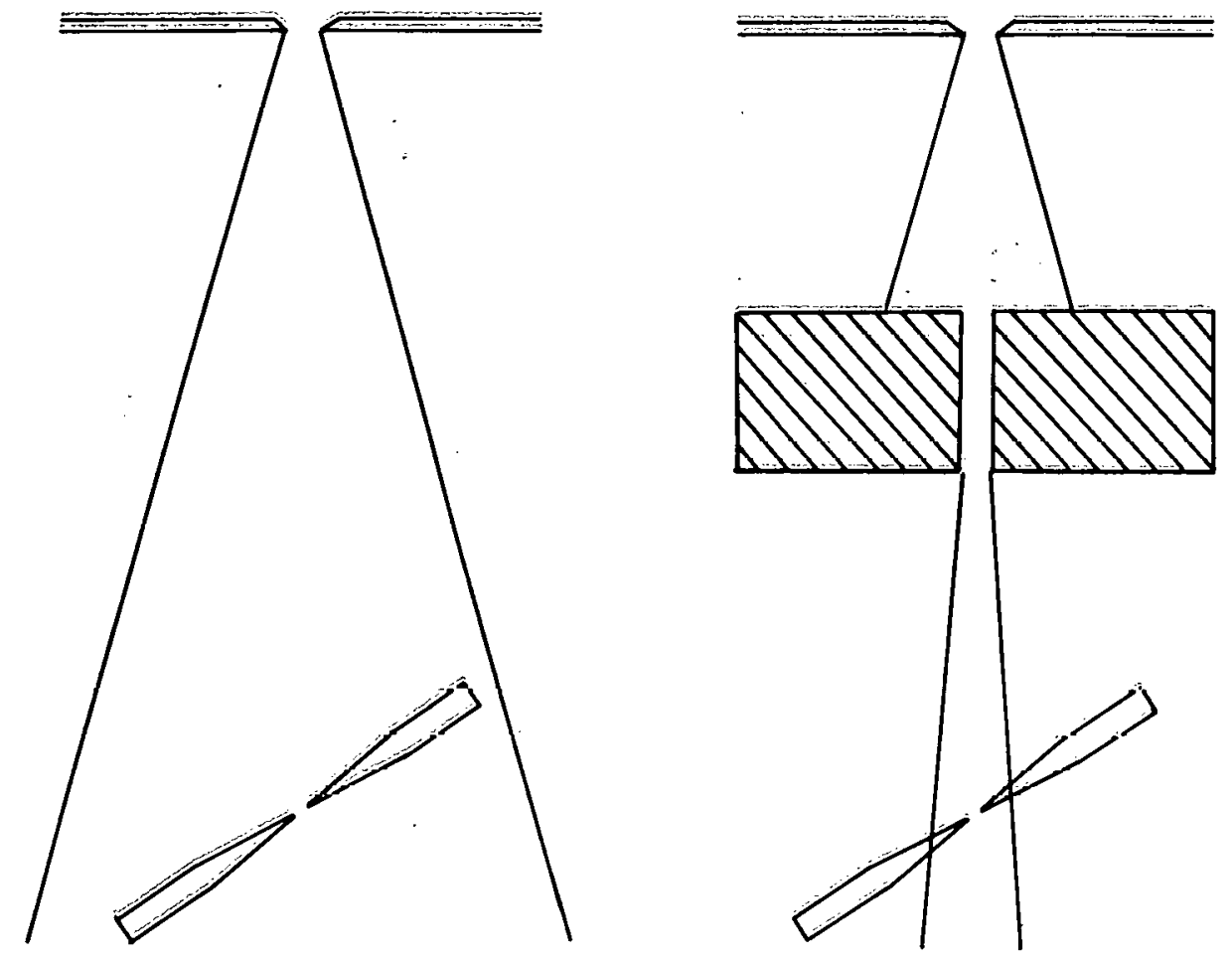

Fig. 46; Schematic Diagram Illustrating Keduction of Fluorescence

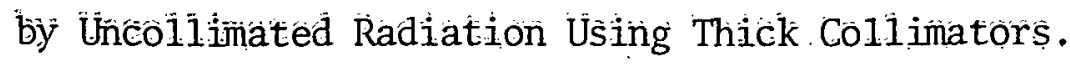




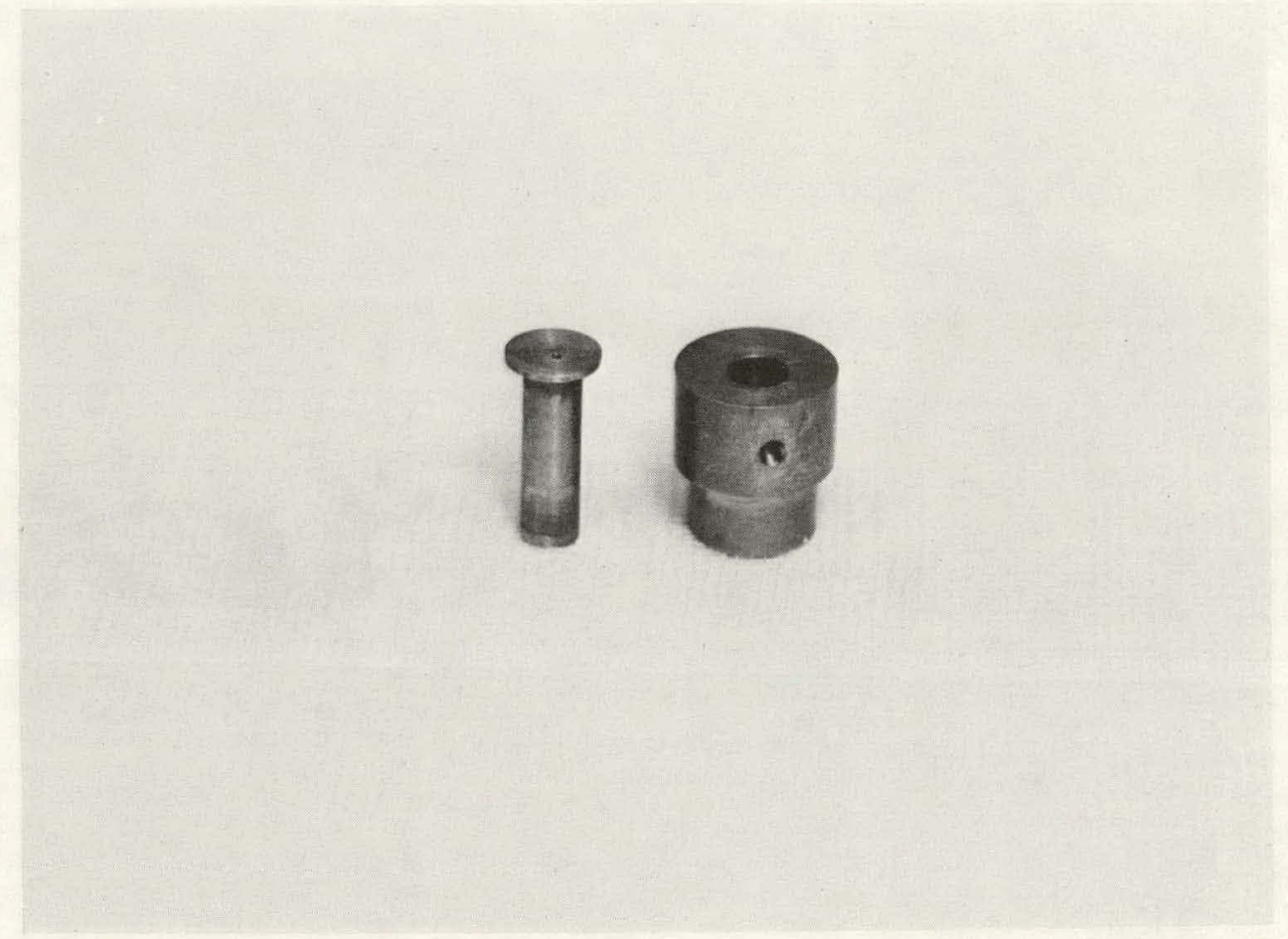

Fig. 47. Eccentric Cylinders Used as Collimators in JEOL JSEM 200. 


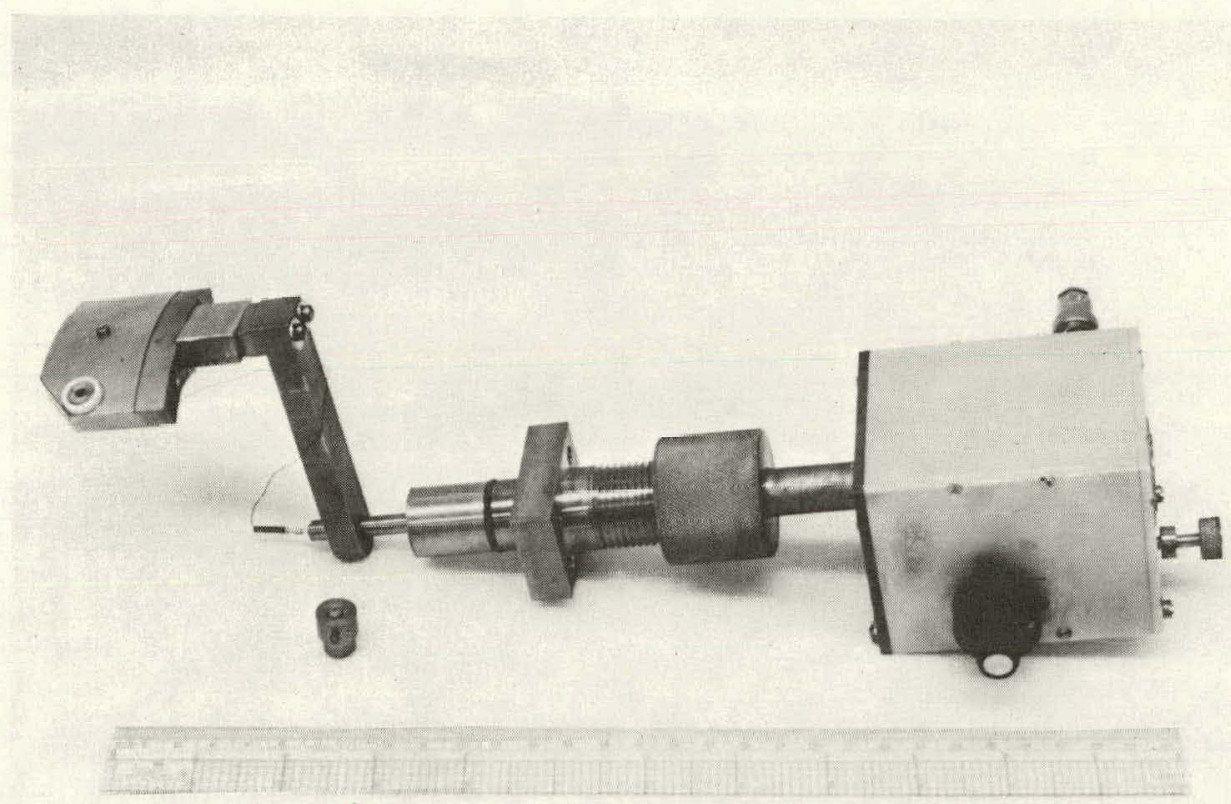

Fig. 48. Adjustable Bremsstrahlung Aperture Drive for JEOL JSEM 200. 


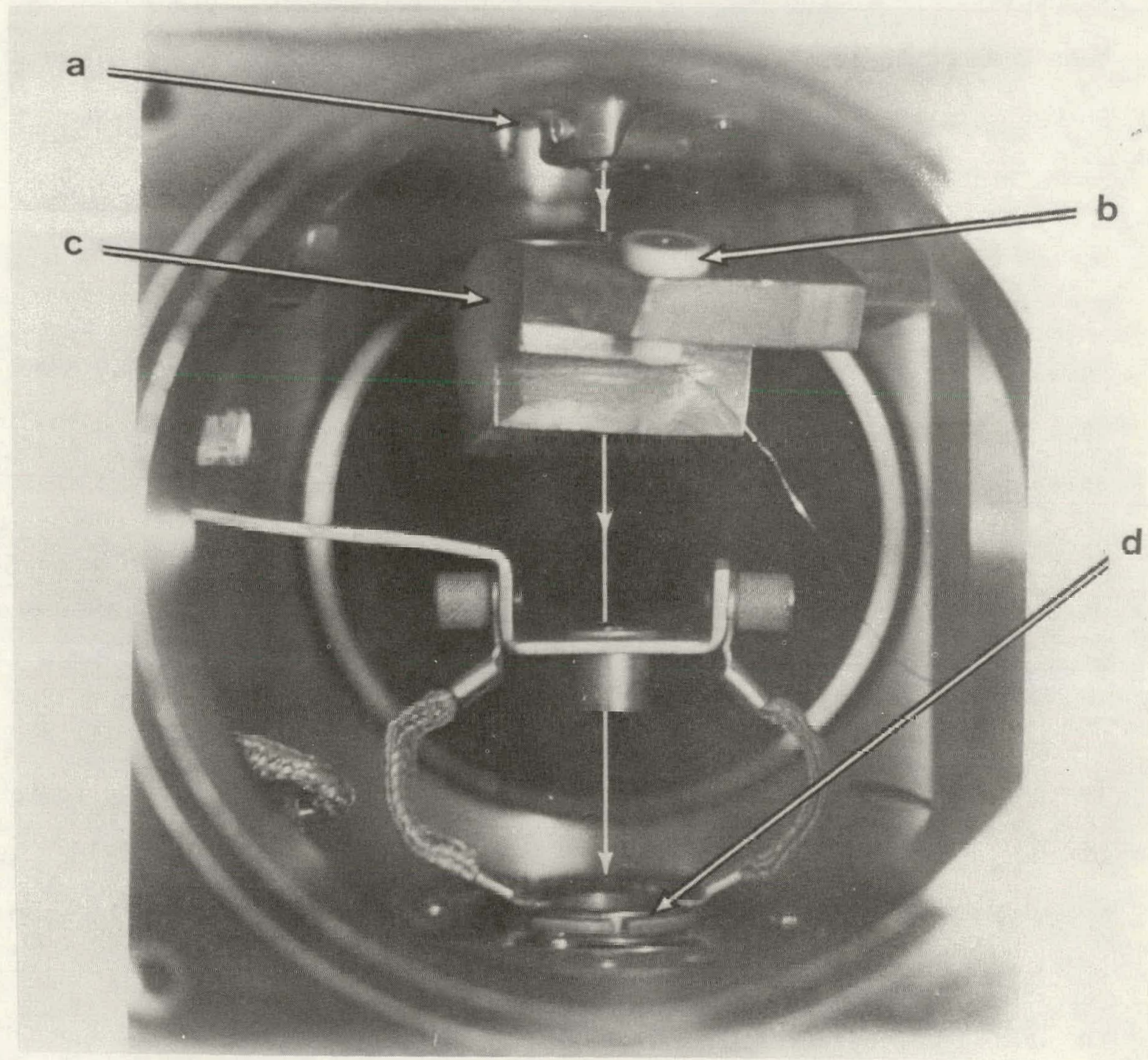

Fig. 49. Adjustable Bremsstrahlung Aperture System Mounted on Microscope Column. (Vertical arrow indicates electron-optical axis.) (a) Eccentric aperture system (see Fig. 47), (b) Faraday cup, (c) adjustable Bremsstrahlung aperture system (Fig. 48), (d) anti-contamination cold trap. 
The resulting changes produced by using such a system are shown in Figs. 41, 42, and 43 for the sample (c) and in-hole (d) spectra, respectively. In the worst case $(200 \mathrm{keV})$ the in-hole spectrum has been reduced to less than $2 \%$ of the total sample emission. The 100 and $50 \mathrm{keV}$ results show even greater improvements. Figure 50 compares the bremsstrahlung corrected results for the variation in the Ni/Al ratio with thickness to the theoretical calculations. Agreement between the experimental data and calculations is now substantially better. The variation in the intensity ratio with increasing thickness is reproduced well in terms of the general analytical shape of the curve. There is sti11, however, a discrepancy in absolute value between the two curves which appears to be roughly constant at a given energy. This can, in part, be attributed to contamination of the sample and will be discussed further in the next section.

The procedure now adopted for $x$-ray measurements in the microscope is to record two spectra for each data point - the first from the region of interest in the sample, and the sernnd the bremsstrahlung (or in-hole) spectrum. The bremsstrahlung spectrum is then subtracted from the sample spectrum before any further analysis is performed. It is important that the uncollimated radiation first be reduced to a low intensity before subtraction. This is simply due to the likelihood of large errors being introduced from the subtraction of twn spectra of similar intensity. The effects of this are illustrated in Figs. 51 and 52. In Fig. 51 the upper curve represents the experimental variation in the $\mathrm{Ni} / \mathrm{Al}$ ratio measured 

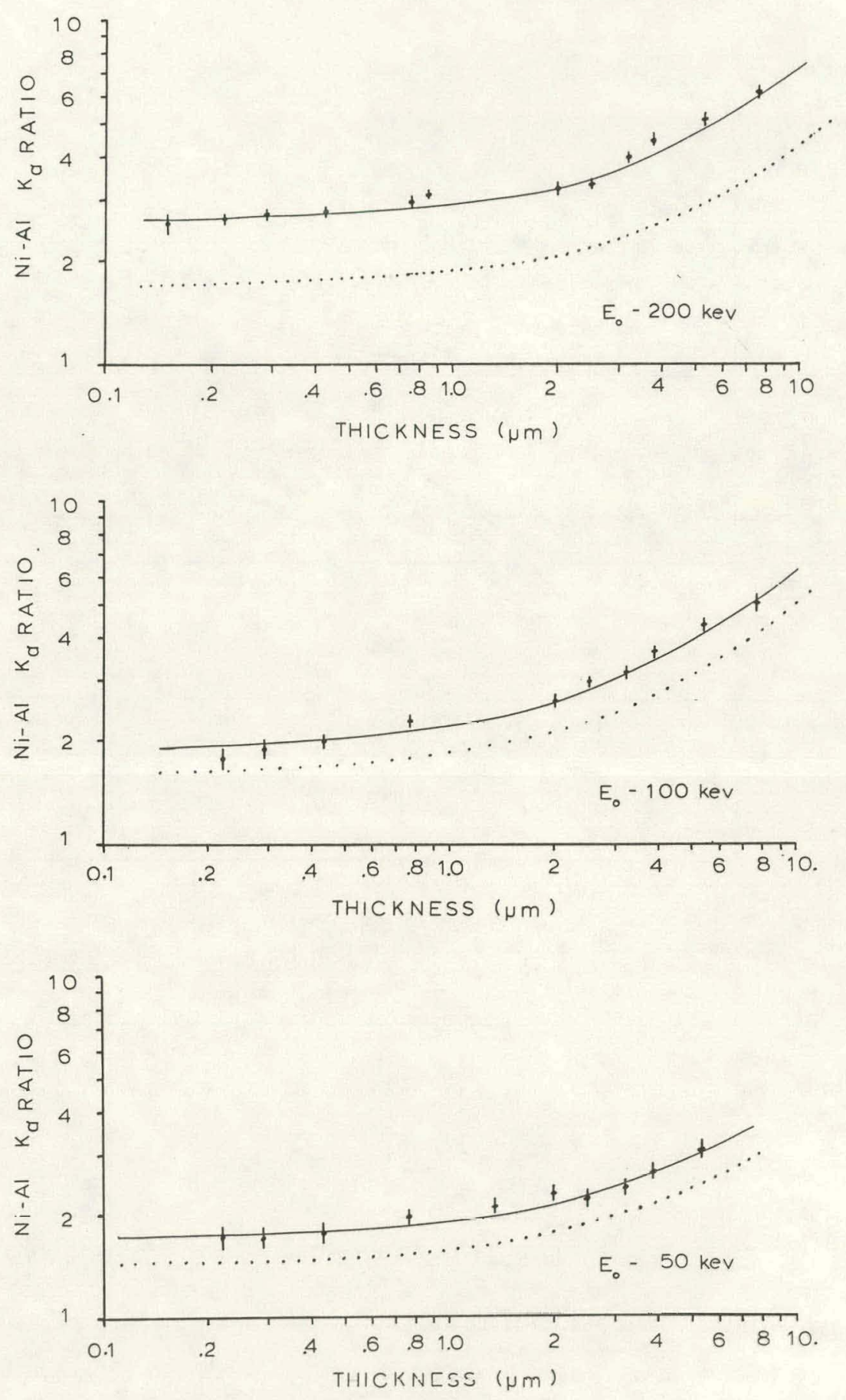

Fig. 50. Corrected Results for the Ni/Al Ratio as a Function of Thickness after Installation of Bremsstrahlung Aperture System, Broken curves indicated calculated variation. 


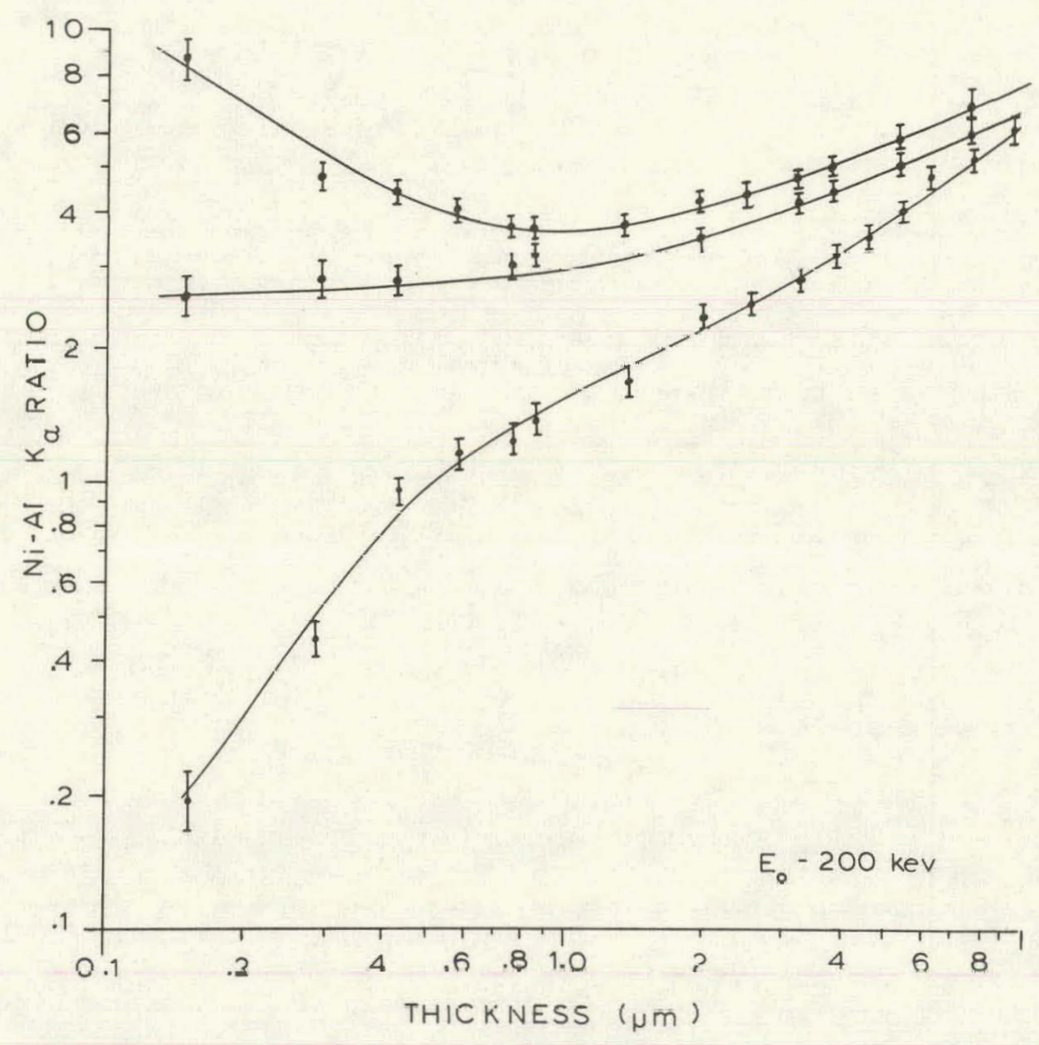

Fig. 51. Errors Resulting from Improper Correction of Bremsstrahlung Fluorescence Problem at $200 \mathrm{keV}$. 

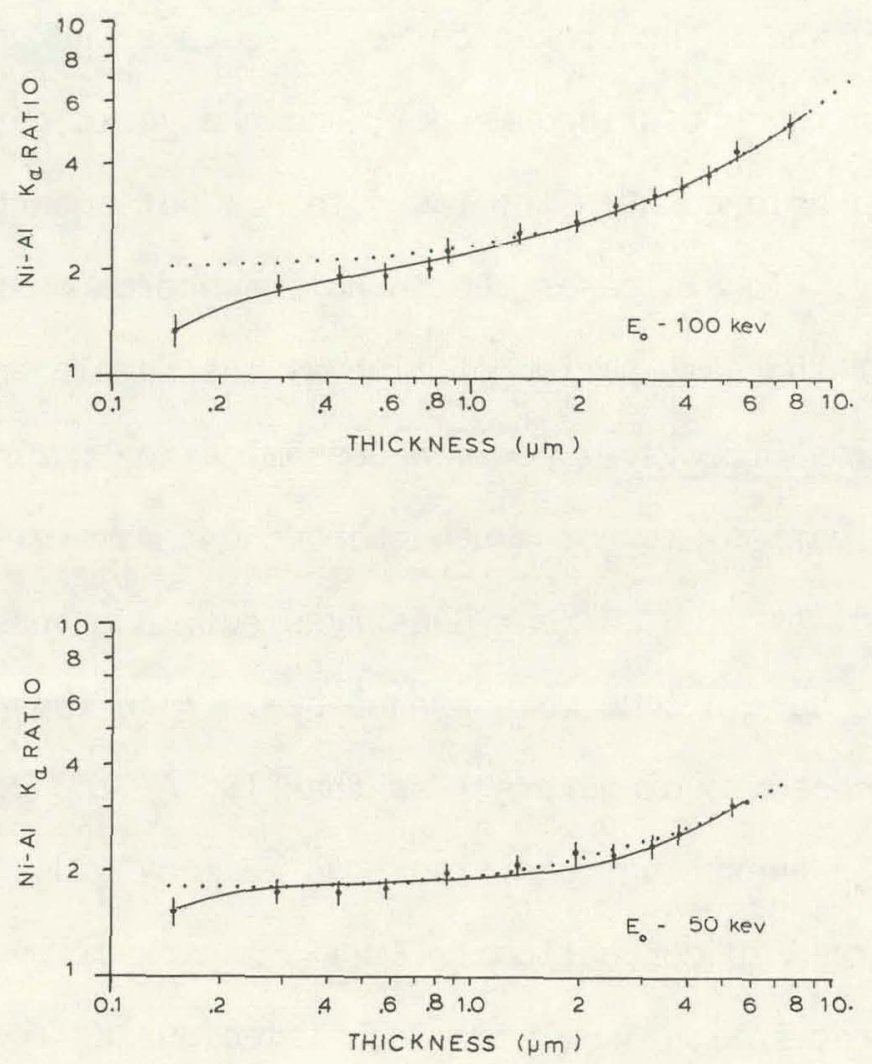

Tig. 52. Errors Resulting from Improper Correction of Bremsstrahlung Fluorescence Problem at 100 and $50 \mathrm{keV}$. 
at $200 \mathrm{keV}$ with no bremsstrahlung correction. The lower curve shows the effect of simply subtracting the in-hole count without first reducing the bremsstrahlung flux. Finally, the middle curve was obtained with the $x$-ray aperture correctly inserted and the reduced in-hole spectrum subtracted from all measurements. Figure 52 plots the similar results for 100 and $50 \mathrm{keV}$ where the broken curve represents the data after being corrected in the prescribed manner, and the solid curve by simply subtracting the in-hole count. The raw data has not been plotted in this figure for clarity. In all cases the in-hole spectrum must, of course, be accumulated for the same period of time as the sample spectrum. Contamination Effects: Specimen contamination during electron microscope investigations is not a new phenomenon; however, with the advent of STEM and its high current density electron probes, the problems generated have become more pronounced. When the probe is stationary and focused to diameters less than $100 \AA$, the contamination rates under certain operating conditions can be many orders of magnitude greater than observed in conventional TEM imaging experiments. Two mechanisms of contamination have been identified during the last few years; ${ }^{84,85}$ volume diffusion of hydrocarbons from the microscope vacuum system, and surface diffusion of adsorbed material on the sample surface. The volume diffusion component can be reduced by improvements to the microscope vacuum system such as those foumd in the newer UIV-STEM instruments where the vacuum levels at the specimen are $\leqslant 2 \times 10^{-9}$ torr (versus $\sim 2 \times 10^{-7}$ torr typical of most new CTEM instruments). The surface 
diffusion component is harder to identify and control, being a function of specimen preparation and handling before and during insertion into the microscope environment.

Fourie ${ }^{85}$ has studied the formation and shape of contamination in cryopumped TEM and UHV STEM systems. In this work he found that for large probe diameters $(0.6$ to $3.0 \mu \mathrm{m})$ the contamination forms in the shape of a disc of material, the exact profile of which varies with incident electron flux, sample composition, and thickness. In general, the contamination layer was least at the center of the irradiated area, while at the edges the accumulation of material was greatest as shown in Fig. 53. For the probe sizes used in this work $(\leqslant 200 \AA)$ the shape of the contamination was different, taking on the form of small spiraling cones. This is illustrated in the micrographs of Figs. 53 and 54. Figure 54 is a TEM micrograph showing a region of $\beta$-NiAl after an $x$-ray experiment performed in the STEM mode. The dark spots on the foil (both on the electron entrance and exit surfaces) are the contaminated regions formed during a $200-\mathrm{s}$ analysis with a $100 \AA$ stationary STEM probe. The micrographs in Fig. 55 show the spiral nature of these contamination cones in more detail. This micrograph was taken in the SEM mode of operation in the JSEM 200. Figure 55(a) views the cone end in the orientation in which it was formed, while in Fig. 55(b) the sample was tilted through $\sim 20^{\circ}$. The spiral shape is most likely due to the electron probe wandering due to charging effects caused by the poor conductivity of the contamination layer. 


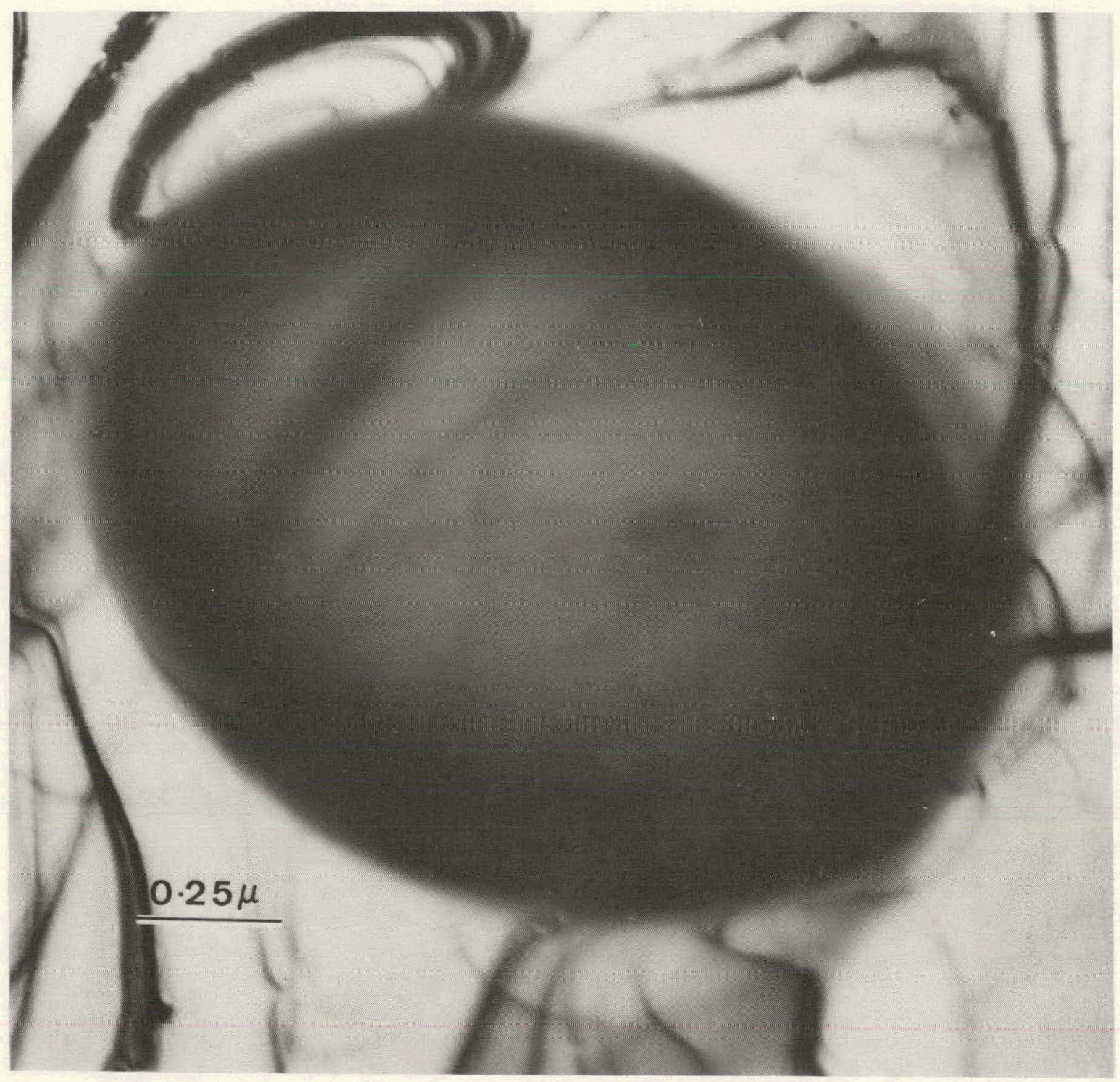

Fig. 53. Contamination Formed During CTEM Operation. 


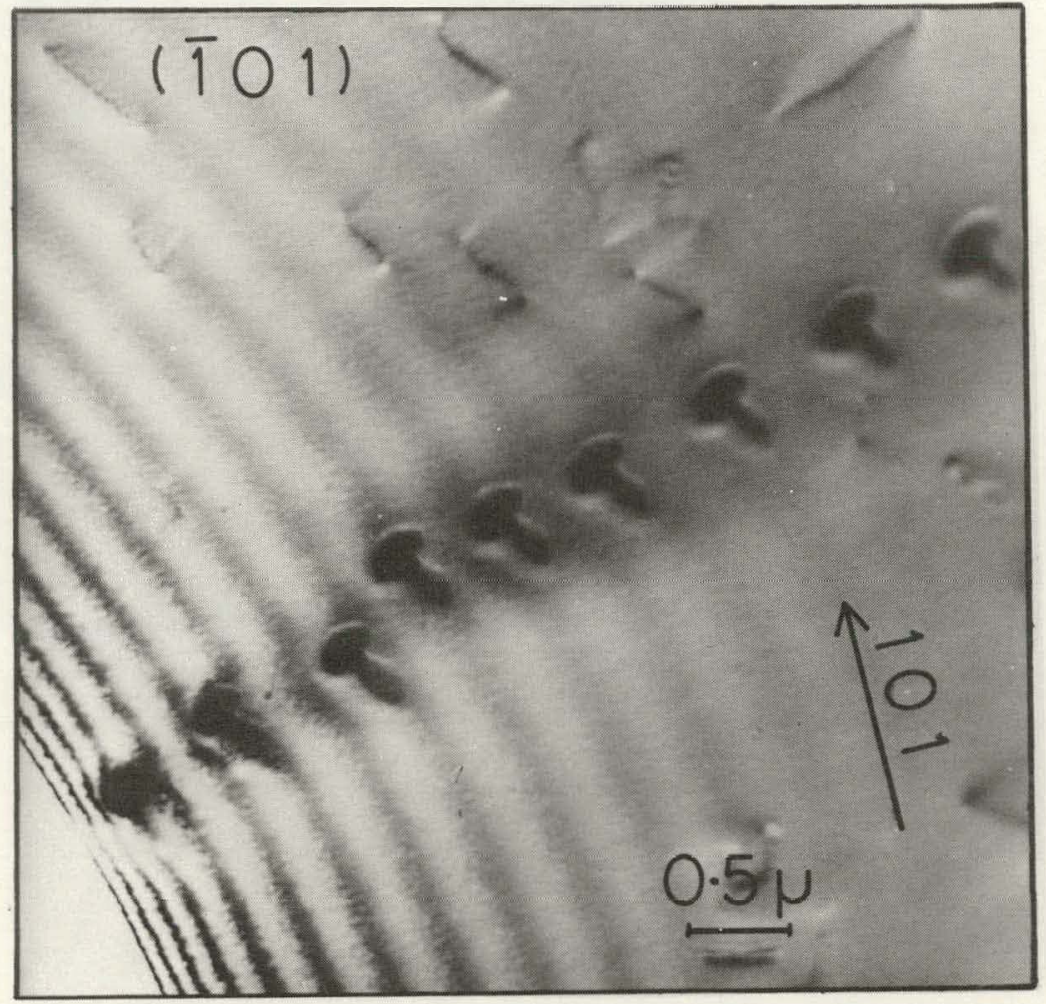

Fig. 54. Contamination Formed During STEM Operation. 

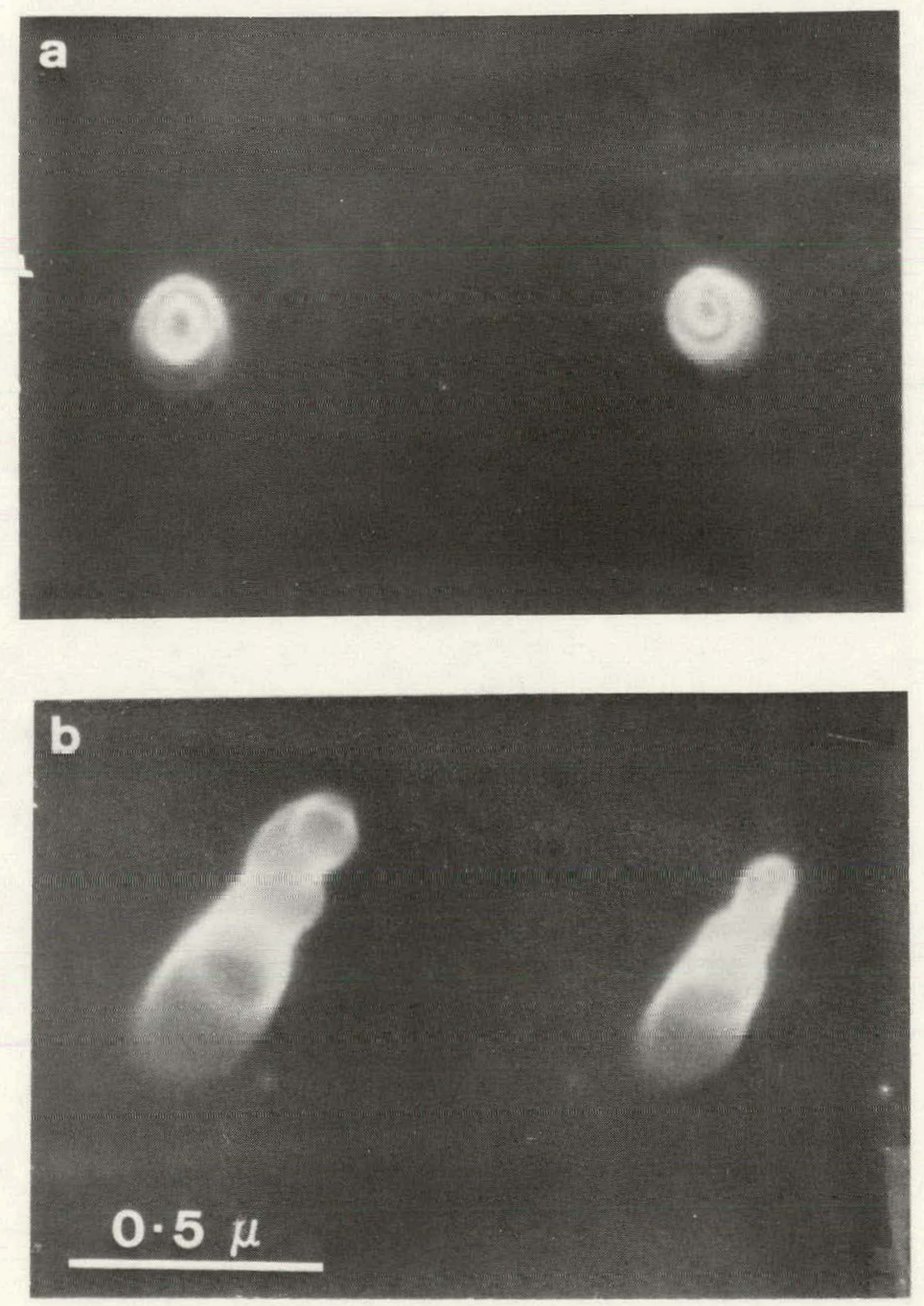

Fig. 55. SEM Micrograph of STEM Contamination Cone Showing Its Spiraling Nature. 
At least three detrimental aspects are immediately apparent from these micrographs. First, there is the obvious result that once the con= tamination forms it is no longer possible to image the particular region of the sample. Second, the wandering of the electron probe as well as scattering effects in the contamination layer effectively broadens the probe size before it interacts with the specimen, thus decreasing the $x$-ray spatial resolution provided by the small probe diameter. Finally, the volume of material deposited on the sample provides yet another source of scattering and absorption which will ultimately decrease the sensitivity of analytical information released from the sample. The remaining part of this discussion will focus on this last point in greater detail,

The first problem to consider is related to the generation of systems peaks by the contamination layer. If the deposited material were purely hydrocarbon, then for the case of $x$-ray detection using standard Si(Li) detectors no additional characteristic emission will be recorded. However, some instruments use a silicon-based diffusion pump oil and 0-ring greases which can lead to the accumulation of silicon as well as hydrocarbons on the sample. This is shown in Fig. 56 which is an expanded region of the $\mathrm{x}$-ray emission spectrum from $\beta$-NiAl from 0.5 to $2.0 \mathrm{keV}$. Three peaks are clearly resolved: Ni $\mathrm{L}_{\alpha}(\sim 0.85 \mathrm{keV})$, Al $K_{\alpha}(\sim 1.49 \mathrm{keV})$ and $\mathrm{Si} K_{\alpha}(\sim 1.74 \mathrm{keV})$. The presence of the silicon peak correlates with the degree of contamination observed to exıst in the local region being analyzed. When the diffusion pump oil was changed from a silicon-based system to one using fluorinated hydrocarbons the Si $K_{\alpha}$ peak was eliminated, until outgassing from "O-ring" greases and roughing pump lines resaturated the diffusion pump oil with silicon contaminants. 

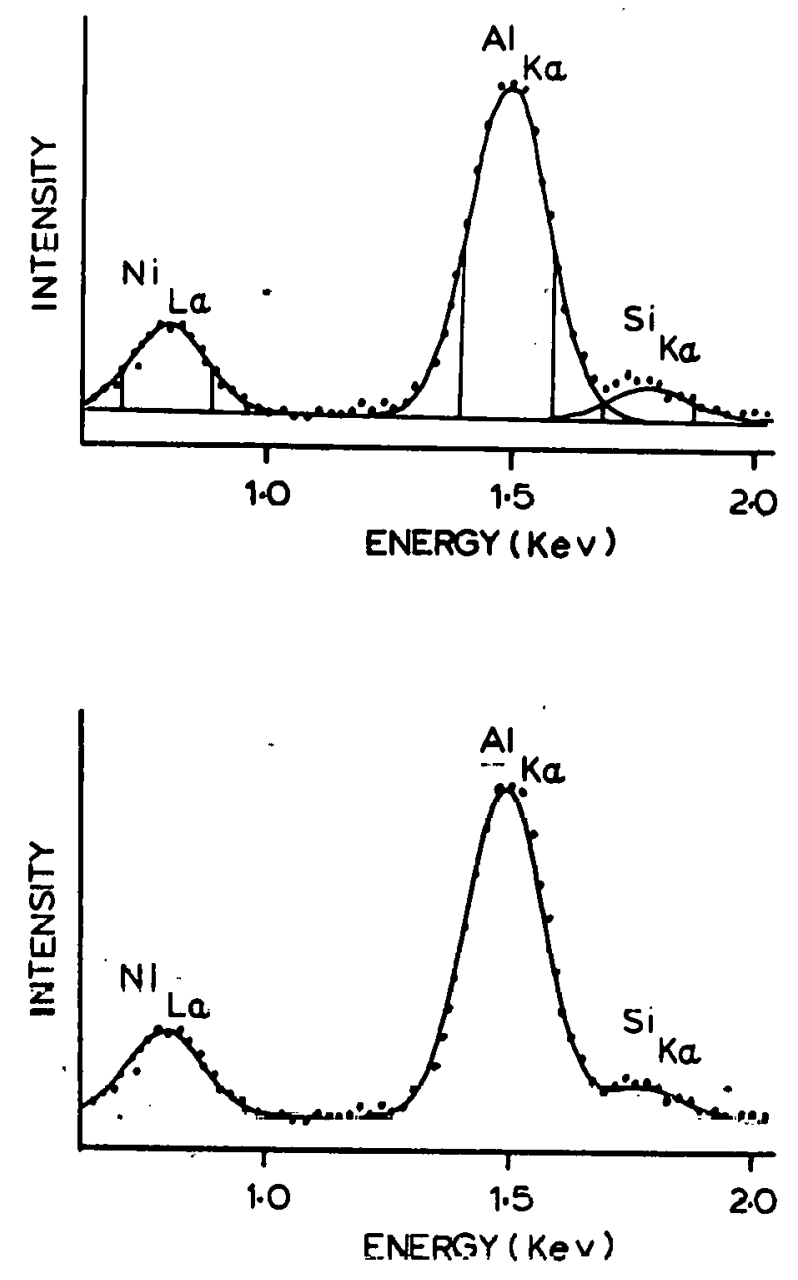

Fig. 56. Silicon Contamination Peak Recorded on a Pure Ni/Al Specimen. 
The accumulation of considerable amounts of foreign material on the sample surface also raises the question of $\mathrm{x}$-ray absorption of $\mathrm{x}$ rays within this deposited layer. Figure $57(a)$ once again compares the measured Ni/Al $K_{\alpha}$ ratio to calculations at $200 \mathrm{keV}$. There is clearly a moderate discrepancy between the two curves. Since all measurements in this figure were obtained under the same operating conditions and measurement times (200 s), the amount of contamination deposited on the sample will be constant to a first approximation. This would then lead to a constant discrepancy between the experimental and calculated results due to absorption of the lower energy $\mathrm{Al} \mathrm{K}_{\alpha} \times$ rays. To test this hypothesis the $\mathrm{Ni} / \mathrm{Al} \mathrm{K}_{\alpha}$ ratio was measured as a function of time (hence also contamination) at constant thickness and incident energy. The results of this experiment, Fig. 57 (b), confirm that absorption effects can be appreciable. To account for most of the discrepancy in Fig. 57(a), the amount of absorption at $200 \mathrm{~s}$ seems to be about the correct urder of magnitude, when comparing Fig. 57 (b) to the appropriate point of $57(\mathrm{a})$. One further aspect of sample contamination is left to consider namely, the effects on mass sensitivity. In an early study of TEM-based microanalysis, Russ ${ }^{86,87}$ presented results which seemed to indicate that the optimum accelerating voltage for $x$-ray work was in the vicinity of $100 \mathrm{keV}$. This conclusion was based on experimental measurements of the peak-to-background $(\mathrm{P} / \mathrm{B})$ ratio for various elements which exhibited a broad maximum in this incident energy range and then a decrease in $F / B$ at higher energies. This is in sharp contrast with the theoretical calculations presented in Sect. 2.2.6 which predict a continuous increase 
(a)

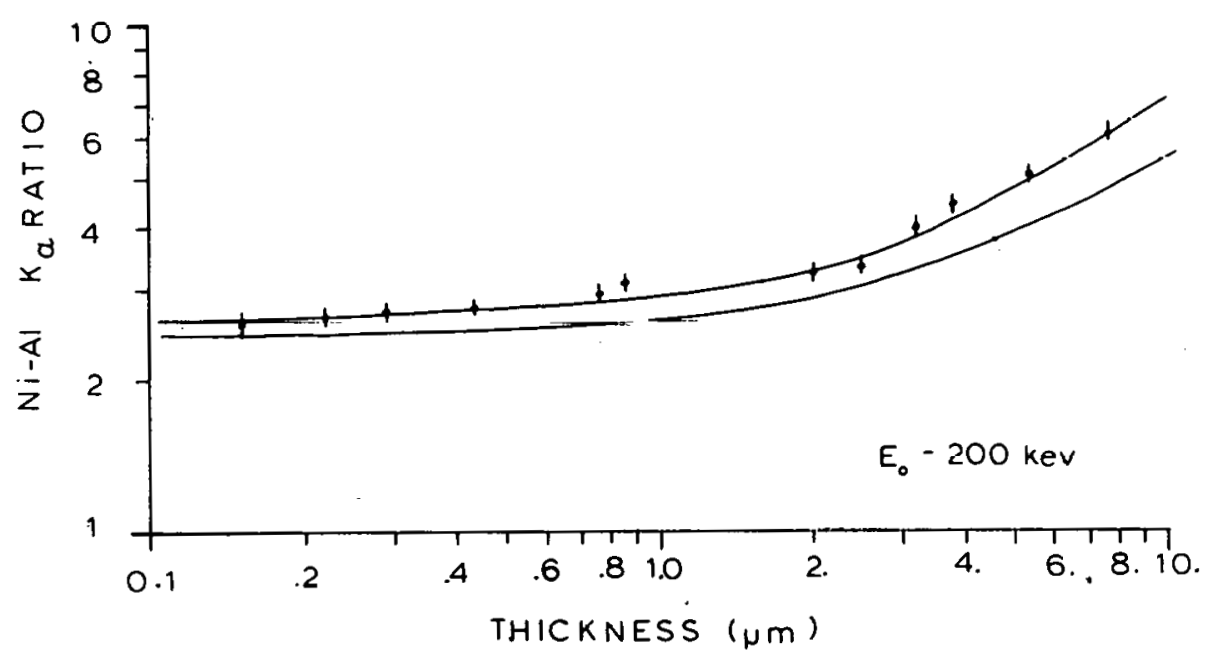

(b)

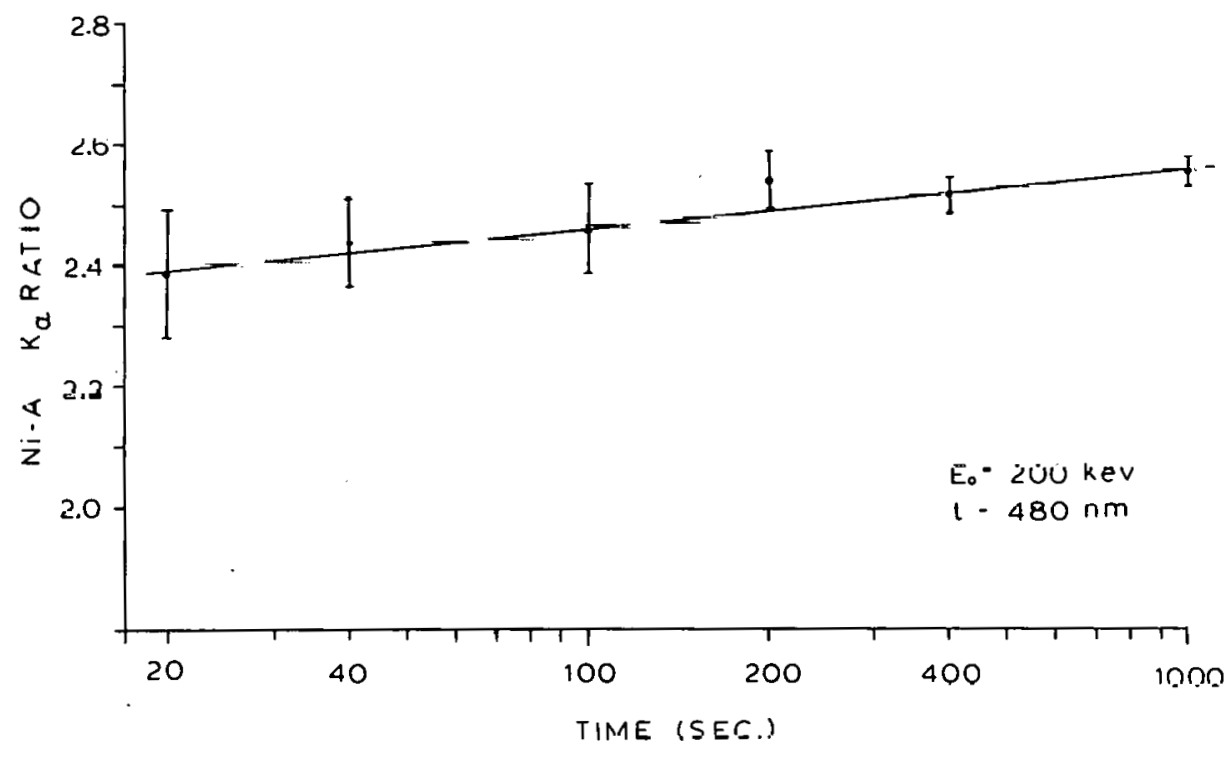

Fig. 57. Comparison of Experimental and Calculated Ni/Al Ratio (a). Effects of contamination accumulation on experimental $\mathrm{Ni} / \mathrm{Al}$ ratio (b). 
in the $\mathrm{P} / \mathrm{B}$ ratio with electron energy. Further experimental work by Zaluzec and Fraser ${ }^{4.4}$ has shown that these discrepancies may be attributed to a combination of $\mathrm{x}$-ray fluorescence and sample contamination effects.

The effects of $x$-ray (bremsstrahlung) fluorescence on quantitative analysis has been discussed at length previously; however, the effects on mass sensitivity have been postponed until this time. Referring to Sect. 2.2.6, theoretical calculations indicate that for thin foils of all elements the characteristic $P / B$ ratio should increase with accelerating voltage monotonically. This result assumes that all $\mathrm{x}$-ray emission from the sample was derived from purely electron excitation. The effects of bremsstrahlung fluorescence on the experimentally determined $\mathrm{P} / \mathrm{B}$ ratio are demonstrated in Fig. 58. Figures 58(a) and 58(b) plot the results of measurement of the $\mathrm{NiK}_{\alpha}$ and $\mathrm{Al} \mathrm{K}_{\alpha} \mathrm{P} / \mathrm{B}$ ratio in $\mathrm{B}$-NiAl as a function of incident beam energy without the correction for bremsstrahlung fluores * cence. These data are similar to those obtained by Russ. in an earlier study. When the appropriate modifications are made to the electronoptical colum the results shown in Figs. 58(c) and 58(c) are obtained. The effects of contamination on the recorded P/B ratio are complex and difficult to control. In all cases the amount of material accumulated on the sample is a function of the total electron flux irradiating the area. Hence it will be assumed in the following discussion that contamination increases monotonically with time at constant probe current. In addition, it will be also assumed that approximately equivalent results can be obtained by holding the product of beam current and irradiation time constant. The trends presented in this section should 


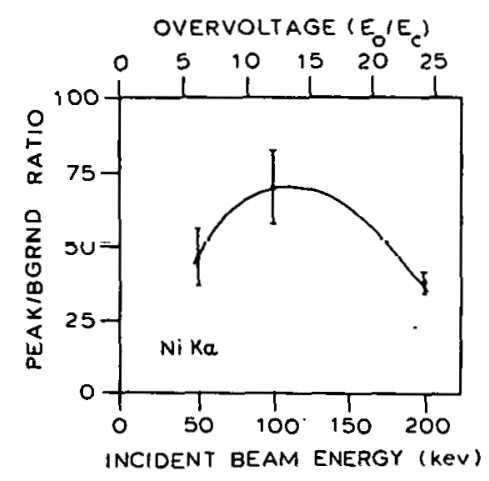

(a)

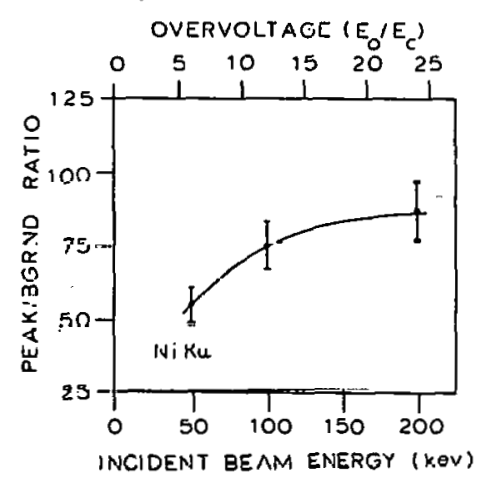

(c)

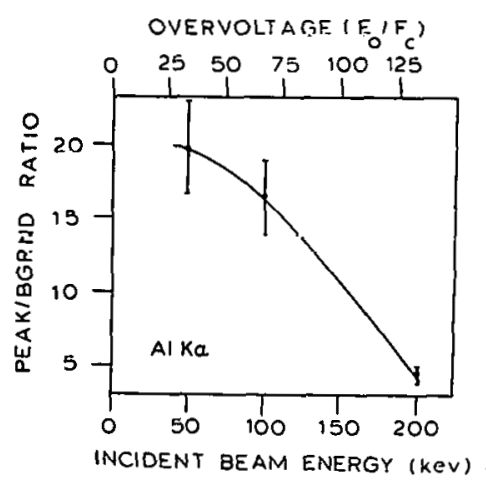

(b)

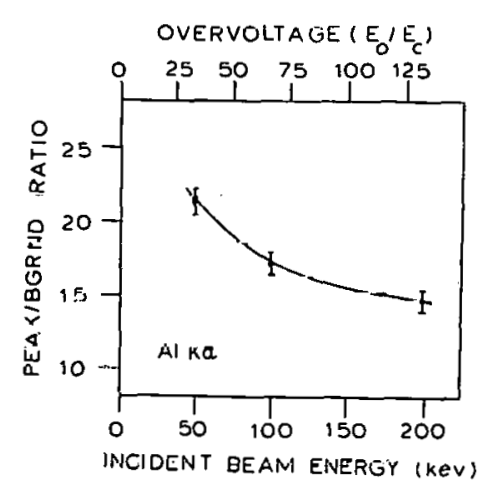

(d)

Fig. 58. P/B Ratios for $\mathrm{Ni}$ and $\mathrm{Al}$ in $\mathrm{Ni} / \mathrm{Al}(\mathrm{a}, \mathrm{b})$ Without Correction for Bremsstrahlung Fluorescence; (c,d) with Correction for Bremsstrahlung Fluorescence. 
be considered only as qualitative indications of the effects of contamination since quantitative measurements of the contamination are presently impossible to obtain. Lastly, in order to minimize the complications which result from the effects of absorption of low-energy $x$ rays in the contamination layer the results shown in the following figures will center mainly on the Ni $K_{\alpha} P / B$ ratio.

Ideally, from the theoretical calculations presented in Sect. 2.2.6, the $\mathrm{P} / \mathrm{B}$ ratio should to a first approximation remain constant as a function of both sample thickness and time, and in the absence of fluorescence effects should increase monotonically with incident beam energy. Experimental measurements, unfortunately, do not exhibit such a simple behavior; they are sumarized in Figs. 59(a) and 59 (b). The discrepancies are, however, systematic in nature and can be related to the nonlinear variation in the continuum intensity generated as a function of time. This can be seen in Figs. $60(\mathrm{a})$ and $60(\mathrm{~b})$. The intensity of the $\mathrm{Ni} \mathrm{K}_{\alpha}$ peak in this figure varies linearly with both sample thickness and irradiation time. The continum intensity, which in this case is the intensity measured in the region from 9.5 to $10.0 \mathrm{keV}$ (i.e., a region isolated from all characteristic peaks), shows a highly nonlinear response. The most important parameter which influences this intensity is the total sample thickness, all other parameters being roughly constant [see Eq. (2.2)]. Thus, the changes in continuum intensity can be directly correlated with the accumulation of contamination on the sample. With this in mind, the systematic changes in Figs. 59(a) and 59(b) can be qualitatively explained. 

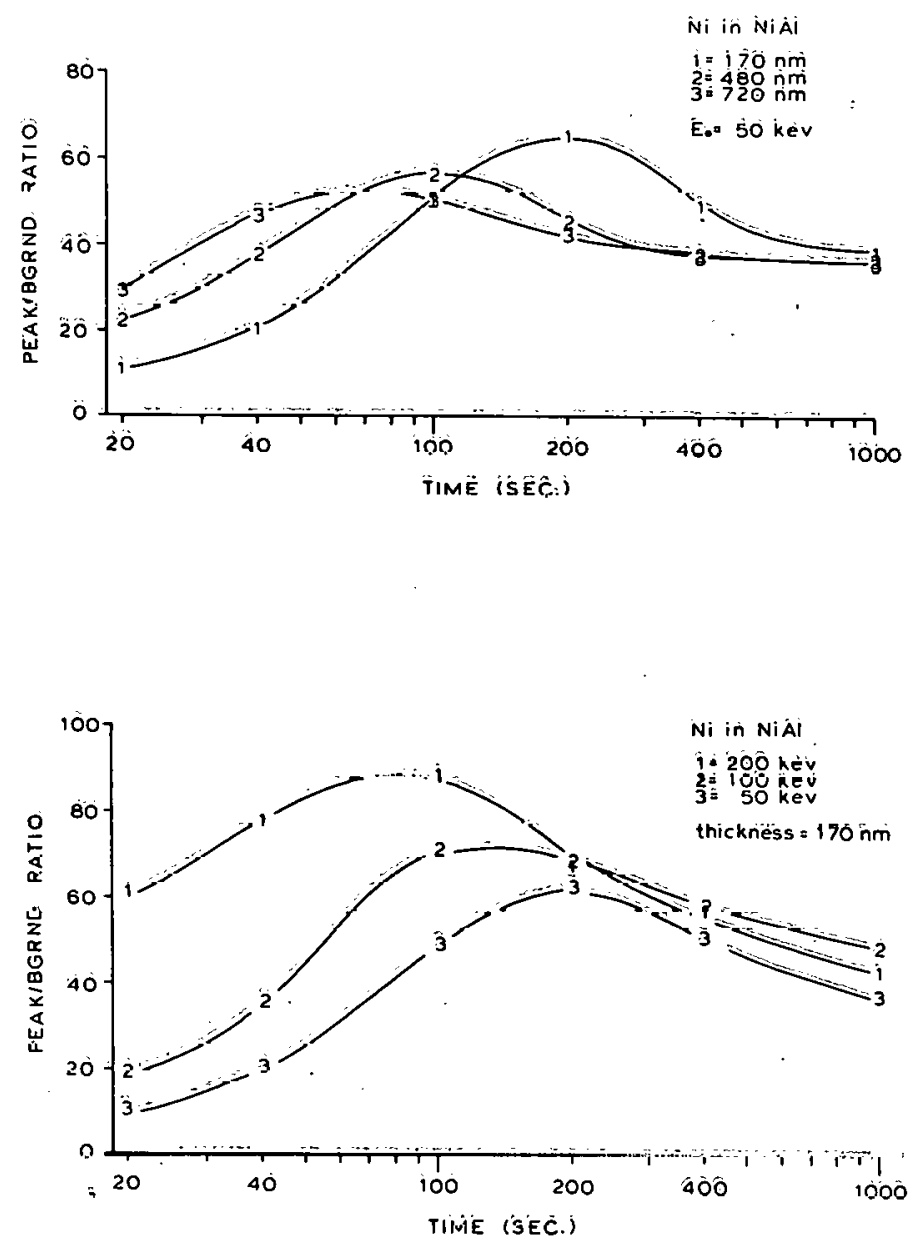

Fig: 59. Variation in Expèrimental $\mathrm{P} / \mathrm{B}$ Ratio as a Function of Contamination and Operating Conditions. 

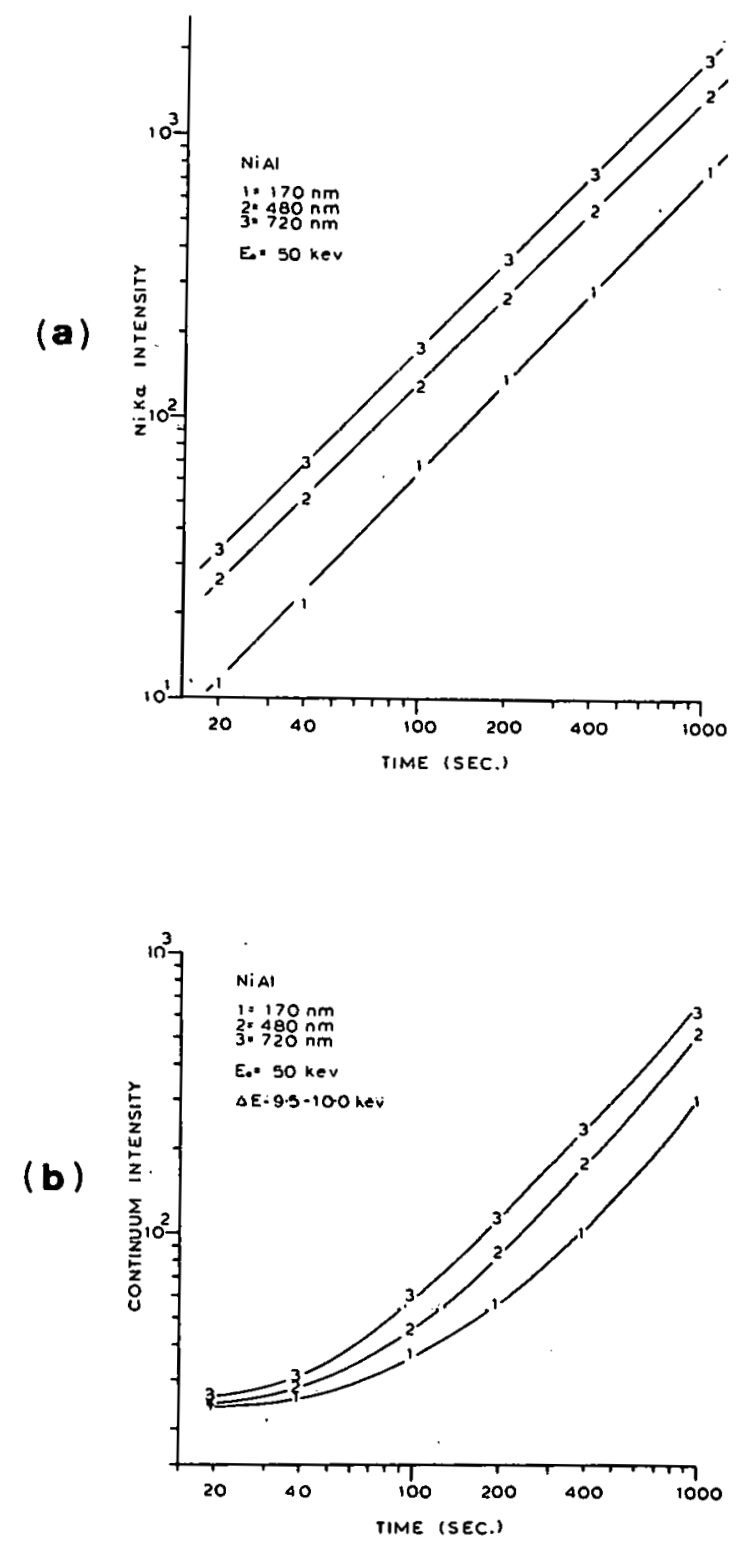

Fig. 60. Variation in Ni $K_{\alpha}$ Intensity with Time and Thickness (a). Variation in continum intensity with time and thickness (b). 
It should now become obvious that plotting the measured $P / B$ ratio as a function of incident energy is inconclusive in the presence of contamination, since 1iterally any value of $P / B$ can be obtained by judiciously choosing the amount of contamination accumulated. Such an effect is illustrated by the experimental data shown in Figs. 61(a) and 61(b) for $\mathrm{Ni} K_{\alpha}$ and $\mathrm{Al} K_{\alpha}$ in $\beta$-NiAl. Thus, a direct comparison with theoretical calculations is difficult. A qualitative comparison can be made, at best, by holding the amount of contamination constant at each measurement point. This can be accomplished by varying the time of analysis at each incident beam energy appropriately (i.e., it is assumed by decreasing the incident energy by one-half requires a doubling data acquisition time). The results of such an experiment are shown in Fig. 61(c), and the qualitative agreement with the calculations of Fig. 35 is excellent.

\subsubsection{Data Reduction for Quantitative Analysis}

The reduction of $x$-ray emission data obtained using a TEM based system can become a complex process bearing in mind the details discussed in the previous sections. In addition to the instrumenta1 and theoretical correction factors which must be evaluated to obtain quantitative results, one must also consider the task of extracting characteristic intensity measurements out of a spectrum consisting of many poaks superimposed upon a slowly varying background. The major difficulty is in complex multi-element analysis, where spectral interference occurs, making it necessary to employ deconvolution techniques to extract 


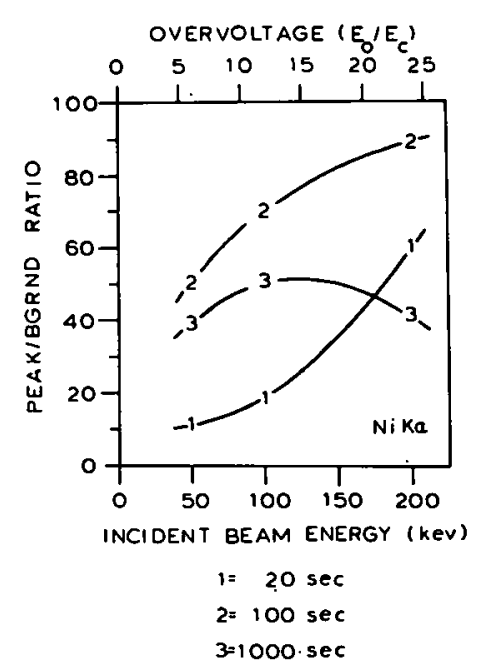

(a)

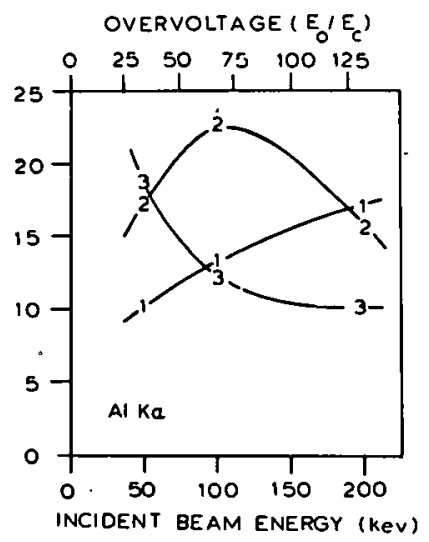

$1=20 \mathrm{sec}$
$2=100 \mathrm{sec}$
$3=1000 \mathrm{sec}$

(b)

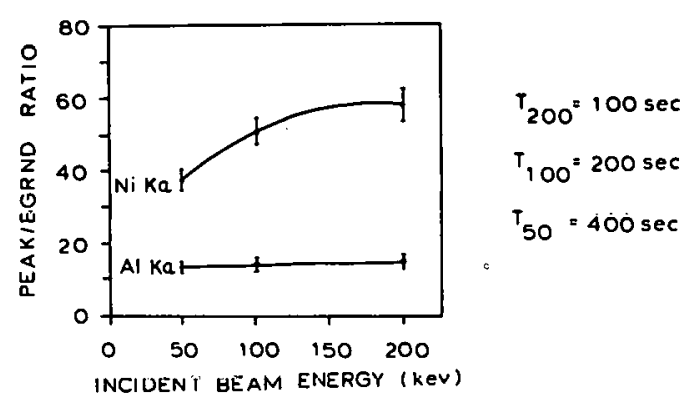

(c)

Fig. 61. Variation in $\mathrm{P} / \mathrm{B}$ Katio as a Function of Time for $\mathrm{Ni}$ (a) and Al (b). Comparison of experimental results at approximately constant contamination (c). 
information obscured by peak overlaps. Clearly, as the level of sophistication of analysis increases, there arises a need for corresponding increases in computational facilities, versatility and speed necessary to perform these tasks.

Consequently, a microcomputer system consisting of a DEC LSI-II microprocessor, with $32 \mathrm{~K}$ words of MOS memory, an AED 5100 dual-density floppy disc mass storage device, an ASR-33 Teletype terminal, and a 4010-1 Tektronics graphics terminal was assembled and interfaced to an ORTEC 6200 multichannel analyzer (MCA). In addition to this hardware expansion a series of operator interactive computer programs have been developed to facilitate simple on-line analysis of $x$-ray spectra. The software developed during this research has undergone a continuous process of updating and revision in order to keep up with changes in experimental techniques and is documented in Appendices A through F. Included in these listings are subroutine libraries (NXRYL, NGRAPH) which serve as building blocks for the general-use programs (NEDS, NXRYRT, NMCS) directed toward specific applications of data reduction and analysis of EDS, ELS, and TSED data. The software has been written in FORTRAN IV to optimize access in a multi-program/user environment and furthemore to facilitate modifications to existing programs as future developments occur. It is appropriate to note at this point that several subroutines found in the $\mathrm{x}$-ray library (NXRYL) have been adapted in whole or in part from the MAGIC series of $x$-ray analysis programs developed by Colby ${ }^{6.3}$ for quantitative analysis of bulk specimens using electron microprobes: 
Three programs documented in the appendices are specifically devoted to x-ray analysis - namely, MCA, NEDS, and NXRYRT. The program MCA is used to read data from the multichannel analyzer and creates a semi-permanent record onto a floppy disc file. NEDS is subsequently used to process recorded spectra, extracting characteristic intensities from the raw data. Finally NXRYRT is used to convert relative intensity ratios into composition measurements using a standardless approach. An outline of a procedure for $x$-ray analysis using the "thin-film" standardless technique is given next, and this can be used for the majority of $\mathrm{x}$-ray work done on the current breed of TEM/STEM/AEM instruments. This will be subsequently followed by a discussion of the application of the absorption correction and finally on quantitative analysis using thinfilm standards.

Standardless Analysis: Having optimized the various geometrical and instrumental factors outlined in Sect. 2.2.6, one proceeds to record a spectrum from the specimen, which should be measured over a sufficient period of time to ensure statistical significance of all characteristic peaks under investigation. In addition to these considerations, one should ensure that the sample is appropriately oriented so that anomalous emission due to electron channeling is minimized. ${ }^{88-90}$ Once the sample spectrum has been recorded and stored, then a second spectrum which is due to the effects of uncollimated radiation must be measured. This can be simply obtained by translating the electron probe off the specimen (for example, by moving the probe into a hole in the TEM disc) and 
accumulating the resulting spectrum under identical operating conditions, This "in-hole" spectrum should not exceed $\sim 5 \%$ of that obtained when the electron probe is positioned on the thinnest region of the sample to be analyzed, since the form of these two spectra may be quite different because of differences in the mechanism of x-ray generation. Thus, subsequent subtraction of spectra may result in substantial errors if they are of comparable intensities.

The correction to the sanmie spertmm for uncollimated radiation is straightforward - namely, a simple chamel-by-chamel subtraction. In order for this subtraction technique to be considered valid, two criteria must be satisfied. First the in-hole spectrum must be small with respect to the sample spectrum; otherwise, large systematic errors can develop. Secondly the in-hole spectrum which is subtracted must reflect the identical electron dose relative to the corresponding sample spectrum. For the case of CTEM/STEM. instruments with conventional thermionic emission or $\mathrm{LaB}_{6}$ fillaments the emission current has sufficient long-term stability that measurements at constant detector live time will to a good approximation be equivalent to constant electron dose. This is not generally valid for field emission sources where the emission current can vary substantially. with time and hence some appropriate beam current monitoring system must be developed to compensate. 91

Once the sample spectrum is corrected for fluorescence by uncollimated radiation then standard techniques for spectral deconvolution can be employed. ${ }^{92}$ In order to facilitate this procedure the program NEDS 
was developed for routine analysis. The program inputs from a floppy " disc file both a sample and in-hole spectrum, then performs a channelby-channel subtraction. Next all characteristic lines are identified by the operator through the use of KLM markers. In this context, KLM markers refer to lines superimposed on a sample spectrum (displayed on a graphics terminal) which indicate the positions of characteristic $x$-ray emission lines ( $K_{\alpha}, K_{\beta}, L_{\alpha}, L_{\beta}, M_{\alpha}, M_{\beta}$, etc.) for a particular atomic species. Once all characteristic lines have been identified a background curve is calculated by fitting a polynomial expression of the form:

$$
\left.\underset{i}{N(E)}=\left\{A \cdot\left(\frac{E_{0}-E_{i}}{E_{i}}\right)^{2}+B \cdot\left(\frac{E_{0}-E_{i}}{E_{i}}\right)+C\right\} \cdot\left\{\begin{array}{c}
3 \\
j=1
\end{array}\right] \exp \left(-\left(\frac{\mu}{\rho}\right)_{j} \cdot \frac{\rho_{j} \cdot t_{j}}{\cos \alpha}\right)\right\}
$$

to regions of the spectrum selected by the operator. Here $N(E)$ is the number of continum photons of energy, $E_{i}, E_{0}$ the incident electron energy, A, B, and C fitting constants, and the last term is the detector efficiency factor previously discussed. After background subtraction the program proceeds to fit Gaussian profiles to all characteristic lines identified by the operator using a chi-squared minimization criterion. For the case of overlapped peaks the program systematically searches parameter space for those combinations of Gaussian peak shapes whose values most closely duplicate the profile of the convoluted spectrum (constrained to the response function of the detector system). Because of this multipeak overlap analysis is not necessarily unique. When finished the program produces an output listing of all analyzed lines 
in a format suitable for processing using the NXRYRT program. A flow diagram together with an example of a fitted spectrum can be found in Appendix A for the program NEDS.

Following data reduction using NEDS or a similar program the next step in quantitative analysis involves the reduction of measured intensities into composition values. Currently the most popular procedure employs the thin-film standardless approach using relative intensity ratios. Here the intensity ratio of all characteristic lines in a given spectrum is calculated relative to the most prominent peak. These intensity ratios are then converted into composition ratios (using Eq. 2.26) and the sample composition determined assuming that

$$
\sum_{i} C_{i} \equiv 1
$$

A flow diagram for the program NXRYRT which converts intensity ratios into composition ratios can be found in Appendix $D$.

'Ihree examples of standardless analysis using NXKYK'I' for samples of $\beta-\mathrm{NiAl}, \beta-2 \mathrm{rNb}$ and $\mathrm{Ni}_{4} \mathrm{Mo}$ are given in Table 1 . As can be seen the calculated compositions for both the $\beta-\mathrm{NiAl}$ and $\beta-\mathrm{ZrNb}$ alloys are in good agreement with the compositions obtained from bulk analyses. The last example $\mathrm{Ni}_{4} \mathrm{Mo}$ compares quantitative analysis of the sample using several of the characteristic emission lines which can be simultaneously recorded in a single $x$-ray spectrum. In this case the most intense lines were $\mathrm{NiK}_{\alpha}(7.48 \mathrm{keV})$ and $\mathrm{MoL}_{\alpha}(2.29 \mathrm{keV})$; however, it was also possible to measure the $\mathrm{MoK}_{\alpha}(17.4 \mathrm{keV})$ as well as the $\mathrm{NiL}_{\alpha}(0.85 \mathrm{keV})$ lines and 
Table 1. Comparison of Thin-Film X-Ray Microanalysis of TEM Specimens to Chemical Analysis Starting Material

\begin{tabular}{|c|c|c|c|c|c|}
\hline \multirow[b]{2}{*}{ Sample } & \multirow{2}{*}{$\begin{array}{l}\text { Incident } \\
\text { Energy }\end{array}$} & \multirow{2}{*}{$\begin{array}{l}\text { Intensity } \\
\text { Ratio }\end{array}$} & \multicolumn{3}{|c|}{ Composition } \\
\hline & & & $\begin{array}{l}\text { Calculated } \\
\text { Ratio }\end{array}$ & $\begin{array}{c}\text { Calculated } \\
\text { (wt } . \%)\end{array}$ & $\begin{array}{c}\text { Bulk } \\
(\text { wt .: })\end{array}$ \\
\hline \multirow{2}{*}{ B-NiAl } & $200 \mathrm{keV}$ & $\begin{array}{r}\frac{\mathrm{NiK}_{\alpha}}{\mathrm{A}_{1} \mathrm{~K}_{\alpha}}=2.05 \\
\pm 0.02\end{array}$ & $\begin{array}{r}\frac{\mathrm{Ni}}{\mathrm{AI}}=2.28 \\
\pm 0.02\end{array}$ & $\begin{array}{l}\mathrm{Ni}=69.5 \\
\mathrm{Al}=30.5\end{array}$ & $\begin{array}{l}\mathrm{Ni}=68.5 \\
\mathrm{Al}=31.5\end{array}$ \\
\hline & $100 \mathrm{keV}$ & $\frac{\mathrm{NiK}_{\alpha}}{\mathrm{AlK}_{\alpha}}=1.79$ & $\begin{array}{r}\frac{\mathrm{Ni}}{\mathrm{A} 1}=2.33 \\
\pm 0.02\end{array}$ & $\begin{array}{l}\mathrm{NI}=69.9 \\
\mathrm{Al}=30.1\end{array}$ & $\begin{array}{l}\mathrm{Ni}=68.5 \\
\mathrm{Al}=31.5\end{array}$ \\
\hline$B-\mathrm{ZrNb}$ & $200 \mathrm{keV}$ & $\begin{aligned} \frac{\mathrm{NbK}_{\alpha}}{\mathrm{ZrK}_{\alpha}}= & 0.163 \\
& \pm 0.003\end{aligned}$ & $\begin{array}{r}\frac{\mathrm{Nb}}{\mathrm{Zr}}=0.176 \\
\pm 0.003\end{array}$ & $\begin{array}{l}\mathrm{Nb}=15.0 \\
\mathrm{Zr}=85.0\end{array}$ & $\begin{aligned} \mathrm{Nb}^{*,+} & =15.4 \\
\mathrm{Zr} & =84 . \\
& +3 \%\end{aligned}$ \\
\hline \multirow{3}{*}{$\mathrm{Ni}_{4} \mathrm{Mo}$} & \multirow{3}{*}{$120 \mathrm{keV}$} & $\begin{aligned} \frac{\mathrm{MoK}_{\alpha}}{\mathrm{NiK}_{\alpha}}= & 0.142 \\
& \pm 0.003\end{aligned}$ & $\begin{aligned} \frac{\mathrm{Mo}}{\mathrm{Ni}}= & 0.489 \\
& \pm 0.01\end{aligned}$ & $\begin{array}{l}\mathrm{Mo}=32.8 \\
\mathrm{Ni}=67.2\end{array}$ & $\begin{aligned} \mathrm{Mo}^{*}= & 29.0 \\
\mathrm{Ni} & =71.0 \\
& \pm 1 \%\end{aligned}$ \\
\hline & & $\frac{\mathrm{MoL}_{\alpha}}{\mathrm{NiK}_{\alpha}}=0.227$ & $\begin{aligned} \frac{\mathrm{Mo}}{\mathrm{Ni}}= & 0.416 \\
& \pm 0.005\end{aligned}$ & $\begin{array}{l}\mathrm{Mo}=29.4 \\
\mathrm{Ni}=70.6\end{array}$ & $\begin{aligned} \mathrm{Mo}= & 29.0 \\
\mathrm{Ni}= & 71.0 \\
& \pm 1 \%\end{aligned}$ \\
\hline & & $\frac{\mathrm{MoL}_{\alpha}^{\dagger}}{\mathrm{NiL}_{\alpha}}=2.73$ & $\begin{array}{r}\frac{\mathrm{Mu}^{\ddagger}}{\overline{\mathrm{N}}^{\prime}} \equiv 0.658 \\
\pm 0.010\end{array}$ & $\begin{array}{l}\text { Moキ }=39.7 \\
\mathrm{Ni}=60.3\end{array}$ & $\begin{aligned} \mathrm{Mo} & =29.0 \\
\mathrm{Ni} & =71.0 \\
& \pm 1 \%\end{aligned}$ \\
\hline
\end{tabular}

* Wet chemical analysis of bulk specimen.

${ }^{\dagger}$ Electron microprobe $x$-ray analysis of bulk specimen.

‡Significant contamination of sample during analysis will affect this result. 
thus quantitative analysis using each of these lines was attempted. Analysis using the NiL $\alpha$ is clearly extremely poor when compared to the other two determinations. This can be attributed directly to absorption effects of the $\mathrm{NiL}_{\alpha}$ line in the contamination layer which formed during the time of data accumulation. The results have been included for comparison. Considering the accuracies to which standardless analysis is valid ( $\sim 5 \%$ for $\mathrm{K}-\mathrm{K}$ and $\mathrm{K}-\mathrm{L}$ lines, $\sim 10 \%$ for $\mathrm{L}-\mathrm{L}$ lines), these results are in good agreement with bulk composition mcasurcment3.

Absorption Correction for Standardless Analysis: An extended discussion on the inclusion of absorption effects was presented earlier in Sect. 2.2.3. Unfortunately there is no simple analytic solution that can be used to include all the aspects which were considered therein and hence numerical integration becomes the only viable alternative. If, however, one assimes that the generation function $\phi_{A}(z)$ is nearly constant, then Eq. (2.33) can be solved analytically as follows:

$$
\begin{aligned}
I_{A}^{K_{\alpha}} & =\phi_{A}^{K_{\alpha}}(0) \cdot \int_{0}^{t_{0}} \exp \left(-\left(\frac{\mu}{\rho}\right)_{A B}^{K_{\alpha, A}} \cdot \dot{\rho} \cdot \frac{\sin \left(\beta_{i}\right)}{\cos \left(\beta_{i}-\theta_{E}\right)} \cdot t\right) d t \\
& =\phi_{A}^{K_{\alpha}}(0) \cdot\left\{\frac{1-\exp \left(-\left(\frac{\mu}{\rho}\right)_{A B}^{K_{\alpha}, A} \cdot \bar{\rho} \cdot \frac{\sin \left(\beta_{j}\right)}{\cos \left(\beta_{i}-\theta_{E}\right)} \cdot t_{0}\right)}{\left(\frac{\mu}{\rho}\right)_{A B}^{K_{\alpha}, A} \cdot \bar{\rho} \cdot \frac{\sin \left(\beta_{j}\right)}{\cos \left(\beta_{i}-\theta_{E}\right)}}\right\}
\end{aligned}
$$

where in this equation $\phi_{A}^{K \alpha}(0)$ is the generation function evaluated at zero thickness (i.e., zero energy loss). 
Using this equation one can then write that the generated intensite ratio with correction for absorption is:

$$
\frac{\phi_{A}^{K_{\alpha}(0)}}{\phi_{B}^{K_{\alpha}}(0)}=\frac{I_{A}^{K_{\alpha}}}{I_{B}^{\alpha}} \cdot\left\{\frac{\left(\frac{\mu}{\rho}\right)_{A B}^{K_{\alpha, A}}}{\left(\frac{\mu}{\rho}\right)_{A B}^{K_{\alpha, B}}}\right\} \cdot\left\{\frac{1-\exp \left(-\left(\frac{\mu}{\rho}\right)_{A B}^{K_{\alpha, B}} \cdot \frac{\sin \left(\beta_{i}\right)}{\cos \left(\beta_{i}-\theta_{E}\right)} \cdot t_{0}\right)}{i-\exp \left(-\left(\frac{\mu}{\rho}\right)_{A B}^{K_{\alpha, A}} \cdot \frac{\sin \left(\beta_{i}\right)}{\cos \left(\beta_{i}-\theta_{E}\right)} \cdot t_{0}\right)}\right\}
$$

This equation is of course only valid for a homogeneous sample of thickness $t_{0}$. The application of an absorption correction to an inhomogeneous sample is nearly impossible without some prior knowledge as to the relative composition and thickness of each phase in the excited volume. Thus, one usually resorts to analysis of thinner regions of the sample which can be then considered locally homogeneous.

Once the intensity ratio has been corrected for absorption effects, then the data can be converted into relative composition ratios using the standardless analysis approach previously discussed. If, however, the application of Eq. (2.55) yields corrections in excess of $10 \%$, then serious consideration should be given to either extended calculations of the variation of $\phi_{A}^{K \alpha}$ with thickness (Sect. 2.2.3) or to repeating the measurements in a thinner region of the sample. Since a $10 \%$ criterion is used as the maximm acceptable absorption correction, the correction due to characteristic $x$-ray fluorescence can be ignored.

Quantitative Analysis Using Thin Standards: Up to this point quantitative analysis has been approached on the basis of calculating correction factors for the generation efficiency of various $x$-ray lines. At best these factors are accurate to $\pm 5 \%$ and therefore it becomes 
appropriate to examine quantification using thin standards in an attempt to improve the accuracy of an analysis. Consider the intensity ratio of the $K_{\alpha}$ line of element $A\left(\dot{I}_{A}{ }^{\alpha}\right)$ from an unknown sample (AB) relative to the intensity of the same line in a standard $\left(I_{S T D} K_{\alpha}\right)$. This can be written in the thin-film approximation as:

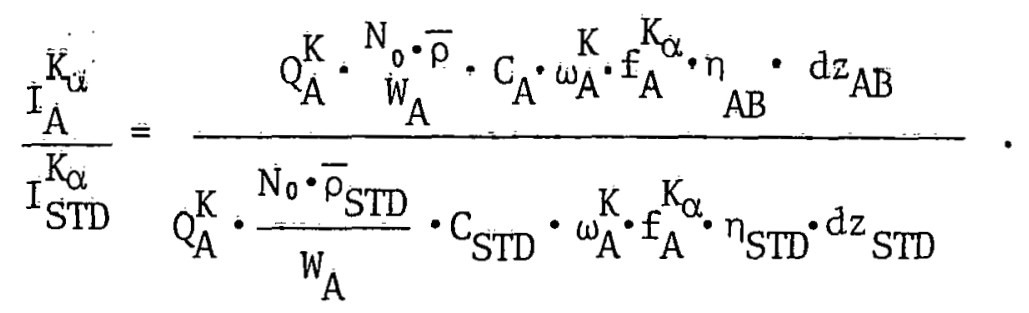

Assiming both the standard and the unknown sample are analyzed at the same incident beam energy and experimental geometry, then Eq. (2.56) can be simplified into

$$
\frac{I_{A}^{K_{\alpha}}}{I_{S T D}^{K_{\alpha}}}=\frac{\bar{\rho}}{\bar{v}_{S T D}} \cdot \frac{n_{A B}}{n_{S T D}} \cdot \frac{d z_{\Lambda B}}{d z_{S T D}} \cdot \frac{L_{A}}{C_{S T D}} .
$$

This equation now relates the characteristic intensity ratio to the composition ratio multiplied by three constant terms. These constants namely the ratios of the relative sample densities $\left(\bar{\rho} / \rho_{\mathrm{STD}}\right)$, the incident electron fiux during each measurement $\left(n_{A B} / n_{S T D}\right)$, and the sample thickness $\left(\mathrm{d} \hat{z}_{\mathrm{AB}} / \mathrm{dz} \mathrm{s}_{\mathrm{STD}}\right)$ - can each be measured independently. Thus it is possible to improve the accuracy of thin-film $x$-ray microanalysis assuming one can prepare suitable thin-film standards. 


\subsection{Electron Diffraction Techniques}

Various methods of obtaining structural information about solids using electron diffraction exist; probably the most well known is the selected-area-diffraction technique (SAD). In this method an aperture placed in the image plane of the objective lens of a TEM effectively defines an area on the specimen from which an electron diffraction pat= tern is obtained. The spatial resolution of this technique is $\sim 5000 \AA$ in diameter with the information being recorded in parallel on a photographic emulsion. The advent of STEM instruments with scan coils located both above and below the specimen as well as modified electron optics providing extremely sma11 electron probes ( $\leqslant 50 \AA$ in diameter) has led to the development of complementary techniques to the SAD method. Among these modes are the scanning transmission ${ }^{93}$ (STED), transmission scanning ${ }^{94}$ (TSED), and stationary diffraction pattern ${ }^{95}$ (SDP) techniques. In this section the TSED and SDP techniques will be outlined and the relative advantages and disadvantages discussed.

\subsubsection{Stationary Diffraction Pattern Technique}

The electron-optical diagram of the stationary diffraction pattern technique in a JEOL JSEM 200 is shown in Fig. 62. A set of scan coils above the specimen deflects the incident electron beam off the optic axis at the back focal plane of the prefield of a highly excited objective lens. Thịs dual-purpose lens acts not only as a final probe forming condensor lens but also serves to deflect the electron beam so 


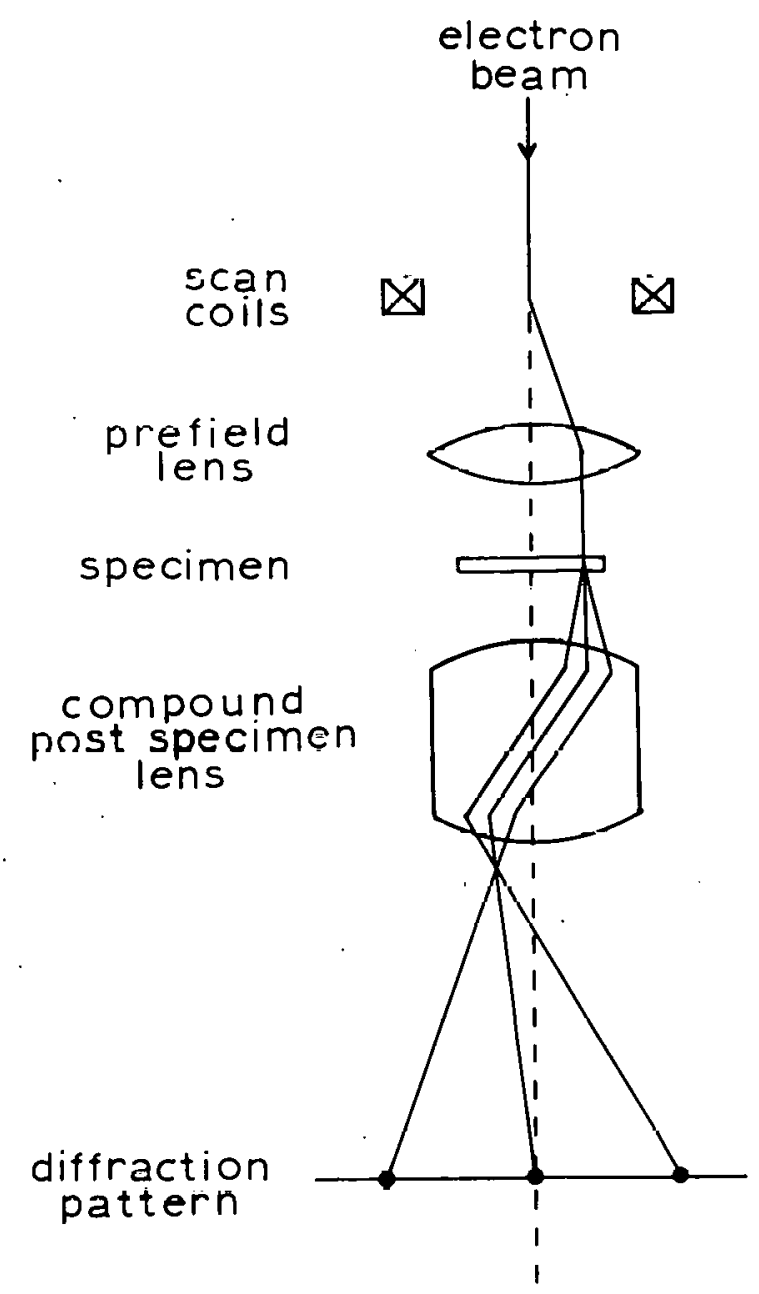

Fig. 62. Ray Diagram for the Stationary Diffraction Pattern Technique. 
that it is once again parallel to the electron-optical axis. The remaining post-specimen field of the compound objective lens system magnifies the resulting electron diffraction pattern so it can be conveniently viewed on a phosphorescent screen at the detector plane. Since the electron probe is incident on the sample plane parallel to the electron-optical axis, a stationary pattern is formed at all times at the final viewing screen.

The principal advantage of this technique is that diffraction patterns from areas as small as $200 \AA$ can be obtained by simply reducing the size of the roster on the specimen. There are, however, two distinct disadvantages of this method. First, the contamination rate usually increases due to the increased current density found in the focused STEM probe. Secondly, the diffraction patterns formed when operating in the highest spatial resolution mode in STEM have poor angular resolution due to the highly convergent beams $\left(10^{-2}\right.$ to $10^{-3}$ radians) used to form the small electron probes. This effect is illustrated in the diffraction patterns of Fig. 63 for incident beam convergences varying from nearly parallel illumination $\left(\sim 6 \times 10^{-4}\right.$ radians, CTEM imaging conditions) to highly convergent i11umination ( $22 \times 10^{-2}$, high resolution STEM imaging) .

This beam convergence can be changed in either of two ways by using a smaller condenser aperture, or by decreasing the excitation of the condenser lens current. Decreasing the aperture size is the simplest means by which the angular resolution can be increased; 

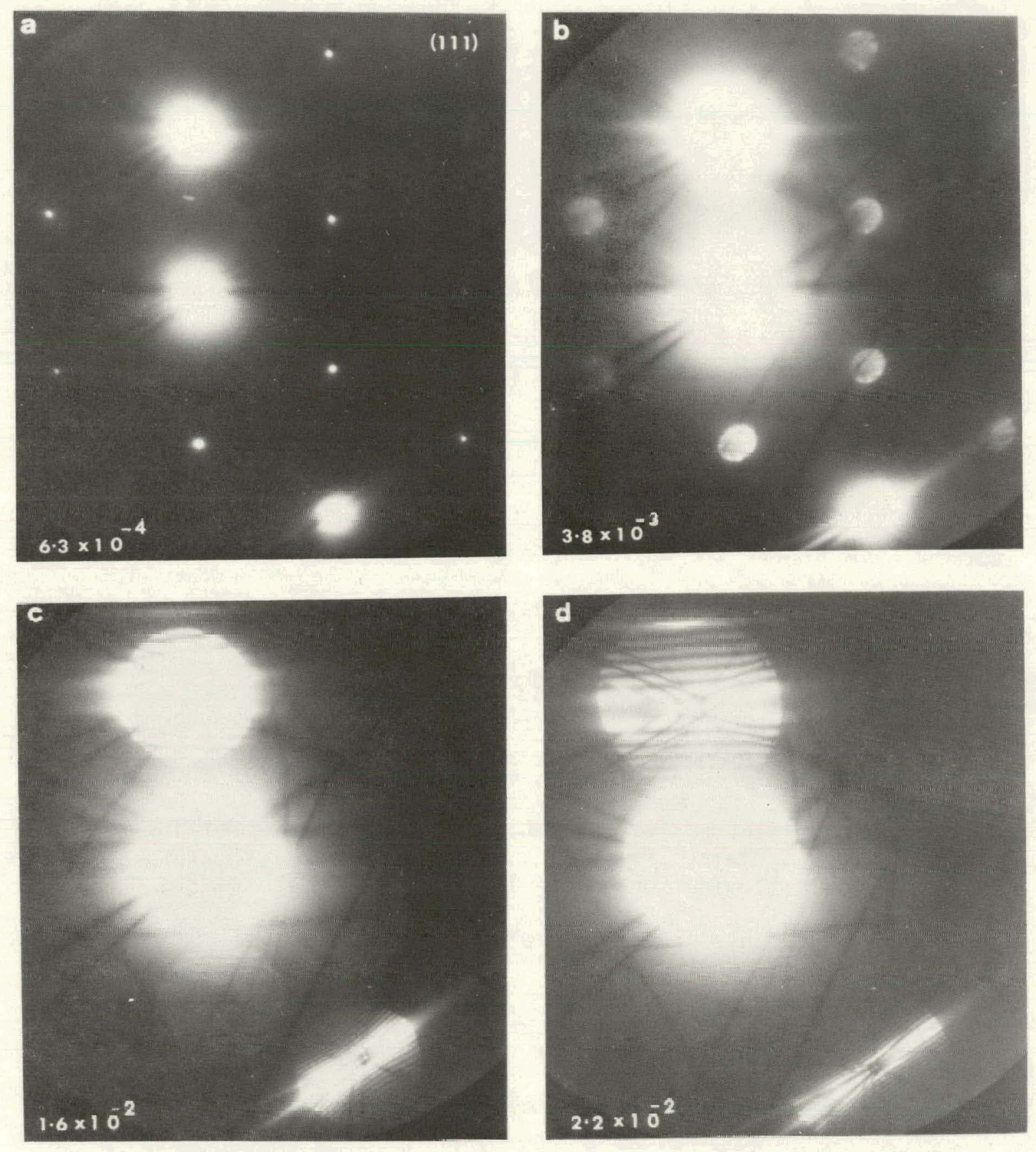

Fig. 63. Comparison of Electron Diffraction Patterns for Various Values of Incident Beam Convergence (as Indicated on Each Figure). 
however, this markedly reduces the incident beam current and hence the diffracted intensities. Similar results can also be obtained by changing the excitation of the second condenser lens which has a much smaller effect on the probe current; however, there is a substantial change in the probe diameter as the condenser current is varied. Figure 64 shows the measured variation in probe diameter as a function of second condenser lens current in the JEOL JSEM 200 operating at $200 \mathrm{keV}$ with a condenser aperture of $100 \mu \mathrm{m}$. At an excitation of $43 \mathrm{~mA}$ the probe diameter was $\sim 1 \mu \mathrm{m}$ at a convergence of $\sim 10^{-4}$ radians, while at STEM imaging conditions $(\sim 52 \mathrm{~mA})$ the probe diameter was $\sim 100 \AA$ at a convergence of $\sim 10^{-2}$ radians. Probe size measurements in Fig. 64 were made by scanning the incident beam perpendicular to a cleavage surface of a crystal of spherolite and simultaneously recording the signal rise profile of the transmitted beam intensity. Although convergent beam diffraction patterns (CBDP) have poor angular resolution, they contain a substantial amount of information; examples of this can be found in the literature. ${ }^{83}, 89,96,97$ Furthermore, it is not always necessary to operate a STEM instrument in the highest resolution mode; thus one can judiciously adjust the parameters of probe size, convergence, and current in order to optimize the relevant experimental information obtained during an analysis. Figure 65 illustrates this point, showing a TEM micrograph of a V-Ti-C alloy containing carbide precipitates. With the STEM probe adjusted to $\sim 300 \AA$ in diameter CBDPs were measured from both the carbide precipitate 


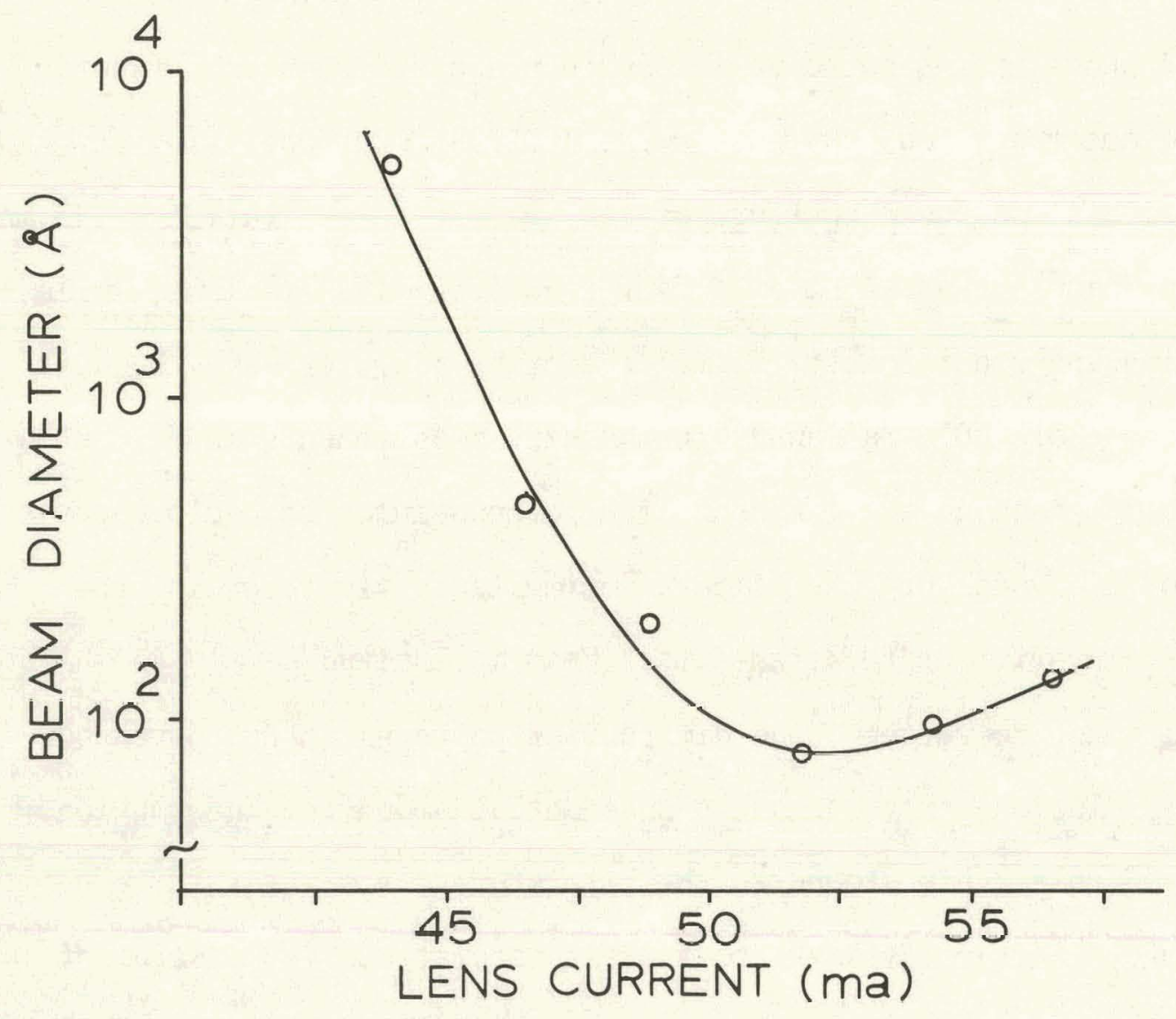

Fig. 64. Variation in Electron Beam Diameter as a Function of Condenser Lens Excitation. 


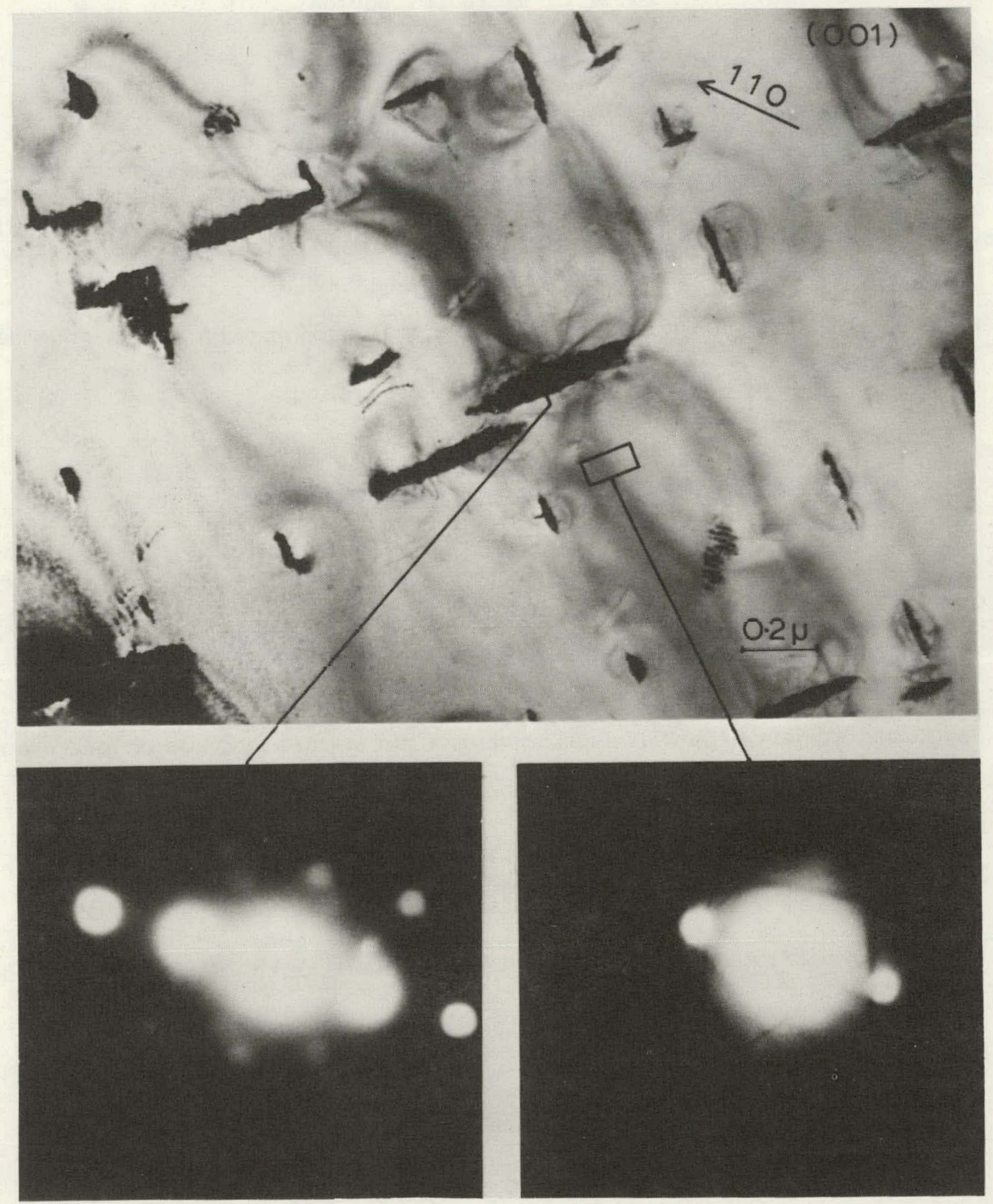

Fig. 65. Example of Analysis of V-Ti-C Precipitate Using Stationary Diffraction Technique. 
as well as the matrix regions as indicated. The CBDP obtained from the precipitate reveal a number of extra reflections, which are also easily detected using SAD techniques. However, using SAD it was not possible to determine uniquely whether the extra reflections were a result of faulting and/or twinning in the precipitate or due to an extension of the unit cell. The CBDP obtained from the nearby matrix region clearly shows the presence of Kikuchi lines which are not detected in the precipitate region indicating that faulting and/or twinning is the most likely source of the satellite intensity.

\subsubsection{Transmission Scanning Electron Diffraction (TSED) \\ Transmission scanning electron diffraction (TSED) 94, 95,98 refcrs} to the process of post-specimen scanning and the subsequent electronic recording of the intensity distribution of diffraction patterns for static incident electron probes. Since this technique involves postspecimen scanning, any intensity distribution which can be observed in the TEM can be measured; thus when operating in the CTEM imaging mode this technique is referred to as transmission scanning electron microscopy (TSEM) . ${ }^{95,98}$ The most important aspect of TSED/TSEM is that the intensity distribution is measured electronically rather than photographically yielding a greater sensitivity as we11 as a wider range of linear response $\left(10^{5}\right.$ versus $\left.10^{1}\right)$ to changes in intensity. The major drawback of the technique is that the information is at present recorded serially. Thus each point must be measured sequentially and hence 
scanning rates necessary to accumulate sufficient statistics may be slow. This lengthy recording time may then introduce other errors such as stage drift.

Figure 66 is a schematic diagram of the electron optical system used for TSED/TSEM measurements in the JSEM 200. In this system a set of post-specimen scan coils located between the intermediate and projector lenses is used to translate the intensity distribution of interest across an aperture in the final viewing screen of the microscope. The sweep rate is determined by a time base in the ORTEC 6200 multichannel scaler (MCS) which produces a digital voltage ramp of 1024 points/scan whose dwell time can be adjusted from $1 \mu \mathrm{s}$ to $10 \mathrm{~s}$ per channel. This digital ramp is routed into a control circuit which splits the signal into a ratioed set of $\mathrm{x}$ and $\mathrm{y}$ scans to which a dc level shift is introduced. Using this signal splitting circuit (Appendix G) digital line scans of continuously variable amplitude, position and orientation can be generated along any desired direction. The detector system consists of the scintillator/photomultiplier unit normally used for STEM imaging. However, in this case the output of the photomultiplier tube (PMT) is routed through a two-stage linear preamplified/amplifier system having a variable gain of 5 to 50 times rather than the STEM electronics. The signal is then digitized using a voltage-to-frequency converter and subsequently stored in the MCS unit. After the data have been accumulated in the MCS memory, software programs can be used to transfor these data to the online microcomputer system for storage (using the program MCA) or analysis (using the program NMCS). 


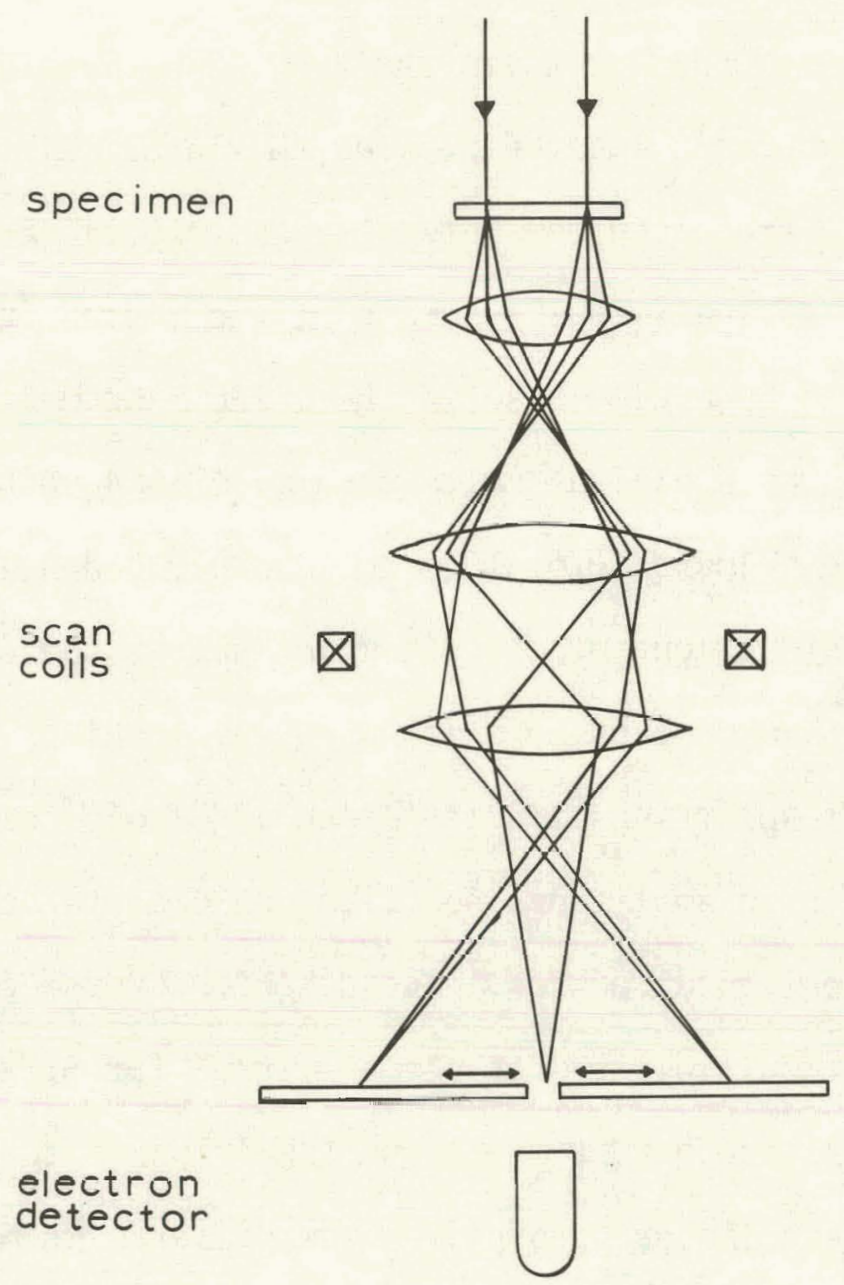

Fig. 66. Ray Diagram for Transmission Scanning Electron Diffraction/Microscopy (TSED/TSEM). 
Figures 67 and 68 are examples of TSED and TSEM measurements made on a TEM/STEM instrument equipped with the identical scanning and detection system as described above. ${ }^{98}$ Figure 67 shows the measured intensity distribution along a $<220>$ direction in reciprocal space of a single crystal of copper in the (001) orientation having a thin surface oxide 1ayer. Two superimposed traces are shown in Fig. 67(a). Trace A is plotted such that the transmitted beam intensity can be conveniently observed, whereas the second trace $B$ is the same data replotted using a scale factor 100 times greater along the $y$-axis; in this curve the Bragg intensities as well as the weak oxide reflections are clearly discernible. The ratio of the weakest oxide reflection to that of the transmitted beam intensity is nearly 2000/1. Figure 68 shows an example of a TSEM measurement of the oscillatory fringe contrast from a semicoherent $\theta^{-}$plate in $\mathrm{AI}-4 \% \mathrm{Cu}$.

\subsection{Electron Energy Loss Spectroscopy (ELS)}

As electrons pass through TEM specimens they suffer losses in energy due to various inelastic scattering events. These changes in energy have two consequences. First, they are responsible for image distortions due to chromatic aberations which can complicate or obscure image detail. Second, the energy losses, which the transmitted beam incurs, are related to specific inelastic scattering processes and thus contain information concerning the nature of the atomic, electronic, and elemental structure of the sample. Furthermore, electron energy loss spectroscopy (ELS) has the highest yield of information per inelastic 

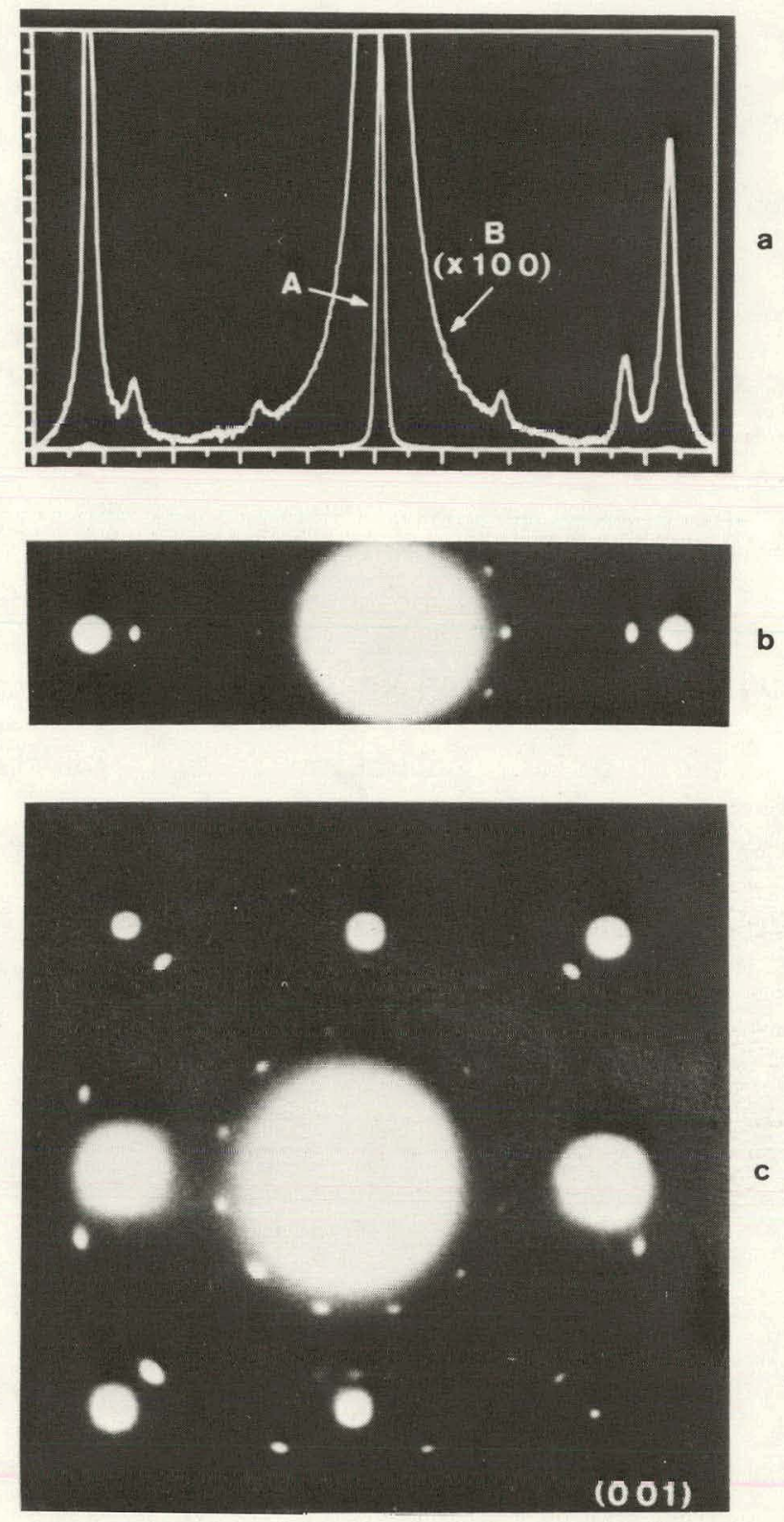

Fig. 67. Example of TSED Measurement along $<220\rangle$ Direction of the (001) Pattern Shown in (c). 

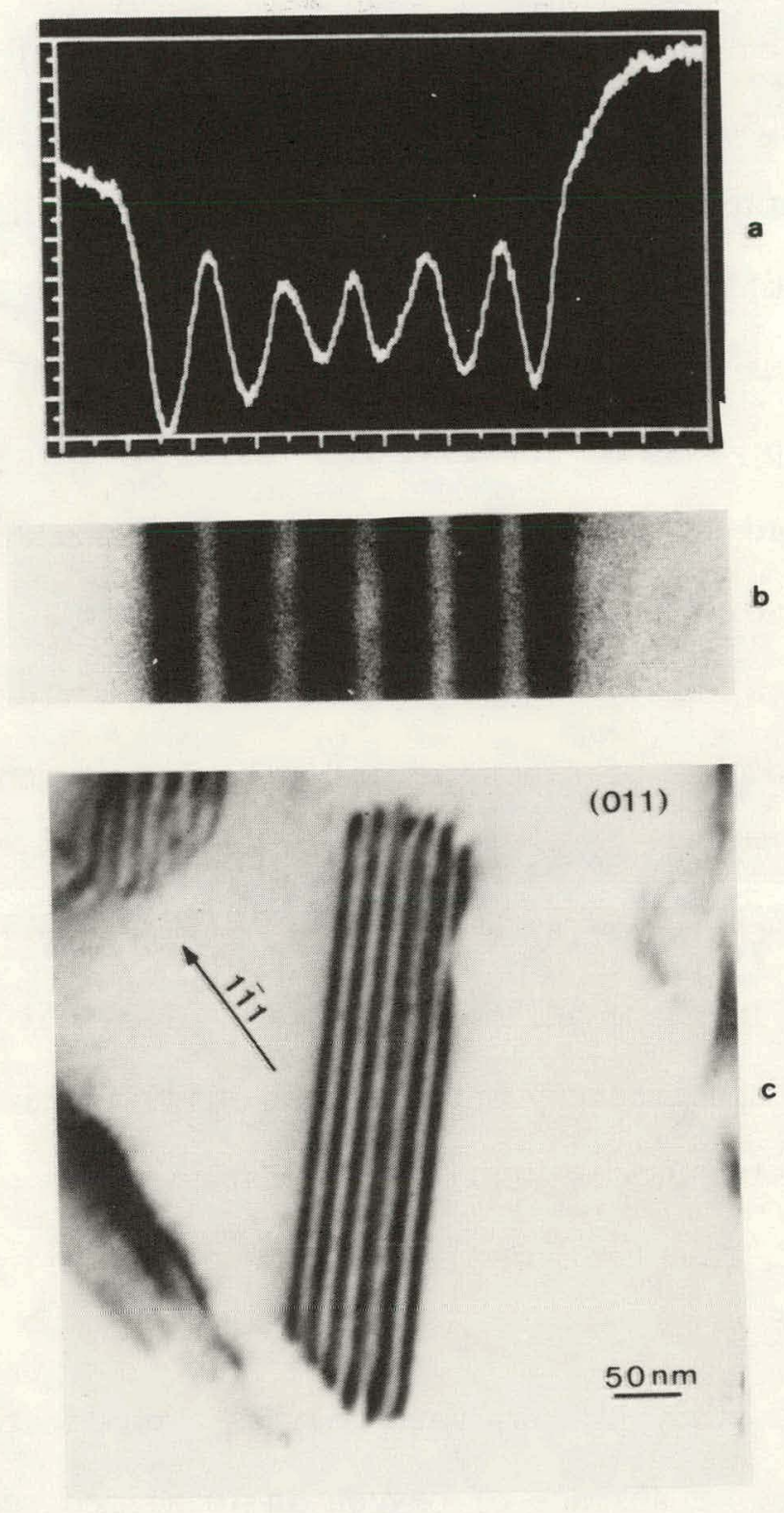

Fig. 68. Example of TSEM Measurement of Fringe Pattern of $\theta^{\prime}$ Precipitate Shown in (c). 
scattering event due to the fact that one is now measuring the primary interaction process rather than a secondary process such as $x$-ray or Auger emission. For materials related research, one of the most important aspects of ELS is the ability to perform light element analysis such as the detection of the interstitial elements $\mathrm{C}, \mathrm{N}$, and $\mathrm{O}$. These measurements cannot realistically be made using conventional $\mathrm{x}$-ray EDS techniques and thus ELS complements the capabilities of an analytical electron microscope systcm. Spatial resolution of this technique is essentially limited only by beam broadening effects and hence for suitable specimens resolutions less than $5 \mathrm{~nm}$ are possible.

Microchemical analysis using energy loss spectroscopy is obtained by measuring the number of electrons which have undergone characteristic energy losses due to the excitation of inner she1l levels. These loss electrons exhibit characteristic profiles in an energy luss speclrull, which are similar in shape and meaning to the classical absorption edge profiles in $x$-ray spectroscopy and are thus similarly labeled. Quantitative analysis using ELS is still in the early stages of development and at the present time the formalism proposed by Egerton and Whelan ${ }^{99}$ seems most promising,

During this study ELS was used only as a qualitativo tool to detect the presence or absence of oxygen in localized regions of the specimen; however, quantitative analysis using ELS is an interesting and important aspect of AEM. 
CHAPTER 3

3. EXPERIMENTAL OBSERVATIONS AND RESULTS

\subsection{Instrumentation}

A JEOL JSEM 200 transmission electron microscope was the primary instrument used during this investigation. This microscope was equipped with a side-entry goniometer stage allowing specimen tilting so that crystallographic analysis can be performed. In addition, the instrument had facilities for scanning transmission electron microscopy (STEM), secondary electron (SEM) and backscattered electron imaging (BEI). Specimen holders capable of double-tilt, tilt-rotate, single-tilt cooling $\left(-196^{\circ} \mathrm{C}\right.$ to $\left.25^{\circ} \mathrm{C}\right)$ and heating $\left(25^{\circ} \mathrm{C}\right.$ to $\left.300^{\circ} \mathrm{C}\right)$ were also available. The electron source was a standard thermionic tumgsten hairpin filament and the microscope was capable of operating at accelerating voltages of 200 , 150,100 , and $50 \mathrm{kV}$. Unless otherwise specified, all micrographs and measurements presented in this section were made at an incident beam energy of $200 \mathrm{keV}$ in conventional TEM imaging and diffraction modes. Because of the nature of the experiments to be performed in the present work, substantial modifications have been made to the electronoptical column of the JSEM 200 in order to upgrade this CTEM/STEM instrument into an analytical electron microscope (AEM). A solid state $\mathrm{Si}(\mathrm{Li}) \mathrm{x}$-ray detector was interfaced to the microscope column and an $\mathrm{x}$ ray collimator designed and attached to the detector head. A special bremsstrahlung aperture (see Sect. 2.2.7) was subsequently installed and 
$\dot{a}$ double-tilt stage modified to reduce system peaks (see Sect. 2.2.7). To lessen the number of backscattered electrons from the anticontamination device, objective aperture blade and pole-piece material from hitting the sample, graphite blocks or coatings were judiciously placed in the specimen chamber. The vacuum system was improved by the addition of a turbo-molecular pump (500 liters/s) to further increase the vacurm in the specimen region and extra liquid-nitrogen cold traps were designed fur the sample chamber, high resolution diffraction chamber, and each of the roughing pump lines to reduce backstreaming and contamination. Special isolation valves were fitted to the specimen prepump and column roughing line to further inhibit backstreaming from these sources once the sample was introduced into the column vacuum. Bayard-Albert ionization gauges were attached to the system to monitor the vacuum levels in the specimen chamber and turbopump lines. After these modifications, the column vacuum near the specimen was typically $\sim 8 \times 10^{-6}$ torr and $\sim 2 \times 10^{-7}$ just above the turbopump. In order to improve the diffraction capabilities in STEM, it became necessary to remove and bore out a fixed aperture in the intermediate lens pole piece. The diameter of this fixed aperture was increased from $3 \mathrm{~mm}$ to $1 \mathrm{~cm}$ yielding nearly a threefold increase in the angle subtended in the STEM diffraction mode. A Faraday cage was designer to fit in the viewing chamber of the column and a ten times preamplifier added to the STEM detector to improve the signal-to-noise performance in the SIEM mode. Finally, an on-line computer system was interfaced to the microscope via the multichannel analyzer. The mini-computer was 
subsequently connected to the central computer of the Materials Researci: Laboratory to allow paper tape and/or direct telephone line transfer of data.

A second instrument recently acquired by the Materials Research Laboratory was used for the electron energy loss measurements presented in this study. The microscope was a Vacuum Generators HB5, dedicated scanning transmission electron microscope (DSTEM), operating at accelerating voltages up to $100 \mathrm{kV}$. The electron source was a cold field emission tungsten single crystal tip and under normal operating conditions the vacuum levels in this UHV instrument were $\sim 2 \times 0^{-11}$ torr in the gun chamber and $\sim 2 \times 10^{-9}$ at the specimen. Electron energy loss measurements were made on a $\pi / 2$ magnetic sector energy analyzer having an energy resolution of $1.2 \mathrm{eV}$ at an input divergence of $10^{-4}$ radian. These data were recorded on an ORTEC multichannel scaler and subsequently analyzed on the mini-computer system previously discussed (Sect. 2.2.8) using the software described in the appendices of, this thesis.

\subsection{Sample Preparation}

The samples used for this study were prepared from an ingot of Zr-15\% $\mathrm{Nb}$ kindly provided by Drs. Paton and Williams of Rockwell International Science Center, Thousand Oaks, California. The asreceived material was homogenized for five days at $1000^{\circ} \mathrm{C}$ in the $B$ field and then quenched to precipitate the omega phase. The bulk sample was cut into wafers approximately $0.5 \mathrm{~mm}$ thick using a high-speed watercooled diamond slicing wheel and disc shaped specimens $3 \mathrm{~mm}$ in diameter 
were subsequently prepared using electrodischarge machining. These discs were cleaned in an $\mathrm{HF}-\mathrm{HNO}_{3}$ solution ${ }^{8}$ to remove contamination from the surface then washed in reagent grade methanol. In order to ensure retention of the omega phase in these discs, all samples were heat treated and quenched a second time. The 3-mm discs were wrapped in zirconium foil and placed in quartz tubes. Large aggregates of sponge titanium were also introduced into the quartz capsules in order to assist the zirconium foils as oxygen getters during heat treatment. 'The capsules were evacuated to a dynamic pressure of $9 \times 10^{-6}$ torr and backfilled with high-purity argon gas. The backfilling and evacuation procedure was repeated five times in order to flush as much residual. gas out of the tube as possible. The capsules containing a 0.25 atm of argon were then sealed and the samples homogenized for $2 \mathrm{~h}$ at $1000^{\circ} \mathrm{C}$, followed by quenching into an ice water bath. Following heat treatment, the discs were analyzed using wet chemical, vacuum fusion mass spectrographic and electron microprobe techniques. The results of these analyses are summarized in Tablc 2.

Initial polishing attempts concentrated on the documented chemical polishing technique of Sass et al. ${ }^{7-12}$ in their studies of $\mathrm{Zr}-\mathrm{Nb}$ alloys. This technique was difficult to use and met with only limited success. Furthermore, when the sample thickness fell below approximately $200 \mathrm{\mu m}$, hydride plates werc obscrved to form spontaneously throughout the sample similar to that reported by Flewitt et al. 100 in alloys of $40-85 \% \mathrm{Nb}$ with Zr. Cooling the solutions to $-30^{\circ} \mathrm{C}$ did not reduce this precipitation and on continued polishing the hydrides were observed to grow, with perforation 
Table 2. Chemical Analysis of $\mathrm{Zr}-15 \% \mathrm{Nb}$ Samples after Heat Treatment

\begin{tabular}{|c|c|}
\hline Element & $\begin{array}{l}\text { Concentration } \\
\text { (atomic parts per million) }\end{array}$ \\
\hline $\mathrm{Zr}$ & $84.6(\text { wt } \%)^{*,+, \neq ~}$ \\
\hline $\mathrm{Nb}$ & 15.4 (wt \%) \\
\hline $\mathrm{H}$ & $86^{\S}$ \\
\hline 0 & $235^{\S}$ \\
\hline $\mathrm{Na}$ & $5^{\#}$ \\
\hline $\mathrm{Mg}$ & $\leqslant 3^{\#}$ \\
\hline $\mathrm{A} 1$ & $70^{\#}$ \\
\hline $\mathrm{Si}$ & $50^{\#}$ \\
\hline $\mathrm{C} 1$ & $4^{\#}$ \\
\hline $\mathrm{Ca}$ & $\leqslant 0.3^{\#}$ \\
\hline $\mathrm{Ti}$ & $50^{\#}$ \\
\hline V & $\leqslant 0.2^{\#}$ \\
\hline $\mathrm{Cr}$ & $10^{\#}$ \\
\hline $\mathrm{Fe}$ & $50^{\#}$ \\
\hline $\mathrm{Ni}$ & $\leqslant 2^{\#}$ \\
\hline $\mathrm{Pd}$ & $7^{\#}$ \\
\hline $\mathrm{Ag}$ & $30^{\#}$ \\
\hline $\mathrm{Cd}$ & $\leqslant 3^{\#}$ \\
\hline $\mathrm{Sn}$ & $\leqslant 2^{\#}$ \\
\hline IIf & $20^{\#}$ \\
\hline $\mathrm{Pb}$ & $0.3^{\#}$ \\
\hline $\mathrm{Bi}$ & $0.3^{\#}$ \\
\hline
\end{tabular}

*Average of three determinations.

${ }^{\dagger}$ Wet chemical analysis.

$\ddagger_{\text {Electron microprobe analysis. }}$

$\S_{\text {Vacuum fusion analysis. }}$

\#Mass spectrographic analysis. 
usually occurring at one of these plates presumably due to preferential polishing. Optical and TEM micrographs of this structure are shown in Fig. 69.

In an attempt to reduce this hydride precipitation, a solution developed by Schober and Sorajic, ${ }^{101}$ which avoids the introduction of hydrogen into the sample, was used. This electrolyte, 0.05 moles/liter of $\mathrm{Mg}\left(\mathrm{ClO}_{4}\right)_{2}$ in $\mathrm{CH}_{3} \mathrm{OH}$, has been successfully used to reduce the hydride formation. Samples were prepared in a dual jet electropolishing unit operating at a potential difference of $100 \mathrm{~V}$ drawing a current of $0.05 \mathrm{~A}$. The sample was mounted in a Teflon holder with all electrical connections made using platinum wire; the cathode used was stainless steel and the solution kept at $-30^{\circ} \mathrm{C}$. Due to the nature of the electrolyte a thick viscous film was observed to form during electropolishing. Schober recommended washing in a dilute $\mathrm{H}_{2} \mathrm{SO}_{4}$ solution after perforation; however, it was found that by adjusting the tlow rate of the polishing solution through the twin jet systom to $n \cdot 10 \mathrm{ml} / \mathrm{s} / \mathrm{Im}^{2} \mathrm{~L}^{2}$ it was possible to continuously wash the film from the sample surfare. Perforation was monitored by the detection of transmitted light from a He-lNe (100 mW) laser via a photo-resistor/1ight pipe system. This photo-resistor was Electronically coupled so that when light was transmitted through a small hole produced by the electropolishing both the flow of polishing solution as well as all electrical power were disconnected. Samples were immediately washed in reagent grade methanol for approximately 2 min followed by a gentle drying using a forced-air blower. The air flow in this case was always directed parallel to the sample surface 

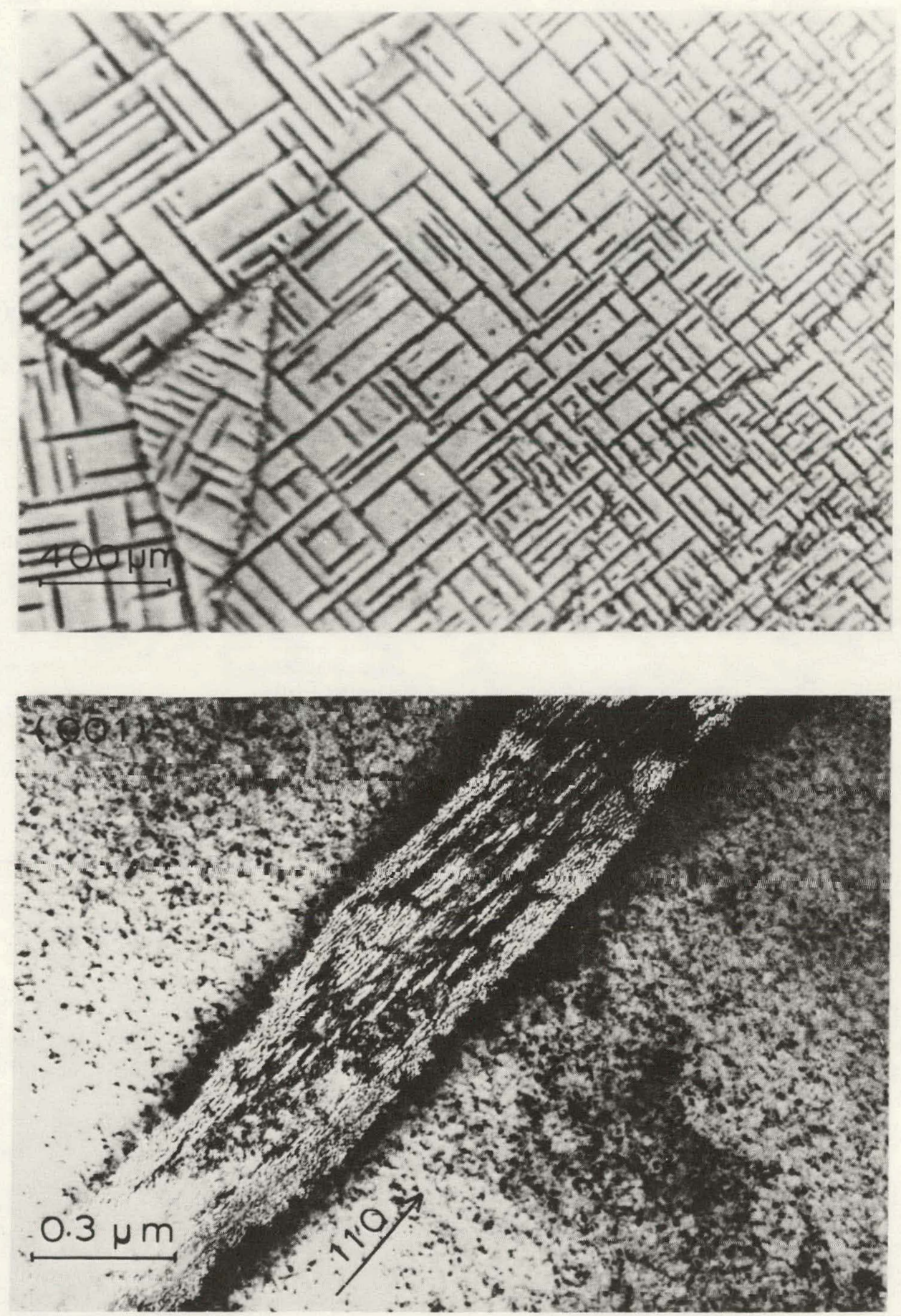

Fig. 69. Optical (a) and CTEM (b) Micrographs of Hydride Precipitates in $\mathrm{Zr}-15 \% \mathrm{Nb}$. 
in order to avoid possible damage to the thin specimen area. Typical thin regions usually contained one or two large grains allowing easy manipulation for crystallographic analysis.

\subsection{Microstructural Observations}

During the course of this study a variety of microstructures in addition to the as-quenched $\omega+\beta$ morphology were noted in the $\mathrm{Zr}-15 \% \mathrm{Nb}$ a1loy. In at least one case the experimental results were initially misinterpreted as the formation of a long-period ordered structure of the $\omega$ phase, and hence a complete characterization of each phase observed became an important part of this research. A summary of these microstructures and their analysis is described in this section.

\subsubsection{As-Quenched Morphology of the Omega Phase}

The as-quenched microstructurc of the $\mathrm{Zr}-15 \% \mathrm{Nb}$ alloy containing the omega phase is shown in Fig. 70. Since there are no discernible IIIicrostructural features in a bright-field TEM image, the presence of omega must be confirmed through the use of electron diffraction, as is shown in the (011) diffraction pattern of Fig. $70(\mathrm{~b})$. The most striking feature of the microstructure of this alloy is the absence of any observable dislocation structure. This result has been reproduced in some 30 as-quenched samples containing the w phase indicating an apparent dislocation density of less than $10^{5} / \mathrm{cm}^{2}$. This low dislocation density correlates with the presences of the omega phase, since for a $\mathrm{Zr}-\mathrm{Nb}$ alloy which does not contain omega, the dislocation density is on the order of $10^{8} / \mathrm{cm}^{2}$. This can be seen in Fig. 71 which is the microstructure of a 

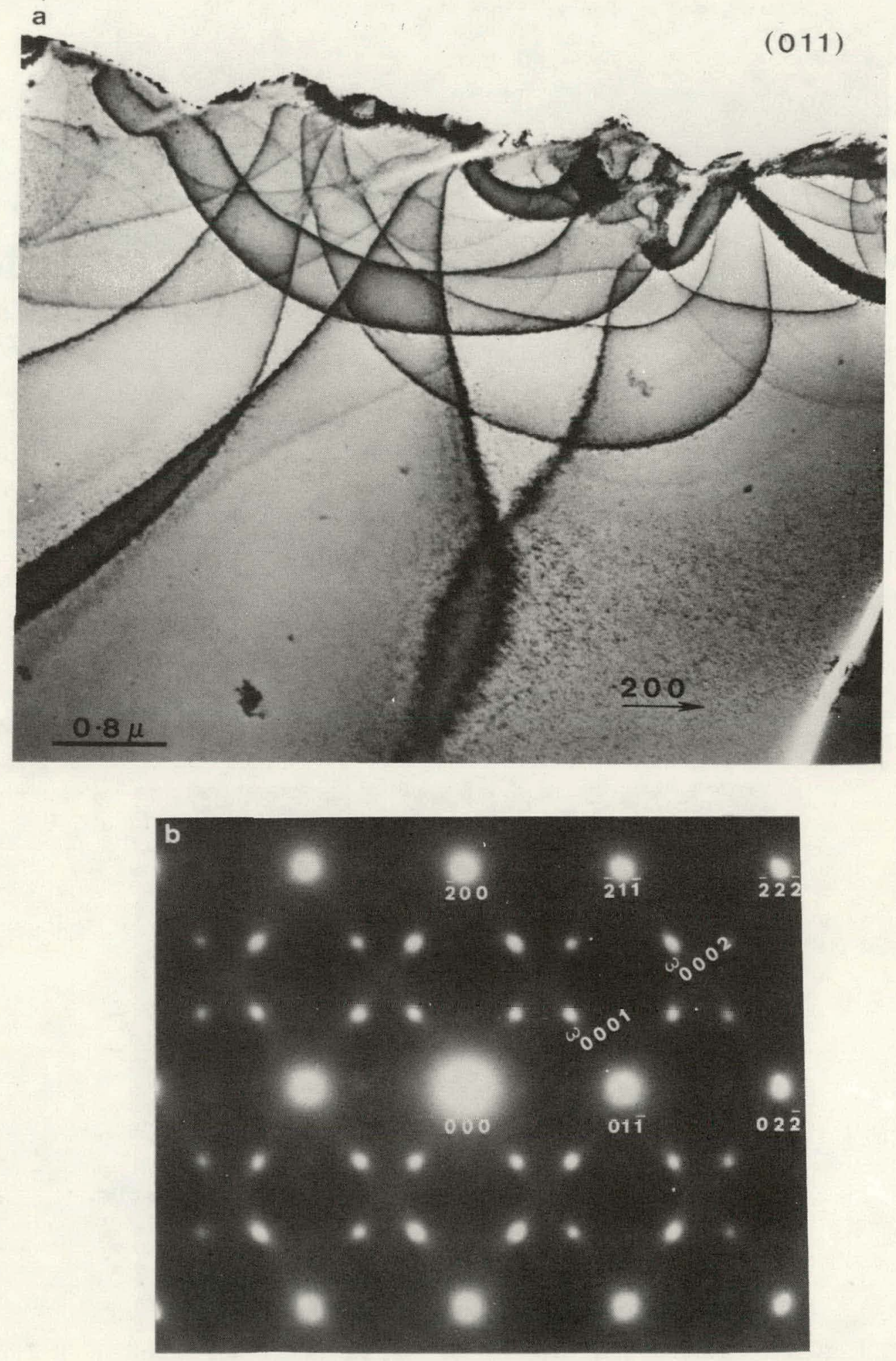

Fig. 70. Microstructure of As-Quenched Morphology in $\mathrm{Zr}-15 \% \mathrm{Nb}$, and Corresponding to Diffraction Pattern. 

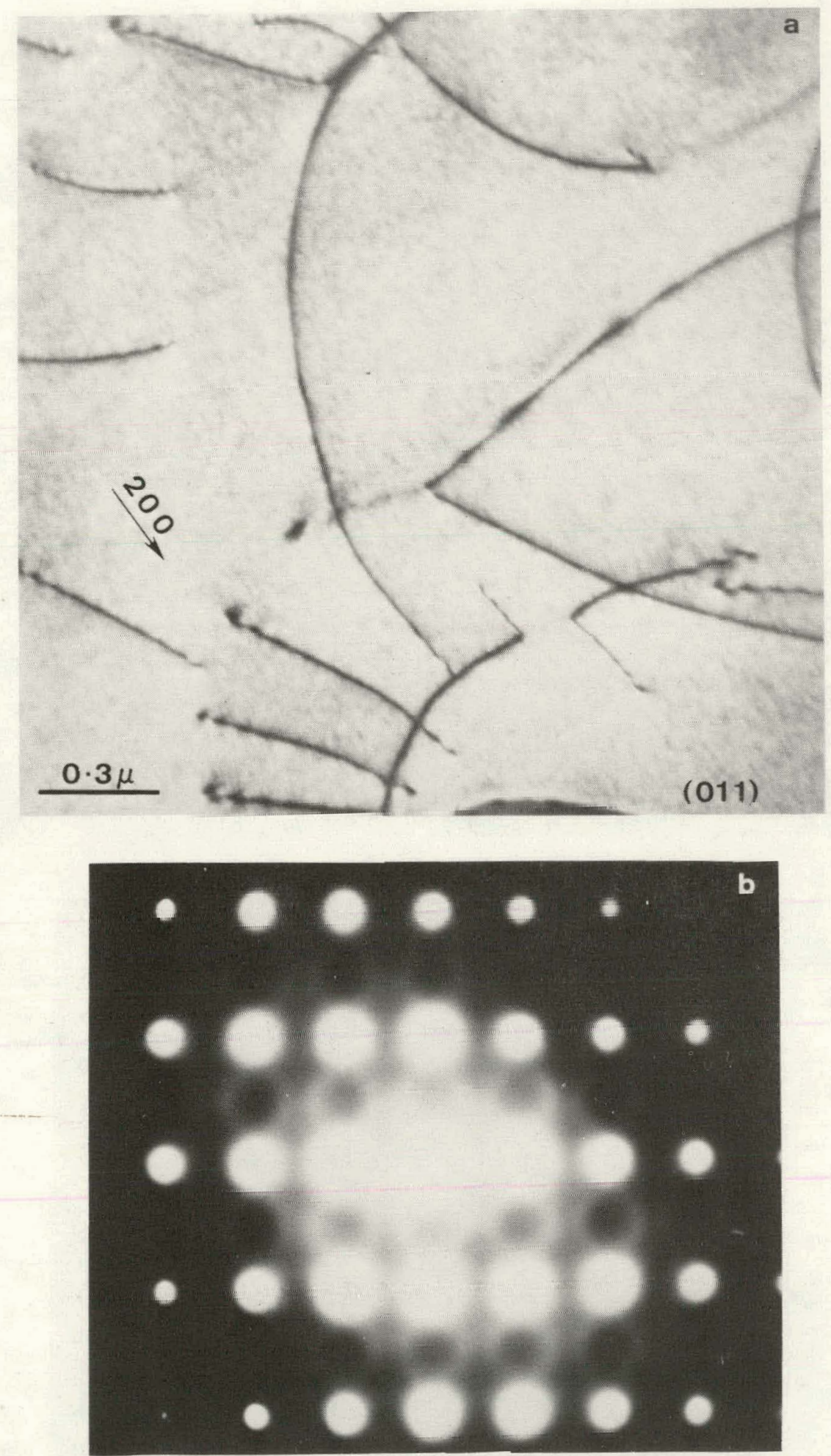

Fig. 71. Microstructure of $\mathrm{Zr}-80 \% \mathrm{Nb}$ and Corresponding Diffraction Pattern. 
$\mathrm{Zr}-80 \% \mathrm{Nb}$ alloy having undergone identical sample preparation steps (heat treatment, polishing, etc.) as the $15 \% \mathrm{Nb}$ alloy. The absence of the omega phase is clearly indicated in the (011) electron diffraction pattern of Fig. 71(b).

Figure $72(a)$ is a TEM bright-field micrograph of the as-quenched omega structure at higher magnification and as pointed out no distinctive features are present. Dark-field micrographs such as Fig. 72(b) taken using an $\omega_{0002}$ reflection [indicated in Fig. 71(b)] show the presence of omega domains less than $30 \AA$ in diameter distributed uniformly in the bcc matrix. Throughout this work no alignment of the omega domains along $<111>$ directions of the bcc lattice has been observed. The fact that in the most recent publication of Kuan et al. ${ }^{12}$ it was not possible to duplicate the alignment of omega particles along <111> directions is significant in that there is now no conflict between these results and that of Sass et a1. ${ }^{7-1}$ A summary of electron diffraction patterns from the $15 \% \mathrm{Nb}$ alloy over a standard stereographic triangle is given in Fig. 73.

\subsubsection{Spontaneous Relaxation Effects}

Although the microstructure shown in Fig. 70 is relatively simple, this observation was more the exception rather than the rule for this alloy. The majority of the samples were observed to contain thin foil artifacts which exhibited planar lath-1ike features. This phenomenon has been termed "spontaneous relaxation"102-105 and has been attributed to a relaxation of the bulk specimen constraints during thinning due to 

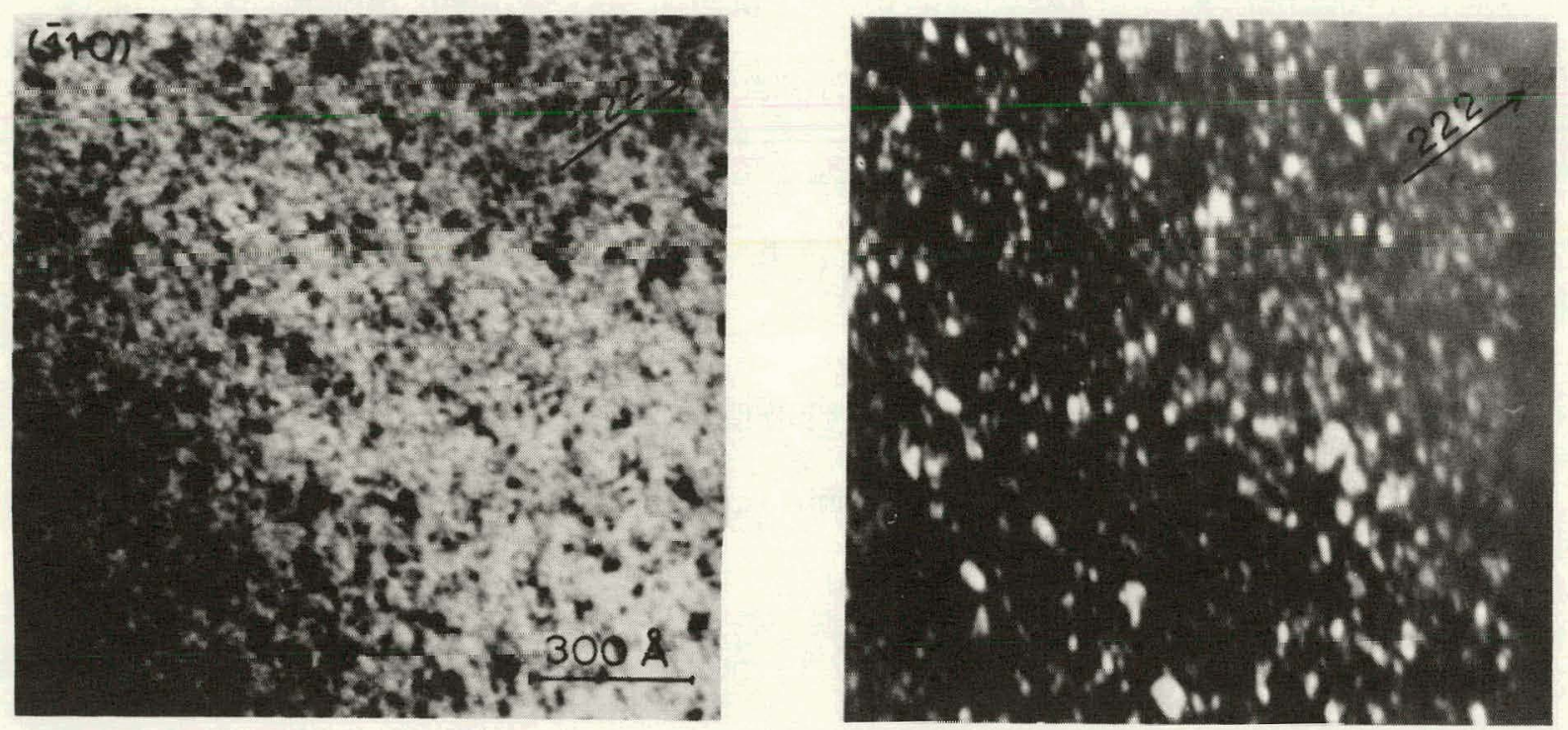

Fig. 72. Bright (a) and Dark-Field (b) CTEM Micrographs of the As-Quenched $\omega$ Structure Showing Approximately $30 \AA \omega$ Domain Size in the Dark-Ficld Images. 


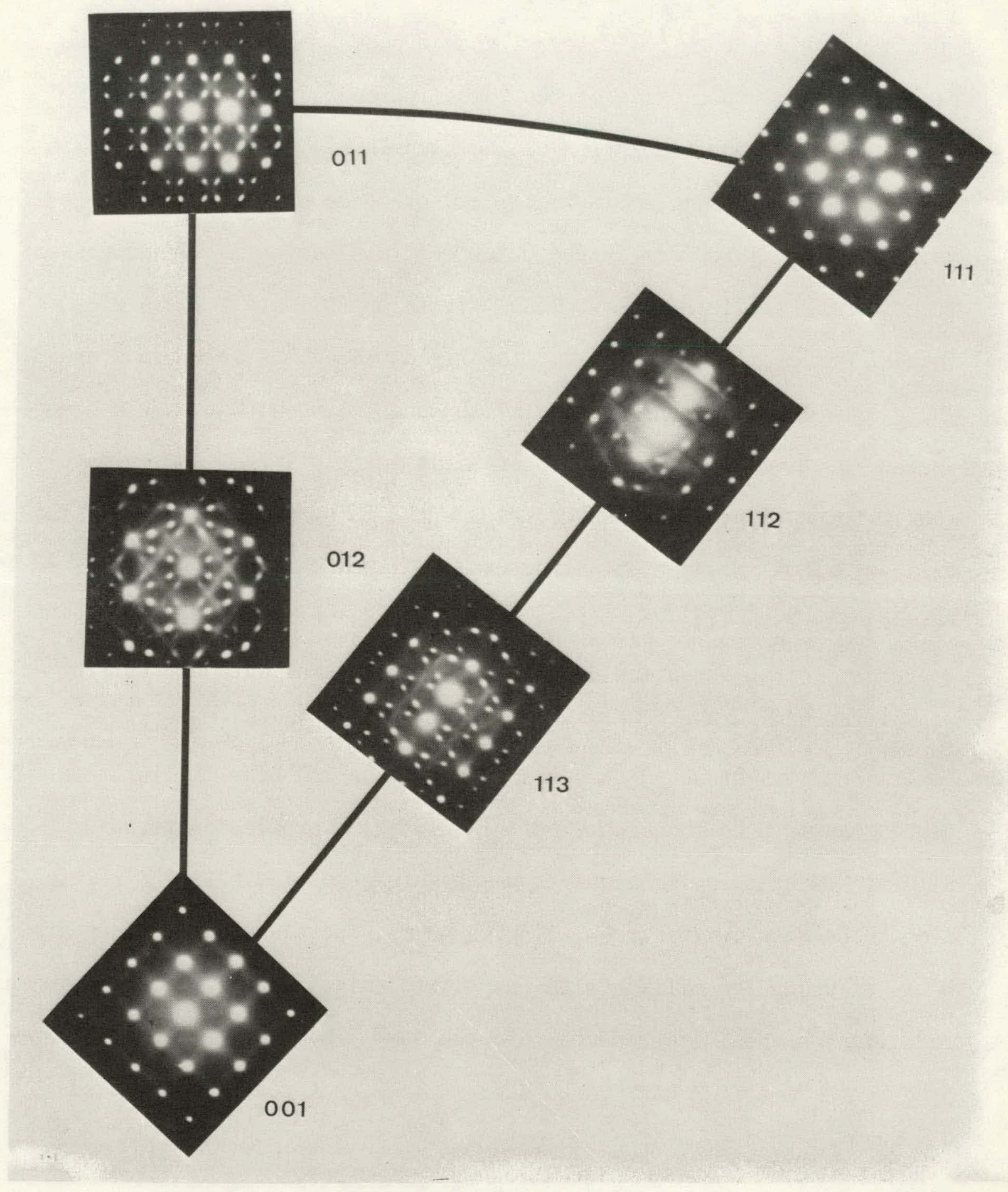

Fig. 73. Summary of Electron Diffraction Patterns from a $\mathrm{Zr}-15 \% \mathrm{Nb}$ Sample Containing the As-Quenched $\omega$ Phase. 
the proximity of the sample surface. An example of this microstructure is shown in Fig. 74. Generally these laths do not occur over the entire specimen region; they do, however, change under the influence of the electron beam. An example of the "dissolution" of these laths with time, and electron irradiation can be seen in Fig. 75 . The region in the lower right of Fig. 75(a) shows the presence of the thin foil artifact, while a moving interface in the upper left was caught in the process of transforming the lath-like region. Within approximately 2 min of initial observation (at $200 \mathrm{keV}$ ) the region was completely transformed. The diffraction patterns of Figs. 75(b) and (c) show that only a change in the relative crystallographic orientation $\left(\sim 10^{\circ}\right)$ from approximately $(001)_{\beta}$ to nearly $(113)_{\beta}$ has occurred as the interface swept across the field of view. No apparent structural differences were observed between the lath-like and matrix regions. However, it was not possih1e to verify this observation using a complete crystallographic tilting experiment duc to the finite lifetime of these thin fuil artifacts. Spurling et a1. ${ }^{102}$ has shown that argon ion beam milling techniques can be used to circumvent the relaxation problem in titanium a11oys. Using this technique it was possible to climinate the relaxation phenomenon in the $\mathrm{Zr}-\mathrm{Nb}$ alloy; however, there are two serious drawbacks of using ion milling with respect to this study. First, considerable radiation damage is introduced into the sample by the ion beam. This produces a "mottled" background superimposed on a coarser undulated 


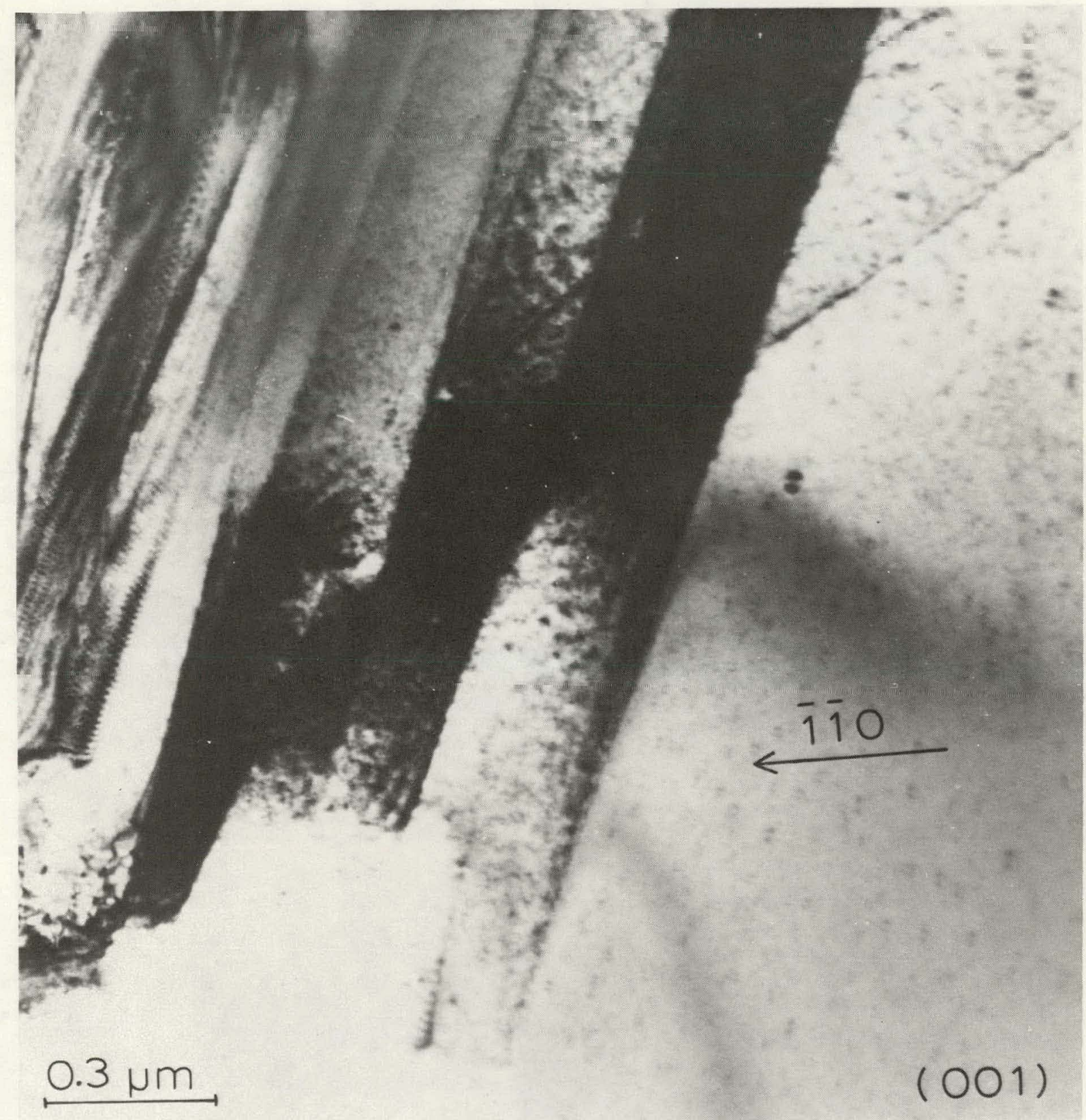

Fig. 74. Spontaneous Relaxation in $\mathrm{Zr}-15 \% \mathrm{Nb}$. 


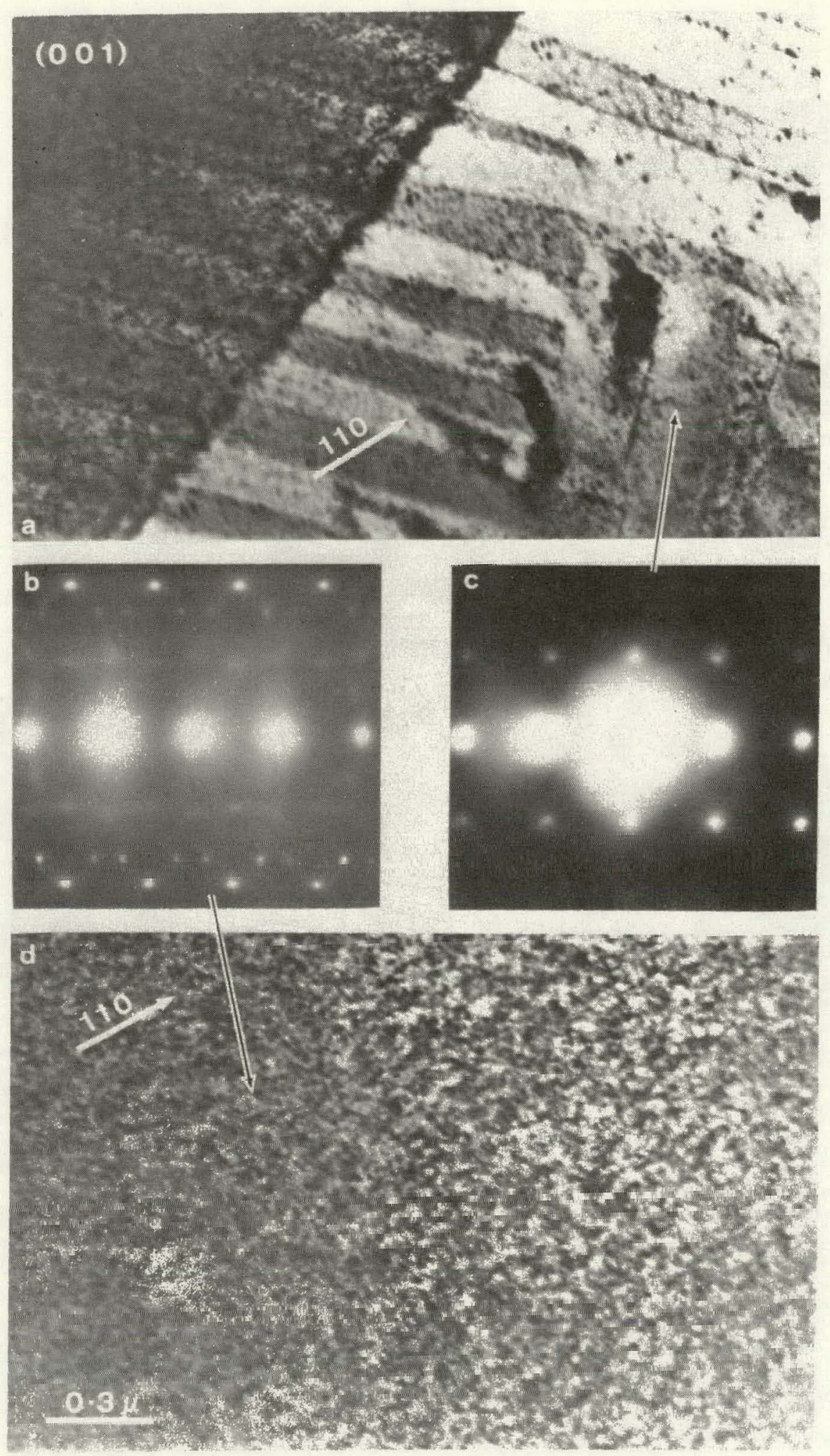

Fig. 75. Example of Dissolution of Spontaneous Relaxation Laths as a Function of Time. 
structure as shown in the micrograph of Fig. 76. This structure precludes any high resolution studies of the $\omega$ phase morphology. The second problem related to ion milling is associated with specimen heating effects. This is due to the fact for typical specimens $\sim 100 \mu \mathrm{m}$ thick the ion beam milling times were on the order of $48 \mathrm{~h}$ for a dual beam system operating at $5 \mathrm{keV}$ at $30 \mathrm{~mA}$ of ion current, and a $20^{\circ}$ glancing angle. Under these conditions sample heating effects in excess of $50^{\circ} \mathrm{C}$ were noted. This has particular importance due to the fact that the omega transformation temperature is in the vicinity of $150^{\circ} \mathrm{C}$. Thus, due to these effects, ion beam thinning of TEM specimens was discarded as a viable solution to the spontaneous relaxation problem in this alloy.

\subsubsection{Phases Resulting from Compositional Inhomogeneities}

It was also observed that occasionally isolated regions of a few specimens contained structures not associated with either the problems previously discussed or the omega phase morphology. One such region is shown in Fig. 77 (a) with its (011) diffraction pattern below. Clearly this structure is not characteristic of the $15 \% \mathrm{Nb}$ alloy, and subsequent $\mathrm{x}$-ray microanalysis measurements showed these regions to be significantly different in composition. The region shown in Fig. 77, for example, was analyzed to contain $50 \% \mathrm{Nb}$. A1l samples found to have such inhomogeneities were discarded from further experimental work. 


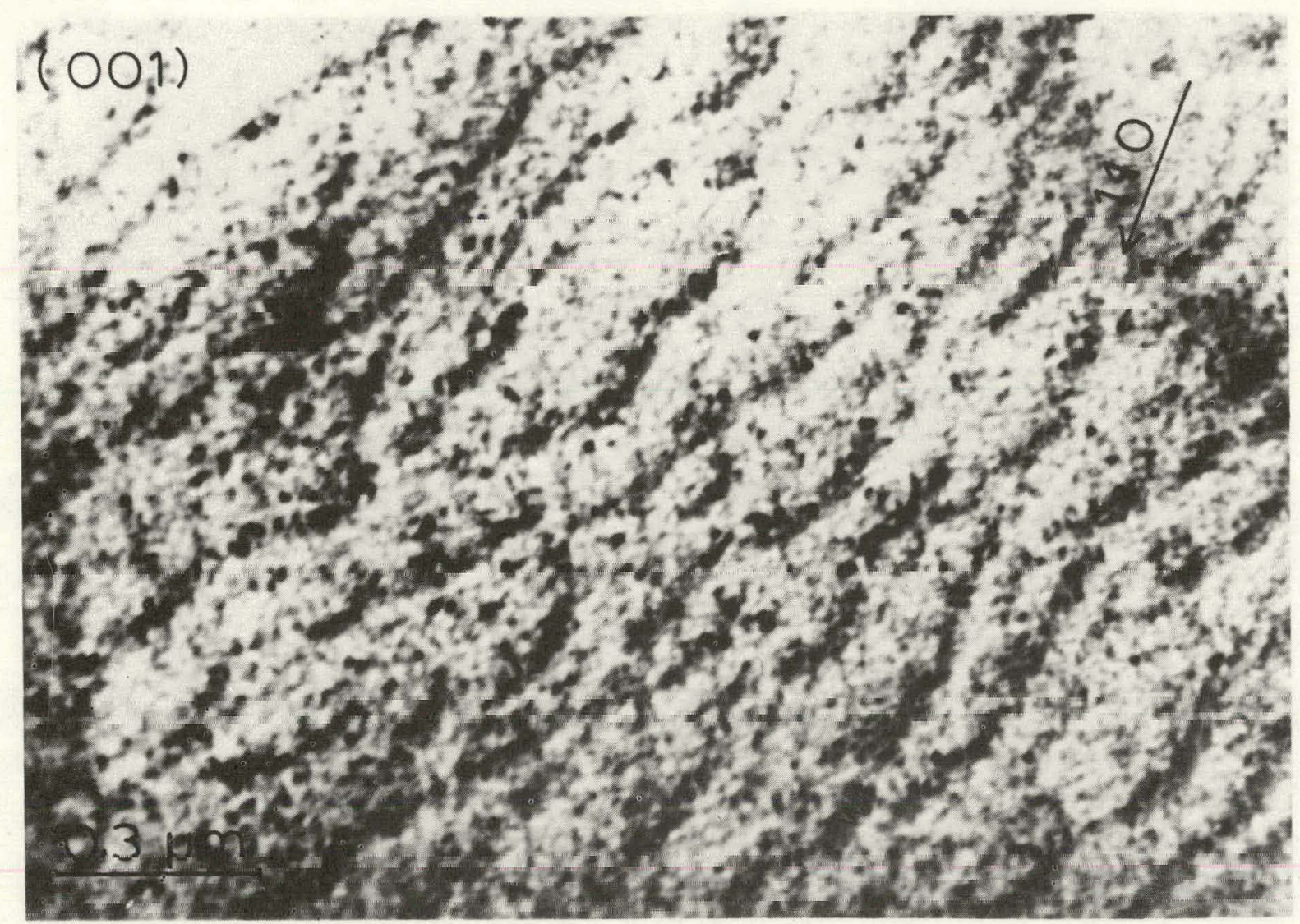

Fig. 76. Example of Typical Argon Ion-Milled Structure in $\mathrm{Zr}-15 \% \mathrm{Nb}$. 


\section{(011)}
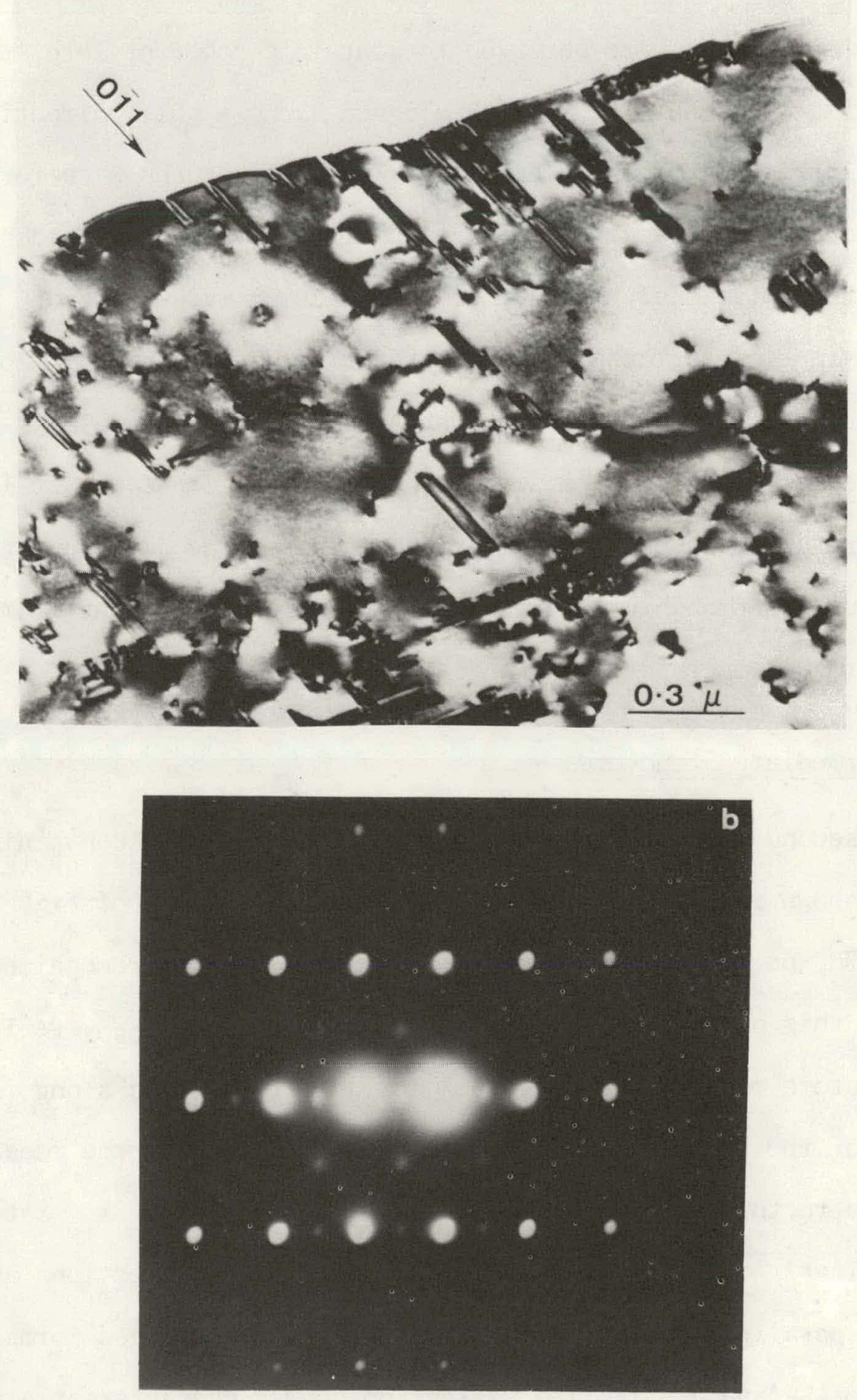

Fig. 77. Microstructure Associated with Compositional Inhomogeneities and Corresponding (011) Diffraction Pattern. 


\subsubsection{Phases Resulting from Incomplete Quenching}

Several samples were observed to contain a coherent lath structure approximately $2500 \AA$ in breadth aligned along a $<011>$ direction of the bcc lattice as shown in Fig, 78. These structures were for the most part too small to analyze using conventional diffraction techniques and thus stationary micro-micro-diffraction was utilized. As can be seen in Fig. 79 these laths alternate between $\beta$ and $\beta+\omega$ phase regions with an occasional hexagonal area being detected. STEM $\mathrm{x}$-ray microanalyses of these laths indicate their composition to be $2 \mathrm{r}-15 \% \mathrm{Nb}$ with no segregation between the alternate phases. The development of this structure is probably the result of incomplete quenching from the B field to room temperature.

\subsubsection{Intermediate Structures}

The second most frequent feature detected in $\beta+\omega$ containing samples is shown in the bright and dark-field micrographs of Fig. 80. As in Fig. 70, no distinct bright-field structure can be recognized; however, in this case dark-field images formed using an $\omega_{0002}$ reflection show a structure of approximately $10 \AA$ periodicity aligned along [200] directions of the bcc lattice. Diffraction patterns from the ideal asquenched $\omega$ structure and this sample are compared in Fig. 81. Satellites are clearly observed to develop just off $\omega_{0002}$ reflections and are aligned parallel to [222] directions. Dark-field images formed using an $\beta \omega_{0002}$ and a satellite reflection show a characteristic fringe 


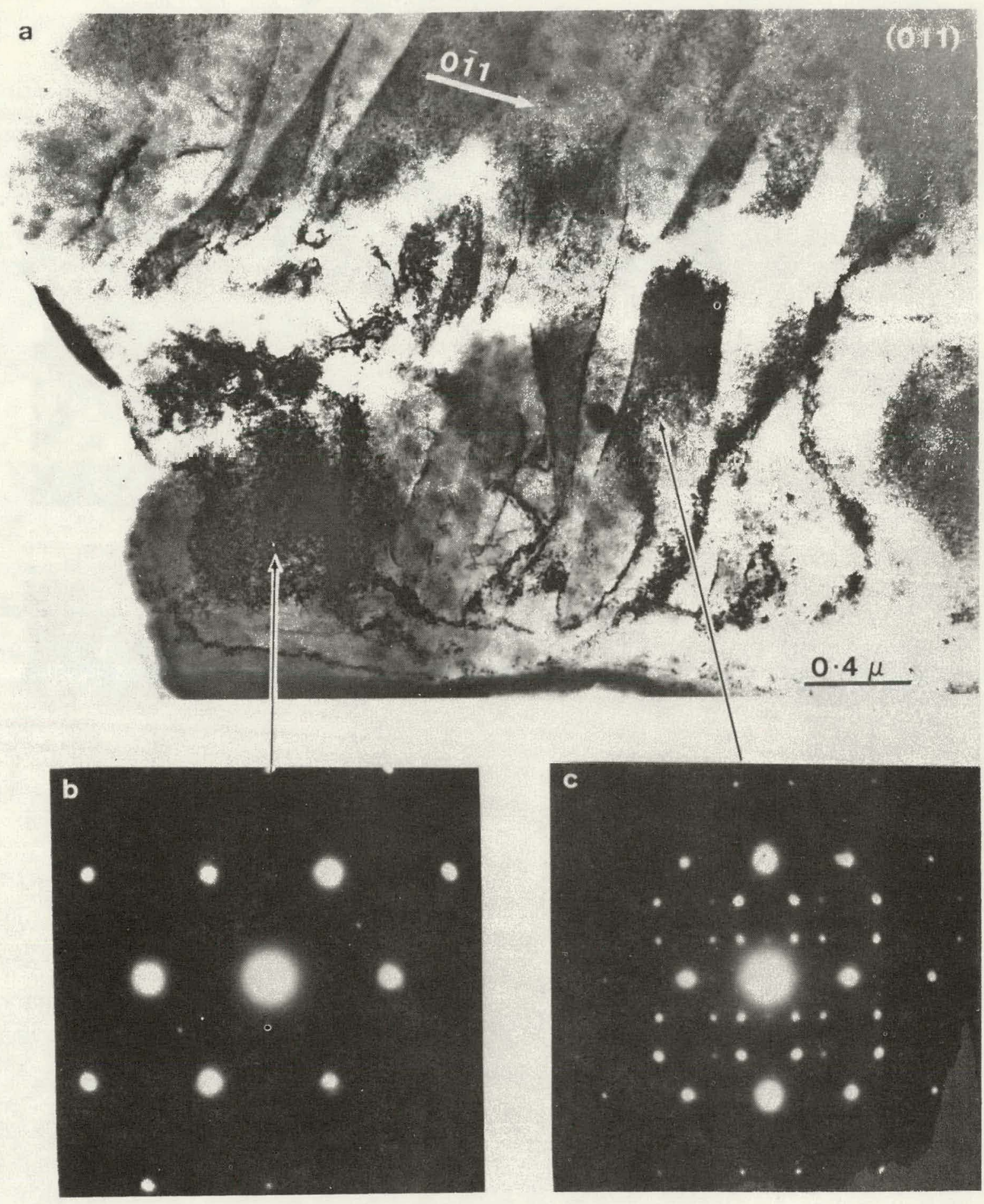

Fig. 78. Lath Structure and Diffraction Patterns Observed in Zr-15\% Nb A1loy. 


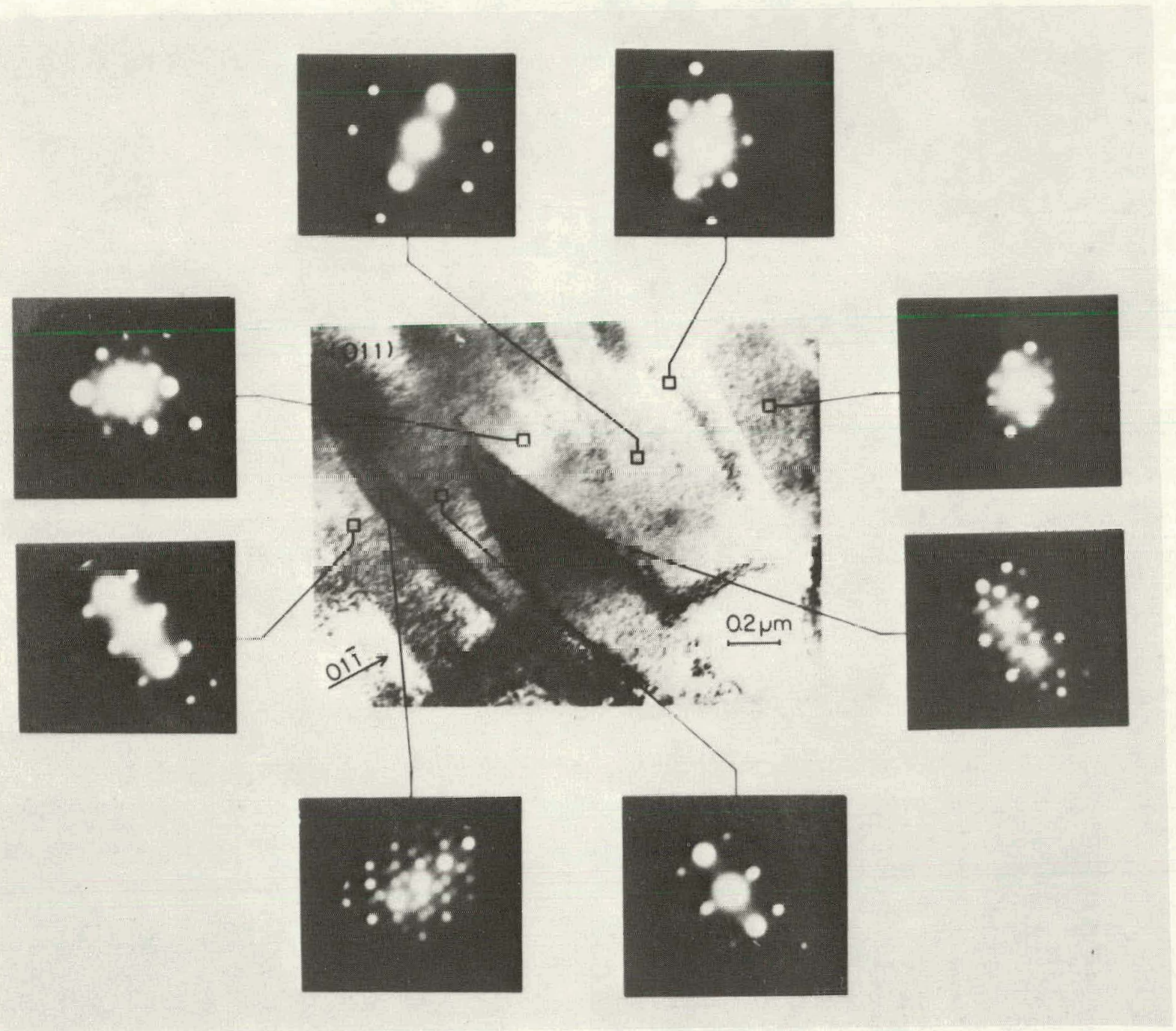

Fig. 79. Analysis of Lath Structure in $\mathrm{Zr}-15 \% \mathrm{Nb}$ Using Stationary Diffraction Pattern Technique. 

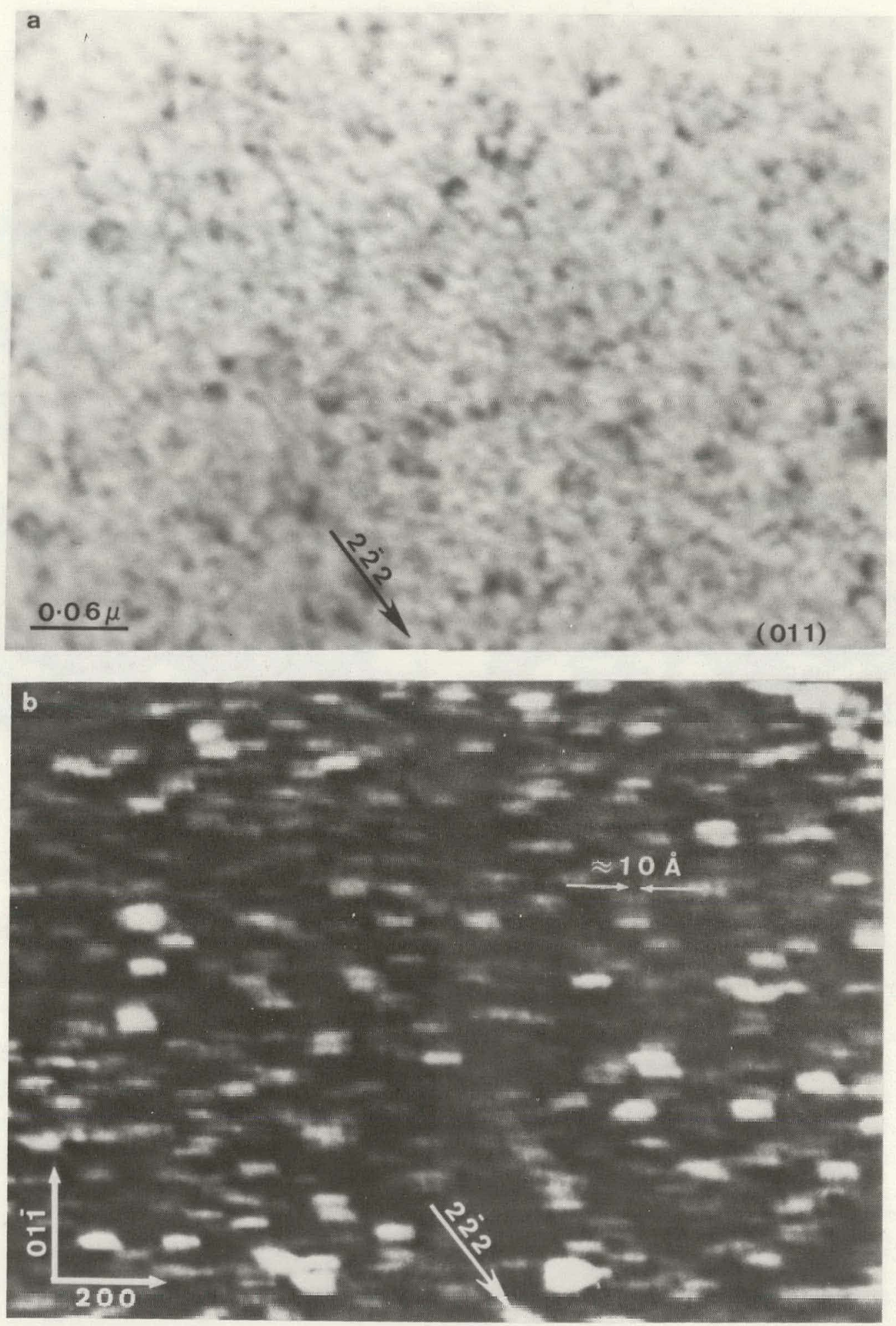

Fig. 80. Bright (a) and Dark-Field TEM Micrographs of Intermediate Structure in $\mathrm{Zr}-15 \% \mathrm{Nb}$. 

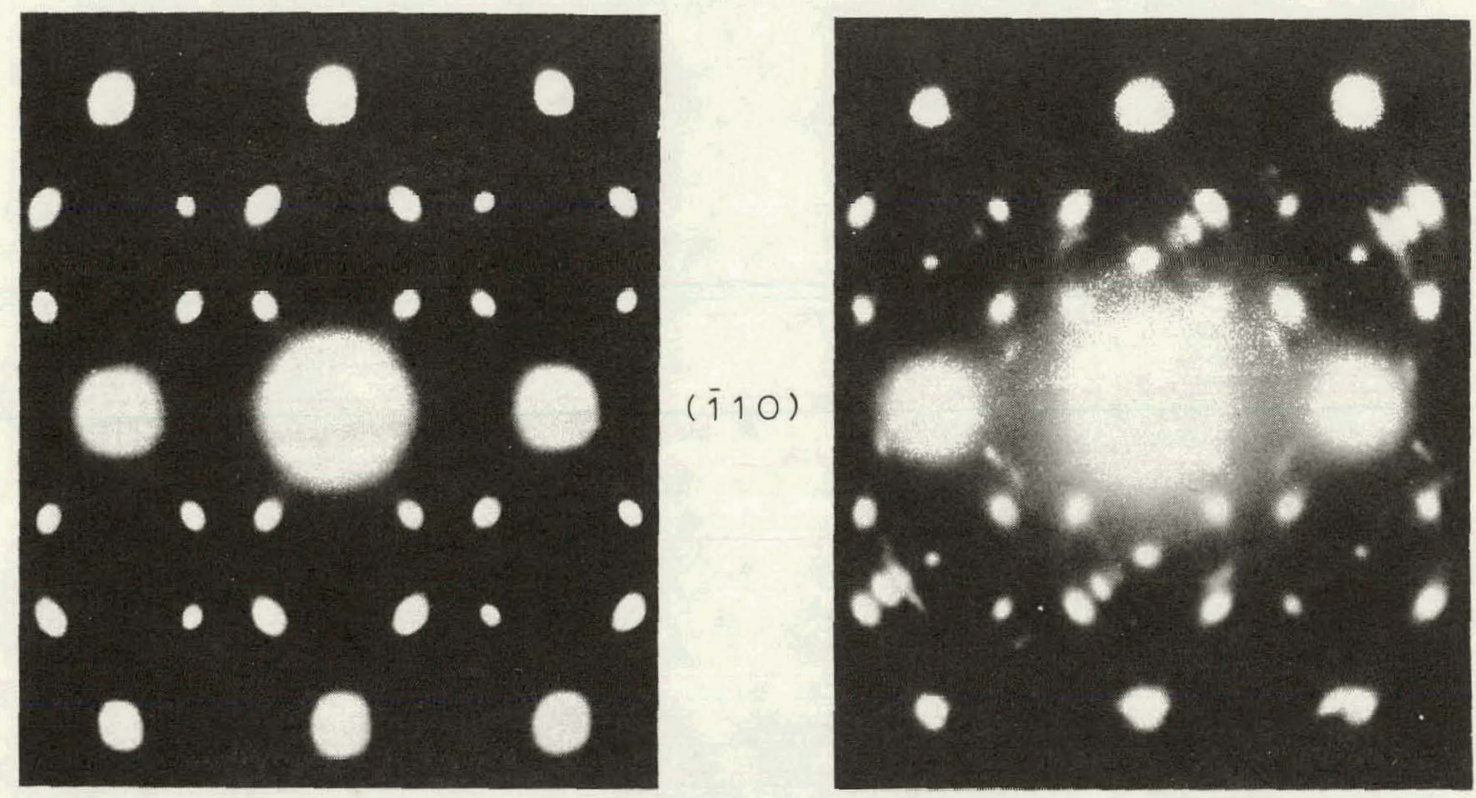

Fig. 81. Comparison of (i10) Electron Diffraction Patterns from As-Quenched $\omega$ (a) and Intermediate Phase (b) Morphology. 
pattern perpendicular to the $[222]_{\beta}$ direction with a spacing of $\sim 25 \AA$ as shown in Fig. 82. The observation of such a structure was initially interpreted as the formation of a long period ordered structure of alternating $\beta$ and $\omega$ phase regions as predicted by Cook's $s^{27-29}$ theory of the omega phase morphology in $\mathrm{Zr}-\mathrm{Nb}$. Thermal cycling experiments on prethinned specimens between -196 and $+30^{\circ} \mathrm{C}$ failed to change the relative intensity of these $\omega$ satellite reflections. Furthermore it was not possible to consistently observe this morphology from one sample to another. These inconsistencies were resolved when the existence of a thin hexagonal film was discovered on samples exhibiting identical $\omega$ satellite reflections. Figure 83 shows one of the few cases in which this film extended beyond the edge of the specimen. The diffraction pattern insert clearly indicates its hexagonal nature. STEM x-ray microchemical analysis of this region indicated a composition identical to the matrix (i.e., $15 \% \mathrm{Nb})$.

Electron energy loss measurements were then subsequently performed in order to check for the possible formation of an oxide layer. The energy loss spectrum labeled $a$ in Fig. 84 was recorded from a contamination zone purposely formed on the film by allowing the STEM probe (in a VG HB5) to remain stationary on a region of the sample for $\sim 0.5 \mathrm{~h}$. In this case a carbon $k$ loss edge is clearly observed. The second spectrum (b) was measured under identical operating conditions by simply translating the electron probe to a "clean" area of the specimen. The positions of the carbon and oxygen $k$ loss edges are indicated on both spectra and it seems that there is no significant oxygen concentration detectable in 


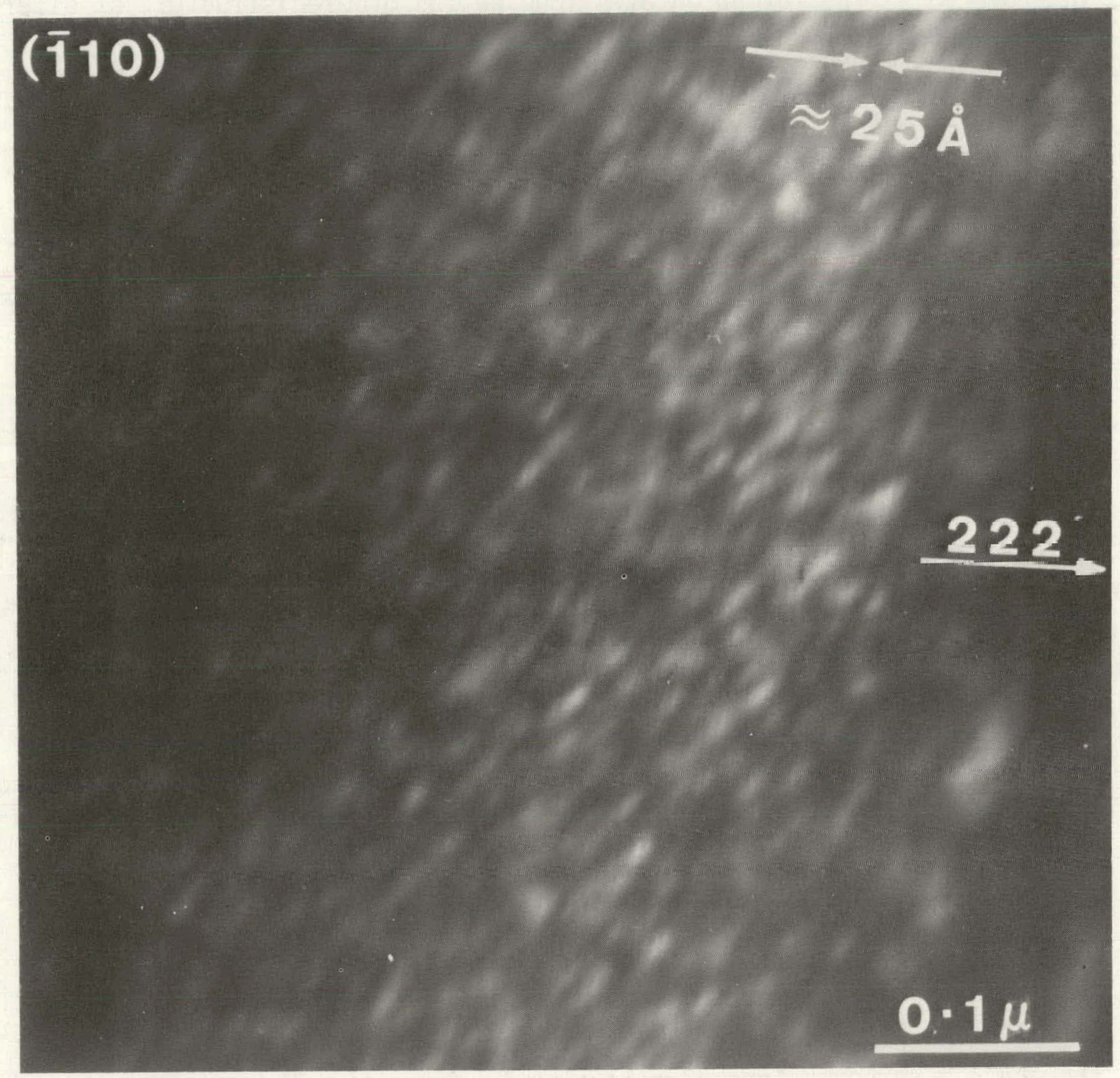

Fig. 82. $25 \AA$ Spacing Fringes Obtained by Imaging Using $\omega_{0002}$ and the Corresponding Satellite Reflection from Fig. 83. 

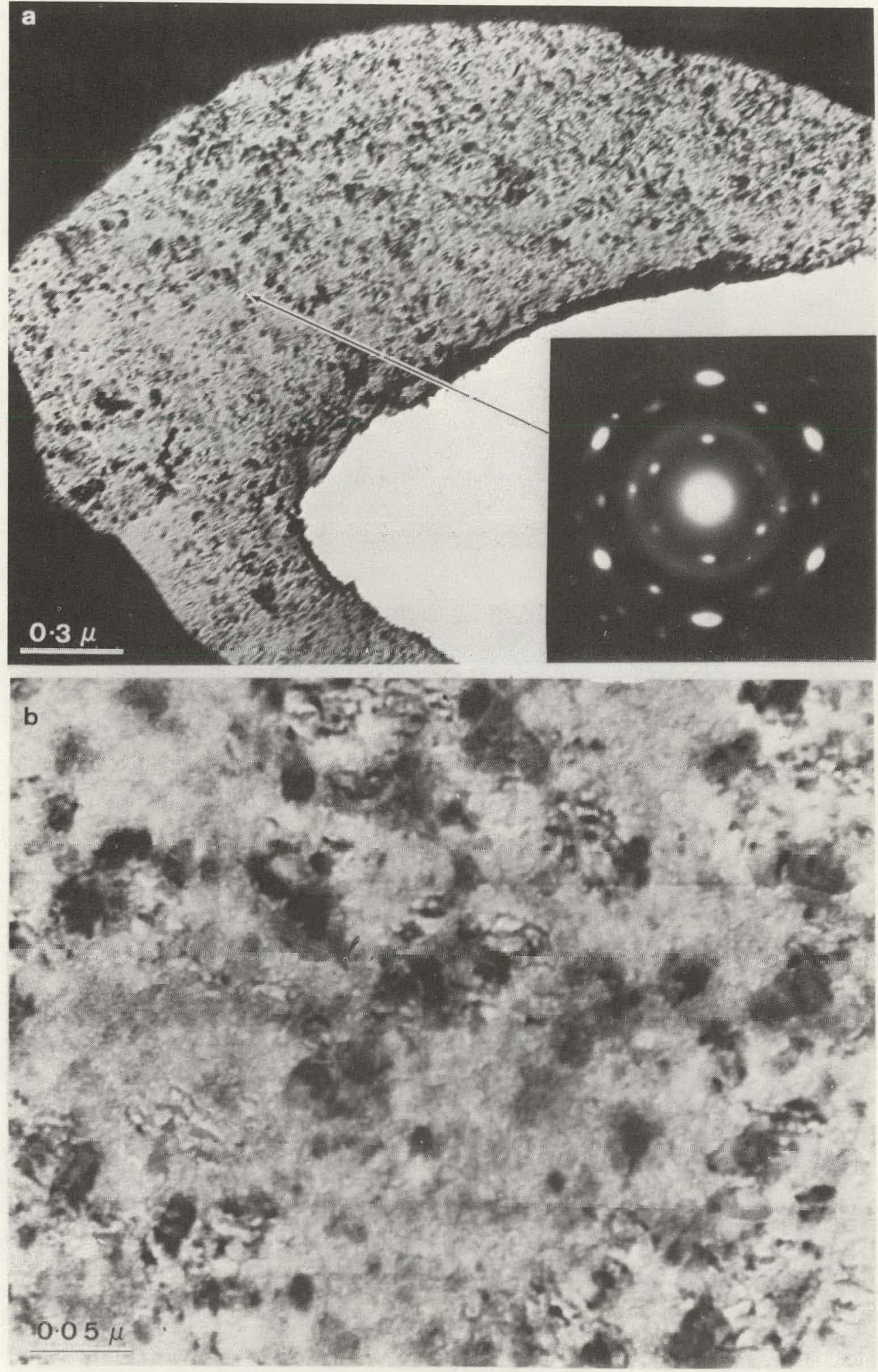

Fig. 83. Microstructure of Thin Crystalline Surface Film. 

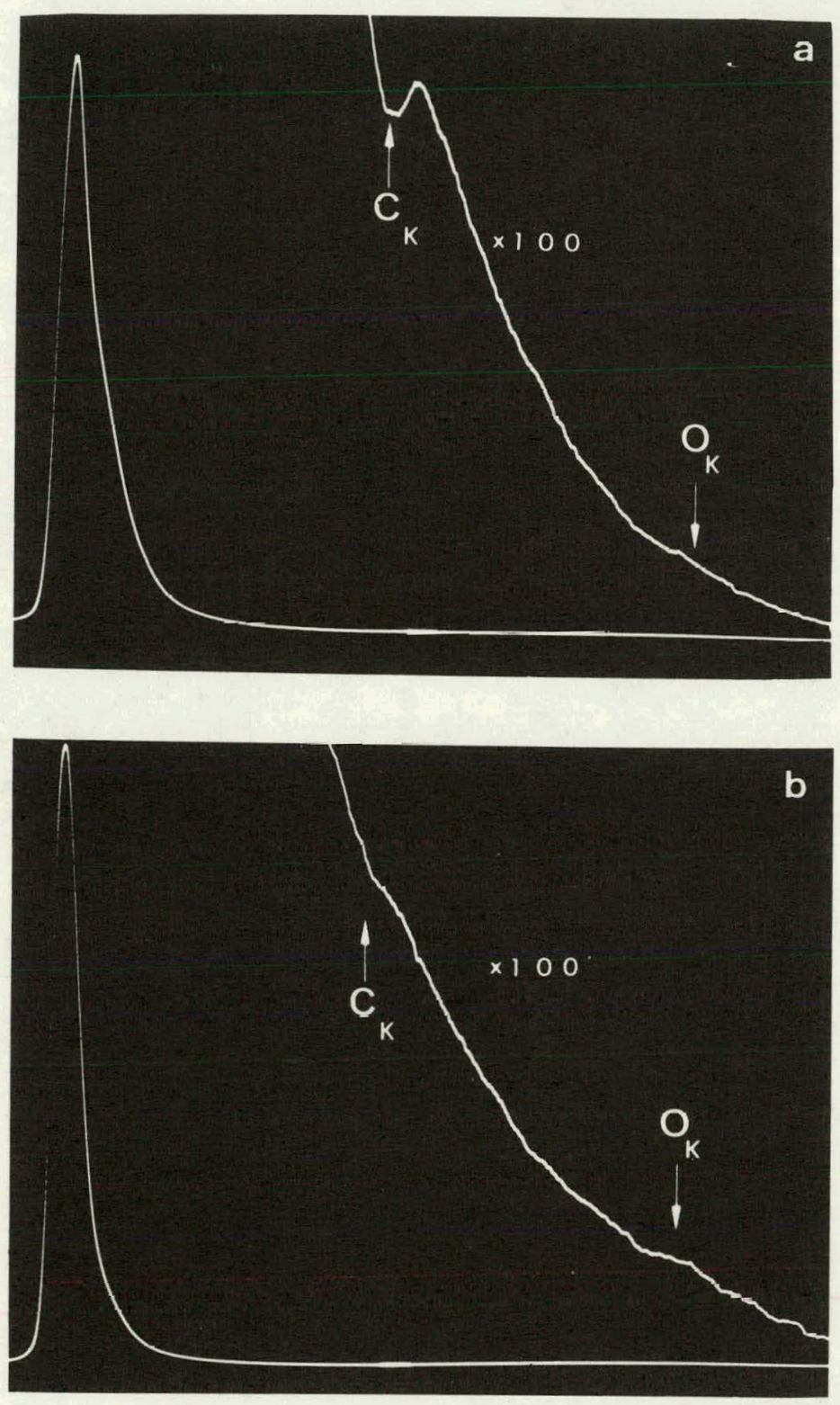

Fig. 84. Electron Energy Loss Measurements from Surface Film of Fig. 83. Spectrum (a) measured from contamination zone due to electron probe; spectrum (b) from "clean" area. Note absence of detectable oxygen $\kappa$ edge in both cases. 
either case. The slight ripple near the position of the oxygen $\kappa$ edge is due to electronic noise and was not reproducible on repeated scans. The development of the $w$ satellites can now be interpreted by reference to Fig. 85. The diffraction pattern of Fig. 85(a) was recorded from the thick (dark) region of Fig. 83(a) and is a (011) $\beta+\omega$ pattern. The hexagonal pattern (b) was recorded from the thin film shown on the same micrograph and is shown in the appropriate experimentally determined orientation with respect to the $\beta+\omega$ matrix. The diffraction pattern labeled (c) was produced by superimposing the negatives of Fig. 85(a) and (b) and the correspondence to the diffraction pattern of Fig. 81 (b) is apparent. These results indicate that the $\omega$ satellite formation can be attributed to the existence of a thin crystalline surface film and not to the development of an ordered $\beta+\omega$ structure.

A second type of lath structure was also observed in some of the samples and is shown in Fig. 86. These laths have a width of approximately $500 \AA$ and no compositional variation was noted between the features. A sequence of diffraction patterns over a standard stereographic triangle is reproduced in Fig. 87. These patterns can be indexed as the $\beta$ and as-quenched $\omega$ structures together with an fcc phase having a lattice parameter of $5.01 \AA$. The orientation relationship between the fcc and bcc phases has been determined to be the following.
(011) $_{\beta} \|(111)_{\mathrm{fcc}}$
and
$[200]_{\beta} \|[\overline{1} 01]_{f c c}$

which differs only slightly from the Kurdjumov-Sacks relation
(011) ${ }_{\beta} \|(111) \mathrm{fcc}_{\mathrm{cc}}$
and
$[1 \overline{1} 1]_{\beta} \|[\overline{1} 01]_{\mathrm{fcc}}$ 

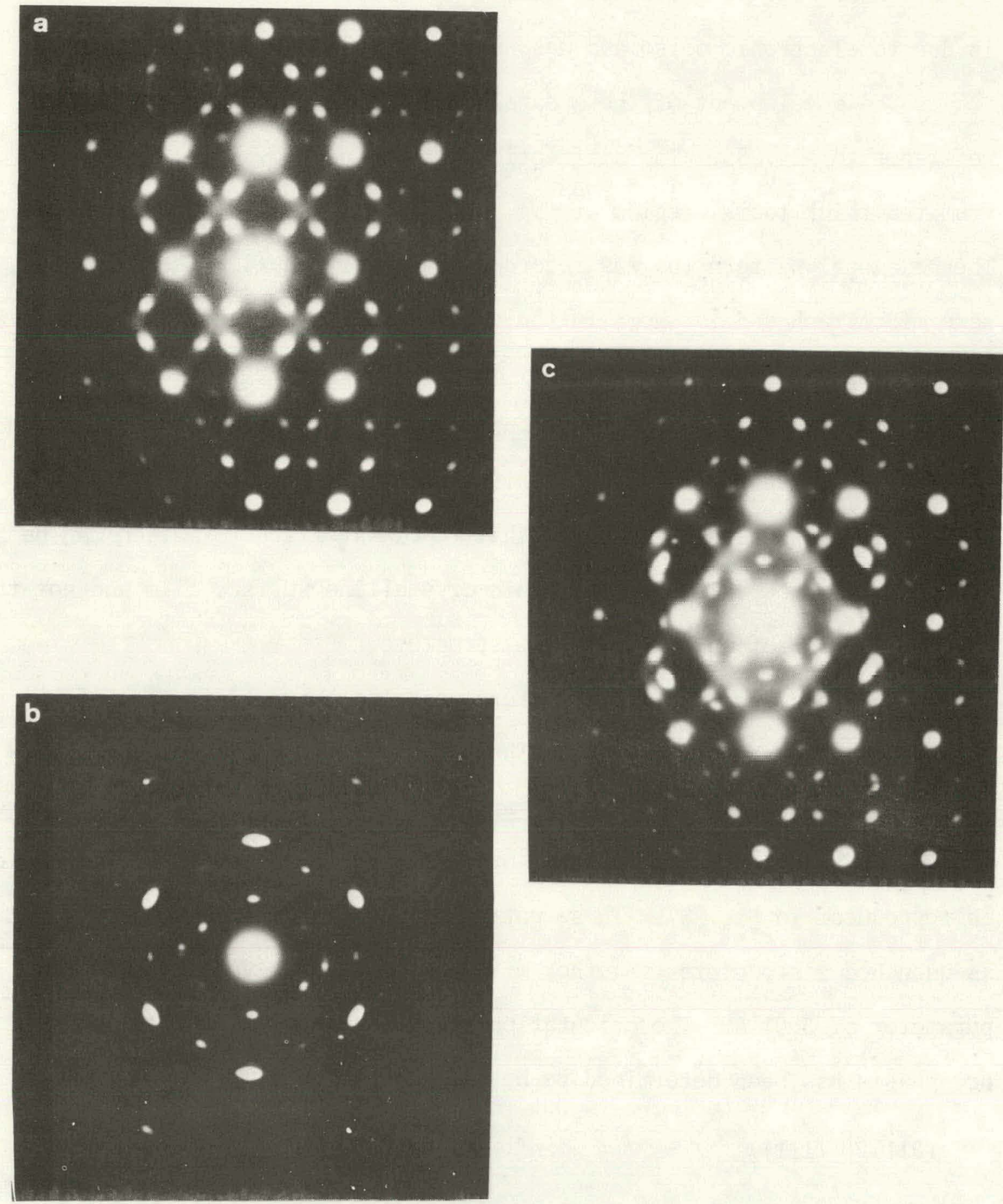

Fig. 85. Development of $\omega$ Satellite Reflections Due to Superposition of Hexagonal Pattern (b) on (011) $\beta+\omega$ Pattern (a) Resulting in Pattern (c). 


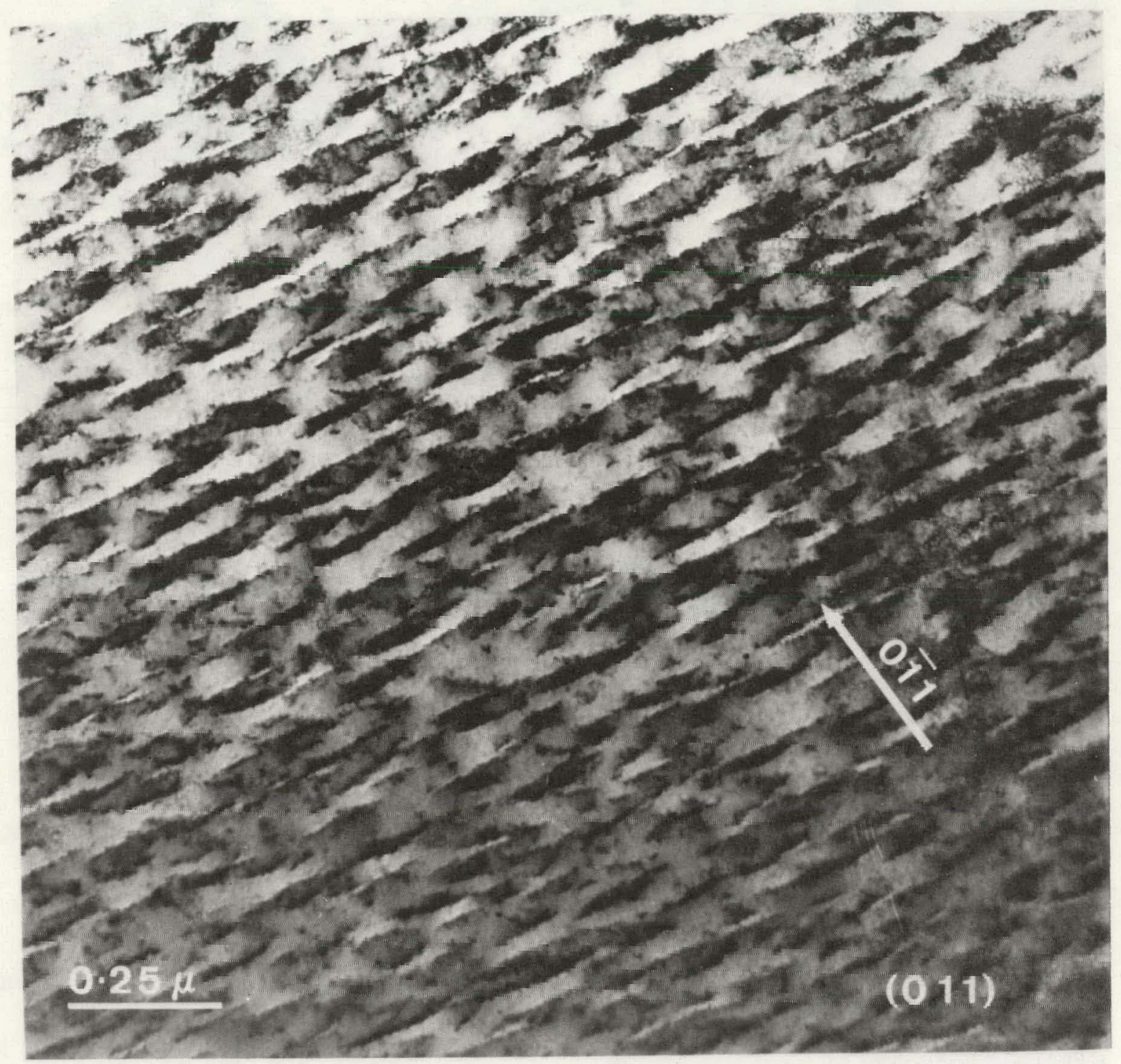

Fig. 86. Coherent Lath Structure Observed in $\mathrm{Zr}-15 \% \mathrm{Nb}$. 


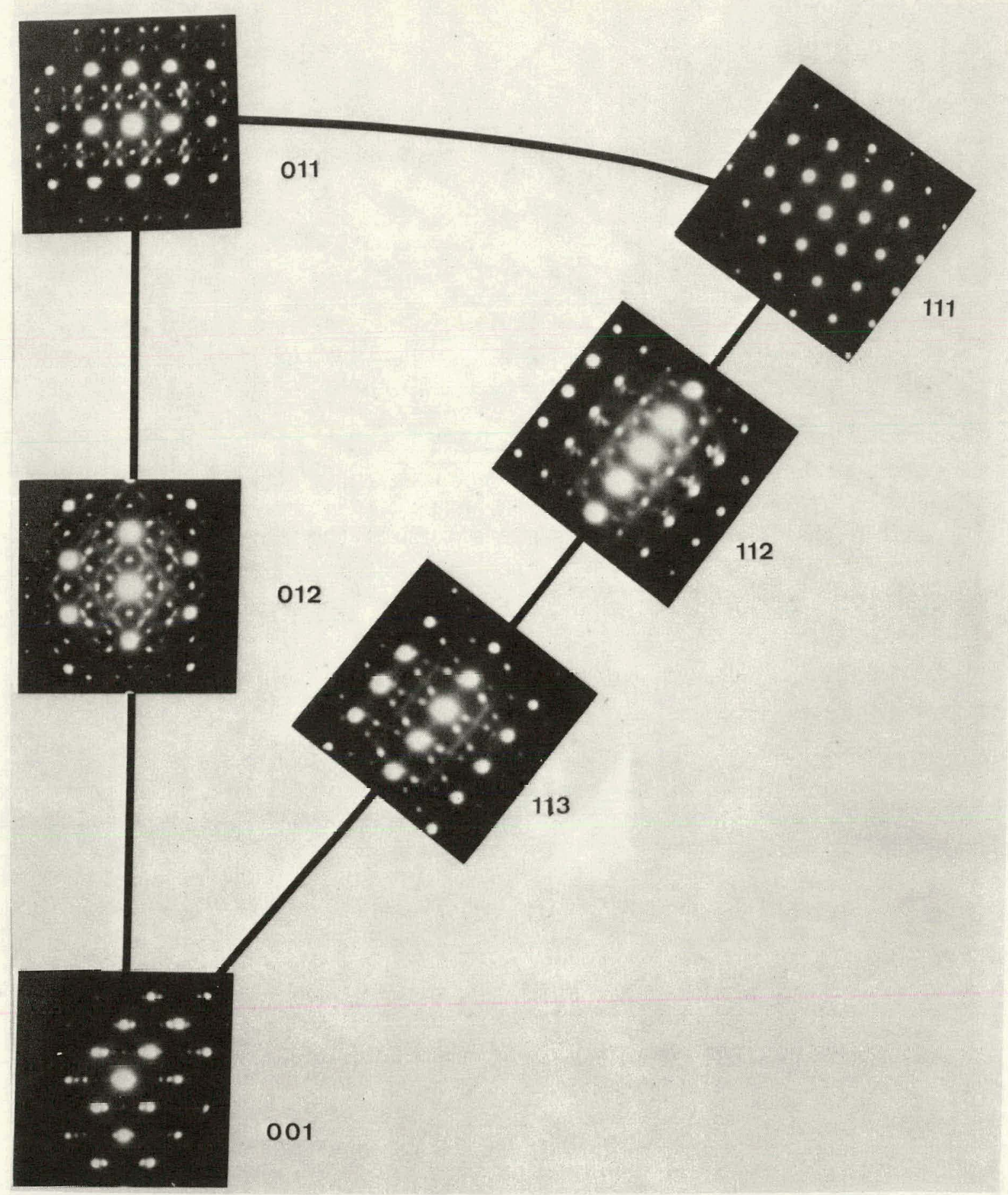

Fig. 87. Summary of Electron Diffraction Patterns from the Coherent Lath Structure of Fig. 86. 
The periodic reflections along the [020] direction of the $(001)_{\beta}$ diffraction pattern are consistent with spiking effects from the ${ }^{(011)} \mathrm{fcc}_{\mathrm{fc}}$ reciprocal lattice plane only $10^{\circ}$ off the $(001)_{\beta}$.

Argon ion-milling of the thinned disc for $1 \mathrm{~h}$ at $4 \mathrm{keV}$ and $30 \mathrm{~mA}$ completely change the observed structure as shown in the TEM micrograph and corresponding diffraction patterns of Figs. 88 and 89 . It was not possible to determine whether these changes were the result of the removal of a surface film or due to specimen heating effects. This structure can be a discontinuous form of the hexagonal film shown in Fig. 83 since the (011) $\beta$ patterns of the two are extremely similar. It is possible that these structures are a form of the $\delta$-hydride which in the zirconium-hydrogen system has an fcc crystal structure and a lattice parameter of $4.79 \AA$.

\subsubsection{Precipitation of Coherent Isothermal Omega}

Specimens aged for one year at room temperature developed a coherent precipitate consistent with the early stages of isothermal (aged) omega. These precipitates are shown in Fig. 90 and appear to be platelets approximately $200 \AA$ in diameter and less than $100 \AA$ thick. Their coherent nature can be identified by the absence of a sharp we11-defined interface with the bcc matrix indicating the presence of a strain field at their perimeter. Interestingly, these platelets form a periodic structure along one of the $\{111\}$ planes in the bcc lattice with an average spacing of $400 \AA$ as shown in Fig. 91. Higher magnification images also indicate the presence of a thin crystalline surface film. This can be 


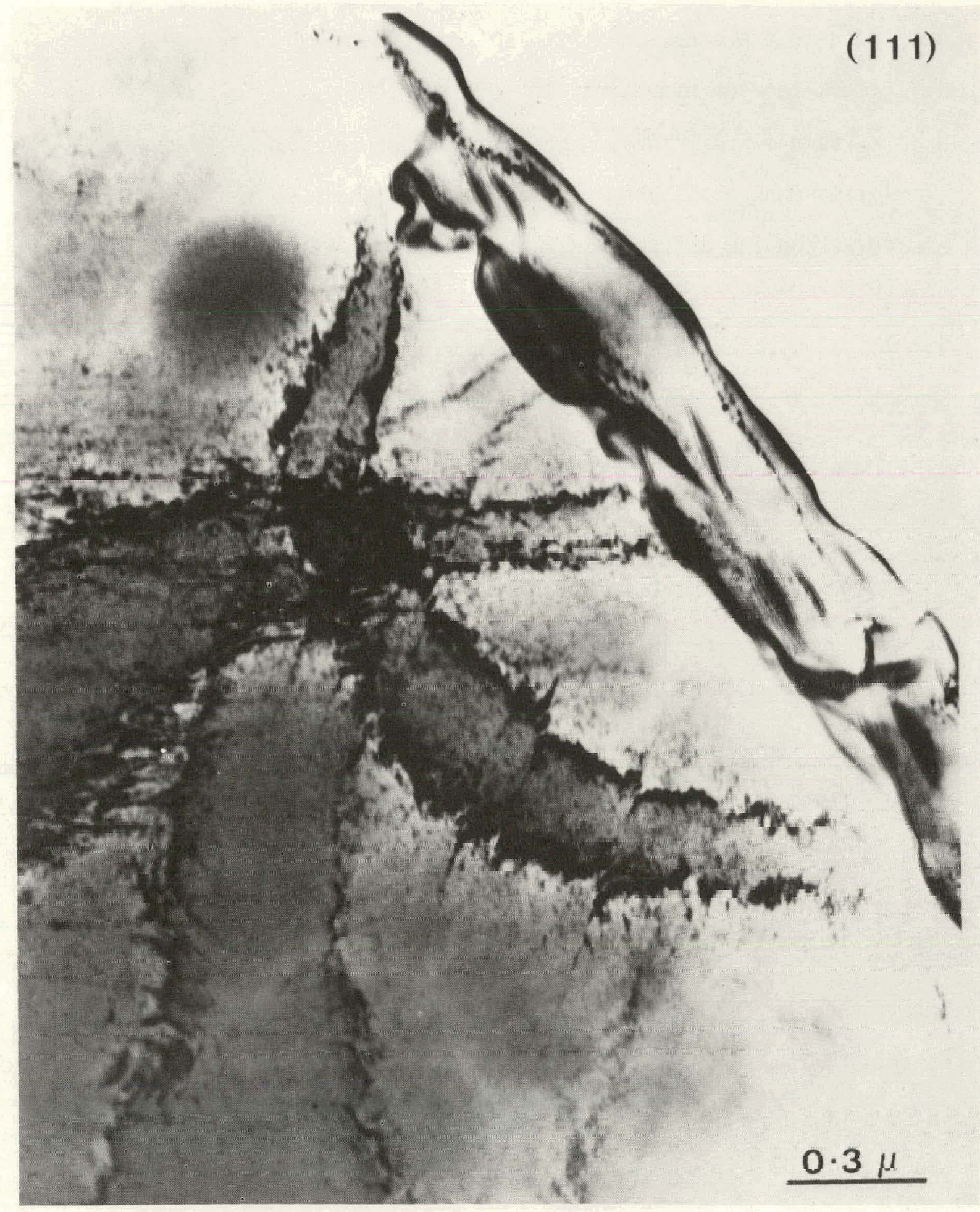

Fig. 88. Microstructural Changes Resulting from Argon IonMilling of Sample Shown in Fig. 86. 


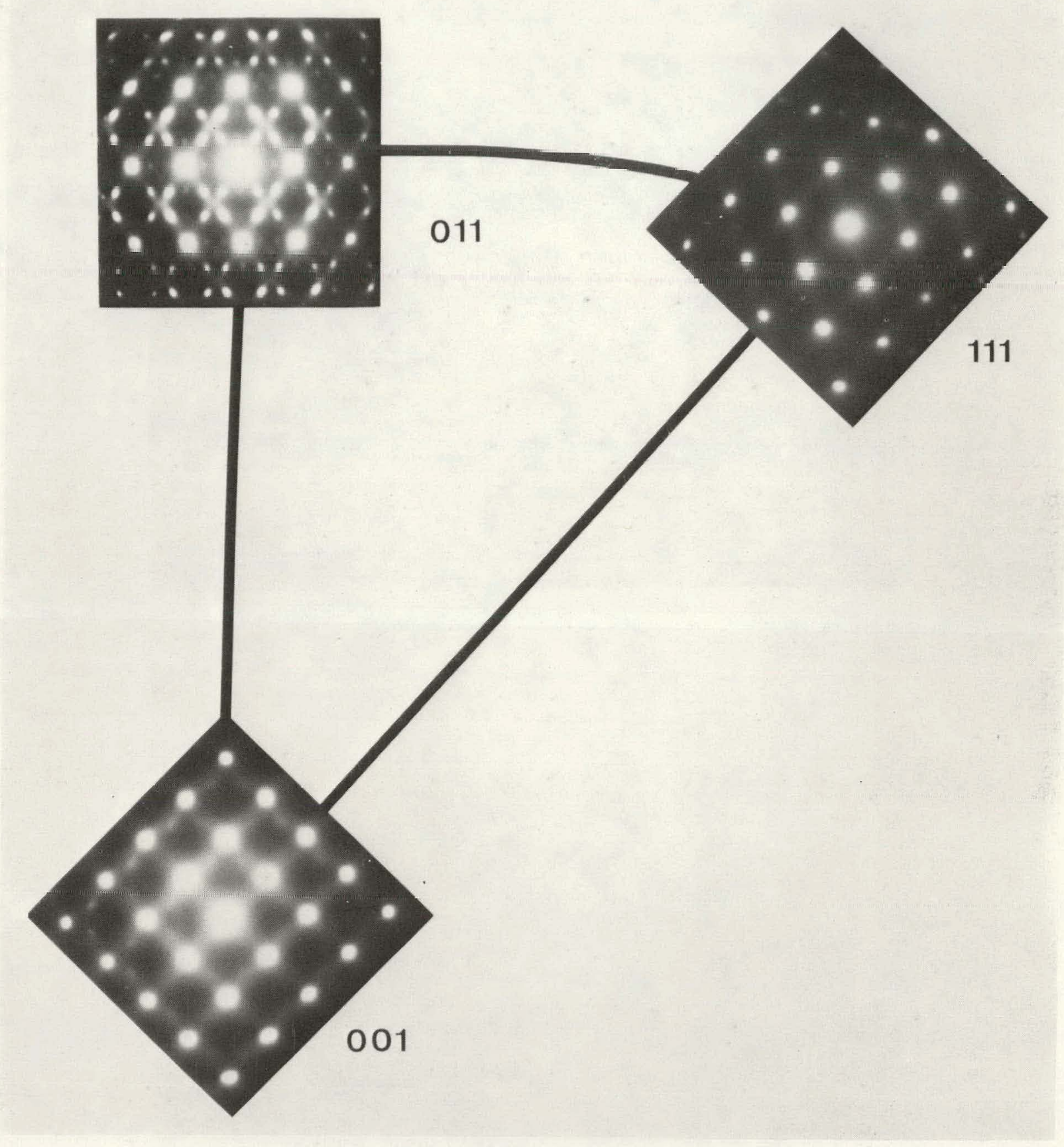

Fig. 89. Corresponding Electron Diffraction Patterns for the Microstructure of Fig. 88. 

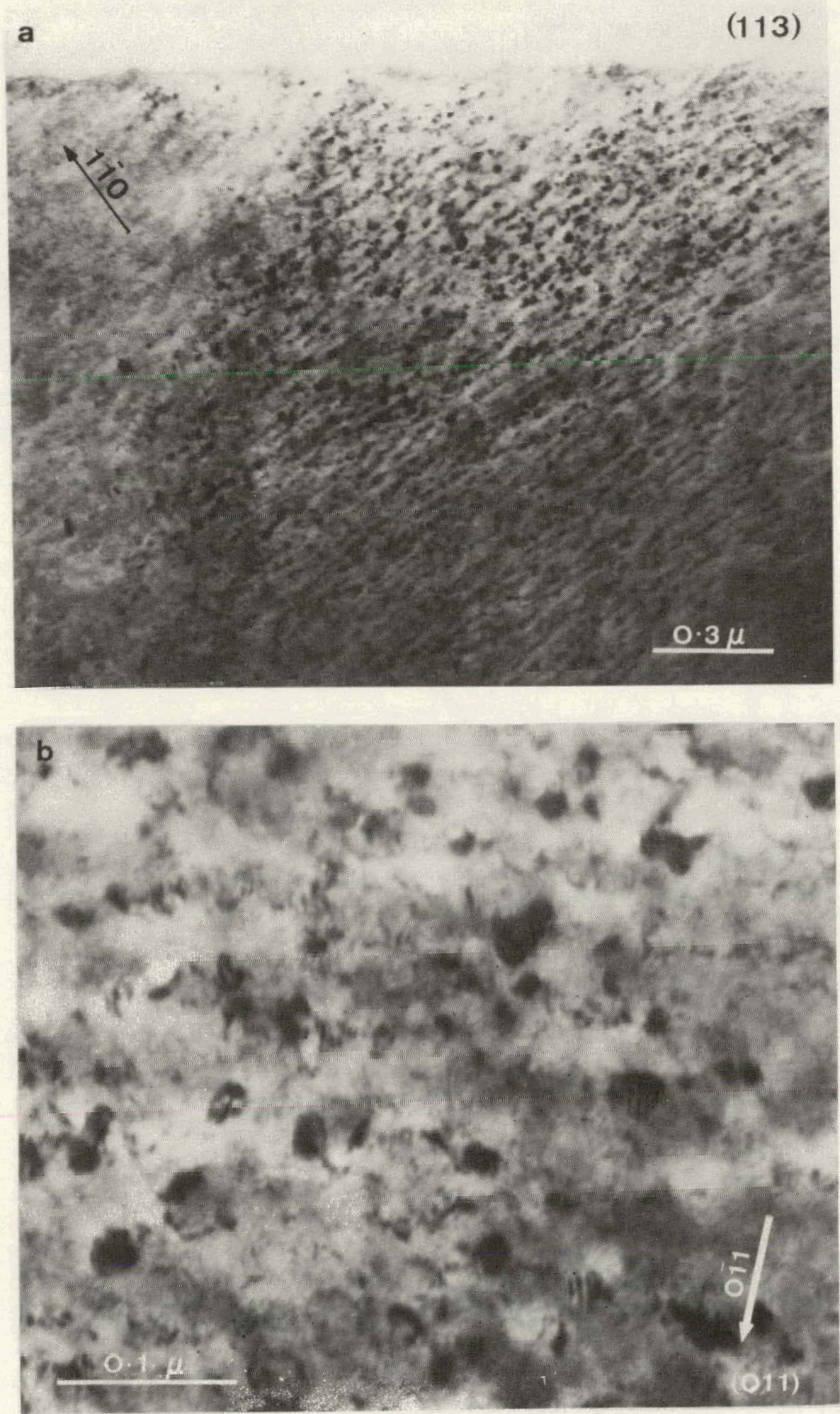

Fig. 90. Precipitation of Coherent Isothermal Omega in $\mathrm{Zr}-15 \% \mathrm{Nb}$, 


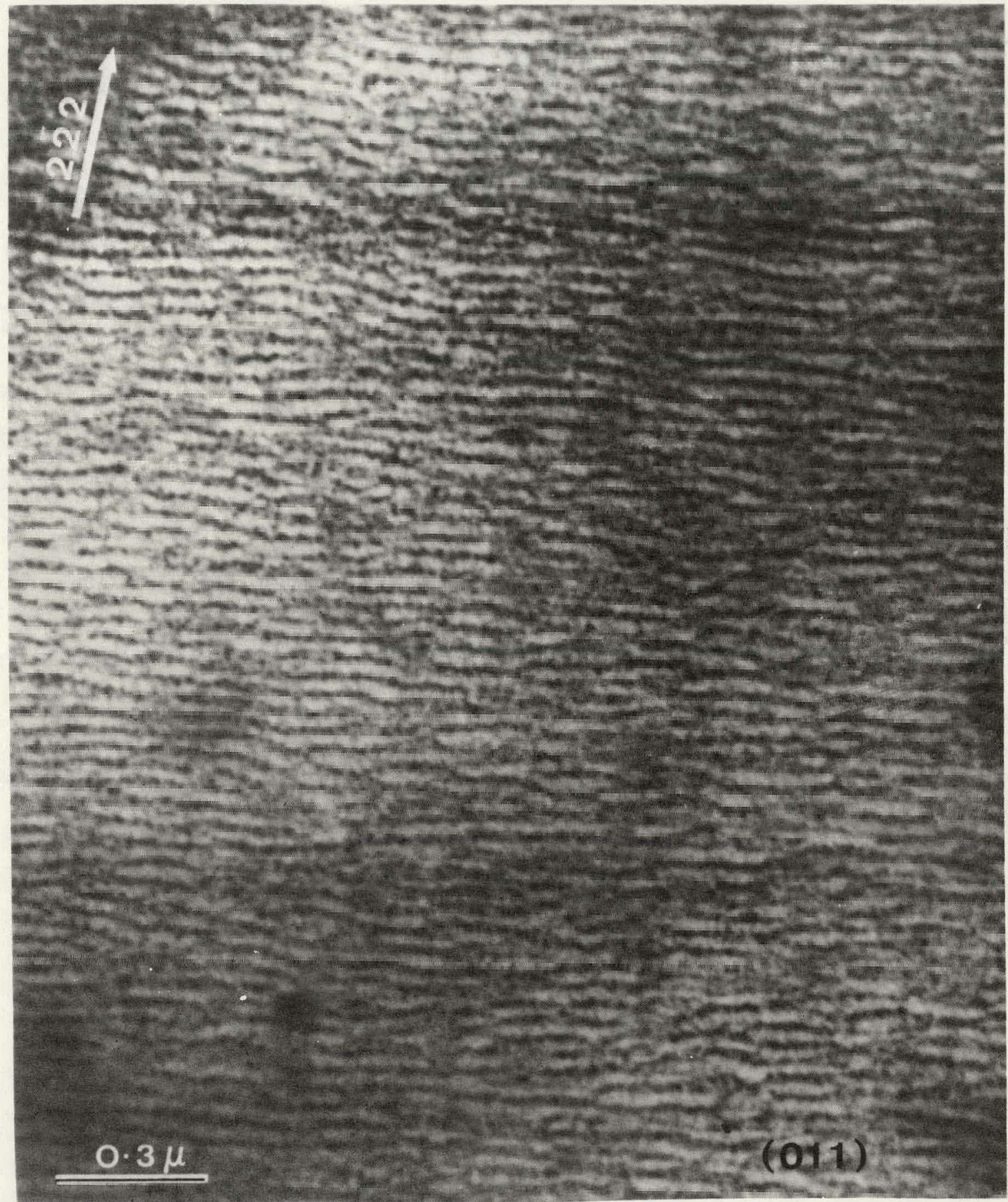

Fig. 91. Periodic Struzture Formed in Samples Containing Coherent Isotherma1 Omega. 
inferred from the discontinuous Moire fringe pattern shown in the TEM bright field image of Fig. 92. Selected area diffraction measurements made as a function of sample thickness (Fig. 93) also indicate the presence of a thin surface film.

The electron diffraction patterns observed over a standard stereographic triangle for this sample are summarized in Fig. 94. The formation of $\langle 100\rangle$ superlattice reflections as well as changes in the bcc lattice parameter $\left(a_{0} \simeq 3.11 \AA\right)$ arc consistent witl the vccurrence of solute segregation followed by the formation of the coherent isothermal (aged) omega phase with a $c / a$ ratio of $\sim 0.615$ and having the orientation relationship

$$
\text { (111) }{ }_{\beta} \|(0001)_{\omega} \quad \text { and }[01 \overline{1}]_{\beta} \|[11 \overline{2} 0]_{\omega}
$$

It was not possible to confirm experimental1y changes in composition of the matrix using $x$-ray microanalysis due to the high contamination rate in the JSEM 200. Electron energy loss measurements did not. indirate the presence of oxygen in the sample. The $\frac{1}{6}\langle 112\rangle$ and $\frac{1}{2}\langle 110\rangle$ reflections in these patterns are due to the thin surface film and can be indexed as an ordered face-centered cubic structure having the following orientation relationship with the bcc lattice.
$(111)_{\beta}$
(011) $_{\text {fec }}$
and
$[1 \overline{10}]_{\beta} \|[200]_{\mathrm{fcc}}$

The corresponding lattice constant of this fec structure is $a_{0}=4.82 \AA$.

\subsection{In-Situ Thermal Cycling Experiments}

Samples characterized as containing only the as-quenched $\omega$ and B phases were used in the thermal cycling experiments described in this 


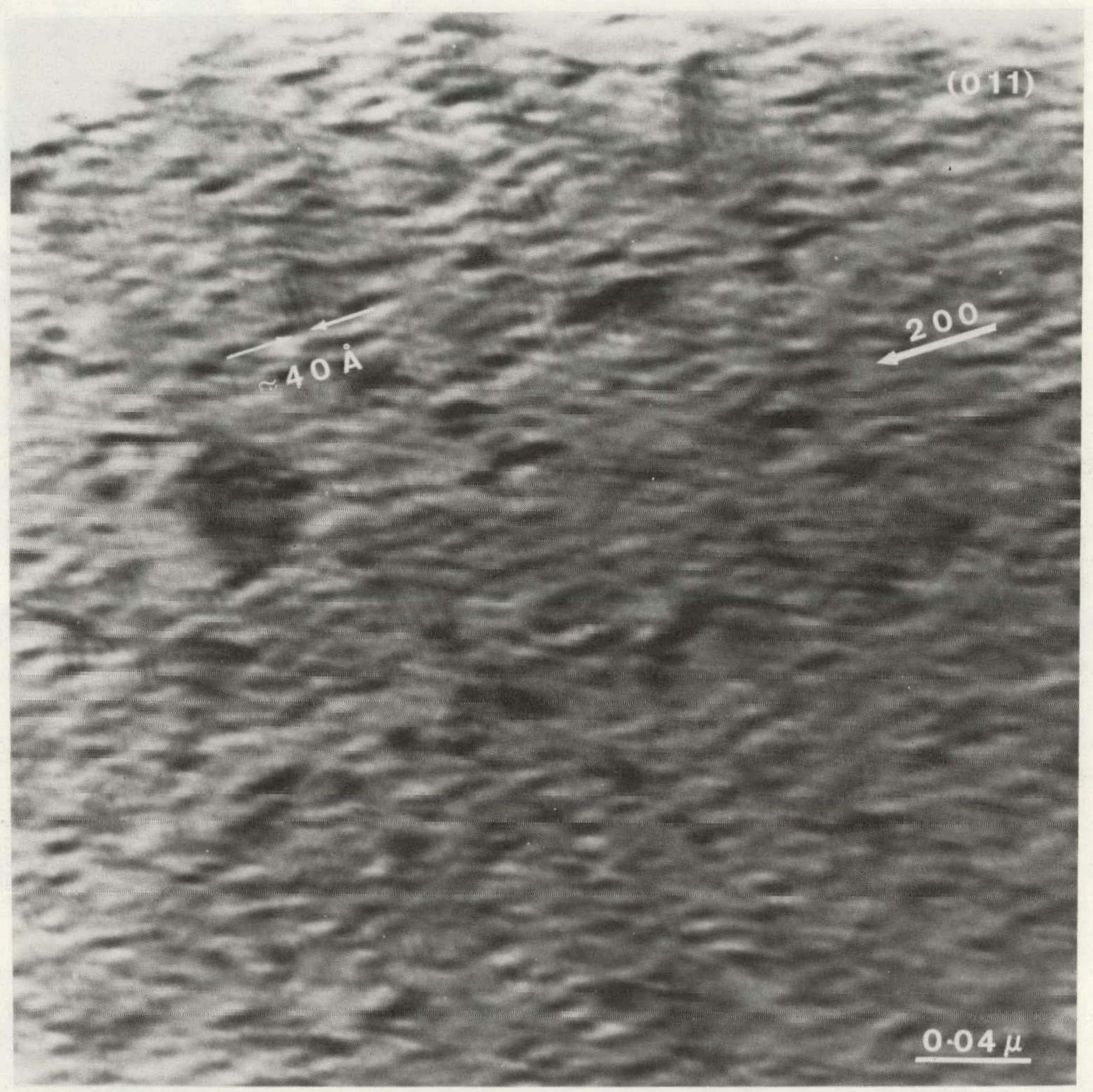

Fig, 92. $40 \AA$ Moiré Fringe Pattern Possibly Due to the Formation of a Thin Surface Film. 


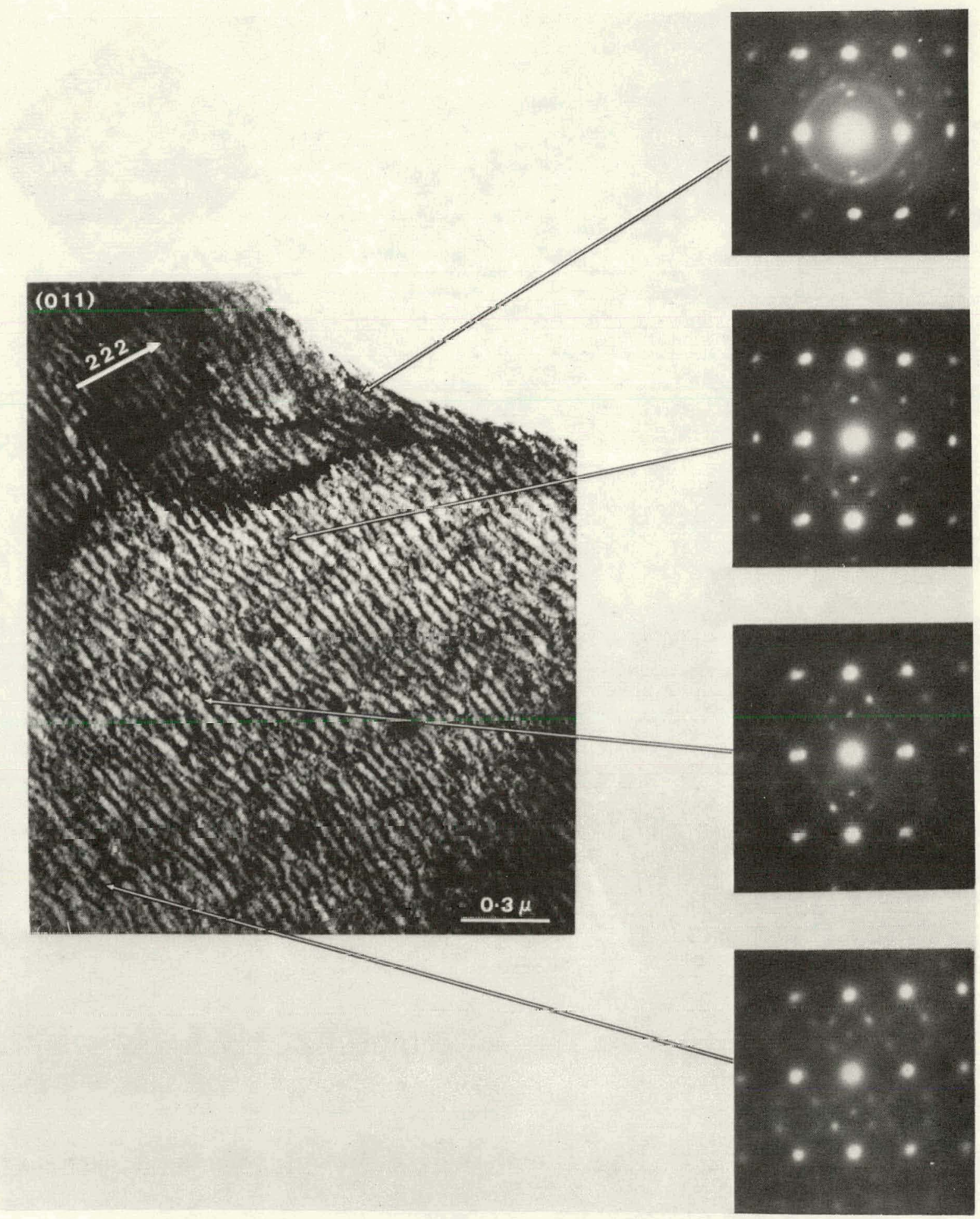

Fig. 93. Variation in Diffraction Patterns as a Function of Sample Thickness Indicating the Possibility of a Surface Film. 


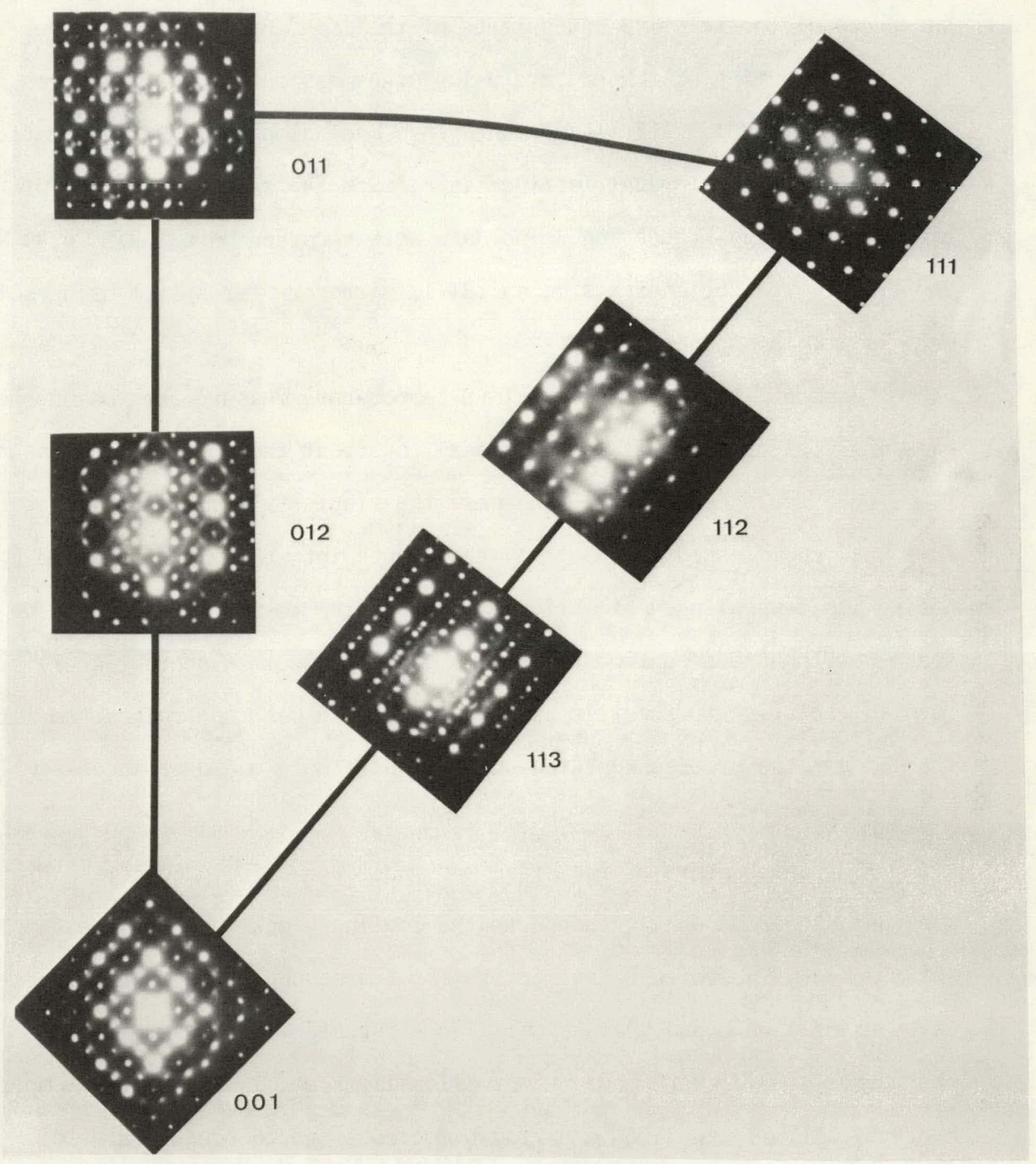

Fig. 94. Summary of Electron Diffraction Patterns from the Microstructure of Fig. 90. 
section. Specimens were mounted in a single-tilt cooling state and measurements of the relative intensities of the $\omega$ and $\beta$ reflections were made as a function of temperature using transmission scanning electron diffraction (TSED). All measurements were made along a $\langle 2 \overline{2} 2\rangle$ systematic row in an $\{011\}$ reciprocal lattice plane, with the sample oriented for exact $<2 \overline{2} 2>$ Bragg scattering. The data were recorded on an ORTEC 6200 MCS and transferred for analysis on an LSI-11 microcomputer system using software listed in Appendix E.

In order to perform these TSED temperature measurements using the existing cooling stage it was necessary to orient the specimen so that an \{011\} pole could be reached using only the single-tilt axis available. This was accomplished by inserting the sample into the microscope and noting its crystallographic orientation relative to the tilt axis. The stage was then removed from the column and the specimen appropriately rotated (relative to the tilt axis). The sample was then reinserted into the TEM and the process repeated until a $\{011\}$ pole could be successfully reached.

The temperature of the stage was monitored using a CopperConstantan thermocouple attached to the cooling block in which the sample was mounted. The voltage drop across the thermocouple junction was measured using a LEED and NORTHRIP millivolt potentiometer. Temperature dependence studies were made unidirectionally from $-196^{\circ} \mathrm{C}$ to room temperature by allowing the liquid nitrogen cooled stage to equilibrate to ambient temperatures via thermal conduction through the stage block. This was necessary because no temperature controlling facilities were 
available for this stage. As a consequence of this, each temperature scan required approximately $12 \mathrm{~h}$ of continuous microscope operation. During this time the specimen position was periodically checked to ensure that a1l measurements were made at constant thickness and orientation. Due to the extremely long duration of each set of measurements, all intensities were normalized to that of the $<222>$ Bragg reflection to compensate for changes in incident beam current. In this experiment, the intensity of the $\left\langle 222{ }_{\beta}\right.$ Bragg peak was monitored rather than the $\langle 000\rangle$ transmitted beam intensity so that the system could operate at the maximum possible sensitivity. Thus the gain of the photomultiplier tube (PMT) was adjusted so that the $\langle 000\rangle$ transmitted beam intensity was just short of saturating the detector. The remaining linear amplifiers were then used to amplify the $<222\rangle_{\beta}$ intensity to the maximum level which can be processed by the voltageto-frequency converter used to digitize the intensity measurements.

Figure 95 shows typical selected area diffraction patterns recorded at a (stage) temperature of -196 and $+22^{\circ} \mathrm{C}$ from approximately an $1000-\AA$ thick region of the $\mathrm{Zr}-15 \% \mathrm{Nb}$ alloy. No substantial differences between these two patterns can be visually detected. TSED measurements of the relative intensity ratio of the $\omega_{0002}$ and $\beta_{222}$ reflections as a function of temperature are given in Fig. 96. A monotonic increase of the $\omega_{0002}$ peak relative to the $\beta_{222}$ intensity with decreasing temperature is apparent. The variation of the intensity ratio of the $\omega_{0002}$ to the $\omega_{0001}$ reflection is essentially constant as indicated in Fig. 97. In-situ thermal cycling experiments were repeated on this sample four times with identical trends 

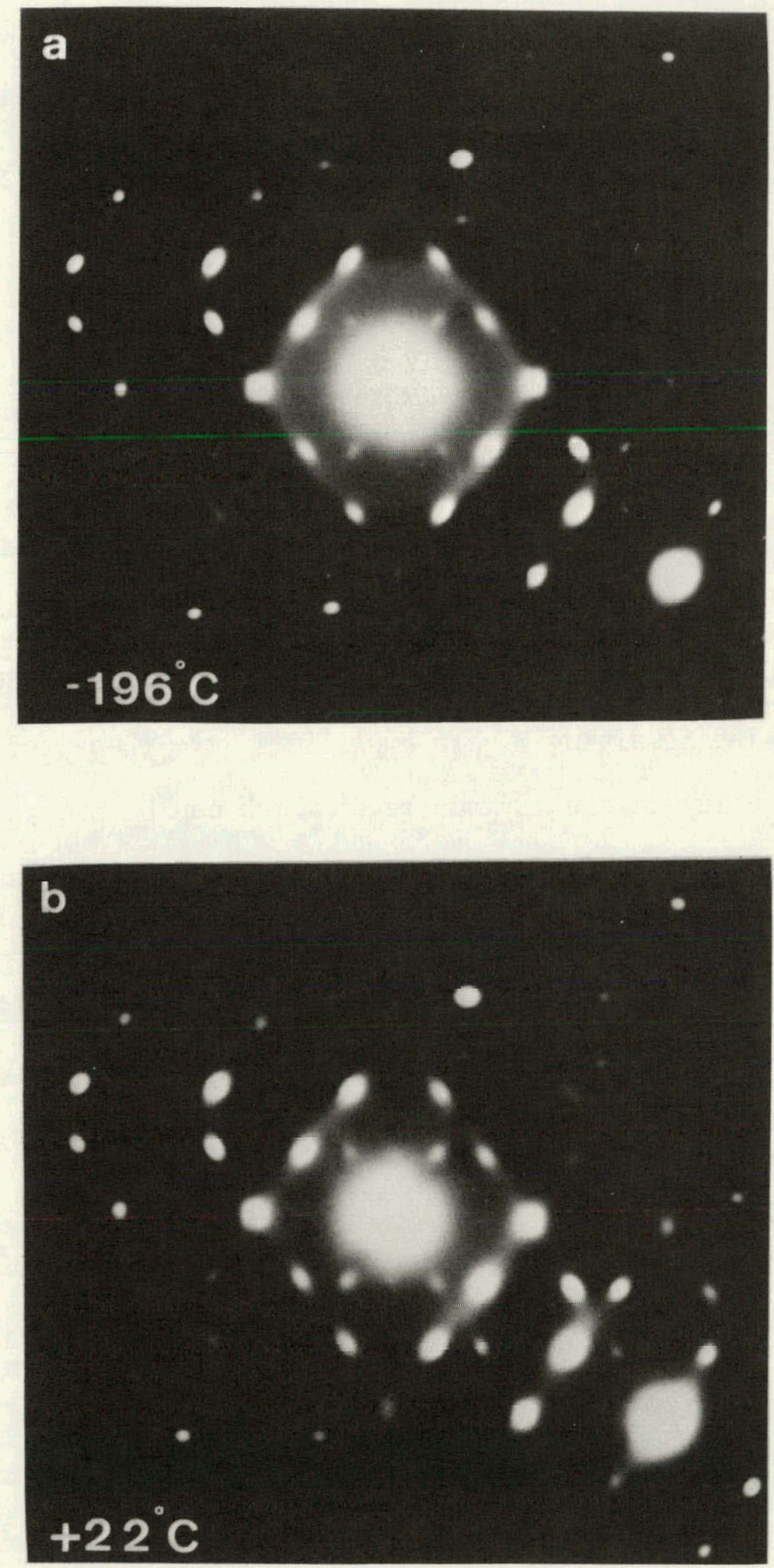

Fig. 95. Diffraction Patterns from the As-Quenched $\omega+\beta$ Morphology at -196 and $+22^{\circ} \mathrm{C}$. 


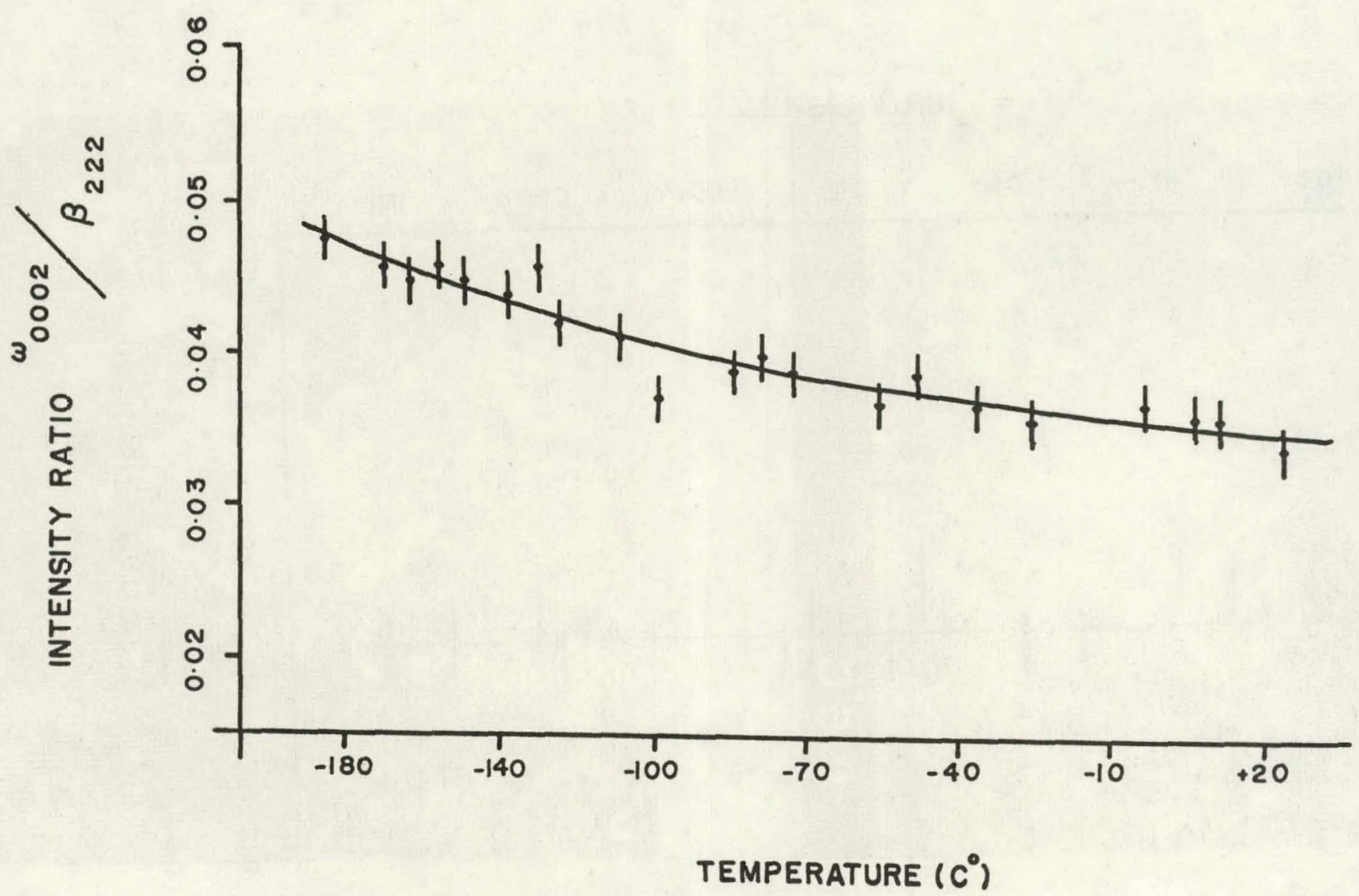

Fig. 96. Experimental Measurements of the Variation of $\omega_{0002} / \beta_{222}$ Intensity Ratio as a Function of Temperature. 


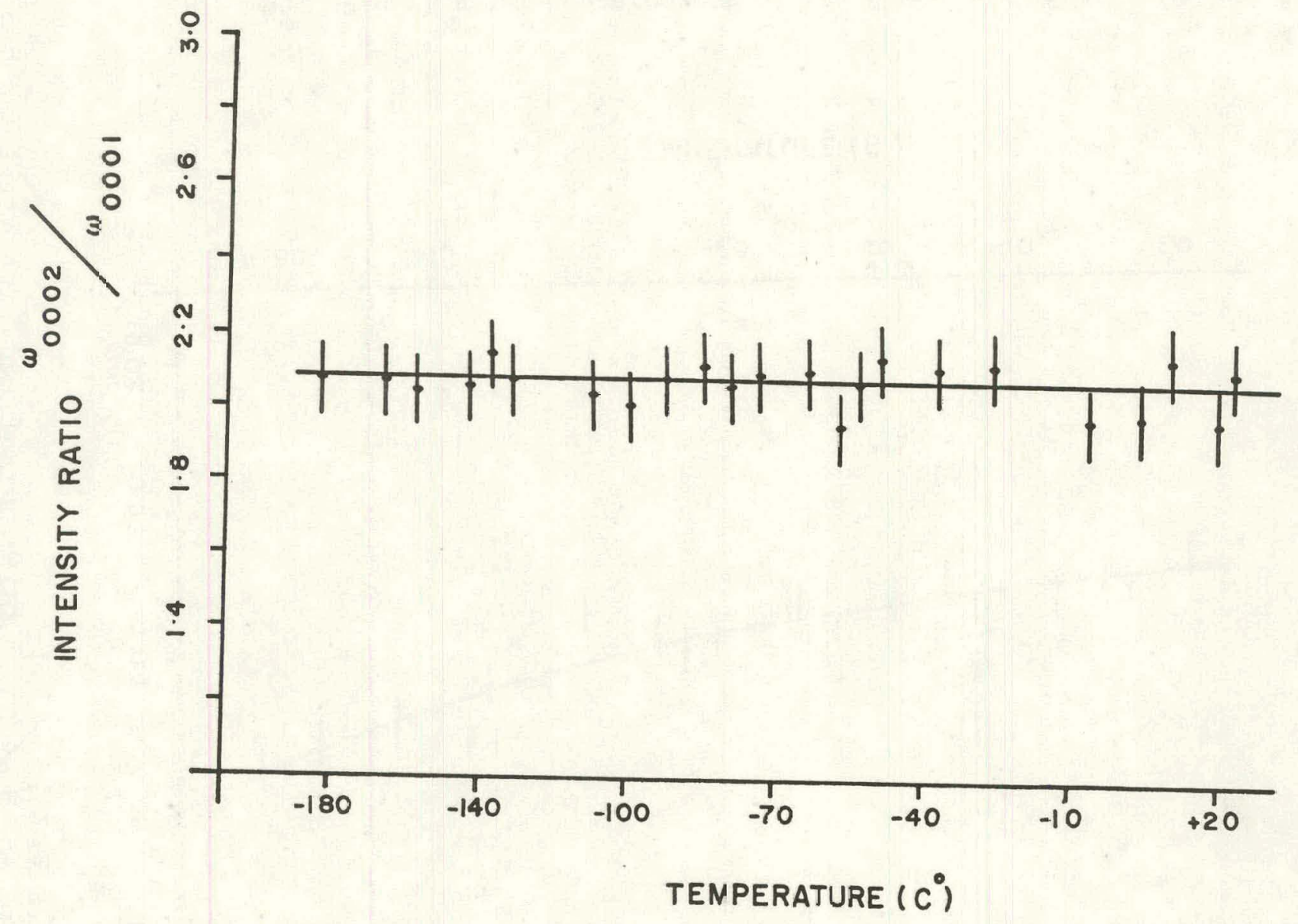

Fig. 97. Experimental Measurements of the $\omega_{0002} / \omega_{0001}$ Intensity Ratio as a Function of Temperature. 
being observed. The acrumulation of contamination on the sample, however, prohibited direct comparisons of these data from one measurement to another. In addition, prethinned samples thermally cycled at $12-\mathrm{h}$ interva1s between -196 and $30^{\circ} \mathrm{C}$ over a period of eight weeks exhibited identical trends when subsequently measured on the JSEM 200.

Structure factor calculations of the intensity distribution along $a<222{ }_{\beta}$ direction for two different $\beta+\omega$ structures are schematically shown in Fig. 98. In this figure the relative intensity of a diffraction maxima (i.e., the square of the calculated structure factor) is linearly proportional to the radius of the circle used to represent a given reflection. The first set of calculations [Fig. 98(a)] illustrate the changes in diffracted intensity as a pure bce structure $(n \equiv 0)$ is subjected to a sinusoidal $\omega$-forming displacement wave [Eq. (1.1)] as a function of order parameter $(n)$. Thus if the bec structure was completely transformed into the $\omega$ phase structure factor considerations predict the ratio of $\omega_{0002} / \omega_{0001}$ to be 9 to 1 . Table 3 lists the $\omega_{0002}$ to $\omega_{0001}$ ratio for specific values of $n$. Figure 98 (b) represents structure factor calculations along a $\langle 222\rangle_{\beta}$ direction for an ordered phase of alternating $\beta$ and $\omega$ regions. In this case it was assumed that these regions had a spatial extent of $24 \AA$ (i.e., approximately four $\beta$ cells = eight $\omega$ ce11s) along the $\langle 222\rangle$ direction. This spatial extent is consistent with the experimental observations of omega domain size observed in this work as well as in the literature. Table 4 1ist.s the calculated structure factor ratios for this periodic arrangement. 


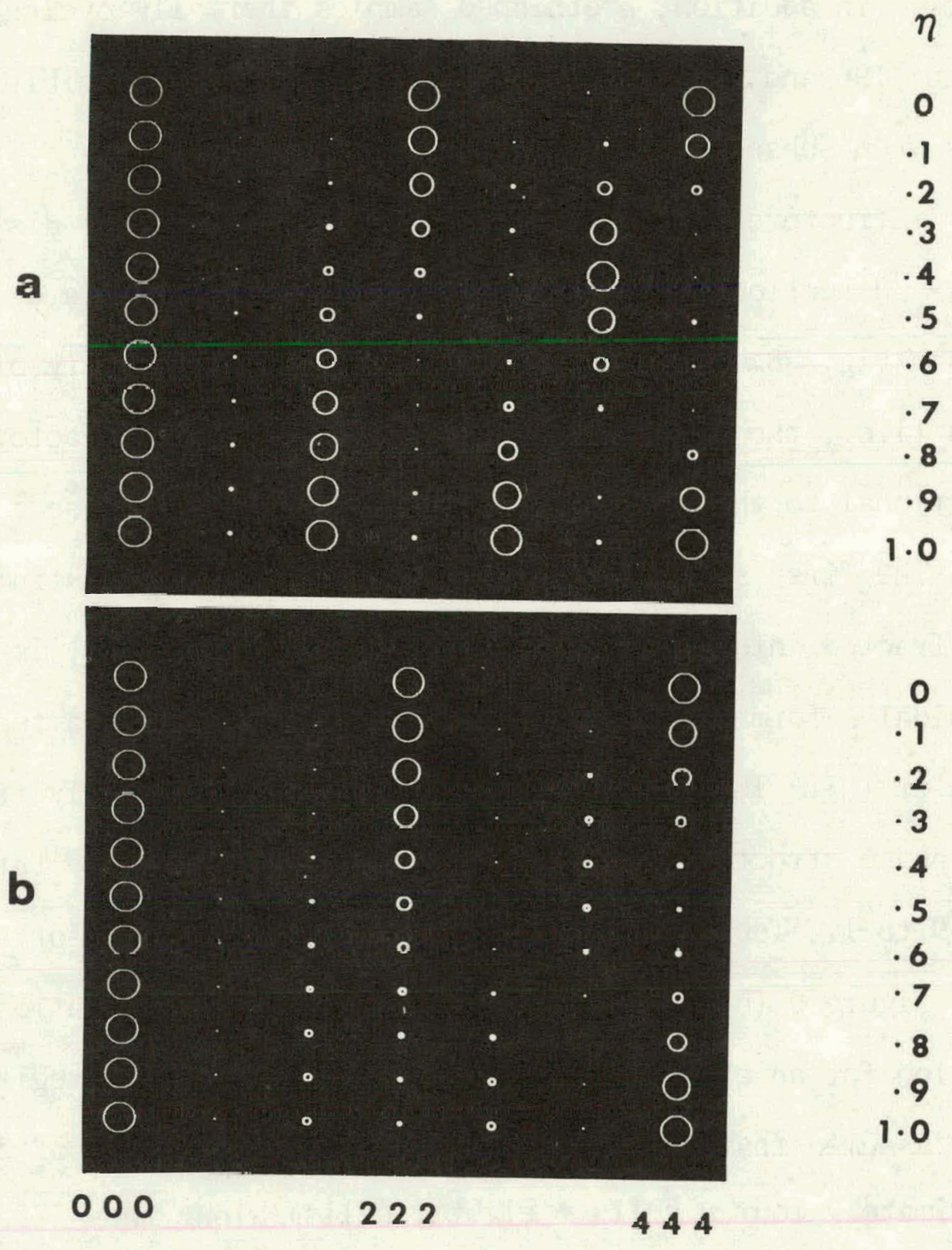

Fig. 98. Schematic Illustration of the Changes Along a $\langle 222>$ Direction in an Electron Diffraction Pattern for: (a) a Homogeneous Sample Changing from the Pure $\beta$ Phase $(\eta=0)$ to the Ideal $\omega$ Phase $(n=1)$ and (b) to a Completely Ordered Structure of the $\beta$ Plus $\omega$ Phase. 
Table 3. Calculated Structure Factor Ratio for Ideal Omega Phase as a Function of Order Parameter ( $n$ )

\begin{tabular}{cc}
\hline $\begin{array}{c}\text { Order Parameter } \\
(n)\end{array}$ & $\begin{array}{c}\text { Structure Factor Ratio } \\
\left(\omega_{0002} / \omega_{0001}\right)^{2}\end{array}$ \\
\hline 0 & -- \\
0.1 & 4.73 \\
0.2 & 5.46 \\
0.3 & 6.18 \\
0.4 & 6.85 \\
0.5 & 7.47 \\
0.6 & 8.00 \\
0.7 & 8.42 \\
0.8 & 8.74 \\
0.9 & 8.93 \\
1.0 & 9.00 \\
\hline
\end{tabular}

Table 4. Calculated Structure Factor Ratio for Long Period Ordered Structure of $\beta+\omega$ Phase as a Function of Order Parameter $(n)$

\begin{tabular}{lcc}
\hline $\begin{array}{c}\text { Order Parameter } \\
(n)\end{array}$ & \multicolumn{2}{c}{ Structure Factor Ratio } \\
\cline { 2 - 3 }$\left(\omega_{0002} / \omega_{0001}\right)^{2}$ & $\left(\omega_{0002} / \beta_{222}\right)^{2}$ \\
\hline 0 & -- & -- \\
0.1 & 4.73 & 0.0041 \\
0.2 & 5.47 & 0.0197 \\
0.3 & 6.18 & 0.0544 \\
0.4 & 6.86 & 0.123 \\
0.5 & 7.47 & 0.250 \\
0.6 & 8.00 & 0.476 \\
0.7 & 8.42 & 0.943 \\
0.8 & 8.74 & 1.41 \\
0.9 & 8.94 & 1.98 \\
1.0 & 9.00 & 2.25 \\
\hline
\end{tabular}


Unfortunately the effects of double diffraction as well as inelastic scattering preclude a direct comparison of these calculations with experimental measurements; however, the following qualitative interpretation of the experimental data can be set forth. Since the ratio of the $\omega_{0002} / \omega_{0001}$ intensity remains essentially constant as a function of temperature, it can be concluded that no change in the degree of completion of the $\omega$ to $\beta$ transformation has occurred due to changes in sample temperature. The monotonic increase in the $\omega_{0002}$ intensity relative to the $\beta_{222}$ reflection can thus bc interpreted as an increase of the total volume fraction of the $\omega$ phase in the sample with respect to the $\beta$ phase. At no time during these measurements was it possible to detect an ordering of the $\omega$ phase relative to the $\beta$ phase either through changes in relative intensities of diffraction peaks or by the development of ordering reflections corresponding to a long period structure. 


\section{CHAPTER 4}

\section{CONCLUSIONS}

The results of this research can be divided into two parts namely the study of the as-quenched omega phase morphology, and the work involved with the application of analytical electron microscopy to materials characterization. With regard to the omega phase, this research has shown that (1) the domain size of the as-quenched omega structure in $\mathrm{Zr}-15 \% \mathrm{Nb}$ is on the order of $30 \AA$, (2) no alignment of omega domains along $<222>_{\beta}$ directions is observed, and (3) samples having undergone thermal cycling experiments in thin foil form did not develop a long-period structure of alternating $\beta$ and $\omega$ phases below the omega transformation temperature. The size of the omega domains noted in this work is consistent with that previously quoted in the. 1iterature, yet for a number of years it has been reported that these domains arrange themselves periodically along specific directions of the bcc lattice. It is difficult to simply resolve this discrepancy, but it was noted during this study that a wide variety of microstructural features can develop as a result of sample preparation and hence it is feasible to postulate that such effects could be responsible for the conflicting results. No coherent long-period $\beta$ and $\omega$ structure immediately below the omega transformation temperature was detected; however, it is possible that thin film constraints may have inhibited the formation of such a metastable structure by shifting stability away from the ordered phase. The CTEM images and diffraction patterns 
observed during this research included moderate inelastic scattering effects and thus it would be interesting to continue studies of this transformation using high resolution imaging combined with energy filtering. At present the only instruments capable of such experiments are the high resolution dedicated scanning transmission electron microscopes (DSTEM) equipped with electron energy loss spectrometers and the possibility of such work is being investigated.

Analytical electron microscopy has been shown to be an extremely powerful tool for the microcharacterization of materials. However, these techniques are not by any means without complications, and it was necessary to investigate the AEM system used in this work so that instrumental artifacts which invalidate the information produced in the microscope environment might be eliminated. Once these factors had been corrected, it was possible to obtain a wealth of information about the microvolıme of material under investigation.

The instmmental problems, which werc associated with $x-$ ray andysis, in the AEM system used during this research included: fluorcsccnce by uncollimated radiation, contamination formed under the influence of the electron probe, and the generation of system peaks in the inmediate vicinity of the specimen. Of these factors, the single most important correction necessary to perform quantitative $x$-ray microanalysis is the reduction of uncollimated radiation. Without such a currection, truc quantitative microanalysis is virtually impossible. The effects of contamination have been shown to be detrimental in all aspects of TEM-based analysis. Imaging, analytical spatial resolution, mass sensitivity, and quantitative 
analysis all suffer in their ultimate performance due to the accumulation of foreign material on the sample surface. It has also. been shown that the removal of system peaks, generated in the instrument, is an important aspect of microchemical analysis which should not be neglected.

Experimental measurements combined with theoretical calculations have shown that the optimum conditions for $x$-ray analysis in an AEM are realized at the highest attainable incident electron beam energy and by using a detector/specimen geometry which maximizes the detector observation angle. Furthermore, once all instrumental corrections have been applied to the AEM system, it is possible to obtain accurate quantitative results in "thin" specimens using the standardless analysis technique and the formalism presented in Chapter 2. The calculation of an absorption correction for a homogeneous semi-thick sample is not difficult., but, as most analyses involve multi-element phases of varying thicknesses, it becomes simpler to analyze thinner regions of the sample where such a correction is not required. Quantitative analysis using thin-film standards is potentially simple, although experimental aspects such as the accurate duplication or measurement of instrumental operating conditions complicates the development of this method.

Finally, two complementary methods of obtaining structural information about a specimen using electron diffraction have been investigated and refined. These methods - namely transmission scanning electron diffraction and the stationary diffraction pattern technique - when combined with appropriate calculations allows one to obtain information about the 
nature of the sample not attainable using conventional selected-areadiffraction techniques.

The ability to perform simultaneously energy dispersive $x$ ray as well as electron energy loss measurements will be one of the most important aspects of AEM to the materials scientist for development in the future. Using these two complementary techniques the potential to perform analysis of all atomic species of $z \geqslant 3$ may be realized, and, in special cases, through systematic studies of plasmon losses it may be possible to analyze for the presence of hydrogenic compounds. Combining these microchemical analysis capabilities with the various electron diffraction techniques for crystallographic analysis makes analytical electron microscopy an essential part of any materials research program. 


\section{LIST OF REFERENCES}

1. D. J. Cometto, G. L. Houze, and R. F. Hehemann, Trans. AIME 233, 30 (1965).

2. J. M. Silcock, Acta Met. $\underline{6}, 481$ (1958).

3. Y. A. Bagaryatskii, G. I. Nosava, and T. V. Tagunova, Akad. Nauk. SSSR 105,1225 (1955).

4. B. A. Hatt and J. A. Roberts, Acta Met. $\underline{8}, 575$ (1960).

5. J. O. Stiegler, J. T. Houston, and M. L. Picklesimer, J. Nucl. Mater. $\underline{11}, 32$ (1964).

6. W. G. Brammer and C. G. Rhodes, Phil. Mag. 16, 477 (1967).

7. S. L. Sass, Acta Met. 17,813 (1969).

8. C. W. Dawson and S. L. Sass, Met. Trans. 1, 2225 (1970).

9. K. K. McCabe and S. L. Sass, Phiz.Mag. 23, 252 (1971).

10. A. T. Balcerzak and S. L. Sass, Met. Trans. 3, 1601 (1972).

11. S. L. Sass, J. Less-Common Metals 28, 157 (1972).

12. T. S. Kuan, R. R. Ahrens, and S. L. Sass, Met. Trans. 6A, 1767 (1975).

13. A.L.J. Chang, W. Krakow, and S. L. Sass, Acta Met. 24, 29 (1976).

14. D. deFontaine, N. E. Paton, and J. C. Williams, Acta Met. 19, 1153 (1971).

15. S. C. Moss, D. T. Keating, and J. D. Axe, Phase Transformation 1973. Pellu State University, 23-25 May, Pcrgamon Press, Oxford, 1973.

16. B. W. Batterman, G. Maracci, A. Merlini, and S. Pace, Phys. Rev. Letters, 31, 227 (1973).

17. W. Lin, H. Spalt, and B. W. Batterman, Phys. Rev. (1976) (submitted).

18. D. deFontaine, Acta Met. 18, 275 (1970).

19. H. E. Cook and D. deFontaine, Acta. Met. 17, 915 (1969). 
20. H. E. Cook and D. deFontaine, Acta Met. 19, 607 (1971).

21. E. S. Fisher and D. Dever, Acta Met. 18, 265 (1970).

22. D. deFontaine, N. E. Paton, and J. C. Williams, Acta Met. 19, 1153 (1971).

23. W. Vandermuelen and A. Deruyttere, Met. Trans. 4, 1659 (1972).

24. B. Borie, S. Sass, and A. Andreassen, Acta Cryst. A29, 585 (1973).

25. B. Borie, S. Sass, and A. Andreassen, Acta Cryst. A29, 594 (1973).

26. H. E. Cook, Acta Met. 21, 1445 (1973).

27. II. E. Cook, Acla Met. 22, 239 (1974).

28. H. E. Cook, Acta Met. 23, 1027 (1975).

29. H. E. Cook, Aeta Met. 23, 1041 (1975).

30. J. Philibert and R. Tixier, NBS No. 298, 13 (1968).

31. J. Philibert and R. Tixier, J. Phys. D 1, 685 (1968).

32. G. Cliff and G. W. Lorimer, Fifth European Conference on EZectron Mireposcopy, 1972 , p. 136.

33. M. H. Jacobs and J. Baborovoska, Fifth European Conference on Electron Microscopy, 1972, p. 140.

34. P. Duncumb, J. deMicroscopie \%, 581 (1965).

35. R. H. Geiss and T. C. Huang, J. Vac. Sci. Tech. 12, 140 (1975).

36. P. Doig and J. W. Edington, Phiz. Mag. 27, 285 (1975).

37. J. Philibert, X-Ray Optics and Microanalysis, Academic Press, New York, 1963, p. 379 .

38. T. Namae, J. EZectron Microscopy 24, 1 (1975).

39. D. J. Marshall and 'I'. A. Ha11, J. thys. D 1,1651 (1968).

40. W. Reuter, Sixth Intern. Conference X-Ray Optics and Mioroanalysis, Academic Press, New York, 1972, p. 121.

41. J. I. Goldstein and D. B. Williams, SEM 1, 651 (1977). 
42. H. L. Fraser, N. J. Zaluzec, J. B. Woodhouse, and L. B. Sis, 33rd Proc. EMSA (1975), p. 106.

43. N. J. Zaluzec and H. L. Fraser, 34th Ann. Proc. EMSA. (1976).

44. N. J. Zaluzec and H. L. Fraser, 8th Int. Conf. on X-Ray Optics and Microanalysis (1977) (in press).

45. J. I. Goldstein, private communication.

46. H. S. Perlman, Proc. Phys. Soc. (London), 76, 623 (1960).

47. A. M. Arthurs and B. L. Moiseiwitch, Proc. Roy. Soc. A247, 550 (1958).

48. N. Mott and H. Massey, Theory of Atomic Colzisions, Oxford Press, London, 1949.

49. C. R. Worthington and S. G. Tomlin, Proc. Phy. Soc. (London), 69, 401 - (1956).

50. E.H.S. Burhop, Proc. Com. Phil. Soc. 36, 43 (1940).

51. J. I. Goldstein, J. L. Costley, G. W. Lorimer, and S.J.B. Reed, SEM 1, 315 (1977).

52. C. J. Powe11, Rev. Mod. Phys. 48, 33 (1976).

53. W. Hink and A. Zieg1er, Z. Physik 226, 222 (1969).

54. L. T. Pockman, D. L. Webster, P. Kirkpatrick, and K. Harworth, Phys. Rev. 71,330 (1947).

55. P. Kirkpatrick and A. V. Baez, Phys. Rev. 71, 521 (1947).

56. L. Landau, J. Phys. Moscow 8, 201 ( ).

57. H. A. Bethe, Ann. Phys. 5, 325 (1930); H.d. Phys. 24, 519 (1933).

58. V. E. Cosslett and R. N. Thomas, Brit. J. App Z. Phys. 15, 1283 (1964); 16, 779 (1965); 15, 883 (1964).

59. A. T. Nelms, National Bureau of Standards, Circular No. 577 (1956).

60. G. D. Archard and T. Mulvey, 3rd Int. Conf. on X-Ray Optics and Microanalysis; Academic Press, 1962.

61. G. Wentze1, 2. Phys. $\underline{43}, 524$ (1927). 
62. N. A. Dyson, X-Rays in Atomic and Nuclear Physics, Longman Group, Ltd., London, 1973.

63. J. Colby, Advances in X-Ray Analysis 11, 287 (1968) (P1enum Press).

64. R. W. Fink, R. C. Jopson, H. Mark, and C. D. Swift, Rev. Mod. Phys. 39, 125 (1967).

65. E.H.S. Burhop, J. Phys. Radizm 16, 625 (1955).

66. V. W. Slivinsky and P. J. Ebert, Phys. Rev. A $\underline{5}, 1581$ (1972).

67. K.F.J. Heinrich, Proc. 11th Conf. MAS (1976), p. 29.

68. Handbook of Chemistry and Physics, 53rd ed. (ed. by R. Weast), Chemical Rubber Company, Cleveland, Ohio, 1973.

69. E. J. McGuire, Phys. Rev. Letters 33A, 288 (1970).

70. J. A. Crowther, Proc. Roy. Soc. A84, 226 (1910).

71. B. Cullity, Elements of X-Ray Diffraction, Chap. 2, Addison Wesley, Reading, MA, 1967.

72. A. Sonmerfe1d, Ann. d. Physik 11, 257 (1931).

73. R. Weinstock, Phys. Rev. 61, 584 (1942).

74. P. Kirkpatrick and L. Wiedmann, Phys. Rev. 67, 321 (1945).

75. JEOL, Ltd., Electron Microscope Division.

76. J. W. Sprys, Rev. Sri., Inst. 46, 773 (1975).

77. N. J. Zaluzec and H. L. Fraser, J. Phys. E. 9, 1051 (1976).

78. E. A. Kenik and J. Bentley, Metals and Ceramics Division, Oak Ridge National Laboratory, private communication (1977).

79. Philips Electroničs̄ Instruments, Inc., Ëlectron Mícroscope Division.

80. Vacuum Generators, Ltd., Electron Microscope Division.

81. J. J. Hren, P. S. Ong, P. F. Johnson, and E. J. Jenkins, 34th Ann. Proc. EMSA, 1976, Claitor's Publishing Company, Baton Rouge, LA, p. 418.

82. H. L. Fraser, M. H. Loretto, and R. E. Smallman, Phil. Mag. 28, 667 (1973). 
83. N. J. Zaluzec and H. L. Fraser, Workshop on Analytical Electron Microscopy, Cornell, 1976.

84. Workshop on Analytical Electron Microscopy, op. cit. General discussio in open form, summarized in Proceedings.

85. J. T. Fourie, SEM/IITRI (ed. by O. Johari), 1976, p. 53.

86. J. C. Russ, SEM/IITRI (ed. by O. Johari), 1976, p. 143.

87. J. C. Russ, SEM/IITRI (ed. by O. Johari), 1976, p. 335.

88. P. Duncumb, Phiz. Mag. 7(84), 2101 (1962).

89. Th. Bronder and J. Jakschik, Phys. Stat. Sol. (a) 31, 503 (1975).

90. C. R. Ha11; Proc. Roy. Soc. A295, 140 (1966).

91. H. L. Fraser and J. B. Woodhouse, Proc. 2nd. Workshop on AEM, Corne11 University (July 1978).

92. R. H. Geiss and T. C. Huang, IBM Technical Report No. 965-IBM-01 (1974)

93. R. H. Geiss, App 2. Phys. Letters 23, 283 (1973).

94. C.W.B. Grigson, Rev. Sci. Inst. 36, 1587 (1965).

95. D. M. Maher, SEM/IITRI (ed. by O. Johari), 1974, p. 215.

96. P. M. Kelly, A. Jostsons, R. G. Blake and J. G. Napier, Phys. Stat. Sol. (a) 31, 771 (1975).

97. B. F. Buxton, J. A. Eades, J. W. Steeds; and G. M. Rockham; Phiz. Trans. Roy. Soc. 281, 171 (1976).

98. R. W. Carpenter and N. J. Zaluzec, Proc. 2nd Workshop on AEM, Cornel1 University (July 1978).

99. R. F. Egerton and M. J. Whelan, Proc. 8th Int. Conf. on EM, Canberra, Australia (1974), p. 384.

100. P. E. Flewitt, P. J. Ash, and A. G. Crocker, Acta Met. 24, 669 (1976).

101. T. Schober and V. Sorajic, MetaZZography 6, 183 (1973).

102. R. A. Spurling, G. G. Rhodes, and J. C. Williams, Met. Trans. 2597 (1974). 
103. M. J. Blackburn and J. C. Williams, Trans. TMS-AIME 239, 287 (1967).

104. H. J. Rack, D. Kalish and R. Fike, Mat. Sci. Eng. 6, 181 (1970).

105. R. Ericksen, R. Taggart, and D. Polonis, Trans. TMS-AIME 245, 359 (1969). 
APPENDIX A

Computer Program NEDS

Subroutine SINPUT

Subroutine SKLM

- Subroutine SBGRND

Subroutine GSETUP

Subroutine GAUSS 
APPENDIX A

\section{Computer Program NEDS}

This appendix contains documentation of the computer program NEDS which has been used to analyze all energy dispersive $x$-ray spectra during this research. The program is currentiy set up to analyze a $10 \mathrm{keV}$ wide spectrum recorded at an energy resolution of $20 \mathrm{eV} / \mathrm{channel}$; however, it

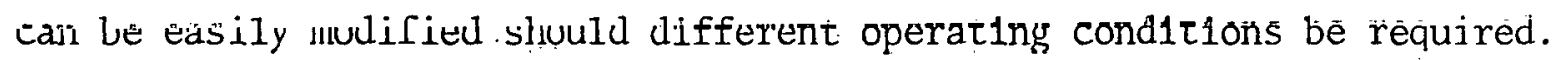
An example of a partial analysis of and EDS spectrum is shown in Figs. 99 and 100. Figure 99(a) shows a typical EDS spectrum recorded from a thin foil in the JEOL JSEM 200, and Fig. 99(b) compares the fitted polynomial background curve [Eq. 2.52)] with the experimental data. The results of a partial analysis of this spectrum is shown graphically in Fig. 100(a), where the program has fitted Gaussian profiles to the $\mathrm{NiL}_{\alpha}, \mathrm{AlK}_{\alpha}$ and $\mathrm{SiK}_{\alpha}$ peaks recorded in the energy range 0.65 to $2.15 \mathrm{keV}$. Figure $100(\mathrm{~b})$ compares the profile (sum) of these three peaks to the experimental data. Figure 101 is a simplified flow diagram for the program NEDS. 

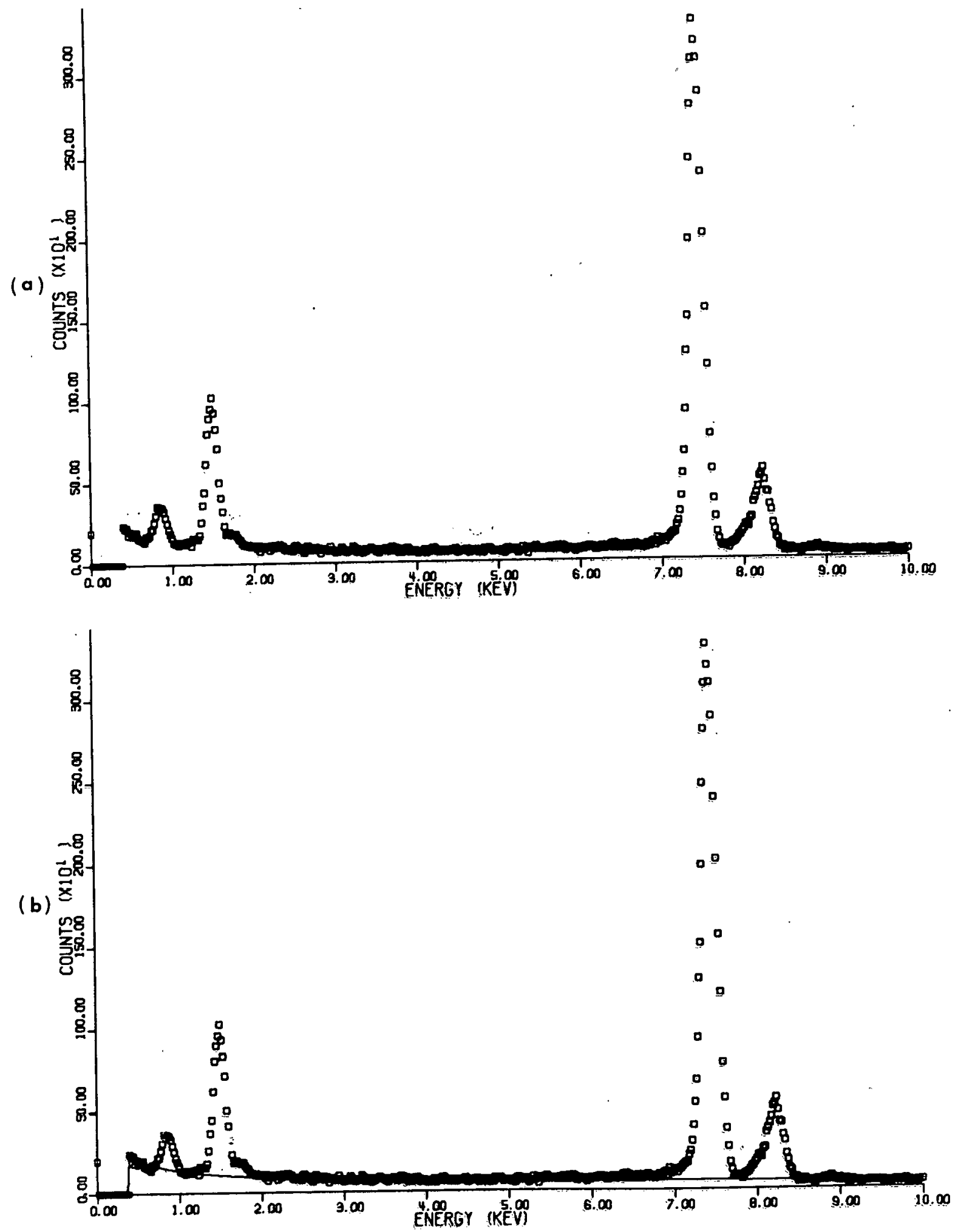

Fig. 99. (a) Typica J X-Ray Spectrm Recorded from the JEOL JSEM 200. (b) Comparison of fitted polynomial background curve (solid line) to experimental data. 
(a)

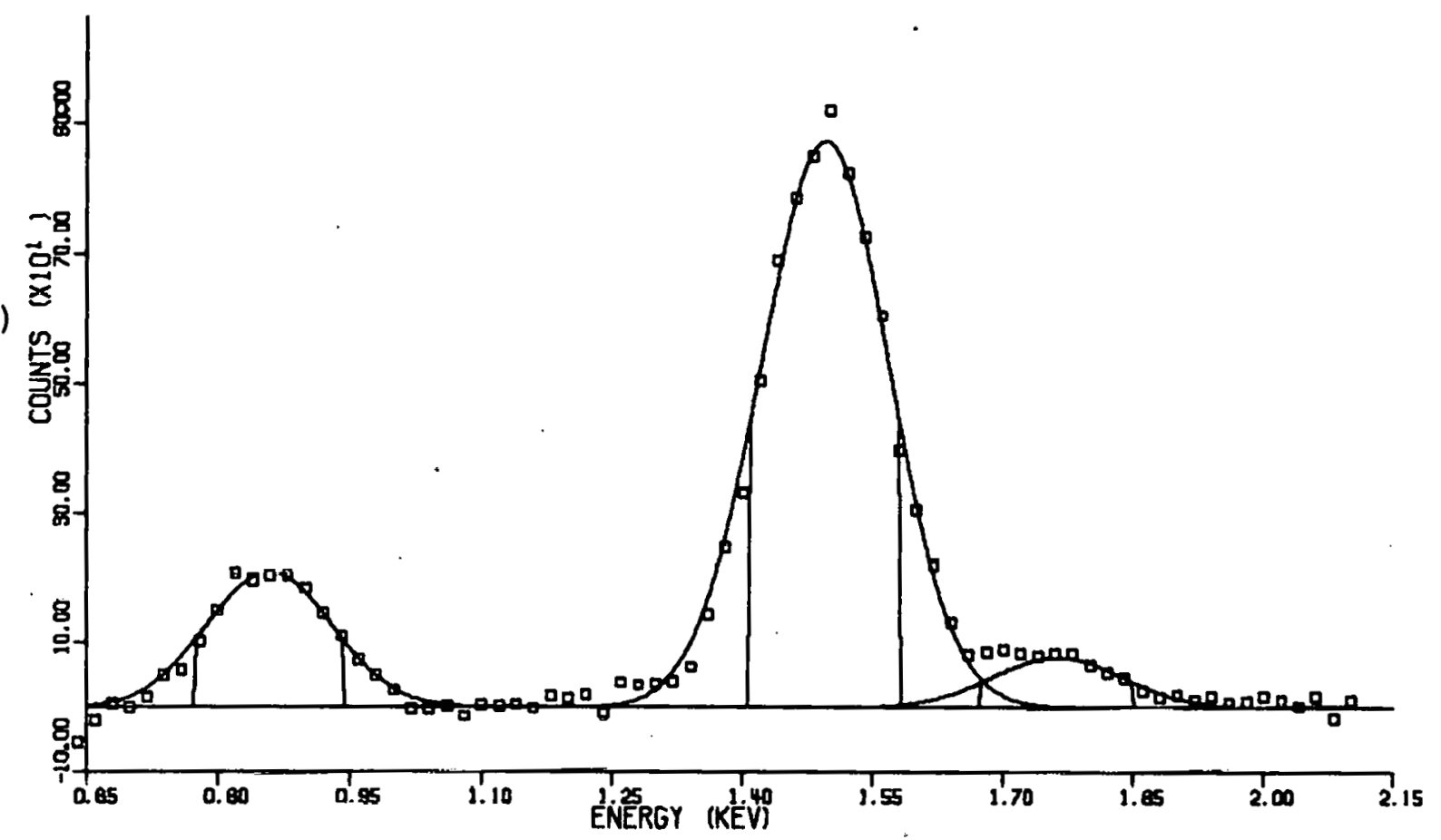

(b)

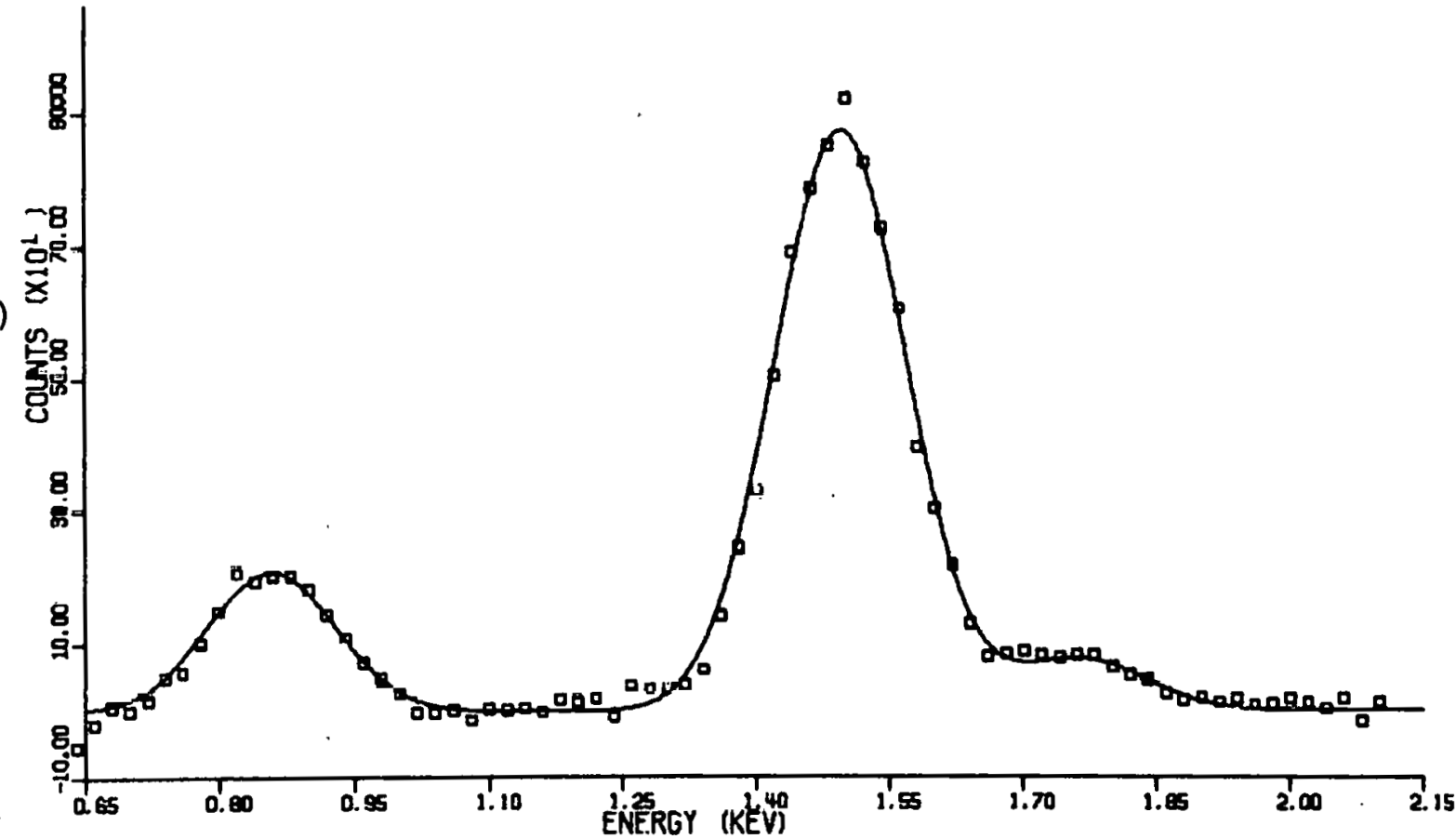

Fig, 100. (a) Fitted Gaussian Profiles for $\mathrm{Ni} \mathrm{L}_{\alpha}(0.85 \mathrm{keV}), \mathrm{A}_{1} \mathrm{~K}_{\alpha}$ $(1.47 \mathrm{keV})$, and $\mathrm{SiK}_{\alpha}(1.75 \mathrm{keV})$ Compared to Experimental Data. FWHM region indicated on each Gaussian curve. (b) Sum of $\mathrm{NiL}_{\alpha}, A 1 K_{\alpha}$, and SiKa profiles (solid line) compared to experimental data. 


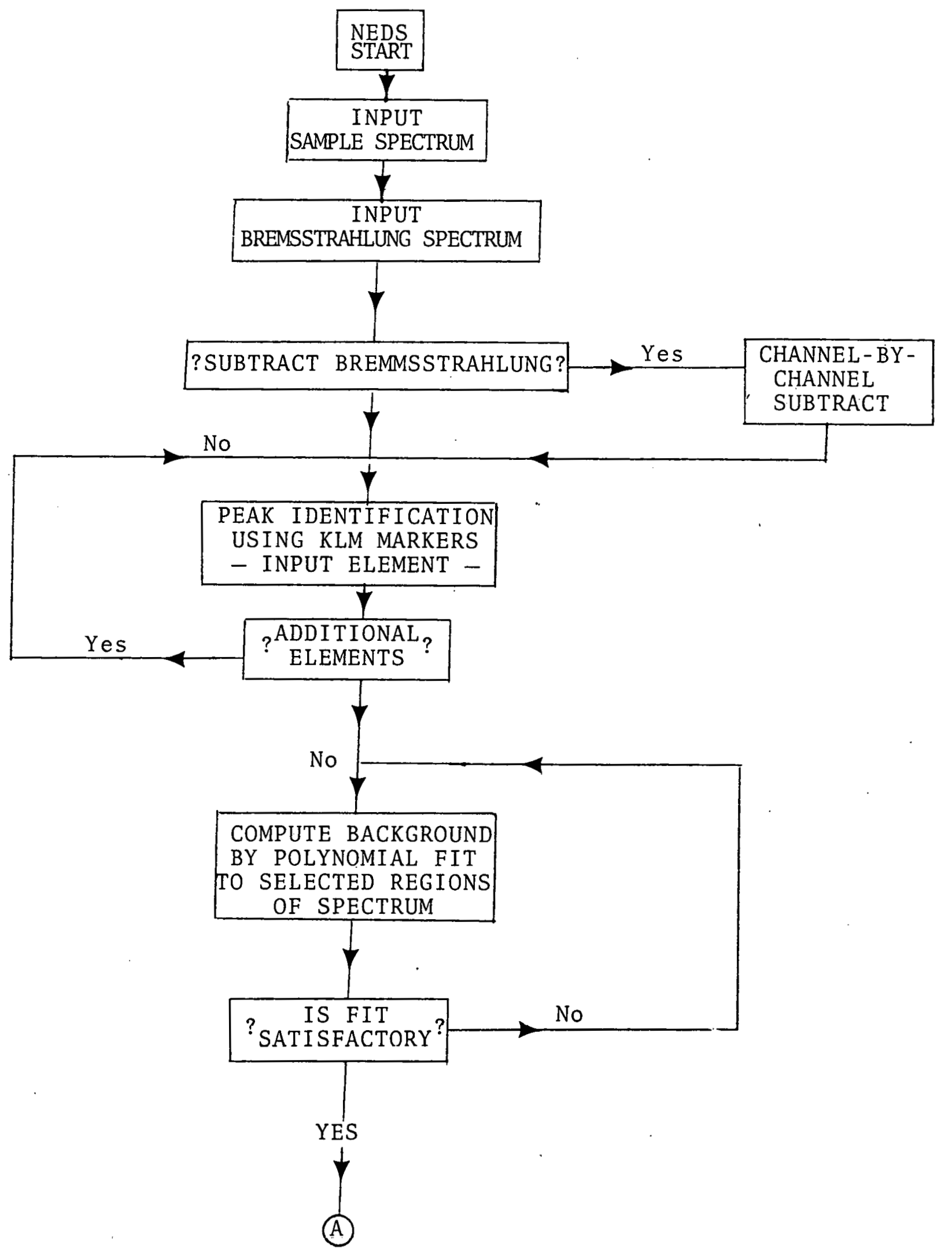

Fig. 101. Flow Diagram for the Program NEDS. 


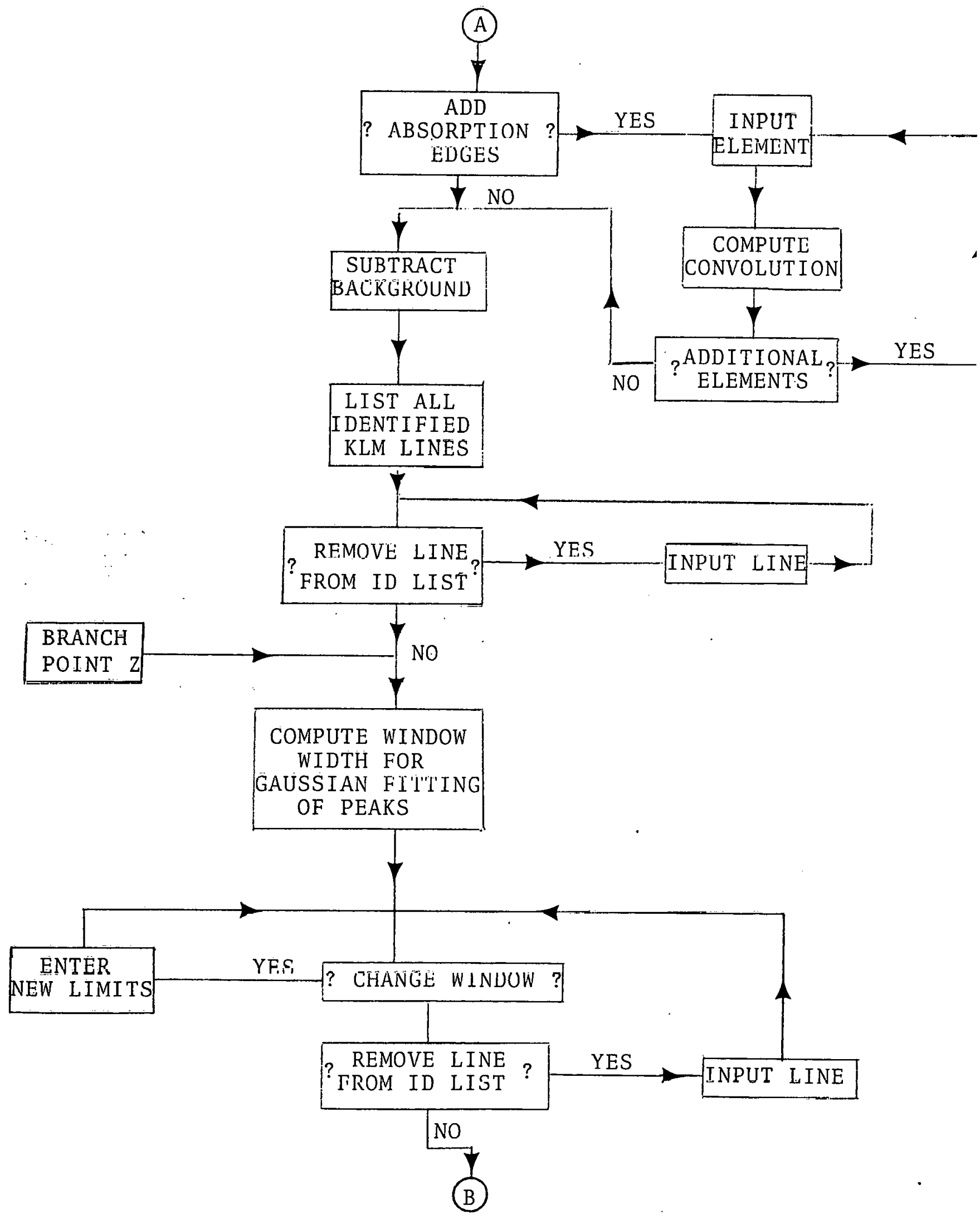

Fig. 101 (continued) 


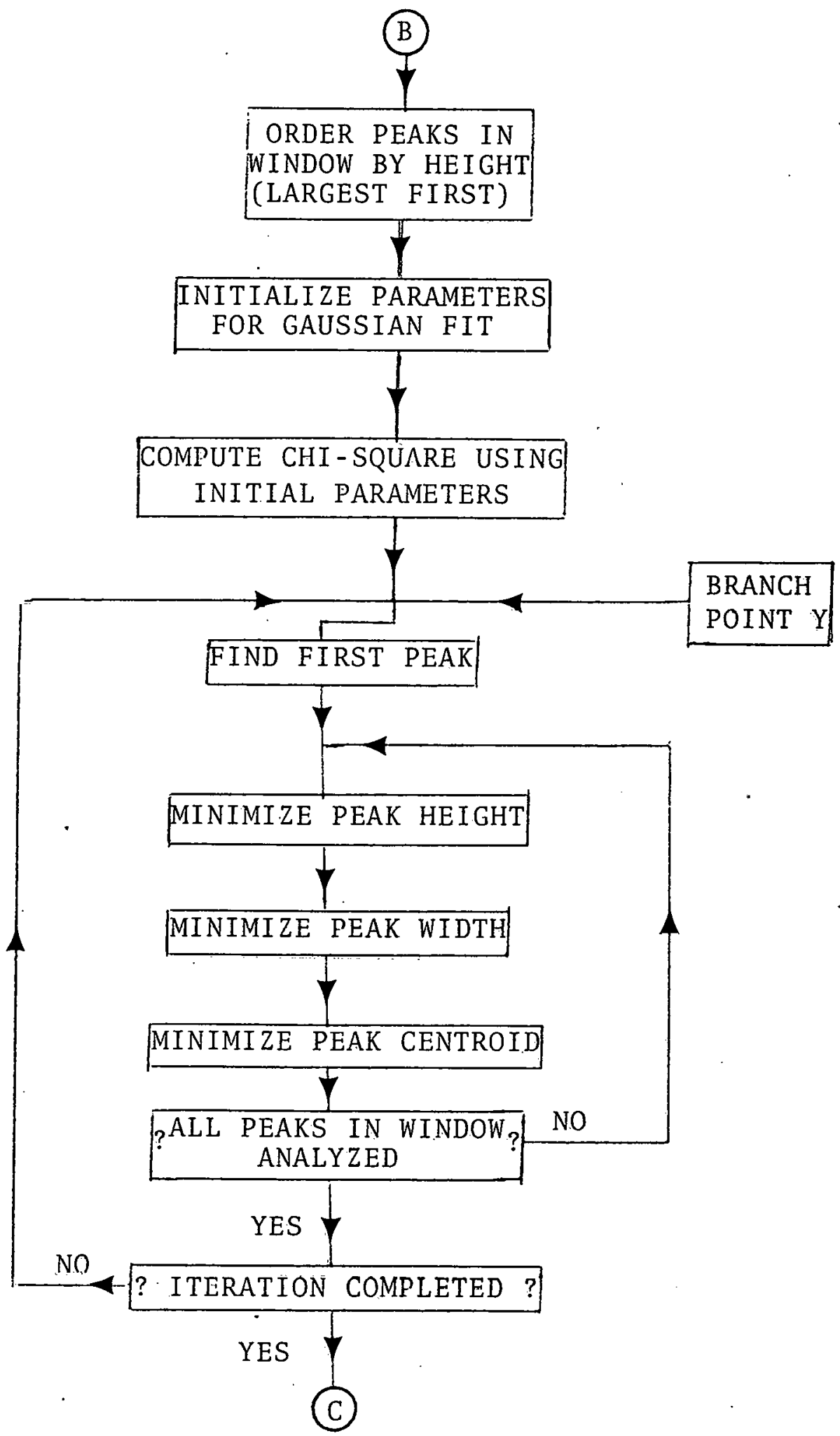

Fig. 101 (continued) 


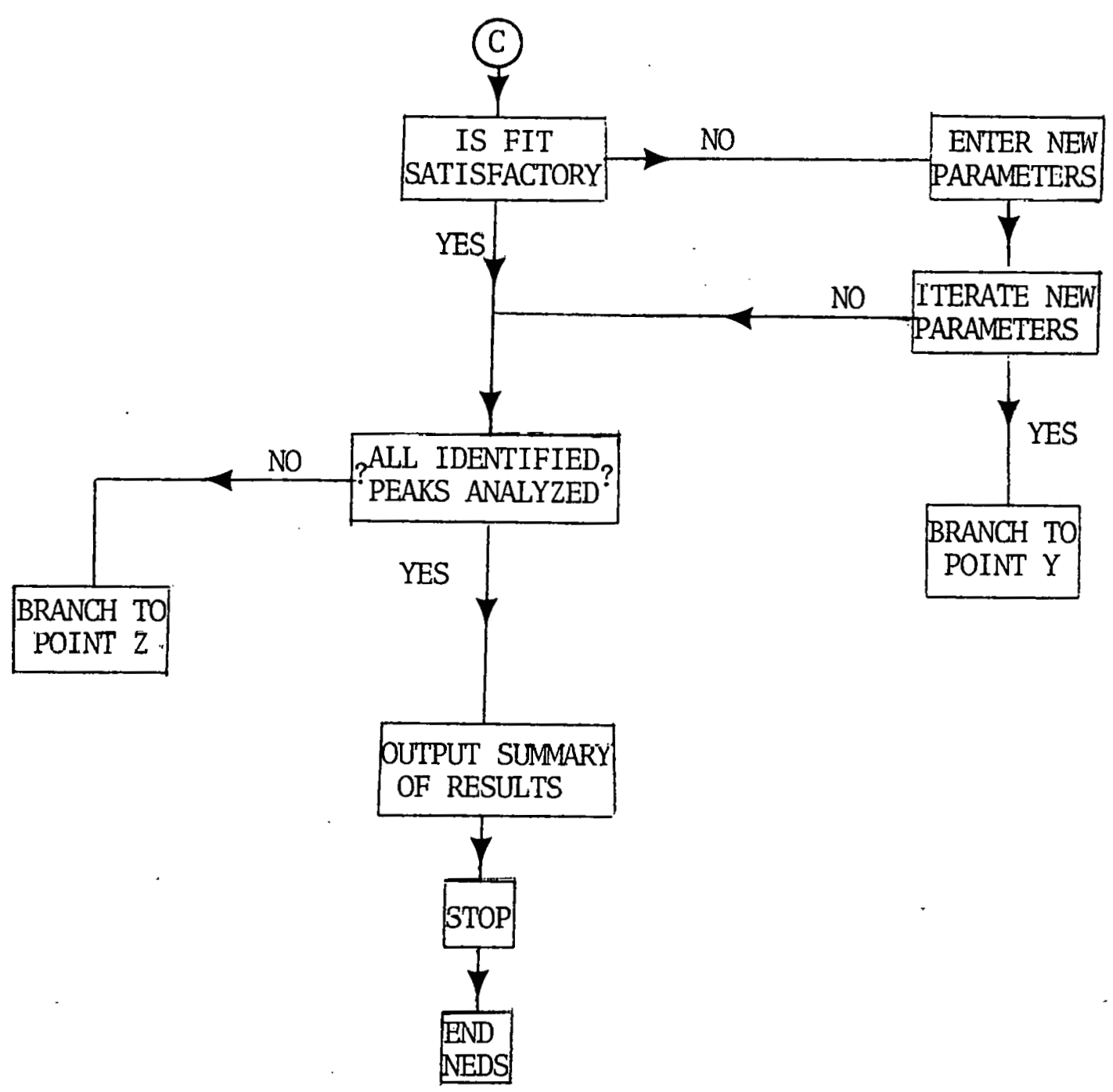

Fig. 101 (continued) 
EIIS IIATA ANALYSIS FFIOGFAM

7702010000

ALL RUESTIONS ANII COMMENTS SHDULLI RE IIIFECTEN TO:

\author{
NESTOF J. ZALUZEC \\ IIEFARTMENT OF METALLUFGY \\ UNIUERSITY OF TLLINOIS \\ UFEANA, ILLINOIS
}

ALL FIIGHTS FESEFUED EY AUTHOR

THIS FROGFAM IS WRITTEN IN FOFITEAN IU FOF USE DN A XEFIOX SIGMA

C OR FIF LSI-11 COMFUTER SYSTEM EQUIFFEII WITH A TEKTFONIX 4010-1

C GRAFHICS IIISFLAY TEFIMINAL. THIS UEFSION IS FOFI USE ON A LSI-11.

C

C IIATA INFUT USES THE FOLLOWING IICE'S

C S=GFIAPHICS TEFIMINAL (INFUT')

C $7=$ GRAFHIICS. TEFMINAL (OUTFUT)

C $10=F$ ILE CONTAINING FFEMGSTFAHLUING SFECTFUM

C $11=$ FILE CONTAJNING SAMFLE (S) SFECTFUM

C 12=FILE USER FOF OUTFUT DF FESUL.TS

c

C TO FIUN ON THE FIF LSI-11 THE FOLLOWING COMMANI WILL INITIATE THE

C SYSTEM COFFECTLY

C

C. FUUN FII1:NEIS.SAY

C

C THIS FROGFAM CAN ANALYZE AT MOST 30 FEAKS

C NOTE THSS IDES NOT CORRESFONI TO 30 ELEMENTS

C ALL INFUT IAATA FFOH TEKMINAL IS IN KEV

C SPECTKUM SET UF TO ANALYZE A 10 KEU IIEGION

C TO CHANGE RESOLUTION OF MCA CHANGE IFES (NOW SET AT ZOEU/CHN)

c

C

$\mathrm{C}$

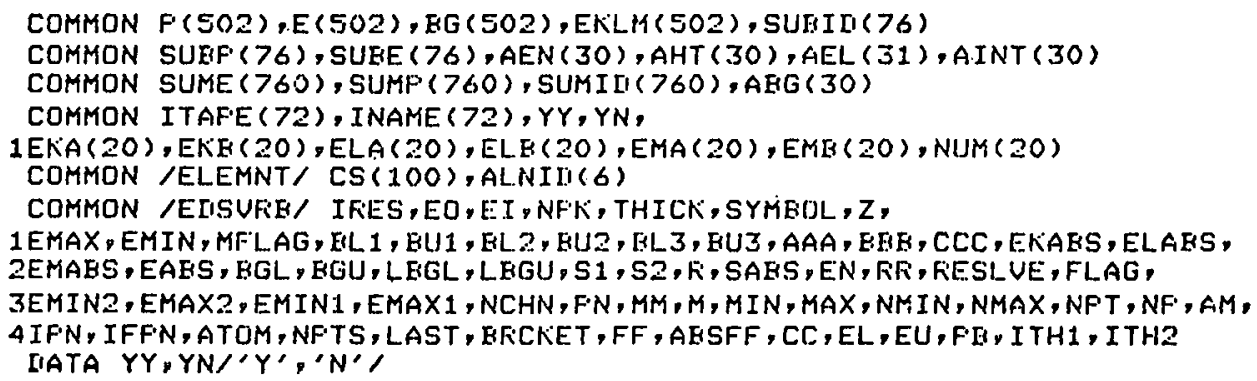




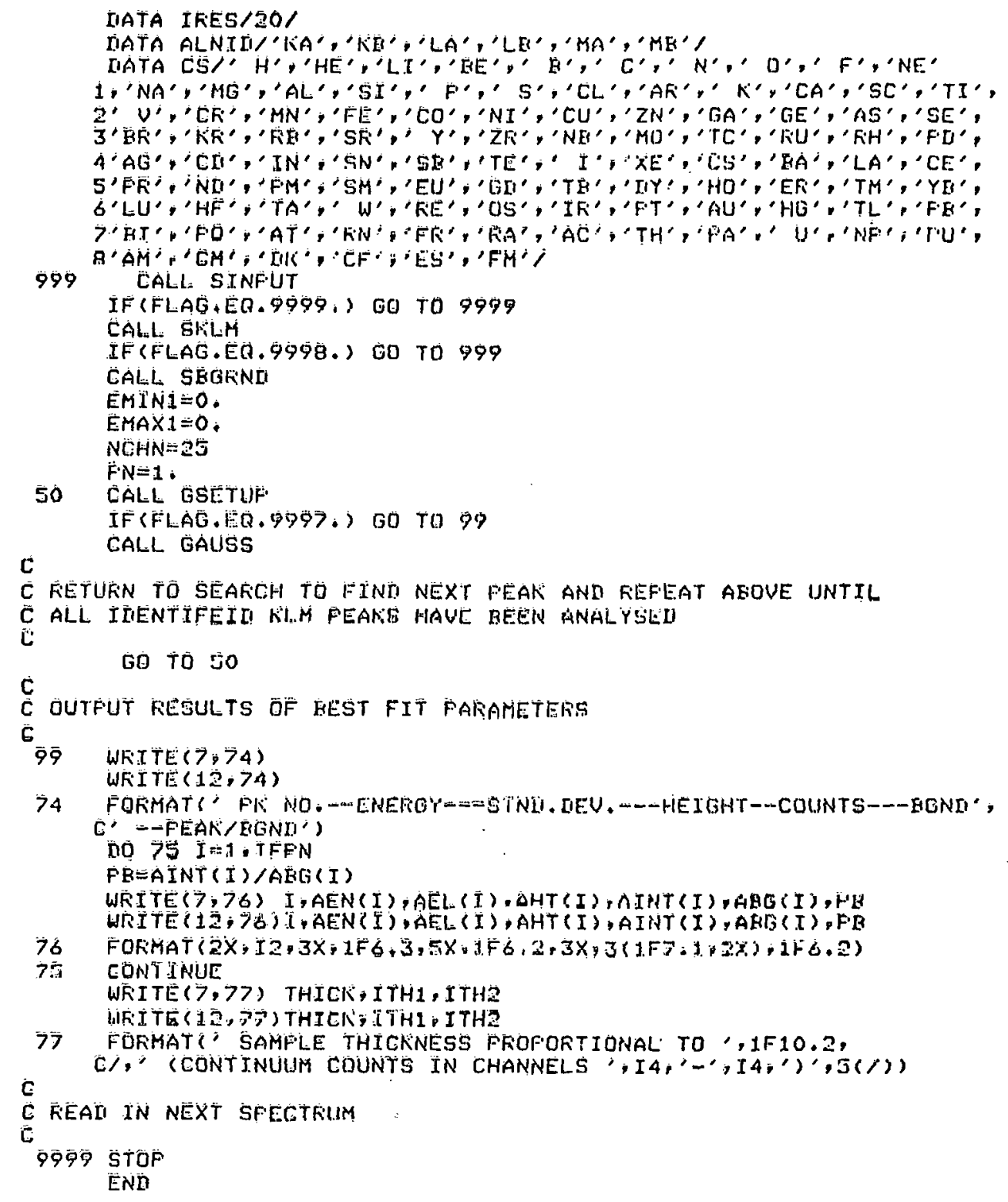




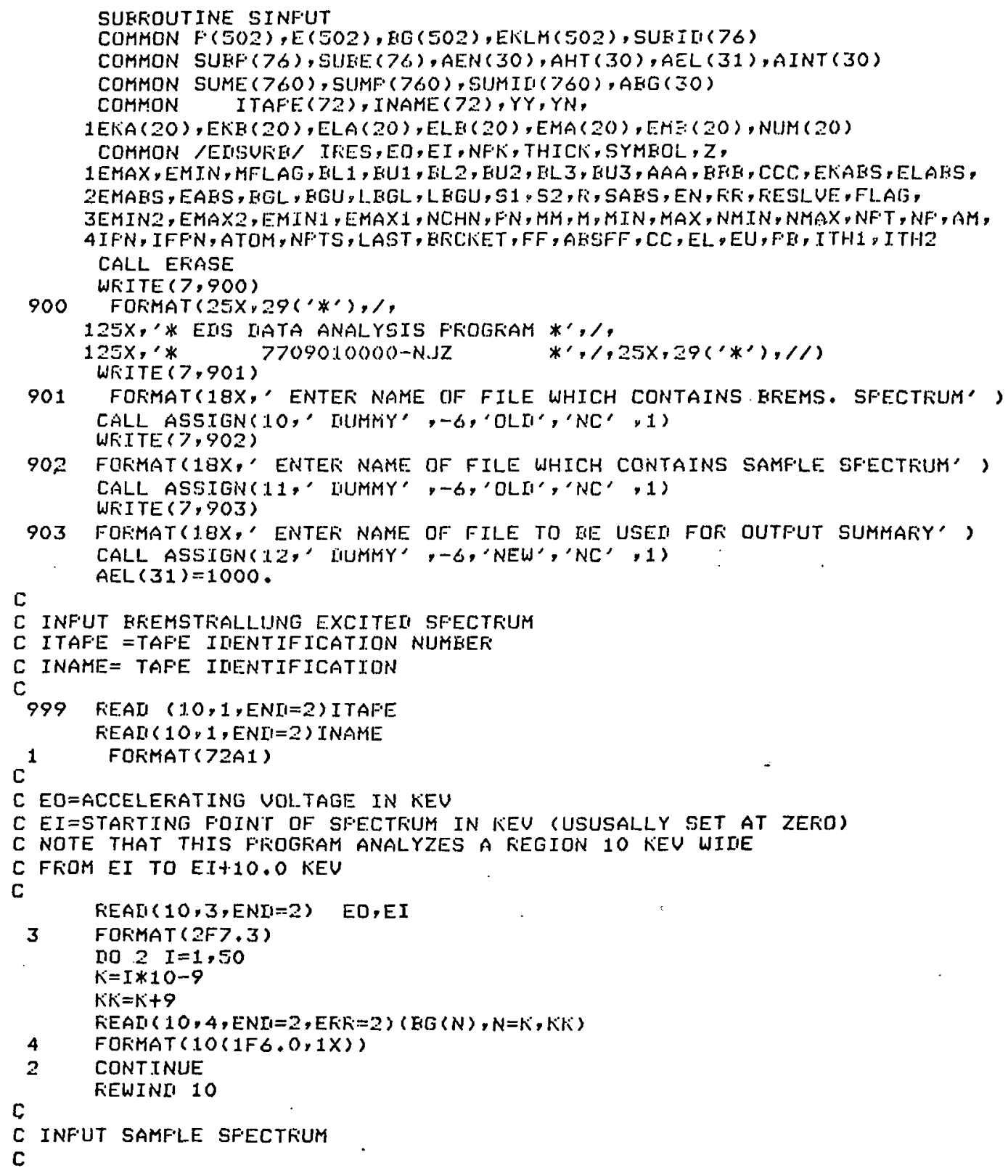




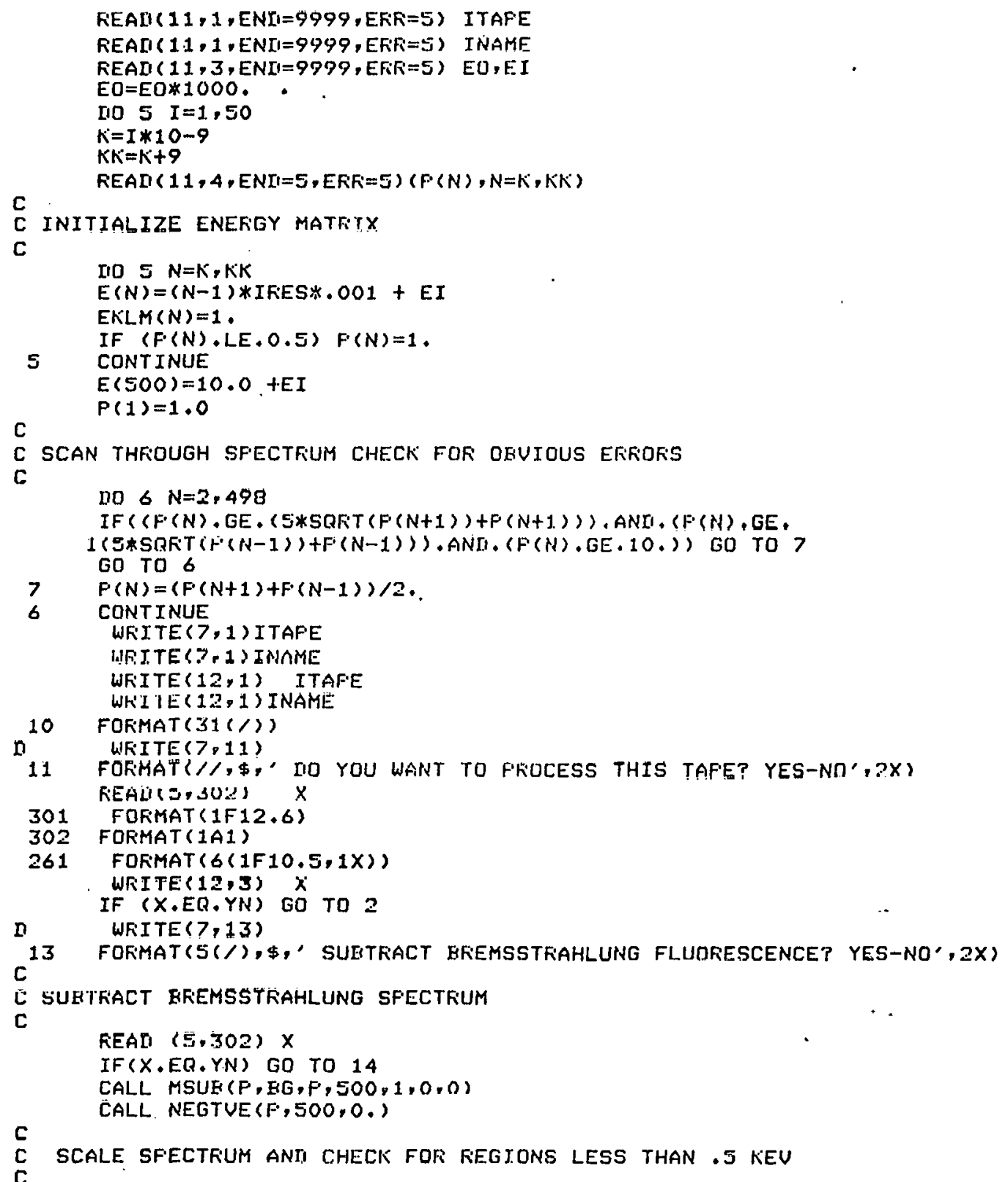




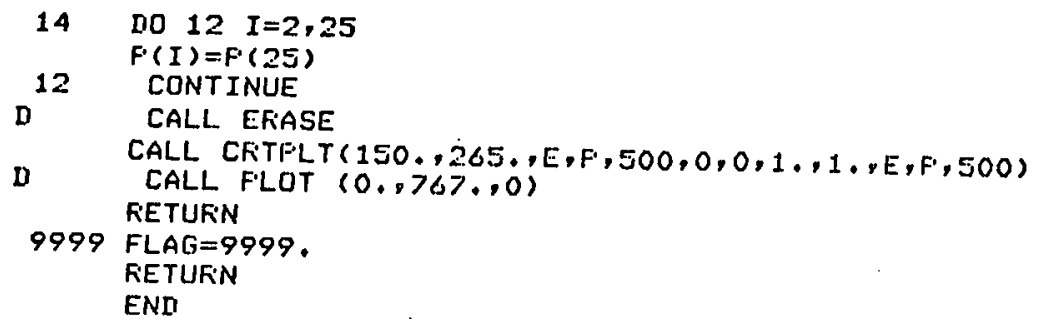




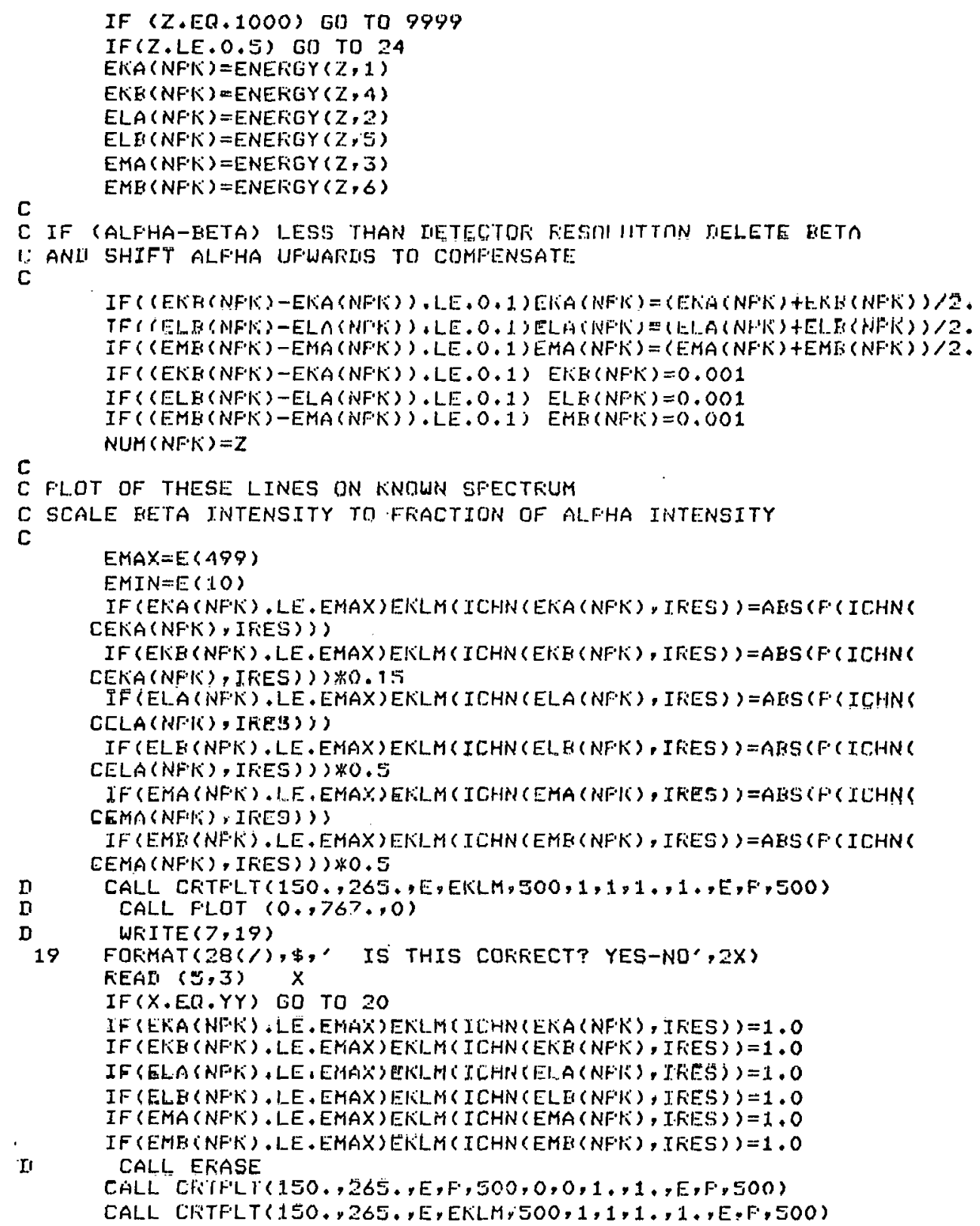




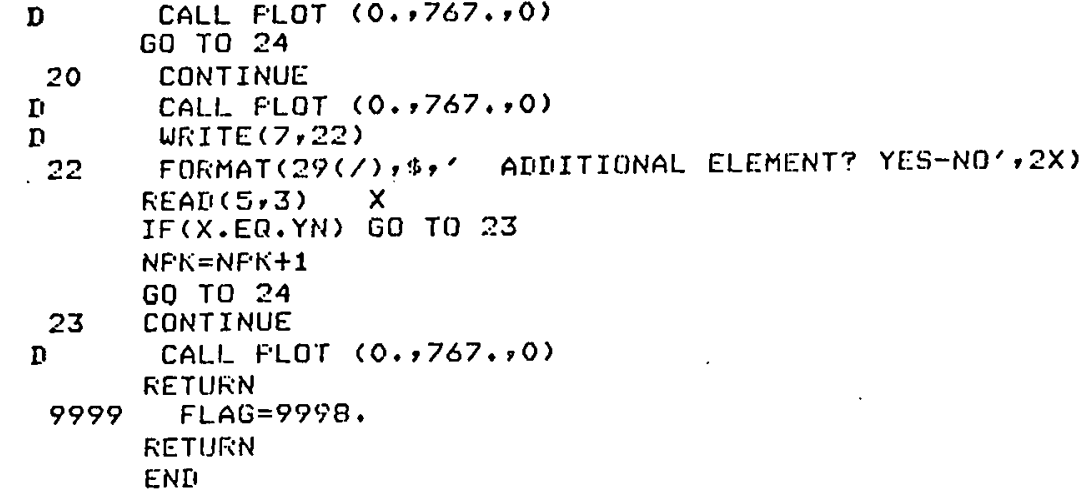




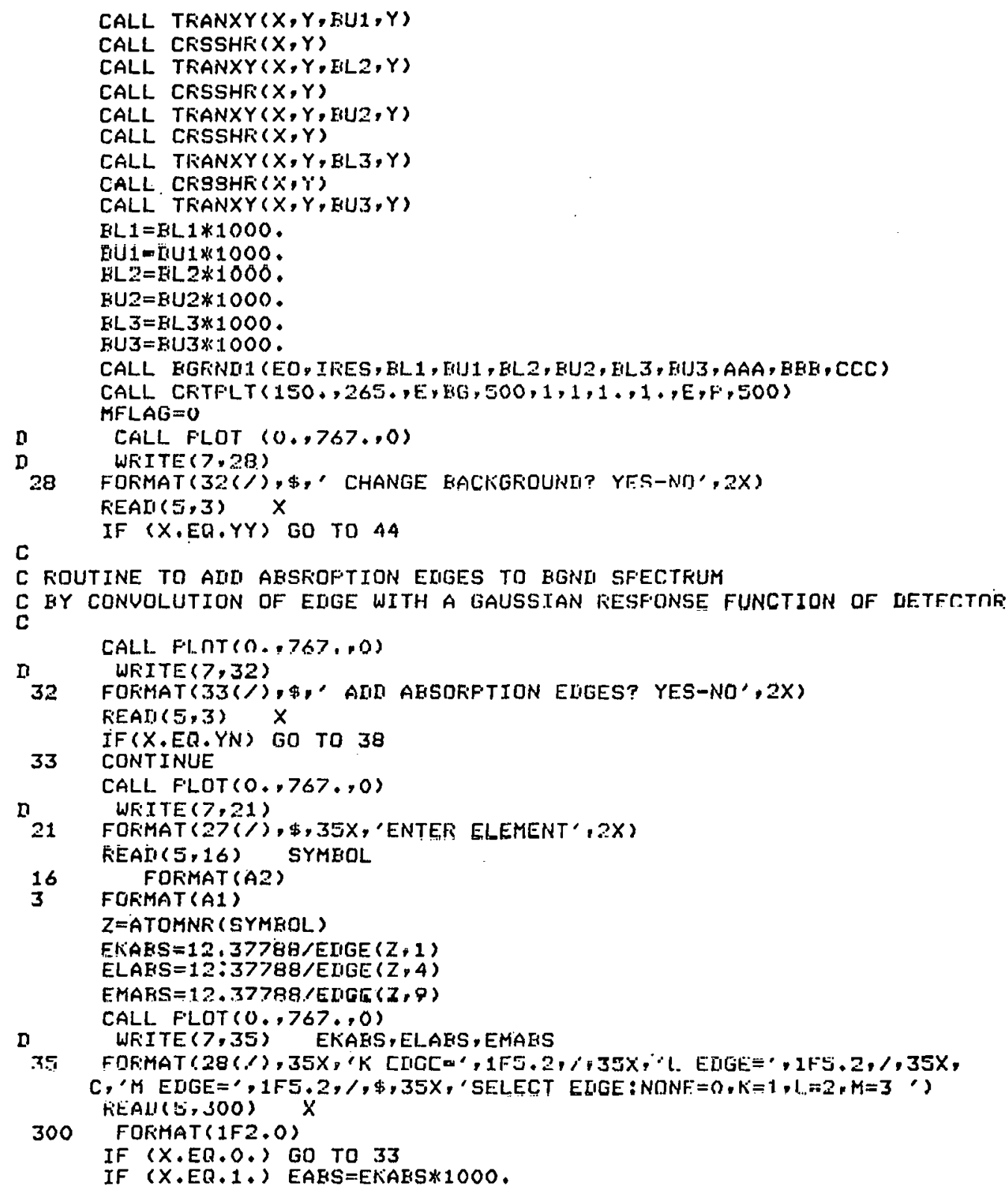




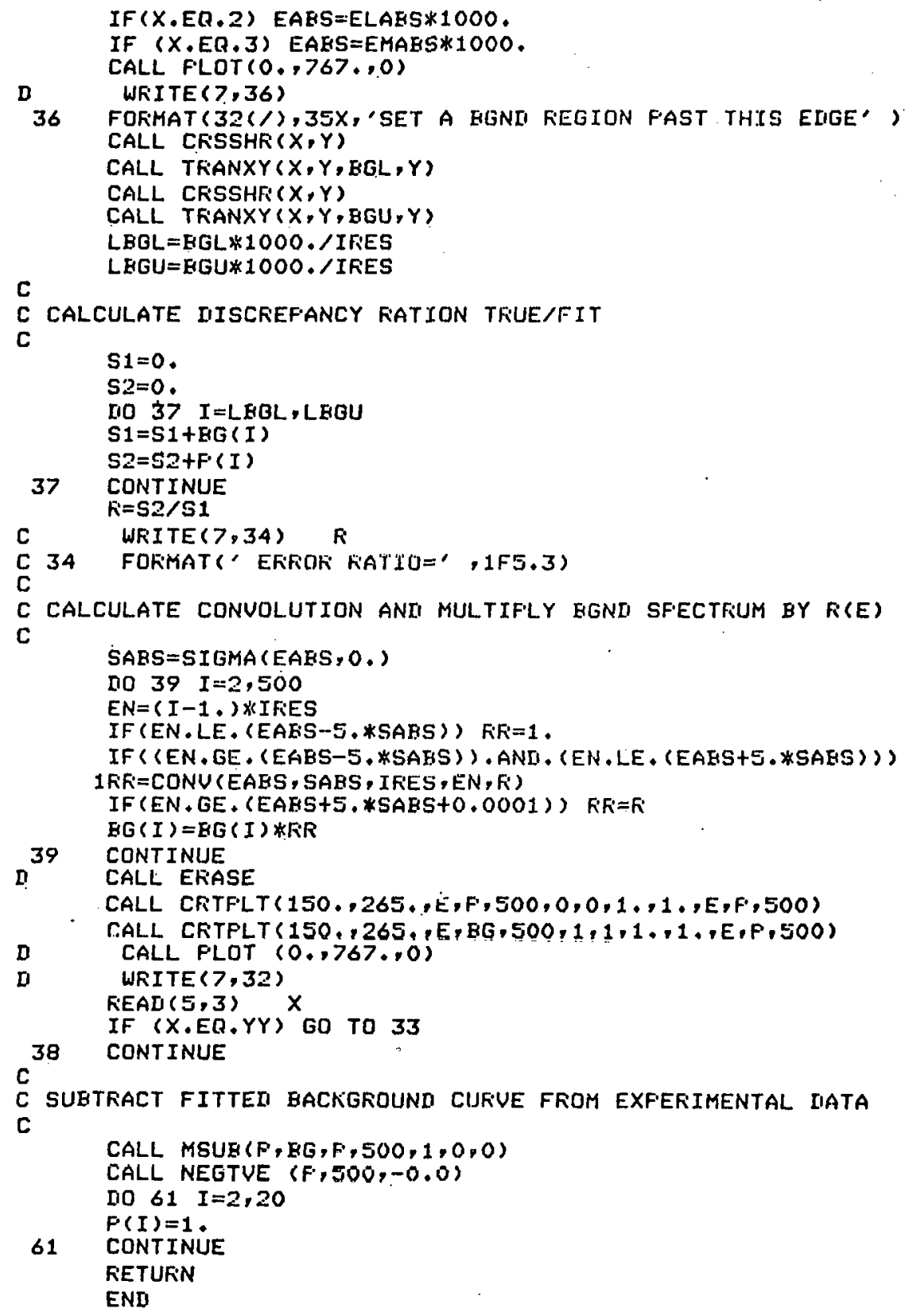




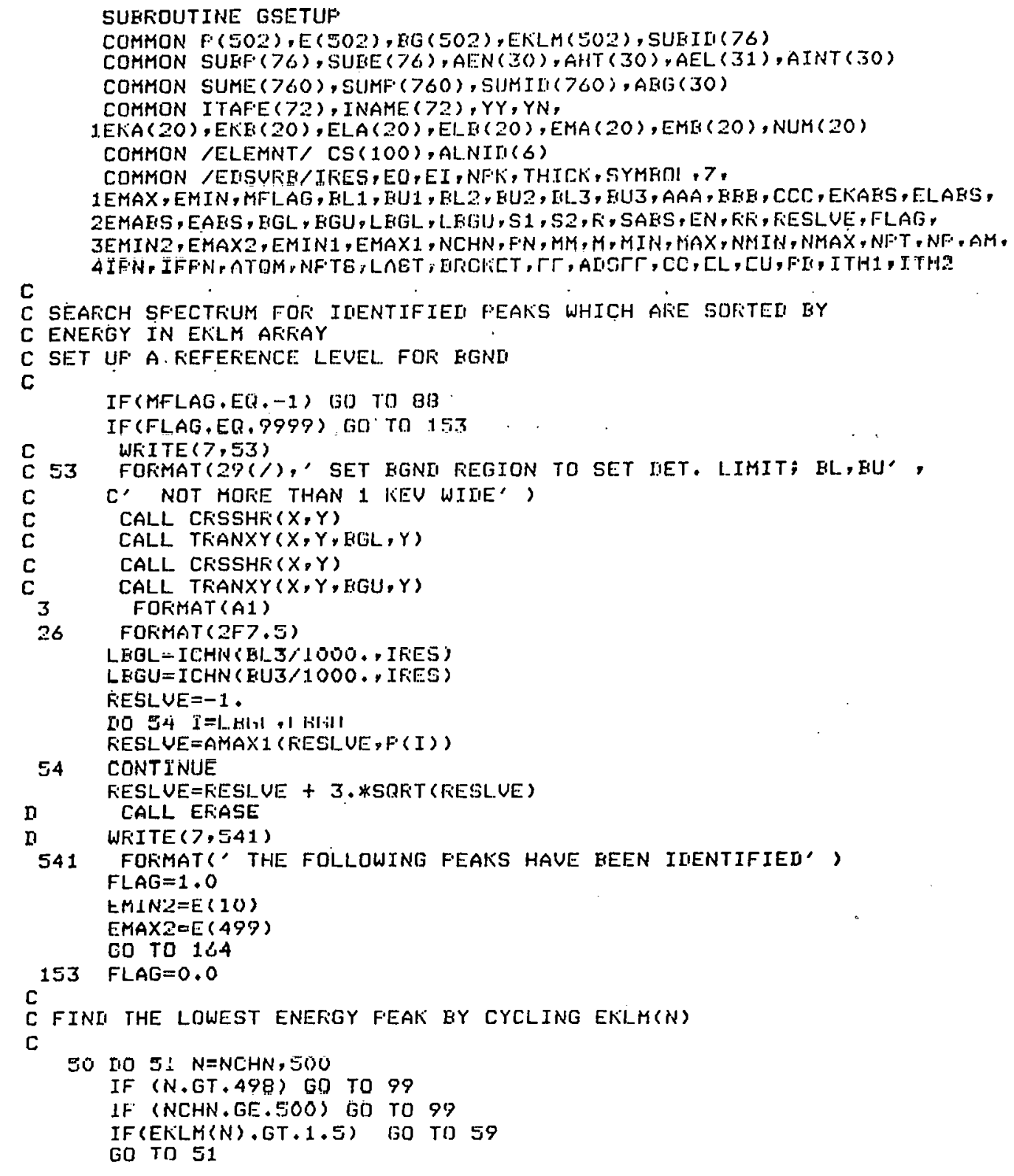




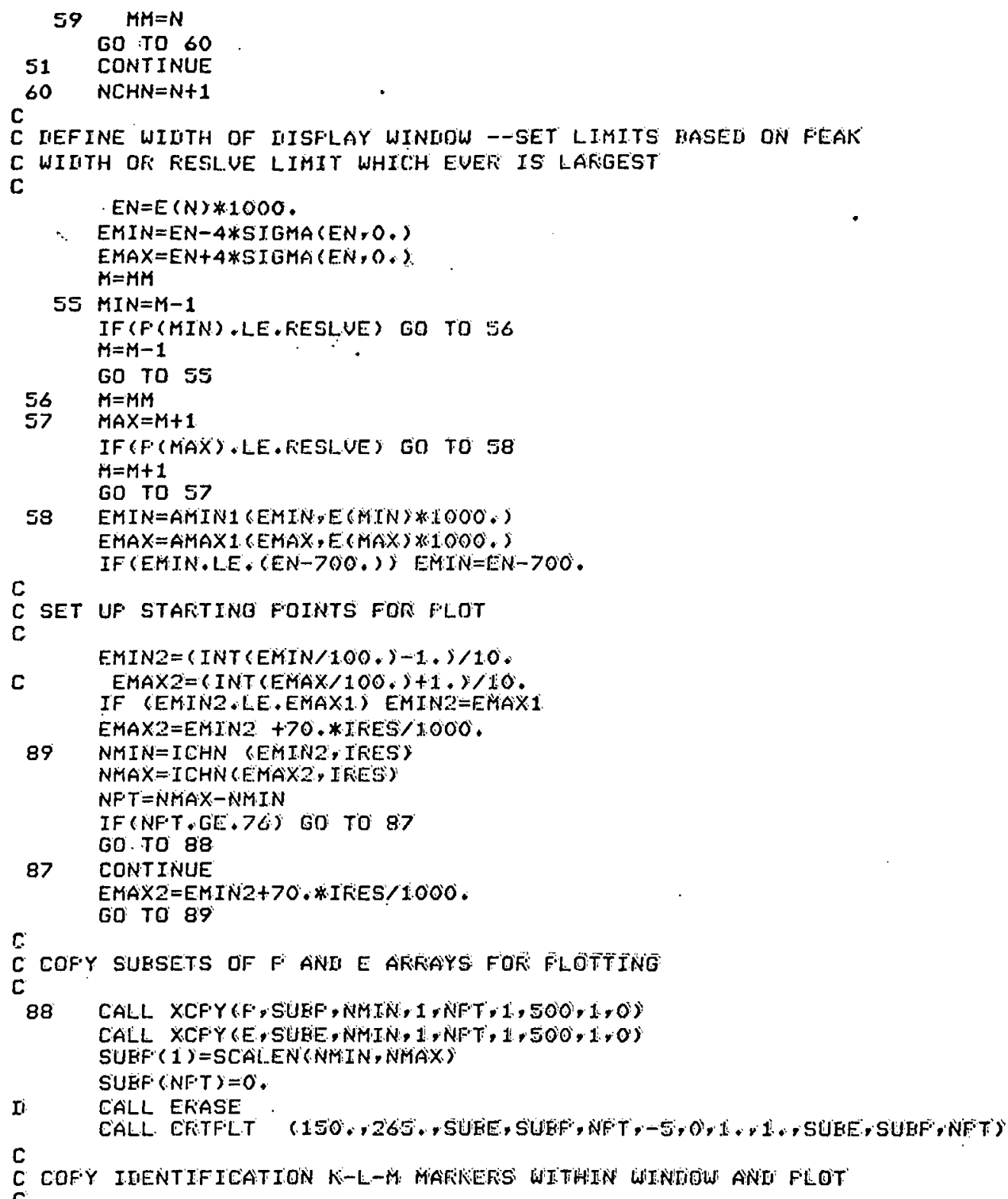


CALL XCFY (EKLM, SUEII, NMIN, 1, NFT, 1,500,1,0)

I) CALL CFTFLT 150,265 , ,SUEE, SUEILI, NFT, $1,1,1,1,1$, SUEE, SUFF, NFT $)$

CALL FLOT $(0,7767, \ldots)$

IF" (MFLAG,EQ,-1) GO TO 157

ᄃ

ORIIER FEAKS WITHIN WINIIOW EY FEAK HEIGHT FOF EVENTUAL

C GAUSSIAN FITTING MOST INTENSE FEAK FIFST

C NF:NUMEER OF FEAKS IN THE WINLOW

C FNAEFEAK NUMEER OF THE FIR'ST FEAK' IN THE WINLOW

C

161

$N F:=0$.

$A M \div 2.0$

$J N=0$

IIO $162 \mathrm{~J}=1$, NFT

$A M=A M A X 1(A M, S U E I M(J))$

$\operatorname{IF}(\operatorname{SUEII}(J), G E, A M), J N=J$

162 CONTINUE

IF (JN.LE.0.5) GO TO 1.63

IFNEF+NPF

$A E N(I F N)=S U B E(J N)$

AHT (IF'N) $=$ SUBIII (JN)

$\operatorname{SUEII}(J N)=1,0$

$N F=N N F+1$

GO TO 161

$163 I F \cdot N=F+N$ $I F F \cdot N=I F \cdot N+N F-1$

157 WFITTE $(7,63)$ NF, EMIN2, EMAX2

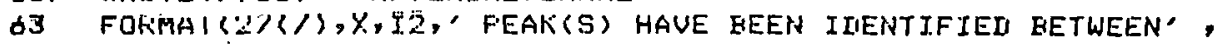
C1F5.2,' TO', IFE.2, , KEV',

c

C WRITE OUT WHICH FEAKS HAUE GEEN FREUIQUSLY IMFNTTFIED

C

$\angle=0$.

61 TOFMAT ( $X, 2(A 2,2 X), 2 X, 1 F>, 4, '$ KEV')

164 IDO $65 \mathrm{I}=1, \mathrm{NFK}$ ATUM=CS $(N U M(I))$

I

IF ( (EKA (I) , GE,EMIN2; , AND , (EKA (I ) , LE,EMAX2) ,

CANI. (EKLM(ICHN (EKA (I), IFES)),GE.2, ))

CWRITE (7,64) ATOM, ALNIII(1),EKA(I)

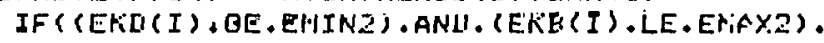

CANI, (EKLH(ICHN (EKB (I), IFEES)),GE,2,))

GWFITE( $>$ IGA) ATOM, NINILI( 2 ), EKKE(I)

$\operatorname{IF}((E L A(I), G E, E M I N 2)$, ANI, (ELA (I),LE,EMAX2).

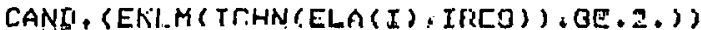

CWFITE $(7,64)$ ATUM, ALNIII (3), ELA(I)

IF ( (ELB (I) , GE,EMIN2) , ANI, (ELE (I) , LE, EM $=X 2)$.

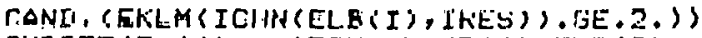

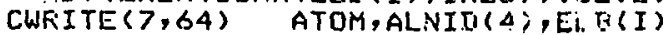

IF ( (EMA II), OE,EHINZ) , ANU, (EMA (I) ,LE,EMAX2),

CANI. (EKLM (ICHN (EMA (I), IFEG)), GE.2.))

CWFITE(7,64) ATOM,ALNIL(5),EMA(I) 


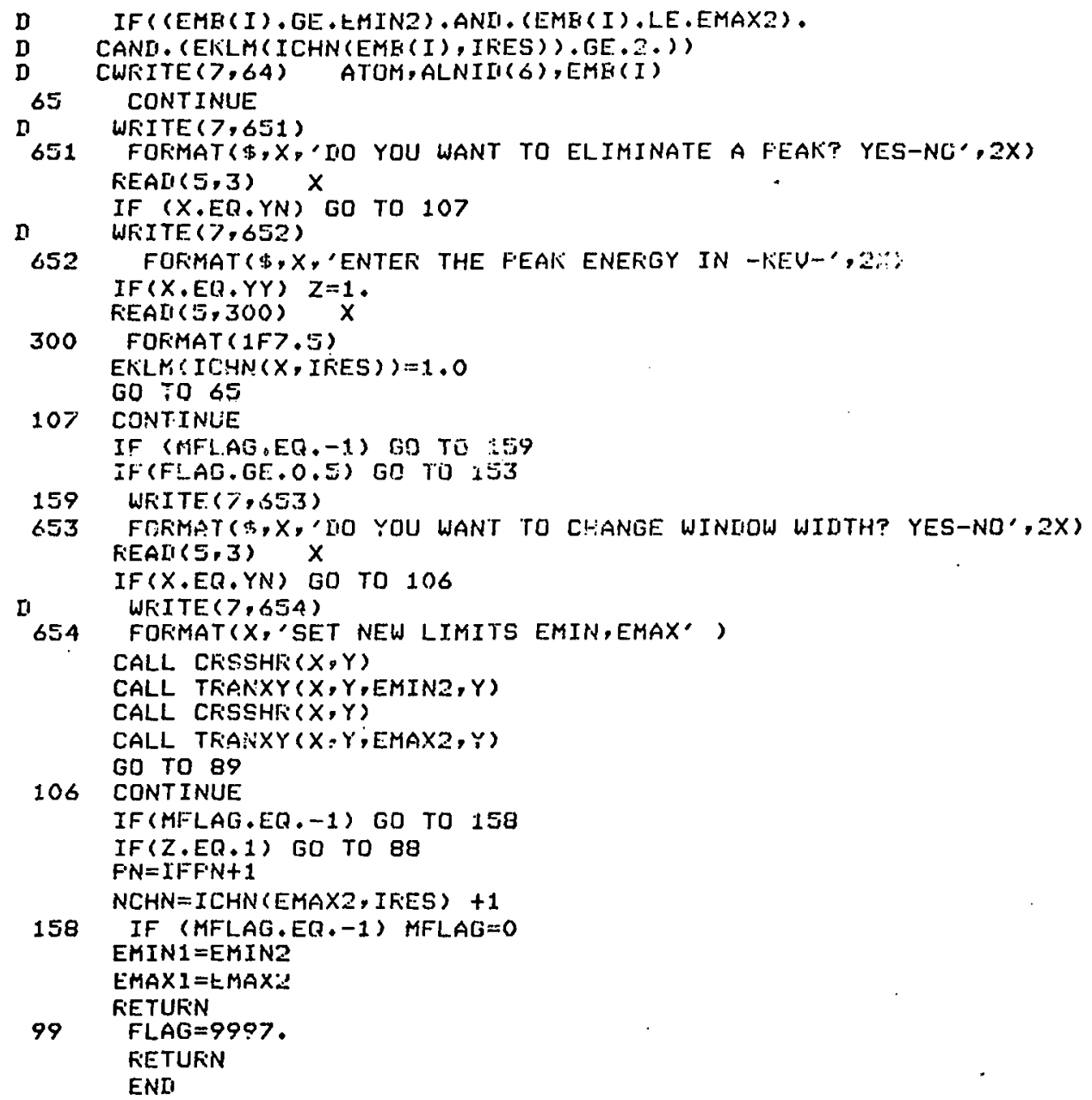




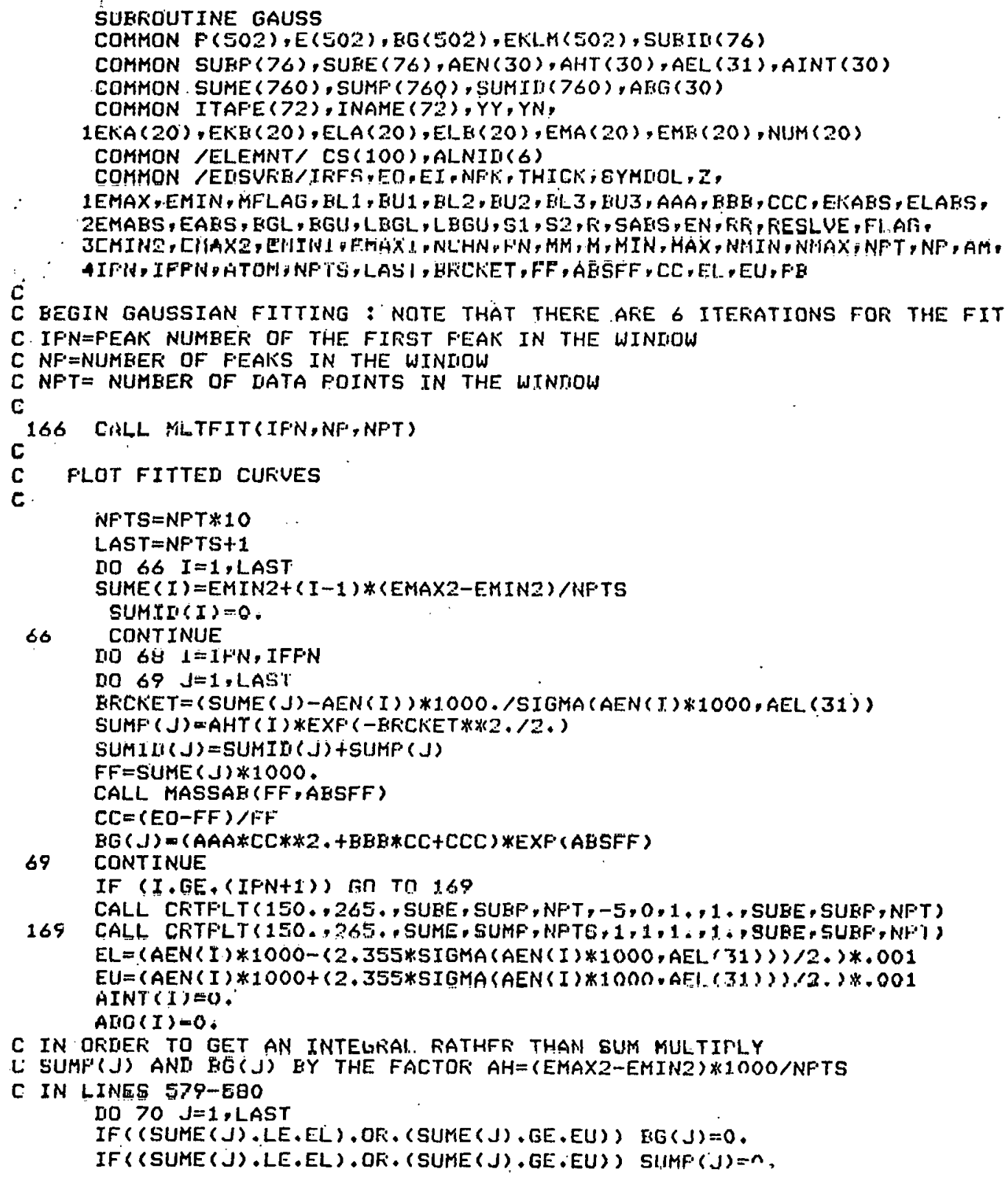


IF (INT $((J-1) / 10),. E Q \cdot((J-1) / 10)$.$) AINT(I)=AINT (I)+SUMP (J)$ $\operatorname{IF}(\operatorname{INT}((J-1) / 10$ ),$E Q \cdot((J-1) / 10)$,$) AEG (I ;=A E G(I)+B G(J)$

70

68

D

67

800

799

WRITE ( 7,804$)$. I, AEN (I), AEL (I), AHT (I)

CALL CFTPLT ( 150,265, , SUME, SUMF, NFTS , 1, 1, 1 , 1, , SUEE, SUEF, NFT)

CONT INUE

CALL PLOT $(0,, 180, .0)$

PAUSE "HIT RETURN TO CONTINUE.

IF (NF.LE, 1) GO TO 67

CALL EFIASE

CALL CRTFLT (150, 265, , SUEE, SUEF, NFT, $-5,0,1,1,1$, SUEE, SUEP, NF:T)

CALL CFTFLT (150, ,265, , SUME, SUMII, NF'TS, 1, 1,1 , 1 , SUEE, SUEF, NFT $)$

CALL PLOT $(0,180,0)$

PAUSE. 'HIT RETUFN TO CONTINUE'

CONTINUE

CALL FLOT $(0, .155, .0)$

WFITE $(7,800)$.

FORMAT $(\$, X, ;$ IS THE FIT SATISFACTOKY? $Y-N$ ")

READ $(5,799) \times$

FORMAT ( $1 A 1)$

IF (X,ER.YY) GO TO 801

CALL PLOT $(0,, 155, ; 0)$

WFITE $(7,802)$

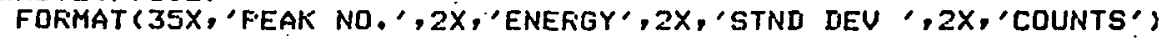

DO 803 I $=$ IFN, IFFN

FORMAT $(39 X, 12,4 X, 1 F 6,3,5 X, 1 F 6,2,2$

REAII (S, BOS) AEN (I), AEL (I), AHT (I)

BOS FORKAT (3F10:0)

803 CONTINUE

$A E L(31)=(A E L($ IFN $) * 2.35482) * * 2,-A E N($ IF' $) * 3489.25-25000.6$

809

WFITE $(7,806)$

806 FOKMAT (\$,' ITERATE NEW UALUES? $Y-N$ ')

READ $(5,799) X$

IF $(X, E Q, Y Y)$ GO TO 850

IF (X.EQ.YN) GO. TO 66

GO TO 809

$850 \quad M F L A G=-1$

801 CONTINUE

D

C

C RETURN TO SEARICH TO FIND NEXT FEAK AND REFEAT AEOUE. UNTIL

C ALL IIIENTIFIED KK-L-M FEAKS HAVE BEEN ANALYSED

FLAG $=9999$.

RETURN

END 
THIS PAGE

\section{WAS INTENTIONALLY LEFT BLANK}




\section{APPENDIX B}

Subroutine Library - NXRYL

Function ATOMNR

Function ATOMWT

Function ENERGY

Function EDGE

Function YIELD

Function BETA

Function CROSS

Function GEN

Function ABGEN

Function FWHM

Function SIGMA

Subroutine PARAM

Subroutine MASSAB

Subroutine QATR
Subroutine BKSCTR

Subroutine KWBGND

Subroutine KBGND

Subroutine MSUB

Subroutine XCPY

Subroutine LOC

Subroutine NEGTVE

Subroutine MLTFIT

Subroutine CHIMIN

Subroutine CHISQ

Subroutine BGRND1

Subroutine BGRND2

Function ICHN

Function CONV

Function SCALEN 


\section{APPENDIX B \\ Subroutine Library - NXRYL}

This appendix contains a listing of a general $x$-ray analysis subroutine library common to the programs NEDS, NXRYRT, and NMCS, as well as important subroutincs dealing with the variuus calculatioñs presented in this research. For example, the calculation of the angular distribution of continuum intensity or the functional dependence of the theoretical peak-to-background ratio for thin foils can be duplicated by reference to these subroutines and Eqs. (2.47) through (2.51). 


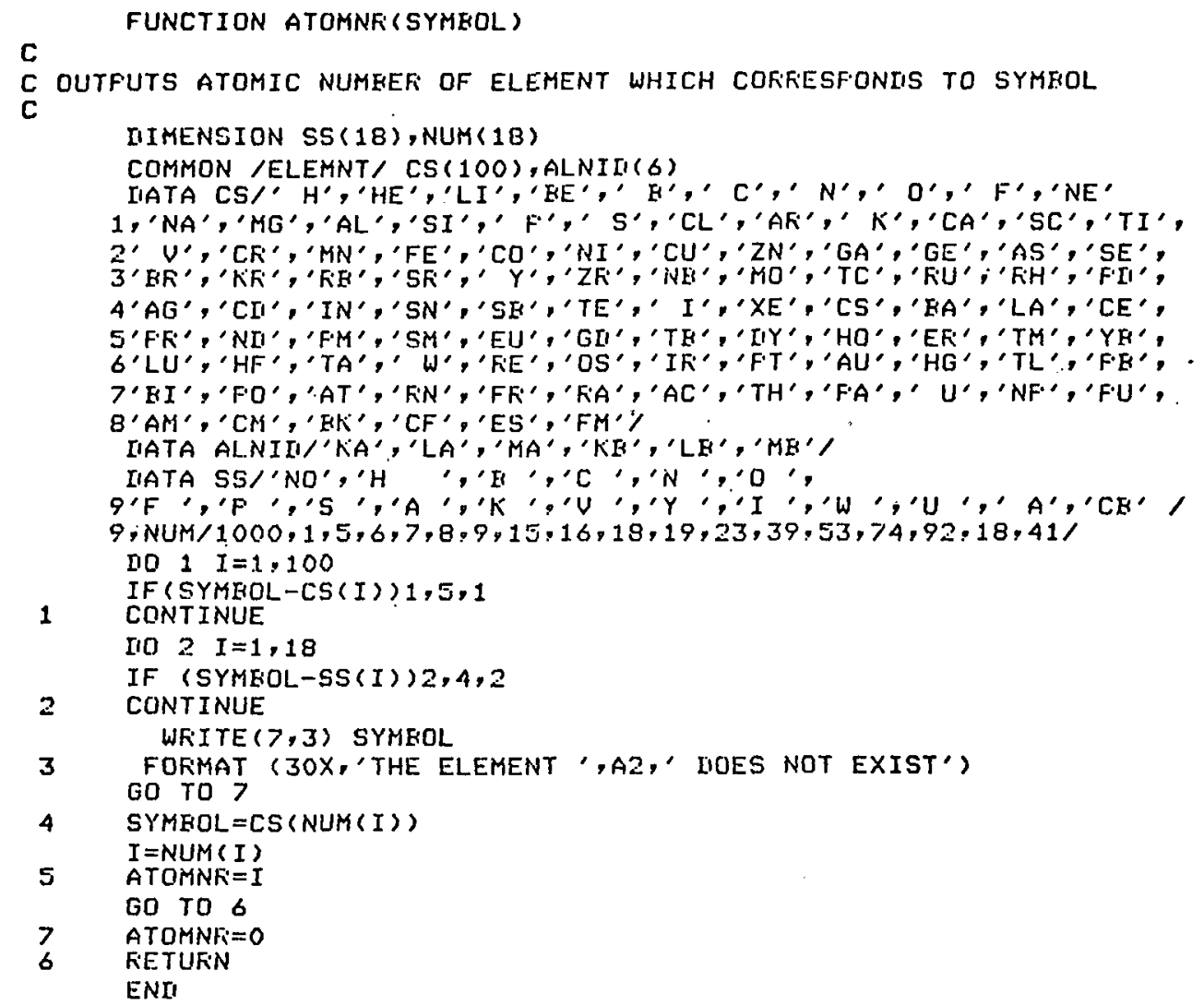

FUNCTION ATOMWT (Z)

C OUTFUTS ATOMIC WEIGHT OF ELEMENT $Z$

c

IIIMENSION A(100)

IIATA A/1.008,4.003,6.939,9.012,10.811,12.011,14.007,15.999,18.998 $120.183,22.990,24.312,26,982,28.086,30,974,32.064,35.453,39.948$, $239.102,40+080,44,956,47.900,50,942,51.996,54,938,55,847,58,933$, $358.710,63.540,65.370,69.720,72.590,74.922,78.960,79.909,83,800$, $485.470,87.620,88.905,91.220,92.906,95,940,99.000,101.070,102.905$, 
$5106.400,107.870,112.400,114.820,118.690,121.750,127.600,126.904$, $6131,300,132.905,137,340,138,910,140,120,140,907,144,240,147.000$, $7150.350,151.960,157.250,158.924,162.500,164.930,167.260,158.734$, $8173.040,174.970,178.490,180.948,183.850,186.200,190.200,192,3$, , $9195.090,196.967,200.590,204.370,207,190,209.980,210.000,210.000$, $1222.000,223.000,226.000,227.000,232.038,231.000,238.030,237.000$, $2244.000,243.000,247.000,247.000,251.000,254.000,253.000 /$

$\mathrm{I}=\mathrm{Z}$

ATOMWT $=A(I)$

FETUFin

Frifi

c

FUNCTION ENEKGY $Z Z, L)$

$c$ DUTFUTS ENEFGY OF XFAY LINE OF ELEMENT $Z$, IF $L=1,2,3,4,5,6$

C THEN OUTFUTS KA,LAYHA,KE,LEY ISE LINES RESFECTIUELY

C OUTFUTS ENERGY IN -KEV-

GO TO $(1,3,5,8,10,12), \mathrm{L}$

$1 \operatorname{IF}(Z-3) 7,2,$,

2 WAVE $=1,1(-1.6745699 E-03+Z *(-6.846) 562 E-05+Z *(6.281489 E-04+$ $1 Z *(1.0809595 E-05+Z *(-3.027904 E-0)+Z *(4.663746) E-09-2.6734801 E-11$ $2 * Z)(\zeta))$

ENEKGY $=12,37788 /$ WAVE

$3 \quad$ IF $(Z-8) 7,4,$,

4 WAUE $=1 . /(1.9140411 \mathrm{E}-02+7 *(-3.8046413 \mathrm{E}-03+2 *(2.6884465 \mathrm{E}-04 t$

$1 Z *(-3.9334536 E-06+Z *(6.4498238 E-08+Z *(-5.1038884 E-10+$

$2 Z * 1.5828(2 E-12))))$

ENERG $Y=12 \cdot 37788 /$ WAUE FIETURN

$5 \quad \operatorname{IF}(Z-40) 7,6,$,

$6 \quad$ WAVE $=1 . /(-.4326787+Z *(.023126494+Z *(-4.6580727 E-04+$

$1 Z *(4.6345531 E-06-1.5432413 E-08 * Z)))$ )

ENEFGY $=12.37788 /$ WAVE

FETURN

$7 \quad$ ENEFEY $=0$.

RETUR'N

$0 \quad \operatorname{IF}(2-11,77,7 ; 9$

$9 \quad$ WAVE $=1 . /(1.90599 E-2-Z *(6.072234 E-3-Z *(1.194595 E-3-Z *(9.651631$

1.E-6-Z*(1.802092E-7-Z*1.037428E-9)))))

EIIETIGY $=12$. 3778B/WAVE

RETUEN

is IF $(Z-20) 7,11,$,

11 WAVE $=1 . /(-2.535951 E-2+Z *(2.33104 E-3-Z *(6.181201 E-5-Z *(4.97452 E$

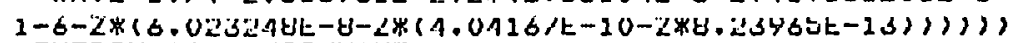

ENEFGY $=12,37788 /$ WAVE

RETUFIN

12

IF $(Z-50) 7,13,$, 
13 WAVE $=1 . /(1.172996 E-1-Z *(9.385426 E-3-Z *(2.797442 E-4-Z *(3.60862$

$19 E-6-Z *(2.812141 E-8-Z * 8.4532 E-11)))))$

ENERGY $=12,37788 /$ WAVE

FEETUFN

END

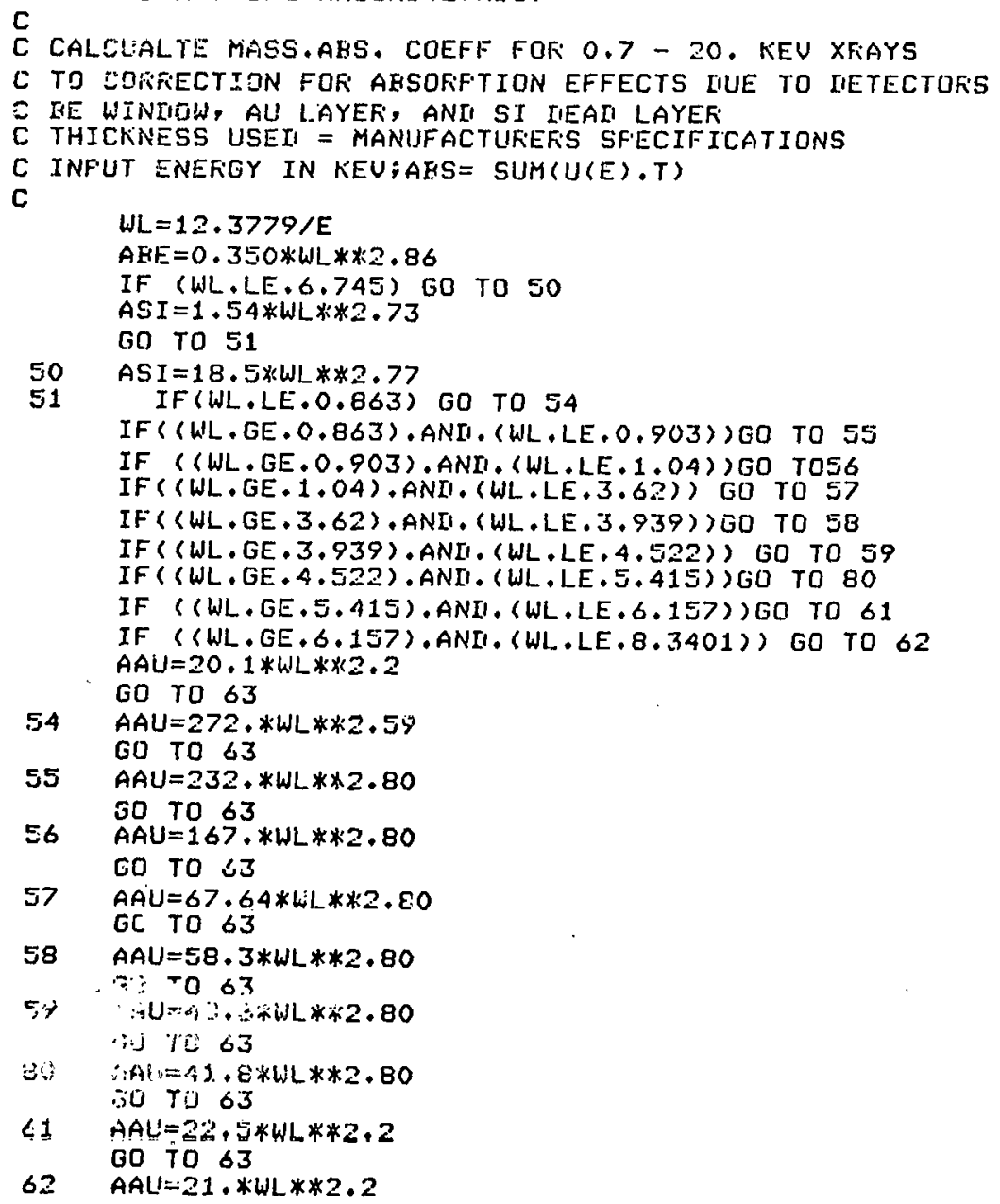




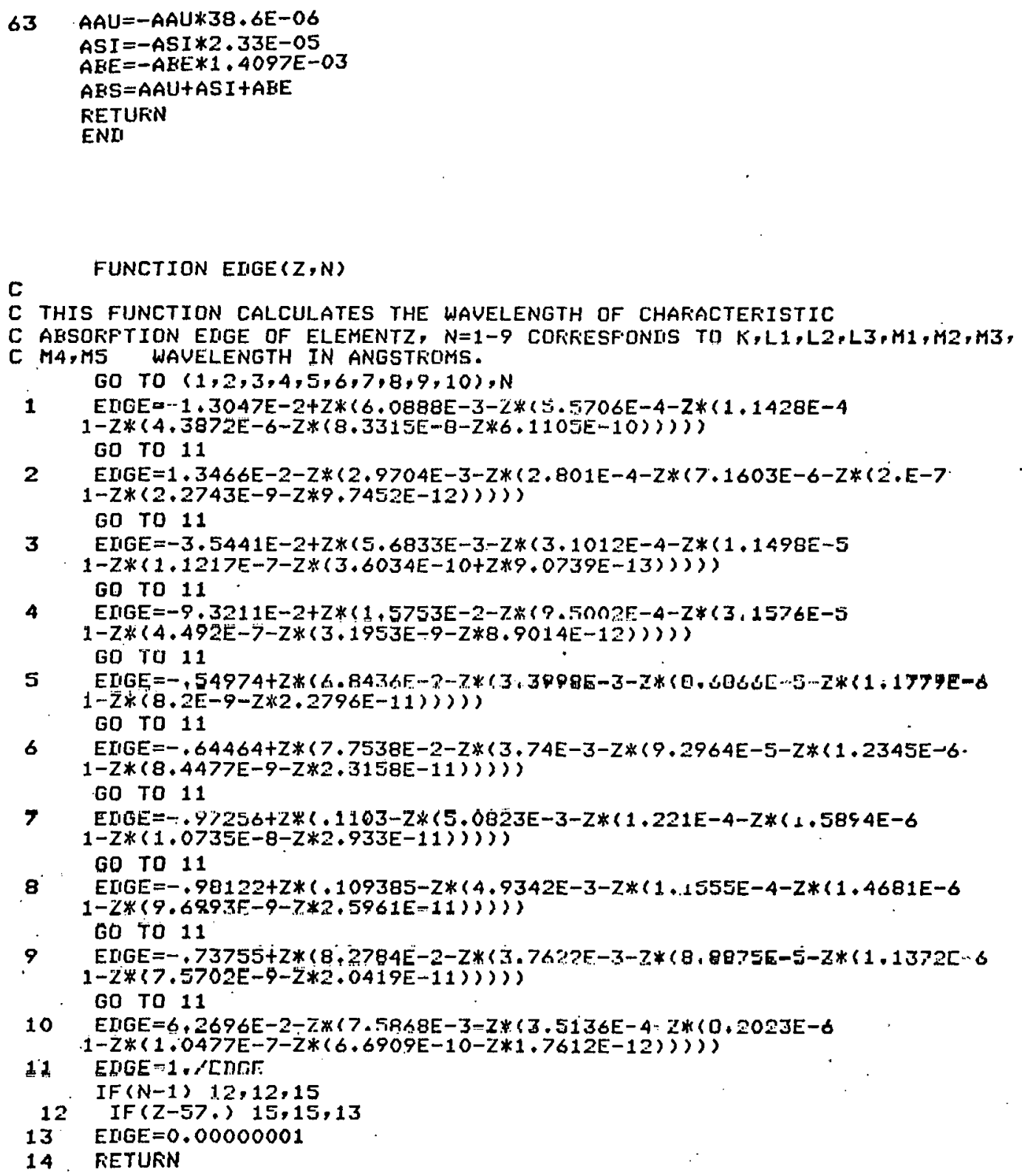




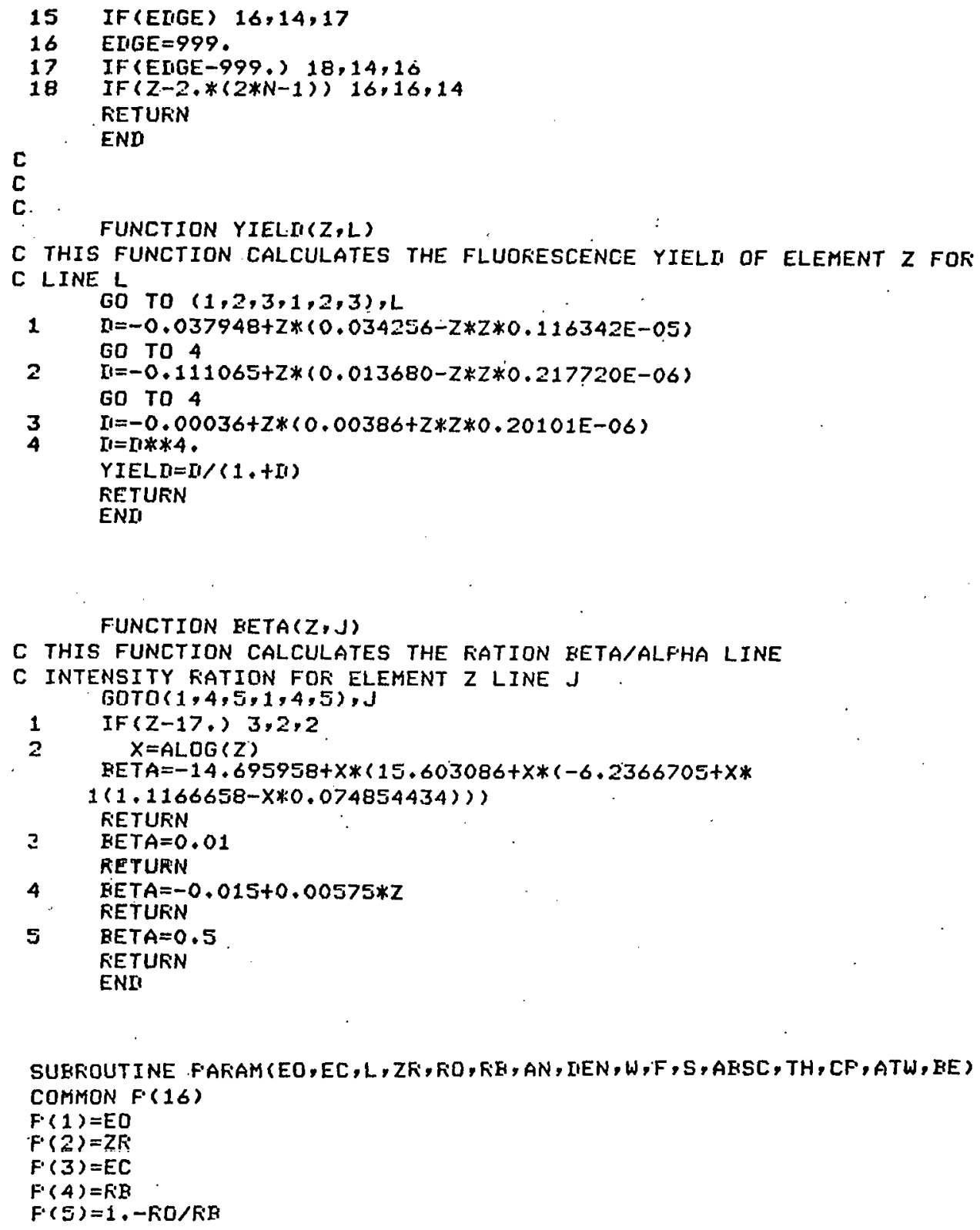




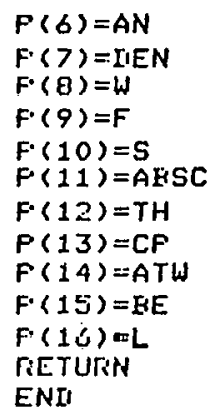

FIINCTION CROSS (TYFE, Z,EO,EC,L)

C CALCULATE THE IONIZATION CFOSS SECTION FOR THE GIUEN FAARA.

C IF TYFE $=0$. CLASSICAL CFOSS SECTION USEI FOF MICFOFFIOEE

C. WITH LUKRECTIONS EY MOTT\&MASSEY, EUFHOF,

C IF TYFE=1 \& WORTHINGTON \& TOHLIN

IF TYFE=1, MOIIFIEI EY ZALUZEC TO ACCOUNT FOF FELATIUITY

C EY CHANGING CONSTANTS (EMFIIICAL FIT)

C IF $L=1$ CALCULATE $K$ SHELL CFOSS SECTION

C IF L=2 CALCULATE I. SHELL CFIOSS SECTION

C EKI=CONSTANT RELATEI TO RAIITUS OF SHELL

C NZ $=$ NUMBER UF ELECTFIONS IN FULL SHELL

C $Z=$ FEIIUCEI FATHLENGTH FARAMETER =FRACTION OF ELECTFON FANGE

C EO= INCILENT ENEKGY (KKEV)

C EC= CFITICAL EXCITATION ENER̈GYY(KEU)

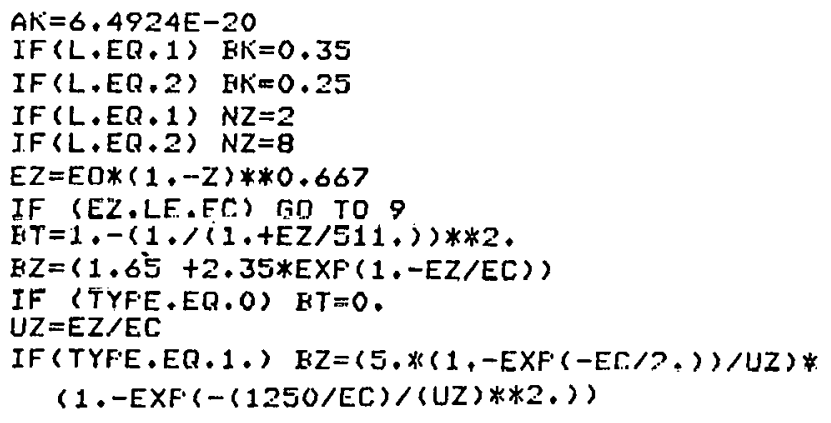




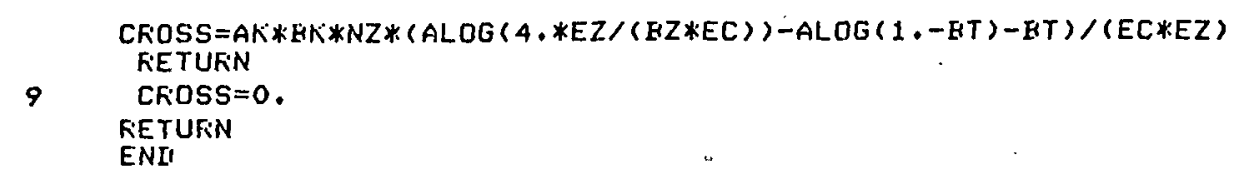

FUNCTION GEN(T)

COMMON F.(16)

$Z=1 . E 04 * T / F \cdot(2)$

$\mathrm{QZ}=\operatorname{CFOOSS}\left(1,, \mathrm{Z}, \mathrm{F}^{\prime}(1), \mathrm{F}^{\prime}(3), \mathrm{F}^{\prime}(16)\right)$

$F Z=F(1) *(1,-F \cdot(5) * E X F \cdot(-F \cdot(10) * F(7) * Z / 4)$,

IF $(F Z, E R, 0) \quad F Z=$,1 .

$Z N=E X F \cdot(-F(10) * F \cdot(7) * Z)$

GEN $=0.005625 * Q Z * F Z * Z N * F(6) * F(7) * F^{\prime}(8) * F(9) * F(13) / F^{\prime}(14)$

RETURN

ENII

FUNCTION AEGEN(T)

COMMON F(16)

$Z=1, E 04 * T / F \cdot(2)$

$\mathrm{BE}=\mathrm{F}(15) / 57.295779$

$T H=F(12) / 57.295779$

$C O K F=S I N(E E) / S I N(T H)$

$A Z=E X F^{\prime}\left(-F(11) * F^{\prime}(7) * \operatorname{COFF} * Z\right)$

AFGEN $=$ GEN $(T) * A Z$

FETUFIN

ENI

SUFROUTINE RATR

FUIFIFISF

TO COMFUTE AN APFFOXIMATION FOK INTEGFAL(FCT(X), SUMMEN OUEF $X$ FROM XL TO XU).

USAGE

CALL RATK ( $X L, X U, E F S, N I I M, F C T, Y, I E F, A U X)$

FAFAMETER FCT RERUIRES AN EXTER'NAL STATEMENT.

IIESCFIFTION OF FAFAMETEFS

$X L$ - , THE LOWER BOUNI OF THE INTEFUAL.

XU - - THE UFPEF EOUNA OF THE INTEFUAL.

EFS - THE UFFEF EOUNI OF THE AESOLUTE FFFIOF.

NIIM - THE DIMENSION OF THE AUXILIAFYY STOKAGE ARFIAY AUX. NIIM-1 IS THE MAXIMAL NUMEEF OF BISECTIONS OF THE INTEFVAL (XL, XU).

FCT - THE NAME OF THE EXTEKNAL FUNCTION SUBFROGFAM USED.

$Y$ - THE RESULTING APFROXIMATION FOR THE INTEGRAL VALUE. 


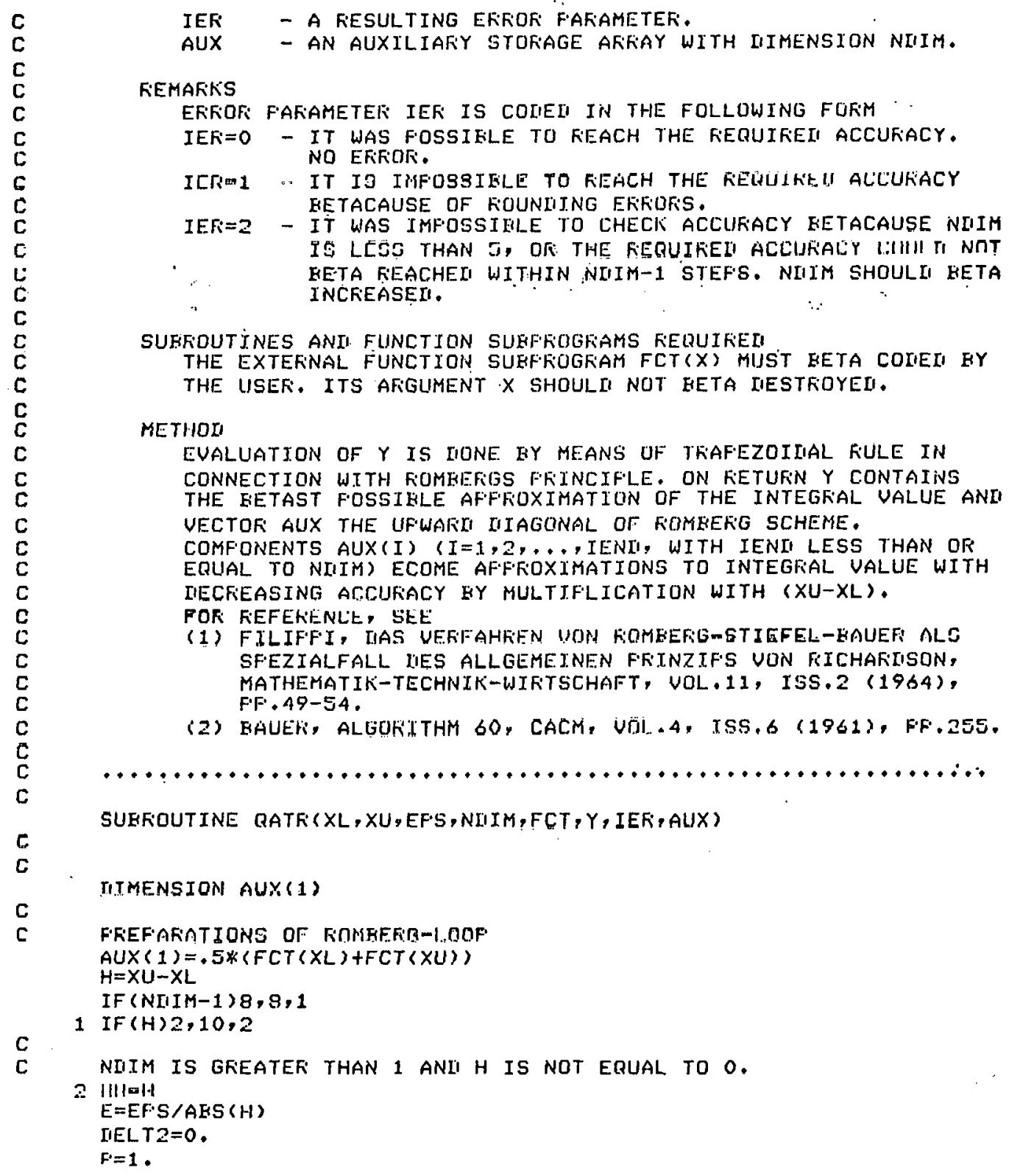




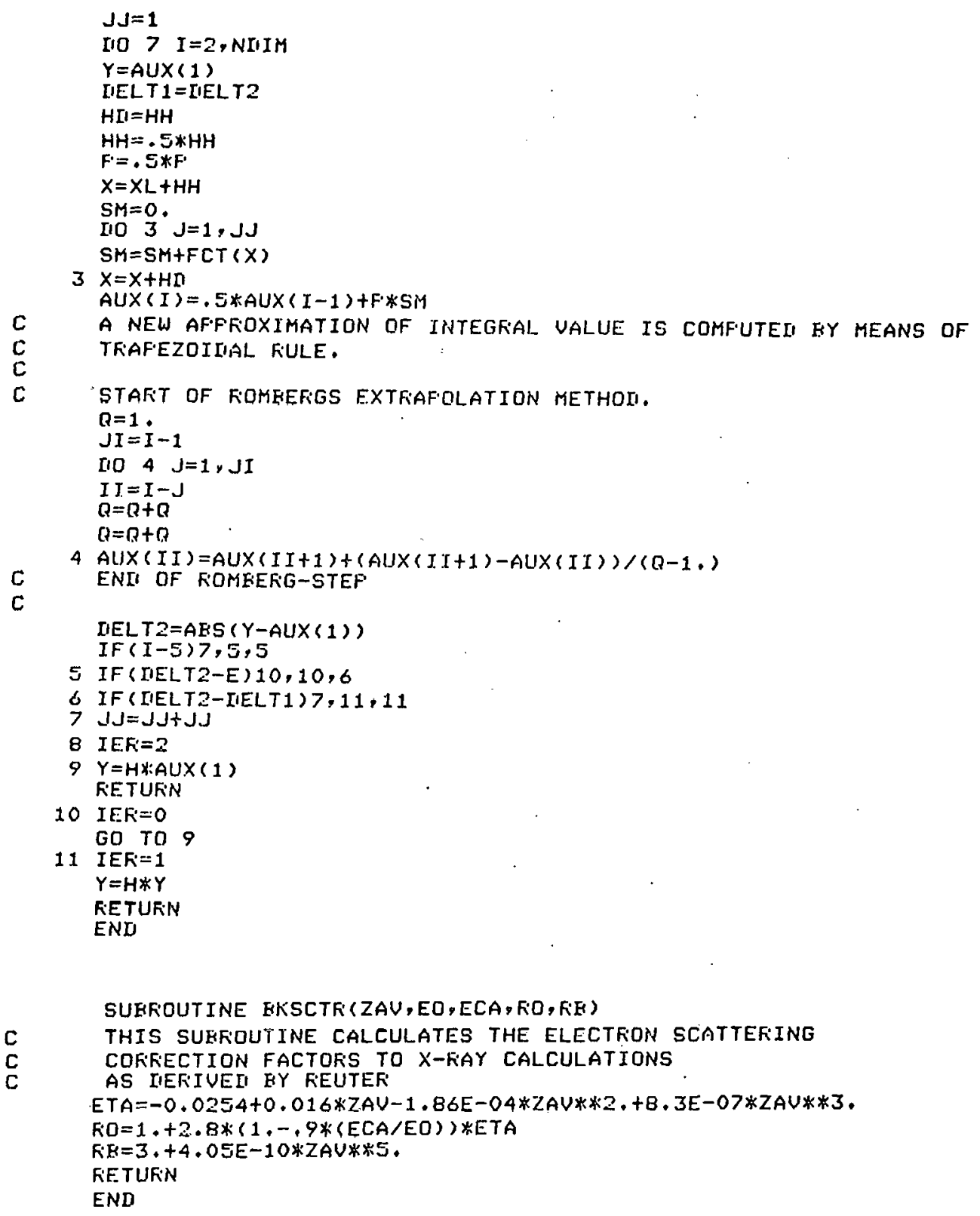




$$
\begin{aligned}
& \text { C } \\
& C \\
& C \\
& C \\
& C \\
& C \\
& C
\end{aligned}
$$

MULTIFLE EY CONUEFSION FACTOR

C $10 * *-50 . /(1.6021 \mathrm{E}-12$ EKGS/EV *4.136E-15 EU SEC $)$

C $10 * *-50 .=k \& W$ CONSTANT

c

$E G=B G * 1.5091 E-24$

FETUFiN

END

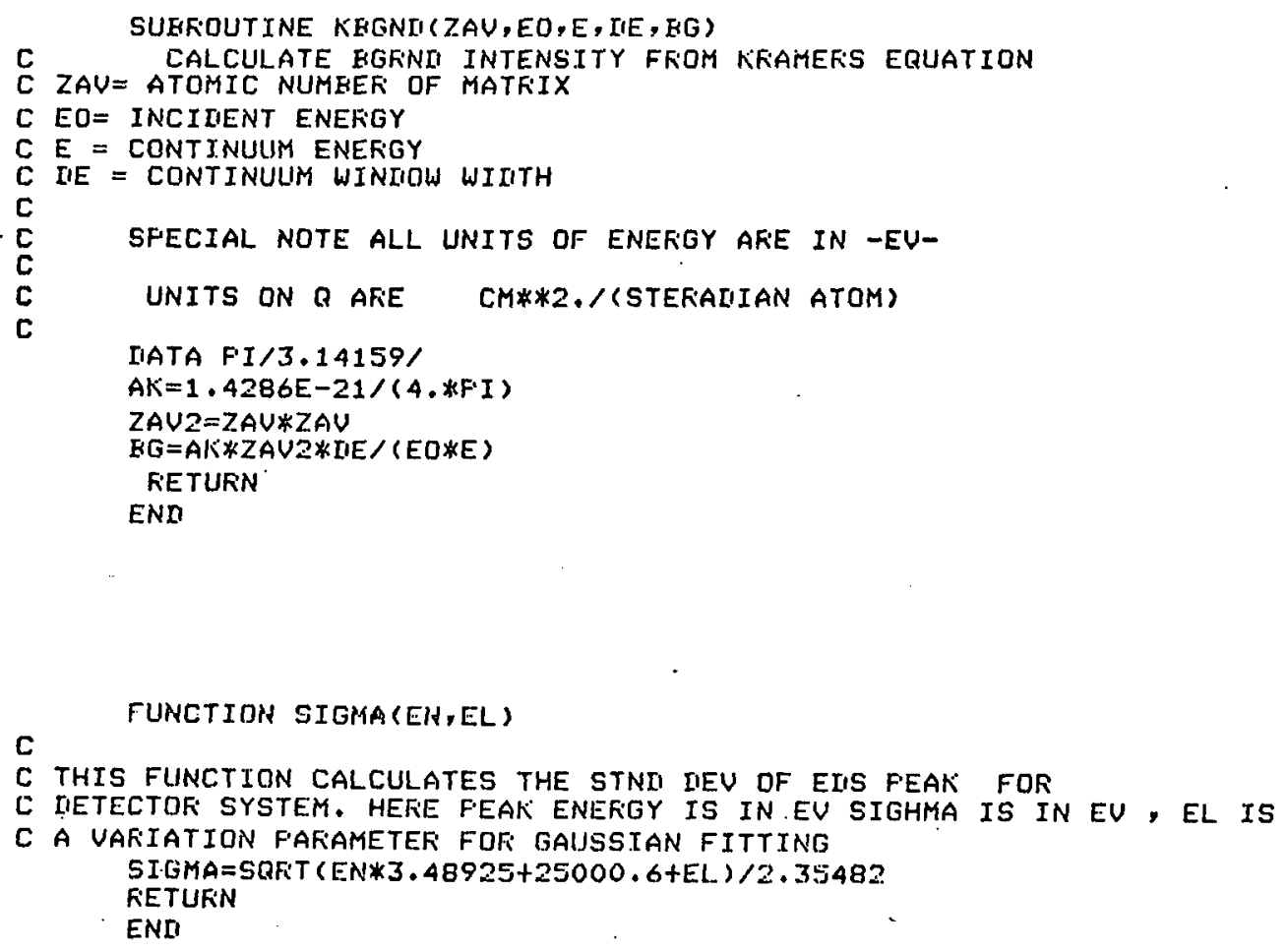

C THIS FUNCTION CALCULATES THE STNII IIEU OF EIIS FEAK FOR

C METECTOK SYSTEM. HEFIE FEAK ENEFGY IS IN.EV SIGHMA IS IN EV, EL IS

C A VARIATION FARAMETER FDF GAIJSSIAN FITTING SI.GMA $=$ SQFiT $(E N * 3.48925+25000.6+E L) / 2.35482$ FETUKN

ENI 


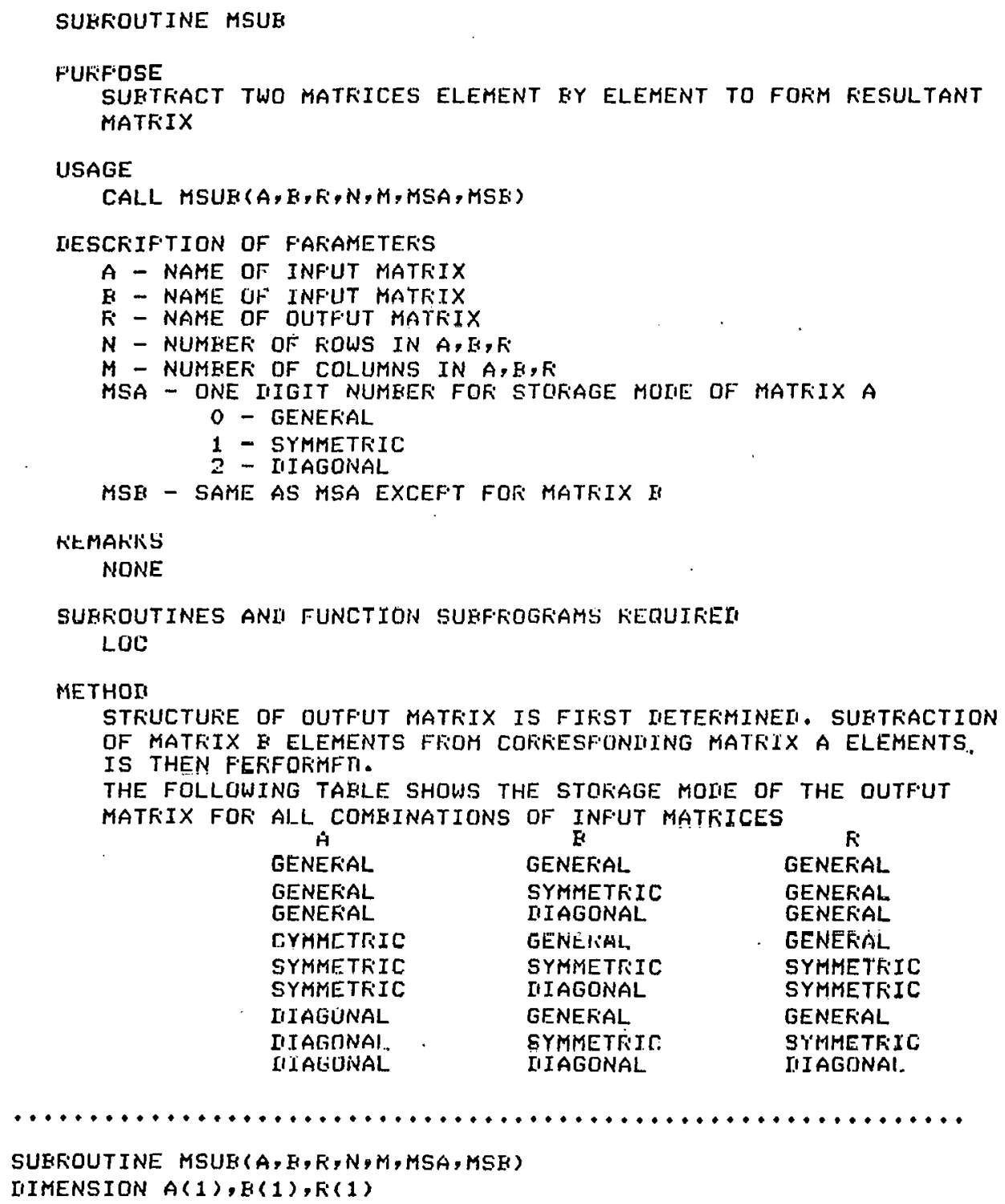

Fi

GENEFIAL GENERAAL GENEFAL GENEFAL SYMMETFIC SYMMETFIC GENER:AL SYMHETFIIC III AGONAI. 


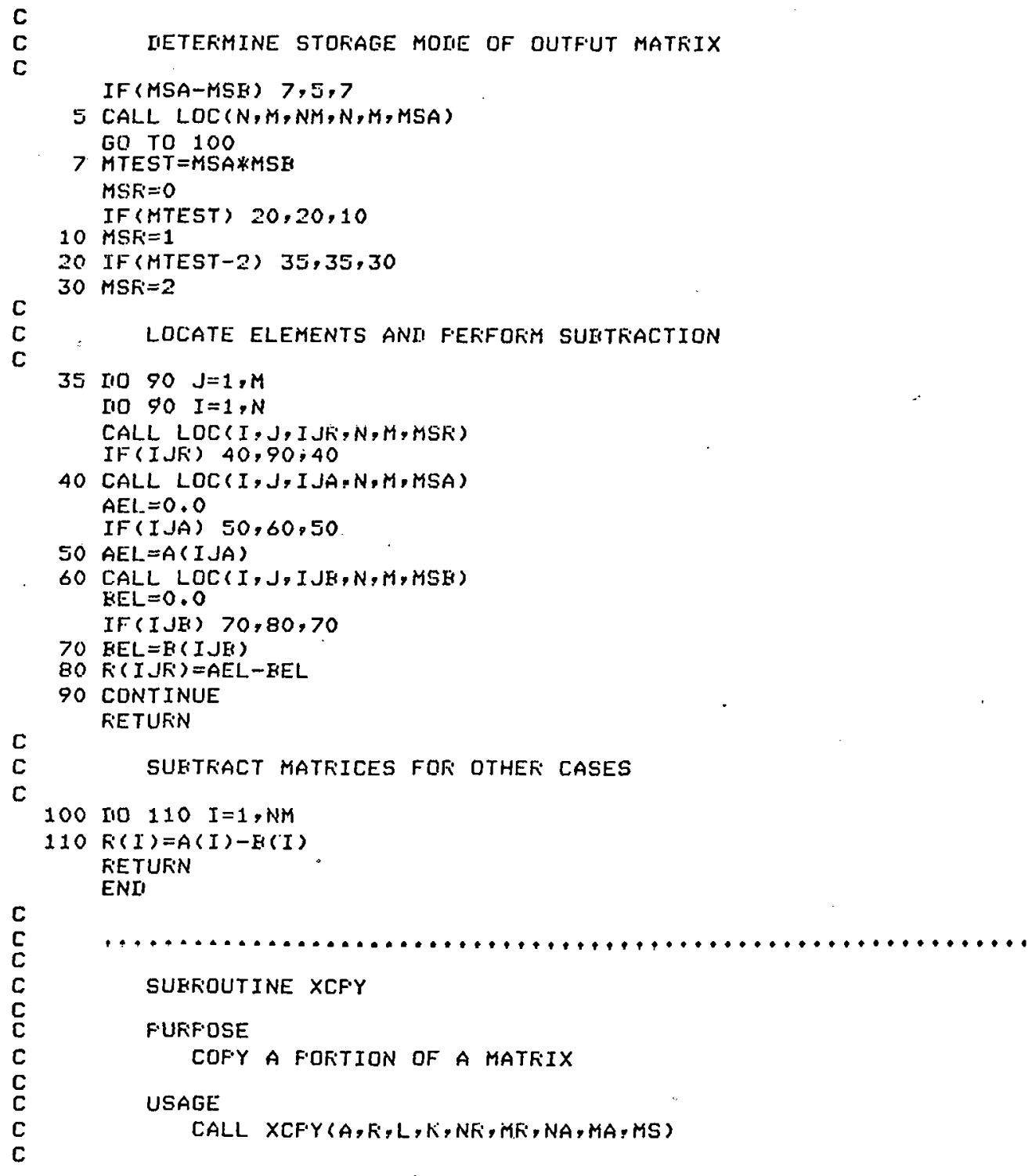




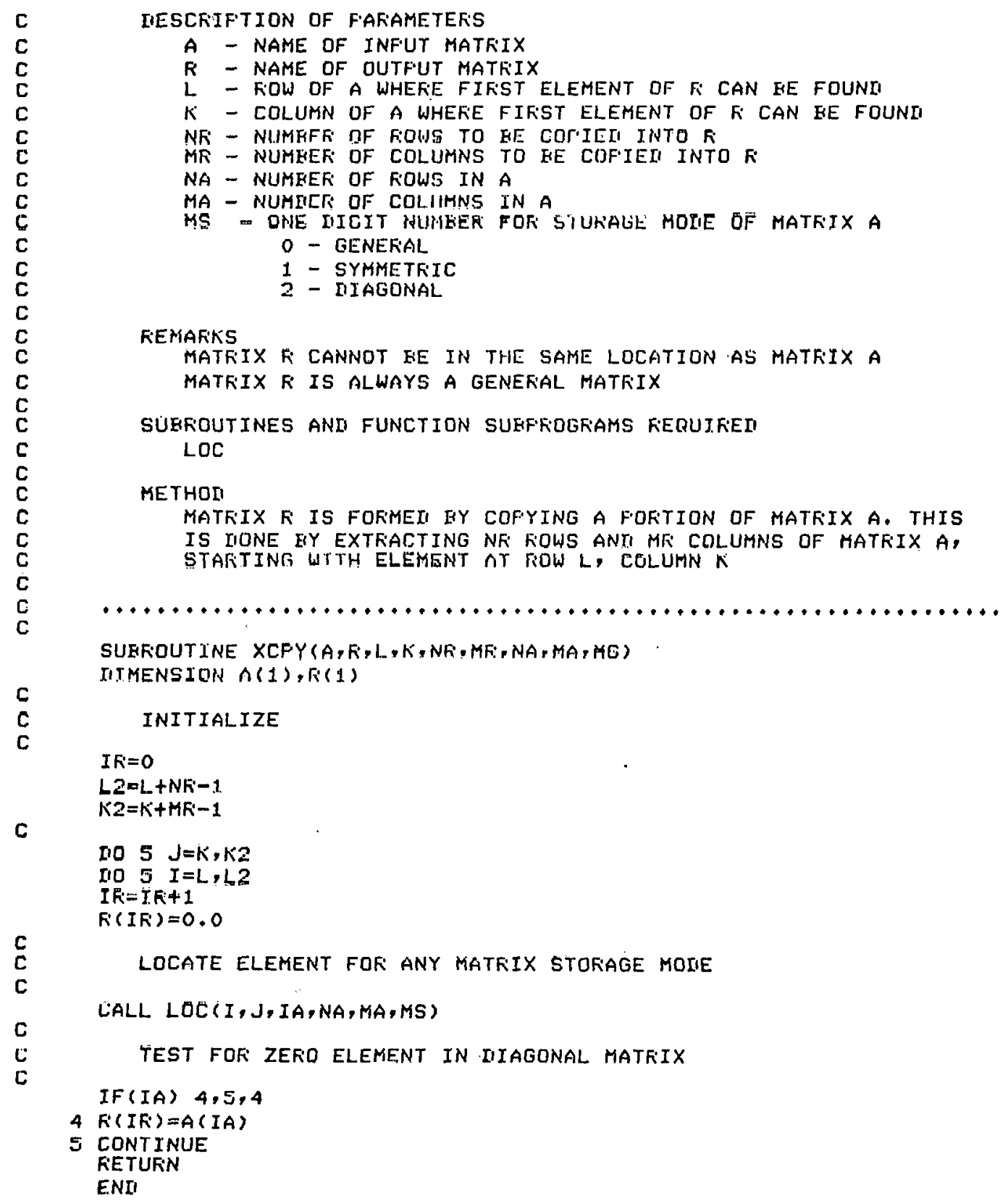




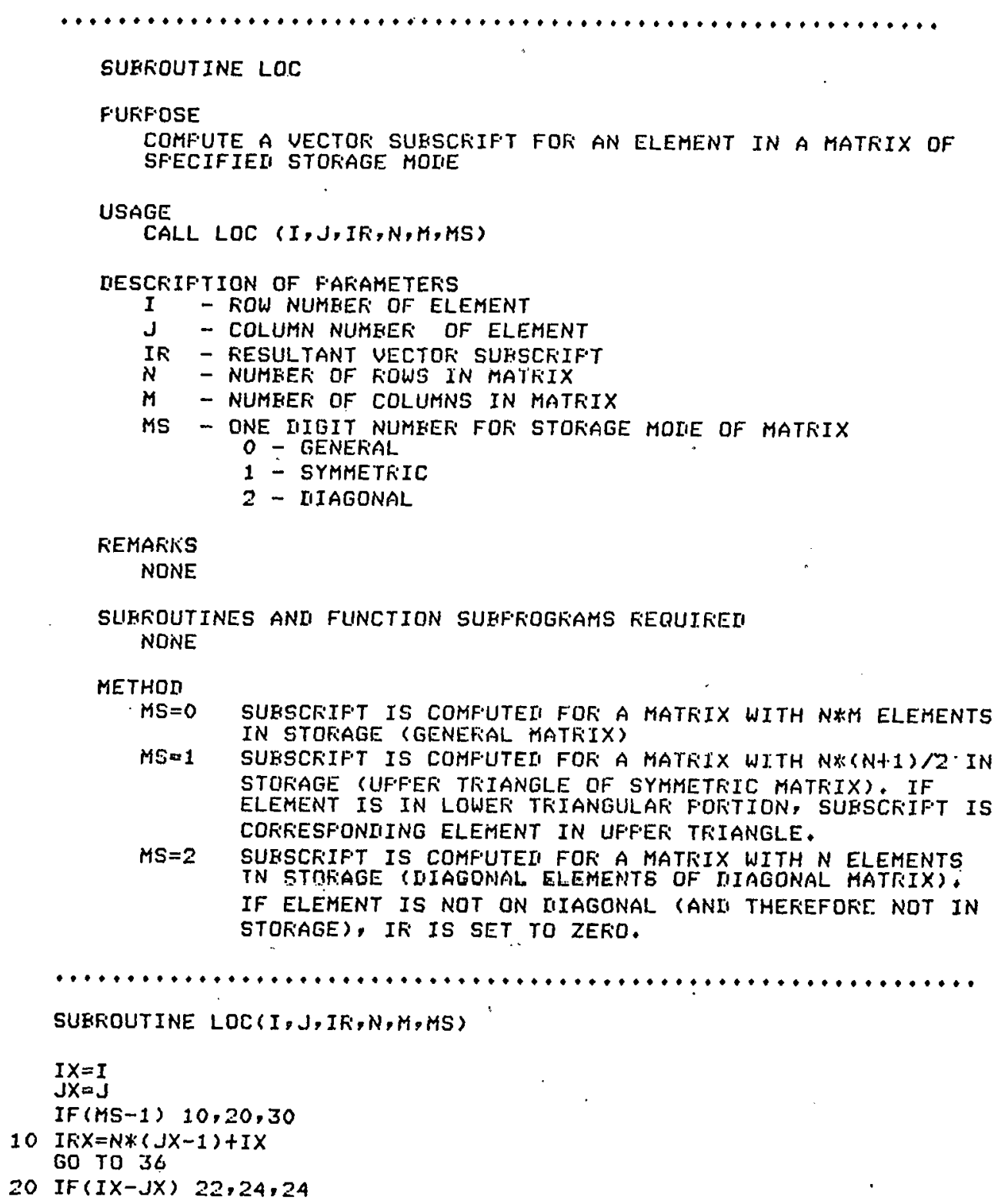

IX $=I$

JXaj

IF (MS-1) $10,20,30$

10 IF $X=N *(J X-1)+I X$

GO TO 36

$20 \operatorname{IF}(I X-J X) \quad 22,24,24$ 


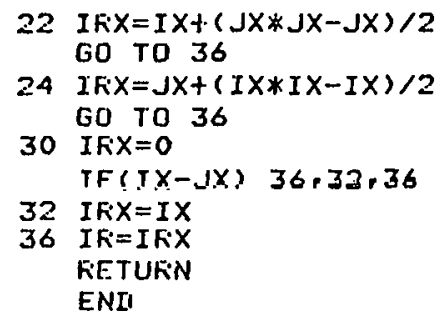

SUEROUTINE NEGTUE (XA.NFT, RIMTT)

C THIS SUEROUTINE SETS THE MAXIMUN NEGTUE VALUE

C STOKEI IN THE AFFAY XA WITH NFT NUMEEF OF FOINTS IIIHENSION XA(1)

INO $1 \mathrm{I}=1$, NF.T

1

IF $(X A(T) \cdot L E \cdot(-1, * R I M I T)) \quad X A(I)=-1$ * *KIIMIT

CONTINUE

FETURN

ENS

FUNCTION ICHN(EN, IRES)

C THIS FUNCTION CALCULATES THE CHNTEL NO. WHICH COFFESFONIS TO

C THE ENEFGY EN, AT RESOLUTION KES,EN IN - KEVICHN $=$ INT (EN*1000 $/$ IRES $)$ RIETURN

ENI

C

FUNCTION CONU (EAES, SAES, IKES, EN, Fi)

C THIS FUNCTION CONUOLUTES A STEF FUNCTION WHICH CHANGES

C IT UALUE FFIMH I TO E. AT AN ENEFEY ENIC, CONVOLUTION IS WITH A C STANIIAEII GAUSSIAAN CHAFACTEFISTIC OF IIETECTOR IFESFONSE.

C ALL ENERGIES IN -EV-

$S 1=0$.

$S 2=0$.

$M 1 M=$ EAES- 3 . ASAES

MAX $=$ EAESH.,$*$ SAES

$M I D=E A E S$

IIO 1 I =MIN,MII, IFES

$S 1=S 1+E X F(-((E N-I) / S I G M A(E N, 0)) * * 2,, / 2$, 


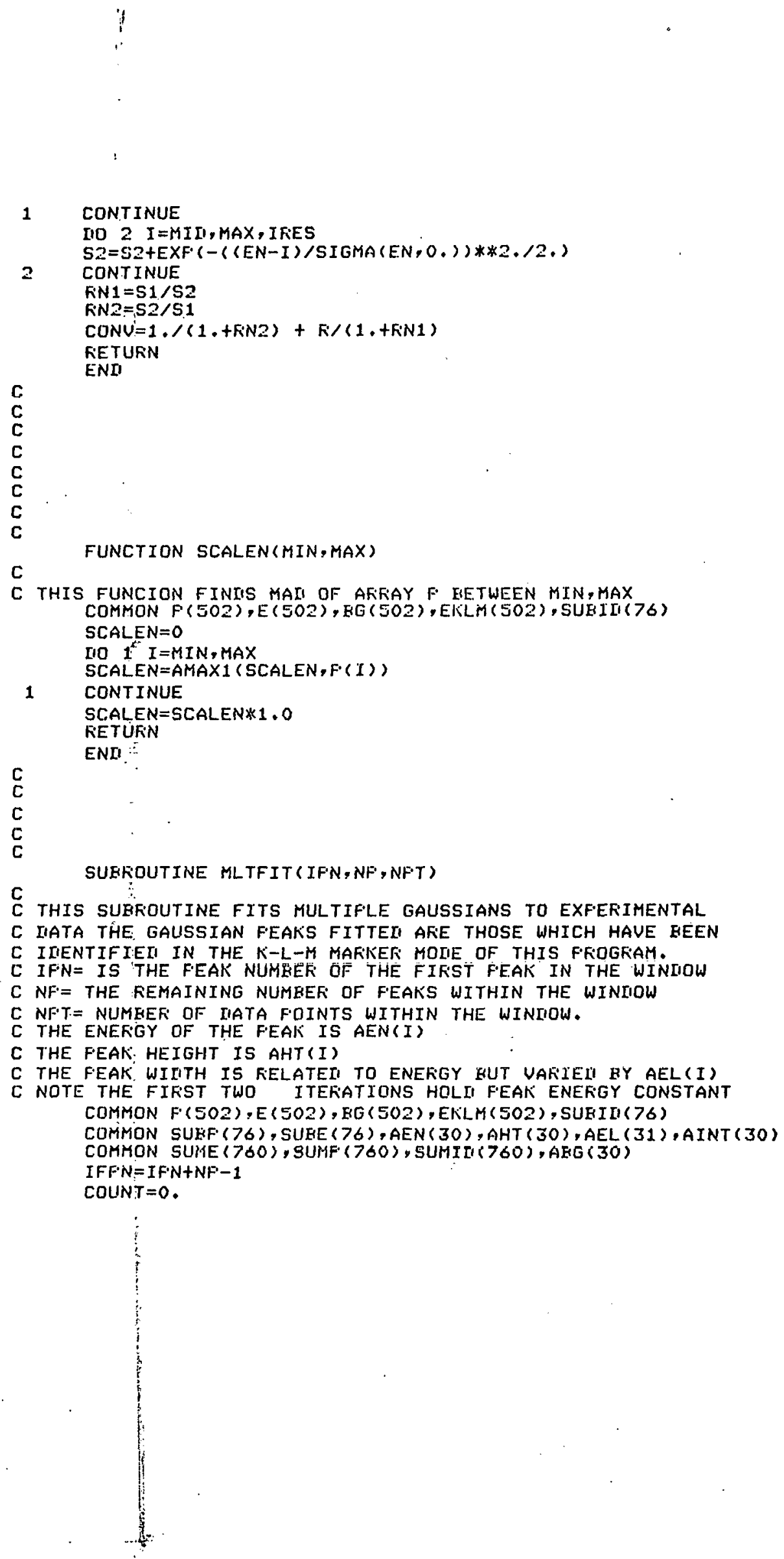




\section{0}

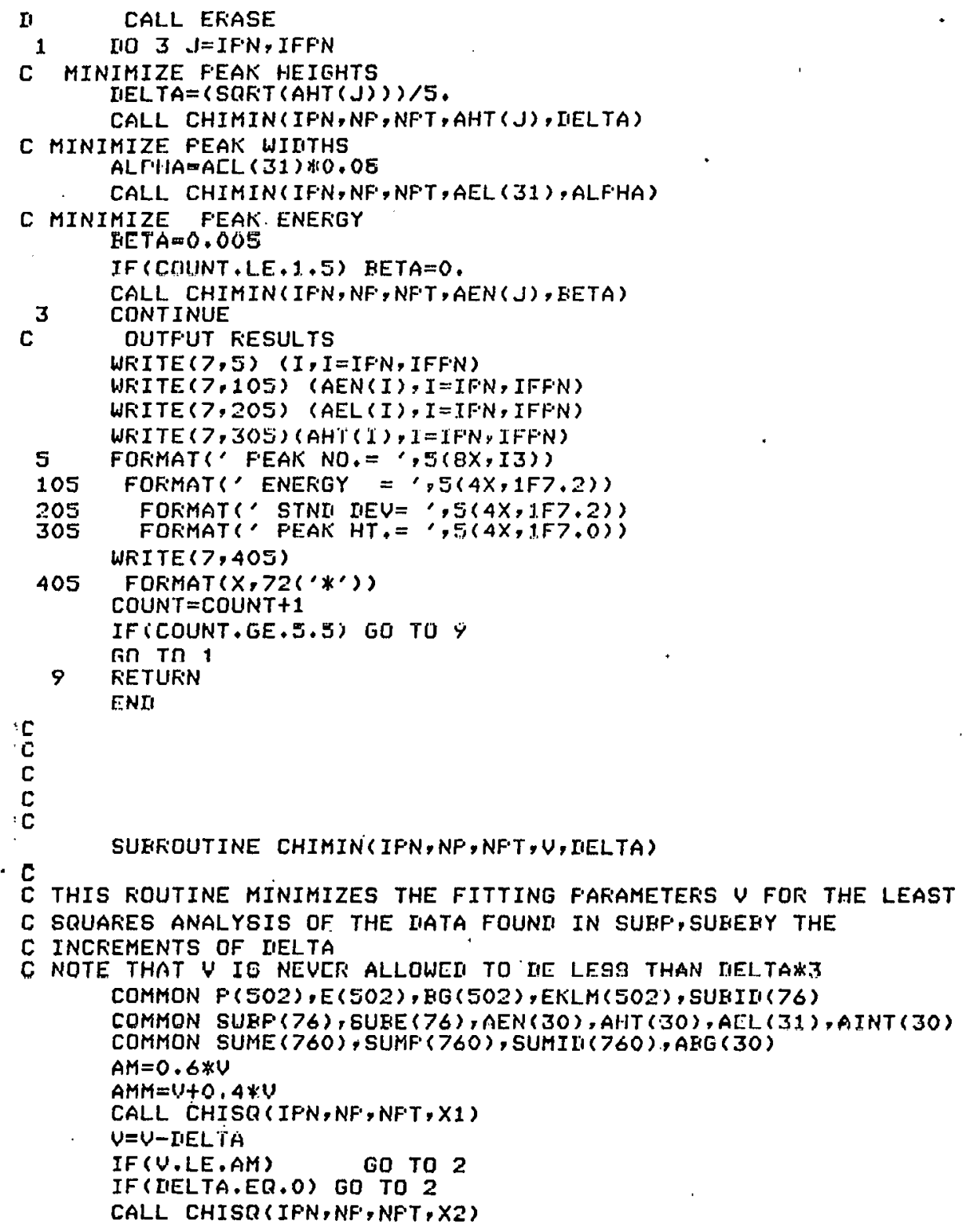




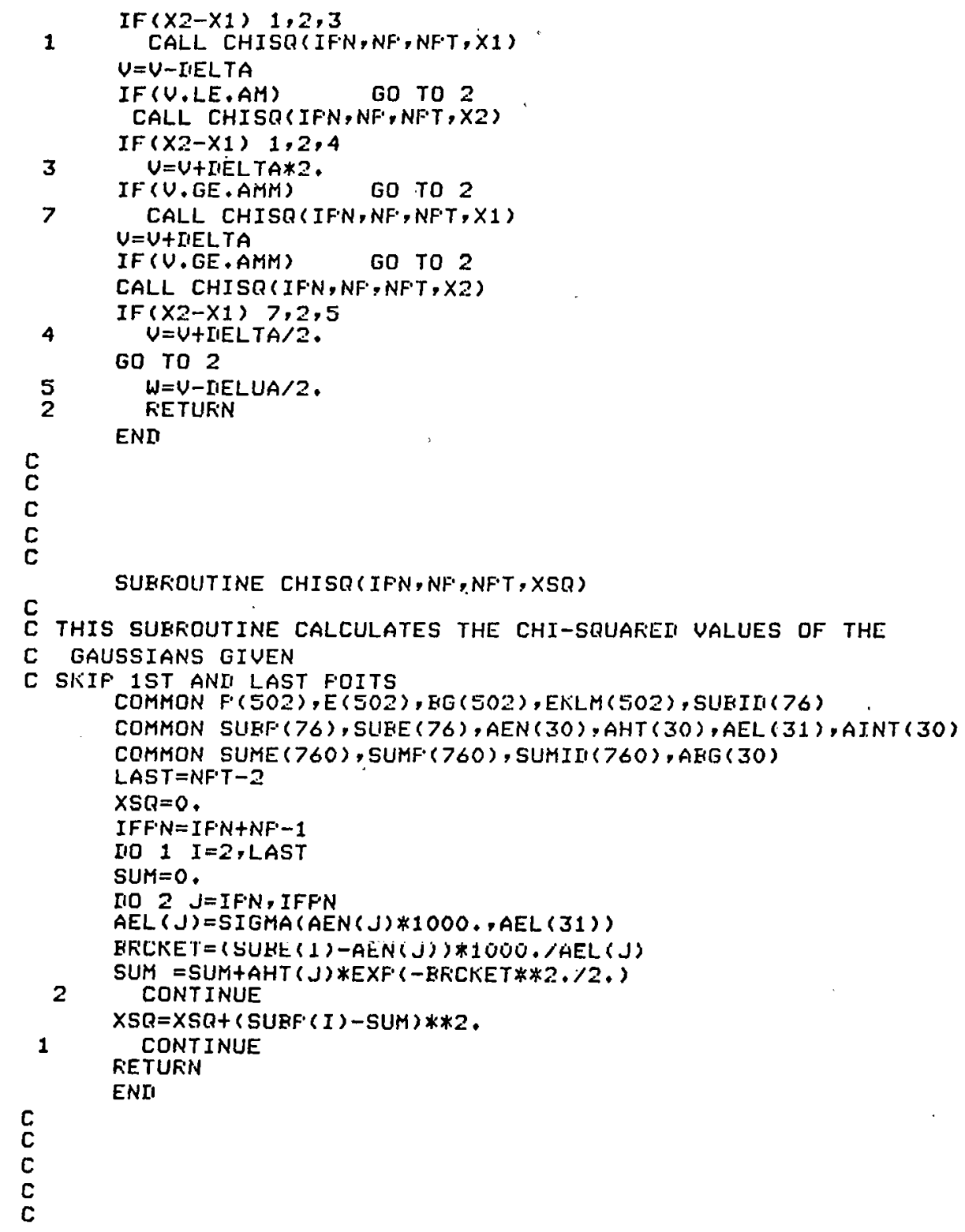


SURKOUTINE FGRNI1 (EO, IFES, EL1, BU1, FL2, BU2, FL 3, BU3, AAA, BEIT, CCC)

C CALCULATES EACKGFIOUNI BY FOL YNOMIAL FIT TO 3 REgIONS/

C INCLUIING EFFECTS OF IIETECTOF EFFICIENCY

C ALL ENEKGIES IN -EV-

COMMON F. (502), E(502), KG(502), EKLM(502), SUEIII(76)

C CALCULATF RII F.AFAMETER:G FDFi LNCII CIICIIO'I RHIVGE

CALL HGFINL' (EO, IKES, EL1, EU1, E11,E12,E13,E1,E23,E2,E33,E3)

CALL EGFNI2 (EO, IFES, FL2, EU2,F11,F12,F13,F1,F23,F2,F33,F3)

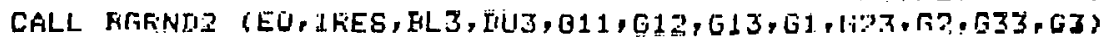

$H \perp 1=E \perp 1++11+611$

$H 12=E 12+F 12+G 12$

$H 13-E 13+F 13+G 13$

HIEE $1+F 1+G 1$

$H 21=H_{12}$

$\mathrm{H} 22=\mathrm{H} 13$

$H 23=E 23+F 23+G 23$

$H 2=E 2+F 2+G 2$

$\mathrm{H} 31=\mathrm{H} 13$

$\mathrm{H} 32=\mathrm{H} 23$

$H 33=E 33+F 33+G 33$

$H 3=E 3+F 3+G 3$

IIELTA1 $=\mathrm{H} 11 *(\mathrm{H} 22 * \mathrm{H} 33-\mathrm{H} 32 * 1223)$

IIELTA2 $=H 12 *(H 21 * H 33-H 31 * H 23)$

DIELTA3 $=H 13 *(H 21 * H 32-H 31 * H 22)$

IIELTA-IIELTA1 IICLTA2+IIEL TH3

$A 1=H 1 *(H 22 * H 33-H 32 * H 23)$

$A:=H 12 *\left(H 2 * \mathrm{H}_{3} 3-\mathrm{H} 3 * \mathrm{H} 23\right)$

$A 3=H 13 *(H 2 * H 32-H 3 * H 22)$

$A A A=(A 1-A 2+A 3) /$ IIELTA

F1 $1=H 11 *\left(\mathrm{H} 2 * \mathrm{H} 33=\mathrm{H} 3 * \mathrm{H}_{2} 3\right)$

$H: 2=H 1 *(H 2+* H 33-H 31 * H 23)$

[i3 $=H_{1} 3 *\left(H_{2} 1 * H_{3} 3-H_{3} 1 * H_{2}\right)$

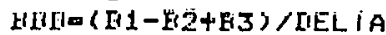

$\mathrm{C} 1=\mathrm{H} 11 *(\mathrm{H} 22 * \mathrm{H} 3-\mathrm{H} 32 * \mathrm{H} 2)$

$\mathrm{C} 2=\mathrm{H}_{12} *\left(\mathrm{H} 21 * \mathrm{H}_{3}-\mathrm{H} 31 * \mathrm{H}_{2}\right)$

$\mathrm{C} 3=\mathrm{H} 1 *\left(\mathrm{H} 21 * \mathrm{H} 32-\mathrm{H} 31 * \mathrm{H}_{2} 2\right)$

$\mathrm{CCC}=(\mathrm{C} 1-\mathrm{C} 2+\mathrm{C} 3) / \mathrm{IIEL}$ TA

CAAL I FI!OT (EבS.,100,,0)

C WFITE $(7,88)$

C 88 FOFMAT' ' CALCULATEI FOLYNOMIAL COEFFICIENTS:' ')

WRITE $(7,89)$ AAA, BEE,CCC

C 89 FORMAT $(2 X, 3(1 X, 1 F E 10,3))$

IIO $90 \quad I=2,500,1$

$F F=(I-1) * I F E$,

CALL MASSAH (FF, AESFF)

CC- - EQW KT I /FF

$E G(I)=(A A A * C C * * 2+B E E * C C+C C C) * E X F$ (AESFF)

$\operatorname{IF}(\mathrm{BG}(I), L E, 0) \quad \mathrm{EG}(I)=1.0$

IF (I.LE.25,) $F G(I)=E G(26)$ 


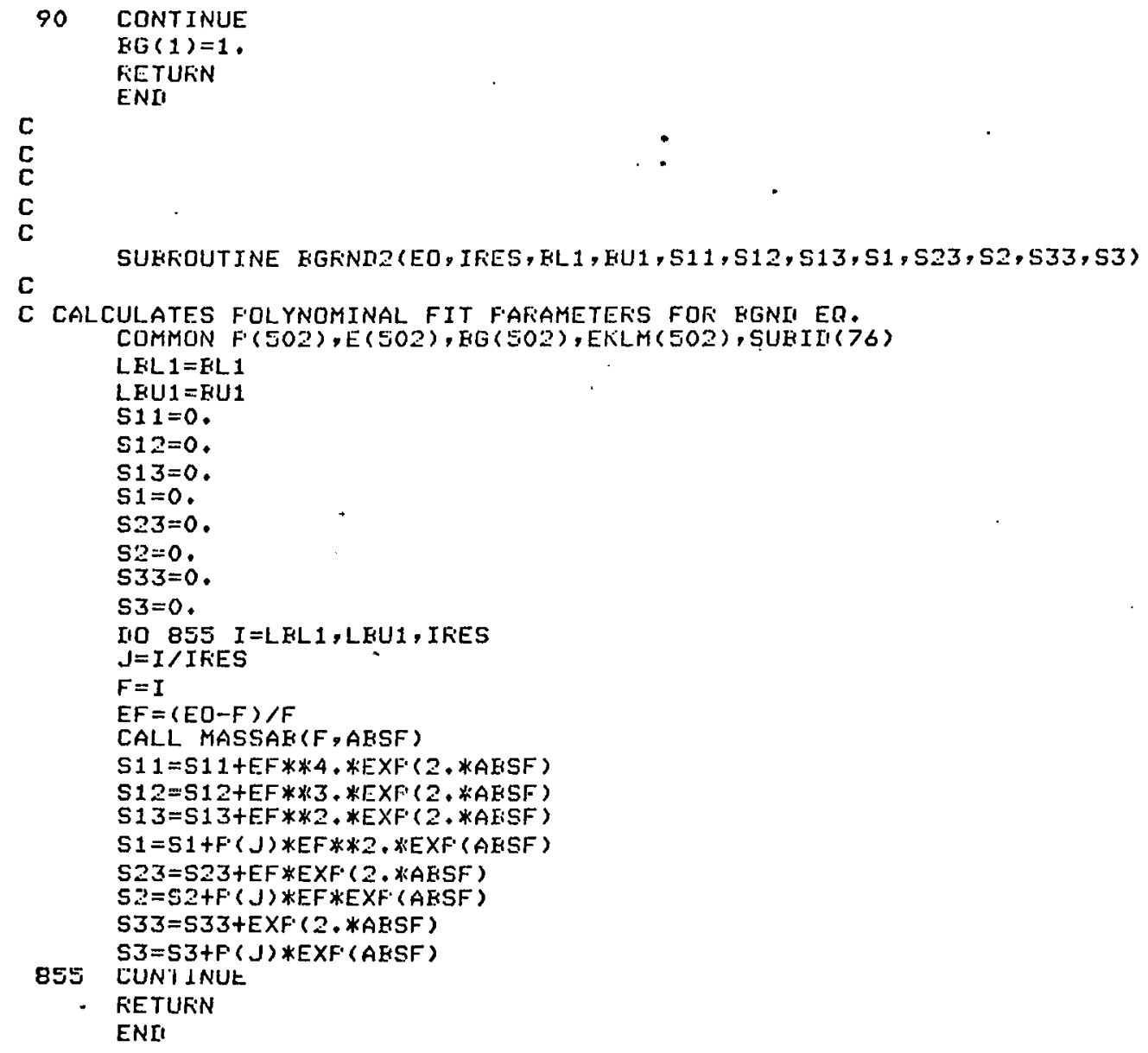

- RETURIN

ENII 
THIS PAGE

\section{WAS INTENTIONALLY LEFT BLANK}




\author{
APPENDIX C \\ Subroutine Library - NGRAPH \\ Subroutine CRTPLT \\ Subroutine SYMPLT \\ Subroutine PLRPLT \\ Subroutine ERASE \\ Subroutine PLOT \\ Subroutine XYTRAN \\ Subroutine TRANXY \\ Subroutine CRSSHR \\ Subroutine POINT \\ Subroutine SPOINT \\ Subroutine LINE \\ Subroutine SYMBOL \\ Subroutine SCALE \\ Subroutine RSCALE \\ Subroutine OFFSET \\ Subroutine AXIS \\ Subroutine TIC.S \\ Subroutine LABEL \\ Subroutine TINPUT \\ Subroutine TOUTPT
}




\section{APPENDIX C \\ Subroutine Library - NGRAPH}

This appendix documents the graphics subroutine library written for use on the DEC LSI-11 microcomputer and Tektronics 4010-1 yraph1es cerminal for use in conjunction with the data analysis programs described elsewhere in these appendices. These routines can also be used on a stand-alone basis for graphics display of calculations performed on the computer system. 
GFAFHICS LIFRARY FOF FIIF LST-11 MICFOCOMFUTER ANY OUEST TONS ANIYOR COMMENTS SHOULI HE IMTEETEI TO

NESTOK .. ZALUZEC

DEFARTMENT OF METALLUEGY

MATEKIALS FESEAFCH LAEQRAPIOFY

UNTUERSITY OF TLLINOIS

UREANA , ILLINQIS G180'1

SURFOUTINE CFTFLT (XZ,YZ,XA, YA,NFT, NMARE IFFLT, XMYYM, SXA, SYA, NS, $1 \mathrm{ILX}, N I L X, I L Y, N I L, Y)$

THIS IS THE GFAFHICS CONTKOL SUEROWTINE WHICH WILE

FFOTUCE A CQMFLETE GFAFH ON' THE TEKTHUNIX 4010-1 TEFMINAL.

INCLUIING AXII,TIC MAKKS ANI LAEELS

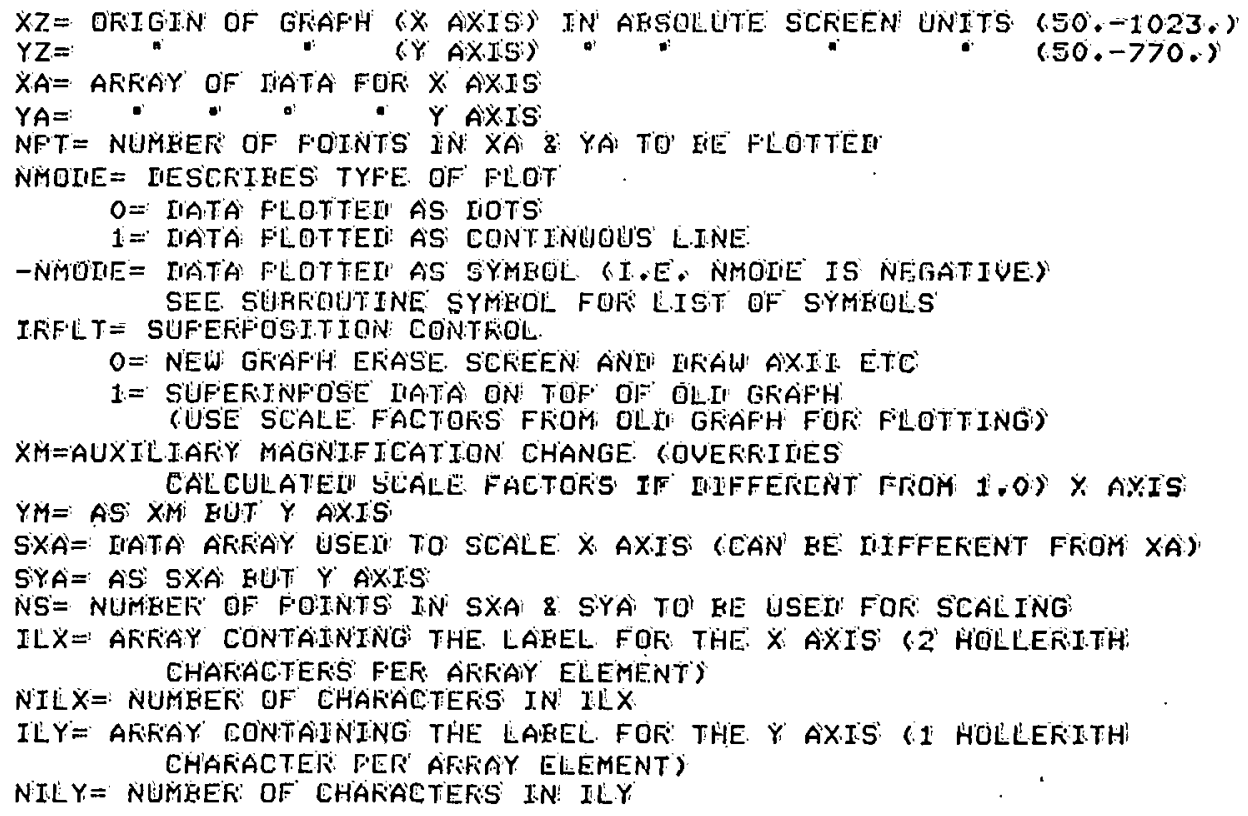


IIMENSION XA(1), YA(1), SXA (1), SYA(1), ILX(1), ILY(1)

COMMON /CFTLST/ XMIN, IIX,YMIN, IYY, OFFX, OFFY, XL,YL

c
C
C
c

c GFIFH LENGTH $X$ AXIS $=800$ UNITS

c GRAFH LENGTH $Y$ AXIS $=500$ UNITS

C

$X L=800$.

$Y L=500$.

c. Galculate scale fattokis

E

CALL SCALE (XMIN, IIX,NS, SXA,XL)

CALL FSCALE (YMIN, IIY, NS, SYA, YL)

$n X=n \times * X M$

II $Y=$ IIY $* Y M$

$c$
$c$

CALL OFFSET (XMIN, IIX, YMIN, IY, XZ,YZ,XL,YL)

C CHECK FOF SUFEFFOSITION

IF (IRFLT,ER.1) GO TO 2

CALL EFAGE

CALL AXIS(1)

CALL TICS

c

CALL LAEEL (ILX,NILX,ILY,NILY)

C LIFAW IATA

2

IF (NMOTE. ER. O) CALL FOINT (NFT, XA,YA)

IF (NMOIIE,ER, 1) CALL LINE (NF.T,XA,YA)

IF (NMOLE.LT.O) NSY YY $=-1$ *NMOTE

IF (NMDOE.LT,O) CALL SFOINT(NFT \&XA,YA,NSYM, B.)

FETUEN

ENII

c

SUEROUTINE SYMFLT $(X Z, Y Z, X A, Y A, N F T, N E M, I F F L T, O K, Y M, X, N X, Y, N Y)$

c

C

$\stackrel{c}{\mathrm{C}}$

c

C

C

c

c

THIS SUEFOUTINE IS SIMTI TAR TU CFTFLT HOWFUFF TNSTENII

OF PLOTTINL $Y$ US, $X$ IT IIRAWS A SYMEOL THE SIZE OF WHICH

IS FROFORTIONAL TO THE INTENSITY OF $Y$ US. $X$ FOSITION

ITS SFECIFIC USE IS IN STKUCTURE FACTOF CALCULATIONS

IN ELECTRION IIIFFRIACTION F'ATTERNS

$X Z, Y Z, X A, Y A, N F T, I F F L T, Y M r$ AE TIEFINEI IN CHIILT

NSM= TYFE OF SYMFOL TO BE FLOTTED

IIK = IS THE IIARK LEVEL INIIICATOO

I.E. A VALUE OF YA.(I) IIK IS CONSIDEREI TO EE ZERO INTENSITY 


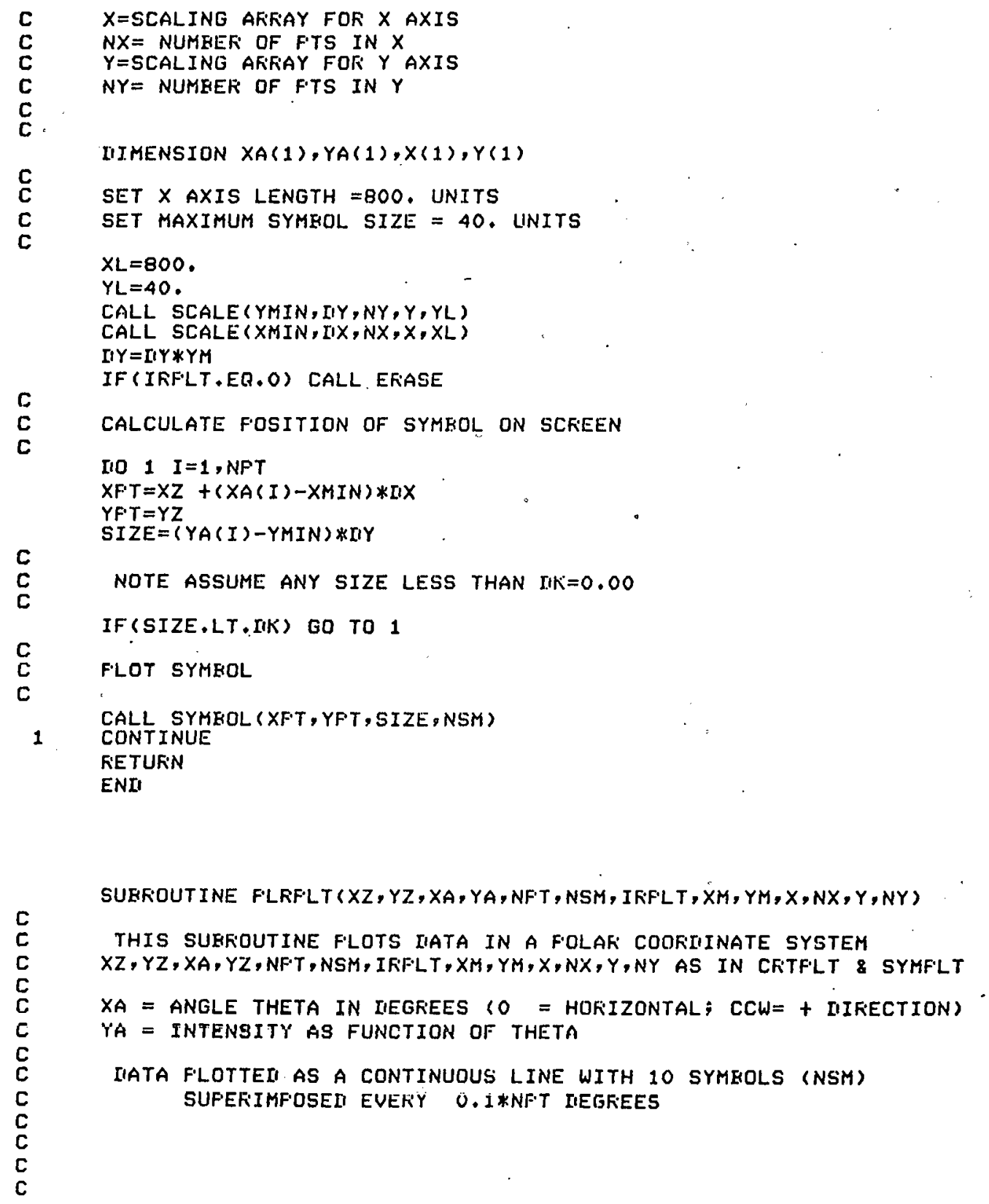




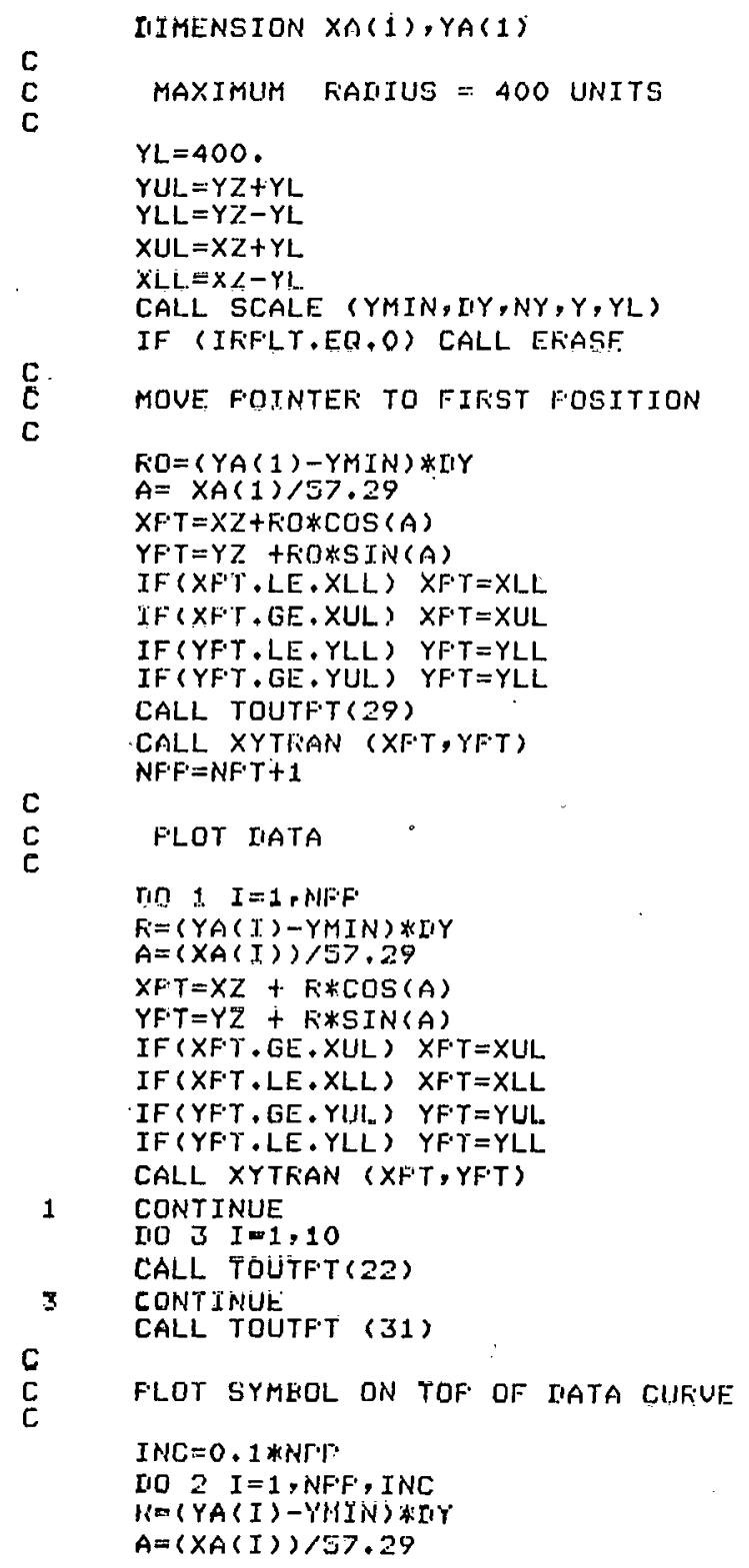




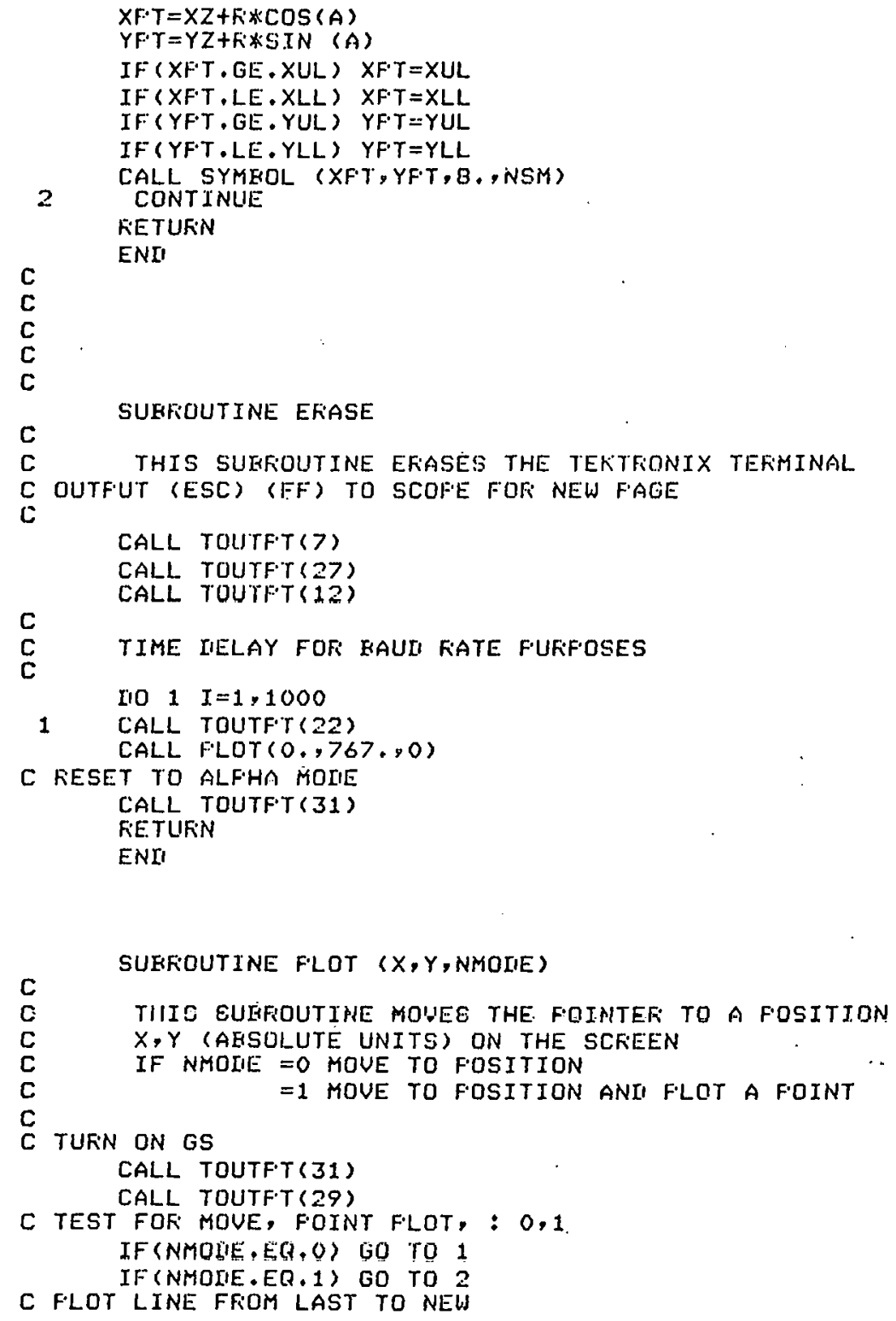




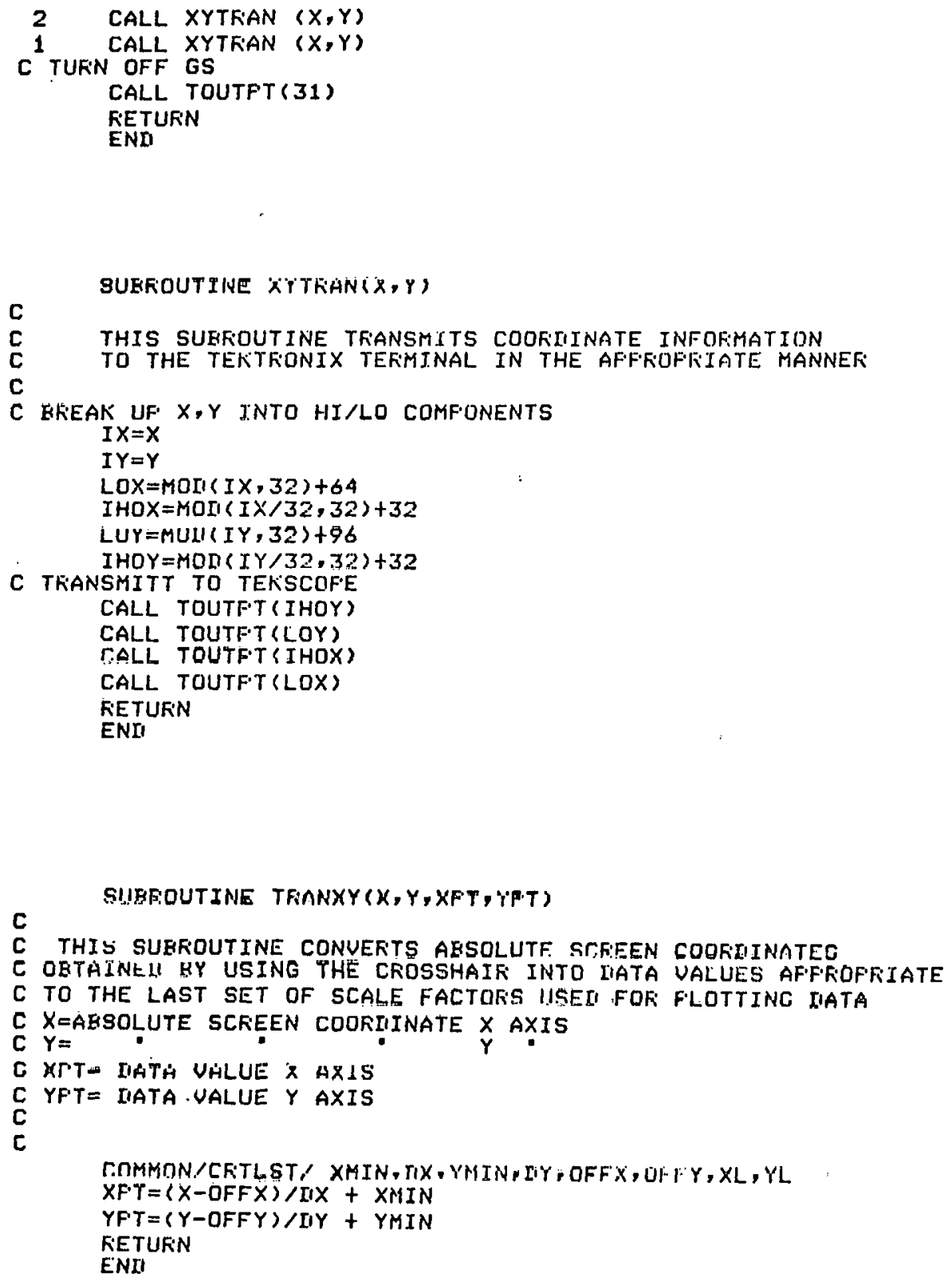




\section{C}

SUEROUTINE CFSSHR $(X, Y)$

C THIS SUEROUTINE LIGHTS UF THE CFIOSSHAIF

C FOSITION THE CFOSSHAIF EY MOUING THE THUMBWHEELS

C ON THE TERMINAL THEN STRIKE ANY FIINTING KEY (OF HIT RETURN)

C THE VALUE OF THE ABSOLUTE SCFEEN COOFIIINATE AFE THEN TFANGMITTED

C TO $X \& Y$

C $X=X$ AXIS AESOLUTE COOKRINATE

C $Y=Y$ AXIS AESOLUTE COOFIIINATE

CALL TOUTFT $(27)$

CALL TOUTPT (26)

CALL TINFUT (IC, IHX, ILX, IHY,ILY)

$X=32 *(I H X-160)+(I L X-160)$

$Y=32 *(I H Y-160)+(I L Y-160)$

FETURN

END

\section{C}

SUEROUTINE FOINT (NFT, AX, YA)

C THIS SUEFOUTINE FLOTS AN ARFAY OF FOINTS WHERE

C NFT =NUMEER OF FOINTS IN THE AFIRAY

$C A X=$ AFRAY OF IIATA FOR THE $X$ AXIS

C YA= ARRAY OF TATA FOR THE Y AXIS

$\mathrm{C}$

C SFECIAL NOTE: THE VALUES OF XMIN,IIX,YMIN,IIY, OFFX,OFFY,XL,YL

C WHICH AFE FEQUIFED FOF FLOTTING AKE INTEFINALLY SET EY

C CALLING THE SUBROUTINES SCALE ANI OFFSET

C

C

COMMON /CFTLST/ XMIN,IIX,YMIN,IIY,OFFX,OFFY,XL,YL

IIIMENSION AX(NFT), YA(NFT)

XENII $=O F F X+X L$

YENI! = DFFY $+Y L$

IIO $1 I=1$, NFT

$X F T=O F F X+(A X(I)-X M I N) * I I X$

$Y F T=D F F Y+(Y A(I)-Y M I N) * I Y$

$X F T=I N T(X F \cdot T)$

YF'T =INT (YF'T)

IF (XF'T,GE,XENII) XF'T=XENI

IF (YFT, GE, YENII) YF.T $=Y E N I$

IF (XFT. LE, OFFX) XF'T =OFFX

IF (YF'T.LE.OFFY) YF' $=O F F Y$

CALL TOUTF'T (29)

CALL XYTKAN (XF'T,YFT)

CAILL XYTFAN (XF'T,YFT)

CALL TOUTFT ( 31 )

1 CONTINUE

RETURN

END 
SUEROUTINE SFOINT (NFT, AX, YA,NTYFE,SIZE)

C

C THIS SURFOUTINE FLOTS AN AFFIAY OF FOINTS WHEKE

C NFT=NUMEEF OF FOINTS IN THE AFIFAY

C AX = AFIFAY OF IIATA FOF THE $X$ AXIS

C YA= AFFEY OF IIATA FOF THE $Y$ AXIS

C NTYFE $=S Y M B O L$ TO EE FLOTTEI AT THE FOINT

C SIZEESTZE OPF THE SYMEOLL TO EE FLOTTEL!

c

C SFECIAL NOTE: THE UALUES OF XMIN,IIX,YMIN,LY, OFFX,OFFY,XL,YL

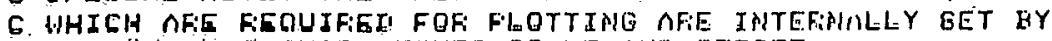

C CALLING THE SUEFOUTINES SCALE ANI OFFSET

c

COMMON /CFITLST/ XMIN,IIX, YMIN, IIY, OFFX, OFFY, XL, YL

IIIMENSION AX (NF.T), YA(NF.T)

$X E N I I M O F F X+X L$

YENII $=O F F Y+Y L$

IIO $1 \quad I=1$, NF.T

$X F T=O F F X+(A X(I)-X M I N) * I X X$

$Y F T=O F F Y+(Y A(I)-Y M I N) * I Y$

$X F T=I N T(X F T)$

$Y F T=I N T(Y F \cdot T)$

IF (XF'T, GE, XENII) XF' $Y=X E N D$

IF (YF.T , GE , YENII) YF:T $=Y E N I$

IF (XF.T,LE, OFFX) XF'T=OFFX

IF (YFT.LE.OFFY) YFT $=$ OFFY

CALL SYMEOL ( $X F$ T, YF.T, SIZE, NTYFE)

1 CONTIRUE

FIETUFIN

END

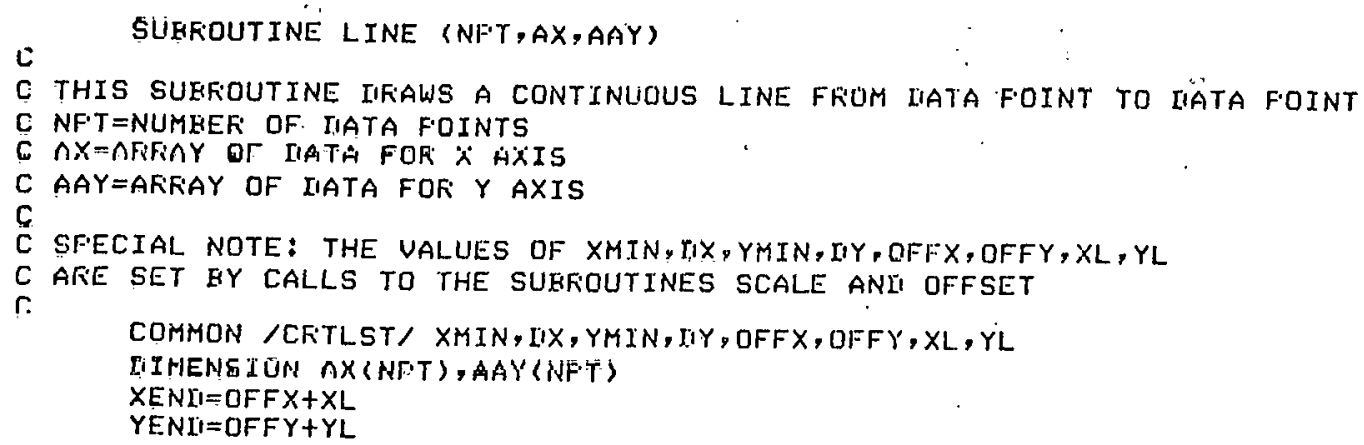


$X L A S T=O F F X+(A X(1)-X M I N) * I I X$

$Y L A S T=O F F Y+(A A Y(1)-Y M] N) * I I Y$

IF (XLAST.GE . XENII) XLAST $=X E N I$

IF (YLAST.GE, YENI) YLAST $=Y E N I$

IF ( $X L A S T$. LE , OFFX) XILAST $=0 F F X$

IF (YLAST.LE. OFFY) YLAST $=$ OFFY

CALL TOUTF.T(29)

CALL XYTRAN(XLAST, YLAST)

IIO $1 \quad I=1, N=Y$

$X F \cdot T=O F F X+(A X(I)-X Y I T N) * T I X$

$Y F \cdot T=D F F Y+(A A Y(I)-Y M I T) * I Y Y$

$I F(X F \cdot T, G E, X E N I I) \quad X F \cdot T=X E N I$

IF (YF.T.GE, YENII) YF.T =YENI

$I F(X F Y, \cdot L E, O F F X) \quad X F Y=O F F X$

IF (YFY.LE. UFFY) YFT=OFFY

CALL XYTFAN (XF.Y, YFT)

1 CONTINUIE

C INSEFT TJ IHE IIELAY FOF BAUII FATE FUEFFOSES

IIO $2 \quad I=1,10$

2 CALL TOUTFT(22)

CALL TOUTFT(31)

FEETLIFN

ENI

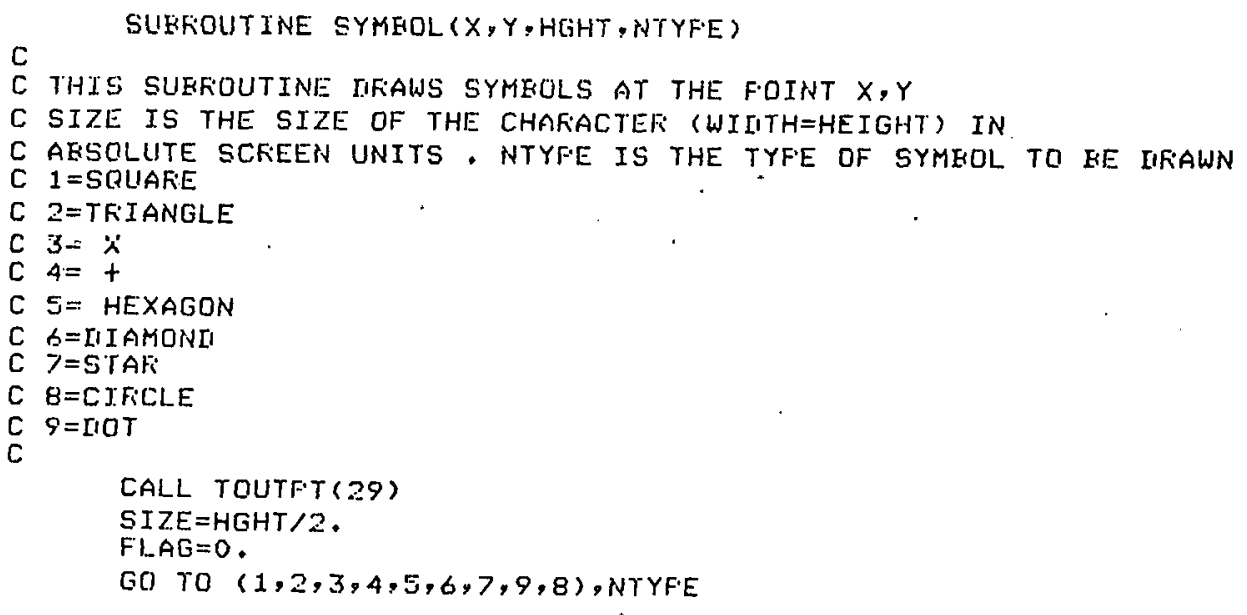




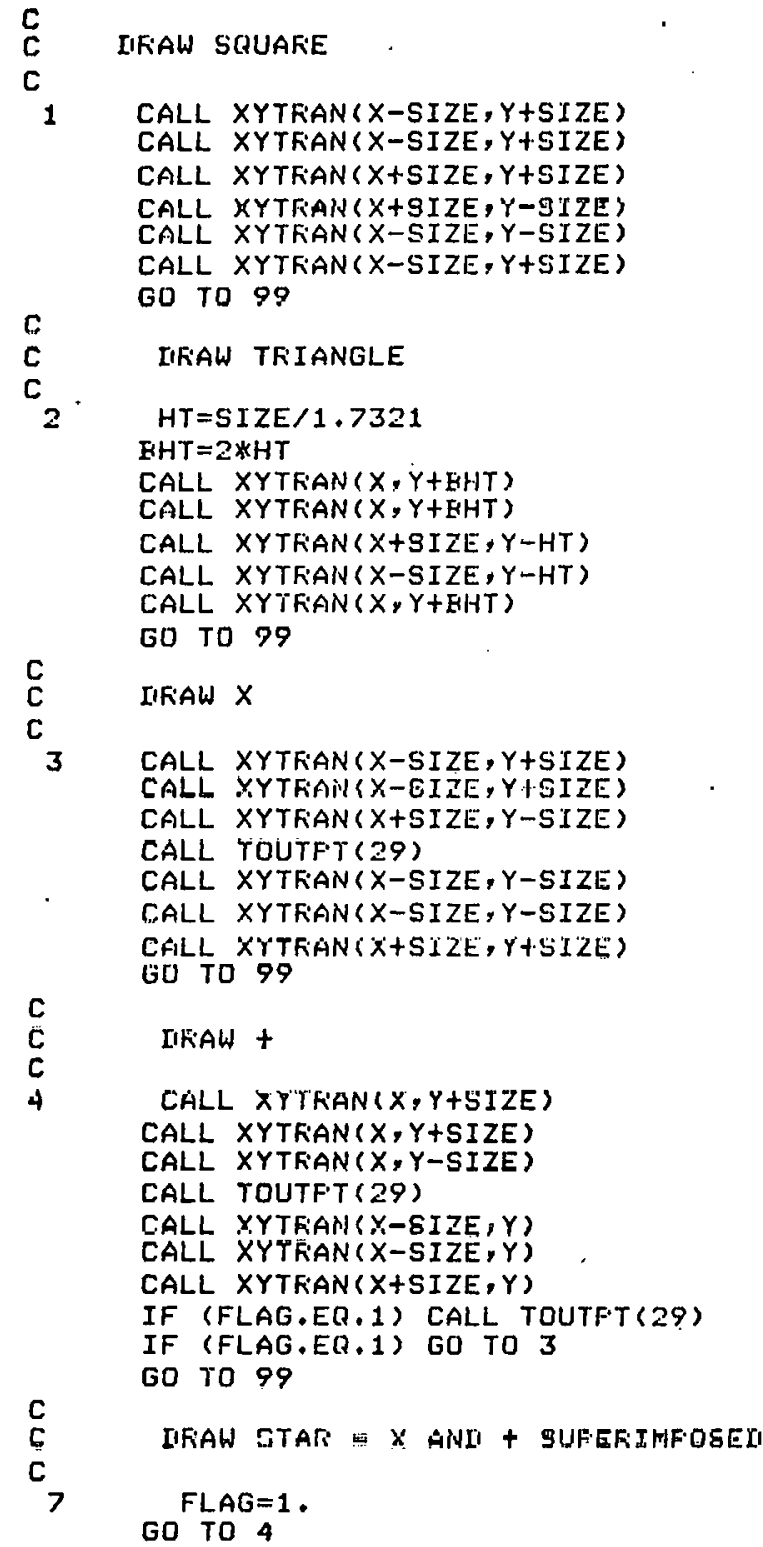




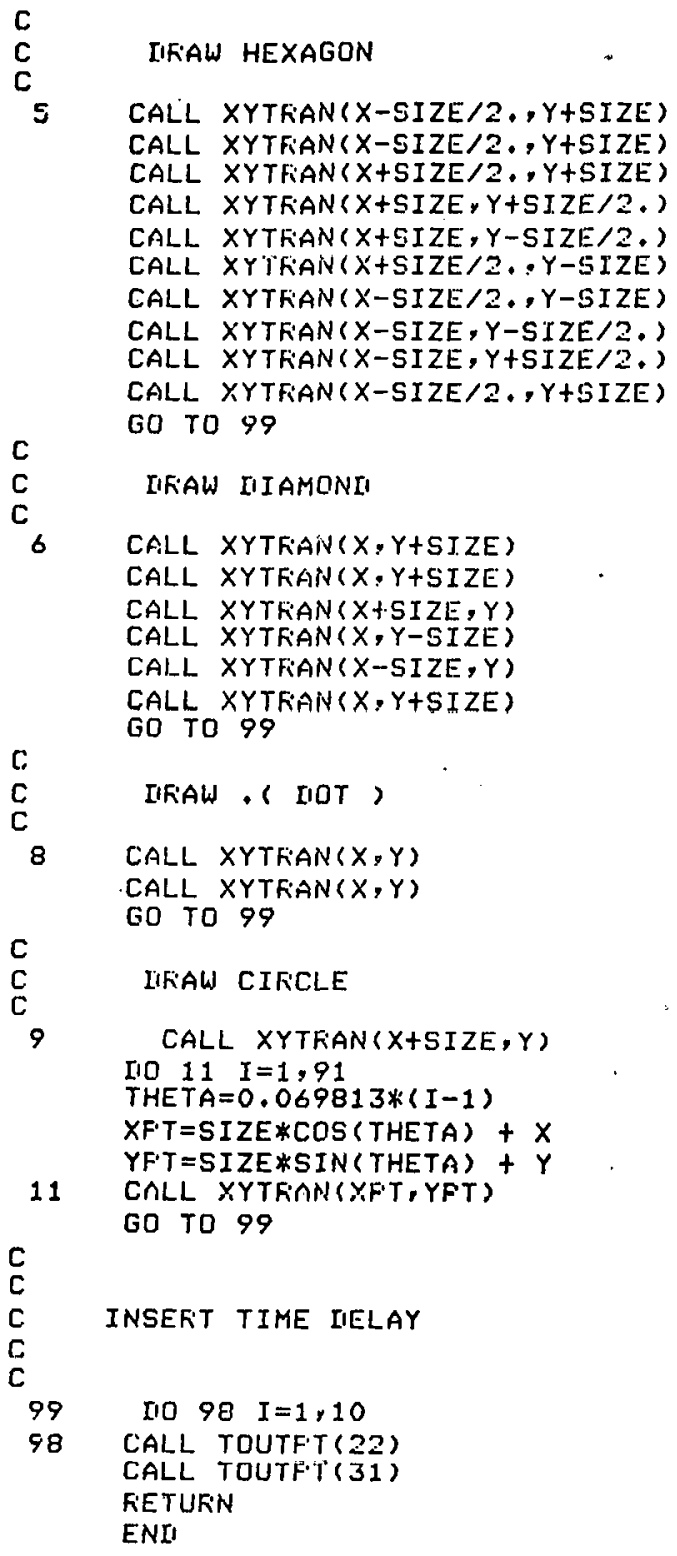




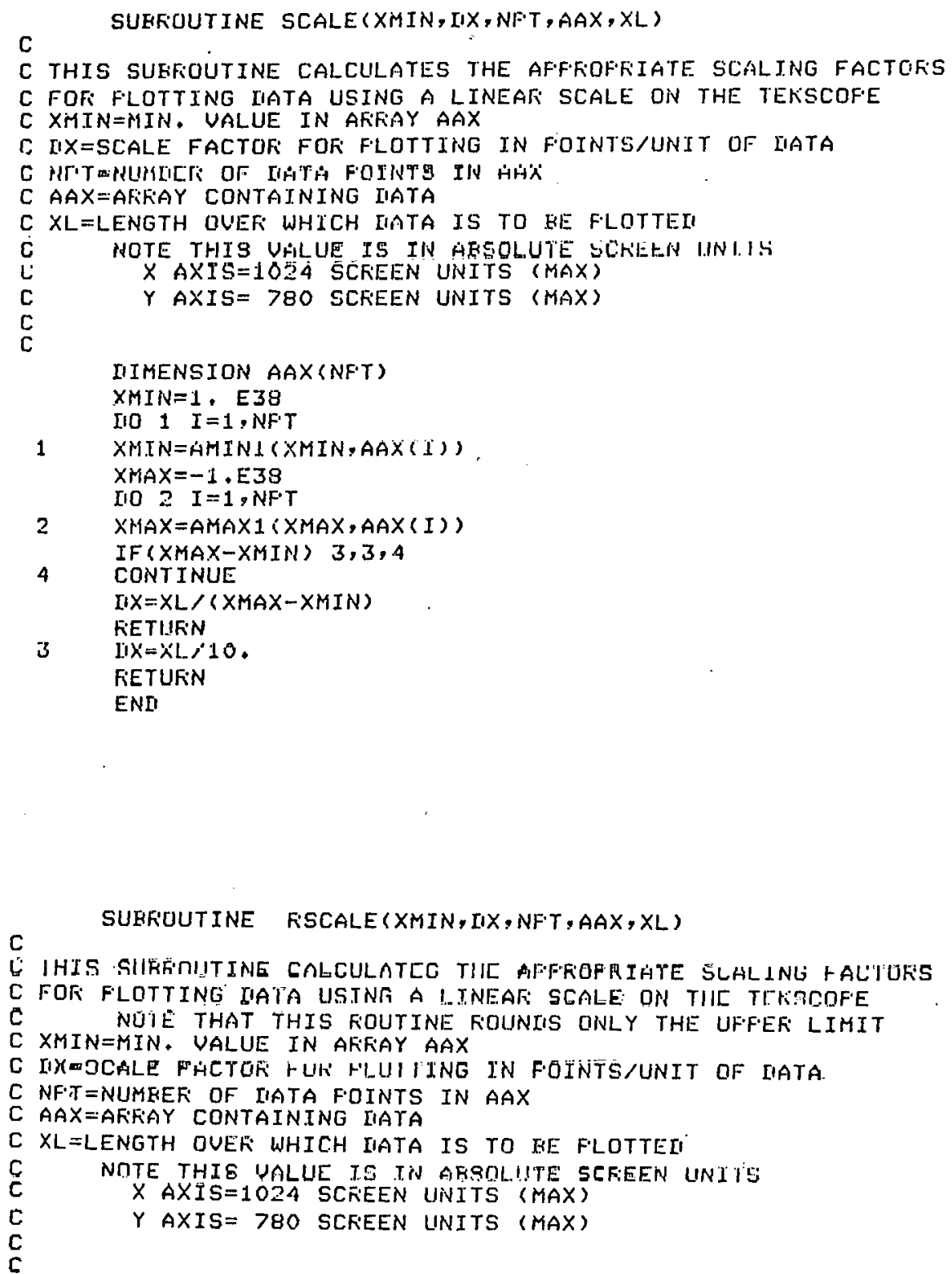


IIIMENSION AAX (NFT)

$X M I N=1$. $E 38$

INO $1 I=1, N F T$

1

$X M I N=A M I N 1(X M I N, A A X(I))$

$X M A X=-1, E 33$

IIO $2 I=1, N F T^{\top}$

$2 \quad X M A X=A M A X 1(X M A X, A A X(I))$

IF (XMAX-XNIIN) $3,3,4$

4 CONTINUE

IF (AES (XMAX) , GE, 100,) GO TO 5

IF (AES $(X M A X), L T, 1.0)$ GO TO 9

XMAX $=10$. WINT (XMAX/10, +1.)

GO TO 6

$9 \quad \mathrm{CNT}=-1$

11 XMAX $=X M A X * 10$.

IF (AES (XMAX).L.T.1.0) GO TO 10

XMAX $=$ INT ( XMAX +1.)*10.*XCNT

GD TO \&

$10 \quad$ CNT $=$ CNT -1 .

GO TO 11

$5 \quad C N T=1$

$7 \quad X M A X=X M A X / 10$.

IF (ABS (XMAX).LT.100.) GO TO 8

CNT $=$ CNT +1 .

GO TO 7

8 XMAX $=$ INT (XMAX +1.)*10.**CNT

6 CONTINUE

IIX $=X L /(X M A X-X M I N)$

RETUKIN

$3 \quad \mathrm{LIX}=\mathrm{XL} / 10$

FETUFN

ENI

$\mathrm{C}$

SUEFOUTINE OFFSET $(A, E, C, D, E, F, G, H)$

C THIS SURFOUTINE STORES THE VALUES OF XMIX, IXX,YMIN, IIY, OFFX, OFFY, XL, YL

C IN THE AFF'FOFFIIATE OKIIEK

C XMIN =MIN VALUE OF IIATA AFFIAY FOF $X$ AXIS

C IIX =SCALE FACTOK FOK $X$ AXIS

C YMIN=MIN. VALUE FOF Y $Y$ AXIS

C IIY SCALE FACTUK FOK Y AXIS

$C$ OFFX=OFFSET OF ORIGIN USEII FOF SHIFTING THE OFIGIN ALONG $X$ AXIS

C OFFY $O F F S E T$ OF ORIGIN ALONG $Y$ AXIS

C NOTE THAT OFFX ANII OFFY MUST FE IM IINITE OF

C ABSOLUTE SCREEN COOFIITNATS 


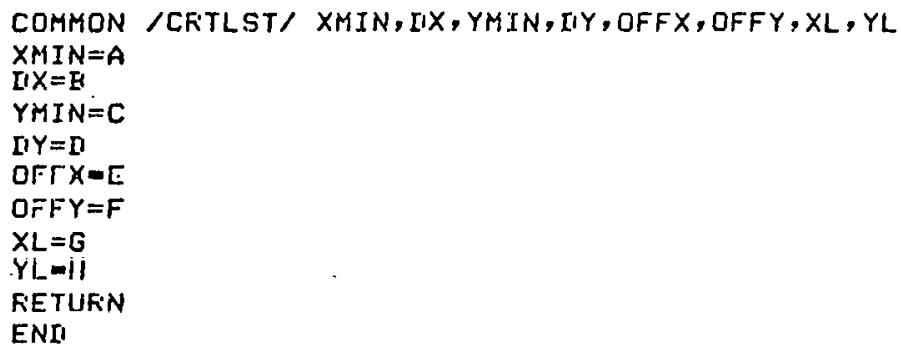

C

SUEFOUTINE AXIS(ITYFE)

C THIS SUEROUTINE IHFAWS $X$ ANII $Y$ AXIT EASEII ON UALUES

C OBTAINEI FKOM THE OFFSET SUEFIDUTINE

C $X L=L E N G T H$ OF $X$ AXJS IN AESOLUTE SCFEEN UNITS

C YL=LENGTH OF $Y$ AXIS IN AESOLUTE SCREEN UNITS

C ITYFE= TYFF UF AXIS $0=O F \cdot E N \quad 1=C L O S E I$

$\mathrm{C}$

C NUTE ILL MAFKS ARE NOT IIRAWN WITH THIS ROUTINE

$\mathrm{C}$

C

COMMON /CFTLST/ XHIN, IIX, YMIN, IYY, OFFX, OFFY, XL, YL $X E N I=O F F X+X L$

$Y E N I I=O F F Y+Y L$

CALL TOUTF'T(29)

CALL XYTKAN (XENI, OFFY)

CALL XYTFAN (XENII,OFFY)

CALL XYTRIAN (OFFX, OFFY)

CALL XYTKAN (OFFX, YENII)

IF (ITYFE.EQ.O) GO TO 1

CALL XYYRAN XXENTI, YENII)

1

CALL XYTFIAN $(X E N L, O F F Y$ )

C INSEFT TIME IIELAY FOK EAUII FATE FUFFOSES

IIO $2 \mathrm{I}=1,10$

2 CALL TOUTFT(22)

CALL TOUTFT $(31)$

FAFTIIFN

ENII 


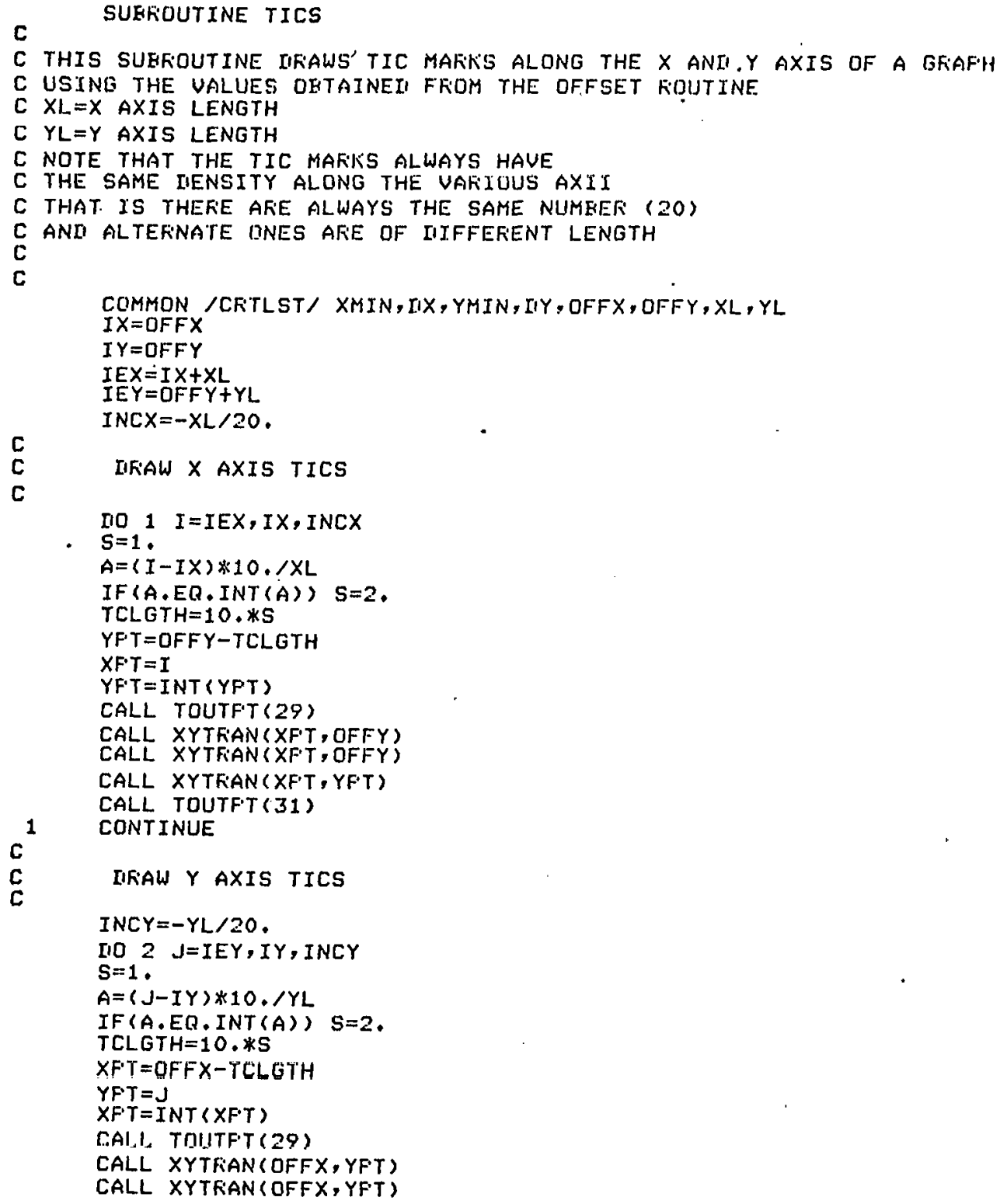




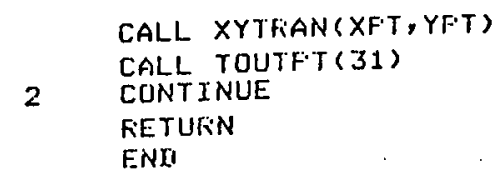

SUEFOUTINE LAEEL (ILX,NILX, ILY,NILY)

C C THIS SUBFOUTINE LAEELS THE AXII OF THE GFIAFH

C ILX =AFFFAY OF CHAFACTEFS FOF LALELING $X$ AXIS STOREII AS 2 HOLLEFITH CHAFACTEFS FEF AR'RAY ELEMEN'T C NILXENUMEEF OF CHAFIACTEFS IN ILX

C ILY=ARFAYY OF CHAFACTERS FOR LAEELING Y AXIS

$\ddot{C}$ STOFEEI AS 1 HOLLEFITH CHAFACTEF FEF AFFIAY ELEMENT C NILY=NUMEEF OF CHARIACTERS TN ILY

C

$\mathrm{C}$

$\mathrm{C}$

C. $X I, \equiv X$ AXIS LENGTH

C $Y L=Y$ AXIS LENGTH

C THE FOUTINE LAEELS THE MIN ANI MAX OF EACH AXIS ANI OUTFUTS

C THE YALUE OF THE INCFEMENT (MTNIIF TTC.) TN IINTTS MF THE QFIIGINAL DIATN

C NOTE THAT THIS FOUTINE HAS ALSO BEEN SETUF TO WRITE ASCI I

C CHARACTEFS TO LAEEL THE TITLES OF THE AXII

r. FME FEATIABTI ITY ALL LAEELING IS IIONE USING THE NOKMAL TTY OUTFUT

C THE GFAFHICS ROUTINE ARE USEI IN OFIIEF TO FOSITION THE OUTFUT

r. WTTH FESFECT TO THE COQRIINATE BYGIEM

c

COMMON /CFTLST/ XMIN,IIX, YMIN, DY, OFFX, OFFY,XL,YL IIIMENSION ILX(1),ILY(1)

$X E N I=\cap F F X+X L-75$. 


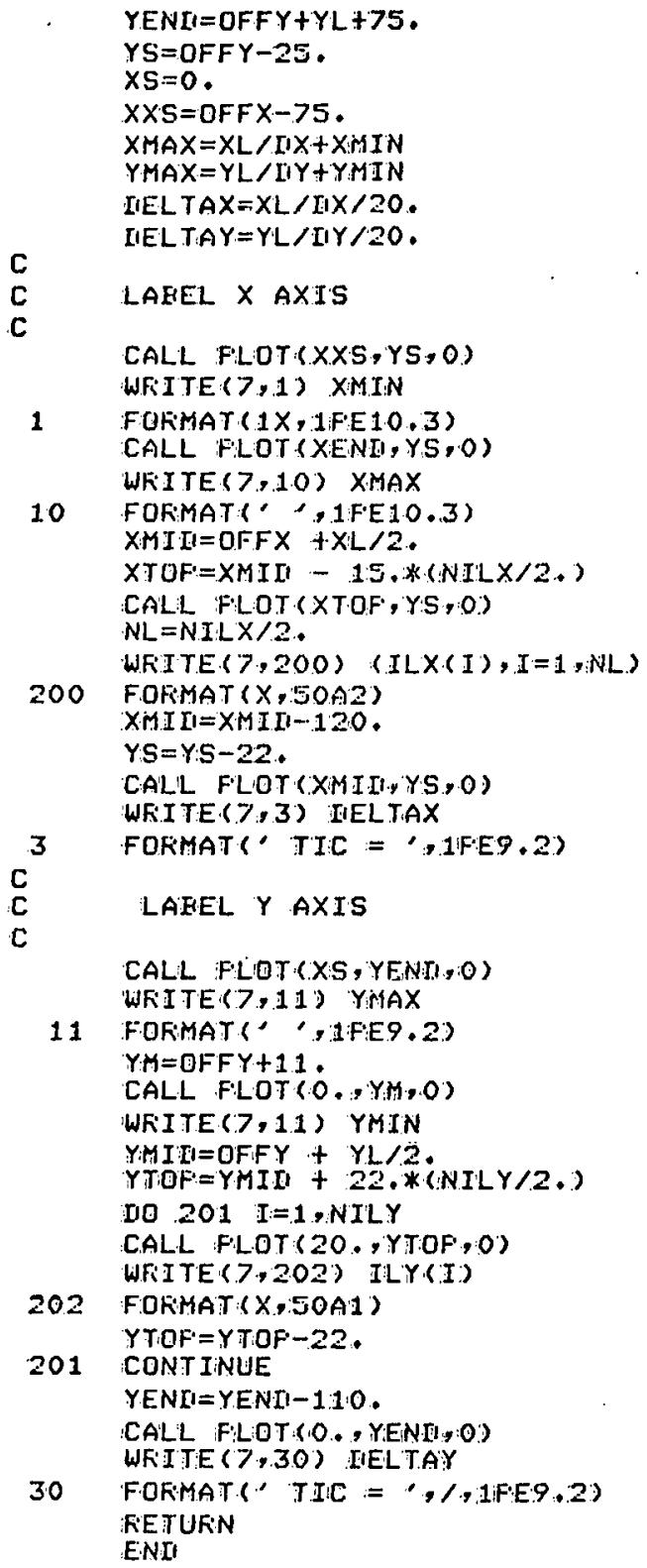




\begin{tabular}{|c|c|c|}
\hline & $\begin{array}{l}\text { - TITLE } \\
\text {-GLOEL } \\
\text { - MCALL } \\
\text {. VZ. } \\
\text {. KEGIEFF }\end{array}$ & $\begin{array}{l}\text { TOUTFT } \\
\text { TOUTFT } \\
\text {. FEGIEF, ..V2... }\end{array}$ \\
\hline $\begin{array}{l}\text { TOUTFT: } \\
\text { WAIT: }\end{array}$ & $\begin{array}{l}\text { TST } \\
\text { TSTT }\end{array}$ & $\begin{array}{l}(F \cdot 5)+ \\
\text { (\$1 1.7564 }\end{array}$ \\
\hline $811:$ & $\begin{array}{l}\text { EMI } \\
\text { ER: } \\
\text { MnUU }\end{array}$ & $\begin{array}{l}\text { WRIT } \\
\text { WAIT } \\
\text { Q(R'S)+, D1 17756d }\end{array}$ \\
\hline & $\begin{array}{l}\text { RTS } \\
\text {.ENR }\end{array}$ & $\begin{array}{l}\text { P'L } \\
\text { TOUTPT }\end{array}$ \\
\hline
\end{tabular}

- TITLE TINFUT

-GLOEL TINFUT

. MCALl . FEGIIEF, . va. .

..V2.

-REGIIEF

TINFUT: TST (FS)+

MOU \$O,COUNT

MTFS 200

TEST: TETH E\$177J60

EMI FEAI

HF TEST

READ: MOU (OA:177562,Q(RS)+

ENC COIINT

EMP OE,COUNT

ENE TEST

MTFS $F$ FC

COUNT: .WORN O

- ENII TINFUT

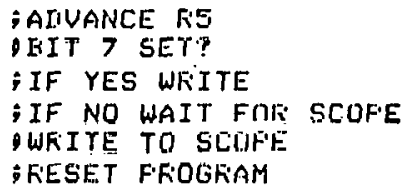

; IGNORE ARG NUMEER

- FLOCK INTERFUF'TS

- IIOES CÖNSOLE HAUI A CHARACTER?

- FiEAII IF IT TIOES

- TFiY aga.tN

¿FUT NUABEF IN ARG.

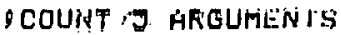

SNOFMAL INTERFUFT CONUITIONS

-FESET FROGRAM CONIITIONS 
APPENDIX D

Computer Program NXRYRT 


\section{APPENDIX D \\ Computer Program NXRYRT}

This appendix contains documentation of the computer program NXRYRT which can be used to convert experimentally determined $x$-ray intensity ratiós into composition ratios using a standardless analysis approach. In addition, the program can be used to predict experimental intensity ratios once the sample composition is entered. Comment lines are appropriately dispersed within the text and a simple flow diagram precedes the documentation (Fig. 102). An example of analysis using this program is given in Chapter 2, Section 2.2.8. 


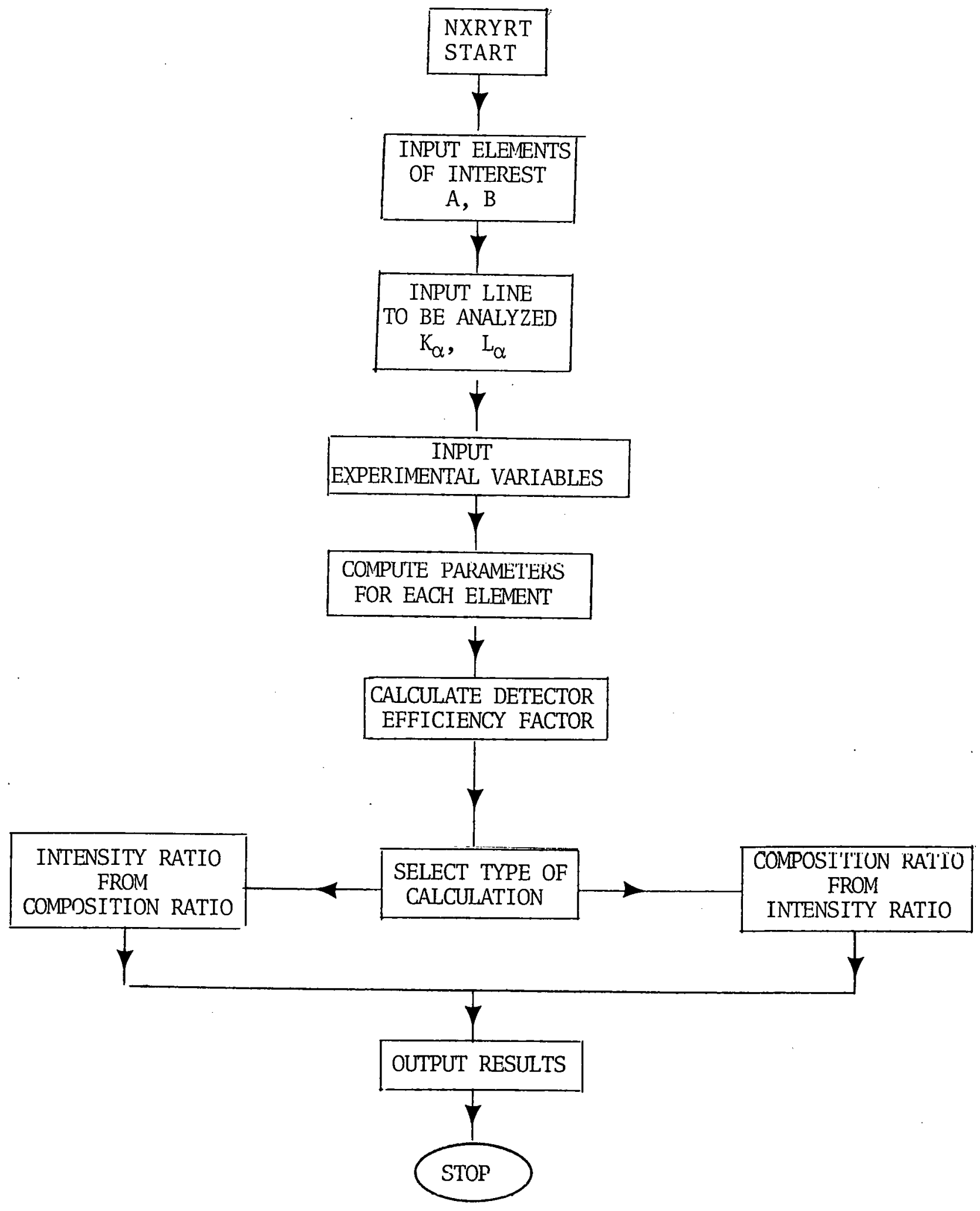

Fig. 102. Flow Diagram for the Progrann NXRYRT. 


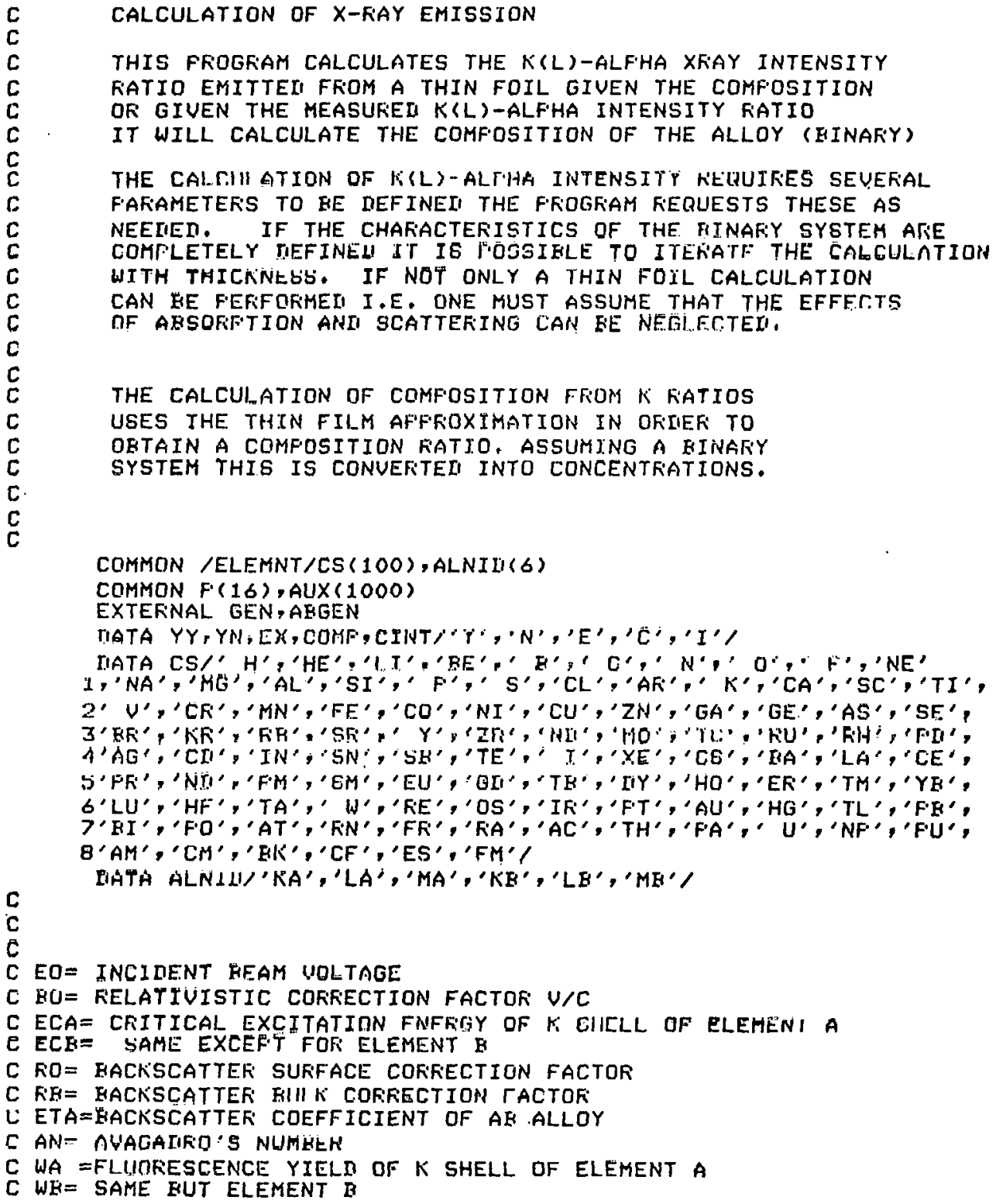




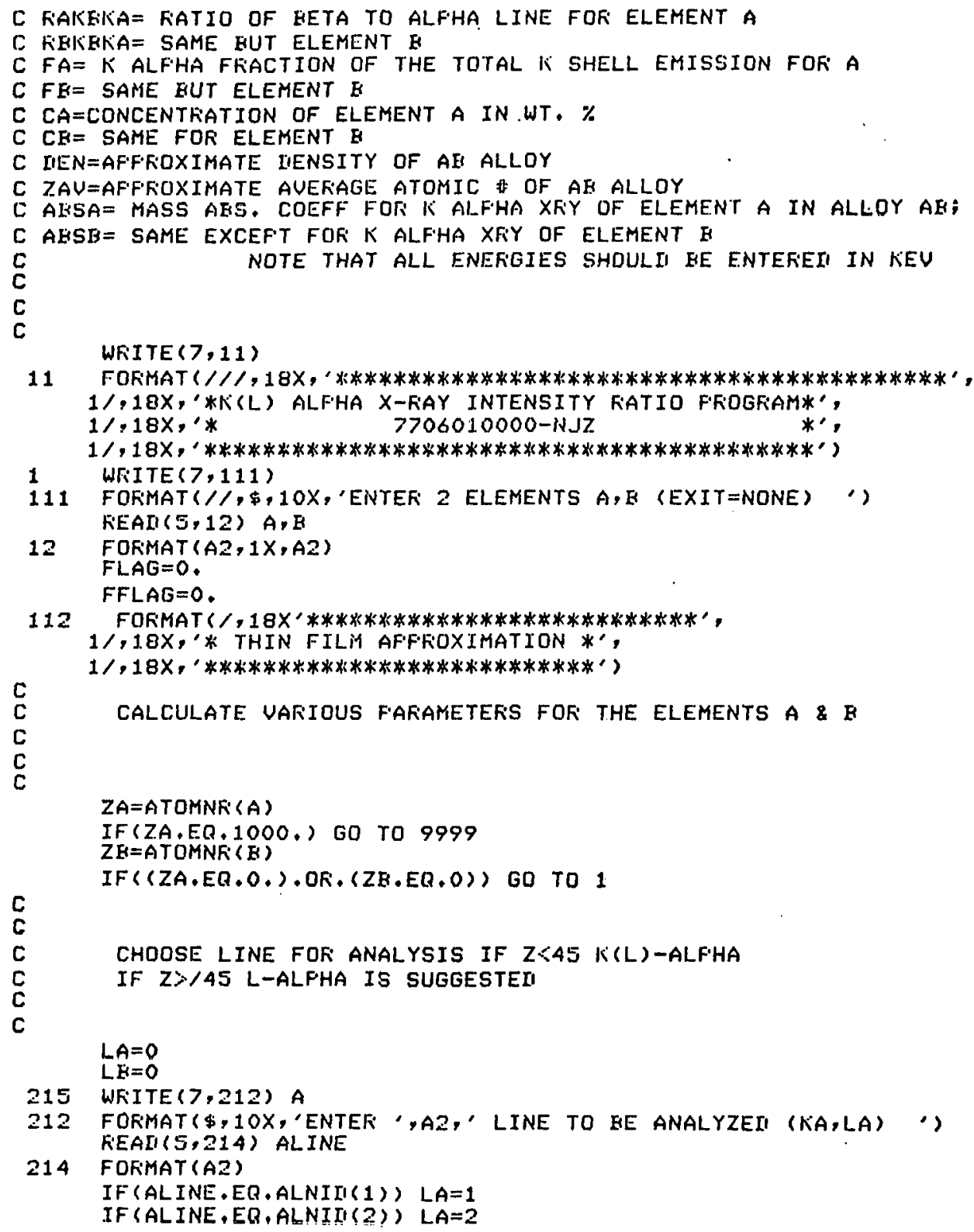


IF (L.A.EQ.O) GO TO- 215

IF ( (ZA.LT.26.).ANII. (LA.EQ.2)) GO TO 217

IF $((Z A \cdot G T, 45$. . ANI, (LA.ER.1)) GO TO 217

216 WFITE $(7,212)$

FEAII (5,214) ALINE

IF (ALINE.EQ. ALNIII(1)) $L E=1$

IF (ALINE, EQ. ALNIIU(2)) LE=2

IF (LE.EQ.O) GO TO 216

IF ( (ZE,L.T.26.) , ANI, (LA,ER.2)) GO TO 219

IF ( (ZE, GT, 15.) , ANII, (LA,ER, 1)) GQ TO 219

OO TO 200

217 WKITE $(7,218)$ A,ALNTIILA)

218 FOFMAT $X$, 'ANALYSIS OF ', $A 2, X, A 2$, ' IS NOT F'FACTICAL ON $A$ ', 1 'SI (LI) X-FAY IIETECTOR SYSTEM') GO TO 215

219 WFITEC(7,218) B,AI_NILILE)

GO TO 216

220 CONTINUE

$E . C A=12.37788 / E$ IIGE $(Z A, L A)$

$E C F=12.37788 / E I I G E(Z F, L E)$

ElIA=ENEFILYY $(Z A, L A)$

$E K E=E N E F G Y(Z E, I ., F)$

$W A=Y I E L I(Z A, L A)$

WE $=Y I E L I(Z F, L E)$

FAKEKA $=$ BETA $(Z A, L A)$

FEKKEKN-EIETA $(Z[I, L E)$

$F A=1 . /(1 .+F A K E K A)$

$F E=1 . /(1 .+F E K E K A)$

ATWA $=A T O M W T(Z A)$

ATWE=ATOMWT (ZB)

C

$A N=6.023 E 23$

REAII IN ACCELEFATING VOLTAGE

WRITE $(7,15)$

FOFMAT $\$, 10 X$, 'FNTEF: ACCELERATINO VOLTAGE IN KEY ")

REALI $(5,16)$ EO

FOFMATY $1 F 7.0$ ?

18 FUKMAT $(1 F\rangle .0)$

C

C

C

C

CHOOSE TYFE OF CALCULATION

179 WFITE $(7,102)$

182 FOKMAT (10X,' INIICATE TYFE OF CALCULATION', 12X, 'COMFOSITION FFOM IEEASUFEII $K(L)$-ALFHA R'ATIOS', $1^{\prime}=$ COMFOSITION', $/$,

$1 \$, 2 X, ' K(L)$-ALFHA INTENSITY RATIO FROM COMFOSITION', 


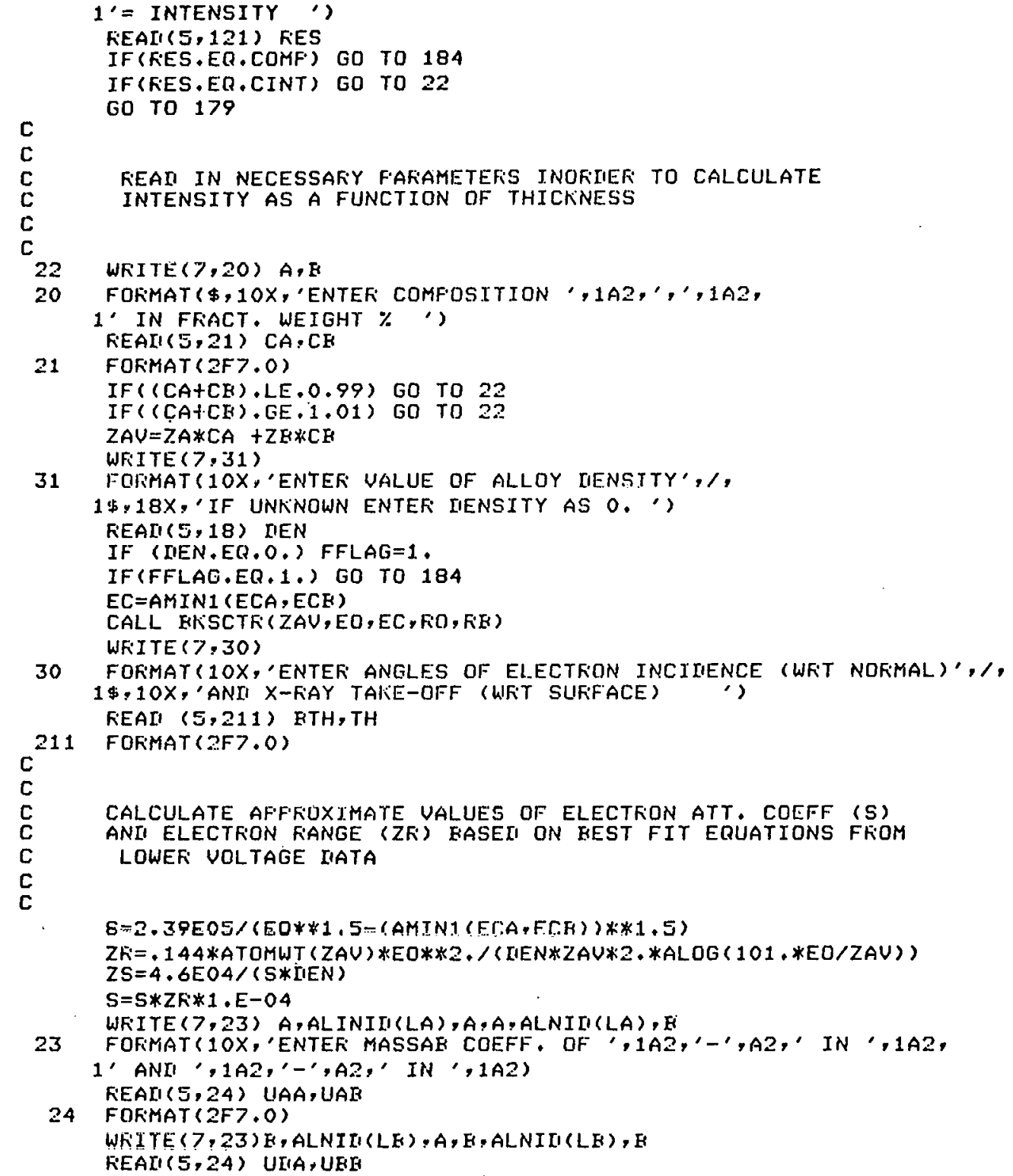




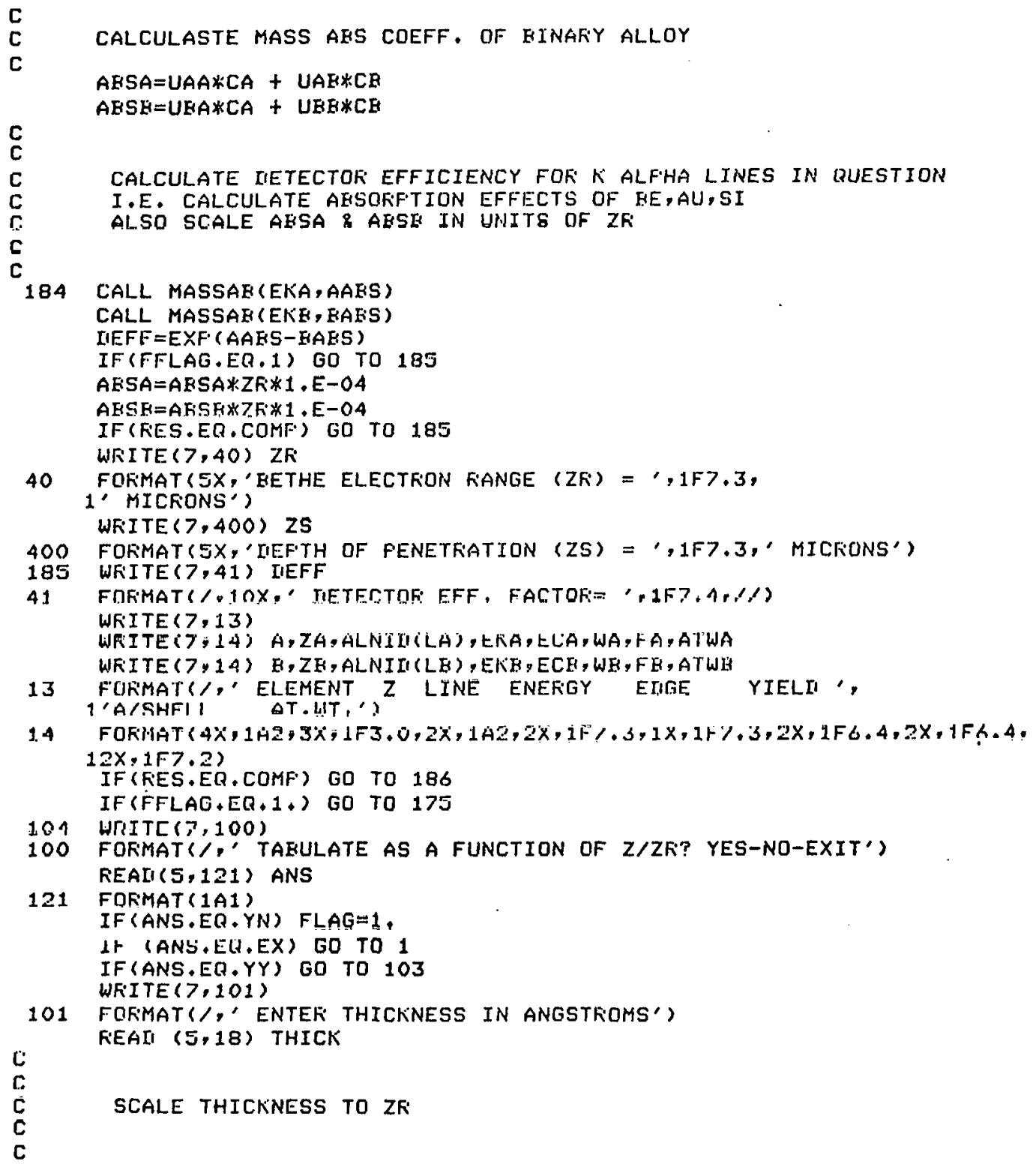




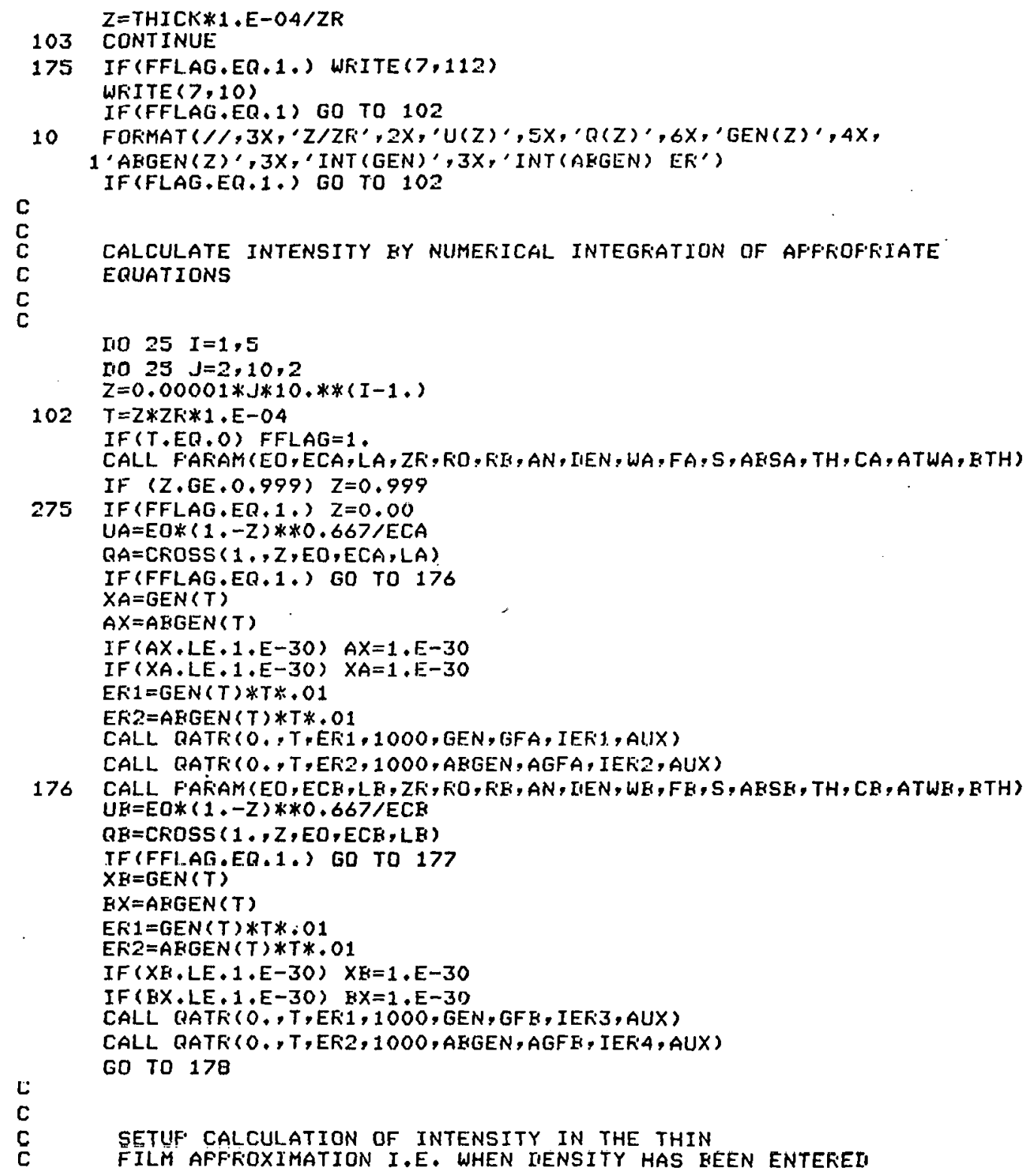

C

C SETUF CALCULATION OF INTENSITY IN THE THIN

C FILM AFFFOXIMATION I.E. WHEN IIENSITY HAS GEEN ENTEREI 


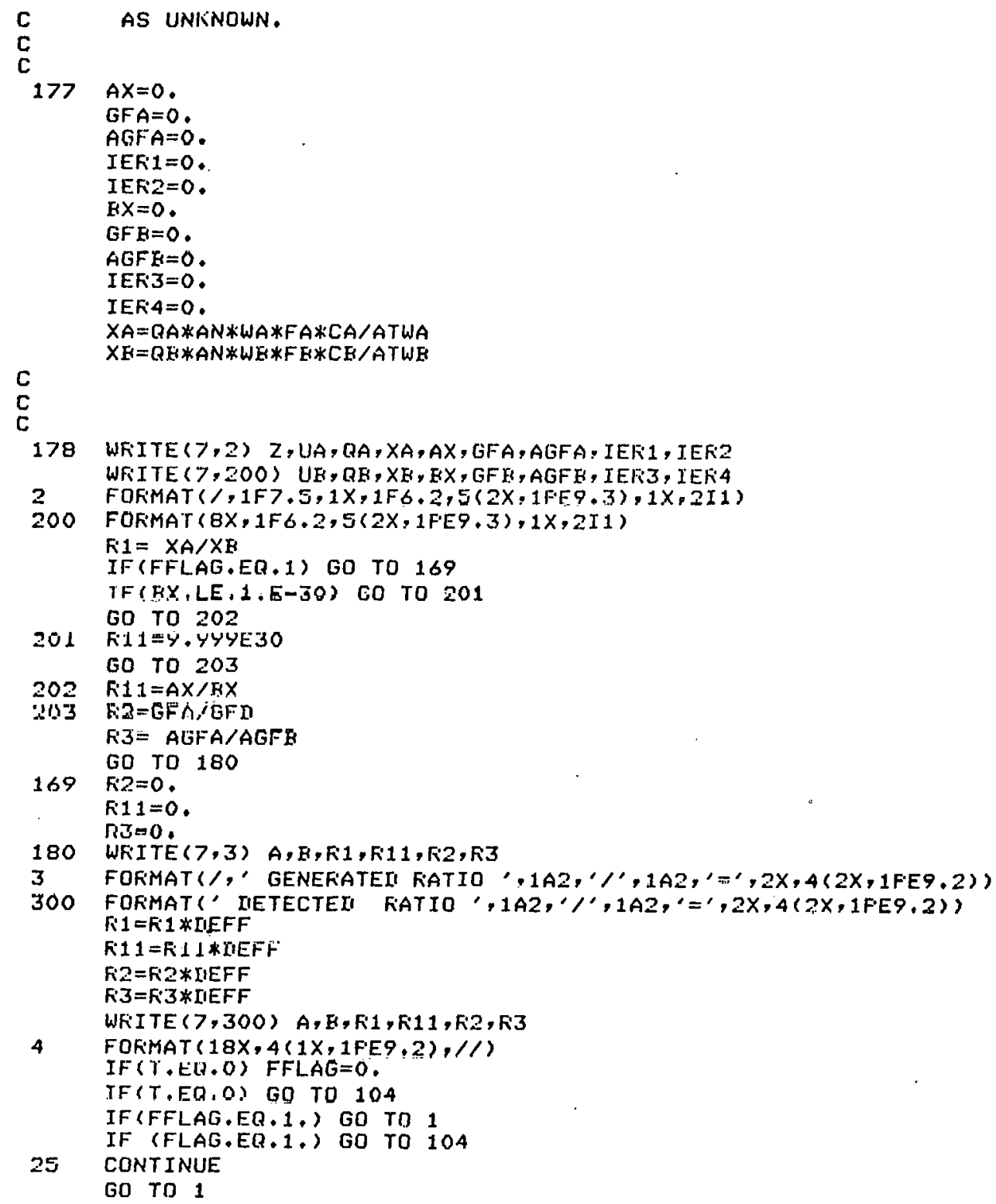




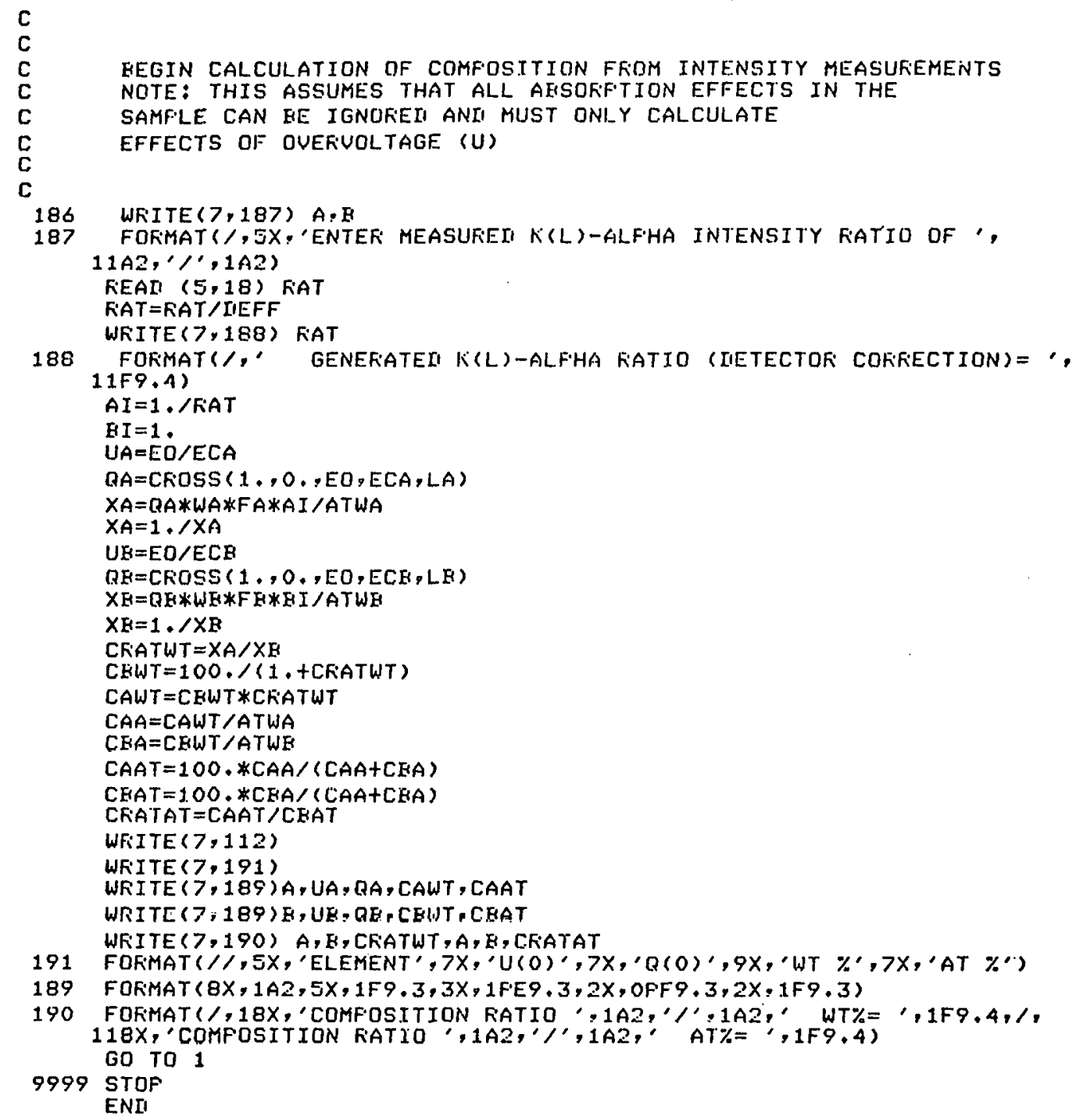




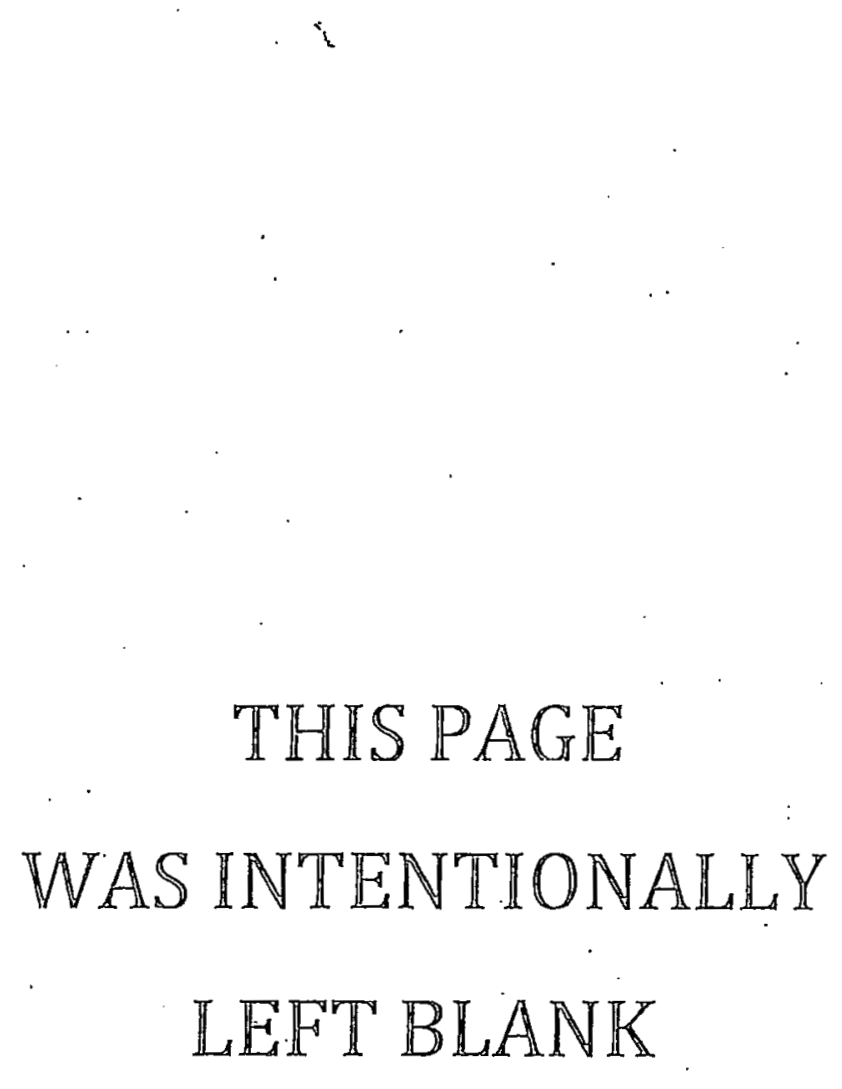




\section{APPENDIX E}

Computer Program NMCS 
APPENDIX E

Computer Program NMCS

This appendix documents the computer program NMCS which is used for data analysis of spectra recorded in a multichannel scaler. Thus the program can be used for simple analysis and/or display of TSED, ELS, and EDS data. Comment lines are appropriately dispersed within the text; Lle program is set up to respond to a simple command structure which includes the following instructions: graph, data, integrate, window, smooth, exit, and function. The graph command allows the replotting of the data at scale factors other than calculated by the graphics library, while the data and integrate instructions provide a means of reading data points or integrals from specific regions of the spectrum. Window allows regions of the spectrum to be expanded to fill the screen, and smooth performs a five-point smoothing of the data. The function instruction allows the data arrays to be operated on by the following functions: $\exp (Y), \ln (Y)$, and $Y^{\text {POWER }}$ along both axes if desired. This is particularly useful in the case of ELS data which when graphed on a log-log plot yields a linear background dependence and can thus be analyzed using the integrate command. 


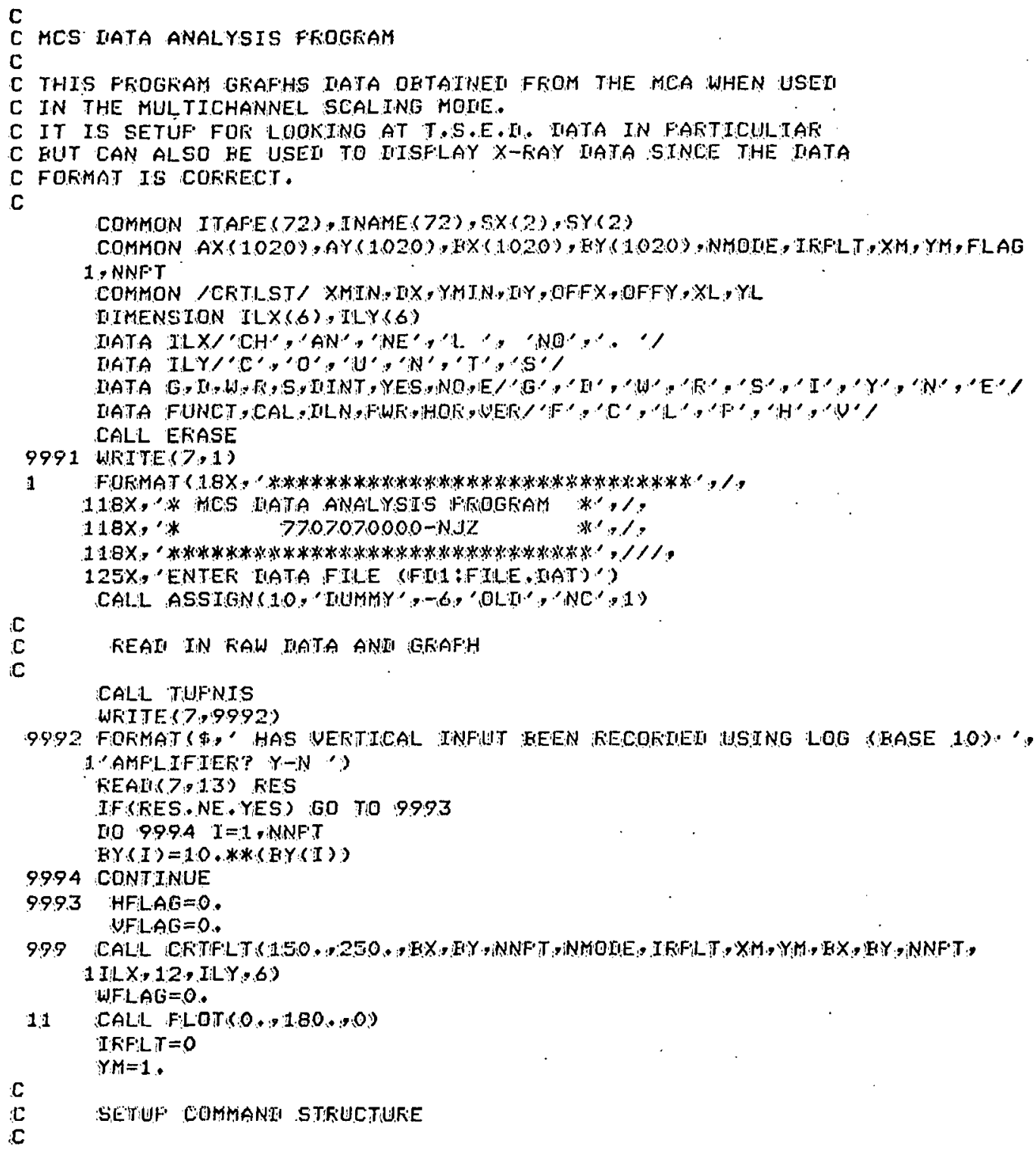




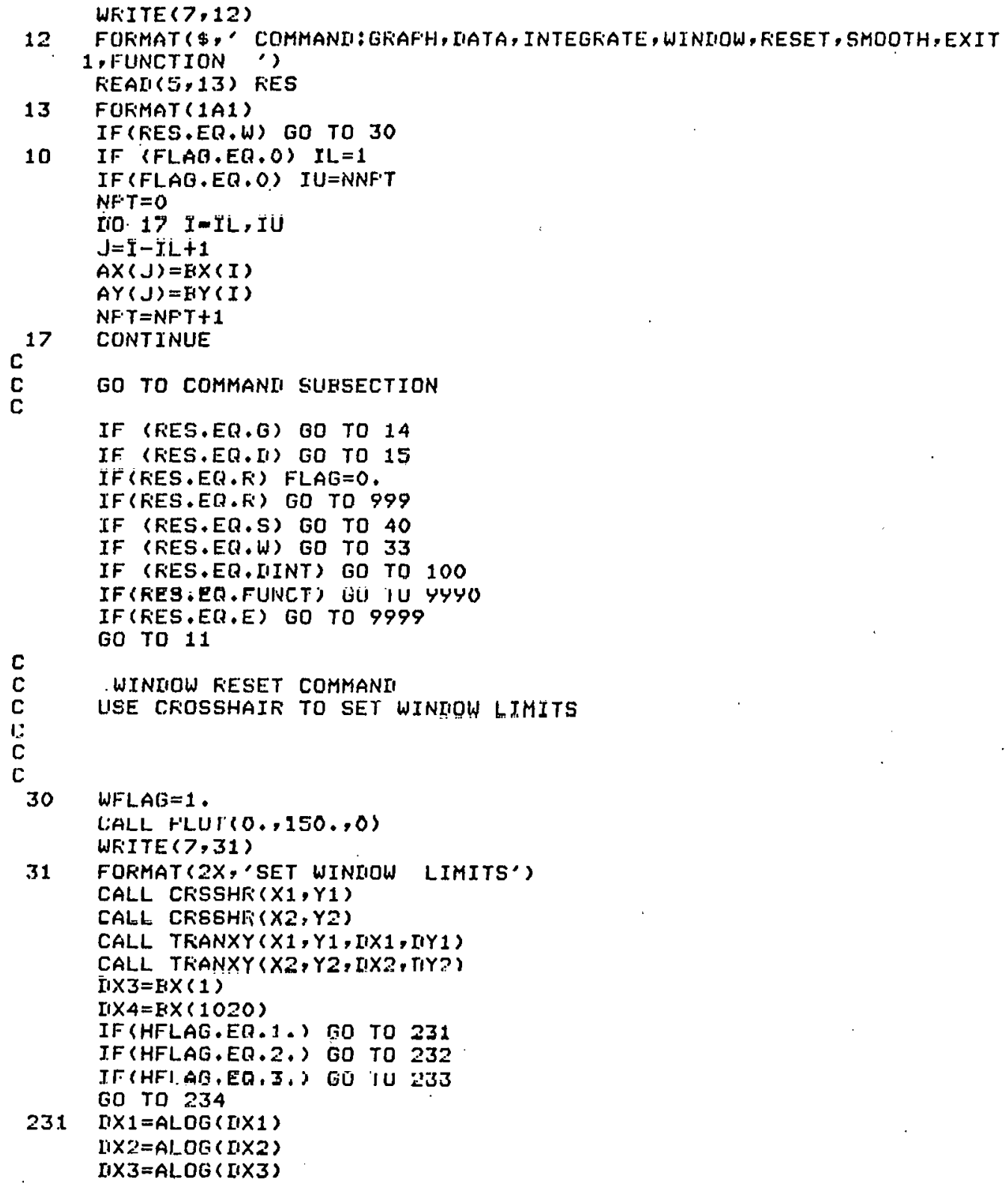


IIX4 $\times$ ALOG $(I \times 4)$

GO TO 234

$232 \quad I \times 1=E \times F(11 \times 1)$

$I \times 2=E \times F(\{1 \times 2)$

$[1 \times 3=E X F((1 \times 3)$

Ii $\times 4=E \times F \cdot(I 1 \times 4)$

GO TO 234

$233 \quad$ KWF $=1$. /F OWEK

$I I \times 1=I \times 1 * * F$ WWF

$[1 \times 2=I \times 2 * * F W W F$

IIX3 $=[1 \times 3 * * F$.WF

II $\times 4=[1 \times 4 * *$ FiWF:

$234 S L F=(I \times 4-I \times 3) / 1019$.

$I L=I N T(([1 \times 1-[1 \times 3) / S L F)$

$I U=I N T((I \times 2-[1 \times 3) / S L F)$

$F L A G=1$.

c

GO TO 18

IIATA FOINT COMMANI

FEAII IIATA FFIOM GRAFH USING CFOSSHAIF FOUTINES

NOTE MUST HAUE FFEUIOUSLY SET A WINIIOW TO FIUN

15 IF(WFLAG,EQ, 0) GO TO 11

WRITE $(7,151)$

151 FOFMAT (\$," ENTEF NUMEEK OF RIATA FOTNTS $(\leftrightarrow=6)$ ")

REAII $(5,152)$ NIIF

152. FOFMAT(I1)

CALL CFTFLT (150, ,250, , AX, AY, NFT, NMOHE, O, XM, YLAST, AX, AY, NF'T,

$1 I L X, 12, I L Y>$

CALL FLOT $(0,0158, .0)$

IIO $154 \mathrm{~J}=1$, NUF

CALL CFSSHF $(X, Y)$

$Y H=158,-J * 22$.

CALL FLOT $(0, \% Y H, O)$

CALL TFANXY $(X, Y, X F \cdot T, Y F T)$

$X P=X F T$

$Y F=Y F \cdot T$

IF (HFLAG,EQ, 1) XF. $T=A L O G(X F \cdot T)$

IF(UFLAG.ER,1) YFT =ALOG(YF.T)

IF (HFLAG.EQ.2) XF'T $=E X F \cdot(X F \cdot T)$

IF (UFLAG,ER, 2) YF.T=EXF'(YFT)

IF (HFLAG.EQ, 3) XFT =XFT**(1,/FOWER )

JF(UFLAG,EQ, 3) YFT $=Y F T * *(1, / F$ OWEF $)$

$I X=(X F T-I I X Z) / S L F$

IF (HFLAG.EQ.O) XFT $=E X(I X)$

C

IF (UFLAG.EQ.O) YFY $=E Y(I X)$ 


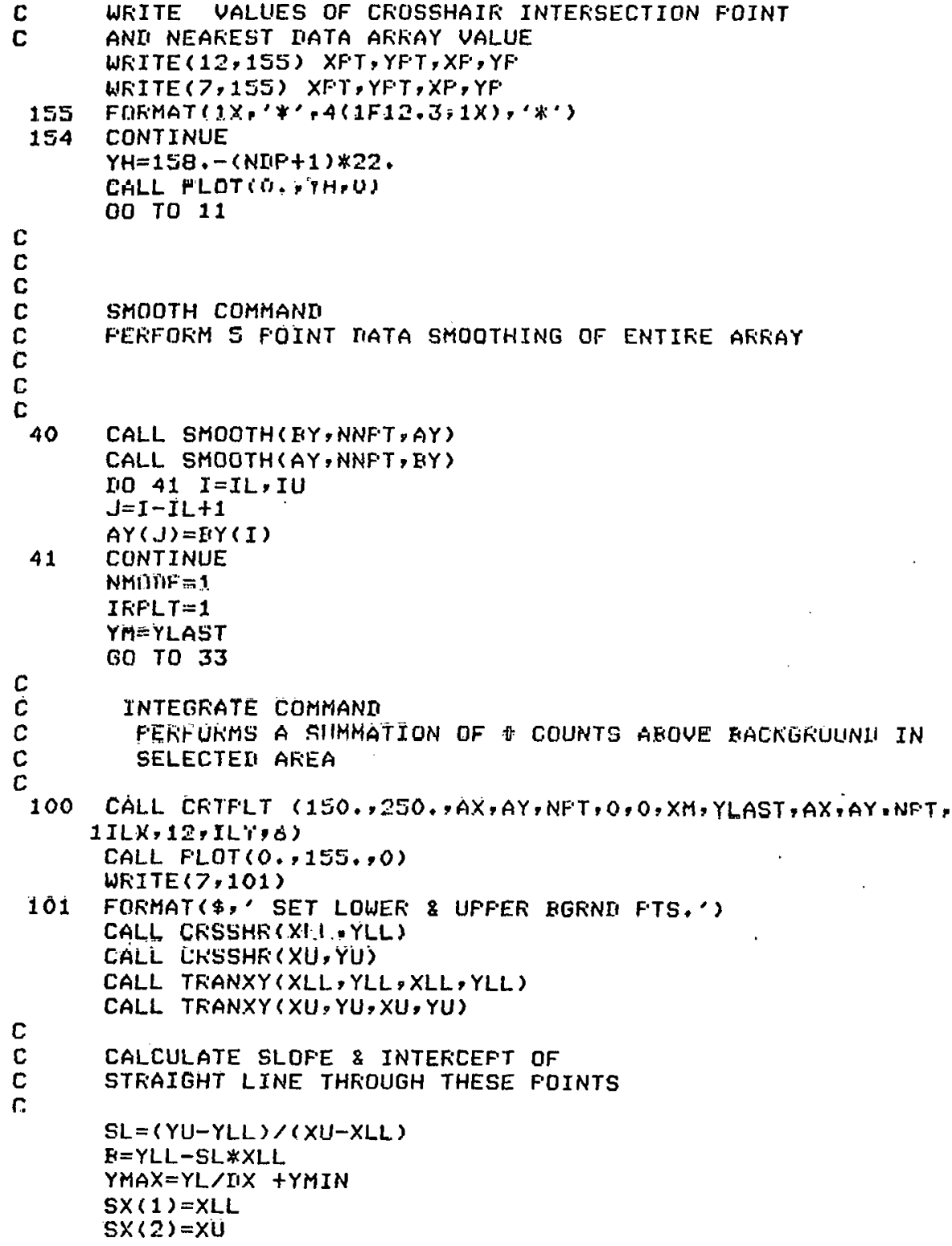




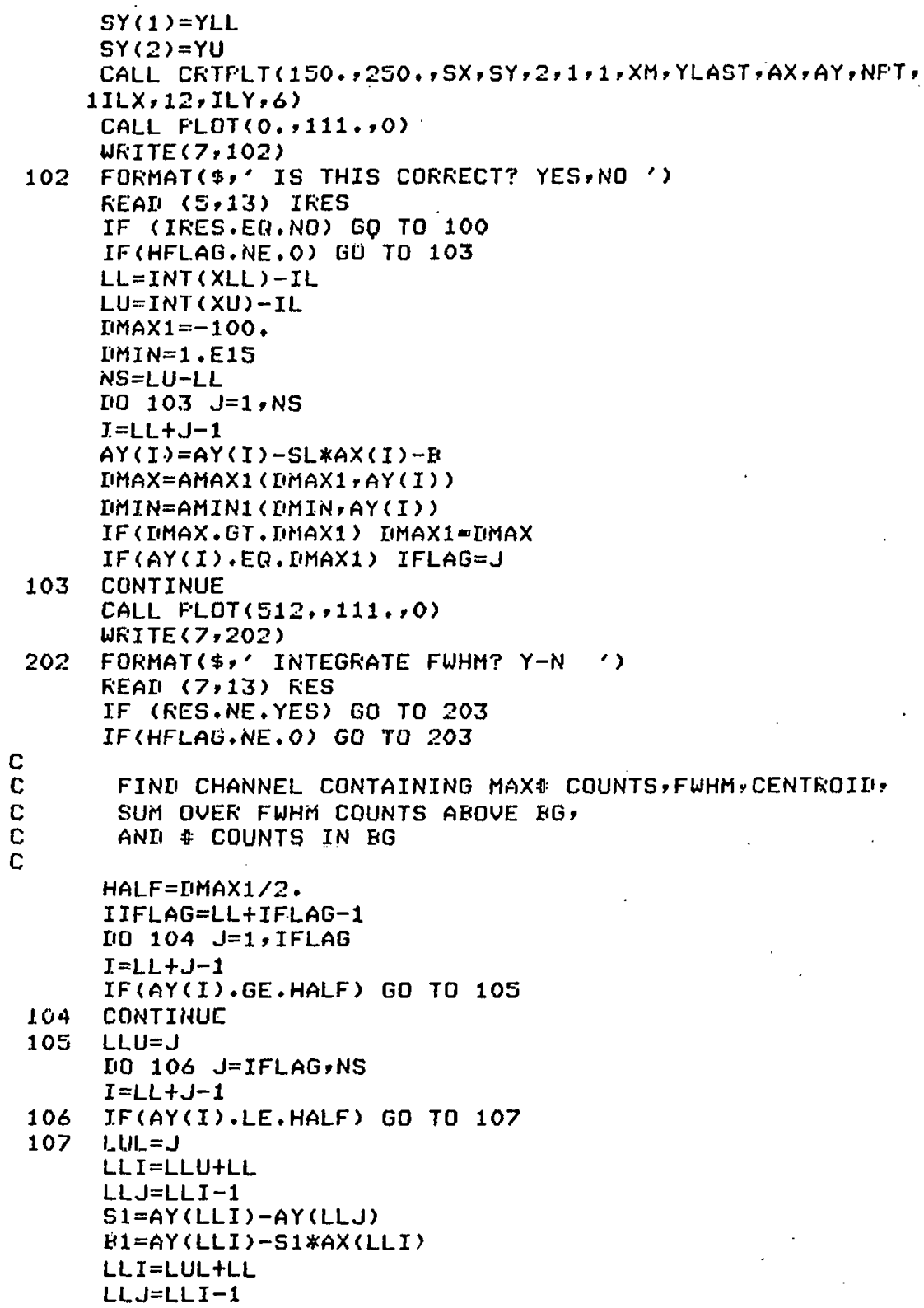




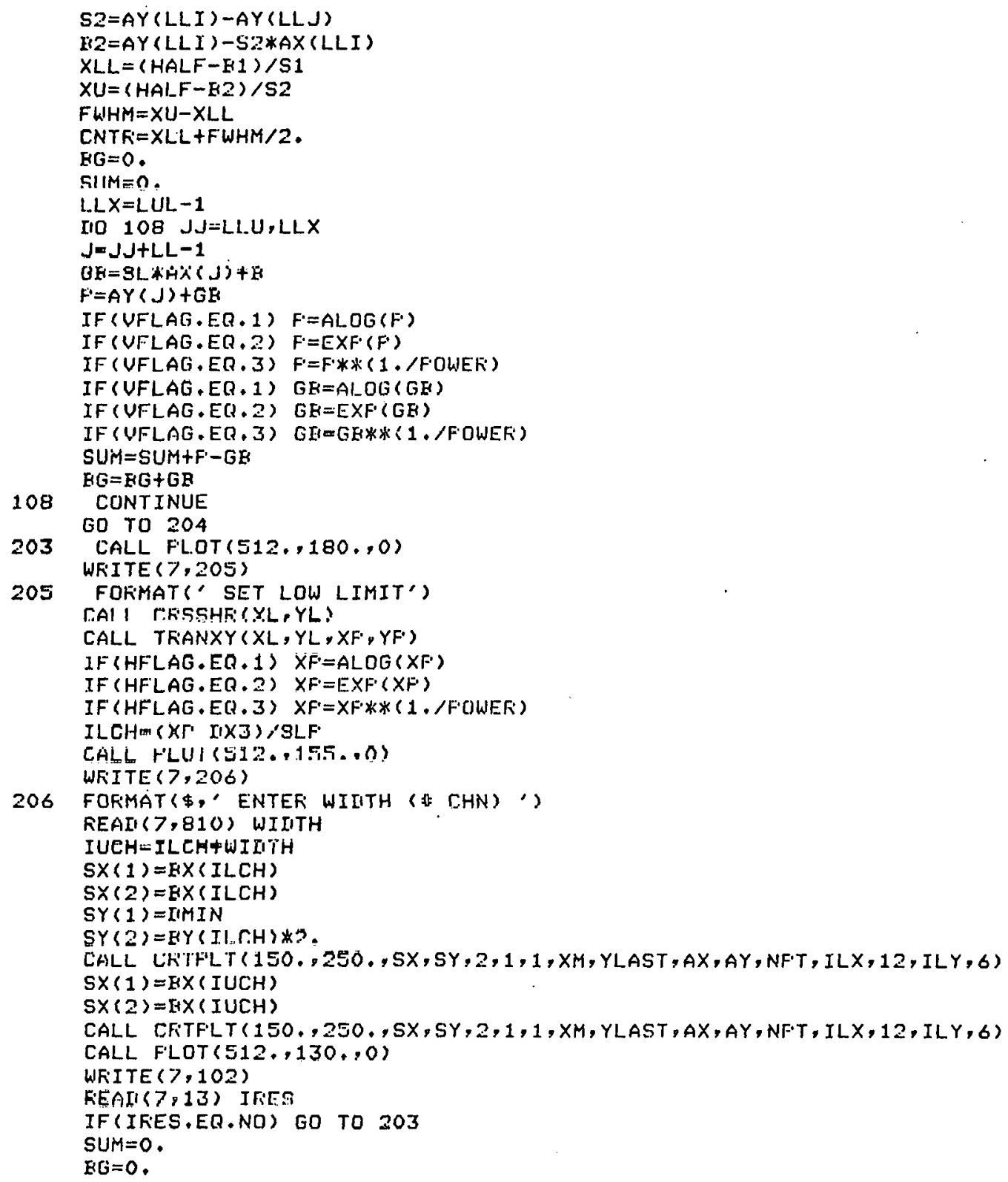




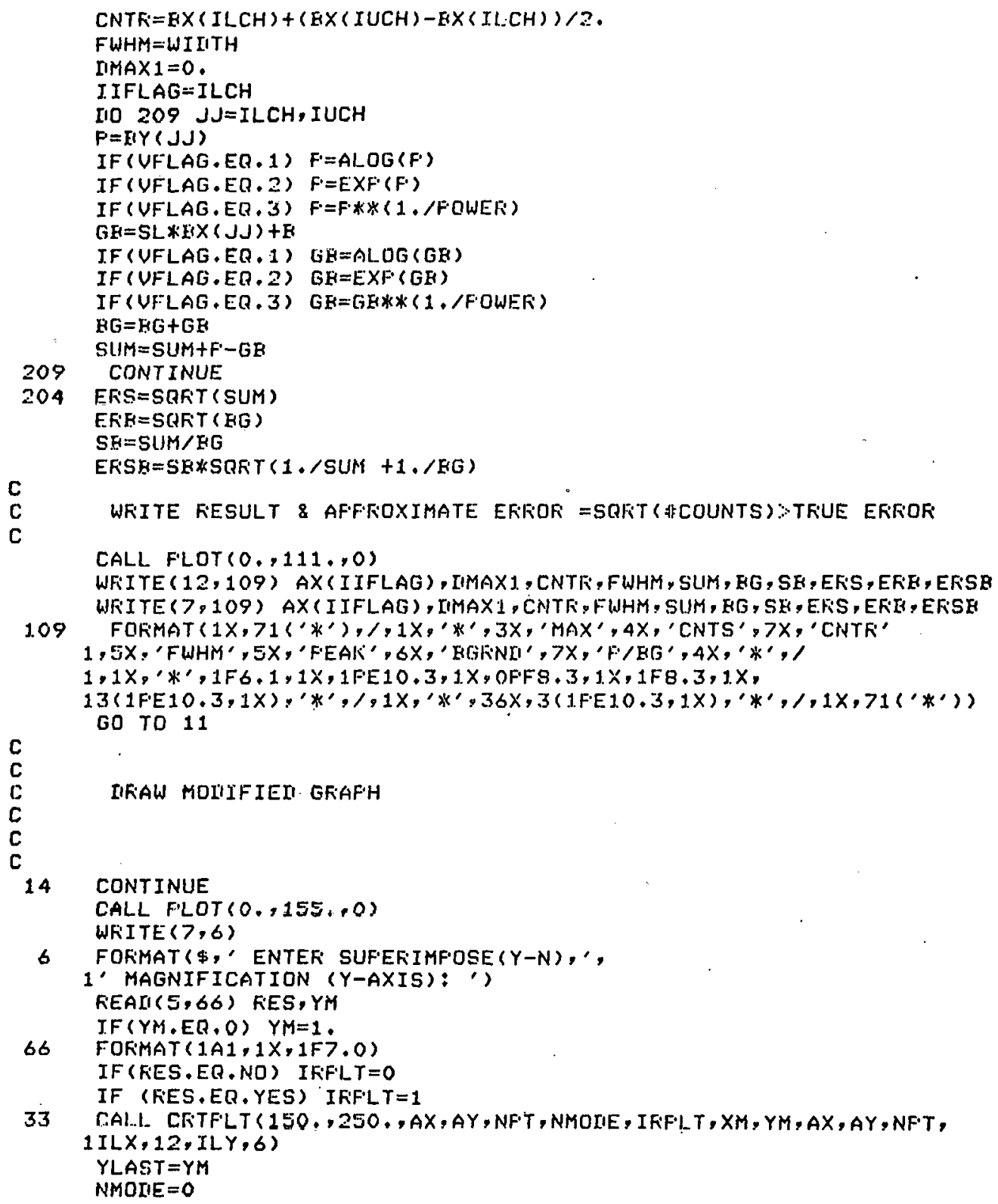




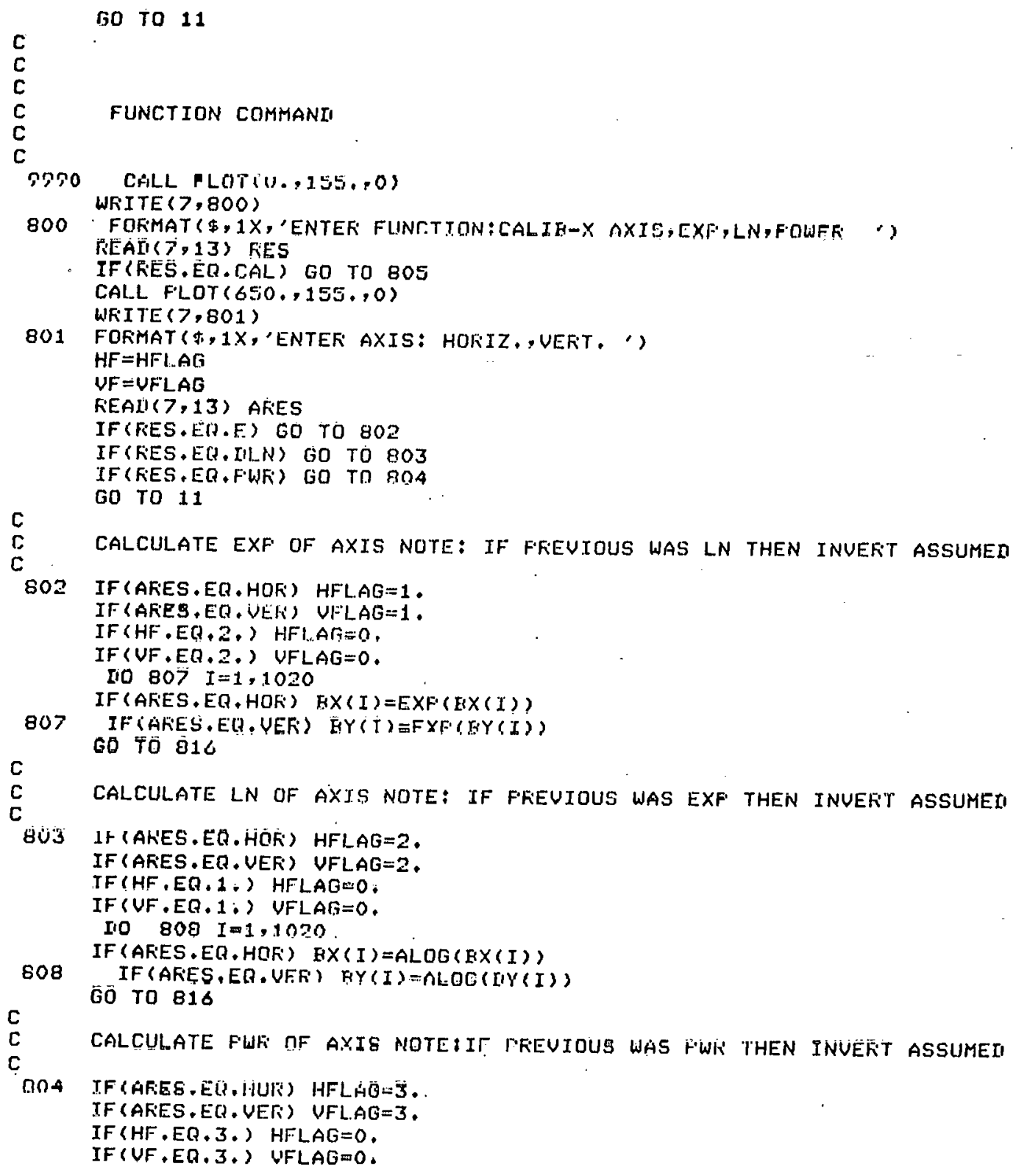




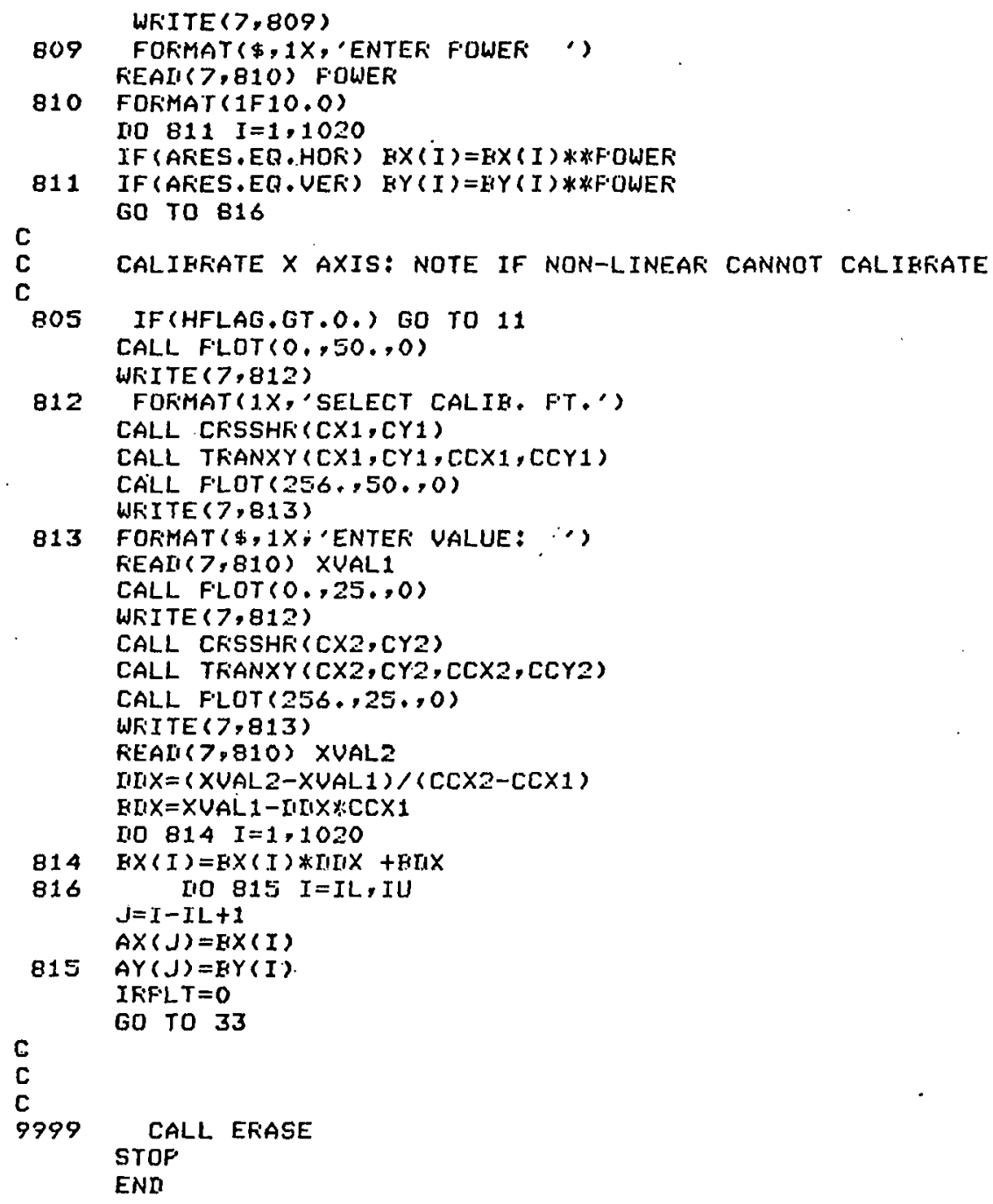




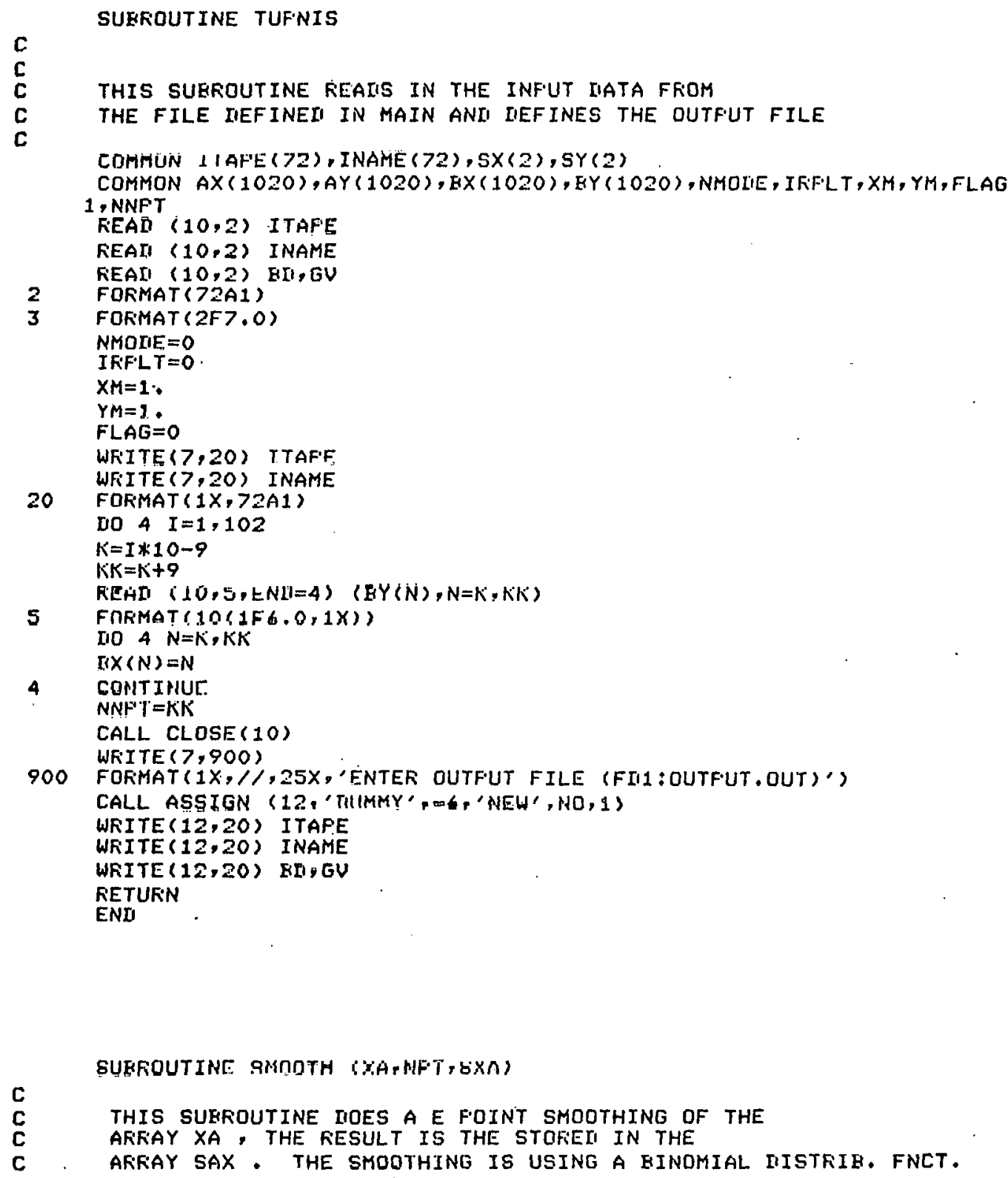


C NOTE CALLING SMMOTH TWICE IS EQUIUALENT TO A

C 5 FOINT SMMOTHING

IIIMENSION XA(1), SXA(1)

$I U=N F T-2$

IIO $1 \mathrm{I}=3$, IU

$1 \quad \operatorname{SXA}(I)=0.5 * X A(I)+0.25 * X A(I-1)+0.25 * X A(I+1)$

FETUEN

ENI 


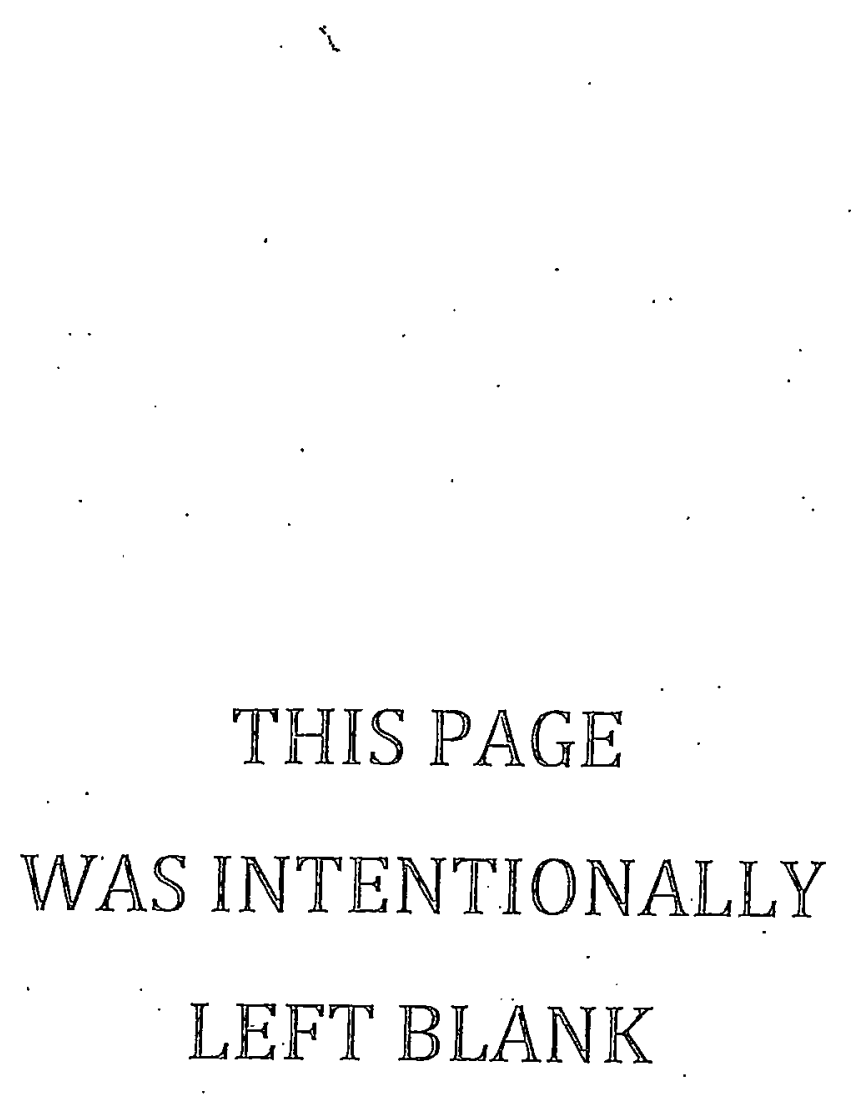




\section{APPENDIX F}

\section{Computer Program MCA}




\section{APPENDIX F \\ Computer Program MCA}

This appendix documents the macro-program MCA which is used to read data from the ORTEC multichannel analyzer and which stores the data into a specified file on a floppy disc. The program was developed by Peggy Mochel and John Woodhouse of the Materials Research Laboratory in conjunction with these research and is included for completeness. 


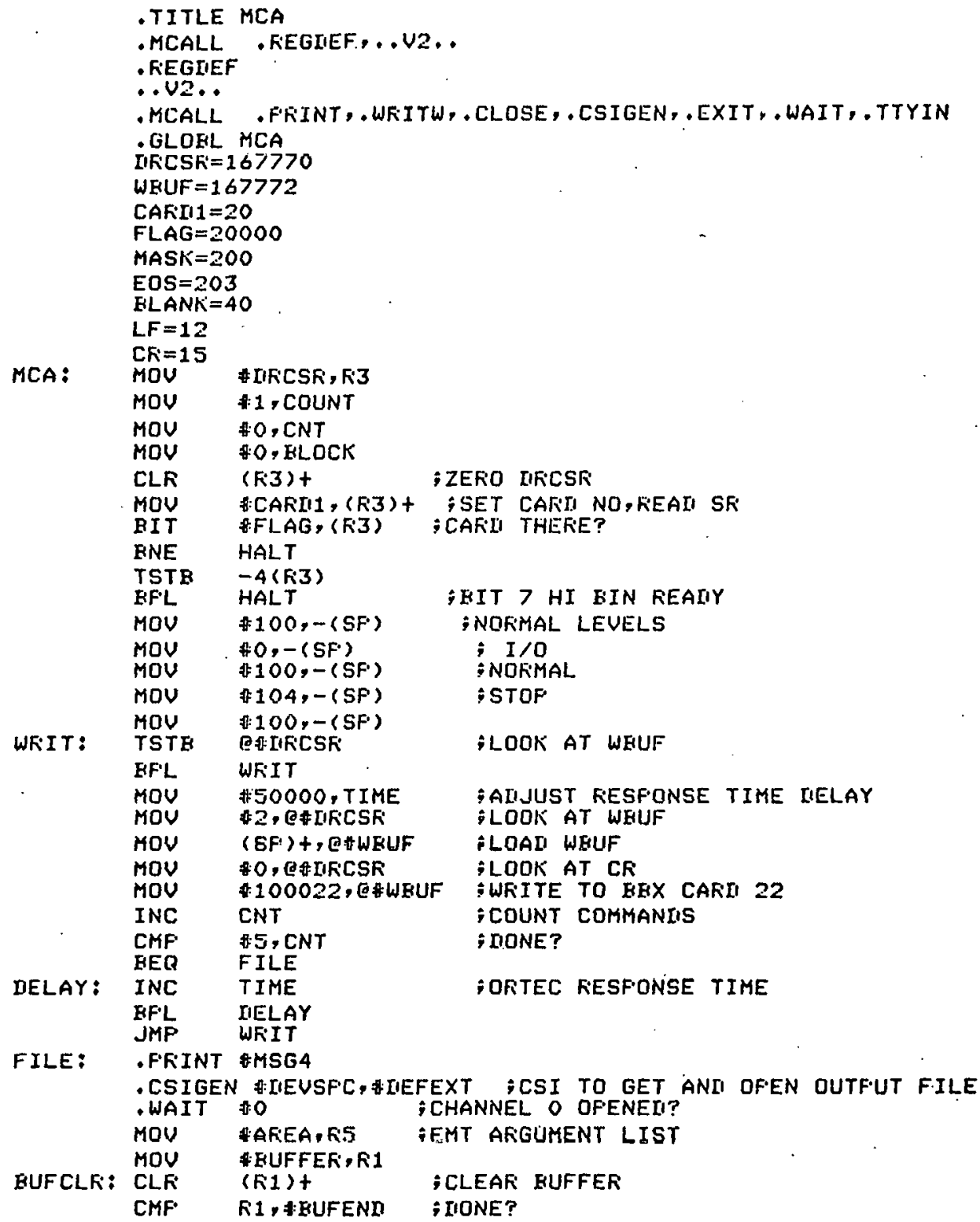




\begin{tabular}{|c|c|c|}
\hline & $\begin{array}{l}\text { ELO } \\
\text { MOU }\end{array}$ & $\begin{array}{l}\text { "LOOF IF NOT } \\
\text {;1ST EUFFER AIIIKESS }\end{array}$ \\
\hline & -FFIINT & EMSG6 \\
\hline \multirow[t]{3}{*}{ SETUF: } & $\begin{array}{l}\text { TTYIN } \\
\text { MOUE }\end{array}$ & $\begin{array}{l}\text { ¿GET TTY JNFUT } \\
\text { :FUT IT IN EUFFER }\end{array}$ \\
\hline & CMFE & ;WAS IT LF \\
\hline & $\begin{array}{l}\text { BNE } \\
\text {-FRINT }\end{array}$ & iIF NOT GET ANOTHEF \\
\hline \multirow{3}{*}{ SET2: } & - TTYIN & \\
\hline & $\begin{array}{l}\text { MNUPA } \\
\text { CMFE }\end{array}$ & 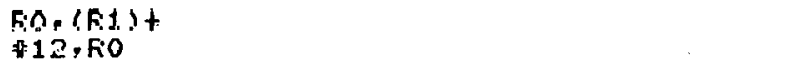 \\
\hline & ENE & SET2 \\
\hline \multirow[t]{4}{*}{ SET3: } & $\begin{array}{l}\text {-FFINT } \\
\text {-TTYIN }\end{array}$ & AMSG8 \\
\hline & MOUE & FO, $(K 1)+$ \\
\hline & $\begin{array}{l}\text { CMFB } \\
\text { FNE }\end{array}$ & $\begin{array}{l}\text { \#12, KOO } \\
\text { SET3 }\end{array}$ \\
\hline & $J M P$ & EEGIN \\
\hline HALT: & $\begin{array}{l}\text {.FEINT } \\
\text {,EXIT }\end{array}$ & \#MSG1 \\
\hline \multirow{9}{*}{ BEGIN: } & MOV & $\{20,0 \# 167772$ \\
\hline & $\begin{array}{l}\text { MOV } \\
\text { BIT }\end{array}$ & $\begin{array}{l}\# 0,0 \# 167770 \\
\$ 40000,(2 \$ 167774\end{array}$ \\
\hline & ENE & ;YES,TKY AGAIN \\
\hline & $\begin{array}{l}\operatorname{MOU} \\
\text { INL: }\end{array}$ & $\begin{array}{l}\text {-NO,LOOK AT IIATA } \\
\text { FLODEK FOTR START OF TEXT }\end{array}$ \\
\hline & CMF & ;50 TIMCS \\
\hline & $\begin{array}{l}\text { EEQ } \\
\text { CMP }\end{array}$ & $\begin{array}{l}\text {;THEN QUIT } \\
\text {; START OF TEXT }\end{array}$ \\
\hline & EER & ¿FEMIUVE 4 ZtKLLSOLH,I_F \\
\hline & $\begin{array}{l}\text { CMP } \\
\text { EER }\end{array}$ & ;ELIM, 4 ZEKOS,CF,LF \\
\hline & ER & ¿NO,TKYY AGAIN \\
\hline WEEI: & $\underset{B K}{C L R}$ & FFESET COUNTEK \\
\hline \multirow[t]{6}{*}{ READ: } & MOV & $\$ 20,0 \# 167772$ \\
\hline & $\begin{array}{l}\text { MOU } \\
\text { IIIT }\end{array}$ & $\begin{array}{l}\$ 0,04167770 \\
\$ 40000,0 \# 167774\end{array}$ \\
\hline & BNE & FEAD \\
\hline & $\begin{array}{l}\text { MOU } \\
\text { CMP }\end{array}$ & $\begin{array}{l}\text { I67774 SEES TIATA } \\
\text { IS IT ENI OF TEXT }\end{array}$ \\
\hline & $\begin{array}{l}\text { BEQ } \\
\text { MOV } \\
\text { BIC }\end{array}$ & $\begin{array}{l}\text { OYES, CLOSE FILE } \\
\text { IIATA TO K2 } \\
\text {;MASK OFF MSH }\end{array}$ \\
\hline & MOVE & ;FUT EYTE IN . HUFFEK \\
\hline \multirow[t]{3}{*}{ ETIN: } & $\begin{array}{l}\text { CMF } \\
\text { ELO }\end{array}$ & 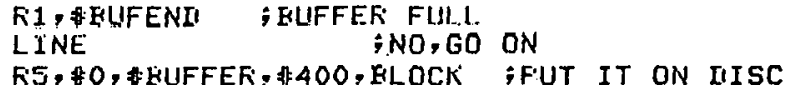 \\
\hline & $\begin{array}{l}\text { INC } \\
\text { CMP }\end{array}$ & $\begin{array}{l}\text { FNCFEMENT FLOCK } \\
\text { :UFFEK LIMIT ON FILE SIZE }\end{array}$ \\
\hline & FEQ & ERR \\
\hline
\end{tabular}




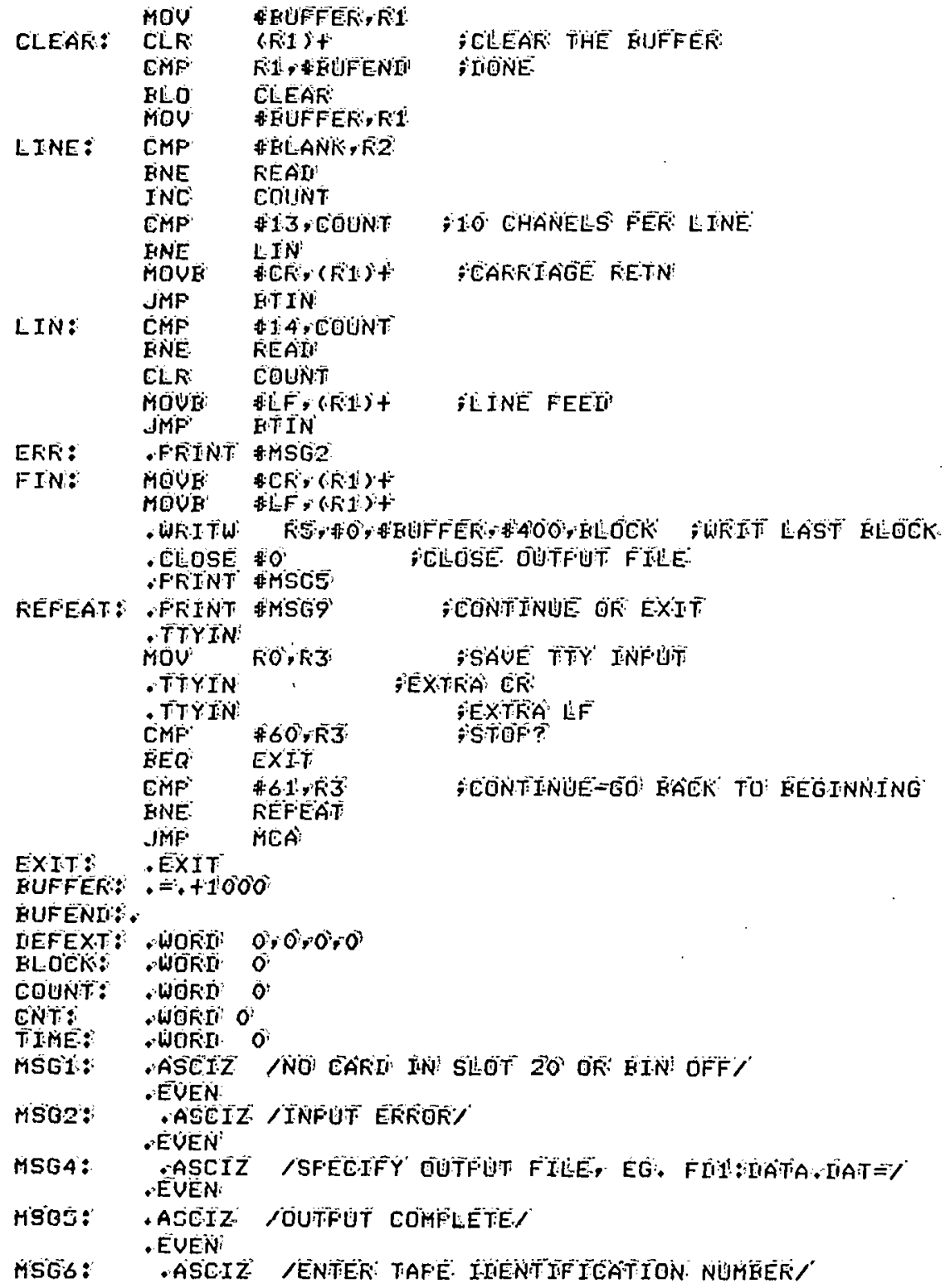




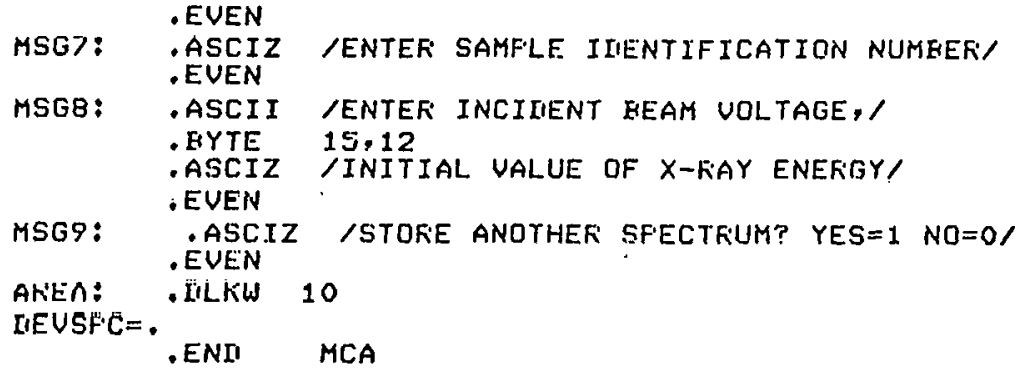


APPENDIX G

Circuit Diagram for TSED/TSEM Measurements 


\section{APPENDIX G}

\section{Circuit Diagram for TSED/TSEM Measurements}

The schematic diagram of Fig. 103 shows the electronic circuit used to control the scanning system for TSED/TSEM measurements. A digital ramp from the ORIEC 6200 MCS, which is synchronized with the sequential channel advance, is routed through the first stage of the scanning system providing a continuously variable scan amplitude adjustment, The signal is then split into a ratioed $x$ and $y$ signal which effectively produces a continuously variable rotation capability. The final stage of the circuit adds a variable dc offset to both the $x$ and $y$ scans, which is finally routed to the intermediate alignment coils on the JSEM 200. The net result of this system is that scans of continuously variablc amplitudc, oricntation and position can be prolucel aboul any point for intensity measurements. Figure 104 summarizes the remaining components of the TSED/TSEM system. 


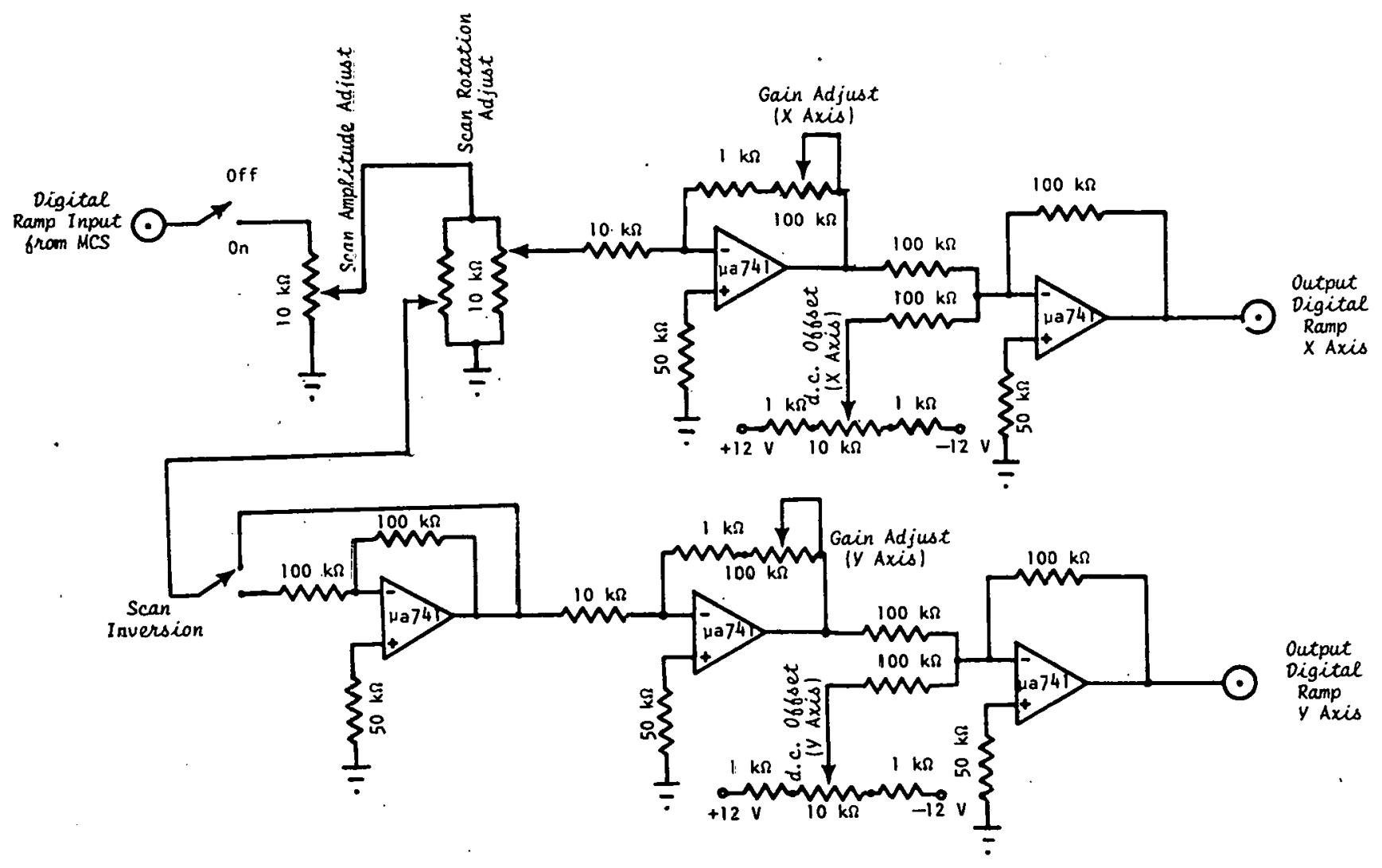

Fig. 103, Circuit Diagram for TSED/TSEM Scanning System. 


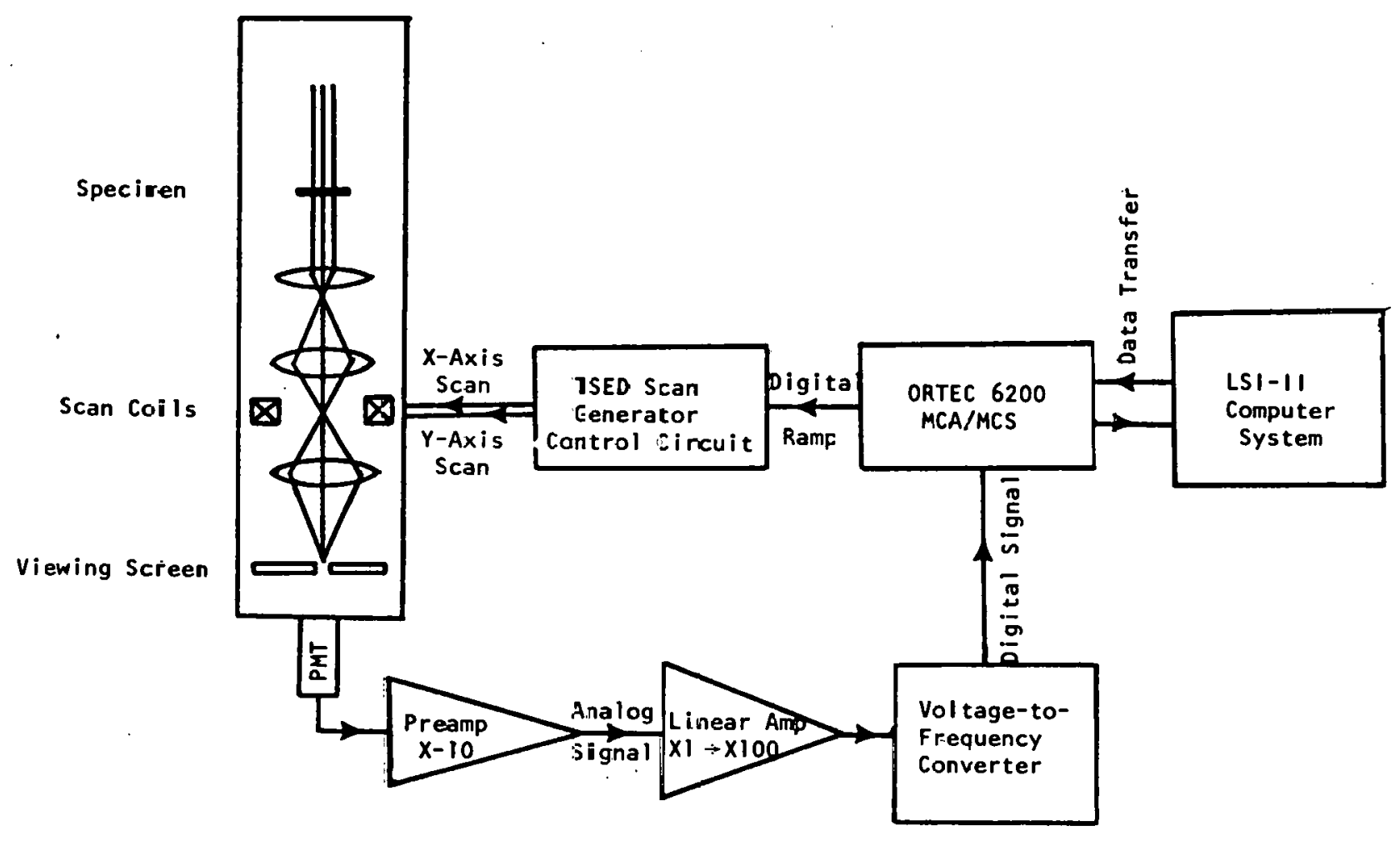

Fig. 104. Blcck Diagram of TSED/TSEM System. 
ORNI/T.1-6705

\section{INTERNAL DISTRIBUTION}

1-2. Central Research Library
3. Document Reference Section
4-13. Laboratory Records Department
14. Laboratory Records, RC
15. ORNL Patent Section
16. P. Angelini
17. J. Bentley
18. E. E. Bloom
19. R. W. Carpenter
20. R. S. Crouse
21. K. Farrel1
22. G. R. Gessel
23. M. L. Grossbeck
24. R. W. Hendricks
25. H. M. Henson (K-25)
26-28. M. R. Hill
29. D. O. Hobson
30. E. A. Kenik
31. C. C. Koch
32. E. H. Lee
33. J. M. Leitnaker
34. J. S. Lin
35. C. T. Liu
36. K. C. Liu
37. J. E. Mack

38. P. J. Maziasz

39. C. J. Mchargue

40. J. Narayan

41. T. S. Noggle

42. S. M. Ohr

43. N. H. Packan

44. H. E. Reesor

45. J. E. Selle

46. P. S. Sklad

47. J. O. Stiegler

48. S. M. Tiegs

49. T. N. Tiegs

50. P. F. Tortorelli

51. R. A. Vandermeer

52. F. W. Wiffen

53. M. K. Wilkinson

54. R. O. Williams

55-64. N. J. Zaluzec

65. A. L. Bement, Jr. (Consultant)

66. R. W. Balluffi (Consiltant)

67. W. R. Hibbard, Jr. (Consultant)

68. E. H. Kottcamp, Jr. (Consultant)

69. M. J. Mayfield (Consultant)

70. J. T. Stringer (Consultant)

EXTERNAL DISTRIBUTION

71. Alcoa Corporation Research Laboratory, New Kensington, PA 15069

72-74. Argonne National Laboratory, Materials Science Division, Argonne, IL 60439

P. Okomato

D. Potter

H. Wiedersich

75. Battelle-Northwest, P.0. Box 999, Richland, WA 99352

S. Bruemmer

76-77. Bell Telephone Laboratories, Murray Hil1, NJ 07974

D. C. Joy

D. Maher 
78. Brookhaven National Laboratory, Metallurgy and Materials Science Division, Upton, LI, NY 11973

79. Carnegie-Mellon University, Department of Metallurgy and Materials Science, Pittsburgh, PA 15213

J. Wilifams

80. Case Western Reserve University, Division of Metallurgy and Materials Science, Cleveland, OH 44106

T. E. Mitchell

81. Corne11 University, Ithaca, NY 14850

J. Silcox, Department of Englneering and Applied Physics, Clark Hall

82. E. I. DuPont de Nemours and Company, Savannah River Laboratory, Aiken, SC 29801

E. Sturken

83. EDAX International, P.0. Box 135, Pralrie View, IL 60069

J. Russ

84-85. Ford Motor Company, Systems Research Laboratories, P.0. Box 2053, Dearborn, MI 48121

II. E. Cuok

J. W. Sprys

86. General Electric Company, 2nd Street, Coshocton, OH 43812

R. Wullenburg

87. Georgia Institute of Technology, Department of Chemical

Engineeriug, Atlanta, GA 30332

E. Stark

88. IBM System Products Division, East Fishki11, Hopewell Junction, NY 12533

R. Ander son

89. IBM Research Laboratories, Monterey and Cattle Roads, San Jose, CA 95153

R. Geiss 
90. Illinois Institute of Technology, Department of Metallurgy, Chicago, IL 60616

N. Breyer

91. Inland Steel Company, 3001 East Columbus Drive, East Chicago, IN 46312

P. L. Mangonon

92. Iowa State University, Materials Science and Engineering Department, Ames, IA 50010

M. Wechsler

93. JEOL USA, 477 Riverside Avenue, Medford, MA 02155

Gary Cogswell

94. Kevex Corporation, P.0. Box 4050, Foster City, CA 94404

J. Colby

95. Lehigh University, Department of Metallurgy and Materials Engineering, Bethlehem, PA 18015

J. I. Goldstein

96. Massachusetts Institute of Technology, Department of Materials Science, Cambridge, MA 02139

J. B. Vandersande

97. Michigan Technological University, Department of Metallurgy and Materials Science, Houghton, MI 49931

W. Clark

98. National Bureau of Standards, Chem. Al21 310.02, Washington; DC 20234

Dale E. Newberry

99. National Institute of Health, 3W13, Building 13, Bethesda, MD 20014

C. Fiori

100. National Science Foundation, Division of Materials Research, 1800 G. Street, NW, Washington, DC 20550

R. Renik 
101. North America Rockwe11 Science Center, Thousand Oaks, CA 91360 N. Paton

102. North Carolina State University, Page Ha1l, Raleigh, NC 27607 R. B. Benson, Jr.

103. Rensselaer Polytechnic Institute, Department of Metallurgy and Materials Sclence, Troy, NY 12181

C. Lyman

104. Sandia Laboratories, Albuquerque, NM 87115

T. J. Headley

105. Sherwin-Williams Research Center, 10909 Cottage Grove Avenue, Chicago, IL 60628

R. L. Harper

106. Stanford University, Department of Materials Science, Stanford, CA 94305

107. University of California, Berkeley, Department of Material Science and Engineering, Berkeley, CA 94720

R. Gronsky

108. Univerofty of Calffornia, Los Angeles, Los Angeles, CA 9illi32.

A. Arde11, Boelter Hall

109. University of Cincinnati, Division of Materials Sclence and Metallurgical Engineering, Location 12, Cincinnati, OH 45221

J. Moteff

110. University of Florida, Department of Materials Science and Engineering, Ga1nesville, FL 32611

J. J. Hren

111-120. University of Illinols, Urbana, IL 61801

Department of Metallurgy
H. L. Fraser (3)
E. N. Pugh
C. A. Wert 


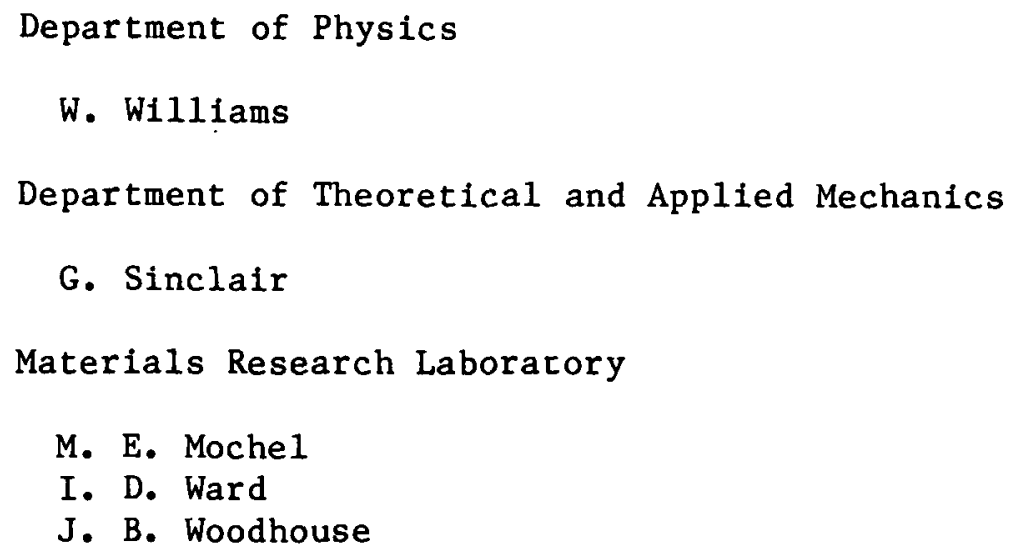

121. University of Michigan, Department of Materials Engineering, Ann Arbor, MI 48109

W. C. Bigelow

122. University of Notre Dame, Department of Metallurgical

Engineering and Materials Science, Notre Dame, IN 46556

C. W. Allen

123. University of Virginia, Department of Materials Science, Charlottesville, VA 22901

K. Lawless

124. U.S. Steel Research Laboratory, Monroeville, PA 15146

R. M. Fisher

125. Vanderbilt University, Department of Mechanical Engineering and Materials Science, Nashville, TN 37235

B. Bayuzik

126. Westinghouse Hanford Company, 326 Building, 300 Area, P.0. Box 1970, Richland, WA 99352
H. Brager
L. E. 'Thomas

127-128. U.S. DOE, Office of Basic Energy Sciences, Division of Materials Sciences, Washington, DC 20545

R. Gottscha11, Ma11 Stop J-309

L. C. Iannie1lo 
129. U.S. DOE, Oak Ridge Operations Office, P.O. Box E, Oak Ridge, TN 37830

Assistant Manager, Energy Research and Development

130-156. U.S. DOE, Technical Information Center, Office of Information Services, P.0. Box 62, Oak Ridge, TN 37830

\section{FOREIGN DISTRIBUTION}

157. AERE Harwe11, Metallurgy Division, Didcot, Berks, England

B. Eyre

158. Alcan Laboratory, Ltd., Atlantic Region Research Center, Banbury, Oxon, England Ox15 7SP

P. L. Morris

159. JEOL Electron Optics Division, 1418 Nakagami, Akishima, Tokyo 196, Japan

Y. Kokubo

160. Laboratorie de Physique du Solide, Université Paris-Sud, Orsay, France

C. Colliex

161. N.V. Philips Glocilampenfabrieken, Electron Optico Department, Building TQ 111-2, Endhoven, The Netherlands

K. Van der Mast

162. Osaka University, Department of Applied Physics, YamadaKam1 Suita, Osaka 565 , Japas

H. Hashimoto

163-164. Oxford University, Department of Metallurgy and Macerials Sclence, Oxford, England

P. B. Hirsch

C. J. Humphreys 
165. Physikalisches Institute der Universitäte Münster, Electronenmikroskopische Abteilung, 44 Münster, West Germany

L. Reimer

166. Siemens A.G., Messgekalewerk, Berlin, Postfach 140, 1000 Berlin 13, West Germany

D. Willasch

167. University of Alberta, Department of Physics, Edmonton, Alberta, Canada

R. Egerton

168-170. Untversity of Birmingham, Department of Physical Metallurgy, Birmingham, England B15 2TT

R. E. Smallman

M. H. Loretto

I. P. Jones

171. UnIversity of Manchester, Department of Metallurgy, Manchester, England M13 9PL

G. W. Lorimer

172. Y. G. Microscopes Ltd., Birches Industrial Estate, Imberhorne Lane, East Grinstead, Suxxex, England

P. Bovey 